\title{
2006 Annual Report
}

\section{Summer Research Institute Interfacial and Condensed Phase Chemical Physics}

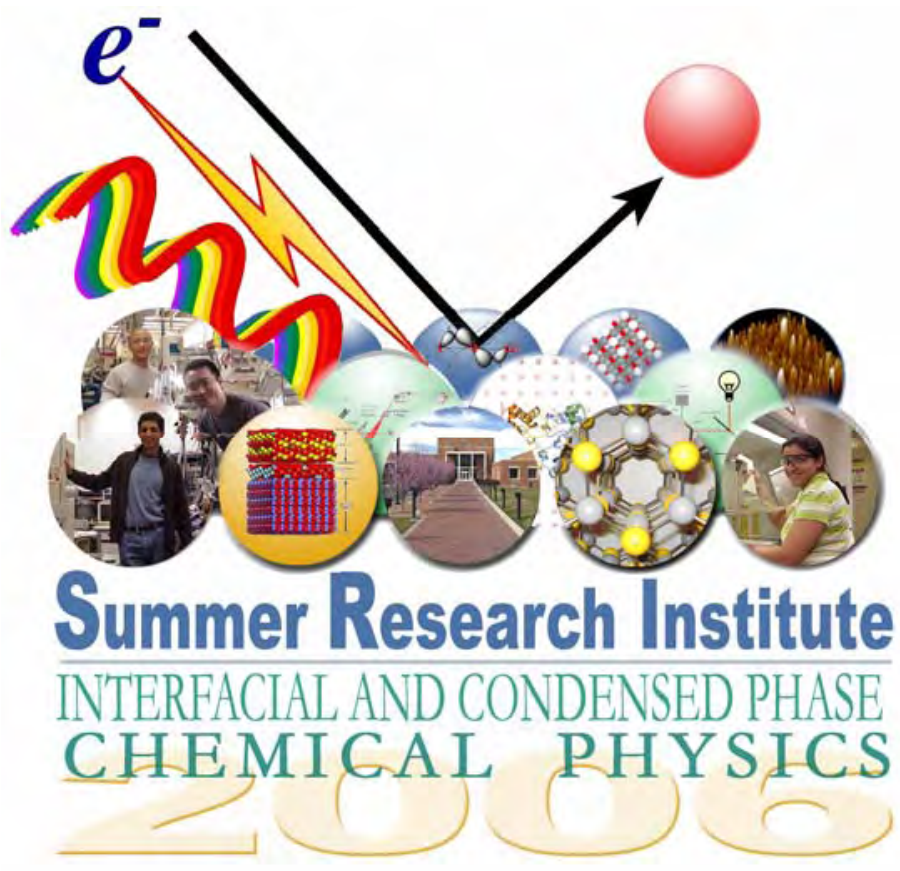

Prepared for the U.S. Department of Energy under Contract DE-AC06-76RL01830 


\title{
DISCLAIMER
}

This report was prepared as an account of work sponsored by an agency of the United States Government. Neither the United States Government nor any agency thereof, nor Battelle Memorial Institute, nor any of their employees, makes any warranty, express or implied, or assumes any legal liability or responsibility for the accuracy, completeness, or usefulness of any information, apparatus, product, or process disclosed, or represents that its use would not infringe privately owned rights. Reference herein to any specific commercial product, process, or service by trade name, trademark, manufacturer, or otherwise does not necessarily constitute or imply its endorsement, recommendation, or favoring by the United States Government or any agency thereof, or Battelle Memorial Institute. The views and opinions of authors expressed herein do not necessarily state or reflect those of the United States Government or any agency thereof.

\author{
PACIFIC NORTHWEST NATIONAL LABORATORY \\ operated by \\ BATTELLE \\ for the \\ UNITED STATES DEPARTMENT OF ENERGY \\ under Contract DE-AC06-76RL01830
}

Printed in the United States of America

Available to DOE and DOE contractors from the

Office of Scientific and Technical Information,

P.O. Box 62, Oak Ridge, TN 37831-0062;

ph: (865) 576-8401

fax: (865) 576-5728

email: reports@adonis.osti.gov

\author{
Available to the public from the National Technical Information Service, \\ U.S. Department of Commerce, 5285 Port Royal Rd., Springfield, VA 22161 \\ ph: (800) 553-6847 \\ fax: (703) 605-6900 \\ email: orders@ntis.fedworld.gov \\ online ordering: http://www.ntis.gov/ordering.htm
}




\title{
2006 Annual Report
}

\section{Summer Research Institute Interfacial and Condensed Phase Chemical Physics}

\author{
NB Avery \\ SE Barlow
}

October 2006

Prepared for

the U.S. Department of Energy under Contract DE-AC06-76RL01830

Pacific Northwest National Laboratory Richland, Washington 99354 
PNNL-16185 
PNNL-16185

\section{Executive Summary}

The Pacific Northwest National Laboratory (PNNL) hosted its third annual Summer Research Institute in Interfacial and Condensed Phase Chemical Physics from May through September 2006. During this period, twenty PNNL scientists hosted twentyseven scientists from twenty-five different universities. Of the twenty-seven participants, one was a graduating senior; twenty-one were graduate students; one was a postdoctoral fellow; and four were university faculty members. 
PNNL-16185 


\section{Table of Contents}

\begin{tabular}{|c|c|}
\hline Executive Summary & iii \\
\hline Contents & $\mathrm{v}$ \\
\hline Acknowledgments & 1 \\
\hline Summary & 3 \\
\hline $\begin{array}{l}\text { Participants and the Institute Structure } \\
\text { Benefits } \\
\text { New in FY06 } \\
\text { Measures of Success } \\
\text { Participant Survey }\end{array}$ & $\begin{array}{l}5 \\
15 \\
16 \\
22\end{array}$ \\
\hline Technical Reports & 25 \\
\hline $\begin{array}{l}\text { Jeremy Cain, Young Liu and Alexander Laskin } \\
\text { "Heterogeneous Reaction Kinetics of } \mathrm{NaCl}+\mathrm{HNO} "\end{array}$ & 27 \\
\hline $\begin{array}{l}\text { Chia-Lin Chang, V. Shutthanandan, and S. Ramanathan } \\
\text { "In-situ studies of initial oxidation of bare InAs surfaces and oxide/InAs interfaces" }\end{array}$ & 31 \\
\hline $\begin{array}{l}\text { Chan Lan Chun, William A. Arnold, and Don Baer } \\
\text { "Metal-doped Iron Particles" }\end{array}$ & 35 \\
\hline $\begin{array}{l}\text { Luis A. Cuadra-Rodriguez, Barney Ellison, and Alla Zelenyuk } \\
\text { "Characterization of Aqueous Particles Internally Mixed With Surfactants” }\end{array}$ & 51 \\
\hline $\begin{array}{l}\text { Jun Cui, K.D. Jordan, L.X. Dang, C. Wick, and V. Glezakou } \\
\text { "I. Theoretical characterization of } \mathrm{H}_{3} \mathrm{O}^{+} \text {at the water/methanol - vapor interface., } \\
\text { II. Theoretical characterization of } \mathrm{NaCl}_{\mathrm{O}_{4} / \mathrm{H}_{2} \mathrm{O} \text { solutions" }}\end{array}$ & 61 \\
\hline $\begin{array}{l}\text { Shiyu Du, Joseph Francisco, and Greg Schenter } \\
\text { "OCl Radical }-\mathrm{H}_{2} \mathrm{O} \text { interaction: ab initio calculation and the development of } \\
\text { empirical potential" }\end{array}$ & 65 \\
\hline $\begin{array}{l}\text { Debamitra Dutta, Brij Mougil and SK Sundaram } \\
\text { "Study of the inflammatory responses of Single-Walled Carbon Nanotubes } \\
\text { (SWCNTs) On Murine Macrophage Cells (RAW 264.7)" }\end{array}$ & 89 \\
\hline $\begin{array}{l}\text { Mikael Elfman and Yanwen Zhang } \\
\text { "A Fast Screening Technique to Evaluate Si Detector Response to Ions” }\end{array}$ & 113 \\
\hline
\end{tabular}


Diego Enry Barreto Gomes, Pedro Pascutti and Theresa Soares, "Progress Report: Attempting to investigate the effects of confinement on Organophosphorus Hydrolase from P. diminuta by Molecular Dynamics simulation.”

Rodrigo Gonzales, Kirsten Johnson, Alexander Laskin and Yury Desyaterik "Characterization of Aerosol Samples Collected During the Milagro-2006 Study in the Mexico City Metropolitan Area”

Matteo Guglielmi, Ursula Röthlisberger, and Roberto Lins

"In Silico Design and Characterization of Rigid Rod $\beta$-barrel Pores"

Rebecca Hopkins, Mary K. Gilles, Alexander Laskin

"Chemical Speciation of Sulfer in Marine Aerosol Particles"

Ngor wai (Corey) Lam, Ivan Keung Chu, and Julia Laskin

"Mechanistic and Energetic Studies of Radical Peptides by means of FT-ICR SID MS"

Vu Lam, Kevin Coffey and Theva Thevuthasan

"Tandem Accelerator and Ion Beam Techniques"

Christopher Lane, Thomas Orlando and Greg Kimmel

"Thermal and Non-thermal Reactions at the Water/TiO 2 (110) Interface"

Chan-Woo Lee, Susan B. Sinnott, Ram Devanathan, Eric D. Wachsman, "First-

Principles Calculations of $\mathrm{La}_{(1-\mathrm{x})} \mathrm{Sr}_{(\mathrm{x})} \mathrm{Co}_{(\mathrm{y})} \mathrm{Fe}_{(1-\mathrm{y})} \mathrm{O}_{3}$ (LSCF) Solids and Surfaces"

Jiangyu Li, Leo Fifield, "Summary of research in the field of PVDF Polymers and Nanocomposites"

Si-Dian Li, Hua-Jin Zhai, Lei-Ming Wang, and Lai-Sheng Wang. "A Photoelectron Spectroscopy and $a b$ initio Computational Study of Boron Oxide Anions $\mathrm{BO}^{-}$and $\mathrm{BO}_{2}{ }^{-“}$

Suiqiong Li, Dr. Zhongyang Cheng, Chongmin Wang, "Characterization of Microstructure and Composition of Magnetostrictive Nanobars as Bio-sensor Platform”

Julie A. Lloyd, Murray V. Johnston and M. L. Alexander

"Assembly of an Ion Trap Mass Spectrometer for LD-CI Experimentation"

Rebecca Lowton, Peter Edwards, and Thomas Autrey "Investigation into the Effect of Milling Ammonia Borane with Magnesium Hydride" 


\begin{tabular}{|c|c|}
\hline $\begin{array}{l}\text { Wagner Alexander Lucena, and Roberto Lins, "Molecular Modeling of the N-acyl } \\
\text { homoserine lactone hydrolase from Bacillus thuringiensis" }\end{array}$ & 205 \\
\hline $\begin{array}{l}\text { Amir Saheb, Mira Josowicz, Jiri Janata and Donald Baer, "Characterization of } \\
\text { Polyaniline-Gold Nanocomposites }\end{array}$ & 221 \\
\hline $\begin{array}{l}\text { Andrzej Sobolewski, and Sotiris Xantheas } \\
\text { "Photochemistry of Liquid Waterand Radiation Induced Solvation” }\end{array}$ & 229 \\
\hline $\begin{array}{l}\text { Jennie L. Thomas, Barbara J. Finlayson Pitts, Douglas J. Tobias and } \\
\text { Liem X. Dang, } \\
\text { "Molecular dynamics of aqueous interfaces: } \\
\text { The surface propensity and thermodynamic properties of aqueous anions" }\end{array}$ & 233 \\
\hline $\begin{array}{l}\text { Guanjun Wang, Lai-sheng Wang, Dr. Xuebin Wang, and Dr. Hin-Koon Hoo } \\
\text { "The Investigation on: I. The Solvation of CN( }\left(\mathrm{H}_{2} \mathrm{O}\right) \text { n- and } \mathrm{N}(\mathrm{CN})_{2}\left(\mathrm{H}_{2} \mathrm{O}\right) \mathrm{n}-; \text { II . The } \\
\text { Configuration of MOOC }\left(\mathrm{CH}_{2}\right)_{\mathrm{n}} \mathrm{COO}^{-}(\mathrm{M}=\mathrm{Na}, \mathrm{K}) \text {; III. Some Oxygen-rich Vanadate } \\
\text { Anion Species by Combination of Photoelectron Spectrometry and Electron-spray" }\end{array}$ & 237 \\
\hline$\frac{\text { Appendix }}{\text { Participant Survey }}$ & 249 \\
\hline
\end{tabular}


PNNL-16185 


\section{Acknowledgments}

Even a modest-sized program such as this one would not happen without the assistance of many people. This year ${ }^{1}$ s scientific oversight committee consisted of Drs. Donald R. Baer, FSD, L. Rene Corrales, FSD and Stephan E. Barlow, EMSL. Ms. Nikki B. Avery was our administrator in 2006 and played the key role in ensuring the institute ran smoothly. Ms. Cynthia A. Irwin, Operations Administrator for Chemical Structure and Dynamics, and Ms. Susan M. Finch of Chemical Sciences provided financial and budgetary support

We also acknowledge Ms. Rebecca J. Janosky of PNNL'1s Science and Engineering Education Office. She handled a myriad of details associated with appointments, including awards, agreements and disbursements, while dealing with a major reorganization. We also wish to acknowledge Ms. Carol A. Elledge, Publications Design Group, Ms. Cori C. Blake, Web Ops, Mr. Mark D. Bayless, Rich Interaction Environments, and Ms. Rose M. Watt, Graph and Multimedia Design Group, respectively, for their expertise and timeliness in creating and developing the institute ${ }^{1} \mathrm{~s}$ website, application website and finalizing the institute ${ }^{1}$ s brochure. Mr. David Spiel provided expert photography support.

Special and belated acknowledgement is owed to my wife, Mrs. Janice Barlow. She initiated the Summer Institute barbeques that have become something of a tradition. Each year we have hosted, and deeply enjoyed two of these get-togethers at our home.

This year we inaugurated a collaboration with the National Science Foundation (NSF), discussed below. However here we need to acknowledge the roles of Dr. Katherine Covet of the NSF and Dr. Allison A. Campbell and Ms. Terry J. Law of EMSL in making this happen.

Finally, we wish to acknowledge the U.S. Department of Energy ${ }^{1}$ s Office of Basic Energy Sciences from whom we received the guidance to initiate the Summer Research Institute in Interfacial and Condensed Phase Chemical Physics and from whom support was ultimately derived.

The real stars of the Interfacial and Condensed Phase Chemical Physics Research Institute were our participants. The remainder of this document is largely devoted to them.

Stephan E. Barlow

Director, ICPCPSRI

October 2006 
PNNL-16185 


\section{Summary}

PNNL's Management and Operations Contract with the U.S. Department of Energy (DOE) ${ }^{1}$ identified an Office of Basic Energy Science (BES) Mission Stretch Goal: "To establish PNNL in a national leadership role in the area of theoretical and experimental condensed phase and interfacial chemical physics research.” Successful achievement of a national leadership role will be evidenced by: "Establishment of a summer school (at least two weeks in duration) to educate graduate students and young scientists in state-of-the- art theory, simulation, and experimental measurement, with a total attendance of at east 60 over the contract period."

Interfacial and Condensed Phase Chemical Physics is not so much a field of study as it is a cross cutting concept. Important issues exist in nearly every scientific discipline from Astronomy to Zoology that fall with the purview of this concept. Likewise all of the technology and engineering of the modern world and hopes for future development depend to one degree or another on our ability to understand and manipulate condensed phases and interfacial processes.

The guidance from DOE HQ Office of Science gave us considerable latitude in implementing the institute's advanced program that focuses on graduate students, postdoctoral fellows and university faculty. The institute caters to more senior people than generally accommodated by PNNL's summer internship programs. We believe that the best education people at this level can receive is to actively engage in research that is relevant to their own career development goals. To accomplish this objective, the institute has a different character and puts different requirements on its participants than do either the standard internship programs or other institutes, workshops, etc. Perhaps the best overall description of the institute is a "visiting scientist program for early career researchers." In this spirit, each young scientist's particular program was individually tailored. The descriptions given below are generally true for most of the young scientists but may or may not apply to any particular individual.

\section{Participants and the Institute Structure}

Prospective visitors applied electronically at the Summer Research Institute's web site: $w w w . p n l . g o v / s i /$. Submission of applications, screening and correspondence were all handled online. This greatly facilitated the review and screening process as well as record keeping. Preliminary screening was handled by the Director of the Institute who acknowledged receipt of the applications and informed the candidates of any additional information or actions that were necessary to complete the application process. A number of candidates were found to be unsuited to participate in the institute and they were informed as quickly as that determination could be made. Reasons for rejection at this stage included:

1 Appendix H of Contract Number: DE-AC06-76RL01830, Modification M375. 
- Candidate's area of interest was outside of the scope of the program, e.g., high energy physics or genetics.

- Prospective PNNL hosts were already committed or unavailable.

- Candidate was finishing a Masters or PhD degree and did not have a new position.

- Candidate failed to finish the application process.

Candidates who passed this first series of hurdles and who identified a willing PNNL host were then required to submit a summary of proposed research or activity that they would conduct at PNNL during the summer. Normally, this proposal was prepared with the assistance of both the PNNL host and the candidate's academic advisor (as appropriate). Once the application deadline had passed, all completed applications were reviewed by a PNNL science panel consisting of Dr. Donald R. Baer and Dr. L. Rene Corrales of PNNL's Fundamental Science Directorate and Dr. Stephan E. Barlow of PNNL's Environmental Molecular Sciences Laboratory (EMSL). All successful candidates received the unanimous approval of the panel. Individual acceptance and rejection letters were prepared and distributed by the Director. Interestingly, all of the candidates who were accepted agreed to come. Table 1 provides some statistics on applications and acceptances.

\begin{tabular}{|l|l|}
\hline Table 1. 2005 Participant Numbers \\
\hline Applications and inquiries & 58 \\
\hline Acceptances & 29 \\
\hline Participants & 27 \\
\hline Graduating Seniors & 1 \\
\hline Graduate Students & 21 \\
\hline Postdoctoral Fellows & 1 \\
\hline Junior Faculty & 1 \\
\hline Senior Researchers & 3 \\
\hline US Citizens/Foreign National & $8 / 19$ \\
\hline PNNL Hosts & 21 \\
\hline
\end{tabular}

The application and acceptance procedure requires participation from all concerned parties: the PNNL hosts, the visitors and the visitors' supervisors at his or her home institution (if applicable). Further, PNNL hosts were expected to provide appropriate mentoring for the visitors as well as ensure all necessary resources were available to accomplish the proposed work. The visitors bear the bulk of the application responsibility in order to come to PNNL for an extended time away from home. The visitors' supervisors were expected to continue financial support during this visit to PNNL since the Summer Research Institute only covered travel to and from PNNL and partial living expenses.

Each visitor had a specific program tailored around his or her schedule and research goals. To accommodate individual schedules, the 2006 summer appointments began in early May and the final appointment concluded in September. Prior to their arrival, each participant was paired with a PNNL host and worked out a research program for the visit. 
All of this activity was coordinated with the visitor's supervisor. Most often, our visitor was playing a role in a collaboration or prospective collaboration between the PNNL host and his or her supervisor. (For the cases of graduating seniors or faculty members slightly different procedures apply.) Table 2 lists our visitors, PNNL hosts and home institution supervisors. Table 3 shows the University Statistics

Table 2.

$$
\begin{aligned}
& \text { Interfacial and Condensed Phase Chemical Physics Summer } \\
& \text { Research } \\
& \text { Institute PNNL visitors, academic advisors, PNNL hosts }
\end{aligned}
$$

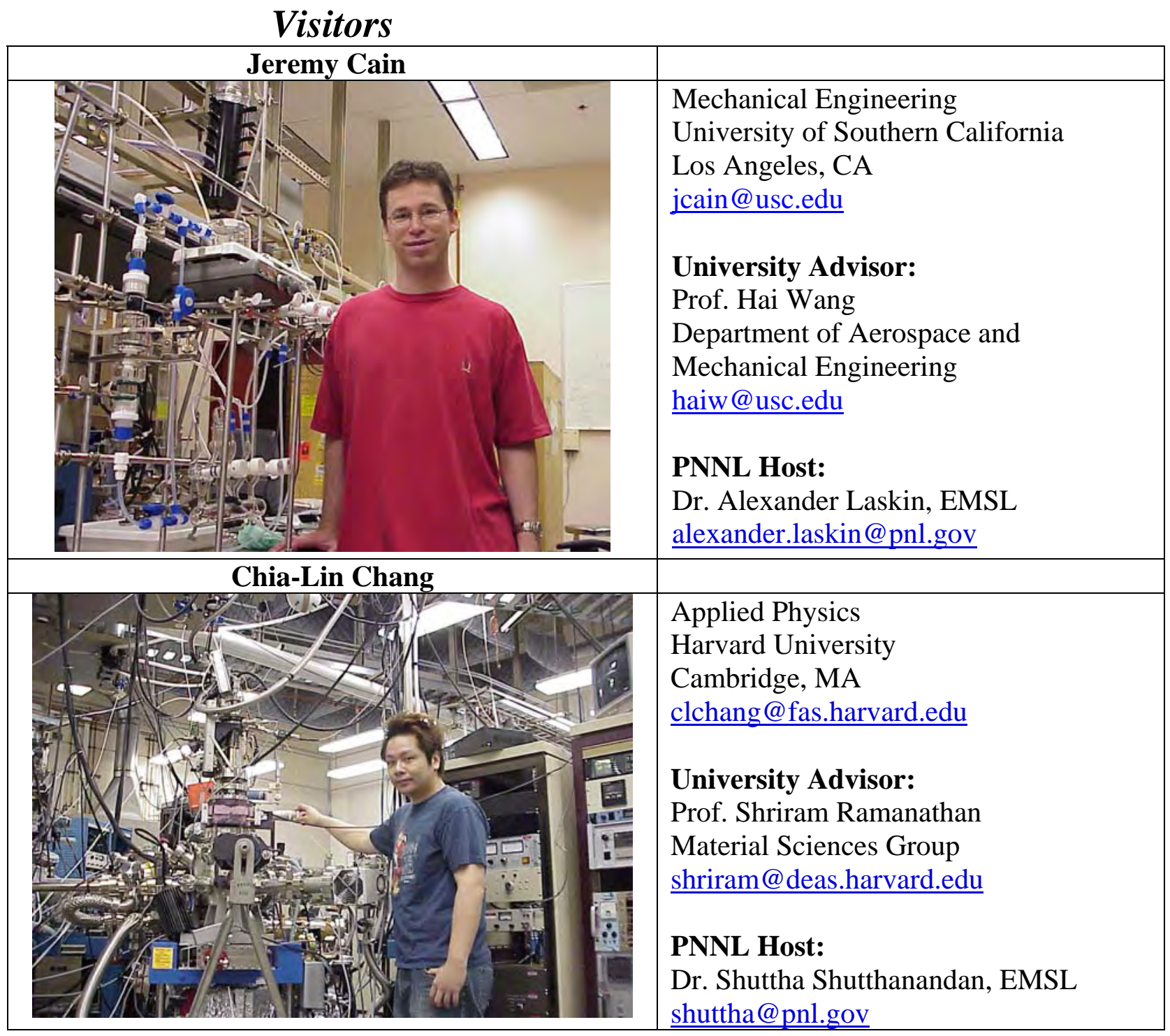




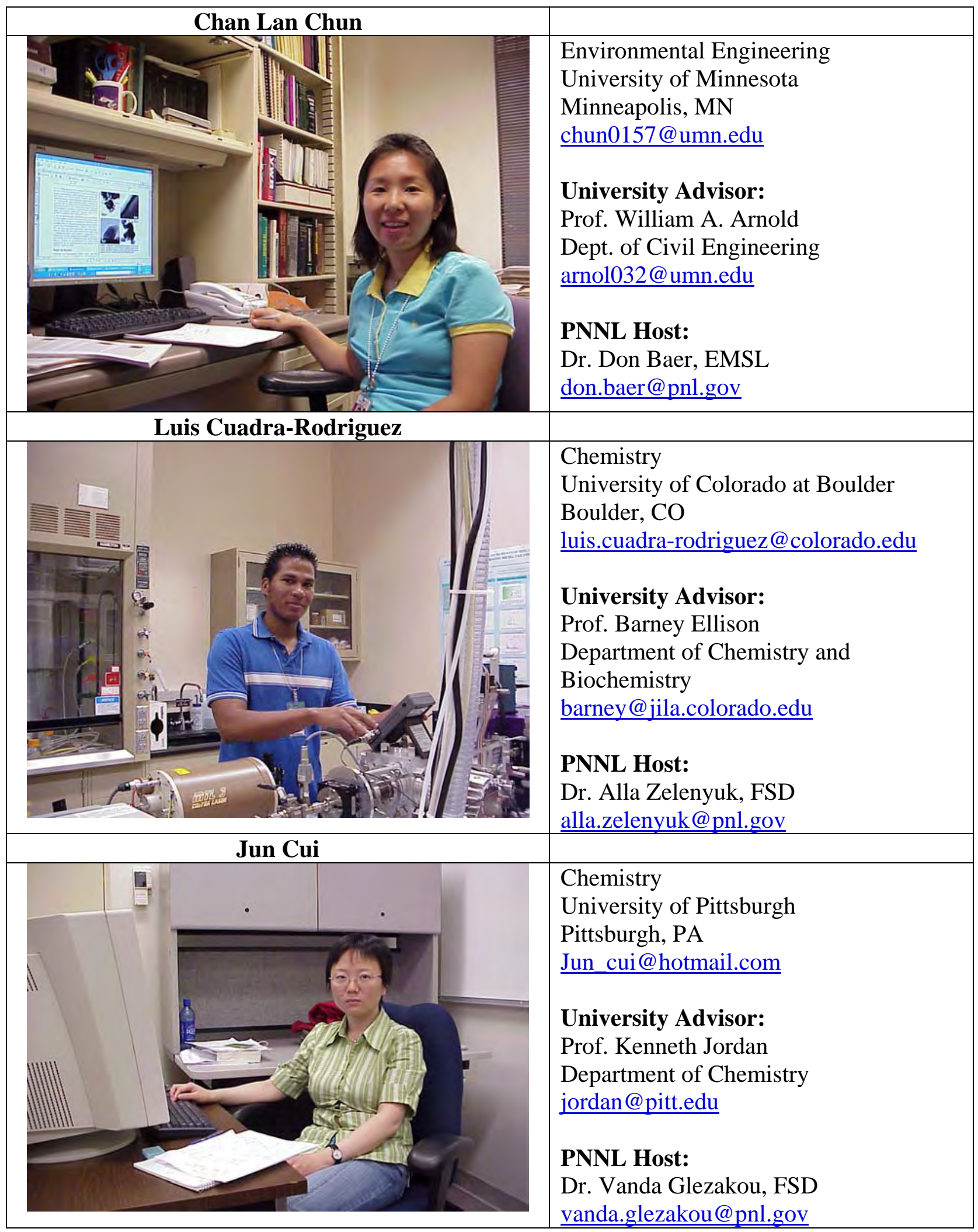




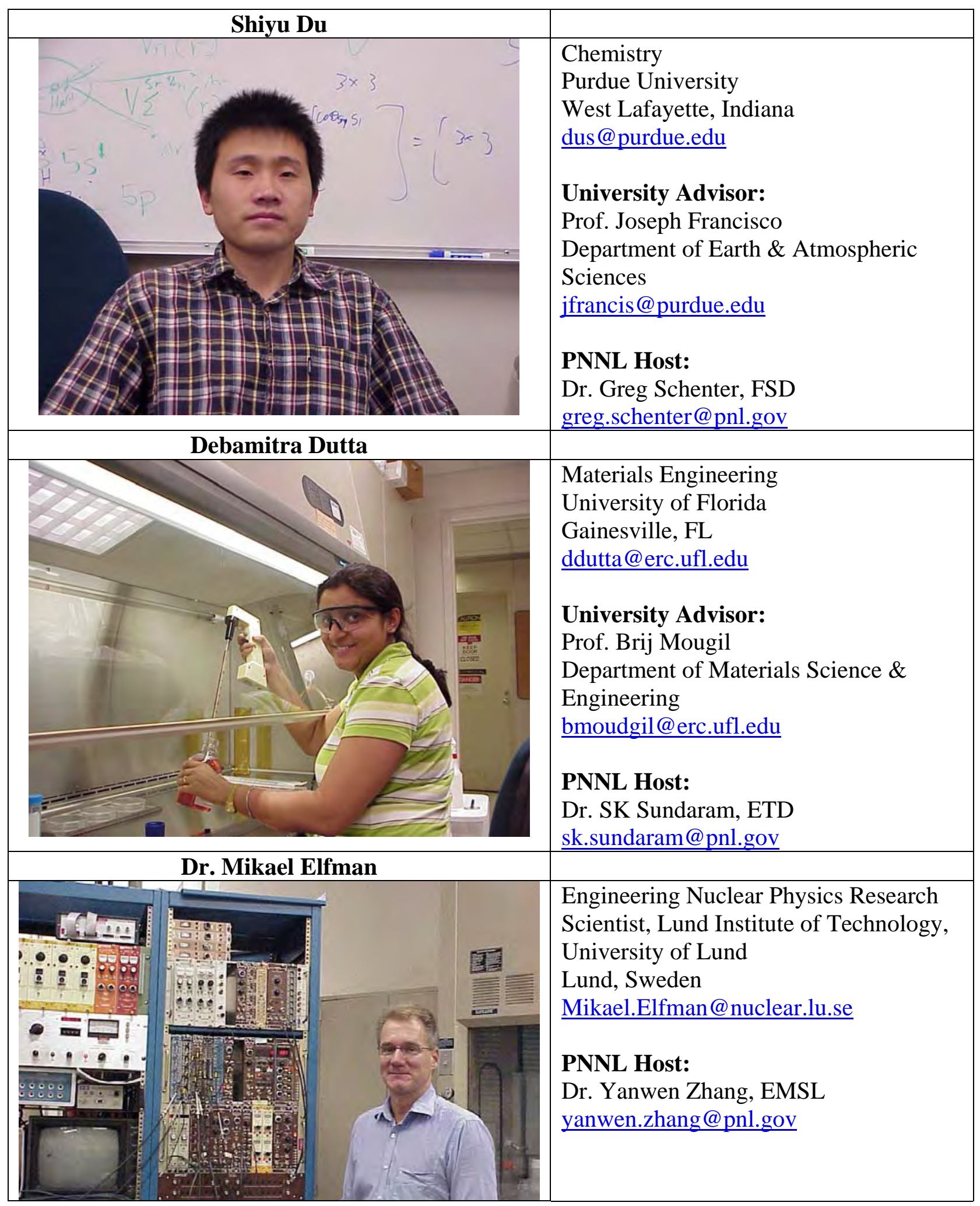




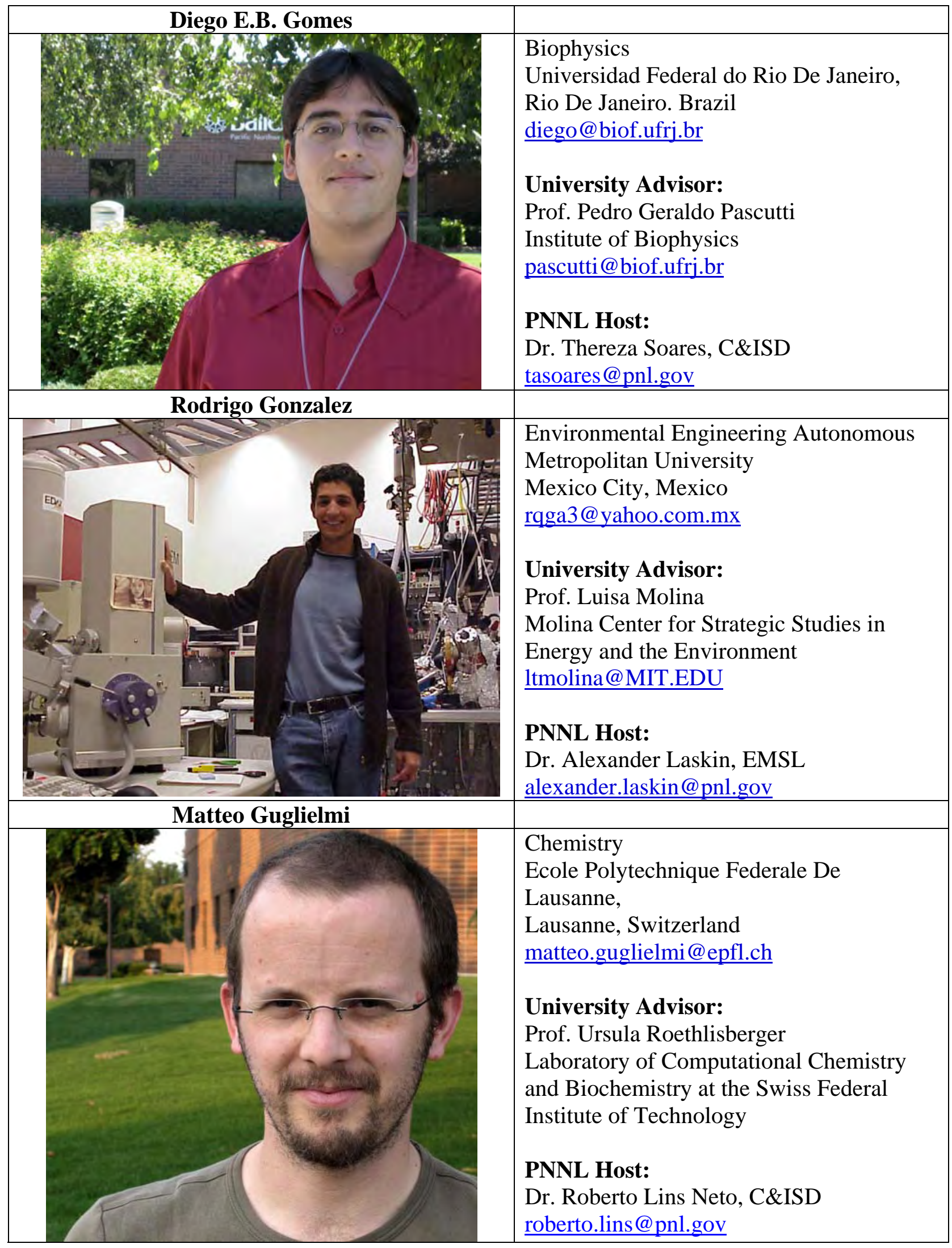


PNNL-16185

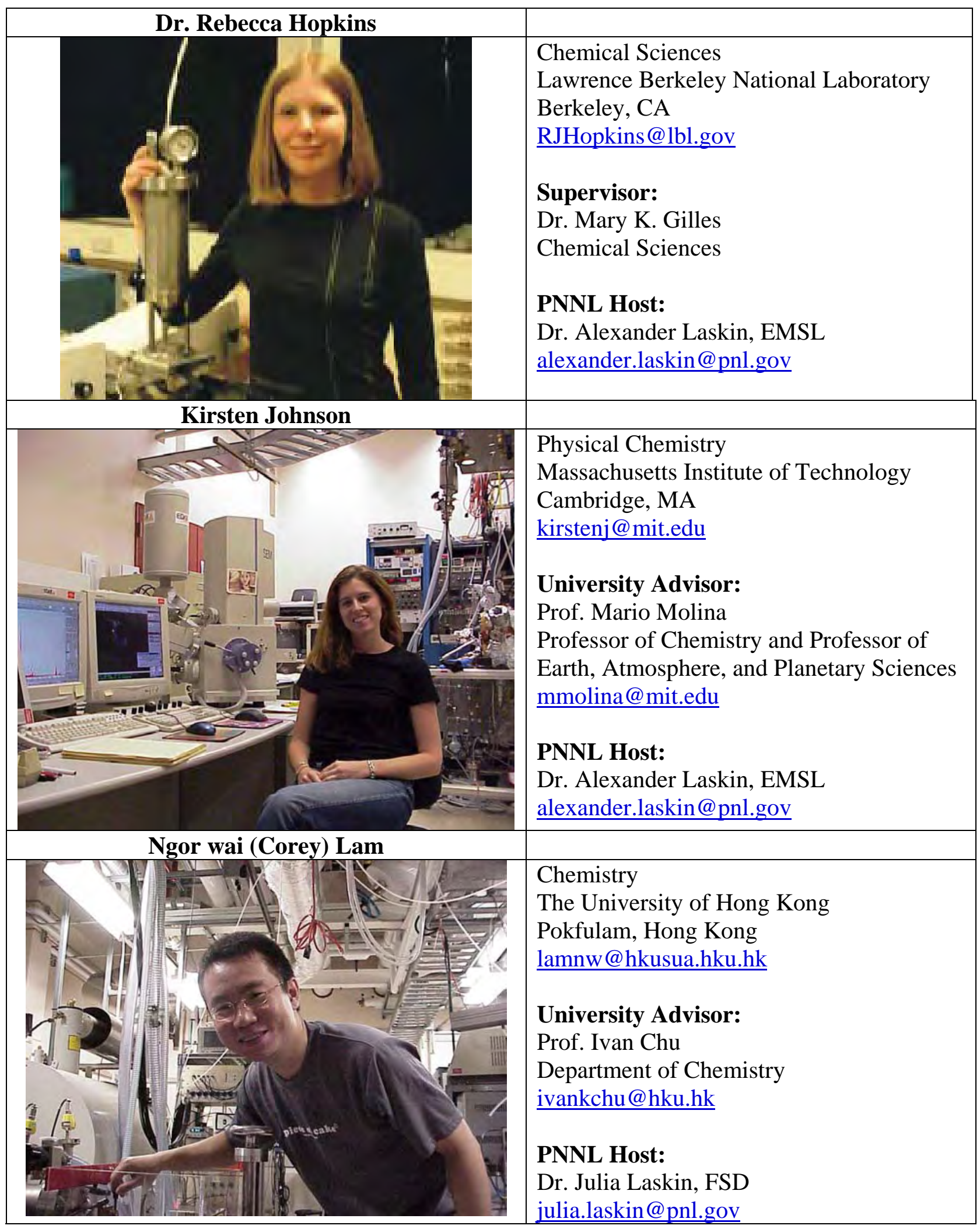




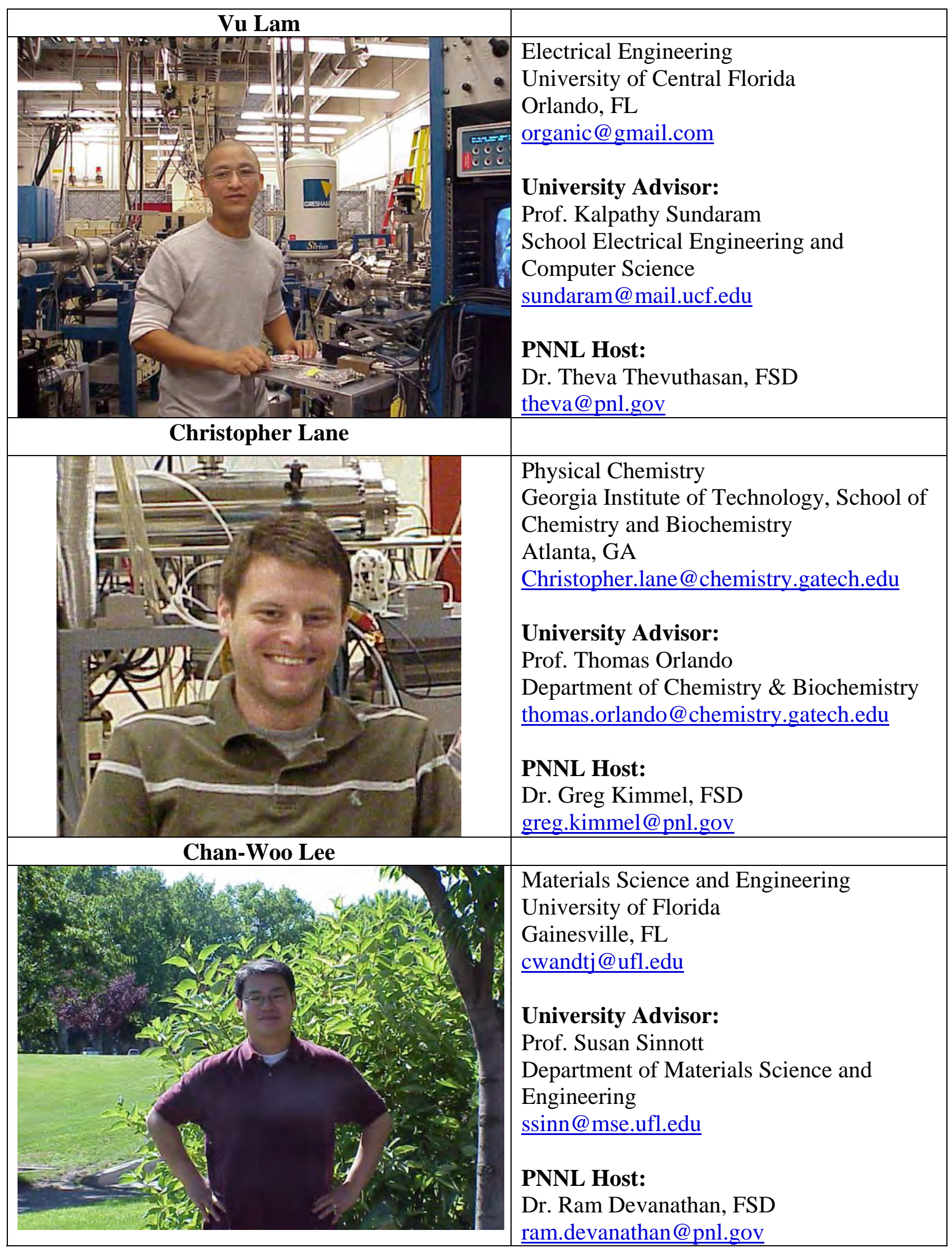




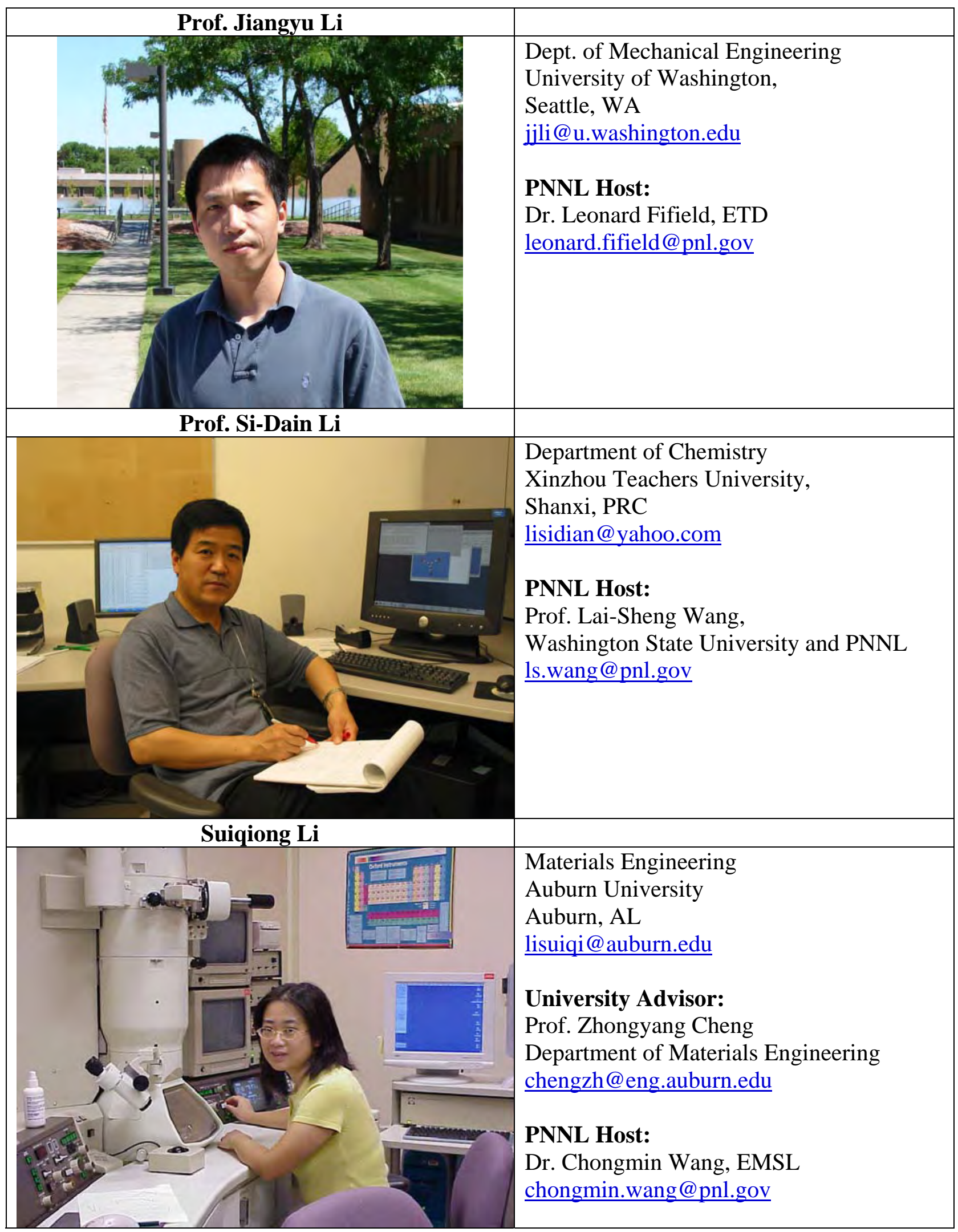




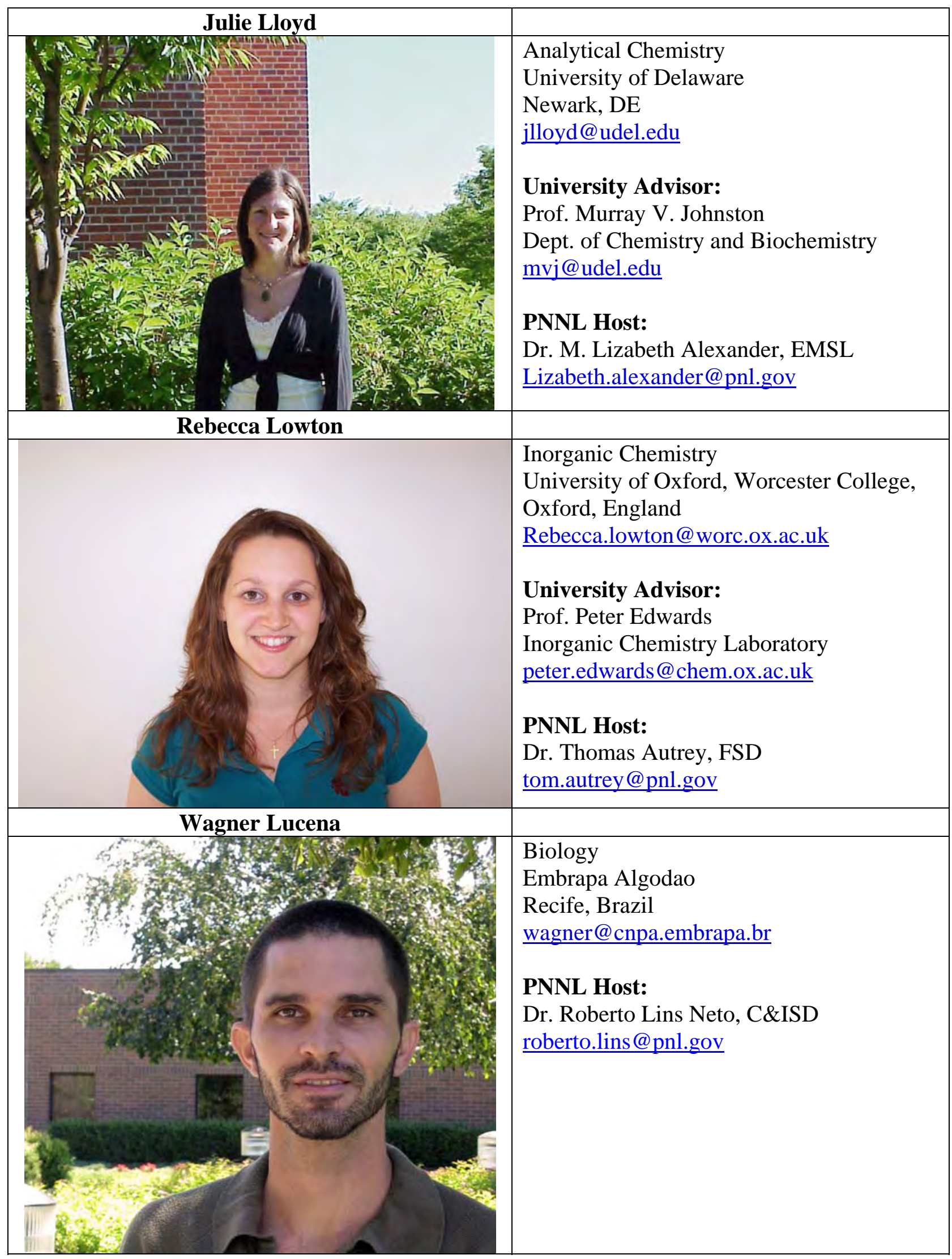




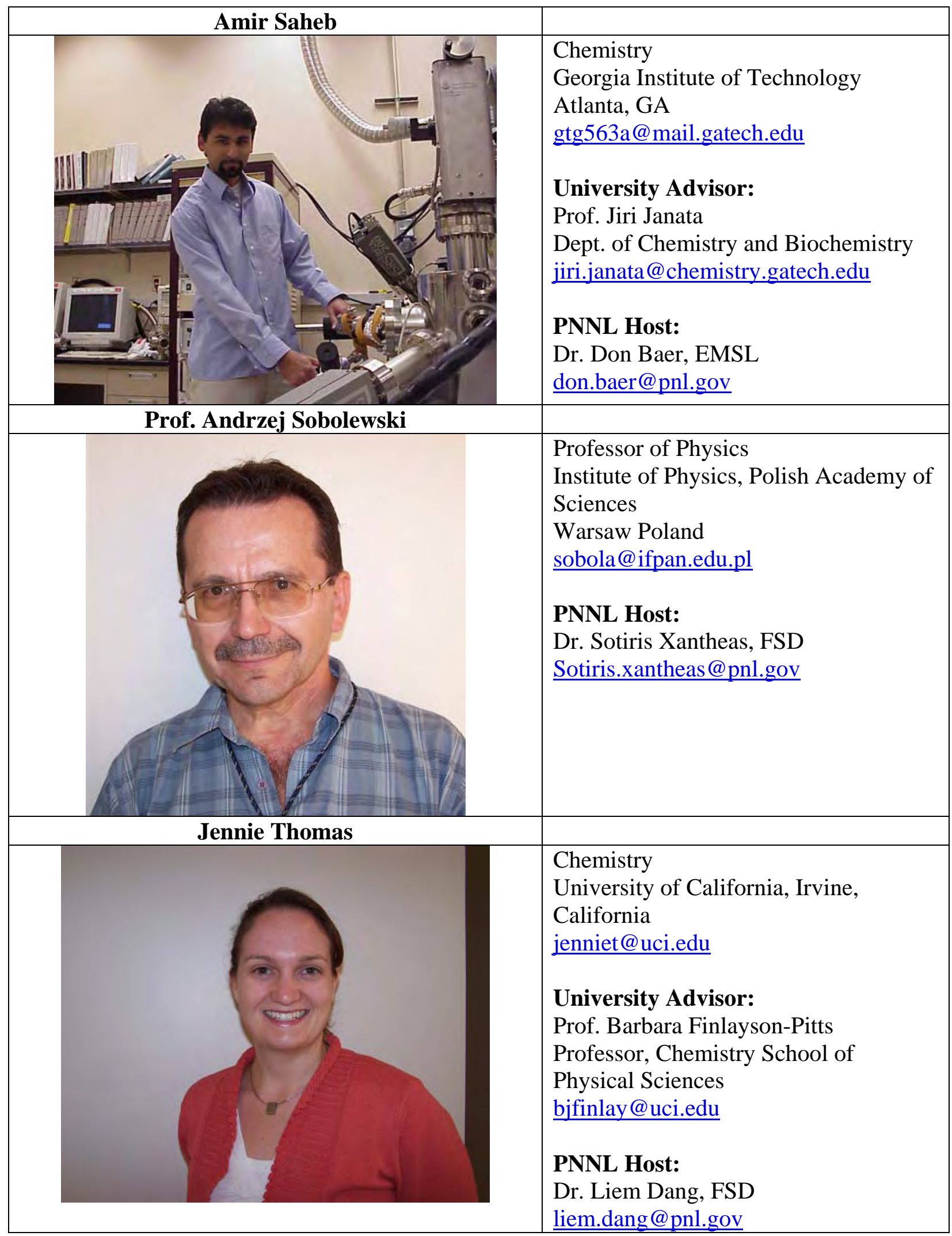




\begin{tabular}{|l|l|}
\hline Guanjun Wang & $\begin{array}{l}\text { Physical Chemistry } \\
\text { Fudan University } \\
\text { Fudan, PRC } \\
\text { 031022047@fudan.edu.cn } \\
\text { University Advisor: } \\
\text { Dr. Mingfei Zhou } \\
\text { Department of Chemistry } \\
\text { mfzhou@fudan.edu.cn } \\
\text { PNNL Host: } \\
\text { Dr. Lai-Sheng Wang, FSD } \\
\text { ls.wang@pnl.gov }\end{array}$ \\
\hline
\end{tabular}

\begin{tabular}{|l|l|}
\hline Table 3. 2005 University Statistics & \\
\hline Auburn University, Auburn, AL, USA & 1 \\
\hline Autonomous Metropolitan University, Mexico City, MEXICO & 1 \\
\hline Ecole Polytechnique Federale De Lausanne, SWITZERLAND & 1 \\
\hline Embrapa Algodão, Campina Grande, BRAZIL & 1 \\
\hline Fudan University, Fudan, PRC & 1 \\
\hline Georgia Institute of Technology, Atlanta, GA, USA & 2 \\
\hline Harvard University, Cambridge, MA, USA & 1 \\
\hline Institute of Physics, Polish Academy of Sciences, Warsaw, POLAND & 1 \\
\hline Lawrence Berkeley National Laboratory, Berkeley, CA, USA & 1 \\
\hline Lund Institute of Technology, Lund SWEDEN & 1 \\
\hline Massachusetts Institute of Technology, Cambridge, MA, USA & 1 \\
\hline University of Oxford, Worcester College, Oxford, ENGLAND & 1 \\
\hline Purdue University, West Lafayette, IN, USA & 1 \\
\hline The University of Hong Kong, Pokfulam, HONG KONG & 1 \\
\hline University of Central Florida Orlando, Orlando, FL, USA & 1 \\
\hline Universidad Federal do Rio De Janeiro, BRAZIL & 1 \\
\hline University of California, Irvine, Irvine, CA, USA & 1 \\
\hline University of Colorado at Boulder, Boulder, CO, USA & 1 \\
\hline University of Delaware, Newark, DE, USA & 1 \\
\hline University of Florida, Gainesville, FL, USA & 2 \\
\hline University of Minnesota, Minneapolis, MN, USA & 1 \\
\hline University of Pittsburgh, Pittsburgh, PA, USA & 1 \\
\hline University of Southern California, Los Angeles, CA, USA & 1 \\
\hline University of Washington, Seattle, WA, USA & 1 \\
\hline Xinzhou Teachers University, Shanxi, PRC & 1 \\
\hline & 27
\end{tabular}




\section{Benefits}

As described above, participation in the Summer Research Institute required a significant investment on the part of all parties involved. The only reason that a young scientist, PNNL host, or academic advisor would be involved is if they believed that their investment would be of benefit. We believe that the institute's structure promoted the likelihood of that success. PNNL is in a rather remote location far from major urban centers and large academic institutions, and these visitors were a real treat at the laboratory. Their inquisitiveness, creativity and energy helped stimulate PNNL staff to think in new, creative ways.

\section{New in FY 2006}

For readers familiar with the Interfacial and Condensed Phase Chemical Physics Summer Research Institute, most of the procedures and the underlying philosophy are unchanged. However, several important details are new. Some of these changes have been motivated by financial constraints; while other changes were driven by new state laws and federal regulations. New opportunities have also opened up.

This year, all of the participants came to PNNL with support from their home institutions. We can no longer offer "fellowships" or other forms of full support. It is a testimony to our growing reputation, however, that we had no shortage of qualified applicants and therefore nearly doubled the number of visitors that we hosted.

We also received assistance from the NSF, which supported seven of our visitors. We coordinated this assistance with the help of Dr. Katherine Covert of the NSF and the EMSL management. The NSF program was originally set-up by Dr. Covert and Dr. Allison Campbell, the EMSL director, for NSF grant holders to perform on-site work at EMSL. By mutual agreement, SRI participant, who were working in EMSL received the same level of support (travel and housing) as non-NSF supported visitors.

Dr. Rebecca Hopkins, a postdoctoral fellow from Lawrence Berkeley National Laboratory was the summer institute's first non-academic participant. She was also the first SRI participant from another national lab.

FY 2006 saw the inauguration of a new lecture series. The inaugural lecture was given by Prof. John Wheeler of UCSD. We hope in the future to expand this to several talks throughout the summer.

Three of our visitors did not fit into the "graduate student or young scientist" category. Dr. Michael Elfman of the Lund Institute of Technology is an expert in PIXIE and accelerator mass spectroscopy. Prof. Si-Dain $\mathrm{Li}$ is a computational chemist from Xinzhou Teachers University, Shanxi, PRC; he is also president of Xinzhou Teachers University. Prof. Andrzej L Sobolewski, is an associate professor of physics at the Physics Institute of the Polish Academy of Sciences and an expert in photophysics. These visitors were sponsored on a "resource available" basis. Their presence here in no 
way took resources from junior researchers. Except for their more senior status, they otherwise met all of the requirements for participation in the Summer Institute.

\section{Measures of Success}

This is an educational and research enterprise. As such, its real success will only become apparent in the coming years and decades. Further, the relatively short time that our visitors were here can only be a small part of the story. We can already point, however, to a few early successes. First, although applicant pool was limited, we did receive twenty-nine outstanding applications. Twenty-seven of these candidates actually came to PNNL to participate in the institute. Second, all of the candidates, PNNL mentors and academic advisors expressed enthusiasm about the institute at the outset and were generally more enthusiastic by the end of the respective summer appointments. In several cases, plans are being readied for return visits to PNNL, independent of the summer program. Numerous presentations and manuscripts are being readied for submission to peer-reviewed journals.

\section{Follow-up}

In last year's report, we stated that at least half-dozen papers were being prepared for publication as a result of the research performed at PNNL by Summer Institute participants. Currently, (October 2006) there are still four to six manuscripts in various stages of preparation as a consequence of the work in 2004, but sixteen papers, listed in Table 4 have already been published in the peer-reviewed literature. At least thirteen presentations at national or international meetings were also produced-see Table 5. In addition, numerous internal PNNL reports have been generated.

The author lists from 2004 (see Table 4 and 5) also point to an additional and somewhat unexpected benefit of the Summer Research Institute. Those visitors doing laboratory work in EMSL were extremely facile in harnessing internal collaborative opportunities and resources. Thus many of the papers included unanticipated participation by non-host PNNL staff members. 
Table 4. Peer Reviewed Publications from 2004 work through August 2006 Jormarie Alvarez, ${ }^{a}$ R. Graham Cooks, ${ }^{c}$ S. E. Barlow, Daniel J. Gaspar, Jean H. Futrell, and Julia Laskin, "Preparation and in Situ Characterization of Surfaces Using Soft Landing in a Fourier Transform Ion Cyclotron Resonance Mass Spectrometer," Anal. Chem., 77 (2005), 3452-3460

Bogdan Gologan, Jason R. Green, Jormarie Alvarez, ${ }^{a}$ Julia Laskin $^{b}$ and R. Graham Cooks, "Ion/surface reactions and ion soft-landing," Phys. Chem. Chem. Phys., 7 (2005) 1490-1500.

Yingge Du, ${ }^{\mathrm{a}} \mathrm{Y}$, S Atha, R Hull, James F Groves, ${ }^{\mathrm{c}}$ Igor V Lyubinetsky, ${ }^{\mathrm{b}}$ and Donald R Baer, “Focused-Ion-Beam Directed Self-Assembly of $\mathrm{Cu}_{2} \mathrm{O}$ Islands on $\mathrm{SrTiO}_{3}(100)$, Applied Physics Letters 84 (2004) 5213-5215

Anita Lagutschenkov, ${ }^{a}$ George S. Fanourgakis, Gereon Niedner-Schatteburg, ${ }^{\mathrm{C}}$ and Sotiris S. Xantheas, ", "The spectroscopic signature of the "all-surface" to "internally solvated structural transition in water clusters in the $n=17-21$ size regime," J. Chem. Phys., 122 (2005) 194310

Fung Suong Ou ${ }^{\mathrm{a}}$, Laxmikant V. Saraf, and Donald R. Baer, "Use of Hydrogen Bubble to Direct Pattern Microstructures of Porous Silicon” Appl. Phys. Lett., (submitted 2005)

JA Lloyd, Jeffrey M Spraggins, ${ }^{\mathrm{a}}$ Murray V Johnston, ${ }^{\mathrm{c}}$ and Julia Laskin, ${ }^{\mathrm{b}}$ "Peptide Ozonolysis: Product Structures and Proposed Mechanisms for Oxidation of Tyrosine and Histidine" J. Amer. Soc. Mass Spectrom. (submitted)

Steven L. Tait, ${ }^{\mathrm{a}}{ }_{\text {Zdenek Dohnálek. }}^{\mathrm{b}}$ Charles T. Campbell ${ }^{\mathrm{C}}$ and Bruce D. Kay, " $n$-alkanes on MgO100. I. Coverage-dependent desorption kinetics of n-butane," J. Chem. Phys., 122 (2005) 164707.

Steven L. Tait, ${ }^{\mathrm{a}}$ Zdenek Dohnálek. ${ }^{\mathrm{b}}$ Charles T. Campbell ${ }^{\mathrm{C}}$ and Bruce D. Kay, " $n$-alkanes on MgO100. II. Chain length dependence of kinetic desorption parameters for small n-alkanes,” J. Chem. Phys., 122 (2005) 164708

Yiguang Wang, ${ }^{a}$ L Zhang, Y Fan, J. Luo, DE McCready, CM Wang, ${ }^{b}$ and $\mathrm{L} \mathrm{An}{ }^{c}$ "Synthesis, Characterization, and Optical Properties of Pristine and Doped Yttrium Aluminum Garnet Nanopowders” J. Am. Ceram. Soc., 88 (2005) 284-286

Jormarie Alvarez $^{\mathrm{a}}$, JH Futrell, and Julia Laskin ${ }^{\mathrm{b}}$. 2006. "Soft-Landing of Peptides onto Self-Assembled Monolayer Surfaces." Journal of Physical Chemistry A 110(4):1678-1687. doi:10.1021/jp0555044

Hyun-shik Chang $^{\mathrm{a}}$, Gregory V. Korshin ${ }^{\mathrm{c}}$, Zheming Wang, and JM Zachara. 2006. "Adsorption of Uranyl on Gibbsite: A Time-Resolved Laser-Induced Fluorescence Spectroscopy Study." Environmental Science and Technology 40(4):1244-1249.

Yingge Du ${ }^{\mathrm{a}}$, Igor V Lyubinetsky ${ }^{\mathrm{b}}$, DR Baer, and James F Groves ${ }^{\mathrm{c}}$. 2006. "Formation of Cu2O Quantum Dots on $\mathrm{SrTiO}_{3}$ (100): Self-Assembly and Directed Self-Assembly." Journal of Applied Physics. [In Press]

Alan G. Joly ${ }^{\mathrm{b}}$, JR Williams, SA Chambers, G Xiong, WP Hess, and David M Laman ${ }^{\mathrm{a}}$. 2006. "Carrier Dynamics in a-Fe 203 (0001) Thin Films and Single Crystals Probed by Femtosecond Transient Absorption and Reflectivity." Journal of Applied Physics 99(5): Article: 053521 (6 pages). 
Howard L Heinisch $^{\mathrm{b}}$, HL, F Gao, RJ Kurtz, and Edward A Le ${ }^{\mathrm{a}}$. 2006. "Interaction of Helium Atoms with Edge Dislocations in Alpha-Fe." Journal of Nuclear Materials 351(1-3):141-148. doi:10.1016/j.jnucmat.2006.02.02

Fung S Ou${ }^{\mathrm{a}}$, Laxmikant V Saraf ${ }^{\mathrm{b}}$, and DR Baer. 2006. "Patterned Regions of Porous Silicon through Trapped Hydrogen Bubbles." Applied Physics Letters 88(14):143113 (3 pages). doi:10.1063/1.2188039

Lloyd JA, Jeffrey M Spraggins ${ }^{\mathrm{a}}$, Murray V Johnston ${ }^{\mathrm{c}}$, and Julia Laskin ${ }^{\mathrm{b}}$. 2006. "Peptide Ozonolysis: Product Structures and Relative Reactivities for Oxidation of Tyrosine and Histidine Residues." Journal of the American Society for Mass Spectrometry 17(9):1289-1298. doi:10.1016/j.jasms.2006.05.009

a) 2004 ICPCPSRI Visitor b) PNNL Host c) Supervisor

\section{Table 5 Presentations/Conference Papers from 2004 work though August 2006:}

Fung Suong $\mathrm{Ou}^{\mathrm{a}}$, Laxmikant V. Saraf, and Donald R. Baer, ${ }^{\mathrm{b}}$ "Site-specific formation of nanoporous silicon on micro-fabricated silicon surfaces," Presented by Fung Suong Ou at MRS 2004 Fall Meeting, Boston, MA on December 1, 2004. PNNLSA-42481, Pacific Northwest National Laboratory, Richland, WA

$\mathrm{CH}$ Henager, $\mathrm{Jr}^{\mathrm{b}}{ }^{\mathrm{b}} \mathrm{EA} \mathrm{Le}^{\mathrm{a}}$, and RH Jones. 2004. "A Model Stress Analysis of Swelling in SiC/SiC Composites as a Function of Fiber Type and Carbon Interphase Structure." In the 11th International Conference on Fusion Reactor Materials in JOURNAL OF NUCLEAR MATERIALS, no. Pt A, ed. Kohyama, A., 329-333 (2005) 502 - 506. Elsevier, Amsterdam, Netherlands

Julia Laskin $^{\mathrm{b}}$, O Hadjar, P Wang, JH Futrell, Jormarie Alvarez ${ }^{\mathrm{a}}$, Jason R Green $^{\mathrm{a}}$, and R.Graham Cooks ${ }^{c}$. 2006. "Soft Landing of Peptide Ions on Surfaces." Presented by Julia Laskin (Invited Speaker) at Isolated Biomolecules and Biomolecular Interactions (IBBI), Trest Castle, Czech Republic on May 10, 2006. PNNL-SA48630

Julia Laskin $^{\mathrm{b}}$, Jormarie Alvarez ${ }^{\mathrm{a}}$, R Graham Cooks ${ }^{\mathrm{c}}$, SE Barlow, and JH Futrell. 2004.

"Novel Techniques for Studying Ion-Surface Interactions in FT-ICR MS." Presented by Julia Laskin (Invited Speaker) at ASMS Conference, Nashville, TN on May 24, 2004. PNNL-SA-40658.

Julia Laskin $^{\mathrm{b}}$, Jormarie Alvarez ${ }^{\mathrm{a}}$, R Graham Cooks ${ }^{\mathrm{c}}$, JH Futrell, and SE Barlow. 2005. "Collisions of Peptide Ions with Surfaces Studied Using FT-ICR MS." Presented by Julia Laskin at 53rd ASMS Conference on Mass Spectrometry, San Antonio, TX on June 7, 2005. PNNL-SA-45421.

Yingge Du $^{\mathrm{a}}$, S Atha, R Hull, James F Groves ${ }^{\mathrm{c}}$, Igor V Lyubinetsky ${ }^{\mathrm{b}}$, and DR Baer. 2004. "Guided Control of $\mathrm{Cu}_{2} \mathrm{O}$ Nanodot Self-Assembly on $\mathrm{SrTiO}_{2}$ (100)." Materials Research Society Symposia Proceedings 811:451-456.

Fanourgakis GS, Anita Lagutschenkov ${ }^{\mathrm{a}}$, K Kowalski, Gereon Niedner-Schateburg ${ }^{\mathrm{b}}$, and Sotiris S Xantheas ${ }^{\mathrm{b}}$. 2005. "Structural, spectral and excited state features of water hydrogen bonding networks: Results of ab-initio calculations and interaction potentials." Presented by Sotiris S. Xantheas (Invited Speaker) at American Chemical Society, San Diego, CA on March 13, 2005. PNNL-SA-43386. 
Fanourgakis GS, Anita Lagutschenkov ${ }^{\mathrm{a}}$, Gereon Niedner-Schateburg ${ }^{\mathrm{b}}$, and Sotiris S Xantheas $^{\mathrm{b}}$. 2005. "Structural and spectral features of size selected water clusters in the n=7-21 regime: Results from electronic structure calculations and empirical potentials." Presented by Sotiris Xantheas (Invited Speaker) at Symposium on Size Selected Clusters, Brand, Austria on February 28, 2005. PNNL-SA-4383

Anita Lagutschenkov ${ }^{\mathrm{a}}$, GS Fanourgakis, Gereon Niedner-Schateburg, and Sotiris S $\underline{\text { Xantheas }}^{\mathrm{b}}$. 2005. "The Spectroscopic Signature of the "All-Surface" to "Internally Solvated" Structural Transition in Water Clusters in the $n=17-21$ Size Regime." Presented by Sotiris S. Xantheas (Invited Speaker) at EMSI International Workshop on Ions and Molecules at Aqueous Interfaces, Prague, Czech Republic on June 27, 2005. PNNL-SA-44788.

Alan G. Joly ${ }^{b}$, WP Hess, G Xiong, David M Laman ${ }^{\mathrm{a}}$, JR Williams, and SA Chambers. 2005. "Carrier Lifetimes and Dynamics in Epitaxial Grown $\mathrm{Fe}_{203} / \mathrm{Cr}_{203}$ Thin Films Measured by Femosecond Transient Reflectivity and Absorption." Presented by Gang Xiong (Invited Speaker) at APS Meeting, Los Angeles, CA on March 21, 2005. PNNL-SA-42076.

Fung S Ou $^{a}$, Laxmikant V Saraf ${ }^{\mathrm{b}}$, DR Baer, Z Wang, and MH Engelhard. 2004. "Use of Hydrogen Reactivity During Chemical Etching to Achieve Site-Specific NanoPorosity." Presented by Don Baer at Micro Nano Breakthrough Conference, Portland, OR on July 28, 2004. PNNL-SA-42111.

Jeffrey M Spraggins $^{\mathrm{a}}$, Julia Laskin ${ }^{\mathrm{b}}$, and Murray V Johnston ${ }^{\mathrm{c}}$. 2005. "Ozonated Peptides: Insight Into Structure and Fragmentation Energetics Using SID FT-ICR MS." Presented by Julia Laskin (Invited Speaker) at 53rd ASMS Conference on Mass Spectrometry, San Antonio, TX on June 8, 2005. PNNL-SA-45420.

Liu Y, Zhiwei Yang ${ }^{\mathrm{a}}$, PL Gassman, Hai Wang ${ }^{\mathrm{c}}$, and Alexander Laskin ${ }^{\mathrm{b}}$. 2006. "Probing Deliquescence, Efflorescence and Hygroscopic Growth of Aerosols Using MicroFTIR Spectroscopy." Presented by Yong Liu at AGU Meeting, San Francisco, CA on December 12, 2006.

a) 2004 ICPCPSRI Visitor b) PNNL Host c) Supervisor

\section{Follow-up}

As we noted above, one year is a fairly short time for scientific research to be performed, the results digested, written-up and published. None-the-less work from the summer of 2005 has already produced three refereed publications (Table 6) and fifteen presentations (Table 7). There is every reason to expect this number to rise substantially in FY 2007.

Last year we noted that Mr. Anoop M. Mayampurath had been offered employment at PNNL. In the intervening year he has become a very productive and respected member of the EMSL's Instrument Development Lab. We also noted that Dr. Zhenrong Zhang's visit in Bruce Kay's lab has been extended. Since that time, she has become a postdoctoral fellow at PNNL. 


\section{Table 6 Publications from 2005 work through August 2006}

Gutowska A, L Li, Y Shin, CM Wang, XS Li, JC Linehan, RS Smith, BD Kay, Benjamin A Schmid ${ }^{\mathrm{a}}$, WJ Shaw, MS Gutowski, and Thomas Autrey ${ }^{\mathrm{b}}$. 2005. "Nano-Scaffold Mediates Hydrogen Release and Reactivity of Ammonia Borane." Angewandte Chemie International Edition 44(23):3578-3582.

Zhenrong Zhang ${ }^{\mathrm{a}}$, O Bondarchuk, John M White ${ }^{\mathrm{c}}$, BD Kay, and Zdneek Dohnalek ${ }^{\mathrm{b}}$. 2006. "Imaging Adsorbate O-H Bond Cleavage: Methanol on $\mathrm{TiO}_{2}(110) . "$ Journal of the American Chemical Society 128(13):4198-4199

Yiguang Wang ${ }^{\mathrm{a}}$, Linan $\mathrm{An}^{\mathrm{c}}$, Y Fan, L Zhang, Sarah D Burton ${ }^{\mathrm{b}}$, and Z Gan. 2005. "Oxidation of Polymer-derived SiAICN Ceramics." Journal of the American Ceramic Society 88(11):3075-3080. doi:10.1111/j.1551-2916.2005.00542.x

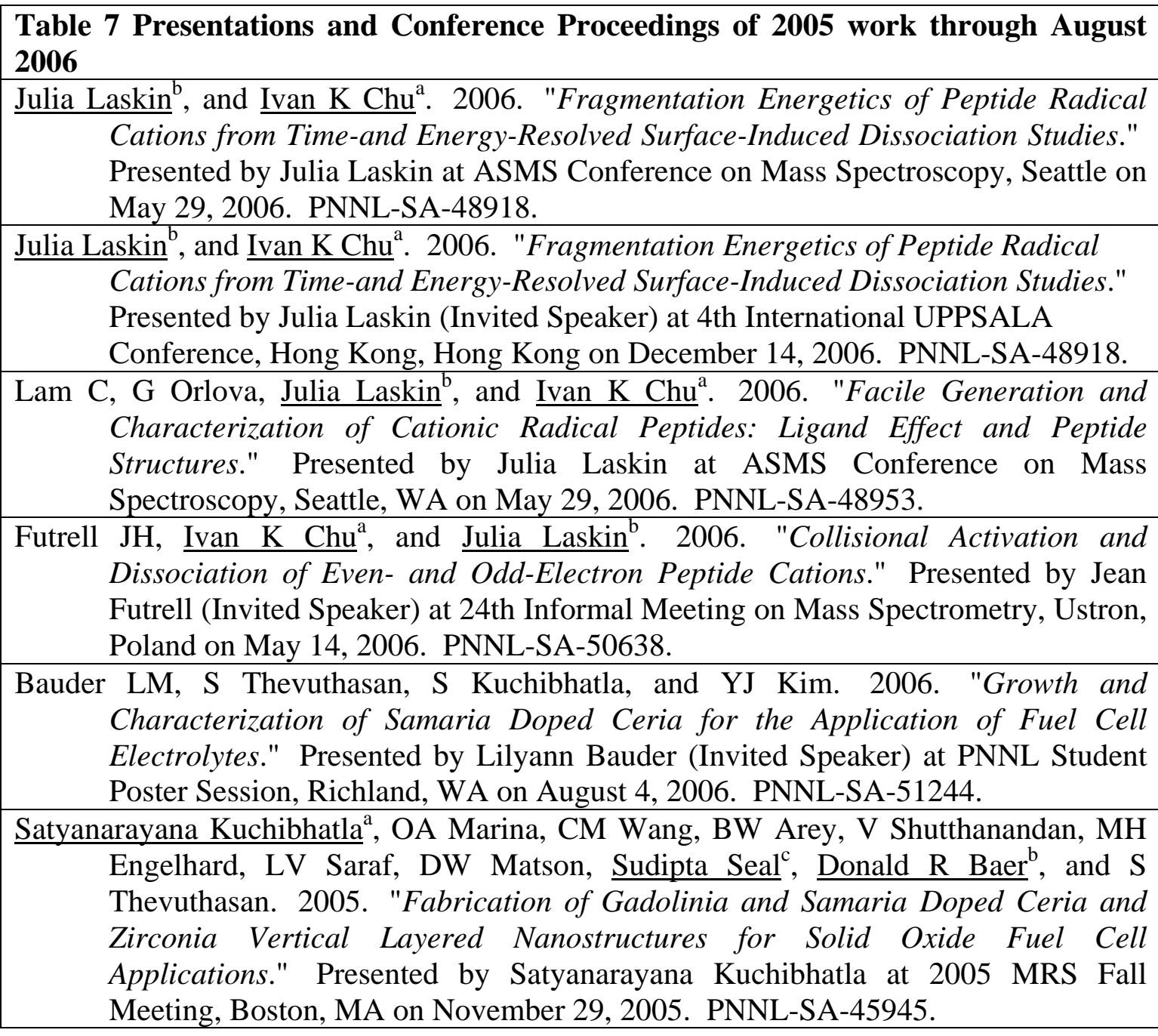


Donald R Baer ${ }^{\mathrm{b}}$, MH Engelhard, AS Lea, DJ Gaspar, KH Pecher, CM Wang, JE Amonette, AA El-azab, Satyanarayana Kuchibhatla ${ }^{\mathrm{a}}$, and Sudipta Seal ${ }^{\mathrm{C}}$. 2006. "The Secret Life of Nanoparticles: characteristics of nanoparticles and nanostructured materials that are frequently forgotten or ignored." Presented by D.R. Baer (Invited Speaker) at 2006 TMS Annual Meeting \& Exhibition, San Antonio, TX on March 12, 2006. PNNL-SA-46479.

Gutowska A, Benjamin A Schmid ${ }^{a}$, L Li, RS Smith, BD Kay, JC Linehan, WJ Shaw, NJ Hess, Y Shin, MS Gutowski, and Thomas Autrey ${ }^{\mathrm{b}}$. 2005. "Hydrogen Storage in HNBH Systems." Presented by Tom Autrey (Invited Speaker) at 3rd International Conference on Materials for Advanced Technologies 2005, Singapore, China on July 5, 2005. PNNL-SA-44161.

Smith RS, Benjamin A Schmid ${ }^{\mathrm{a}}$, NJ Hess, BD Kay, and Thomas Autrey ${ }^{\mathrm{b}}$. 2005. "Mechanistic studies of molecular hydrogen formation from borane ammonia complexes." Presented by Tom Autrey at PacifiChem 2005, Honolulu, HI on December 16, 2005. PNNL-SA-44791.

Benjamin A Schmid $^{\mathrm{a}}$, A Gutowska, L Li, WJ Shaw, JC Linehan, NJ Hess, LL Daemen, C Brown, E Mamontov, and and Thomas Autrey ${ }^{\mathrm{b}}$. 2005. "Novel hybrid materials for hydrogen storage: using mesoporous scaffolds to enhance the reactivity and selectivity of hydrogen release from ammonia borane." Presented by Tom Autrey at PacifiChem 2005, Honolulu, HI on December 19, 2005. PNNL-SA-44871.

Smith RS, BD Kay, LL Daemen, C Brown, E Mamontov, NJ Hess, WJ Shaw, JC Linehan, Benjamin A Schmid ${ }^{\mathrm{a}}$, AC Stowe, MS Gutowski, and and Thomas Autrey $^{\mathrm{b}}$. 2005. "Spectroscopic Studies of Hydrogen Formation From Amineborane Complexes." Presented by Tom Autrey at 230th National American Chemical Society Meeting, Washington, DC on August 28, 2005. PNNL-SA45354.

Hartl MA, LL Daemen, Thomas Autrey ${ }^{\mathrm{b}}$, JC Linehan, NJ Hess, AC Stowe, MS Gutowski, C Brown, E Mamontov, and Benjamin A Schmid ${ }^{\mathrm{a}}$. 2005. "Methyl Torsion Dynamics in Methyl Borate - An Inelastic Neutron Scattering Study." Presented by Monika A. Hartl at 230th National American Chemical Society Meeting, Washington, DC on August 28, 2005. PNNL-SA-45356.

Thomas Autrey ${ }^{b}, M$ Bowden, T Kemmitt, WJ Shaw, NJ Hess, JC Linehan, MS Gutowski, and Benjamin A Schmid ${ }^{\text {a }}$. 2006. "Mechanistic Studies of Hydrogen Release from Solid Amine Borane Materials." Presented by Mark Bowden at Materials Research Society, San Francisco, CA on April 18, 2006. PNNL-SA-49481.

Wei Wei $^{\mathrm{a}}$, Y Sun, John M White ${ }^{\mathrm{c}}$, G Xiong, AG Joly, Kenneth M Beck ${ }^{\mathrm{b}}$, and Wayne P $\underline{\text { Hess }}^{\mathrm{b}}$. 2006. "Real Time Study of $\mathrm{Cu}$ Diffusion Through a Ru Thin Film by Photoemission Electron Microscopy (PEEM)." In The Materials Research Society. PNNL-SA-49613, Pacific Northwest National Laboratory, Richland, WA.

Zhenrong Zhang ${ }^{\mathrm{a}}$, O Bondarchuk, John M White ${ }^{\mathrm{c}}$, J Kim, BD Kay, and Zdenek Dohnalek ${ }^{\mathrm{b}}$. 2006. "Surface Reactions of Alcohols on $\mathrm{TiO}_{2}$ (110) Investigated by in situ Scanning Tunneling Microscopy." Abstract submitted to ACS National Meeting, San Francisco, CA. 


\section{Participant Survey}

The questionnaire in the Appendix survey was sent to all of the summer program participants (visitors, hosts and advisors). From these, approximately seventy individuals we received thirty-two replies. I also attempted to conduct exit interviews with all of the visitors and actually met with all but three. Further, I have had informal discussions with visitors and PNNL hosts/mentors. Even though the Summer Institute is a scientific program, we have made no attempt to be "scientific" about the survey. The survey serves three distinct purposes. First, it helps to identify problems and weaknesses. Second, we also attempt to identify the "really" good things that need to be retained. And third, it helps to provide guidance for future program improvements.

\section{General Impressions}

Overall, the results of our survey and other inquiries produced very similar results to previous years' feedback. That is, most participants-visitors, hosts and home institution supervisors - were more enthusiastic about the program after participating in it than before. I received both written and verbal comments to the effect that the program should be continued into the indefinite future and suggestions that the program should be expanded in several ways.

Praise was universal for the smooth operation of the summer program. Credit for this goes primarily to our administrator, Ms. Nikki Avery. Not only did she organize things to run as smoothly as possible, when the inevitable problem did arise, she was able to resolve them quickly. On the one or two occasions where problems became more protracted, the difficulties could generally be traced to people not contacting her promptly---in future years, our visitors and hosts need to be more aware of this avenue.

Comments that elicited narrative responses are given in the appendix and arranged according to the role of the respondent—Academic Supervisor, PNNL host, visitor.

\section{Difficulties}

Most of the difficulties experienced by our visitors fell into two broad categories: those associated with PNNL and those associated with Richland. Lab-level problems and difficulties mostly arise from our visitors' unfamiliarity with the national lab work environment and PNNL's own idiosyncratic features. Most of these problems required the visitor to become more familiar with PNNL, while others required Nikki Avery's intervention. Future visitors will be explicitly reminded to contact Nikki or the SRI Director when as soon as problems arise.

The most irksome problem confronted our foreign national visitors, who generally cannot get 24/7 access to EMSL laboratories. This is an EMSL management policy that was implemented for reasons of safety and security. Most SRI visitors came by my office at the beginning of their stay and I informed them of this policy. This seemed to reduce the frustration levels that were evident in earlier years. 
Other difficulties experienced by our visitors are simply the result of the physical location of the laboratory on the outskirts of Richland, WA. Housing and transportation can be challenging. Nikki arranged for all of our visitors from outside the US to stay at PNNL's guesthouse. A number of participants from inside the US also stayed there. All were pleased with the accommodations; however it is difficult get from there to local stores without a car. Similarly those staying in town had a three to five mile commute to the lab. The housing and transportation problems have improved somewhat over the last few years, but still remain a challenge.

There were two cases where the PNNL host appeared to be over committed and either did not have enough physical space - a serious problem across PNNL—or was engaged in extensive travel and did not have adequate time. All would be hosts and mentors need to be reminded of their obligations in both of these areas. It is better not to host a summer visitor than to be unable to provide the resources necessary for a successful experience. With that said, the Summer Institute's general experience with PNNL hosts has been very positive.

\section{Future Improvements}

\section{Publicity}

In FY 2006, we substantially increased our publicity efforts, and as reported above nearly doubled our participation. It will be important to maintain this effort and expand it if possible in 2007. Publicity of the very best sort is of course "word of mouth" that is spreading from participants. We now appear in the American Chemical Society's list of summer research opportunities. We should endeavor to reach out to other profesional societies and groups. Further, we need to refine and expand our annual mailing list. We also need to look into getting listed on the DOE educational opportunities website.

\section{Participation and Continuity}

Several participants recommended that the program be both expanded and extended pass the FY 2007 stretch goal year. This year's small experience with more senior visitors indicates that there is a real need for a program similar to this one to serve that group. At this juncture, simply expanding the scope of the ICPCPSRI program may be a viable approach as the marginal costs would be small.

\section{Other Iprovements}

Several comments were received to the effect that more activities to promote interactions among the Summer Institute's participants are needed. Currently the two barbeques are the only such avenue. In the future, we would like to expand the lecture program, possibly by collaborating with the distinguished visitor program. Whatever is done in this regard, it must not place an undue burden on our participant's limited time at PNNL. Currently the only activity mandated by the Summer Institute is the preparation of a final report-this should remain unchanged. 
PNNL-16185 
PNNL-16185

\section{Technical Reports}

The remainder of this document is devoted to the technical reports provided by each young scientist. As noted above, some of these are single paragraph abstracts and, therefore, have rather limited content. Other reports, however, are nearly complete papers. These documents are "as received," apart from minor formatting changes in some cases. We have made no effort to edit or evaluate these reports. Given the limited distribution intended for this report, none of the results in these reports should be considered "published" by virtue of appearing here. 
PNNL-16185 
PNNL-16185

\title{
Heterogeneous Reaction Kinetics of $\mathrm{NaCl}+\mathrm{HNO}_{3} *$
}

\author{
Jeremy Cain and Hai Wang \\ Department of Aerospace and Mechanical Engineering \\ University of Southern California \\ Los Angeles, CA \\ and \\ Dr. Yong Liu and Dr. Alexander Laskin \\ Pacific Northwest National Laboratory \\ Richland, WA
}

Previous investigations into heterogeneous reaction kinetics of $\mathrm{NaCl}^{1}$ and a variety of atmospheric particles ${ }^{2}$ have been performed in a reaction chamber depicted in Figure 1(a). In this reactor setup, aerosol samples are deposited on TEM grids supported by a grid holder, and the holder is placed in a circular chamber with diluted gaseous reactants flowing at a constant flow rate. Even though measurements of reaction parameters have been performed successfully using this configuration, a detailed knowledge of the effects of fluid flow on the reaction kinetics is not known. Laskin et al. ${ }^{1}$ recognized that gaseous diffusion of the reactant to the substrate surface is an important factor in the determination of fundamental reaction rate parameters. In order to obtain accurate rate measurements, it is necessary to control the diffusion boundary layer thickness. For this reason, a new reaction chamber was designed and implemented, as seen in Figure 1(b). The new reactor is based on the concept of stagnation flow, i.e., an axi-symmetric flow of diluted reactant gas jet impinging on the TEM grids fixed atop the grid holder. This design is expected to give desirable flow field and uniform diffusion boundary layer thickness, as well as allow for flow variables to be easily manipulated.

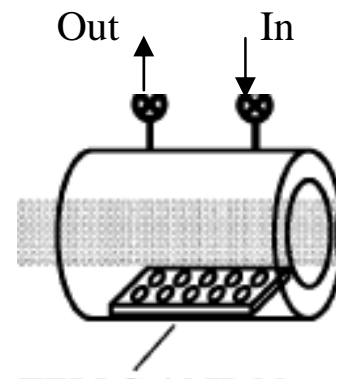

TEM Grid Holder

(a) Old reactor design 
PNNL-16185

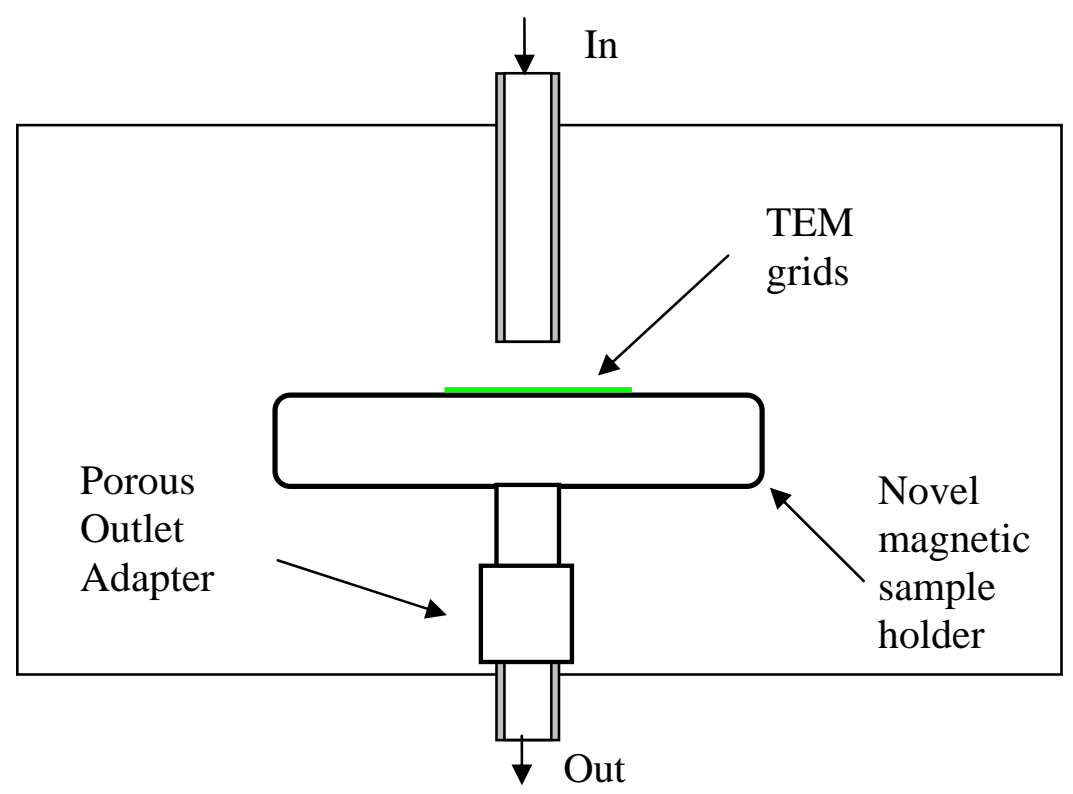

(b) New reactor design using stagnation flow

Fig. 1 - Different reaction chamber designs

The primary purpose of my Summer Institute Study at EMSL was to examine the new reactor, which was designed by computational fluid dynamics (CFD), and apply the knowledge gained from the CDF simulations in both testing the reactor and improving upon its design. Modeling the fluid flow in FLUENT, ${ }^{3}$ a CFD solver, allowed for detailed information such as spatial variations in species concentration like that of Figure 2(a) to be obtained. It was determined through numerical modeling how the transport of reacting gas species would be affected by changes in certain parameters (e.g., tube diameter and velocity). Upon examination of model results, optimal operating conditions were determined, as seen in Figure 2(b), and used in heterogeneous reaction experiments. In the limiting case of complete surface coverage, the diffusion boundary layer thickness increases and concentration gradient decreases radially, with the latter being a much stronger function of radial co-ordinate than the former. The key parameters which change the boundary layer profile are velocity (V), tube offset height (L), and tube inner diameter (d). Numerical simulations are able to tell us the changes in concentration gradient with those in $\mathrm{L}$ and $\mathrm{d}$, as shown in Figure 2(b). Thus, for a desirable concentration gradient tolerance we know how many grids can be tested and under what conditions they may be tested. 
PNNL-16185

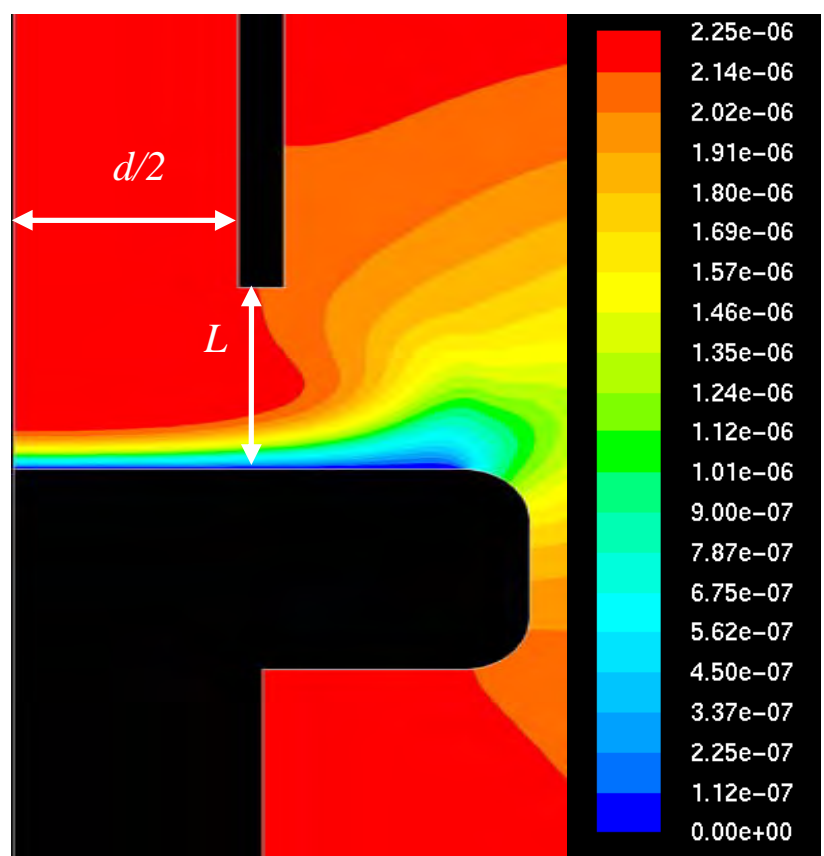

(a) $\mathrm{HNO}_{3}$ mass fraction contours for 2-D axi-symmetric stagnation flow

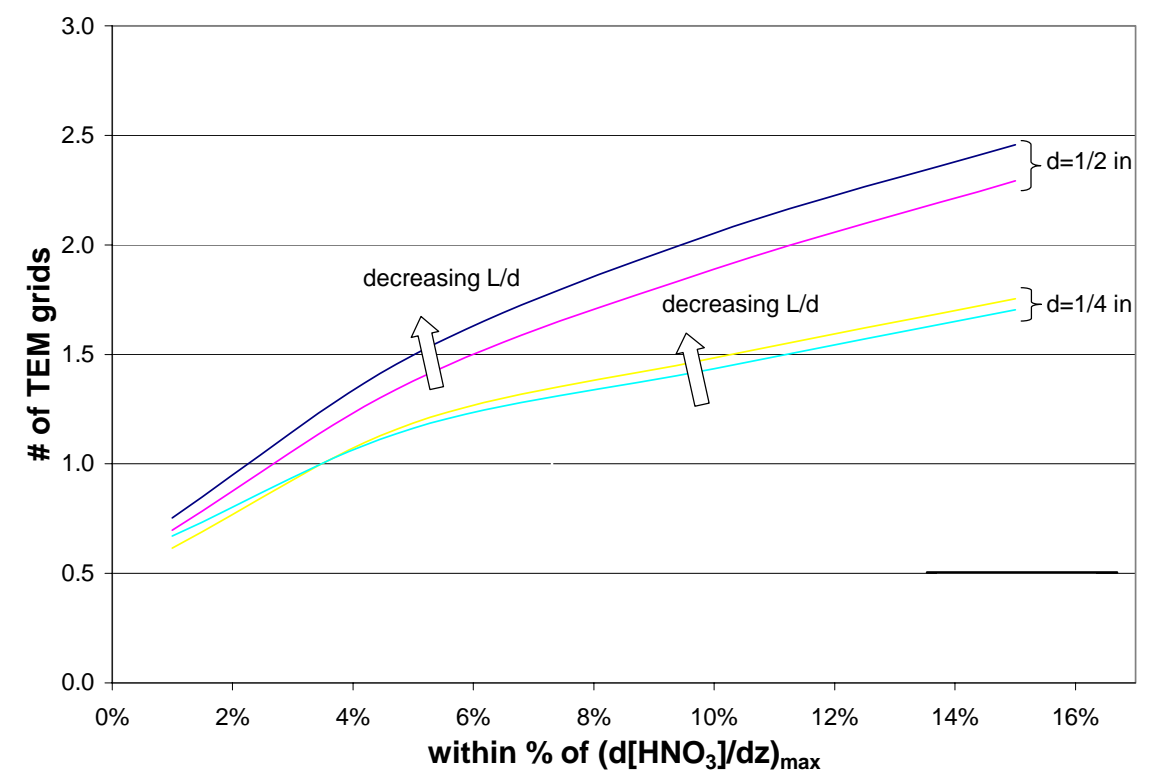

(b) Reactor operating conditions for varying parameters ( $\mathrm{V}=4 \mathrm{~L} / \mathrm{min})$

Fig. 2 - Sample computational modeling results

The new reactor design was then used to study the heterogeneous reaction kinetics of $\mathrm{NaCl}$ with $\mathrm{HNO}_{3}$ at the relative humidity $\mathrm{RH}=80 \%$. Experimental results obtained thus far show the expected dependency of pseudo-first order rate constant as a function of number density of particles on grid surface, as seen in Figure 3. Based on a diffusionkinetic analysis ${ }^{1}$, we have 


$$
\begin{aligned}
\frac{\left[\mathrm{HNO}_{3}\right]_{\infty}}{k_{I}} & =a+b \mathrm{~N}_{s} \\
& =\frac{1}{k_{I I}}\left(1+\delta k_{s}\right)+\left[\delta\left[C l^{-}\right]_{d, 0} e^{-k_{I} t}\left(\frac{4}{3} \pi r_{d}^{3}\right)\right] N_{s}
\end{aligned}
$$

A fit to the data gives the intrinsic second-order rate constant $k_{I I}=5.5 \times 10^{-15}\left(\mathrm{~cm}^{-3} \mathrm{~s}\right)$ with an uncertainty factor equal to about 2.4. The reaction uptake coefficient is then calculated from

$$
\gamma_{\mathrm{net}}=\frac{4 k_{\text {II }}\left[\mathrm{Cl}^{-}\right]_{d} V_{d}}{\bar{c}_{\mathrm{HNO} 3} S_{d}}
$$

Assuming that the droplets are spherical, we obtain $\gamma_{\text {net }}=0.06(x / \div 2.4)$. This value is in a close agreement with the recently report value of 0.05 at $\mathrm{RH}=85 \%$ by Saul et al. ${ }^{3}$ who studied the same reaction kinetics in a substrate-free laminar flow reactor. The close agreement suggests that the current reactor design is valid and is expected to give quantitative heterogeneous kinetic data. Validating the design through conformation of experimental results is necessary before moving to systems which have not been studied before, such as heterogeneous reactions of reactive free radicals with soot.

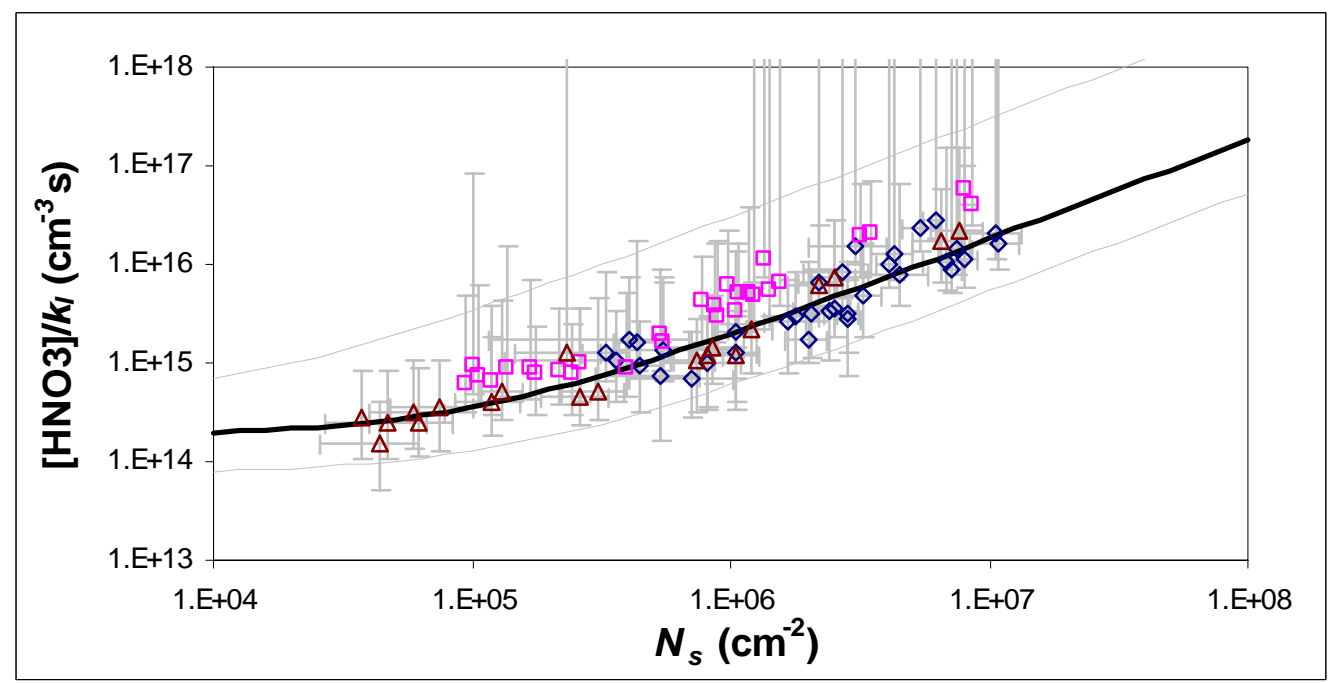

Fig. 3 - Variation of pseudo-first order rate constant as a function of $\mathrm{NaCl}$ particle number density on the substrate surface

\section{References:}

1. Laskin, A.; Wang, H.; Robertson, W.H.; Cowin, J.P.; Ezell, M.J.; Finlayson-Pitts, B.J. J. Phys. Chem. A 2006, 110, 10619.

2. Krueger, B.J.; Grassian, V.H.; Iedema, M.J.; Cowin, J.P.; Laskin, A. Anal. Chem. 2003, 75, 5170.

3. Saul, T.D.; Tolocka, M. P.; Johnston, M. V. J. Phys. Chem. A. 2006, 110, 7614. 
PNNL-16185

\title{
In-situ studies of initial oxidation of bare InAs surfaces and oxide/InAs interfaces
}

\author{
C.L. Chang, S. Ramanathan \\ Division of Engineering and Applied Sciences, \\ Harvard University, Cambridge, MA 02138 \\ and \\ V. Shutthanandan, \\ Pacific Northwest National Laboratory, \\ Richland, WA
}

The scaling of MOSFET (metal-oxide-semiconductor field effect transistors) has become aggressive due to the need for higher performance and integration density in nano-electronic devices [1]. A major effort is to replace $\mathrm{SiO}_{2}$ with higher-dielectric (high-k) constant oxide films as the gate dielectric in future transistor application due to its high leakage currents and intrinsic reliability concerns [2]. Thus integration of high-k dielectrics into CMOS processes is a major challenge. Beyond the substitution of conventional $\mathrm{SiO}_{2}$ by high-k dielectrics for geometric scaling, another avenue to overcome the issue of surface mobility degradation is to use substrates with higher hole and electron mobility instead of silicon. Also, high mobility channel layers could alleviate the saturation of Si MOSFET drain current upon dimension shrinkage by providing a higher source injection velocity. InAs is a good candidate worthy of further investigation for this purpose.

Interface between high-k dielectrics and the substrate is of concern since unwanted interface layers with lower permittivity will degrade the device performance. The purpose of annealing in oxygen environment is to let the oxygen vacancies within the dielectrics be filled to reduce the leakage current. But it could be possible that introduced oxygen will not only fill up anion vacancies, but also diffuse deeper toward the oxide/InAs interface to form undesired oxide interfacial layers. To confirm the oxygen diffusion mechanism during the annealing process, two experiments with yttria-doped zirconia (YDZ) grown on InAs and bare InAs have been annealed in oxygen at various temperatures and studied.

YDZ films were grown by electron beam evaporation method on InAs substrate at room temperature. High resolution Rutherford backscattering spectrometry (RBS) and nuclear reaction analysis (NRA) measurements were carried out in random geometries at the Environmental Molecular Science Laboratory (EMSL) accelerator facility, Pacific Northwest National Laboratory (PNNL). Both in situ NRA and RBS measurements are performed after annealing experiments. ${ }^{18} \mathrm{O}$ is introduced at a pressure of $2 \times 10^{-6}$ Torr during heat treatment at five different temperatures: $200^{\circ} \mathrm{C}, 300^{\circ} \mathrm{C}, 400^{\circ} \mathrm{C}$, and $500^{\circ} \mathrm{C}$ for 30 minutes. SIMNRA [3] was used to simulate the experimental RBS spectra as shown in Fig. 1, and the total anion concentration is $3.15 \times 10^{17} \mathrm{~cm}^{-2}$. NRA data on an as-loaded sample shows that the as-deposited YSZ film a small fraction of vacancies. In table 1 it shows that some ${ }^{16} \mathrm{O}$ leaves the $\mathrm{YDZ}$ film until $200^{\circ} \mathrm{C}$, but is similar from $200^{\circ} \mathrm{C}$ to $500^{\circ} \mathrm{C}$. Whereas ${ }^{18} \mathrm{O}$ increases proportional to the rising temperature above $200^{\circ} \mathrm{C}$ as in table 1 . Beyond $200^{\circ} \mathrm{C}{ }^{16} \mathrm{O}$ doesn't appear to exchange with ${ }^{18} \mathrm{O}$ as the results suggest. The oxygen occupancy ratio is defined as total oxygen areal density $\left({ }^{16} \mathrm{O}\right.$ and $\left.{ }^{18} \mathrm{O}\right)$ divided by the total anion sites; and oxygen occupancy ratio reaches unity at $500^{\circ} \mathrm{C}$. 
PNNL-16185

\begin{tabular}{|c|c|c|c|}
\hline Annealing Condition & ${ }^{16} \mathrm{O}$ NRA $(\mathrm{Nt})_{i}\left(\mathrm{atoms} / \mathrm{cm}^{2}\right)$ & ${ }^{18} \mathrm{O}$ NRA $(\mathrm{Nt})_{i}\left(\mathrm{atoms} / \mathrm{cm}^{2}\right)$ & Occupancy ratio \\
\hline As-loaded & $3.05 \mathrm{E}+17$ & 0 & $96.7 \%$ \\
\hline $200^{\circ} \mathrm{C}$ & $2.72 \mathrm{E}+17$ & $5.76 \mathrm{E}+14$ & $86.5 \%$ \\
\hline $400^{\circ} \mathrm{C}$ & $2.72 \mathrm{E}+17$ & $1.78 \mathrm{E}+16$ & $92.1 \%$ \\
\hline $500^{\circ} \mathrm{C}$ & $2.88 \mathrm{E}+17$ & $2.88 \mathrm{E}+16$ & $100 \%$ \\
\hline
\end{tabular}

Table 1. Comparison of ${ }^{16} \mathrm{O}$ and ${ }^{18} \mathrm{O}$ by NRA as a function of annealing temperature.

\section{Energy [keV]}

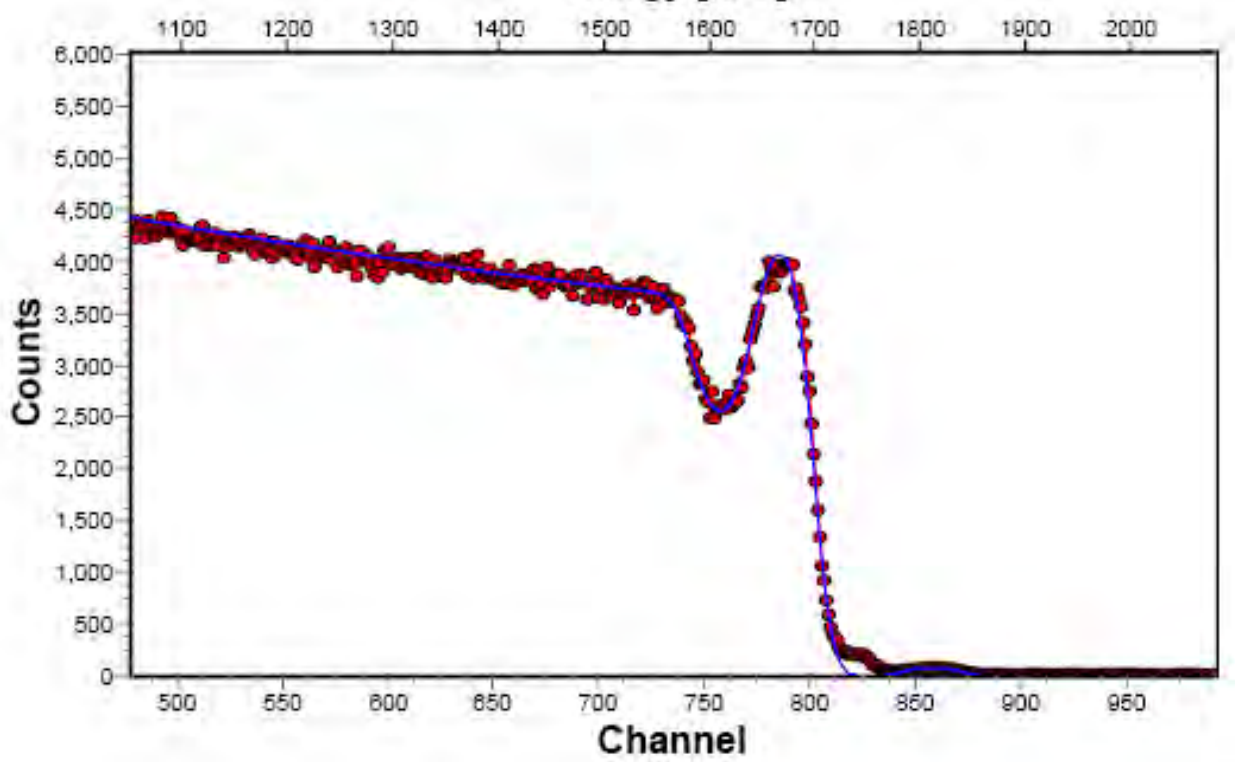

Fig. 1 RBS spectrum for YDZ on InAs simulated by SIMNRA[3].

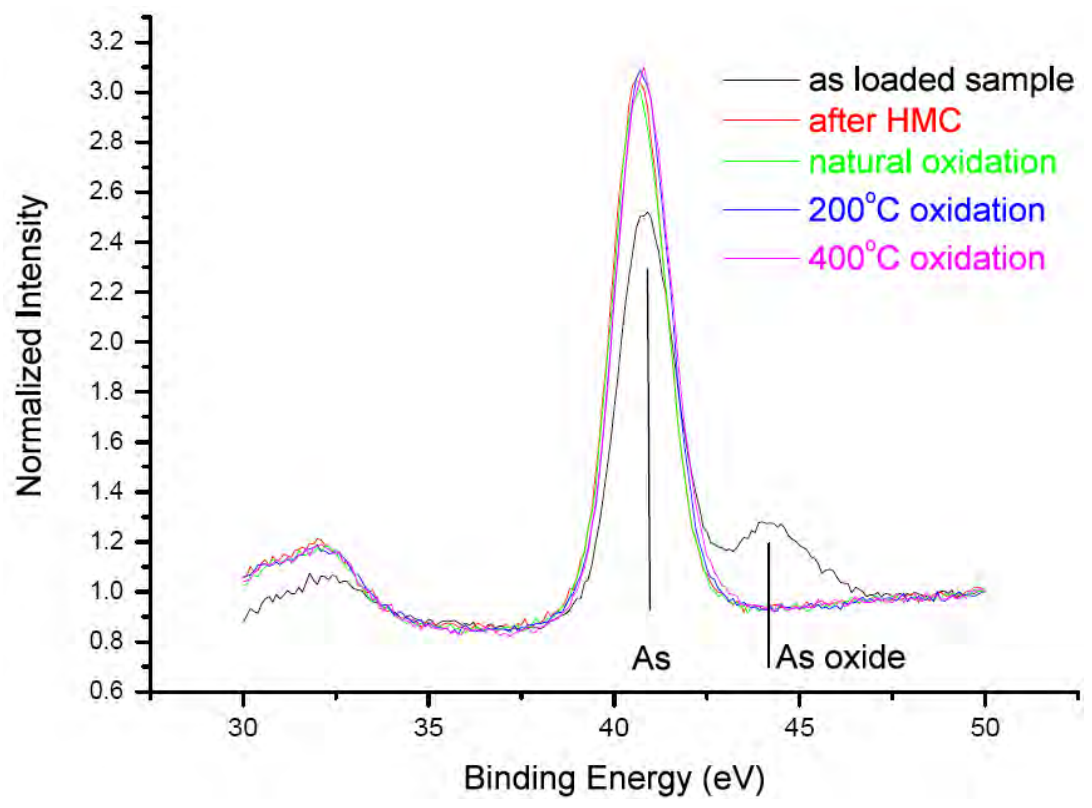

Fig. 2 (a) XPS for arsenic. 
PNNL-16185

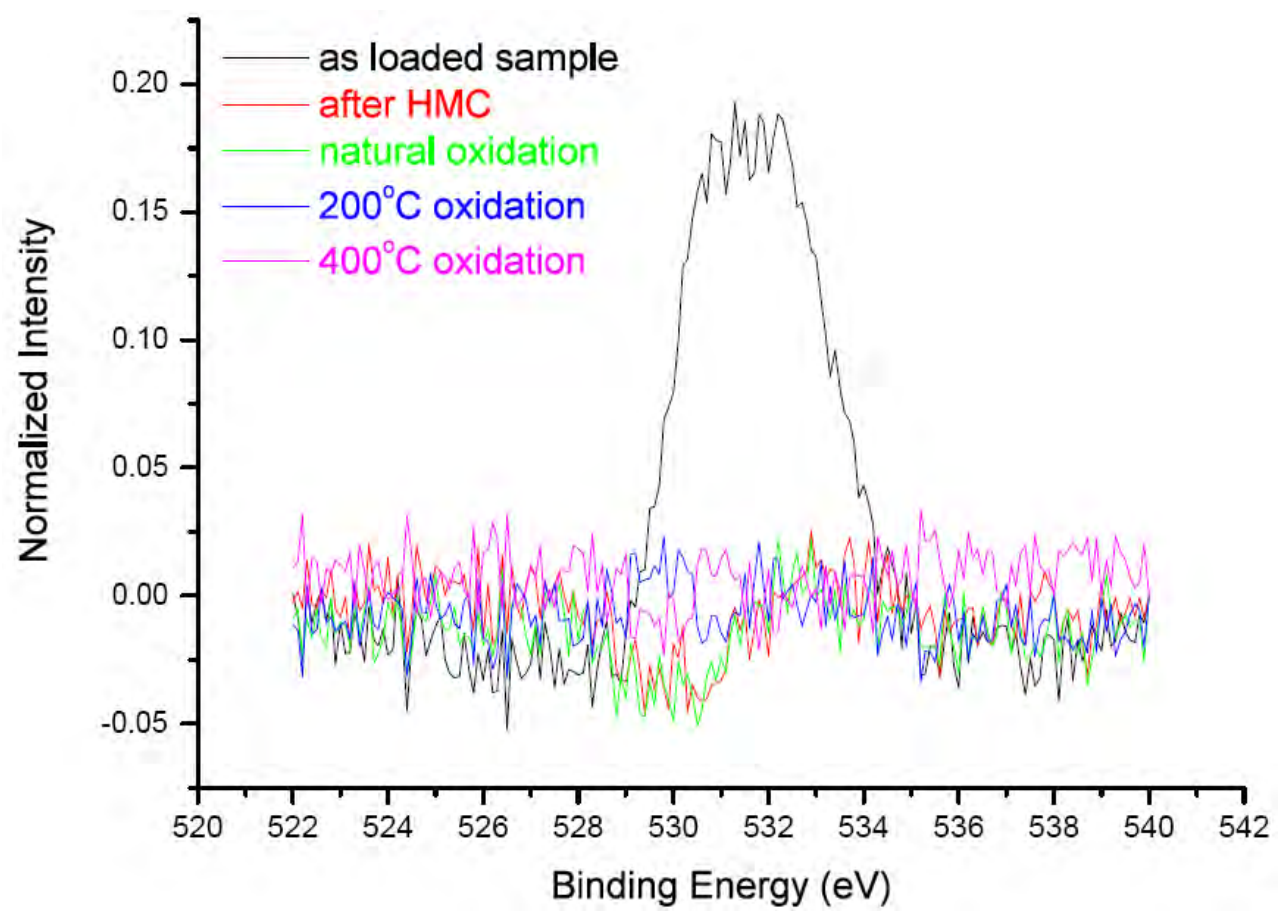

Fig. 2(b) XPS for oxygen

Furthermore, the oxidation of InAs bare substrates under identical annealing conditions has also been investigated. Both in situ XPS and NRA measurements are carried out to acquire information on the surface oxide. After the complete removal of native oxide on InAs by HMC (Hydrogen Molecule Method), the XPS shows no oxide formed on InAs surface as seen in Fig. 2(a) for arsenic and in Fig. 2(b) for oxygen peak. Following the HMC, the same annealing conditions as above are performed. No oxide peak shows up as we can see in Fig. 2(a) and the amount of oxygen is not increasing as temperature is increasing up to $500^{\circ} \mathrm{C}$. The oxygen concentration information from NRA measurement confirms this result. This supplementary experiment tells the information that under the same oxygen annealing condition the bare InAs is resistant to oxidation. From this information we could infer that during annealing, oxygen will fill up the anion vacancies instead of diffusing deeper toward YSZ/InAs to form the undesired interfacial oxide layers.

Both oxygen diffusion mechanism for YSZ on InAs and bare InAs in oxygen annealing are characterized and studied by in situ high resolution RBS, NRA and XPS measurements. The result suggests the oxygen will fill up the anion vacancies instead of forming the unwanted oxide interfacial layers between YSZ and InAs.

1. Y. Taur, "CMOS design near the limit of scaling," IBM Journal of Research \& Development, 40 (2002), 213-222.

2. M. K. Weldon et al., "The surface science of semiconductor processing: gate oxides in the evershrinking transistor,” Surface Science, 500 (2002), 859-878.

3. SIMNRA User's guide, edited by M. Mayer, Max-Planck-Institut fur Plasmaphysik, Germany. 
PNNL-16185 
PNNL-16185

\title{
Metal-doped iron particles
}

\author{
Chan Lan Chun and William A. Arnold \\ University of Minnesota \\ Minneapolis, $\mathrm{MN}$ \\ and \\ Don Baer, EMSL \\ Pacific Northwest National Laboratory \\ Richland, WA
}

\section{Report:}

1. Major routes of particle synthesis

- Solution based synthesis (Starting material: dry Toda iron): Chanlan Chun in EMSL

- Sputtering based synthesis: Samples from Dr. Qiang group (U of Idaho)

- Hydrogen-reduction process (Starting material: 6-line ferrihydrite): Dean Matson and Chanlan Chun in PSL

2. Samples ( $\sqrt{ }$ : completely done, $\mathrm{W}$ : waiting list, and N/A: not applicable) 
PNNL-16185

\begin{tabular}{|c|c|c|c|c|c|c|c|c|}
\hline Process & Sample ID & Description (washing) & Synthesis & ICP & TEM & XRD & XPS & 4-Cl-NB \\
\hline \multirow{13}{*}{$\begin{array}{l}\text { Solution } \\
\text { (Toda iron) }\end{array}$} & DT-UT & Untreated Toda iron & $\sqrt{ }$ & & $\sqrt{ }$ & $\sqrt{ }$ & $\sqrt{ }$ & $\sqrt{ }$ \\
\hline & C-M & Toda iron w/o dopants after Milli-Q washing & $\sqrt{ }$ & $\sqrt{ }$ & & $\sqrt{ }$ & $\sqrt{ }$ & \\
\hline & S-M-Cu-1 & 0.17 \% Cu-doped Toda iron (Milli-Q water) & $\sqrt{ }$ & $\sqrt{ }$ & & $\sqrt{ }$ & & $\sqrt{ }$ \\
\hline & S-M-Cu-10 & $0.62 \%$ Cu-doped Toda iron (Milli-Q water) & $\sqrt{ }$ & $\sqrt{ }$ & & $\sqrt{ }$ & & $\sqrt{ }$ \\
\hline & S-M-Ni-1 & 0.12 \% Ni-doped Toda iron (Milli-Q water) & $\sqrt{ }$ & $\sqrt{ }$ & & $\sqrt{ }$ & $\sqrt{ }$ & $\sqrt{ }$ \\
\hline & S-M-Ni-10 & $0.43 \%$ Ni-doped Toda iron (Milli-Q water) & $\sqrt{ }$ & $\sqrt{ }$ & $\sqrt{ }$ & $\sqrt{ }$ & $\sqrt{ }$ & $\sqrt{ }$ \\
\hline & S-M-Pd-1.5 & 0.08 \% Pd-doped Toda iron (Milli-Q water) & $\sqrt{ }$ & $\sqrt{ }$ & $\sqrt{ }$ & $\sqrt{ }$ & & $\sqrt{ }$ \\
\hline & C-A & Toda iron w/o dopants after acid washing & $\sqrt{ }$ & $\sqrt{ }$ & $\sqrt{ }$ & $\sqrt{ }$ & $\sqrt{ }$ & \\
\hline & S-A-Cu-1 & $0.12 \%$ Cu-doped Toda iron (Acid ) & $\sqrt{ }$ & $\sqrt{ }$ & & $\sqrt{ }$ & & \\
\hline & S-A-Cu-10 & $0.75 \%$ Cu-doped Toda iron (Acid ) & $\sqrt{ }$ & $\sqrt{ }$ & $\sqrt{ }$ & $\sqrt{ }$ & $\sqrt{ }$ & \\
\hline & S-A-Ni-1 & $0.19 \%$ Ni-doped Toda iron (Acid ) & $\sqrt{ }$ & $\sqrt{ }$ & & $\sqrt{ }$ & $\sqrt{ }$ & \\
\hline & S-A-Ni-10 & $0.62 \%$ Ni-doped Toda iron (Acid ) & $\sqrt{ }$ & $\sqrt{ }$ & $\sqrt{ }$ & $\sqrt{ }$ & $\sqrt{ }$ & \\
\hline & S-A-Pd-1.5 & 0.07 \% Pd-doped Toda iron (Acid ) & $\sqrt{ }$ & $\sqrt{ }$ & & $\sqrt{ }$ & $\sqrt{ }$ & \\
\hline \multirow{10}{*}{$\begin{array}{l}\text { Hydrogen } \\
\text { Reduction }\end{array}$} & HR-Fe & Iron without dopants & $\sqrt{ }$ & $\sqrt{ }$ & $\sqrt{ }$ & $\sqrt{ }$ & $\mathrm{W}$ & $\sqrt{ }$ \\
\hline & HR-FePd0.5 & $0.5 \%$ Pd-doped iron & $\sqrt{ }$ & $\sqrt{ }$ & $\sqrt{ }$ & $\sqrt{ }$ & W & $\sqrt{ }$ \\
\hline & HR-FePd1 & $1 \%$ Pd-doped iron & $\sqrt{ }$ & $\sqrt{ }$ & $\sqrt{ }$ & $\sqrt{ }$ & W & $\sqrt{ }$ \\
\hline & HR-FePd2 & $2 \%$ Pd-doped iron & $\sqrt{ }$ & $\sqrt{ }$ & $\sqrt{ }$ & $\sqrt{ }$ & $\mathrm{W}$ & $\sqrt{ }$ \\
\hline & HR-FeCu0.5 & $0.5 \% \mathrm{Cu}$-doped iron & $\sqrt{ }$ & & & $\sqrt{ }$ & & \\
\hline & HR-FeCu1 & $1 \% \mathrm{Cu}$-doped iron & $\sqrt{ }$ & & & $\sqrt{ }$ & & \\
\hline & HR-FeCu2 & 2 \% Cu-doped iron & $\sqrt{ }$ & & & $\sqrt{ }$ & $\mathrm{W}$ & \\
\hline & HR-FeNi0.5 & $0.5 \%$ Ni-doped iron & $\sqrt{ }$ & & & $\sqrt{ }$ & & \\
\hline & HR-FeNi1 & $1 \%$ Ni-doped iron & $\sqrt{ }$ & & & $\sqrt{ }$ & & \\
\hline & HR-FeNi2 & $2 \%$ Ni-doped iron & $\sqrt{ }$ & & & $\sqrt{ }$ & $\mathrm{W}$ & \\
\hline \multirow{6}{*}{ Sputtering } & SP-FeCu2 & Uniformly 2 \% Cu-doped iron & $\sqrt{ }$ & N/A & $\sqrt{ }$ & & $\sqrt{ }$ & \\
\hline & SP-FeCu5 & Uniformly 5 \% Cu-doped iron & $\sqrt{ }$ & N/A & & & $\sqrt{ }$ & \\
\hline & SP-FeCu15 & Uniformly $15 \%$ Cu-doped iron & $\sqrt{ }$ & N/A & $\sqrt{ }$ & & $\sqrt{ }$ & \\
\hline & SP-FeNi2 & Uniformly $2 \%$ Ni-doped iron & $\sqrt{ }$ & N/A & $\sqrt{ }$ & & $\sqrt{ }$ & \\
\hline & SP-FeNi5 & Uniformly $5 \%$ Ni-doped iron & $\sqrt{ }$ & N/A & $\sqrt{ }$ & & $\sqrt{ }$ & \\
\hline & SP-FeNi15 & Uniformly $15 \%$ Ni-doped iron & $\sqrt{ }$ & $\mathrm{N} / \mathrm{A}$ & $\sqrt{ }$ & & $\sqrt{ }$ & \\
\hline
\end{tabular}

\section{Section 1. Metal-doped iron produced by solution process}

\section{Synthesis of metal-doped irons}

- Synthesis of metal-doped irons was performed in an anoxic chamber using deoxygenated Milli-Q water at $22 \pm 2{ }^{\circ} \mathrm{C}$.

- Dry Toda iron was used as a starting material.

- Toda iron was washed with deoxygenated Milli-Q water or $0.01 \mathrm{~N} \mathrm{H}_{2} \mathrm{SO}_{4}$ solution before metal doping process

- Metal plating solutions were prepared by dissolving metal sulfates in $0.01 \mathrm{~N} \mathrm{H}_{2} \mathrm{SO}_{4}$ solution. The initial concentrations of metal solution were ranged from 0.6 to $10 \mu \mathrm{M}$.

- After doping, particles were flash dried using acetone.

- Sample description 
PNNL-16185

\begin{tabular}{|l|l|}
\hline ID & Description \\
\hline C-M & Toda iron washed using Milli-Q water (sonication) \\
\hline C-A & Toda iron washed using $0.01 \mathrm{~N}_{2} \mathrm{SO}_{4}$ solution \\
\hline S-M-Cu-10 & Toda iron doped with $\mathrm{Cu}$ (high concentration, $\mathrm{CuSO}_{4}$ ) after Milli-Q water washing \\
\hline S-A-Cu-10 & Toda iron doped with $\mathrm{Cu}$ (high concentration) after acid washing \\
\hline S-M-Cu-1 & Toda iron doped with $\mathrm{Cu}$ (low concentration) after Milli-Q water washing \\
\hline S-A-Cu-1 & Toda iron doped with $\mathrm{Cu}$ (high concentration) after acid washing \\
\hline S-M-Ni-10 & Toda iron doped with $\mathrm{Ni}$ (high concentration, $\mathrm{NiSO}_{4}$ ) after Milli-Q water washing \\
\hline S-A-Ni-10 & Toda iron doped with $\mathrm{Ni}$ (high concentration) after acid washing \\
\hline S-M-Ni-1 & Toda iron doped with $\mathrm{Ni}$ (low concentration) after Milli-Q water washing \\
\hline S-A-Ni-1 & Toda iron doped with $\mathrm{Ni}$ (low concentration) after acid washing \\
\hline S-M-Pd-1.5 & Toda iron doped with Pd (high concentration, PdSO ${ }_{4}$ ) after Milli-Q water washing \\
\hline S-A-Pd-1.5 & Toda iron doped with Pd (high concentration) after acid washing \\
\hline
\end{tabular}

\section{Materials Characterization}

- The materials were characterized using inductively coupled plasma (ICP), X-ray diffraction (XRD), and transmission electron microscopy (TEM). X-ray photoelectron spectroscopy (XPS) analysis is not scheduled yet.

- $\quad$ ICP samples were prepared by dissolving the solid particles in a solution of 3.6 $\% \mathrm{HNO}_{3}$ and $5.3 \% \mathrm{HCl}$, followed by dilution in Milli-Q water. The diluted solutions were analyzed for $\mathrm{Fe}, \mathrm{Pd}, \mathrm{Ni}, \mathrm{Cu}$, and $\mathrm{S}$ using Perkin-Elemer Optima 3000DV.

- XRD was performed using a PANalytical X'pert MPD diffractometer equipped with an X'Celeator detector and cupper source. Each sample was protected from oxygen by applying a few drops of $50 \%$ glycerol and $50 \%$ methanol. Data was collected over the range of $5-80^{\circ} 2 \theta \alpha$ at a scan rate of $2 \%$ min.

- High-resolution TEM was performed using a JEOL JEM 2010F operated at 200 $\mathrm{kV}$. All images were digitally collected using CCD camera and were analyzed using Gatan Digital Micrograph 3.3.1. Samples were mounted on carbon-coated TEM grids which were then mounted on airtight TEM sample holder in the anoxic glove box for minimizing oxygen exposure during transfer from the anoxic chamber to the microscope.

\section{Results and Discussion ICP Analysis}

- The amount of metal present in the final solid was low in comparison with materials synthesized by other processes (hydrogen reduction and sputtering process) This is because of high surface area of dry Toda iron and low solubility of metals in $0.01 \mathrm{~N} \mathrm{H}_{2} \mathrm{SO}_{4}$.

- The amount of metal deposited on iron is below monolayer (0.02ML $~ 0.16 \mathrm{ML})$ 
Table 1. Metal content onto dry Toda iron

\begin{tabular}{|c|c|c|c|c|c|}
\hline $\begin{array}{c}\text { Metal } \\
\text { Additive }\end{array}$ & ID & $\begin{array}{c}\text { Plating Solution } \\
\text { Concentration }^{a} \\
(\mathrm{mM})\end{array}$ & $\begin{array}{c}\text { Deposited } \\
\text { Metal Mass }^{\mathrm{b}} \\
(\mu \mathrm{moles} / \mathrm{g} \mathrm{Fe})\end{array}$ & $\begin{array}{c}\text { Deposited } \\
\text { Metal Content }^{\mathrm{C}} \\
(\mathrm{mol} \%) \\
\end{array}$ & $\begin{array}{c}\text { Relative } \\
\text { concentration to } \\
\text { monolayer(ML) }\end{array}$ \\
\hline \multirow{4}{*}{ Copper } & S-M-Cu-1 & 1.40 & 17.4 & 0.17 & $0.04 \mathrm{ML}$ \\
\hline & S-M-Cu-10 & 7.20 & 89.6 & 0.62 & $0.13 \mathrm{ML}$ \\
\hline & $\mathrm{S}-\mathrm{A}-\mathrm{Cu}-1$ & 1.40 & 17.4 & 0.12 & $0.03 \mathrm{ML}$ \\
\hline & S-A-Cu-10 & 7.20 & 89.6 & 0.75 & $0.16 \mathrm{ML}$ \\
\hline \multirow{4}{*}{ Nickel } & S-M-Ni-1 & 2.02 & 18.7 & 0.12 & $0.02 \mathrm{ML}$ \\
\hline & S-M-Ni-10 & 10.19 & 62.9 & 0.43 & $0.09 \mathrm{ML}$ \\
\hline & S-A-Ni-1 & 2.02 & 20.8 & 0.19 & $0.04 \mathrm{ML}$ \\
\hline & S-A-Ni-10 & 10.19 & 70.0 & 0.62 & $0.12 \mathrm{ML}$ \\
\hline \multirow{2}{*}{ Palladium } & S-M-Pd-1.5 & 0.58 & 7.23 & 0.077 & $0.02 \mathrm{ML}$ \\
\hline & S-A-Pd-1.5 & 0.58 & 7.22 & 0.071 & $0.02 \mathrm{ML}$ \\
\hline
\end{tabular}

a. Aqueous metal concentration used in doping process

b. Deposited additive mass resulting from displacement plating

$=\frac{\text { Initial Plating Solution Concentration }- \text { Plating Solution Concentration after Displacement }}{\text { Toda Iron Mass }} \times$ Volume of Plating Solution

c. Content of metal present in each sample determined by dissolving the solid particles in a solution of $3.6 \% \mathrm{HNO}_{3}$ and $5.3 \% \mathrm{HCl}$, followed by ICP analysis

d. Amount of deposited atom equivalent to a single monolayer was calculated: $4.76 \mathrm{~mol} \% \mathrm{Cu} ; 5.00$ mol \% Ni; and 4.10 mol \% Pd. (See Appendix)

$X R D$

- XRD patterns show that the materials are composed of magnetite and iron. In addition, no significant difference in the degree of crystallinity, which would be detected by changes in peak broading and relative intensity, were apparent between samples. (Figure 1)

- According to peak areas of iron (220) face and magnetite (440) face, the ratio of magnetite/iron in metal-doped irons are usually lower than that in untreated Toda iron (Table 2). A possible explanation is that iron oxide may be dissolved more than metal iron by washing process because metal iron was protected from iron oxide shell. This led to change ratio of magnetite to iron.

- No significant difference was found in XRD patterns between Milli-Q water washing and acid washing.

- Glycerol coating did not make a difference for most of samples except for three samples (written in red in Table 2). Those three samples caught a fire while transferred to sample holder, suggesting they are much more likely to start reacting in air in comparison with the undoped material. 


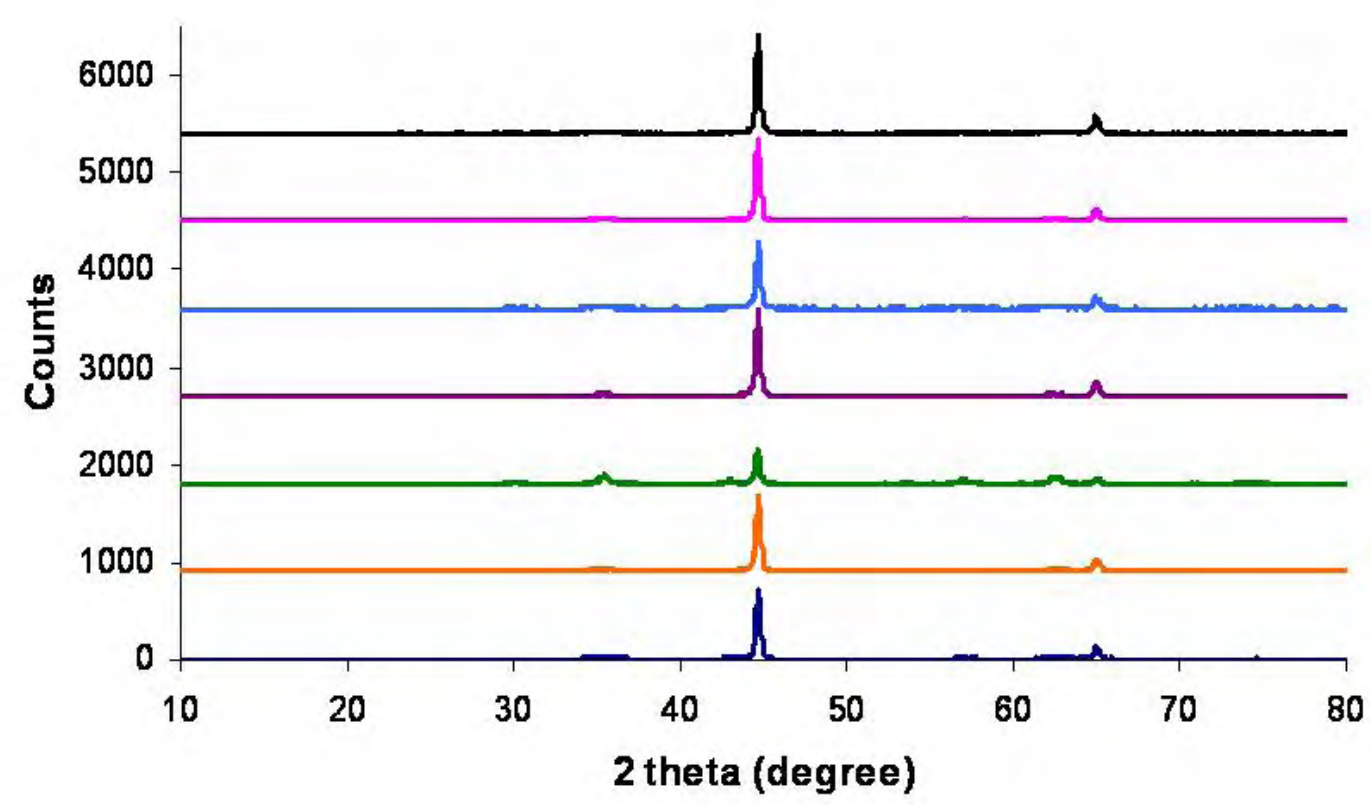

DT-UT

C-M

S-M-C

S-M-N

C-A

S-A-Cl

S-A-Ni

Figure 1. XRD patterns of the metal-doped irons.

Table 2. Ratio of Magnetite/Fe

\begin{tabular}{|c|c|c|c|c|c|}
\hline \multirow{2}{*}{ ID } & \multicolumn{2}{|c|}{ Milli-Q water washing } & \multirow{2}{*}{ ID } & \multicolumn{2}{|c|}{ Acid washing } \\
\hline & $\mathrm{Mag} / \mathrm{Fe}^{\mathrm{a}}$ & Fe as Mag ${ }^{b}$ & & Mag/Fe & Fe as Mag. \\
\hline C-M & 0.28 & 0.12 & C-A & 0.62 & 0.23 \\
\hline S-M-Cu-1 & 0.16 & 0.07 & S-A-Cu-1 & 0.24 & 0.11 \\
\hline S-M-Cu-10 & 0.24 & 0.11 & S-A-Cu-10 & 0.20 & 0.09 \\
\hline S-M-Ni-1 & 0.25 & 0.11 & S-A-Ni-1 & 0.31 & 0.13 \\
\hline S-M-Ni-10 & 0.23 & 0.10 & S-A-Ni-10 & 0.26 & 0.11 \\
\hline S-M-Pd-1.5 & 0.64 & 0.24 & S-A-Pd-1.5 & 0.33 & 0.14 \\
\hline
\end{tabular}

${ }^{\mathrm{a}}$ Ratio of magnetite/Fe calculated using XRD peak area (Untreated Dry Toda iron: 0.40 )

${ }^{\mathrm{b}}$ Fraction of Fe atom as magnetite phase(Untreated Dry Toda iron: 0.28)

\section{TEM Images}

- Metal-doped Toda irons consist of aggregation of small irregular particles which consist of a single crystal $\mathrm{Fe}^{0}$ core with an oxide shell in a similar to untreated Toda iron.

- However, oxide shells of metal-doped Toda irons became rougher and thicker in comparison of those of untreated one. Such changes are due to washing process given that metal-doped irons have similar morphology with Toda iron solely after washing process (Figure S1). Acid washing makes more dramatic change on morphology than Milli-Q washing.

- TEM images of metal-doped irons do not show the location where metals are deposited on iron surfaces, suggesting metal dopants may be incorporated with iron oxide shell during plating process. 

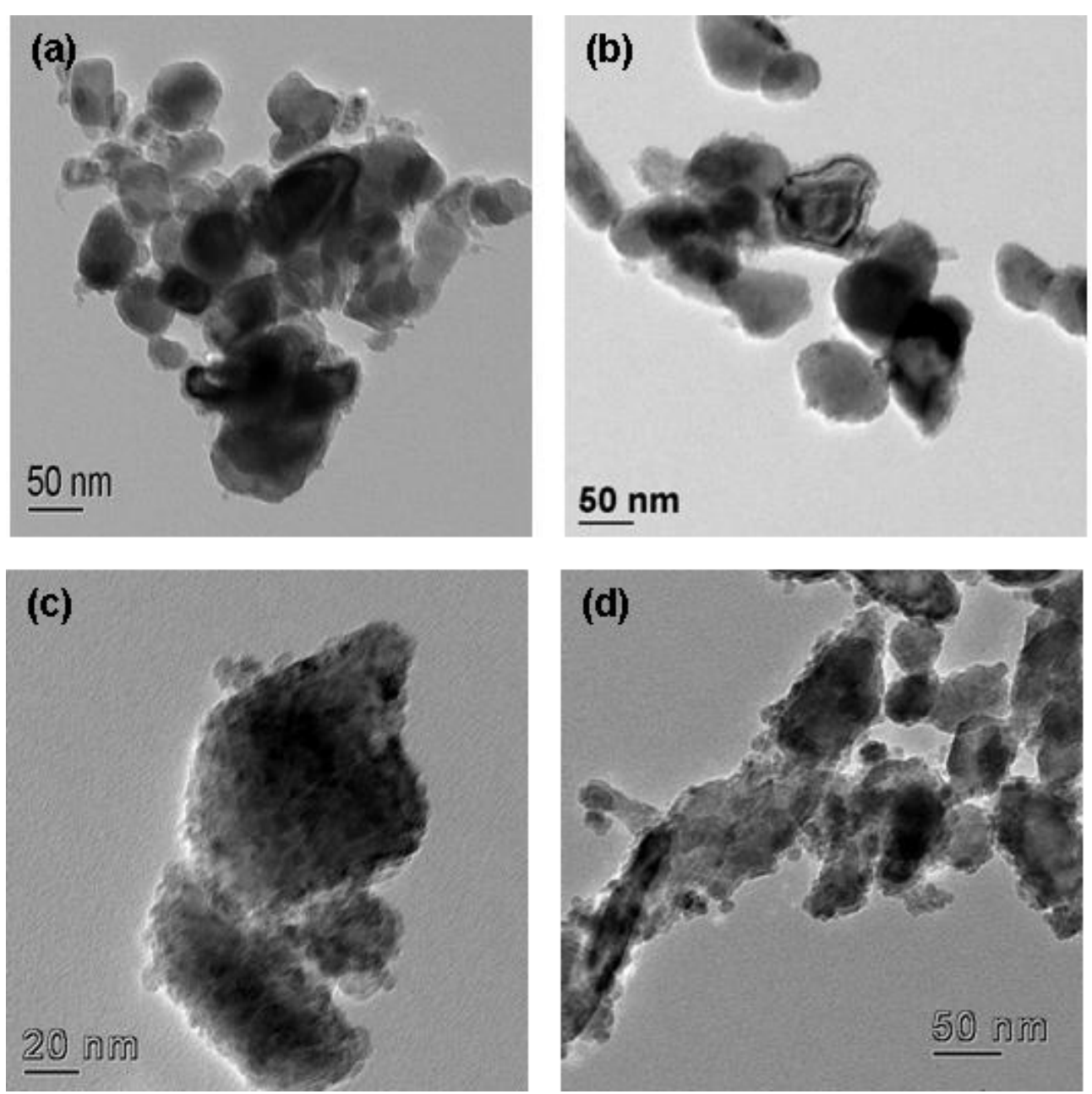

Figure 2. TEM images of (a) untreated dry Toda iron, (b) $0.43 \%$ Ni-doped Toda iron after Milli-Q water washing (S-M-Ni-10), (c) $0.62 \%$ Ni-doped Toda iron after acid washing (S-A-Ni-10), and (d) $0.75 \%$ Cu-doped Toda iron after acid washing (S-A-Cu10)

\section{Reactivity Test}

- In order to test reactivity of metal-doped iron, kinetic experiments were performed using 4-chloro-1-nitrobenzene (4-Cl-NB). 4-Cl-NB has been used as a model compound of insecticide and herbicide. It can be reduced to 4-chloroaniline (4-Cl-An) in the presence of reductants such as reduced iron or iron oxides.

- Batch experiments were carried out in $74 \mathrm{~mL}$ serum bottles containing a $0.4 \mathrm{~g} / \mathrm{L}$ mineral suspension buffered at $\mathrm{pH} 7$ with deoxygenated $25 \mathrm{mM}$ bistripropane (BTP). Initial concentration of 4-Cl-NB was $100 \mu \mathrm{M}$. Samples were withdrawn at selected intervals using a two-syringe technique involving simultaneous injection of deoxygenated BTP buffer to avoid the introduction of headspace. The samples were filtered though $0.2 \mu \mathrm{m}$ PTFE Gelman Acrodisk syringe tip filter to stop the reaction and to remove mineral particles.

- 4-Cl-NB and 4-Cl-An were analyzed using High Pressure Liquid Chromatography (Agilent 1100 Series) equipped with a C18 column (Model?). 
The injection volume was $20 \mu \mathrm{L}$ and the detector wavelength was 245 and $276 \mathrm{~nm}$ for 4-Cl-NB and 4-Cl-An, respectively. The mobile phase was $5 \mathrm{mM} \mathrm{pH}$ 7 phosphate buffer/acetonitrile $(2 / 3 \mathrm{v} / \mathrm{v})$ at a flow rate of $1 \mathrm{~mL} / \mathrm{min}$.

- Figure 3 shows that all metal dopants $(\mathrm{Cu}, \mathrm{Ni}$, and $\mathrm{Pd})$ promoted reduction rates of 4-Cl-NB. Cu-doped iron (S-M-Cu-1) have higher reactivity than Ni-doped one (S-M-Ni-1) even though they have similar deposited metal mass of $\sim 18 \mu$ moles/g Fe (Note that this observation is different from results obtained by David Cwiertny). Pd-doped iron (S-M-Pd-1.5) has relatively low deposited metal mass ( $7 \mu$ moles/g Fe) but its reactivity is similar with $\mathrm{Ni}$-doped ones.

- Similar reactivity between S-M-Cu-1 (17.4 $\mu$ moles/g Fe) and S-M-Cu-10 (89.6 $\mu$ moles/g Fe) reveals that increasing the mass of $\mathrm{Cu}$ deposited on the dry Toda iron does not affect reactivity. Likewise, it is true for Ni-dopes irons (S-MNi-1 and S-M-Ni-10).
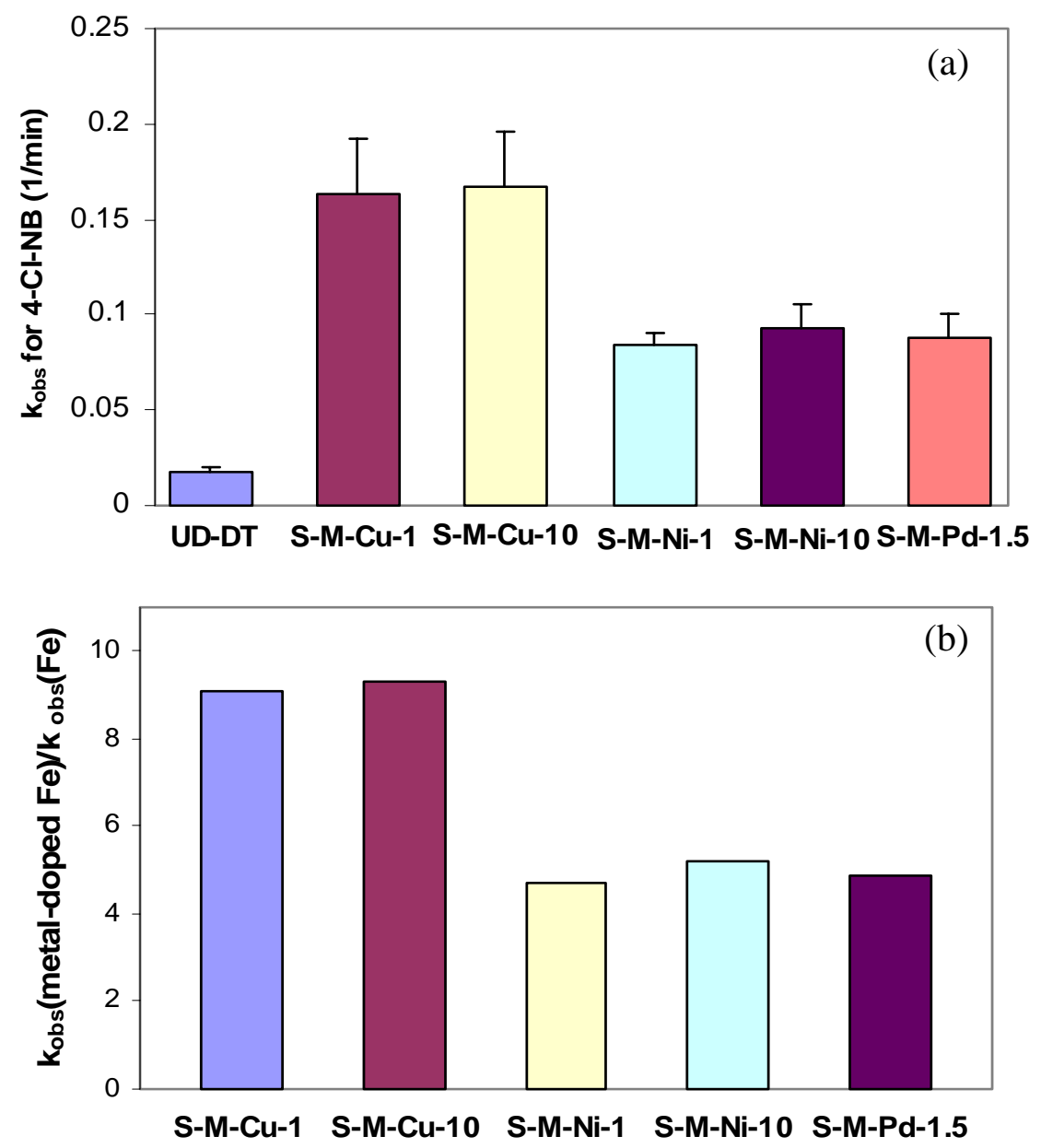

Figure 3. (a) Pseudo-first-order-reduction rate constants of 4-Cl-NB in the presence of dry Toda iron or metal-doped dry Toda irons and (b) rate enhancement provided by metal-doping 
PNNL-16185

\section{Iron Corrosion Test}

- Aqueous Fe concentration generated over time was measured for the rate of iron corrosion in metal-doped iron/water system.

- Batch reactors were prepared in the similar manner to reactivity experiment.

Reactors were filled with deoxygenated $25 \mathrm{mM}$ BTP buffer (pH 7) and a mineral mass loading of $2.5 \mathrm{~g} / \mathrm{L}$. Multiple, identical batch reactors were prepared for each sample and reaction vials were scarified at certain time interval. Iron concentration was analyzed using ICP (Perkin-Elemer Optima 3000DV)

- There is no significant difference on both the amount of Fe(II) generated over $24 \mathrm{~h}$ and corrosion rate among metal-doped dry Toda irons

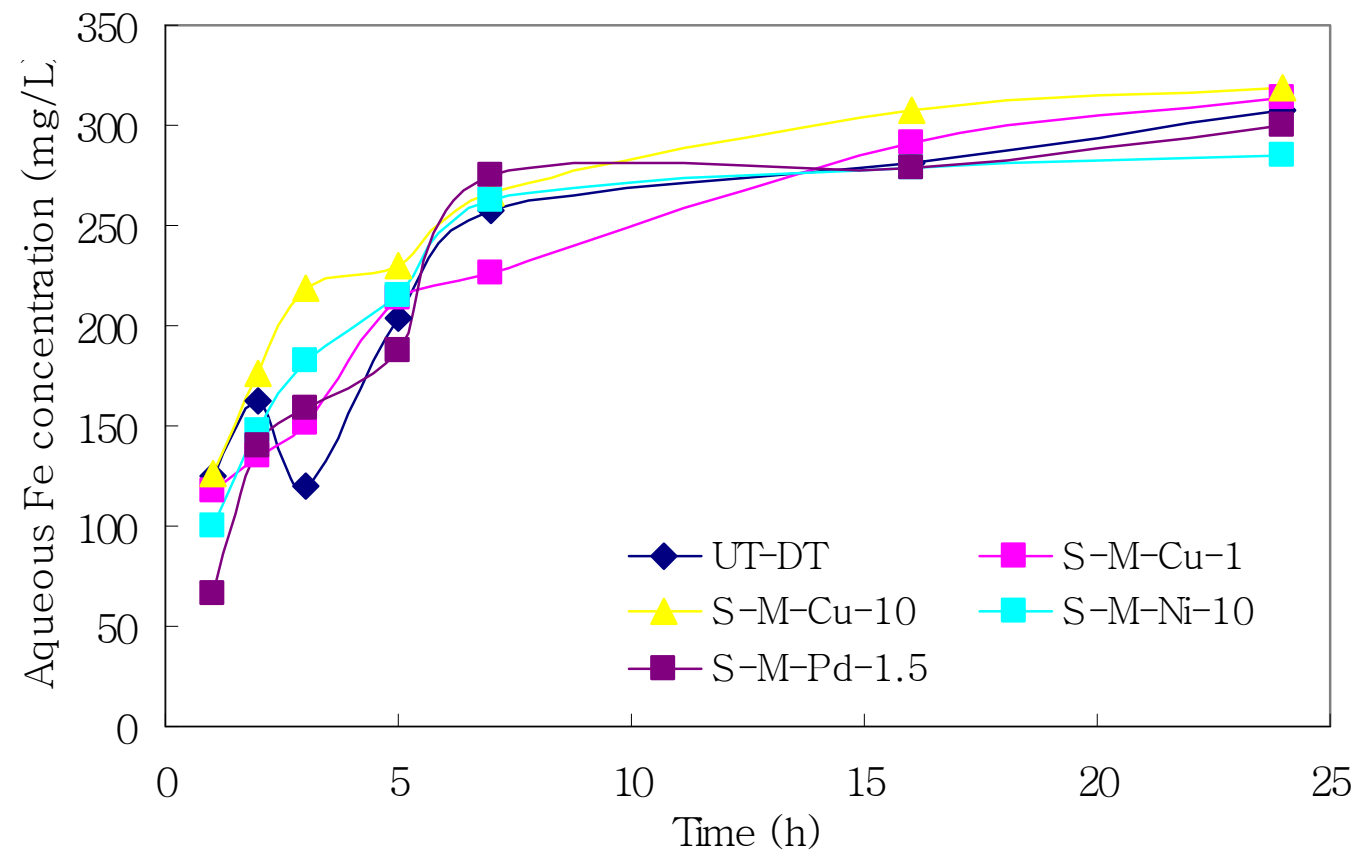

Figure 4. Aqueous Fe concentration as a function of time in metal-doped dry Toda iron/water batch system 
PNNL-16185

\section{Appendix}

\section{A Single Monolayer Equivalent of Metal Atoms}

Assumption

- The metal layer is dominantly in the lowest energy fcc (111) orientation

- BET surface area of Toda iron (SA) $=29 \mathrm{~m}^{2} / \mathrm{g}$

$\mathrm{FCC}(111)$ area $=$ equilateral triangle with the side equal to $4 \mathrm{r}$

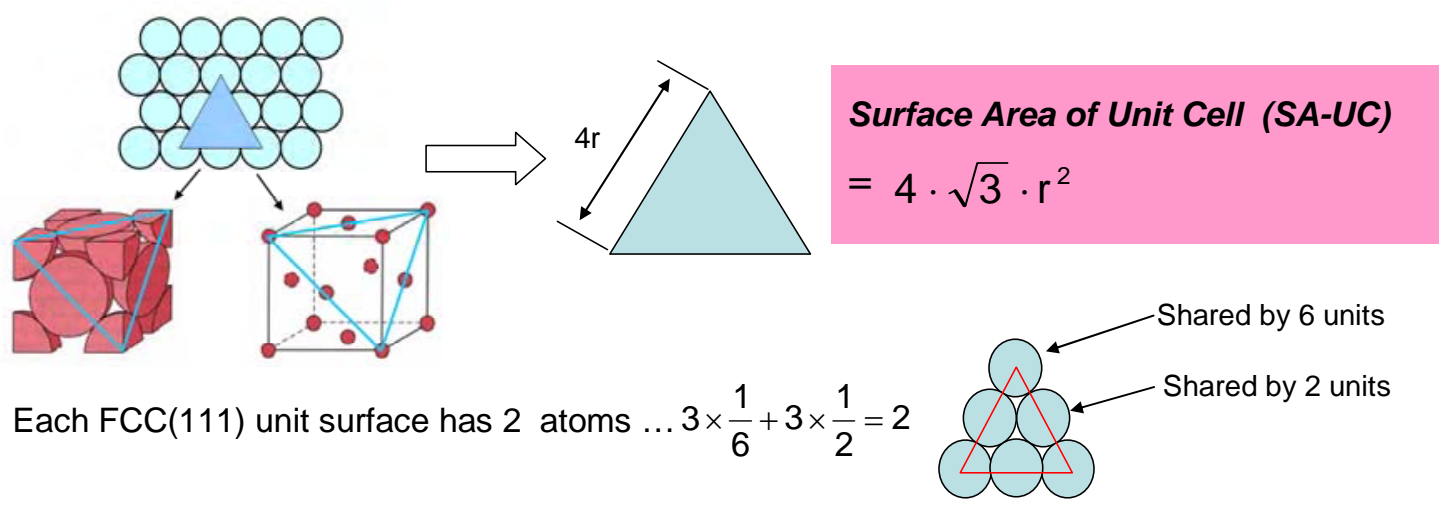

\section{A Single Monolayer Equivalent of Metal Atoms}

$$
=\frac{\mathrm{SA} \mathrm{m}}{2}\left|\frac{\text { unit cell }}{\mathrm{g}-\mathrm{Fe}}\right| \frac{2 \text { atoms }}{\mathrm{SA}-\mathrm{UCm}^{2}}\left|\frac{\mathrm{mol}-\text { metal }}{\text { unit cell }}\right| \frac{6.02 \times 10^{23} \text { atoms }}{\text { uto }}
$$

\begin{tabular}{|c|c|c|c|}
\hline Surface area of Toda iron $\left(\mathrm{m}^{2} / \mathrm{g}\right)$ & 29 & & \\
\hline Dopant & $\mathrm{Ni}$ & $\mathrm{Cu}$ & $\mathrm{Pd}$ \\
\hline Atomic mass & 58.7 & 63.5 & 106.4 \\
\hline Density of Atom $(\mathrm{g} / \mathrm{ml})$ & 8.9 & 8.96 & 12 \\
\hline Radius of Atom (nm) & 0.125 & 0.128 & 0.138 \\
\hline Surface area of Unit Cell $\left(\mathrm{m}^{2}\right)$ & $1.076 \mathrm{E}-19$ & $1.129 \mathrm{E}-19$ & $1.311 \mathrm{E}-19$ \\
\hline Metal mass for monolayer $(\mu \mathrm{mol} / \mathrm{g})$ & 895.007 & 853.129 & 734.770 \\
\hline Metal/Fe for monolayer (mol \%) & 5.00 & 4.76 & 4.10 \\
\hline
\end{tabular}
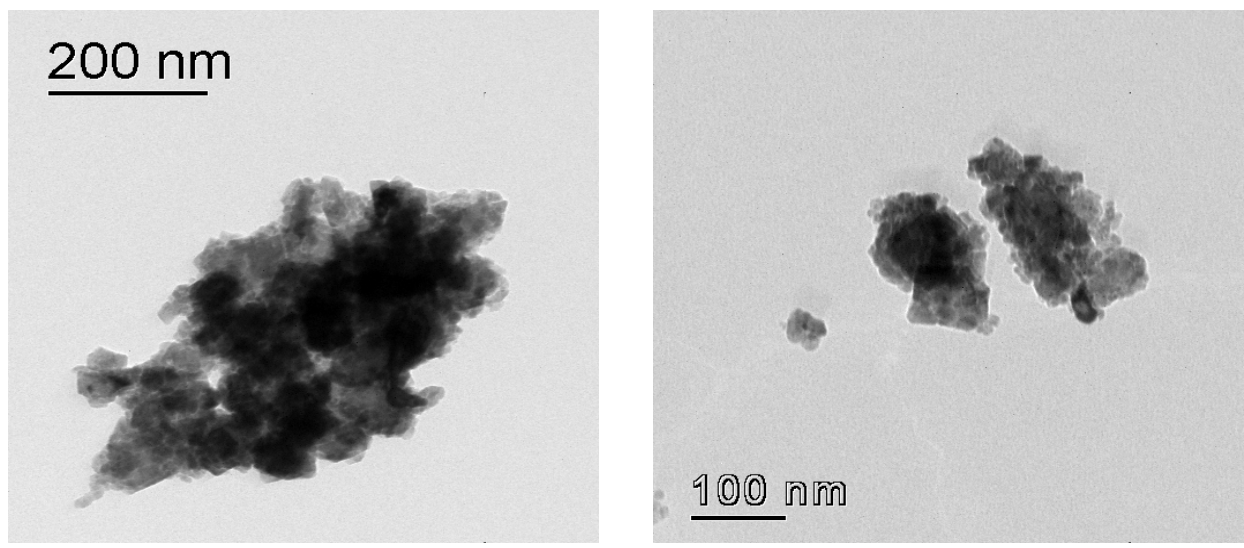

Figure S1. TEM images of dry Toda iron after acid washing 
PNNL-16185

\section{Section 2. Pd-doped iron produced by hydrogen reduction}

\section{Synthesis of Pd-doped Iron}

- Ferrihydrite nanoparticles were prepared using a method adopted from Matson et al. (1994)

- The dried ferrihydrite was heated at $330{ }^{\circ} \mathrm{C}$ in air for 30 minutes to form hematite.

- Sulfate was added to hematite in the form of $\mathrm{H}_{2} \mathrm{SO}_{4}$ (Water content is $10 \mathrm{l} / \mathrm{g}$ )

- Four samples of hematite with various amounts of palladium doping $(0,0.5,1.0$, and $2.0 \%$ moles $\mathrm{Pd} / \mathrm{moles} \mathrm{Fe}$ ) were prepared by adding a palladium(II) acetate dissolved in acetone.

- Pd-doped hematite was heated in a flow of $\mathrm{H}_{2}(10 \mathrm{ml} / \mathrm{min})$ at $450{ }^{\circ} \mathrm{C}$ for 2 hours.

- Sample description

\begin{tabular}{lll}
\hline Sample ID & Dean’s label & Description \\
\hline HR-Fe & $58160-31-4$ & 0 mol \% palladium-doped iron \\
HR-FePd0.5 & $58160-31-1$ & 0.5 mol \% palladium-doped iron \\
HR-FePd1 & $58160-31-2$ & 1 mol \% palladium-doped iron \\
HR-FePd2 & $58160-31-3$ & 2 mol \% palladium-doped iron \\
Ferrihydrite & $52654-90-1$ & Stating material \\
Hematite & - & Oxidized material of ferrihydrite (52654-90-1) \\
\hline
\end{tabular}

Materials Characterization

- The materials were characterized using inductively coupled plasma (ICP), X-ray diffraction (XRD), and transmission electron microscopy (TEM). X-ray photoelectron spectroscopy (XPS) analysis is not scheduled yet.

- $\quad$ ICP samples were prepared by dissolving the solid particles in a solution of 3.6 $\% \mathrm{HNO}_{3}$ and $5.3 \% \mathrm{HCl}$, followed by dilution in Milli-Q water. The diluted solutions were analyzed for $\mathrm{Fe}, \mathrm{Pd}, \mathrm{Ni}, \mathrm{Cu}$, and $\mathrm{S}$ using Perkin-Elmer Optima 3000DV.

- XRD was performed using a PANalytical X'pert MPD diffractometer equipped with an X'Celeator detector and cupper source. Each sample was protected from oxygen by applying a few drops of $50 \%$ glycerol and $50 \%$ methanol. Data was collected over the range of $5-80^{\circ} 2 \theta \alpha$ at a scan rate of $2 \% \mathrm{~min}$.

- High-resolution TEM was performed using a JEOL JEM 2010F operated at 200 $\mathrm{kV}$. All images were digitally collected using CCD camera and were analyzed using Gatan Digital Micrograph 3.3.1. Samples were mounted on carbon-coated TEM grids which were then mounted on airtight TEM sample holder in the anoxic glove box for minimizing oxygen exposure during transfer from the anoxic chamber to the microscope. 
PNNL-16185

\section{Results and Discussion}

XRD Analysis

- XRD patterns show that the materials are composed of magnetite and iron. In addition, no significant difference in the degree of crystallinity, which would be detected by changes in peak broadening and relative intensity, were apparent between samples. (Figure 1)

- According to peak areas of iron (220) face and magnetite (440), the average ratio of magnetite/iron is 1.10 ( 0.40 for Toda iron) and the fraction of Fe atom in magnetite phase is 0.35.(0.28 for Toda iron)

- Unidentified peaks in the pattern of HR-Fe indicate the presence of other phases but those peaks do not match with peaks of possible iron oxides.

- XRD patterns of starting materials are shown in Fig S1.

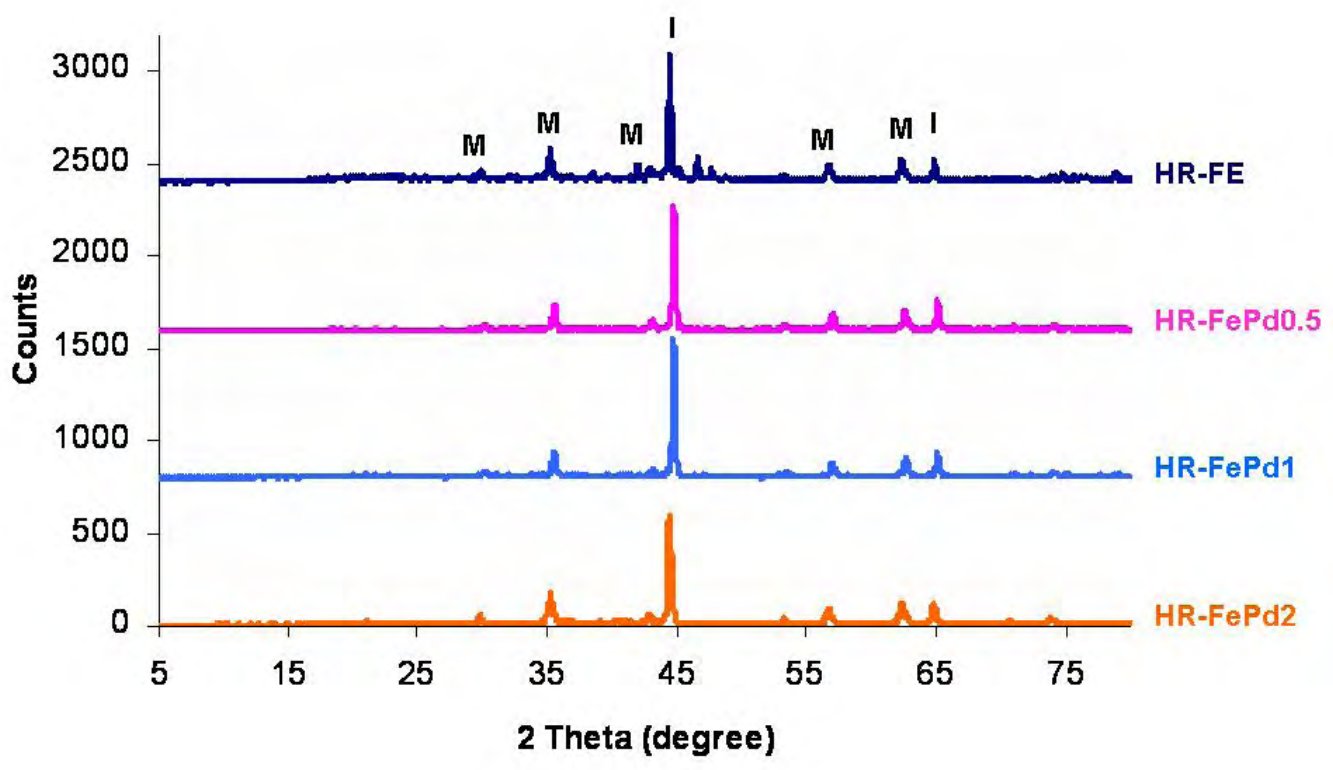

Figure 1. XRD patterns of the $0 \%, 0.5 \%, 1 \%$, and $2 \%$ palladium-doped iron. I and M are used to label iron and magnetite peaks, respectively.

\section{Composition of Pd-doped Iron}

- ICP analysis shows that the amount of palladium present in each of the samples was consistent with the percentage of palladium in the hematite. The concentrations of other metals such as $\mathrm{Cu}$, and $\mathrm{Ni}$ were found to be trace $(\mathrm{Cu} / \mathrm{Fe}$ and $\mathrm{Ni} / \mathrm{Fe}$ are less than $0.01 \mathrm{~mol} \%)$. Sulfur concentration is below the detection limit.

- Quantitative EDX analysis shows that the atomic ratios of Pd/Fe are lower than ICP results.

- Pd doping does not make a difference in the ratio of magnetite/iron. 
Table 1. Summary of Composition

\begin{tabular}{lcccc}
\hline ID & $\begin{array}{c}\text { Pd Content }^{\mathrm{a}} \\
(\mathrm{mol} \mathrm{\% )}\end{array}$ & $\begin{array}{c}\text { Pd Content }^{\mathrm{b}} \\
\text { (atomic \%) }^{\mathrm{e}}\end{array}$ & Magnetite/Fe $^{\mathrm{c}}$ & $\begin{array}{c}\text { Fe as }^{\text {Magnetite }} \\
\text { M }^{\mathrm{d}}\end{array}$ \\
\hline HR-Fe & 0.08 & $\mathrm{ND}^{\mathrm{e}}$ & 1.26 & 0.38 \\
HR-FePd0.5 & 0.44 & 0.23 & 0.86 & 0.30 \\
HR-FePd1 & 1.10 & 0.74 & 1.02 & 0.34 \\
HR-FePd2 & 1.95 & 1.13 & 1.24 & 0.38 \\
\hline
\end{tabular}

${ }^{\mathrm{a}}$ Values were determined using ICP. ${ }^{\mathrm{b}}$ Values were quantified using TEM-EDX for three different locations. ${ }^{\mathrm{c}}$ Ratios of magnetite/iron were calculated using XRD peak areas; Fe(220) and Magnetite (440). ${ }^{\mathrm{d}}$ Fraction of Fe atoms as in magnetite phase ${ }^{\mathrm{e}}$ Not detectable.

\section{TEM Analysis}

- Figure 1a show that iron without Pd doping consists of aggregated of faceted plates and smaller irregular particles.

- The faceted plates are usually composed of a nearly single crystal $\mathrm{Fe}^{0}$ core with a oxide shell (Figure 1c) but smaller irregular particles do not have oxide shell (Figure $1 \mathrm{~b}$ and $1 \mathrm{~d}$ ).

- Particle size varies from 30 to $100 \mathrm{~nm}$
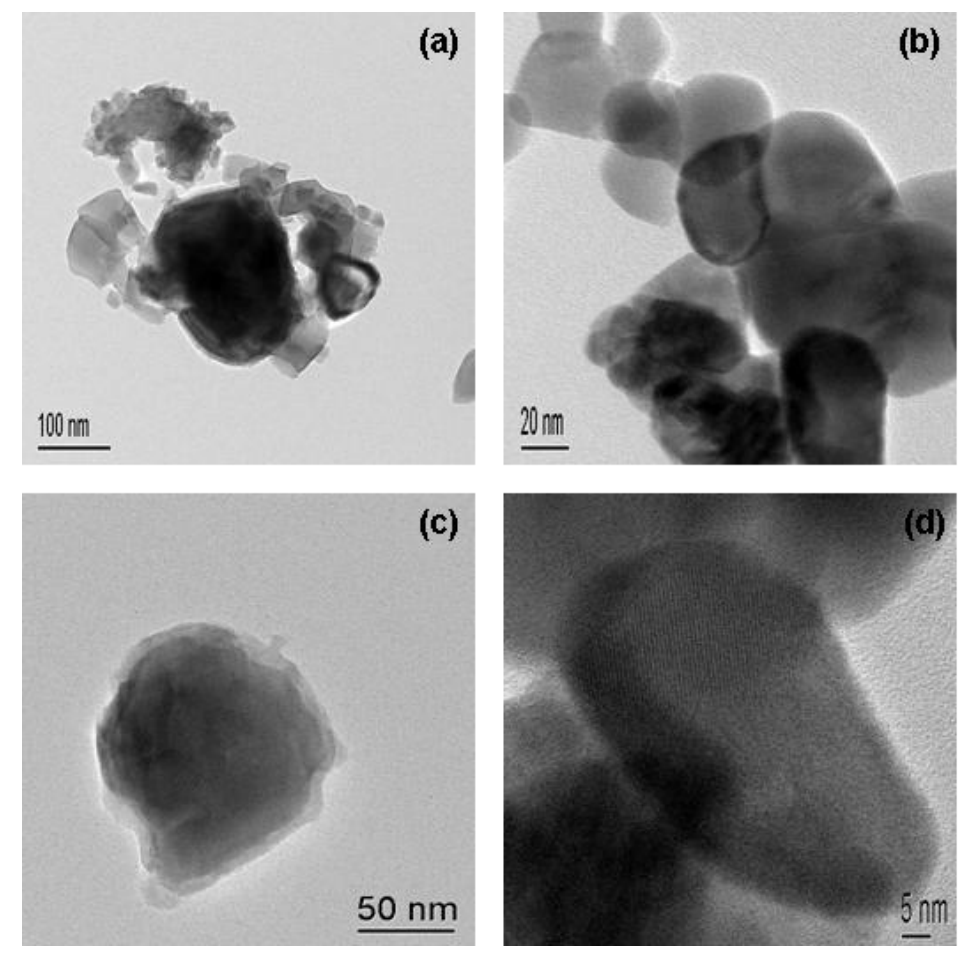

Figure 2. TEM images of iron particles without Pd doping: (a) Low magnification image of iron aggregates, (b) and (d) higher resolution images of irregularly shaped metal iron particles, and (c) higher resolution image of individual iron particle composed of $\mathrm{Fe}^{0}$ core and oxide shell. 
- TEM images of Pd-doped irons shows formation of smaller particles on iron particles. TEM-EDX verifies that such particles are Pd metal. The population of Pd particles increases with Pd loading (Figure 3a, 3b, and 3d). Pd particles are spherical and typically 2-10 $\mathrm{nm}$ in diameter.

- Pd deposition on iron particles is discontinuous and heterogeneous. Even Figure $3 \mathrm{~d}$ and 3e are images of 2\% Pd-doped iron, the region in Figure 3e appears virtually free of deposited Pd but the region in Figure 3d has lots of small Pd particles.

- Pd deposition occurred on iron metal surface as well as on iron oxide shell. However, Pd was deposited predominately on iron oxide shell (Figure 3d and 3f).

- Figure 3c shows that small Pd particles deposited on iron oxide shell also have oxide shell.

- TEM images of starting materials are presented in Figure S2.
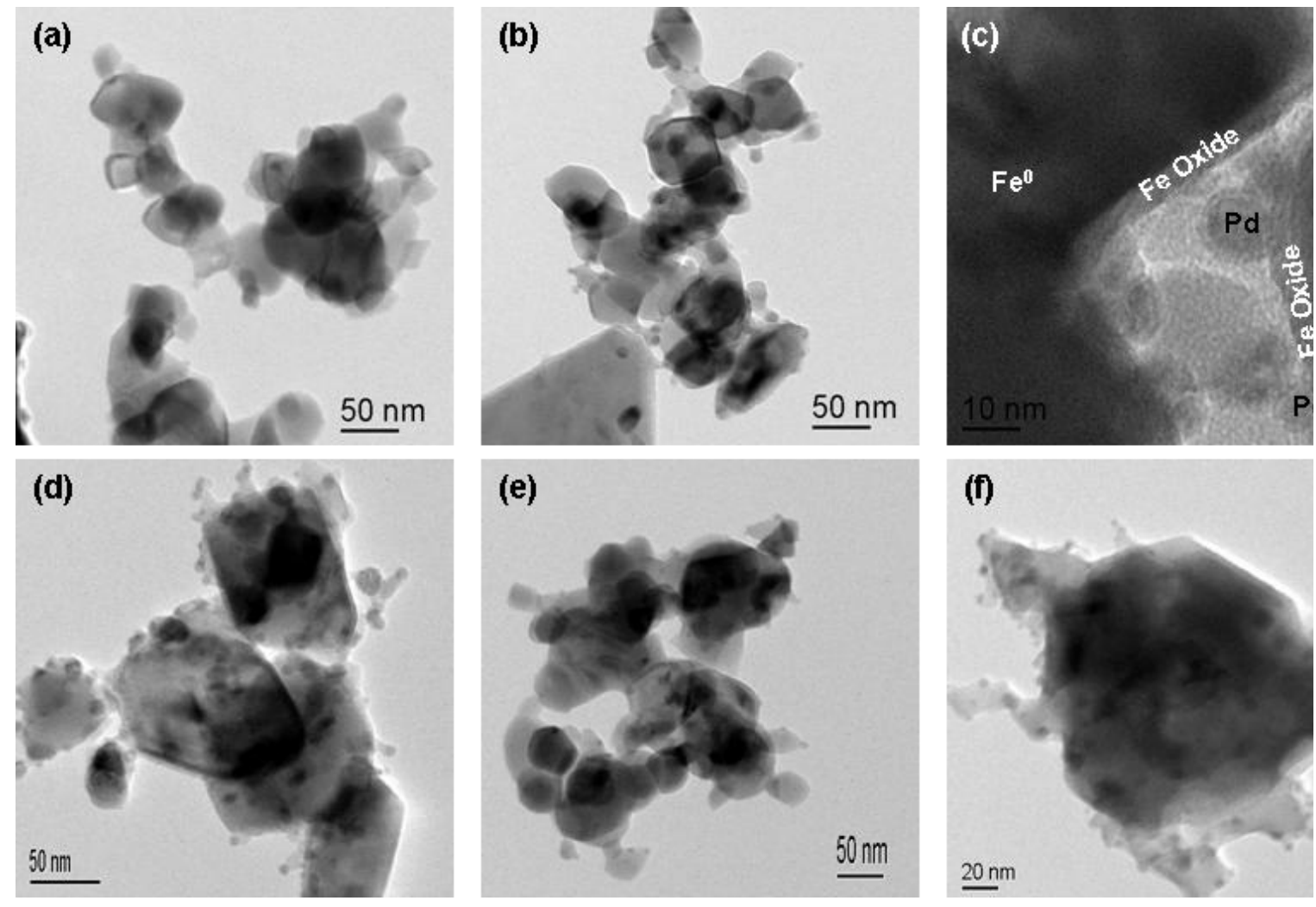

Figure 3. Representative TEM images of Pd-doped iron particles: (a) $0.5 \%$ Pd; (b) and (c) $1 \%$ Pd; (d), (e), and (f) $2 \%$ Pd

\section{Reactivity and Corrosion Test}

- Reactivity was evaluated via kinetic batch experiment using 4-Cl-NB. A detailed description of the 4-Cl-NB batch systems used in this study is included in Section 1. One difference is that initial concentration of 4-Cl-NB was $400 \mu \mathrm{M}$.

- Iron produced by hydrogen process in PSL has much higher reactivity than dry Toda iron. 
- Unlike metal-doped irons produced by solution process, no rate enhancement by metal additive was observed. Due to high reactivity, the observed rate constant is highly mass transfer limited. If that is the case, we cannot see the effect of metal doping on reactivity because the particle size of each sample is similar.
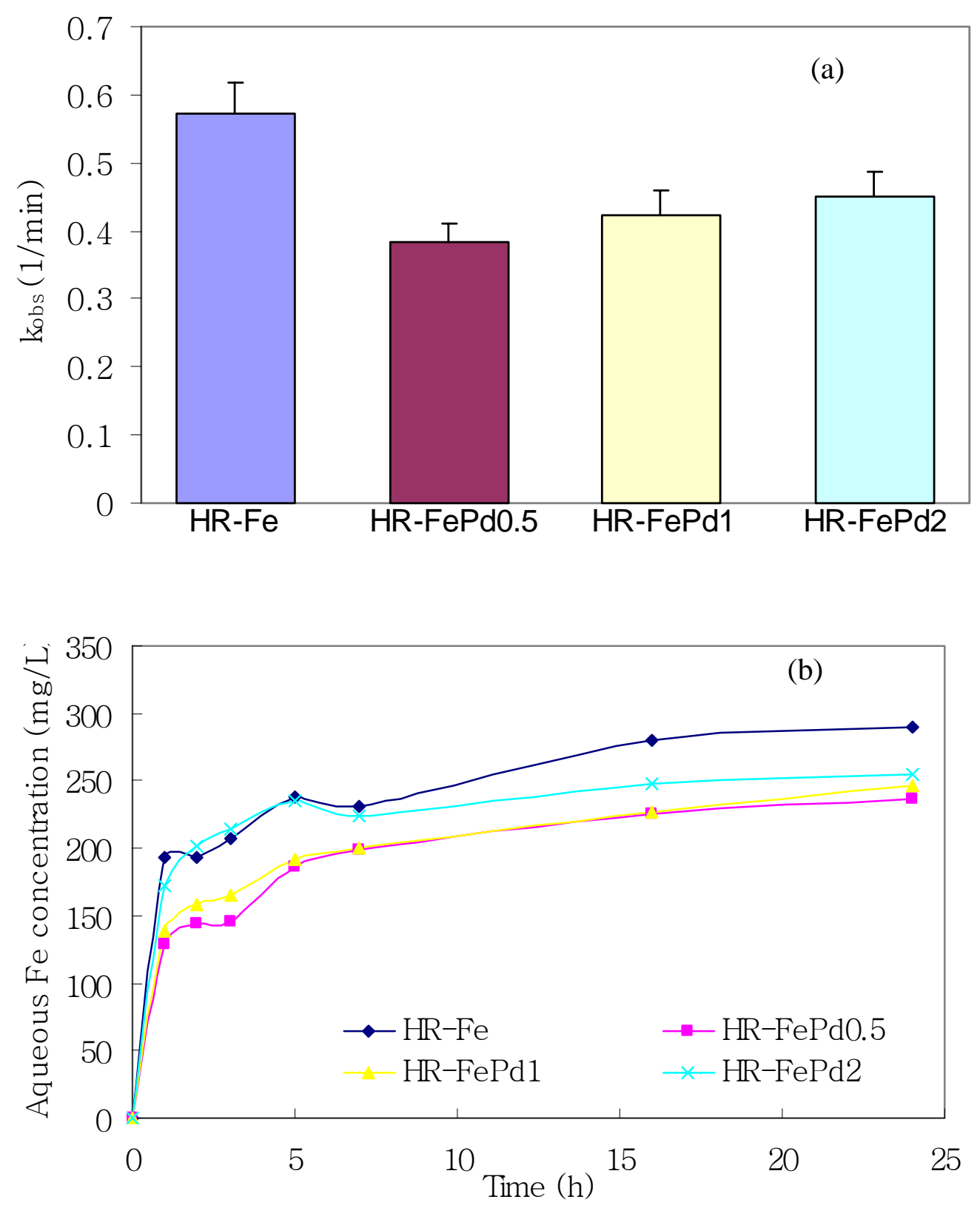

Figure 4. (a) Pseudo-first-order-rate constant of 4-Cl-NB by iron and Pd-doped irons and (b) aqueous Fe concentration as a function of time in iron/water batch systems. 

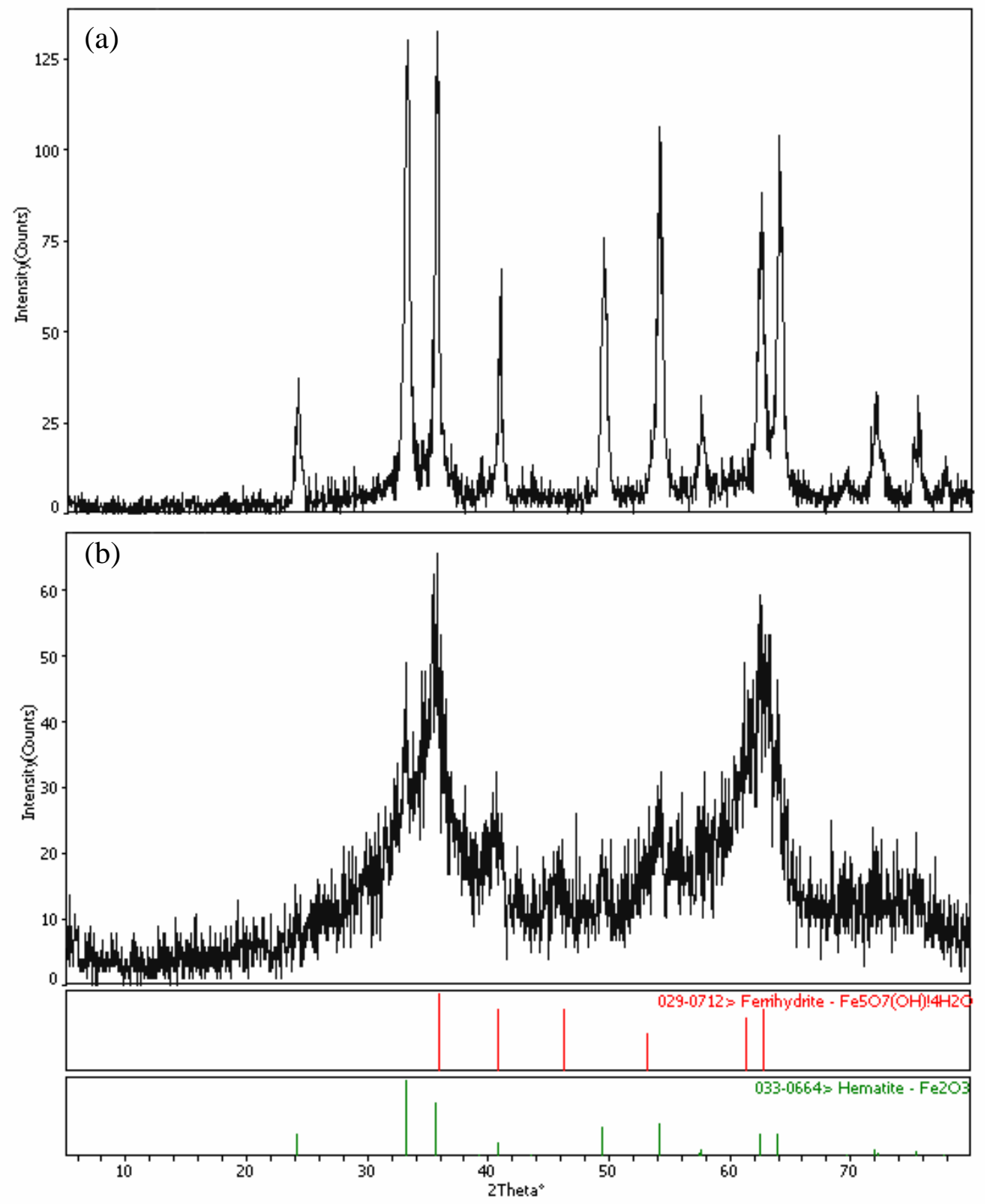

Figure S1. XRD patterns of (a) hematite and (b) ferrihydrite. 

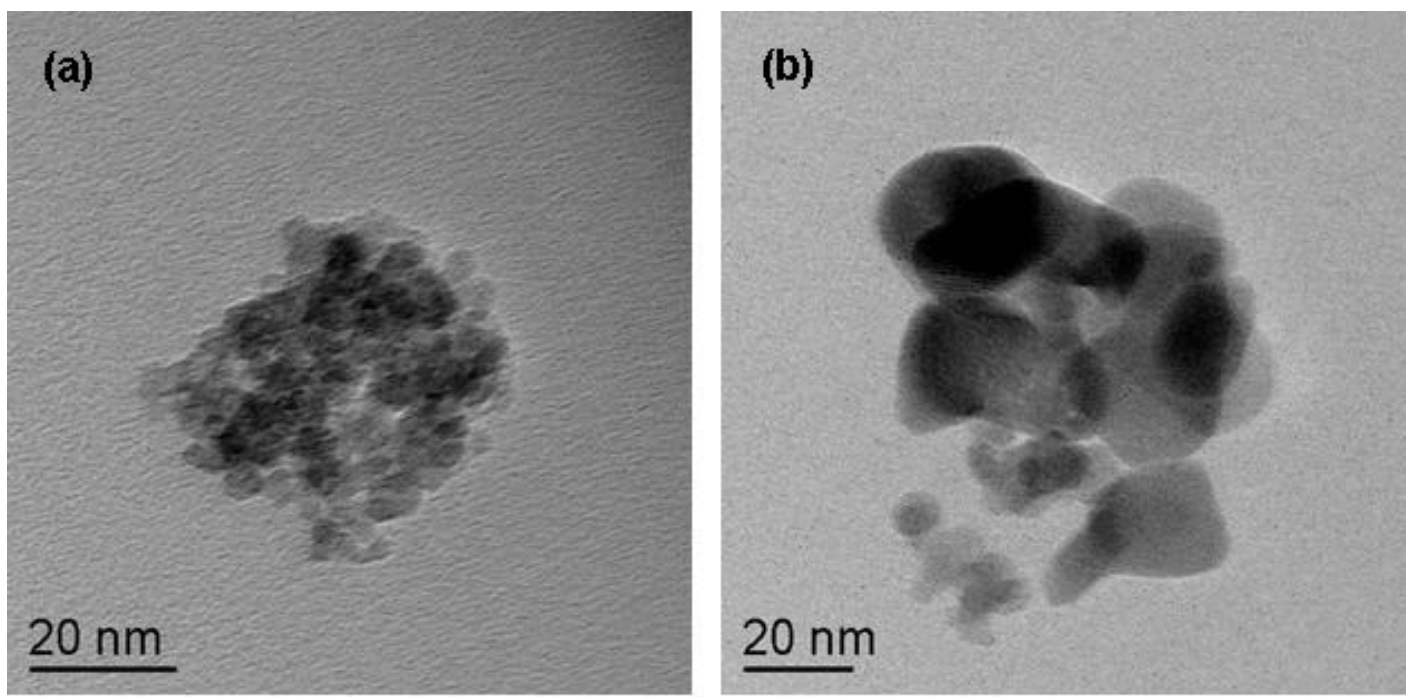

Figure S2. TEM images of (a) ferrihydrite and (b) hematite 
PNNL-16185

\title{
Characterization of Aqueous Particles Internally Mixed With Surfactants
}

\author{
Luis A. Cuadra-Rodriguez and Barney Ellison \\ University of Colorado at Boulder, \\ Department of Chemistry and Biochemistry \\ Boulder, Colorado \\ and \\ Alla Zelenyuk \\ Pacific Northwest National Lab \\ Environmental Molecular Sciences Laboratory \\ Richland, WA
}

In the past few decades aerosols research has gained more attention due to aerosols' climate effect and temperature influence in the atmosphere. Many studies have shown that organic matter composed a significant amount of atmospheric particles. One interesting type of aerosol is the "inverted" micelle that consists of a water core with inorganic salts covered with surfactants. These surfactants matter can affect the chemical and physical properties particles. In this study we studied the effect of surfactants on aqueous particles at low relative humidity at different surfactants concentrations. Size resolved experiments were conducted using SMPS and SPLAT to get mobility and aerodynamic size distributions to get the effective densities of these particles. Both size distributions show that due to surfactants the particles are retaining water and as the surfactant concentration is increased the effective density decreases for a given mobility diameter. Qualitatively this is also confirmed by SEM pictures where as the surfactant concentration is increased more surfactant is associated to a $\mathrm{NaCl}$ particle, which explains the lower effective density. More work need to be done in order to estimate how much water is retained in these types of particles. 
PNNL-16185

\section{Introduction}

Aerosols or particulate matter has gained attention in the last few decades. These can affect climate in different ways. Aerosols can act as cloud condensation nuclei $(\mathrm{CCN})$ that increase the reflectivity of sunlight by clouds, which lead to lower earth temperature. ${ }^{1-2}$ Also, the scattering and absorption of light by fine particles in the atmosphere can increase this cooling effect due to the change in physical and chemical properties of the aerosols. Many field studies have shown that organic compounds are a significant part of the total particulate matter in the atmosphere, accounting between $10 \%-70 \%$ of the fine particulate mass. $^{3}$

Ellison et al. (1999) proposed a mechanism that account for the organic compounds in the aerosols, its reactivity and the effect in its CCN activity by the generation of nascent marine aerosols. ${ }^{4}$ These aerosols are "inverted" micelles composed of a water core and inorganic salts covered with surfactants (hydrophobic tail with a polar head). These surfactants are believed to affect the chemical and physical properties of aqueous aerosols. Recently, Thornton et al.(2006) found that the reactive uptake of $\mathrm{N}_{2} \mathrm{O}_{5}$ by aqueous $\mathrm{NaCl}$ particles coated with sodium dodecyl sulfate is suppressed by a factor of 10 as the surfactant completely cover the aerosol surface. ${ }^{5}$

Even though chemical properties have been studied, almost no study has attempted to study the physical properties of these "inverted" micelles aerosols. In this study we show some physical properties (size distribution, effective density, SEM) for different aqueous aerosols covered with sodium dodecyl sulfate (SDS), sodium oleate (SO) and laurtrimonium chloride (LC). The measurements were done at low relative humidity and suggest that the particles retain water. The amount of water retained still a question that need to be addressed. 
PNNL-16185

\section{Experimental}

Aerosols were generated by aerosolizing aqueous solutions with a TSI atomizer. The aerosol flow was $0.20-0.30 \mathrm{~L} \mathrm{~min}^{-1}$ and was used as the sampling flow to the Scanning Mobility Particle Sizer (SMPS), whose sheath flow was set to $4.0 \mathrm{~L} \mathrm{~min}^{-1}$. All experiments were done at low relative humidity; therefore the SMPS was conditioned with dry air flow before and within measurements. This ensures a low relative humidity environment and cleanliness of the sampling lines. The aerosol flow was passed through two diffusion dryers in series and then into the SMPS. For each solution, mobility diameter size distribution measurements were carried out using the SMPS/CPC system. Subsequently, size selected particles from the SMPS were sampled by SPLAT to get the aerodynamic size distribution. SPLAT details are described in reference 6 . In addition, Scanning Electron Microscopy (SEM) was used to further characterize the particles.

Sodium dodecyl sulfate (99\% pure), sodium oleate (98\% pure), laurtrimonium chloride (98\% pure), sodium chloride (99.5\% pure) were obtained from Sigma-Aldrich Co and used without further purification. Aqueous solutions were made using ultra pure deionized water.

\section{Results and Discussion}

Aqueous solutions. The behavior and properties of surfactants in aqueous particles internally mixed with surfactants was investigated. Aqueous solutions of sodium dodecyl sulfate (SDS), sodium oleate (SO) and laurtrimonium chloride (LC) were made in the $10^{-5}-10^{-1} \mathrm{M}$ range. Mobility diameter size distribution measurements show that for SDS and SO the peak is broadening as the concentration is increased, whereas in LC no significant differences are observed (shown in Figure 1). This could be interpreted as having the particles coated with surfactants as expected, which presumably will increase particle diameter 
and slow water evaporation. Although the measured vacuum aerodynamic diameter distribution for a given mobility diameter shows not many differences for SDS.
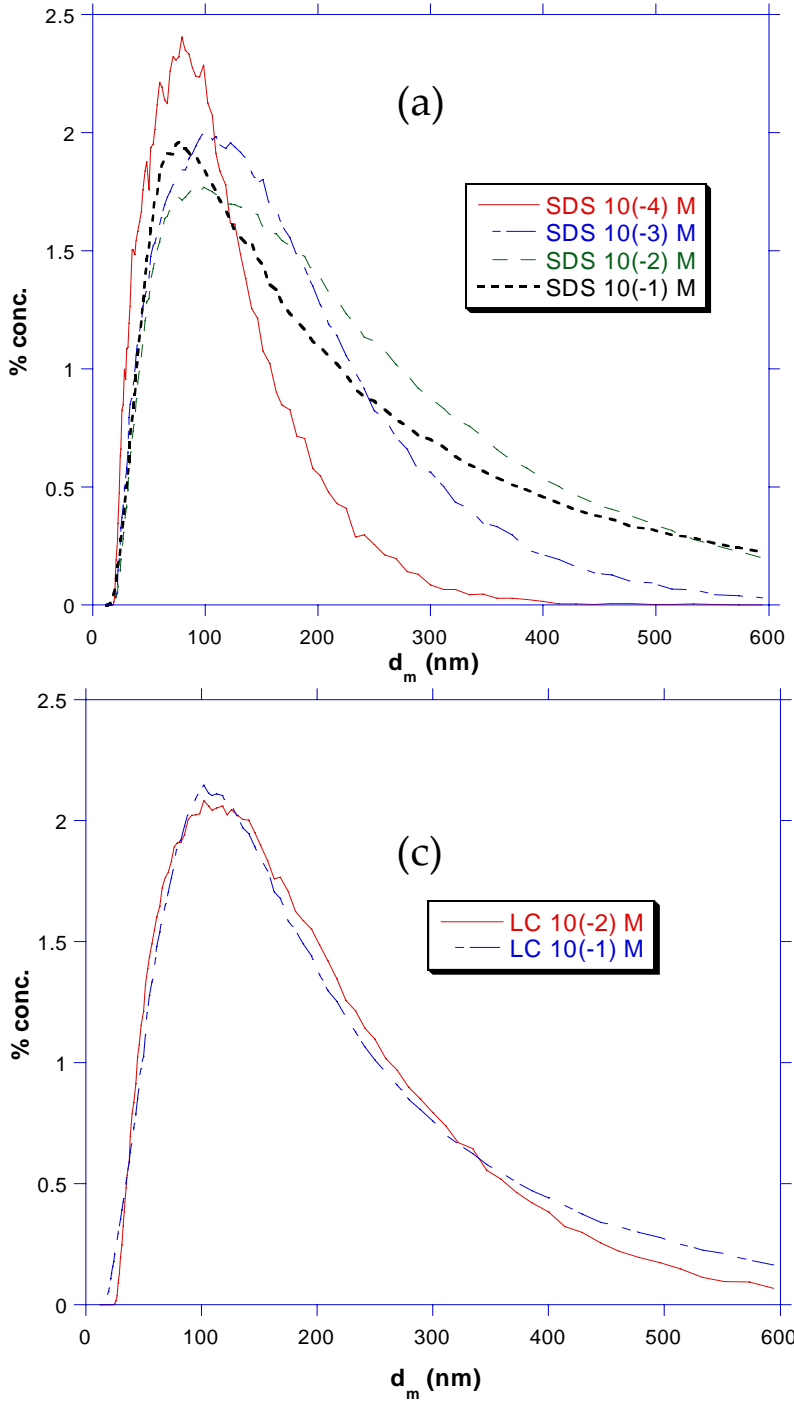

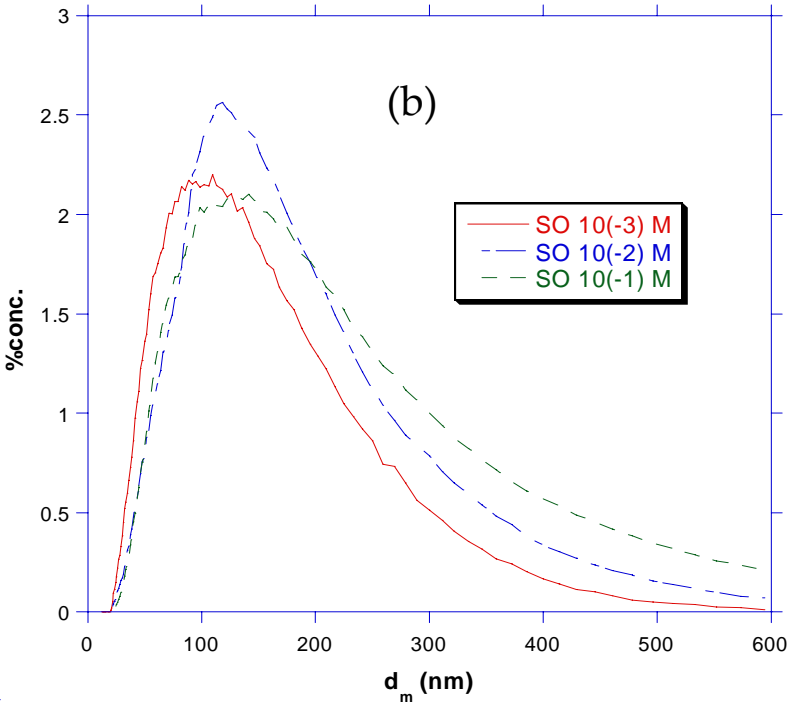

Figure 1. Mobility diameter size distribution for aqueous particles containing (a) SDS; (b) SO and (c) LC at different concentrations.

In Figure 2 it is shown the vacuum aerodynamic diameter distribution for SDS and SO for a mobility diameter of $508 \mathrm{~nm}$ and different concentrations. The distribution for SDS does not differ as much as the one for SO as the concentration is increased. At low concentrations a tail appears in the distribution showing a "bimodal" or spread distribution, even though is the 
same mobility diameter. Evaporation mechanisms and kinetics in the aerodynamic lens for wet particles could be complicated, possibly making particles lose water in different ways which is seen as a "bimodal" distribution. This is shown by Zelenyuk et al. (2006) for $\mathrm{NaCl}$ aqueous droplets. ${ }^{7}$ In addition, the double bond in SO could affect how the particles are coated by the surfactant giving more room for water to evaporate at low concentrations. As the concentration is increased the bimodal feature disappears but still the peak broadens. The SO most intense peaks and SDS peaks show aerodynamic diameter differing by $<5 \%$, whereas the SO smaller peak differs by $24 \%$.

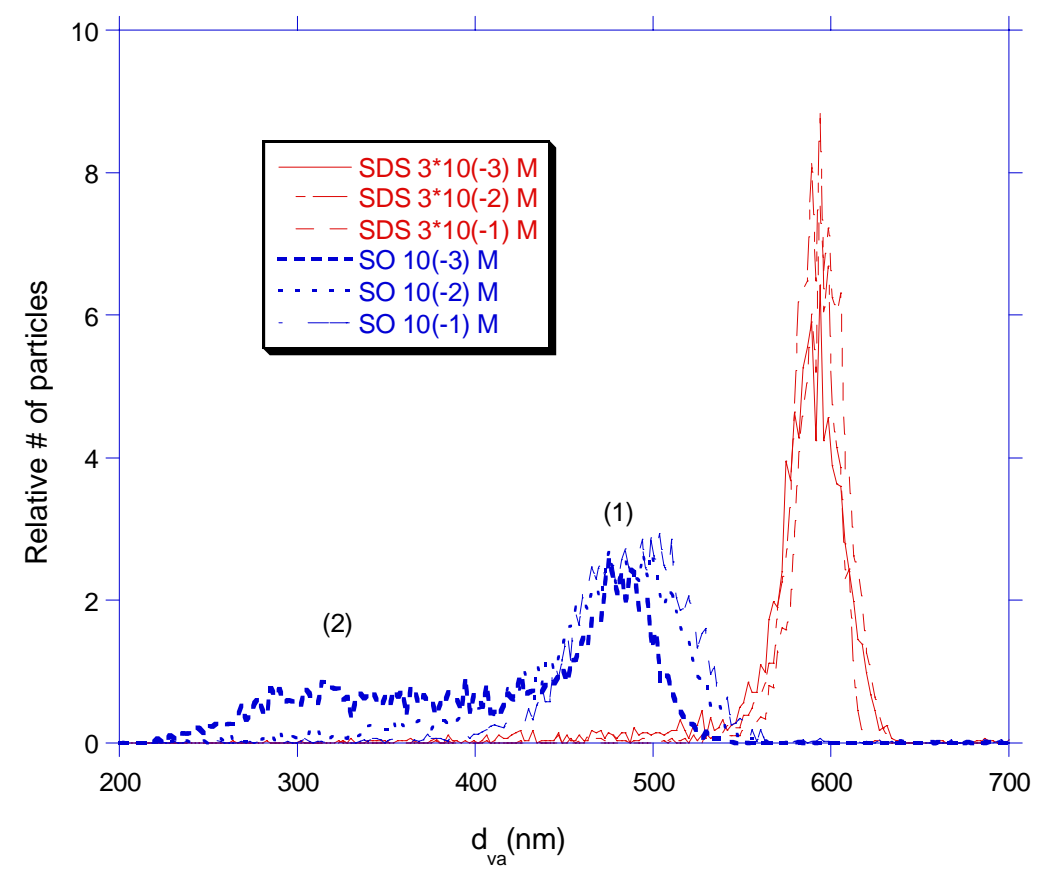

Figure 2. SDS and SO aerodynamic size distribution with a mobility diameter of $508 \mathrm{~nm}$

Furthermore the effective density, which is related to the ratio of $d_{v a}$ to $d_{m}$, also behaves differently for these two systems. Table 1 show $\mathrm{d}_{\mathrm{va}} / \mathrm{d}_{\mathrm{m}}$ for the two systems at different concentrations for $\mathrm{d}_{\mathrm{m}}=508 \mathrm{~nm}$. Again, the main peak for both systems differ only by a few percent that is within experimental error except for second peak for SO. SDS has a bulk density of $0.37 \mathrm{~g} \mathrm{~cm}^{-3}$ and SO has a bulk 
PNNL-16185

density of $1.02 \mathrm{~g} \mathrm{~cm}^{-3}$. If substantial amount of water is retained due to surfactant coverage the effective density should resemble that of water. In the opposite case the effective density should reflect more likely the bulk density. Since SO and water have similar densities is difficult to get information about water retention in those particles. SDS density is significantly different from water. Based on the measured effective density, aqueous SDS particles retain water in the aerosol phase.

Table 1. Summary of $\mathrm{d}_{\mathrm{va}} / \mathrm{d}_{\mathrm{m}}$ for SDS and SO for a mobility diameter of $508 \mathrm{~nm}$

\begin{tabular}{ccccccc}
\hline & \multicolumn{3}{c}{ SDS } & & \multicolumn{3}{c}{ SO } \\
& $3 \times 10^{-3} \mathrm{M}$ & $3 \times 10^{-2} \mathrm{M}$ & $3 \times 10^{-1} \mathrm{M}$ & $1 \times 10^{-3} \mathrm{M}$ & $1 \times 10^{-2} \mathrm{M}$ & $1 \times 10^{-1} \mathrm{M}$ \\
$\mathbf{d}_{\mathrm{va}} / \mathbf{d}_{\mathbf{m}}$ & 1.16 & 1.17 & 1.18 & $0.95(1)$ & & 0.96 \\
& & & & $0.72(2)$ & & 0.97 \\
\hline
\end{tabular}

$\mathrm{NaCl} . \mathrm{NaCl}$ thermodynamic and particle shape information is known, therefore the effect of surfactants on these hygroscopic particles was studied. Solutions of $\mathrm{NaCl} \sim 0.05 \mathrm{M}$ containing SDS and $\mathrm{NaCl} \sim 0.1 \mathrm{M}$ containing SO were analyzed in the range $0 \mathrm{M}-10^{-5} \mathrm{M}$ for surfactants concentration. The mobility size distribution follow the same trend as for the aqueous solutions; as the surfactant concentration is increased from $10^{-5} \mathrm{M}$ to $10^{-2} \mathrm{M}$ the peak broadens to higher sizes. $\mathrm{NaCl}$ measured effective density also represents what has been measured ${ }^{8}$ before including dynamic shape factors. An interesting observation is that SDS changes the particle effective density more than SO; what was expected due to their bulk densities. This is shown in Figure 3, where the $d_{v a} / d_{m}$ is plotted as a function of $\mathrm{d}_{\mathrm{m}}$ for SDS and SO in the $0 \mathrm{M}-10^{-5} \mathrm{M}$ surfactant concentration range. Although $\mathrm{NaCl}$ concentration is different in each surfactant solution, the $\mathrm{NaCl}$ effective density for both concentrations is measured to be close and within experimental error. In addition, several runs of SDS in $\mathrm{NaCl} \sim 0.1 \mathrm{M}$ follow the 
same trend and fit perfectly when the $\mathrm{NaCl}$ :Surfactant ratio is considered. Despite that, this behavior cannot be explained completely by simple means of bulk densities as is the case for inorganic salts aerosols. ${ }^{8}$
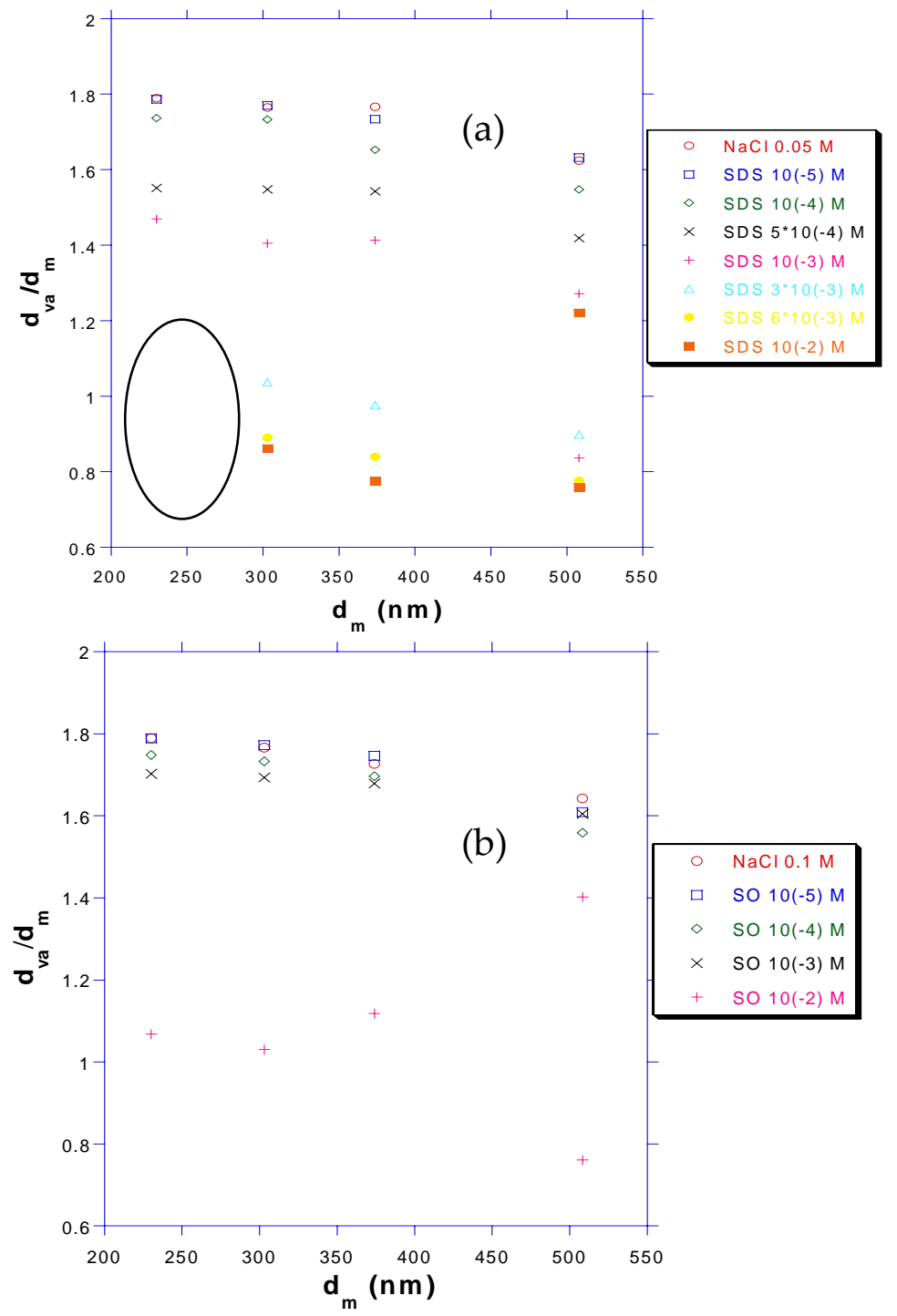

Figure 3. $\mathrm{dva} / \mathrm{dm}$ as a function of $\mathrm{dm}$ for (a) SDS and (b) SO at different concentrations

Moreover, for SDS as the concentration is increased smaller particles "disappeared", marked as a black circle in Figure 3. Again the aerodynamic size distribution changes from resolved peaks for $\mathrm{NaCl}$ solution to almost converging, broad peaks as the surfactant concentration approaches $10^{-2} \mathrm{M}$ 
(figure not shown). This behavior is similar to the aqueous solutions bimodal behavior but in this case the size resolution for multiple charged particles is lost and only one broad peak is observed for every mobility diameter selected.

Scanning Electron Microscopy was used to understand what happened to small particles and also to have another mean to characterize the particles. Mobility diameter of 500 $\mathrm{nm}$ was selected, sample and analyzed using SEM for particles containing $\mathrm{NaCl}$ with $1 \times 10^{-4} \mathrm{M}, 1 \times 10^{-3} \mathrm{M}$ and $6 \times 10^{-3} \mathrm{M}$ SDS. The same was done for a mobility diameter of $224 \mathrm{~nm}$. In Figure 4, the SEM picture is shown for $\mathrm{d}_{\mathrm{m}}=224 \mathrm{~nm}$ and $[\mathrm{SDS}]=6 \times 10^{-3} \mathrm{M}$. Particularly interesting is the fact that large particles are present even though small were selected, which is something we don't understand. But there are not as many as small ones. In this picture is clear that small particles are present, but they seem too small to be detected by SPLAT. Another important feature is that many $\mathrm{NaCl}$ are associated with a "wet mark" around it, implying that the particles were deposited wet on the substrate. This is observed more and more at high concentration of surfactants, which explain the lower effective densities at those concentrations and the water retention.

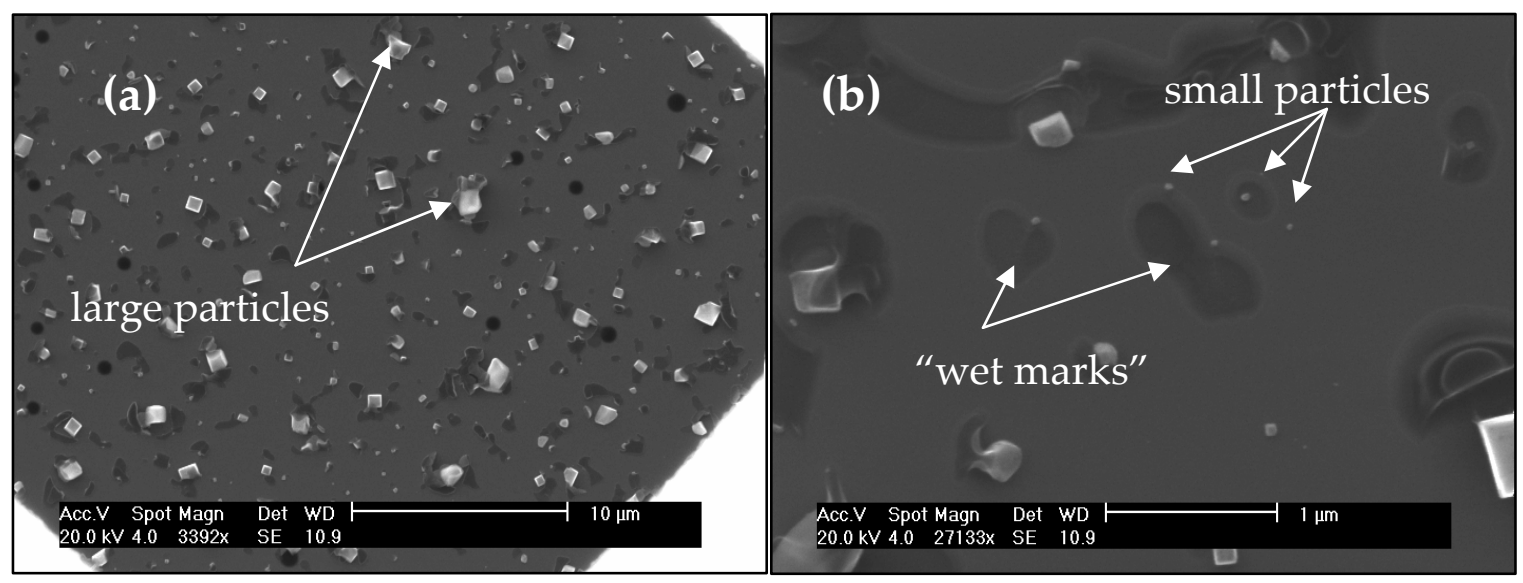

Figure 4. SEM pictures for particles containing $\mathrm{NaCl}$ and [SDS] $=6 \times 10^{-3} \mathrm{M}$ with mobility diameter, $d_{m}$ of $224 \mathrm{~nm}$. (a) 3392x magnification and (b) 27133x magnification. 
PNNL-16185

\section{Conclusion}

Here we have studied the physical properties of aqueous particles internally mixed with different surfactants. All measurements were conducted at low RH and show that every surfactant behaves differently and don not follow a particular trend. Aerodynamic size distribution shows that the particles lose water in different ways for SO when compare to SDS because of the peak broadening at low surfactant concentration. The surfactant surface coverage and structure may play a role in the suppression of water loss. Effective density measurements, mobility size distribution and SEM pictures show that the different systems retain water even though effective density could not be explain by simple means as other systems. SEM pictures help explain qualitatively the effective densities for SDS in aqueous $\mathrm{NaCl}$ and the particles' morphology. An interesting observation is that many $\mathrm{NaCl}$ particles are associated with a "wet mark" that implies the particles were deposited wet on the substrate. Despite all that, the amount of water retain or lose by these type of particles still a challenge that need more measurements to start understanding the "inverted" micelle system.

\section{References}

1) Cruz, C.N.; Pandis, S.N. Atmospheric Environment. 1997, 31 (15): 2205-2214.

2) Penner, J.E.; Hegg, D.; Leaitch, R. Unraveling the role of aerosols in climate change Environmental Science and Technology. August 1, 2001, pp 333 A- 340 A.

3) Tolocka,M.P.; Solomon, P. A.; Mitchell, W.; Gemmill, D.; Wiener,R. W.; Homolya, J.; Natarajan, S.; Vanderpool, R. W. Aerosol Sci.Technol. 2001, 34, 88-96.

4) Ellison, G. B.; Tuck, A. F.; Vaida, V. J. Geophys. Res.-Atmos. 1999, 104, 11633-11641.

5) Mcneill, V.F.; Patterson, J.; Wolfe, G.M.; Thornton, J.A. Atmos. Chem Phys., 2006, 6, 1635-1644.

6) Zelenyuk, A.; Imre, D. Aerosol Science Tech. 2005, 39, 1-15.

7) Zelenyuk, A.; Imre, D.; Cuadra-Rodriguez, L.A. Analytical Chem. 2006, ASAP article; DOI: $10.1021 / \mathrm{ac} 061184 \mathrm{o}$

8) Zelenyuk, A.; Cai, Y.; Imre, D. Aerosol Science Tech. 2006, 40, 197-217. 
PNNL-16185 
PNNL-16185

\title{
I. Theoretical characterization of $\mathrm{H}_{3} \mathrm{O}^{+}$at the water/methanol - vapor interface
}

\section{Theoretical characterization of $\mathrm{NaClO}_{4} / \mathrm{H}_{2} \mathrm{O}$ solutions}

\author{
Jun Cui, and K.D. Jordan \\ Department of Chemistry, University of Pittsburgh \\ Pittsburgh, PA \\ and \\ L.X. Dang, C. Wick, V. Glezakou, \\ Pacific Northwest National Laboratory \\ Richland, WA
}

Proton transport plays an important role in biochemistry, chemistry, and many other fields. Proton transport in methanol/water solution can serve as a valuable model system for understanding proton transport in mixed hydrophobic and hydrophilic environments, thus provide insight into proton transport in biological systems. During my stay in PNNL, I used molecular dynamics simulations to study proton behavior at methanol/water solution - vapor interface. In the simulations, three solutions with varying concentration of methanol were used: $1 \mathrm{M} \mathrm{HCl} /$ water, $0.25 \mathrm{M}$ methanol/1 M $\mathrm{HCl} /$ water, $0.5 \mathrm{M}$ methanol/1 M HCl/water. The Dang-Chang force field ${ }^{1}$ was used for water and the AMBER force field ${ }^{2}$ for methanol. The Dang-Chang model was modified to account for the $\mathrm{H}_{3} \mathrm{O}^{+}$ions. The modified model puts a -0.42 Coulomb point charge on $\mathrm{O}$ atom, a 0.47 Coulomb point charge on each of the three $\mathrm{H}$ atoms. Periodical boundary conditions were applied in all three directions. A box consisting of 1000 molecules was placed into the simulation cell with dimensions $28 \times 28 \times 157 \AA$. AMBER 7 software package was adopted for molecular dynamics simulation. The initial coordinates were produced using the Monte Carlo simulated annealing method. It was found that the 
PNNL-16185

simulations had difficulty in equilibrating. Ab inito calculations revealed that the proton on $\mathrm{H}_{3} \mathrm{O}^{+}$tends to transfer to the $\mathrm{O}$ atom of a methanol molecule. This strong interaction caused the problems in the Monte Carlo simulations.

During my study at PNNL, I also started simulations aimed at understanding the experimental finding that the folding/unfolding temperatures of model proteins depend sensitively on the perchlorate concentration. We wanted to examine the idea that the main influence of the perchlorate is on the structure of the water. I started molecular dynamics simulations of $\mathrm{NaClO}_{4} /$ water solution, studying the hydration structure of $\mathrm{ClO}_{4}{ }_{4}^{-}$. As described above, the AMBER and Dang-Chang force fields were used for the ions and water respectively. All the simulations were carried out in an isobaric-isothermal (NPT) ensemble at $1 \mathrm{~atm}$ and $298 \mathrm{~K}$. The radial distribution functions of three solutions (0.5 M, $0.83 \mathrm{M}, 1.0 \mathrm{M} \mathrm{NaClO}_{4}$ in water) are displayed in the following figures. Figure 1 shows the $\mathrm{Cl}-\mathrm{Cl}$ radial distribution function change with perchlorate concentrations. Figure 2 is the perchlorate concentration dependence of perchlorate $\mathrm{Cl}$-water oxygen. Work on this project is continuing.

\section{References}

(1) Dang, L. X.; Chang, T.-M. J. Chem. Phys. 1997, 106, 8149.

(2) Cornell, W. D.; Cieplak, P.; Bayly, C. I.; Gould, I. R.; Merz, K. M.; Ferguson, D. M.; Spellmeyer, D. C.; Fox, T.; Caldwell, J. W.; Kollman, P. A. J. Am. Chem. Soc. 1995, 117, 5179. 
PNNL-16185

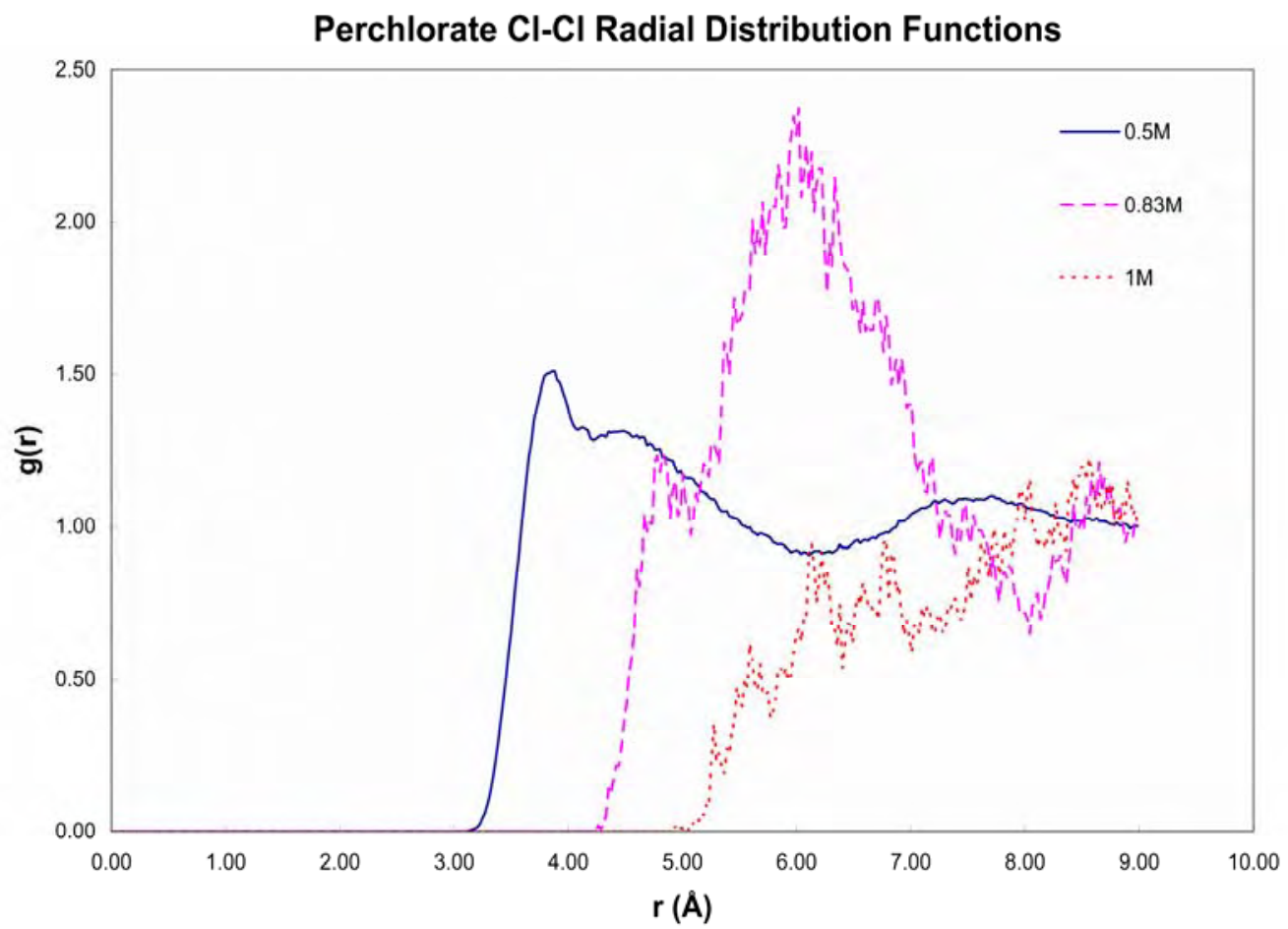

Figure $1 . \mathrm{NaClO}_{4}$ concentration dependence of radial distribution functions between perchlorate chlorine.

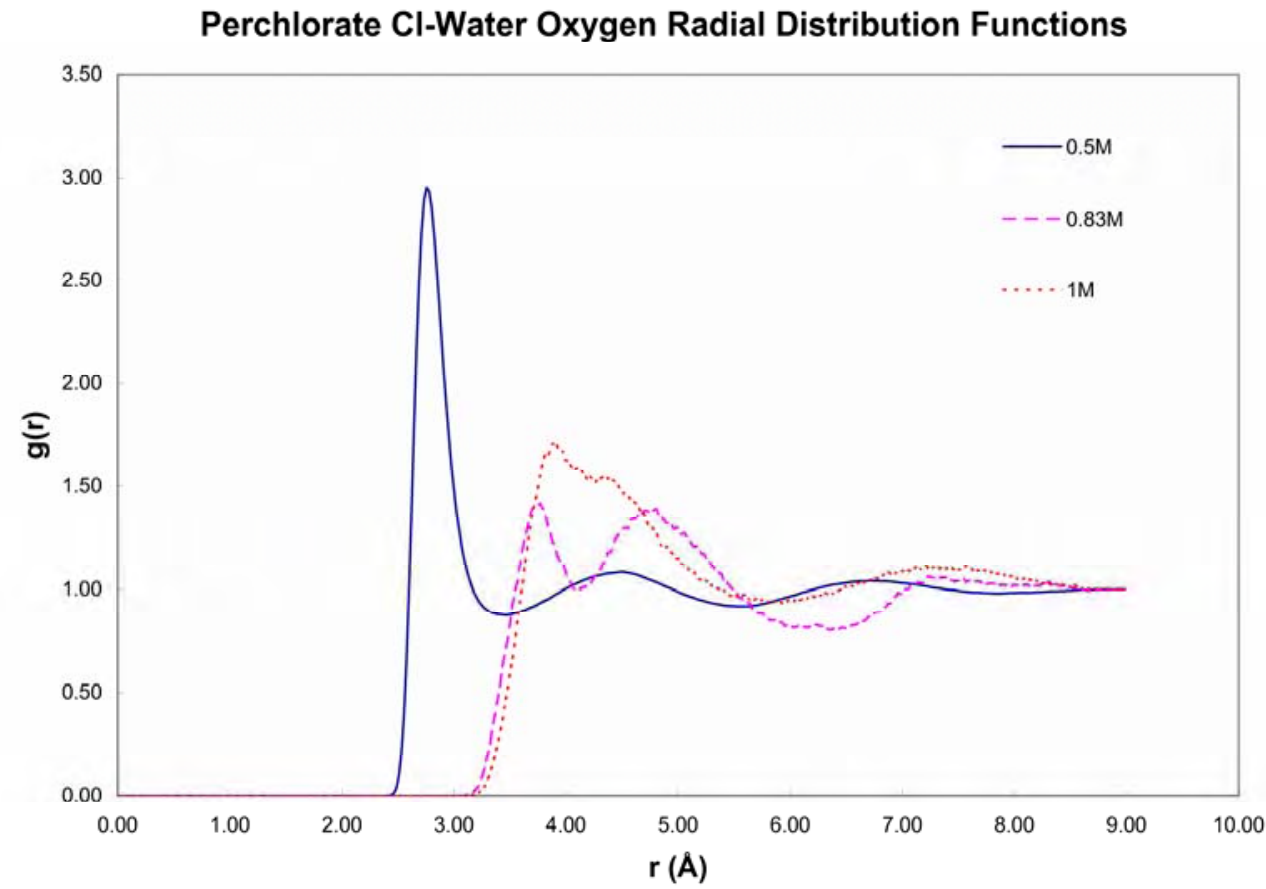

Figure 2. $\mathrm{NaClO}_{4}$ concentration dependence of radial distribution functions between perchlorate chlorine and water oxygen. 
PNNL-16185 
PNNL-16185

\title{
OCl Radical $-\mathrm{H}_{2} \mathrm{O}$ interaction: ab initio calculation and the development of empirical potential
}

\author{
Shiyu Du and Prof. Joseph Francisco \\ Department of Chemistry \\ Purdue University \\ West Lafayette, Indiana USA 47907-1393 \\ and \\ Gregory K. Schenter and Bruce Garrett \\ Chemical Science Division \\ Pacific Northwest National Laboratory \\ Richland, WA 99352
}

\begin{abstract}
$\underline{\text { Abstract }}$
In the ozone removal processes in the atmosphere, the $\mathrm{ClO}$ free radical is found to act in an important role in recent years. In this work, we present our Potential Energy Scan of the $\mathrm{H}_{2} \mathrm{O} \cdot \mathrm{OCl}$ system with high level ab initio methods. Because of the existence of lowlying excited states of the $\mathrm{H}_{2} \mathrm{O} \cdot \mathrm{OCl}$ complex, and their potential impact on the chemical behavior of the $\mathrm{OCl}$ radical in the atmosphere, we perform a Potential Energy Surface (PES) scan at CCSD(T)/aug-cc-pVTZ level of both the first excited and ground states in order to model the physics of the unpaired electron in the OCl radical. Empirical rigid potentials for both ground and excited states were built based on a Thole Type Model. The two minimums of the $\mathrm{H}_{2} \mathrm{O} \cdot \mathrm{OCl}$ complex are recovered by the empirical potential.
\end{abstract}




\section{Introduction}

Free radicals are well known to be the most reacting species in the atmosphere. The hydrogen bonding effect is considered as one of most important association processes in the chemical transformations and more and more hydrogen-bonded complexes are found by both experimental and theoretical studies ${ }^{1-11}$. A better understanding of the reactions, bonding characters, energies and roles of the complexes formed between water molecules and reacting free radicals are worth pursuing.

In the ozone removal processes in the atmosphere, $\mathrm{ClO}$ free radical is found to play an important role ${ }^{12-15}$ in recent years. It has been proposed ${ }^{12-16}$ that the critical steps in some mechanisms in the ozone removal process are that a $\mathrm{ClO}$ dimer is formed and then it is photodecomposed. For the formation of $\mathrm{ClO}$ dimer, Francisco et al. ${ }^{11,16}$, by $a b$ initio study, found a water-ClO complex of a skewed nonplanar structure which is proposed to be the chaperone in the dimer formation process. Fu at el. ${ }^{17}$ found other stationary points in the $\mathrm{ClO} \cdot \mathrm{H}_{2} \mathrm{O}$ complex potential energy surface for both of the $\mathrm{A}^{\prime}$ and A's states and studied the corresponding vibrational frequencies with Density Functional Theory ${ }^{18,19}$ and the Moller-Plessett Perturbation method ${ }^{20-22}$. All the previous studies concentrated on the stationary points. However, as one of the most atmospherically important species, these studies are far from enough because a complete study of the potential energy surface and high level calculations are needed in order to model the chemical process of this radical in the atmosphere and other surroundings in the liquid phase by molecular dynamics simulation. It is also necessary to obtain an empirical potential for the $\mathrm{ClO} \cdot \mathrm{H}_{2} \mathrm{O}$ system.

In this work, we report the potential energy surface scan for both the ground and first excited state by locating the single electron on different orbitals with a high level calculation at the level of couple cluster involving single and double excitations with a perturbation estimation of the triple excitations $[\operatorname{CCSD}(\mathrm{T})]^{23-26}$ with the basis set of the family of augmented correlation-consistent basis sets of triple zeta quality (aug-ccpVTZ). This approach has been shown to be large enough to converge in a previous related study ${ }^{27}$ and a fitted empirical interaction potential we modified from the rigid potential between water molecules “Thole-type" model (TTM) ${ }^{28-31}$ for $\mathrm{H}_{2} \mathrm{O} \cdot \mathrm{OCl}$ 
PNNL-16185

(mTTMC) to the binding energy by $a b$ initio calculations. With the consideration of the single electron effect, we obtain a well behaved empirical potential.

\section{Method}

\section{A. Computational Details}

The ground state and first excited state electronic energy of the $\mathrm{ClO} \cdot \mathrm{H}_{2} \mathrm{O}$ system were both scanned. In order to characterize the potential energy surface, we locate the $\mathrm{ClO}$ radical on different configurations relative to the water molecule. Our ab initio calculations are all at the level of CCSD(T)/aug-cc-pVTZ. In each potential energy scan, we calculate approximately 30 40 points. The interaction energy of any configuration is calculated from the difference between the corresponding $\mathrm{ClO} \cdot \mathrm{H}_{2} \mathrm{O}$ complex energy and the monomer energies in their optimized geometries at the level of CCSD(T)/aug-ccpVTZ. All the $a b$ initio calculations were performed with the Molpro 2002 suite of programs $^{32}$ at $\mathrm{EMSL}^{33}$.

\section{B. Empirical Models}

In this work, we reparameterize the $\mathrm{TTM}^{28-31}$ among recently established models ${ }^{34-37}$ because by modifying this model, we have successfully established an empirical potential for the $\mathrm{OH} \cdot \mathrm{H}_{2} \mathrm{O}$ system. A great similarity between the $\mathrm{ClO} \cdot \mathrm{H}_{2} \mathrm{O}$ and the $\mathrm{OH} \cdot \mathrm{H}_{2} \mathrm{O}$ potentials ${ }^{16}$ is found.

In the TTM model, as discussed in detail in the literature ${ }^{28-31}$, the interactions between two molecules are decomposed by Lennard-Jones type attractive-repulsive interaction energy ( $\left.U^{\text {pair }}\right)$, the induction energy ( $\left.U^{\text {ind }}\right)$ and the electrostatic energy $\left(U^{\text {elec }}\right)$. Since the interaction between water molecules has been well parameterized, we keep all the parameterizations for $\mathrm{H}_{2} \mathrm{O}-\mathrm{H}_{2} \mathrm{O}$ interaction unchanged. As we have found in the potential surface fitting for the $\mathrm{OH} \cdot \mathrm{H}_{2} \mathrm{O}$ system, it is possible to model the single occupied orbital by setting the three diagonal elements of the polarizability tensors $\left(\alpha_{x x}, \alpha_{y y}\right.$, and $\left.\alpha_{z z}\right)$ for the $\mathrm{Cl}$ and $\mathrm{O}$ atoms in the $\mathrm{ClO}$ radical. And another important feature of this model is the introduction of the "M-site" which is a hypothetical site where the electrostatic interaction is located and has been successfully used to obtain good $\mathrm{H}_{2} \mathrm{O}-\mathrm{H}_{2} \mathrm{O}$ and $\mathrm{OH} \cdot \mathrm{H}_{2} \mathrm{O}$ interaction energies. In our mTTMC model, we also add an "M-site" for the 
$\mathrm{ClO} \cdot \mathrm{H}_{2} \mathrm{O}$ complex between the two atoms in $\mathrm{ClO}$ radical besides the one which has been set up for water molecules. One difference between this potential and the $\mathrm{OH} \cdot \mathrm{H}_{2} \mathrm{O}$ system is that we introduce the Lennard-Jones type interaction between the $\mathrm{Cl}$ atom and the $\mathrm{H}$ atom in the water molecule.

\section{Computaional Results}

\section{A. Sampling of Potential Energy Surface}

In the sampling of the $\mathrm{ClO} \cdot \mathrm{H}_{2} \mathrm{O}$ Potential Energy Surface (PES), the coordinate for $\mathrm{OCl}$ radical monomer is shown in Fig $\mathbf{1}$ and that for $\mathrm{H}_{2} \mathrm{O}$ is the same as previous work. ${ }^{28-}$

${ }^{31}$ For the complex, we adopt the same spherical coordinate system that was used for the $\mathrm{H} 2 \mathrm{O} \cdot \mathrm{OH}$ complex. As shown in Fig 2, the water molecule is set in the plane perpendicular to the z-axis. The $\mathrm{O}$ atom $(\mathrm{O} 1)$ in $\mathrm{H}_{2} \mathrm{O}$ is placed at the origin and one of the $\mathrm{O}-\mathrm{H}$ bonds is located in the positive direction on the $\mathrm{x}$-axis. The coordinate of the other $\mathrm{H}$ atom can be calculated from the rigid bond angle $\theta_{\mathrm{w}}$ and $\mathrm{OH}$ bond length $\mathrm{r}^{\mathrm{w}}$ OH of the water molecule that are optimized at the level of $\operatorname{CCSD}(T) / a u g-c c-p V T Z$. The bond distance of $\mathrm{ClO}$ radical, $\mathrm{rOCl}_{\mathrm{OCl}}$ is also optimized at the same level of theory. Since TTM in this work is a rigid model, we fixed the values of $\theta_{\mathrm{w}}, \mathrm{r}^{\mathrm{w}}$ OH and $\mathrm{r}_{\mathrm{OCl}}$ to the values shown in Table 1. The coordinate of the $\mathrm{O}$ atom $(\mathrm{O} 2)$ in $\mathrm{ClO}$ can be determined by the distance between the two oxygen atoms (one of which is in the origin point) $\mathrm{R}_{\mathrm{OO}}$, and the two spherical coordinates $\theta$ and $\varphi$. The coordinates of the $\mathrm{Cl}$ atom can be obtained similarly by first calculating the relative coordinate to $\mathrm{O} 2$ by the $\mathrm{O}-\mathrm{Cl}$ bond distance, and two spherical coordinate $\theta$ ' and $\varphi^{\prime}$ corresponding to $\mathrm{O} 2$ and then adding the coordinate of $\mathrm{O} 2$ relative to the origin point $(\mathrm{O} 1)$. So the rectangular coordinates of the five atoms are calculated as follows:

$\mathrm{O} 1$ (in $\left.\mathrm{H}_{2} \mathrm{O}\right):(0,0,0)$

$\mathrm{H} 1$ (in $\left.\mathrm{H}_{2} \mathrm{O}\right):\left(\mathrm{r}^{\mathrm{w}}{ }_{\mathrm{OH}}, 0,0\right)$

$\mathrm{H} 2$ (in $\left.\mathrm{H}_{2} \mathrm{O}\right):\left(\mathrm{r}^{\mathrm{w}}{ }_{\mathrm{OH}} \cos \theta_{\mathrm{w}},-\mathrm{r}^{\mathrm{w}}{ }_{\mathrm{OH}} \sin \theta_{\mathrm{w}}, 0\right)$

$\mathrm{O} 2$ (in $\mathrm{ClO})$ : $\left(\mathrm{R}_{\mathrm{OO}} \cos \theta \cos \varphi, \mathrm{R}_{\mathrm{OO}} \cos \theta \sin \varphi, \mathrm{R}_{\mathrm{OO}} \sin \theta\right)$

$\mathrm{Cl}$ (in $\mathrm{ClO}):\left(\mathrm{R}_{\mathrm{OO}} \cos \theta \cos \varphi+\mathrm{r}_{\mathrm{OCl}} \cos \theta^{\prime} \cos \varphi^{\prime}, \mathrm{R}_{\mathrm{OO}} \cos \theta \sin \varphi+\mathrm{r}_{\mathrm{OCl}} \cos \theta^{\prime} \sin \varphi^{\prime}, \mathrm{R}_{\mathrm{OO}} \sin \theta+\mathrm{r}_{\mathrm{OCI}} \sin \theta^{\prime}\right)$

The PES is totally determined by five variables, $\mathrm{R}_{\mathrm{OO}}, \theta, \varphi, \theta^{\prime}$ and $\varphi^{\prime}$ for both the ground state and the first excited state. 
The PES scans for the ground state and first excited state are shown in Fig 3- Fig 9 corresponding to all the five variables as listed in Table 2 . $\mathrm{R}_{\mathrm{OO}}$ is scanned more than others because it is the main parameter to determine the long range interaction. The reason we made PES scans for both states is that because of the existence of the single occupied orbital, the excited state obtained by exchanging the appropriate double occupied orbital and the single occupied orbital that is sometimes very close in energy with the ground state. This implies that the excited states can be a very important factor in determining the behavior of $\mathrm{ClO} \cdot \mathrm{H}_{2} \mathrm{O}$. For the same reason, as in our study of the $\mathrm{H}_{2} \mathrm{O} \cdot \mathrm{OH}$ system, we also have considered both states. In this work, the first three scans are for $\mathrm{R}_{\mathrm{OO}}$ and the other four scans are for $\varphi, \theta, \varphi^{\prime}$ and $\theta^{\prime}$ respectively.

As shown in Fig 3, the potentials are obtained by scanning the interaction energy as a function of $\mathrm{R}_{\mathrm{OO}}$ with the configuration as pictured in the insert. The angle of Cl-O2-O1 is kept $70^{\circ}$ which is the minimum obtained from scan \# 4. In this configuration, the ground and excited states have A” and A' symmetry respectively and it is consistent with the corresponding $\mathrm{H}_{2} \mathrm{O} \cdot \mathrm{OH}$ potential. This is probably because the complex in the A" state has larger orbital overlapping on the molecular plane than A'. The binding energies of the two states at the minimum are 3.03 (at $\mathrm{R}_{\mathrm{OO}}=3.05 \AA$ ) and 1.53 (at $\mathrm{R}_{\mathrm{OO}}=3.20 \AA$ ) $\mathrm{kcal} / \mathrm{mol}$ respectively and are consistent with those optimized at the MP2 level by Fu et $\mathrm{al}^{17}$. The energy difference of the two states is lager than those of $\mathrm{H}_{2} \mathrm{O} \cdot \mathrm{OH}$ (3.51 and 1.41 $\mathrm{kcal} / \mathrm{mol}$ ) at the corresponding geometries, but the equilibrium $\mathrm{R}_{\mathrm{OO}}$ values are quite similar with those of $\mathrm{H}_{2} \mathrm{O} \cdot \mathrm{OH}$ which are 3.00 and $3.20 \AA$ respectively. The energy differences of the $A^{\prime}$ and $A$ ” states decrease when $R_{\mathrm{OO}}$ increases and are lower than 0.5 $\mathrm{kcal} / \mathrm{mol}$ when $\mathrm{R}_{\mathrm{OO}}$ is larger than $4.0 \AA$.

The second $\mathrm{R}_{\mathrm{OO}}$ scan (scan \#2) is performed for the configuration at which the $\mathrm{Cl}$ atom in $\mathrm{OCl}$ radical forms a weak bond with the $\mathrm{O}$ atom in water molecule as Fig 4 illustrates. The angle formed by the $\mathrm{OCl}$ radical and the water molecular plane is $87^{\circ}$, which is the lowest in energy among all angles. The Cl-O1 distances at equilibrium are $2.81 \AA$ and $2.86 \AA$ for the ground state (A') and excited state (A”) respectively and the ClH distance of A' state is calculated to be $3.48 \AA$, which is consistent with Fu et al. ${ }^{17}$, s result. The binding energies of the two states are 3.39 and $3.09 \mathrm{kcal} / \mathrm{mol}$. And we find that the energy gap between the two states is less than $0.5 \mathrm{kcal} / \mathrm{mol}$ when $\mathrm{R}_{\mathrm{OO}}$ is larger than 4.2 
$\AA$. The equilibrium distance is much larger and the bonding energy is also lower when comparing to those of $\mathrm{H}_{2} \mathrm{O} \cdot \mathrm{OH}$. These differences are probably due to the much larger electron density on $\mathrm{Cl}$ atom than the $\mathrm{H}$ atom in $\mathrm{OH}$.

Our last $\mathrm{R}_{\mathrm{OO}}$ scan (scan \#3) is plotted in Fig $\mathbf{5}$, where $\mathrm{OCl}$ radical is positioned on the bisector of the $\mathrm{H}-\mathrm{O}-\mathrm{H}$ angle of water molecule. This configuration belongs to $\mathrm{C}_{2 \mathrm{v}}$ point group. The binding energy for ground state $\mathrm{B}_{2}$ and excited state $\mathrm{B}_{3}$ are 1.76 (at $\mathrm{R}_{\mathrm{OO}}=$ $3.05 \AA$ ) and 1.28 (at $\mathrm{R}_{\mathrm{OO}}=3.20 \AA$ ) kcal $/ \mathrm{mol}$. When comparing these results with the $\mathrm{H}_{2} \mathrm{O} \cdot \mathrm{OH}$ complex, the binding energy is about $0.2-0.4 \mathrm{kcal} / \mathrm{mol}$ less but the equilibrium geometries are very close. These are consistent with what is revealed from the calculations for scan \# 1 . This implies that the interaction radius for $\mathrm{O} 2$ and $\mathrm{H}$ atom are close to that of $\mathrm{H}_{2} \mathrm{O} \cdot \mathrm{OH}$ although the interaction energy is lowered. This is probably a result that $\mathrm{O} 2$ has a lower electron density because of much larger electronegativity of chlorine than that of hydrogen. Another interesting fact is that the equilibrium $\mathrm{R}_{\mathrm{OO}}$, in this configuration correspnonds to a transition state in $\mathrm{H}_{2} \mathrm{O} \cdot \mathrm{OH}$. With further calculations, we will see that this is probably also true for $\mathrm{H}_{2} \mathrm{O} \cdot \mathrm{OCl}$.

The angular potential scans are plotted for Fig 6 to Fig 9 which correspond to the scans of the four angular variables respectively. In Fig $\mathbf{6}(\operatorname{scan} \# 4)$, the interaction energies of the ground state A" and excited state A' are calculated by changing the $\varphi^{\prime}$ from $-90^{\circ}$ to $90^{\circ}$ as shown in the insert. The calculations show that the energy difference between the two states is less than $0.5 \mathrm{kcal} / \mathrm{mol}$ when $\varphi^{\prime}$ is in the $\left[-30^{\circ}, 27^{\circ}\right]$ range. As seen from the figure, there are two minimums $\left(\varphi^{\prime}= \pm 70^{\circ}\right)$ and one maximum $\left(\varphi^{\prime}=-3^{\circ}\right)$ on PES for the ground state, whose binding energies are correspondingly $2.98,2.65$ and $1.80 \mathrm{kcal} / \mathrm{mol}$. However, for the excited state, only one minimum $\left(\varphi^{\prime}=30^{\circ}\right)$ is found with a binding energy of $1.75 \mathrm{kcal} / \mathrm{mol}$. This is quite different from the similarity of the A' and A" states on the PES of $\mathrm{H}_{2} \mathrm{O} \cdot \mathrm{OH}$ at the analogous geometry.

The potential scan with $\theta^{\prime}$ as the variable ( $\operatorname{scan} \# 5$ ) is given in Fig 7 . The OCl radical is positioned on the opposite side to the $\mathrm{H}$ atoms of the water molecule. The angle of . OCl to the water molecule plane is set $87^{\circ}$ with $\mathrm{R}_{\mathrm{OO}}=4.4 \AA$ as in scan $\# 2$ which results in the approximate symmetry of the potential curves for both states. The energy splitting is less than $0.5 \mathrm{kcal} / \mathrm{mol}$ except when $\theta^{\prime}$ is ranged from $\theta^{\prime}=100^{\circ}$ to $150^{\circ} \mathrm{kcal} / \mathrm{mol}$ with a maximum of $0.88 \mathrm{kcal} / \mathrm{mol}$ when $\theta^{\prime}=120^{\circ}$. The energy of the ground state $A^{\prime}$ has a 
binding energy $3.39 \mathrm{kcal} / \mathrm{mol}$ when $\theta^{\prime}$ is $93^{\circ}$ and this exactly matches the minimum configuration of scan \#2.

The next angular scan is the binding energy as a function of $\varphi$ (scan \#6) as graphed in

Fig 8. The $\mathrm{R}_{\mathrm{OO}}$ is fixed to be $3.15 \AA$. The $\mathrm{H}$ atom is kept collinear with the two $\mathrm{O}$ atoms all through the scan. The most important feature about this scan is that the energy splitting of the ground (A”) and excited (A') states is all less than $0.5 \mathrm{kcal} / \mathrm{mol}$, which means the excited state is significant to predict the chemical behavior of the structure with the rotation of the OCl radical. The two minima appear when the OCl radical is near collinear to the two $\mathrm{OH}$ bonds in the water molecule which gives a binding energy 1.82 and 1.69 for the two states. The two maxima are found when the OCl radical is on the bisector of $\mathrm{H}-\mathrm{O}-\mathrm{H}$ angle in the water molecule or its extension. This result is consistent with the study of the $\mathrm{H}_{2} \mathrm{O} \cdot \mathrm{OH}$ potential energy surface.

The last potential scan is a $\theta$ scan (scan \#7) as shown in Fig 9. The OCl radical is rotating along the plane passing the bisector of the water molecule and perpendicular to it. The $R_{0 O}$ is set as $3.05 \AA$, the minimum of scan \#3. As we see from the figure, the binding energy decreases with the increasing $\theta$ value and reaches the minimum when $\theta=90^{\circ}$ and all the atoms are coplanar. The energy of A' state is more stable than A" state but their difference is less than $0.5 \mathrm{kcal} / \mathrm{mole}$ when $\theta<75^{\circ}$. A point worthy of noting is that if we consider the results of scan \#3, scan \#6 and this scan, the minimum of scan \#7 or scan \#3 is probably corresponding to a first order stationary point or a transition state which resembles the potential energy surface of $\mathrm{H}_{2} \mathrm{O} \cdot \mathrm{OH}$.

So with the above calculations, we obtained the potential energy surface of $\mathrm{H}_{2} \mathrm{O} \cdot \mathrm{OCl}$, we find the PES resembles that of $\mathrm{H}_{2} \mathrm{O} \cdot \mathrm{OH}$ in some of the configurations. And as expected, the excited states of both of the two complexes have a energy difference less than $0.5 \mathrm{kcal} / \mathrm{mol}$ from the ground states, namely, very important in determining their chemical behaviors.

\section{B. The description of the empirical potentials}

All the fitted parameters for the $\mathrm{ClO} \cdot \mathrm{H}_{2} \mathrm{O}$ complex in mTTMC are listed in Table 3 and Table $\mathbf{4}$ for the ground and first excited states respectively. All the parameters are for the OCl radical since we use the same parameterizations for the water molecule as that in 
TTM2- $\mathrm{R}^{28-31}$ model. The OCl bond length is set as the $\operatorname{CCSD}(\mathrm{T})$ optimized geometry. As shown in the tables, the charges on the $\mathrm{Cl}$ atom and $\mathrm{O}$ atom of the $\mathrm{OCl}$ radical are $\pm 3.22 \times 10^{-20} \mathrm{C}$ and $\pm 4.58 \times 10^{-20} \mathrm{C}$ for the ground and excited states respectively. This is smaller than those of the $\mathrm{OH}$ radical. The ground state dipole moment is calculated as $1.14 \mathrm{D}$, comparable to our $\operatorname{CCSD}(\mathrm{T})$ prediction $1.23 \mathrm{D}$ and the experimental value $1.24 \mathrm{D}^{38}$ These results are reasonable because the $\mathrm{Cl}$ atom has a much higher electronegativity. The polarizability of the $\mathrm{O}$ atom has a larger component than the other two directions for the ground state, which is similar to that of the $\mathrm{OH}$ radical. But for the excited state, the $\mathrm{x}$ and $\mathrm{z}$ components are dominant. We find that the $\mathrm{Cl}$ atom has a much larger polarizabilty with main component in $\mathrm{x}$ direction for both states. The difference of the $\mathrm{Cl}$ atomic polarizability between the two states is in that the excited state has a much lower y component. The ground state molecular polarizability is estimated to be $3.02 \AA^{3}$ by averaging the diagonal components of the polarizability tensors and it is in excellent agreement with the estimation of $3.04 \AA^{3}$ at the level of CCSD(T)/aug-cc-pVTZ. As for the Lennard-Jones potential, we include all the possible combinations between the two bodies. The powers for the O-O interaction are fixed to be 12 and 6, and this interaction shows a consistent $\sigma$ value with that in the $\mathrm{H}_{2} \mathrm{O} \cdot \mathrm{OH}$ potential and a lower $\varepsilon$ than $\mathrm{H}_{2} \mathrm{O} \cdot \mathrm{OH}$ potential. The O1-Cl4 interaction is calculated to be stronger than O-O interaction for both states, which indicates a strong chemical bonding effect between the two atoms. The $\mathrm{O} 2-\mathrm{H} 2(3)$ interaction is much stronger in the ground state than in the excited state. Their magnitudes suggest that the chemical bonding between them only occurs when the system is in the ground state. In this potential, the $\mathrm{H} 2(3)-\mathrm{Cl} 4$ interaction is also considered since the $\mathrm{Cl}$ atom has a large van der Waals radii. The data show that this interaction has large $\sigma$ values but very small $\varepsilon$ values. This means that there is an additional repulsion to reproduce the PES of the $\mathrm{H}_{2} \mathrm{O} \cdot \mathrm{OCl}$ system.

The empirical potentials for scan \#1 shown in Fig $\mathbf{3}$ are consistent with the ab initio values. The fitted ground state energy is $2.92 \mathrm{kcal} / \mathrm{mol}$ at the minimum of $\mathrm{R}_{\mathrm{OO}}=3.00 \AA$, with a deviation of $0.1 \mathrm{kcal} / \mathrm{mol}$ in binding energy and $-0.05 \AA$ in geometry. The energies of fitted excited state also well resemble the A' state, whose minimum is predicted to be $\mathrm{R}_{\mathrm{OO}}=3.10 \AA, 0.1 \AA$ shorter than the $\mathrm{CCSD}(\mathrm{T})$ prediction. The energy difference between 
the ab initio and fitted values is less than or very slightly above $0.3 \mathrm{kcal} / \mathrm{mol}$ when $\mathrm{R}_{\mathrm{OO}}>$ $3.2 \AA$.

The mTTMC model prediction for scan \#2 is also satisfactory as shown in Fig 4. The energy deviations of the empirical potential for the states A' and A" at the minimum are only $0.03 \mathrm{kcal} / \mathrm{mol}$ with $\mathrm{R}_{\mathrm{OO}}$ errors $0.0 \AA$ and $-0.1 \AA$ respectively. When $\mathrm{R}_{\mathrm{OO}}>4.2 \AA$, the energy errors from the quantum chemical calculations are below $0.1 \mathrm{kcal} / \mathrm{mol}$ for the ground state and $0.5 \mathrm{kcal} / \mathrm{mol}$ for the excited state.

The performance of the current OCl potentials on the transition state (scan \#3 in Fig 5) is also in good agreement with the ab initio results. The deviation of the empirical potential is less than $0.15 \mathrm{kcal} / \mathrm{mol}$ when $\mathrm{R}_{\mathrm{OO}}>3.0 \AA$ for both A' and A" states. At the minimum points, the prediction error of the parameterized potential is only -0.05 and $-0.10 \AA$ in $\mathrm{R}_{\mathrm{OO}}$ for the ground and excited states respectively and approximately $0.01 \mathrm{kcal} / \mathrm{mol}$ in binding energy for both.

Our new OCl potential also gives satisfactory prediction on the angular scans.

As shown in Fig 6, the two states of the empirical potential correctly separate in scan \#4. For the ground state, the empirical potential gives two minimums with binding energies of 2.52 and $3.29 \mathrm{kcal} / \mathrm{mol}$ located at $\varphi^{\prime}=-70^{\circ}$ and $80^{\circ}$ (beyond the range of the figure) respectively. These results resemble the ab initio calculations which predict $-70^{\circ}$ and $70^{\circ}$ as two minimums as observed in the figure. As for the excited state, the empirical potential has a binding energy of 1.92 at the minimum, which agrees very well with the $\operatorname{CCSD}(\mathrm{T})$ prediction although the $\varphi^{\prime}$ angle has a 30 degrees difference. When $\varphi$ ' is in the range of $\left[-40^{\circ}, 30^{\circ}\right]$, the energy deviation of both of the two fitted states is less than $0.2 \mathrm{kcal} / \mathrm{mol}$.

Scan \#5 plotted in Fig 7 is also fitted with good accuracy. The energy splitting is well mimicked by the Thole Type model. The deviations are less than $0.3 \mathrm{kcal} / \mathrm{mol}$ when $\theta^{\prime}$ is in the range of $\left[0^{\circ}, 30^{\circ}\right],\left[78^{\circ}, 108^{\circ}\right]$ and $\left[156^{\circ}, 180^{\circ}\right]$. The minimum point $\theta^{\prime}=93^{\circ}$ and the minimum point $\theta^{\prime}=0^{\circ}$ are both correctly located with binding energy error no more than $0.07 \mathrm{kcal} / \mathrm{mol}$ for both of the A' and A" states.

The $\varphi$ scan is well fitted with the mTTMC model for both A' and A" states as seen in Fig 8. The maximum and minimum points found by the empirical potential are consistent with the prediction of the quantum chemical calculations for both states except a $-10^{\circ}$ 
error for the ground state at $\varphi=270^{\circ}$. The prediction fitted ground state has a maximum deviation of $0.15 \mathrm{kcal} / \mathrm{mol}$ at $\varphi=0^{\circ}$, which is probably due to the difficulty in fitting the flatness of the $\operatorname{CCSD}(\mathrm{T}) \mathrm{PES}$ when $\mathrm{OCl}$ is collinear to the $\mathrm{OH}$ bond of the water molecule. But with $\varphi$ in the range of $20^{\circ}$ to $230^{\circ}$, the binding energy deviation is lower than $0.1 \mathrm{kcal} / \mathrm{mol}$. In contrast, the excited state is better fitted when $260^{\circ}<\varphi<360^{\circ}$ with deviation within $0.1 \mathrm{kcal} / \mathrm{mol}$. The error for the excited state is all less than 0.30 $\mathrm{kcal} / \mathrm{mol}$.

For the last scan (scan \#7 in Fig 9), the results of the mTTMC prediction are also satisfactory. Both empirical potentials show a minimum at $\theta=90^{\circ}$ as the $\operatorname{CCSD}(\mathrm{T})$ potential with an error of $0.02 \mathrm{kcal} / \mathrm{mol}$ and $0.01 \mathrm{kcal} / \mathrm{mol}$ respectively for $\mathrm{A}^{\prime}$ and A" states respectively. The error is lower than $0.24 \mathrm{kcal} / \mathrm{mol}$ all over the potential scan for both states.

As discussed above, by reparameterization of the modified TTM potential, we obtained empirical potentials of $\mathrm{H}_{2} \mathrm{O} \cdot \mathrm{OCl}$ system with rather good quality. The overall comparison of the empirical potential and the $a b$ intio results are shown in Fig 10 and Fig 11 for ground and excited states respectively. In these figures, we show the energy difference by $\triangle E=E^{a b}$ initio $-E^{\text {TTM }}$ for all the scans. As seen from the curve, the perturbation of the energy error mostly occurs around 0 for both states. The ground state energy shows a better accuracy except only one point of the repulsive wall in Scan \#1. When the binding energy is around $-2 \mathrm{kcal} / \mathrm{mol}$, some negative error can be seen in Scan \#5 for the ground state. The error of the excited state also behaves very reasonably and all the error is in the range of $\pm 1 \mathrm{kcal} / \mathrm{mol}$. The figures illustrate that the performance of our parameterization is satisfactory in predicting the stabilization of the complex.

In order to quantitatively show the error of the mTTMC potential, we summarize the average difference $(\overline{\Delta E})$, average of the absolute difference $(\overline{|\Delta E|})$, the variance of the signed difference $(\sigma(\Delta E))$, and the maximum difference $(\max (\mid \overline{|\Delta E|}))$ between the CCSD(T) calculation results and the mTTMC potentials as shown in Table 5. The overall average errors are both very close to 0 for ground and excited states. The average absolute error is only $0.09 \mathrm{kcal} / \mathrm{mol}$ for the ground state when the interaction energy is less than $-3 \mathrm{kcal} / \mathrm{mol}$. The corresponding variance of the error with the same cutoff 
energy is also the least, which is $0.12 \mathrm{kcal} / \mathrm{mol}$. This is an indication that the minimum points of the ground state are well reproduced by the mTTMC potential. The largest $\sigma(\Delta E)$ value of $0.25 \mathrm{kcal} / \mathrm{mol}$ and a $0.17 \mathrm{kcal} / \mathrm{mol}$ of $\overline{|\Delta E|}$ can be found when the interaction energy is below $-2 \mathrm{kcal} / \mathrm{mol}$, which reflect the error in Scan \#5 as shown in Fig 10. Approximately 180 points of the ground state potential energy surface are in the range of -2 to $0 \mathrm{kcal} / \mathrm{mol}$. The $\overline{|\Delta E|}$ in this range is only $0.13 \mathrm{kcal} / \mathrm{mol}$, showing very good accuracy. For the excited state, the predicted absolute error can be found slightly larger than the ground state. The largest absolute error, which is $0.94 \mathrm{kcal} / \mathrm{mol}$, in the excited state is found in Scan \#4. The distribution of the points for excited state is also most in the range of -2 and $0 \mathrm{kcal} / \mathrm{mol}$ (up to 206 points). The $\sigma(\Delta E)$ value of the excited state is about $0.3 \mathrm{kcal} / \mathrm{mol}$.

The comparison of the above results with those that we obtained from the mTTM potential for the $\mathrm{H}_{2} \mathrm{O} \cdot \mathrm{OH}$ system show that the newly fitted modified TTM potential for the $\mathrm{H}_{2} \mathrm{O} \cdot \mathrm{OCl}$ is more accurate. This indicates that the TTM functional form with anisotropic polarizabilities behaves better for $\mathrm{H}_{2} \mathrm{O} \cdot \mathrm{OCl}$ than $\mathrm{H}_{2} \mathrm{O} \cdot \mathrm{OH}$.

With the newly built potential, we performed energy minimization of the $\mathrm{H}_{2} \mathrm{O} \cdot \mathrm{OCl}$ complex. Two minima are obtained. The global minimum is predicted to be the one by Fu. et al. ${ }^{17}$ in Fig 12. And the local minimum is close to the geometry calculated by Francisco et al. ${ }^{11,16}$, but with a planar structure as in Fig 13. We reoptimized the two minima of the complex at the level of $\operatorname{CCSD}(\mathrm{T}) /$ aug-cc-pVTZ. The geometry parameters are shown in Fig 12 and Fig 13. The binding energy of the global and local minimums are predicted to be $3.42 \mathrm{kcal} / \mathrm{mol}$ and $3.22 \mathrm{kcal} / \mathrm{mol}$ and their corresponding dipole moments are 5.01 D and 1.18 D respectively. The closeness of the two minima strongly suggests that both of the two $\mathrm{H}_{2} \mathrm{O} \cdot \mathrm{OCl}$ complex configuration may exist with a significant population. This conclusion is in very good agreement with the prediction by our mTTMC model since the energy difference of the local minimum from the global minimum predicted by our empirical potential is lower than $0.02 \mathrm{kcal} / \mathrm{mol}$. This finding may have significant implication for the experimental study of the interaction between the OCl radical and water. 
PNNL-16185

\section{Conclusion}

In this work, we performed ab initio calculations on the PES of the $\mathrm{H}_{2} \mathrm{O} \bullet \mathrm{OCl}$ system at the level of CCSD(T)/aug-cc-pVTZ. From the computational results, we find that it is in

good comparison with that of the $\mathrm{H}_{2} \mathrm{O} \cdot \mathrm{OH}$ complex. The empirical potential has been constructed with the TTM functional form. Our fitted empirical potentials well resemble the ab initio results and the optimized structures of the $\mathrm{H}_{2} \mathrm{O} \cdot \mathrm{OCl}$ complex also agrees well with previous predictions.

\section{References}

1. Stumm, W.; Morgan, J.J. Aquatic Chemistry; Wiley; New York, 1996

2. R. L. Mauldin III, S. Madronich, S. J. Flocke, and F.L. Eisele, G.J. Frost, and A.S.H. Pervot, Geophys. Res. Lett. 24, 3033 (1997)

3. M. Poeselova, P. Jungwirth, D. J. Tobias, and R. B. Gerber, J. Phys. Chem. B. 107, 12690 (2003)

4. P. Cabral do Couto, R. C. Guedes, and B. J. Costa Carbral, J. Chem. Phys., 119, 7344 (2003)

5. A. C. Legon, and D. J. Millen, Chem. Rev. 86, 635 (1986)

6. S. A. Cooke, G. K. Gorlett, C. M. Evans, and A. C. legon, Chem. Phys. Lett. 272, 61 (1997)

7. P. Hobza, J. Sponser, and T. Beschel, J. Comput. Chem. 16, 1315 (1995)

8. A. C. Legon, and D. J. Millen, Acc. Chem. Res. 20, 39 (1987)

9. J. Lundell, and Z. Latajka, J. Phys. Chem. 101, 5004 (1997)

10. A. C. Legon, Chem. Phys. Lett. 237, 291 (1995)

11. Y. Li, and J. S. Francisco, J. Chem. Phys. 115, 8381 (2001)

12. R. A. Cox, and G. D. Hayman, Nature 332, 796 (1988)

13. L. T. Molina, and M. J. Molina, J. Phys. Chem. 91, 433 (1987)

14. M. J. Molina, Science 238, 1253 (1987)

15. S. P. Sander, R. R. Friedl, and Y. L. Yung, Science 245, 1095 (1989)

16. J. S. Francisco and S. P. Sander J. Am. Chem. Soc. 117, 9917 (1995)

17. H. Fu, Z. Zhou, and X. Zhou, Chem. Phys. Lett., 382, 466 (2003)

18. A. D. Becke, J. Chem. Phys. 98, 5648 (1993) 
PNNL-16185

19. C. Lee, W. Yang, and R. G. Parr, Phys. Rev. B 37, 785 (1988)

20. M. J. Frisch, M. Head-Gordon, and J. A. Pople, Chem. Phys. Lett. 166, 281 (1990)

21. M. Head-Gordon and T. Head-Gordon, Chem. Phys. Lett. 220, 122 (1994)

22. C. Moeller and M. S. Plesset, Phys. Rev. 46, 618 (1934)

23. C. Hampel and H. -J. Werner, J. Chem. Phys. 1046286 (1996);

24. M. Schütz and H.-J. Werner, J. Chem. Phys. 114, 661 (2001);

25. M. Schütz and H.-J. Werner, Chem. Phys. Lett. 318, 370 (2000);

26. M. Schütz, J. Chem. Phys. 113, 9986 (2000).

27. D. P. Schofield and H. G. Kjaergaard, J. Chem. Phys. 120, 6930 (2004)

28. C. J. Burnham, J. C. Li, S. S. Xantheas, and M. Leslie, J. Chem. Phys. 110, 4566 (1999)

29. C. J. Burnham and S. S. Xantheas, J. Chem. Phys. 116, 1479 (2002)

30. S. S. Xantheas, C. J. Burnham, and R. J. Harrison J. Chem. Phys. 116, 1493 (2002)

31. C. J. Burnham and S. S. Xantheas, J. Chem. Phys. 116, 1500 (2002)

32. R. D. Amos, A. Bernhardsson, and A. Berning, et al. MOLPRO, a package of ab initio programs

33. See http://www.emsl.pnl.gov for more information

34. M. W. Mahoney and W. L. Jorgensen, J. Chem. Phys. 114, 363 (2001)

35. A. Glattli, X. Daura, and W. F. van Gunsteren, J. Chem. Phys. 116, 9811(2002)

36. H. Yu, T. Hansson, and W. F. van Gunsteren, J. Chem. Phys. 118, 221(2003)

37. H. Yu and W. F. van Gunsteren, J. Chem. Phys. 121, 9549(2004)

38. NIST webbook. See http://webbook.nist.gov/chemistry/form-ser.html. 
Table 1. The geometry of CCSD(T)/aug-cc-pvtz optimized $\mathrm{H}_{2} \mathrm{O}$ and OCl.

$\mathrm{r}_{\mathrm{OH}}^{\mathrm{w}}(\AA) \quad \theta_{\mathrm{w}}$ (degrees) $\mathrm{r}_{\mathrm{OCl}}(\AA)$

0.9616005

104.186

1.5936

Table 2. The variables and parameters of CCSD(T)/aug-cc-pVTZ PES scan of $\mathrm{H}_{2} \mathrm{O} \cdot \mathrm{OH}$.

\begin{tabular}{|c|c|c|c|c|c|}
\hline Scan \# & $\begin{array}{l}\mathrm{R}_{\mathrm{OO}} \\
(\AA)\end{array}$ & $\begin{array}{c}\theta \\
\text { (degrees) }\end{array}$ & $\begin{array}{c}\varphi \\
\text { (degrees) }\end{array}$ & $\begin{array}{c}\theta^{\prime} \\
\text { (degrees) }\end{array}$ & $\begin{array}{c}\varphi^{\prime} \\
\text { (degrees) }\end{array}$ \\
\hline 1 & Variable & 90 & 0 & 90 & 70 \\
\hline 2 & Variable & 87 & 128 & 3 & 52 \\
\hline 3 & Variable & 90 & -52 & 90 & -52 \\
\hline 4 & 3.15 & 90 & 0 & 90 & Variable \\
\hline 5 & 4.40 & 87 & 128 & Variable & -52 \\
\hline 6 & 3.15 & 90 & Variable & 90 & $=\varphi$ \\
\hline 9 & 3.05 & Variable & -52 & $=\theta$ & -52 \\
\hline
\end{tabular}


Table 3. Parameterization of the OCl radical in empirical ground state potential.

\begin{tabular}{|c|c|c|c|c|c|}
\hline \multicolumn{2}{|c|}{$\mathrm{Q}_{\mathrm{Cl}}$ (electronic charge) } & $3.216 \times 10^{-20} \mathrm{C}$ & \multicolumn{2}{|c|}{ Qo (electronic charge) } & \multirow{2}{*}{$\begin{array}{c}-3.216 \times 10^{-20} \mathrm{C} \\
0.074\end{array}$} \\
\hline$\alpha_{\mathrm{O}, \mathrm{xx}}\left(\AA^{3}\right)$ & 0.074 & $\alpha_{O, y y}\left(\AA^{3}\right)$ & 1.477 & $\alpha_{\mathrm{O}, \mathrm{zz}}\left(\AA^{3}\right)$ & \\
\hline$\alpha_{\mathrm{Cl}, \mathrm{xx}}\left(\AA^{3}\right)$ & 4.446 & $\alpha_{\mathrm{Cl}, \mathrm{yy}}\left(\AA^{3}\right)$ & 2.848 & $\alpha_{\mathrm{Cl}, \mathrm{zz}}\left(\AA^{3}\right)$ & 0.148 \\
\hline$\sigma_{\mathrm{OO}}(\AA)$ & 2.986 & $\varepsilon_{\mathrm{OO}}(\mathrm{kcal} / \mathrm{mol})$ & 0.186 & M-O distance in $\mathrm{OH}(\AA)$ & 0.407 \\
\hline$\sigma_{\mathrm{O} 1 \mathrm{Cl} 4}(\AA)$ & 2.567 & $\varepsilon_{\mathrm{O} 1 \mathrm{Cl} 4}(\mathrm{kcal} / \mathrm{mol})$ & 0.961 & $\mathrm{n}, \mathrm{m}^{*}$ & $10.35,4.00$ \\
\hline$\sigma_{\mathrm{O} 2 \mathrm{H} 3}(\AA)$ & 2.001 & $\varepsilon_{\mathrm{O} 2 \mathrm{H} 3}(\mathrm{kcal} / \mathrm{mol})$ & 0.193 & $\mathrm{n}, \mathrm{m} *$ & $9.00,4.00$ \\
\hline$\sigma_{\mathrm{Cl} 4 \mathrm{H} 3}(\AA)$ & 3.295 & $\varepsilon_{\mathrm{Cl} 4 \mathrm{H} 3}(\mathrm{kcal} / \mathrm{mol})$ & 0.050 & $\mathrm{n}, \mathrm{m}^{*}$ & $9.01,5.61$ \\
\hline
\end{tabular}

Table 4. Parameterization of the OCl radical in empirical first excited state potential.

\begin{tabular}{cccccc}
\hline \multicolumn{2}{c}{$\mathrm{Q}_{\mathrm{Cl}}($ electronic charge $)$} & $4.582 \times 10^{-20} \mathrm{C}$ & \multicolumn{2}{c}{ Qo $($ electronic charge } & $-4.582 \times 10^{-20} \mathrm{C}$ \\
$\alpha_{\mathrm{O}, \mathrm{xx}}\left(\AA^{3}\right)$ & 0.593 & $\alpha_{\mathrm{O}, \mathrm{yy}}\left(\AA^{3}\right)$ & 0.074 & $\alpha_{\mathrm{O}, \mathrm{zz}}\left(\AA^{3}\right)$ & 0.593 \\
$\alpha_{\mathrm{Cl}, \mathrm{xx}}\left(\AA^{3}\right)$ & 4.446 & $\alpha_{\mathrm{Cl}, \mathrm{yy}}\left(\AA^{3}\right)$ & 0.297 & $\alpha_{\mathrm{Cl}, \mathrm{zz}}\left(\AA^{3}\right)$ & 0.148 \\
$\sigma_{\mathrm{OO}}(\AA)$ & 2.981 & $\varepsilon_{\mathrm{OO}}(\mathrm{kcal} / \mathrm{mol})$ & 0.216 & $\mathrm{M}-\mathrm{O}$ distance in $\mathrm{OH}(\AA)$ & 0.635 \\
$\sigma_{\mathrm{O} 1 \mathrm{Cl} 4}(\AA)$ & 2.713 & $\varepsilon_{\mathrm{O} 1 \mathrm{Cl} 4}(\mathrm{kcal} / \mathrm{mol})$ & 0.542 & $\mathrm{n}, \mathrm{m} *$ & $13.16,8.78$ \\
$\sigma_{\mathrm{O} 2 \mathrm{H} 3}(\AA)$ & 1.810 & $\varepsilon_{\mathrm{O} 2 \mathrm{H} 3}(\mathrm{kcal} / \mathrm{mol})$ & 0.031 & $\mathrm{n}, \mathrm{m}^{*}$ & $10.40,6.00$ \\
$\sigma_{\mathrm{Cl} 4 \mathrm{H} 3}(\AA)$ & 3.338 & $\varepsilon_{\mathrm{Cl} 4 \mathrm{H} 3}(\mathrm{kcal} / \mathrm{mol})$ & 0.043 & $\mathrm{n}, \mathrm{m}^{*}$ & $13.57,4.80$ \\
\hline \multicolumn{7}{c}{}
\end{tabular}




\begin{tabular}{|c|c|c|c|c|c|c|c|c|c|c|}
\hline \multirow[b]{2}{*}{$\begin{array}{c}E_{c u t} \\
(\mathrm{kcal} / \mathrm{mol})\end{array}$} & \multicolumn{4}{|c|}{ Ground State } & & \multicolumn{4}{|c|}{ Excited State } & \multirow[b]{2}{*}{$N\left(E_{\text {cut }}\right)$} \\
\hline & $\begin{array}{c}\overline{\Delta E} \\
(\mathrm{kcal} / \mathrm{mol})\end{array}$ & $\begin{array}{c}\overline{|\Delta E|} \\
(\mathrm{kcal} / \mathrm{mol})\end{array}$ & $\begin{array}{c}\sigma(\Delta E) \\
(\mathrm{kcal} / \mathrm{mol})\end{array}$ & $\begin{array}{c}\max (|\Delta E|) \\
(\mathrm{kcal} / \mathrm{mol})\end{array}$ & $N\left(E_{\text {cut }}\right)$ & $\begin{array}{c}\overline{\Delta E} \\
\text { (kcal/mol) }\end{array}$ & $\begin{array}{c}\overline{|\Delta E|} \\
(\mathrm{kcal} / \mathrm{mol})\end{array}$ & $\begin{array}{c}\sigma(\Delta E) \\
(\mathrm{kcal} / \mathrm{mol})\end{array}$ & $\begin{array}{c}\max (|\Delta E|) \\
(\mathrm{kcal} / \mathrm{mol})\end{array}$ & \\
\hline-3 & -0.02 & 0.09 & 0.12 & 0.29 & 20 & 0.10 & 0.11 & 0.13 & 0.31 & 7 \\
\hline-2 & 0.00 & 0.17 & 0.25 & 0.73 & 69 & 0.08 & 0.31 & 0.35 & 0.51 & 26 \\
\hline-1 & -0.05 & 0.15 & 0.22 & 0.74 & 154 & -0.04 & 0.21 & 0.30 & 0.94 & 121 \\
\hline 0 & -0.07 & 0.13 & 0.19 & 0.74 & 243 & -0.03 & 0.19 & 0.28 & 0.94 & 232 \\
\hline 1 & -0.06 & 0.13 & 0.20 & 1.13 & 251 & -0.03 & 0.19 & 0.28 & 0.94 & 249 \\
\hline 3 & & & & & & -0.03 & 0.19 & 0.28 & 0.94 & 251 \\
\hline
\end{tabular}

Table 5. Average error $\overline{\Delta E}$, average unsigned error $|\overline{\Delta E}|$, variance of the average error $\sigma(\Delta E)$, and the maximum unsigned error $\max (|\Delta E|)$ in the mTTM fit to the CCSD(T)/aug-cc-pVTZ predicted ground and excited states of the $\mathrm{H}_{2} \mathrm{O} \cdot \mathrm{OCl}$ complex. The results are obtained corresponding to the number of points $N\left(E_{\text {cut }}\right)$ with cutoff energy $E_{\text {cut }}$. 


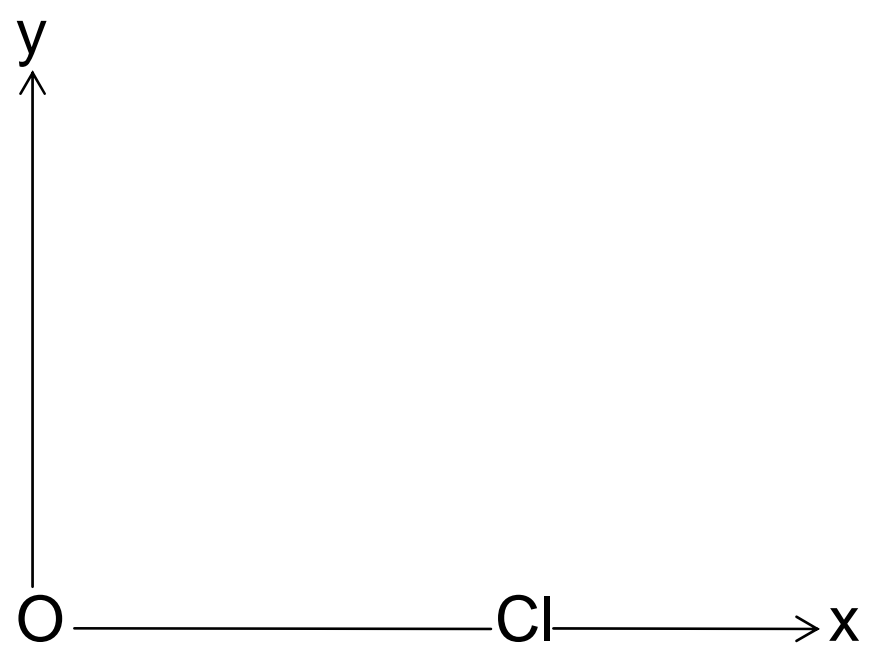

Fig 1. The coordinate setup fro the OCl radical monomer

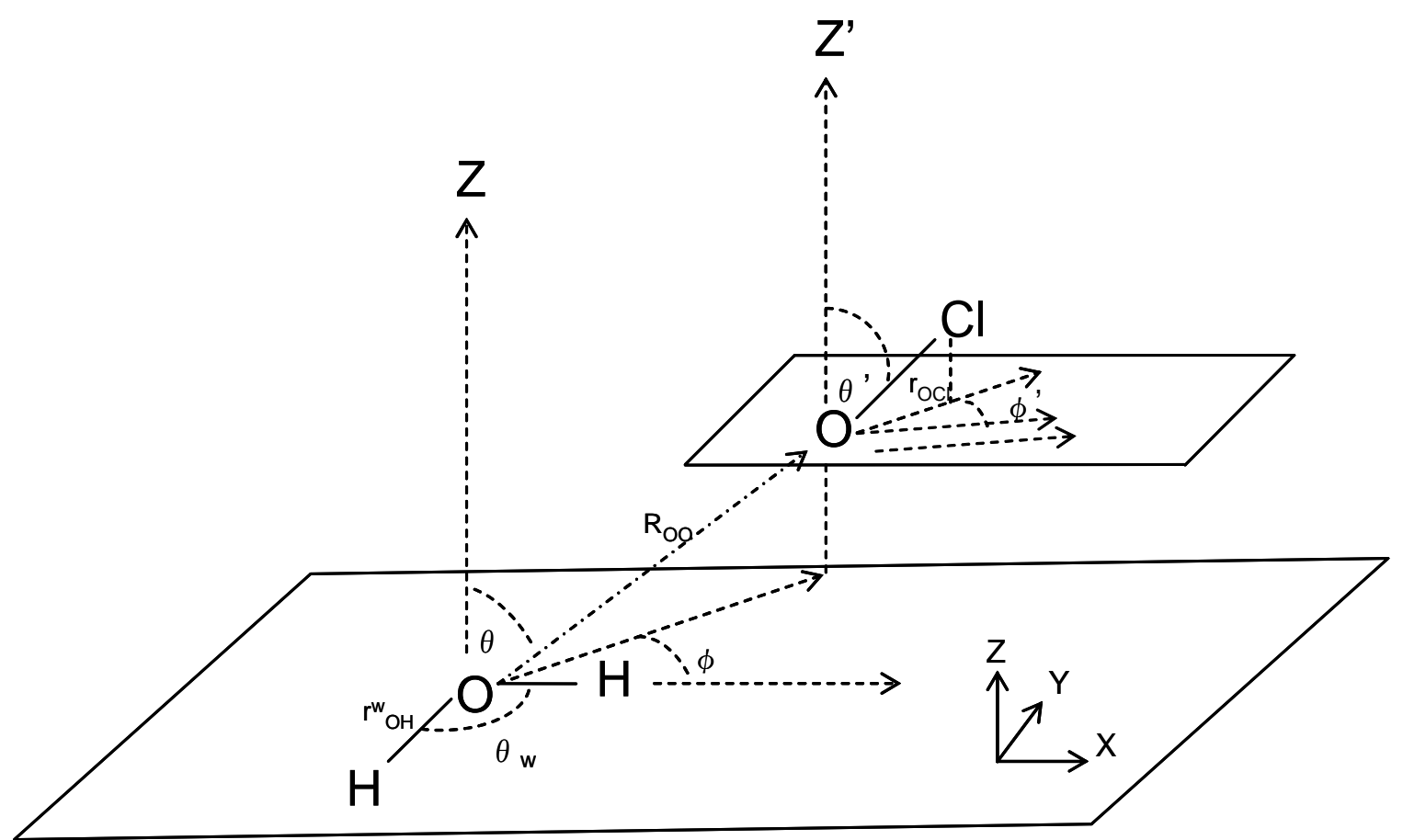

Fig 2. The coordinate setup for the $\mathrm{H}_{2} \mathrm{O} \cdot \mathrm{OCl}$ complex 
PNNL-16185

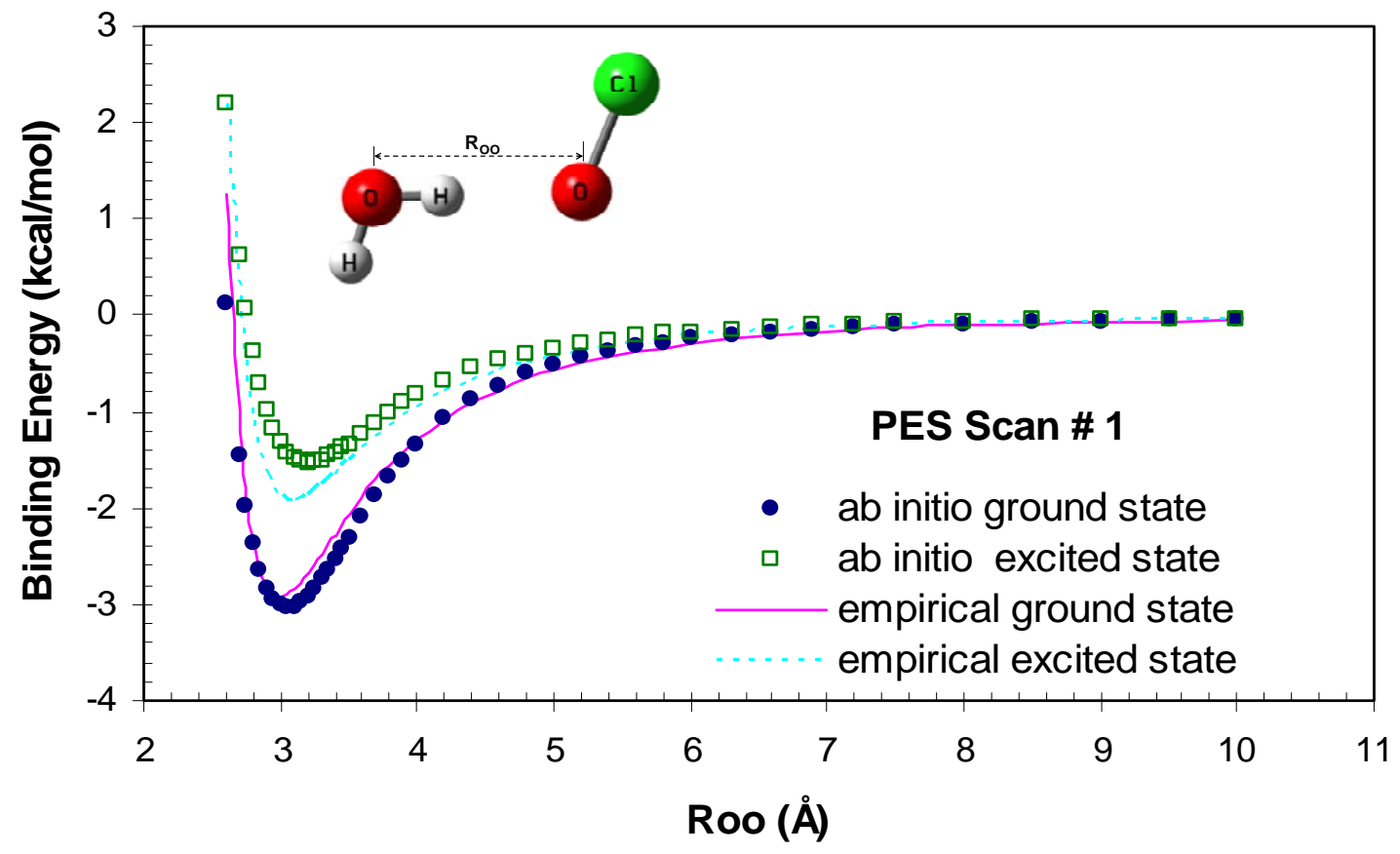

Fig 3. Potential Scan \#1. Interaction energy vs $\mathrm{R}_{\mathrm{OO}}$ for the ground and excited states predicted by CCSD(T) calculations and mTTMC potential. 


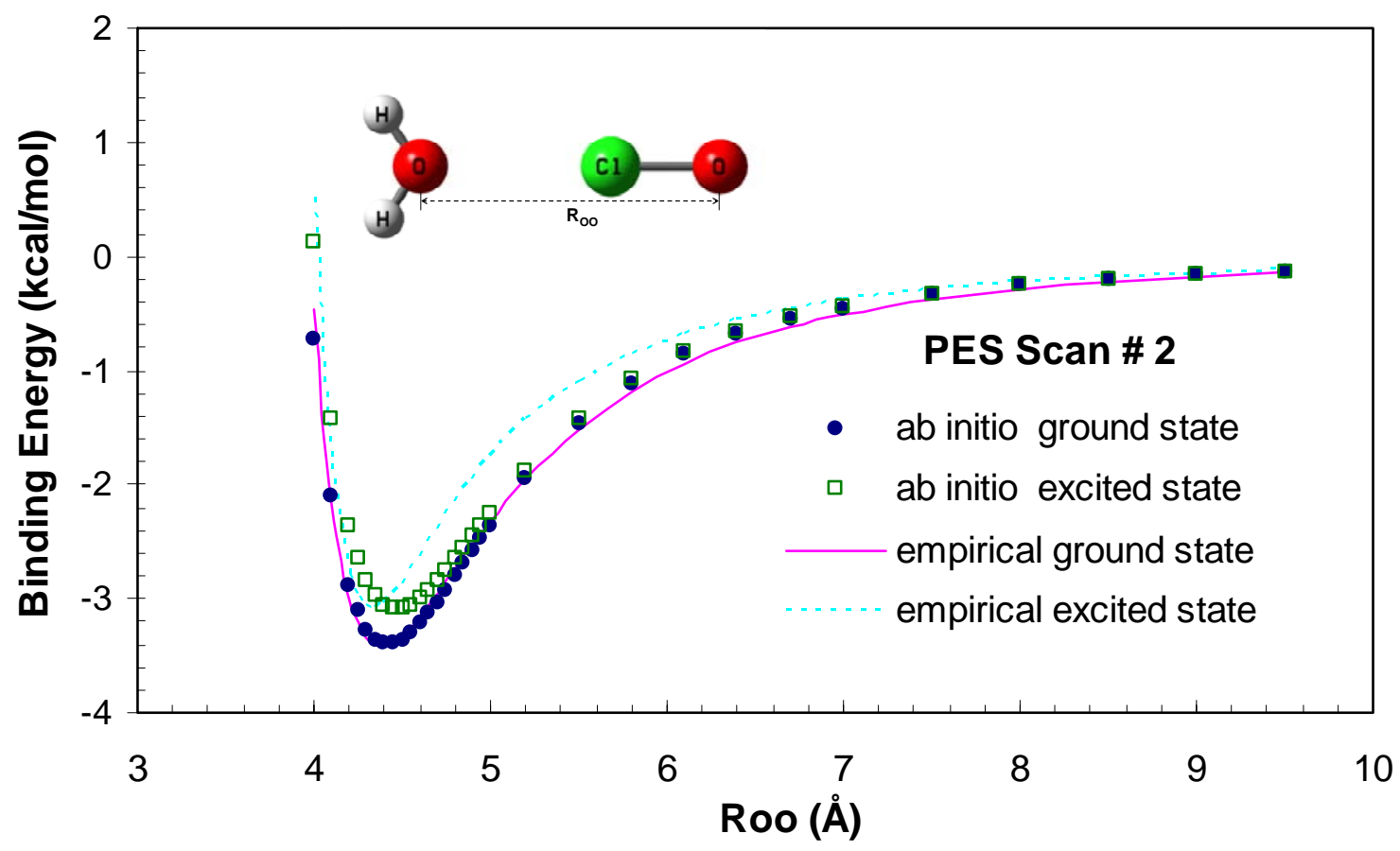

Fig 4. Potential Scan \#2. Interaction energy vs $\mathrm{R}_{\mathrm{OO}}$ for the ground and excited states predicted by CCSD(T) calculations mTTMC potential.

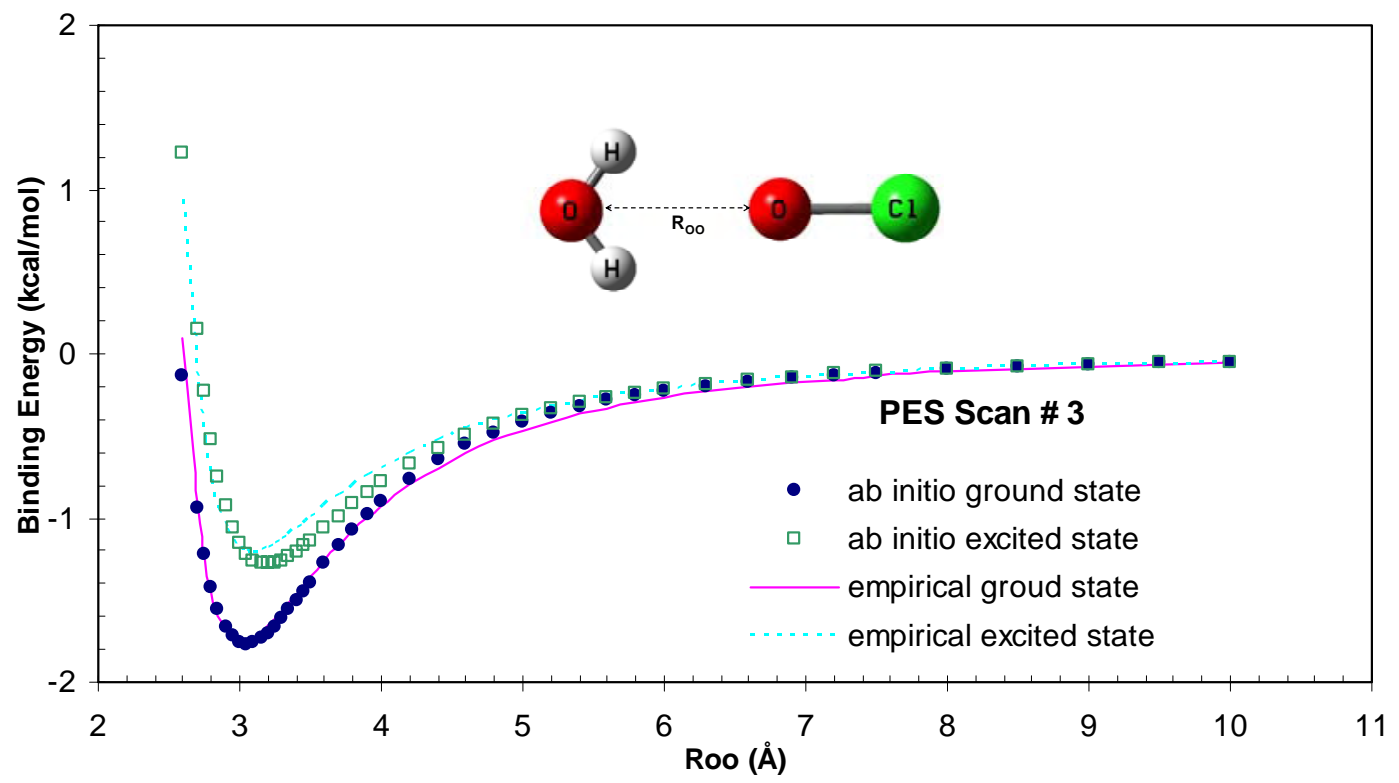

Fig 5. Potential Scan \#3. Interaction energy vs $\mathrm{R}_{\mathrm{OO}}$ for the ground and excited states predicted by CCSD(T) calculations and mTTMC potential. 


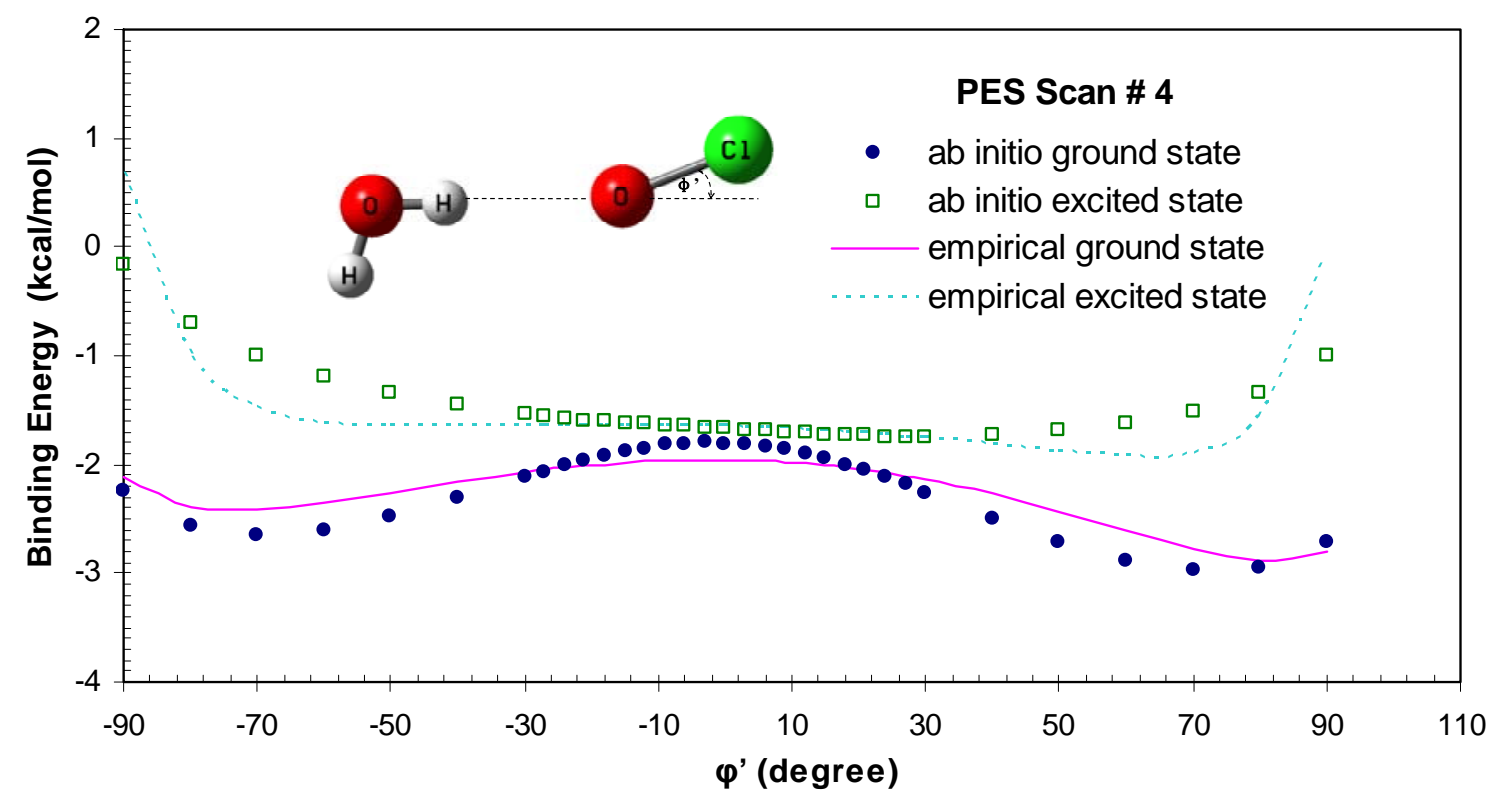

Fig 6. Potential Scan \#4. Interaction energy vs $\varphi^{\prime}$ for the ground and excited states predicted by CCSD(T) calculations and mTTMC potential.

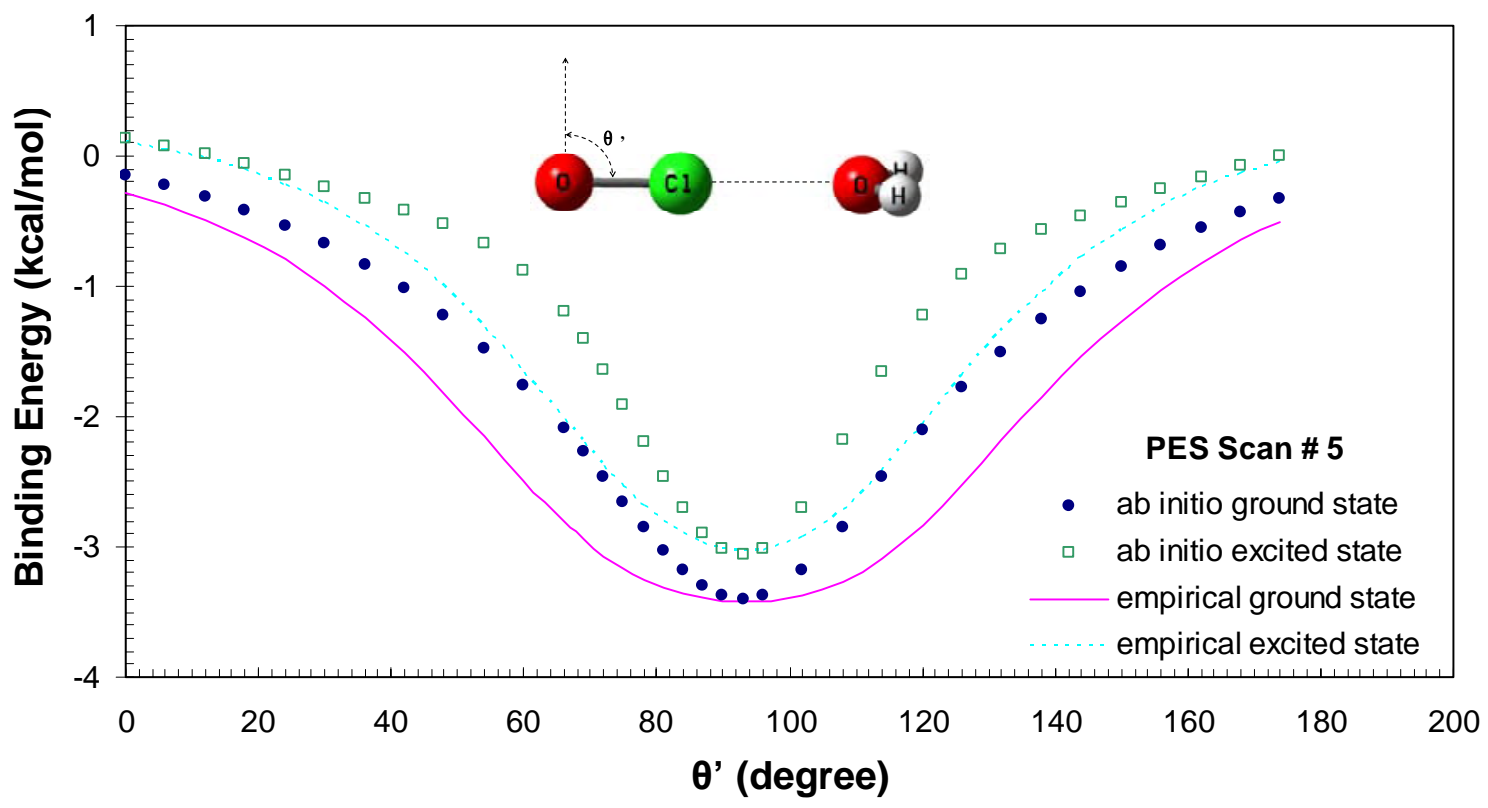

Fig 7. Potential Scan \#5. Interaction energy vs $\theta$ ' for the ground and excited states predicted by CCSD(T) calculations and mTTMC potential. 


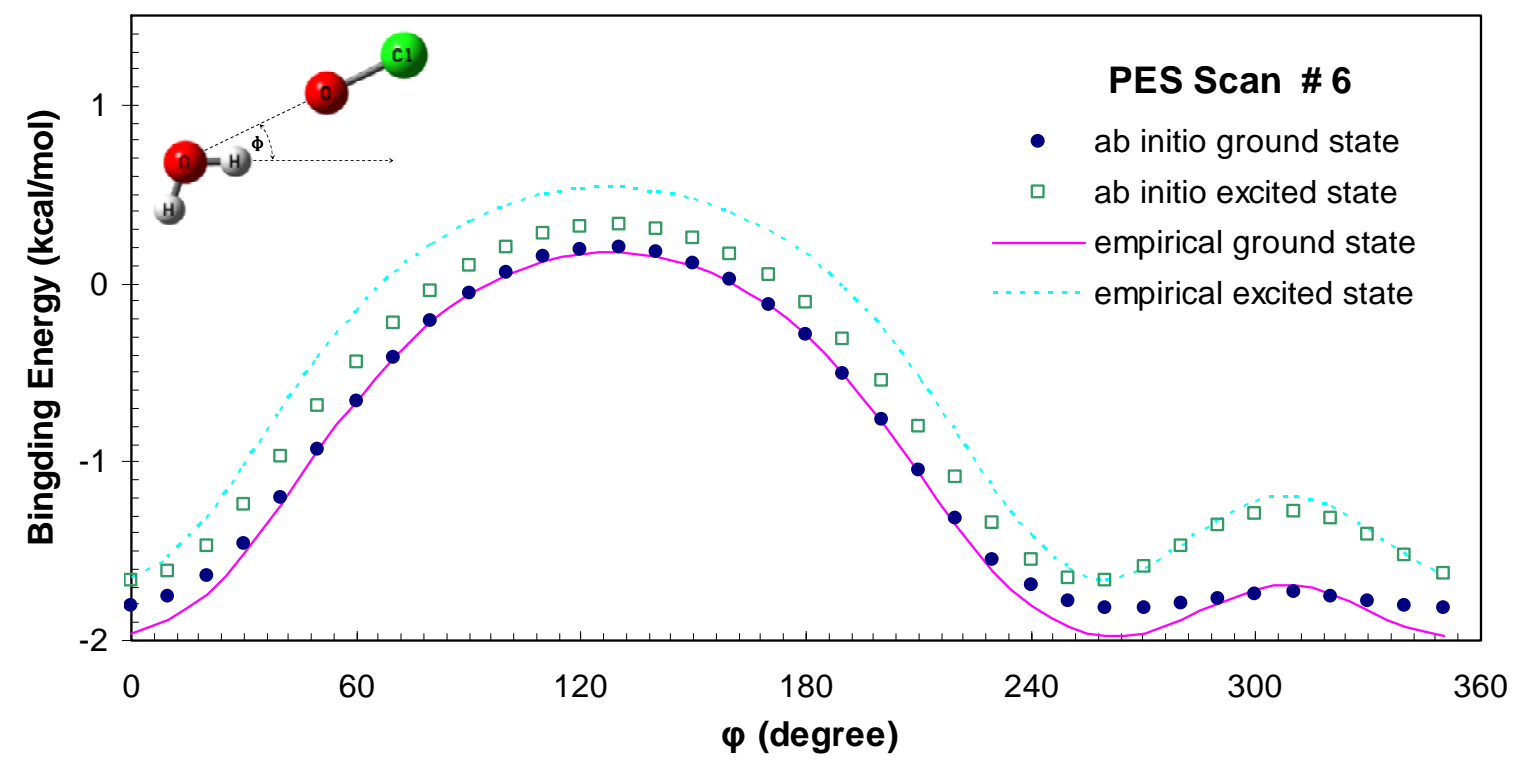

Fig 8. Potential Scan \#6. Interaction energy vs $\varphi$ for the ground and excited states predicted by $\operatorname{CCSD}(\mathrm{T})$ calculations and mTTMC potential.

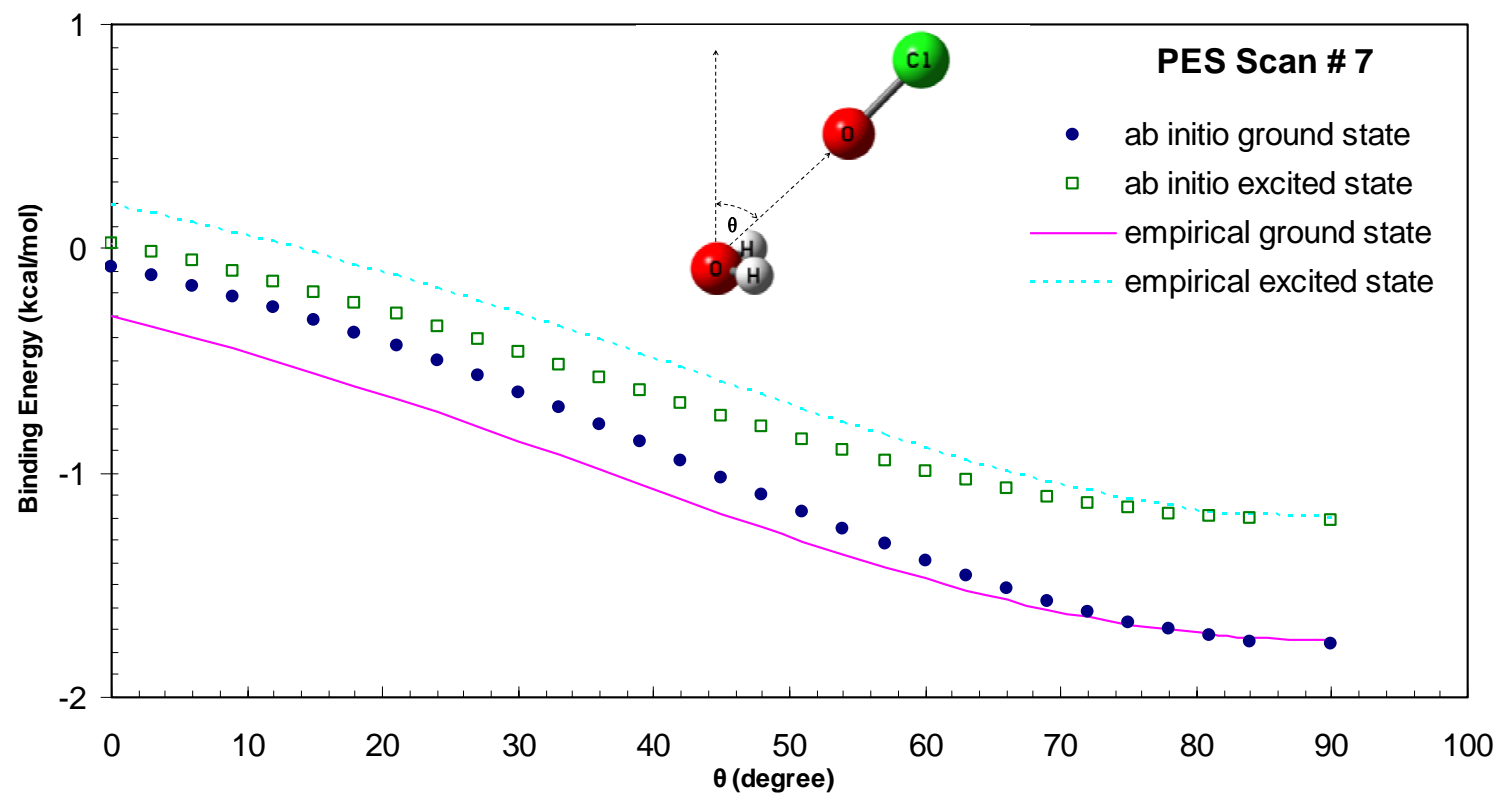

Fig 9. Potential Scan \#7. Interaction energy vs $\theta$ for the ground and excited states predicted by CCSD(T) calculations and mTTMC potential. 
PNNL-16185

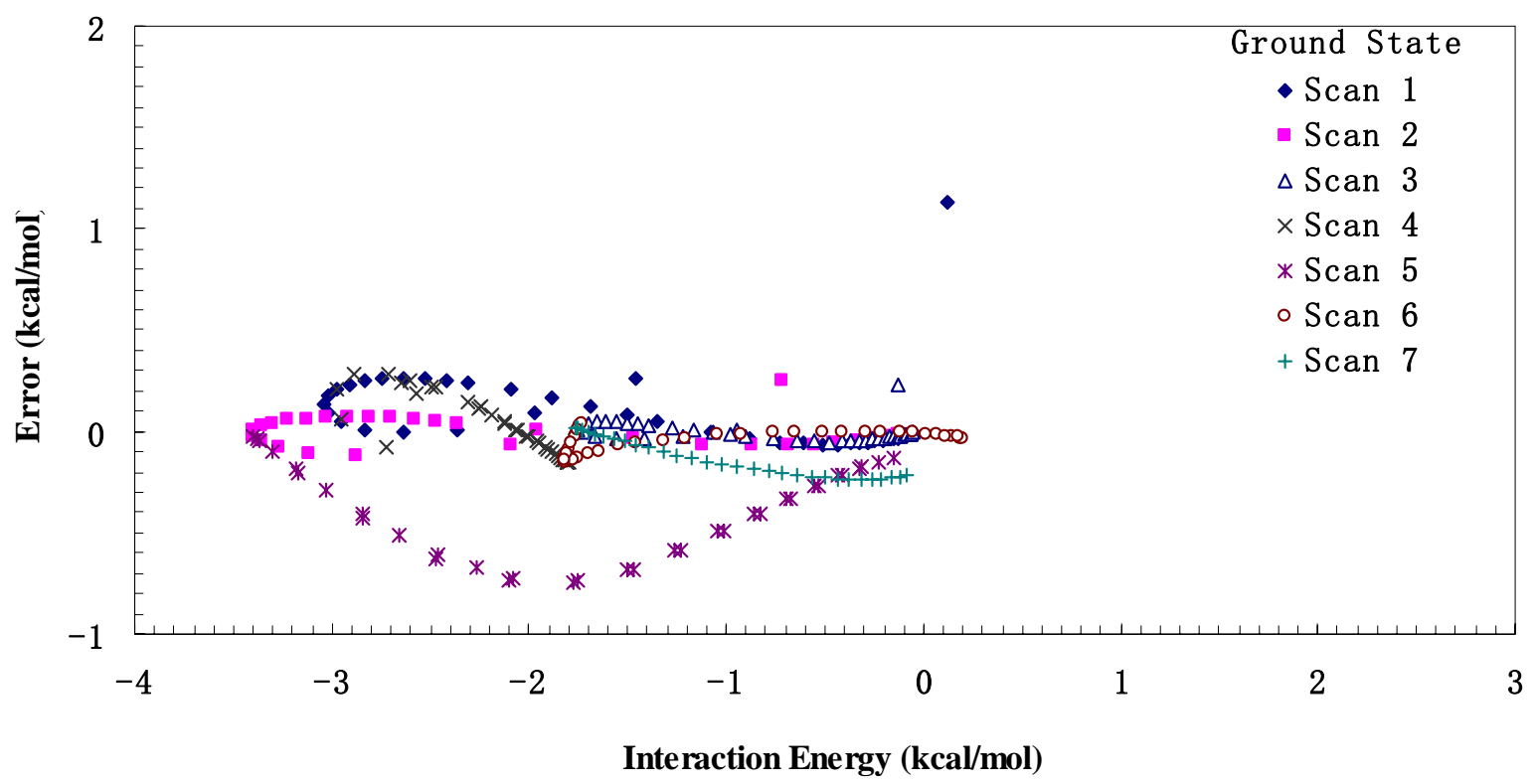

Fig 10. Errors in parameterized ground state potential as a function of the CCSD(T) energy for the PES scans.

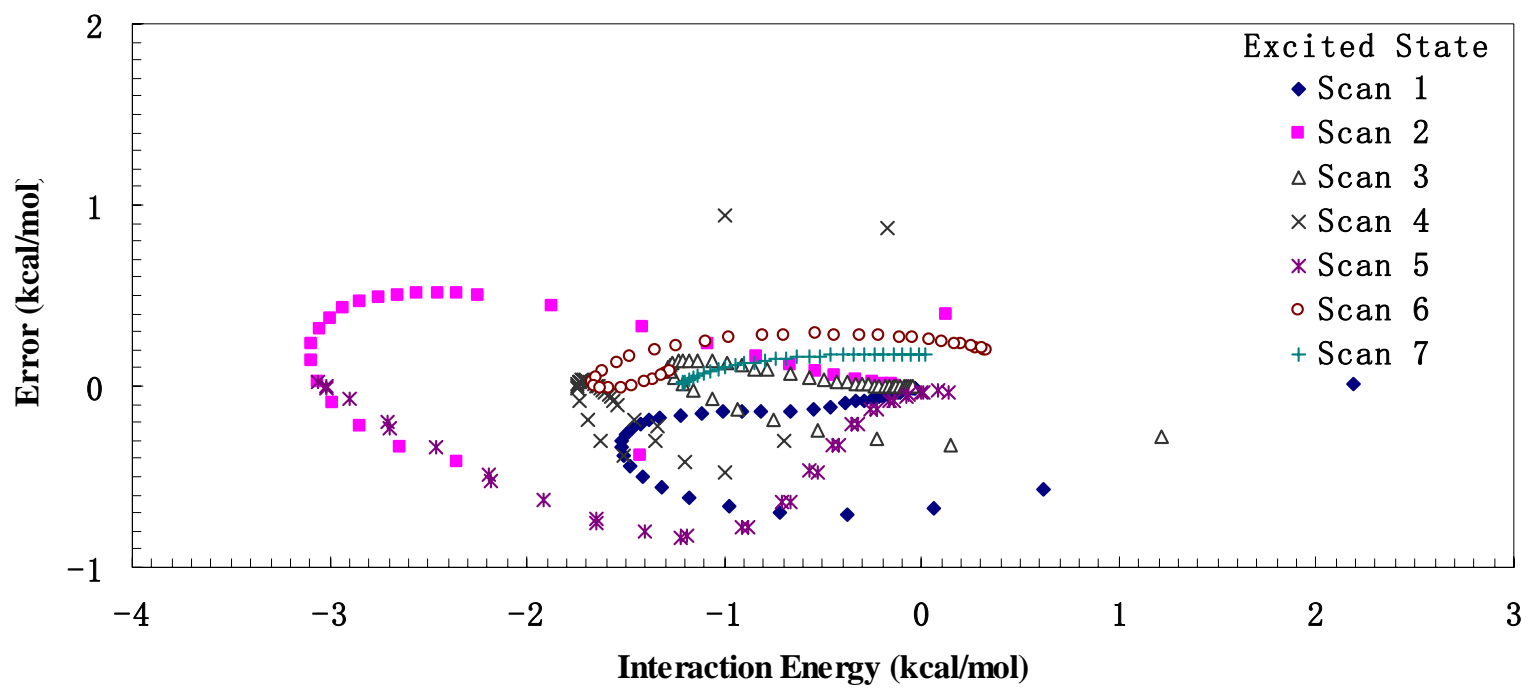

Fig 11. Errors in parameterized excited state potential as a function of the $\operatorname{CCSD}(T)$ energy for the PES scans. 
PNNL-16185

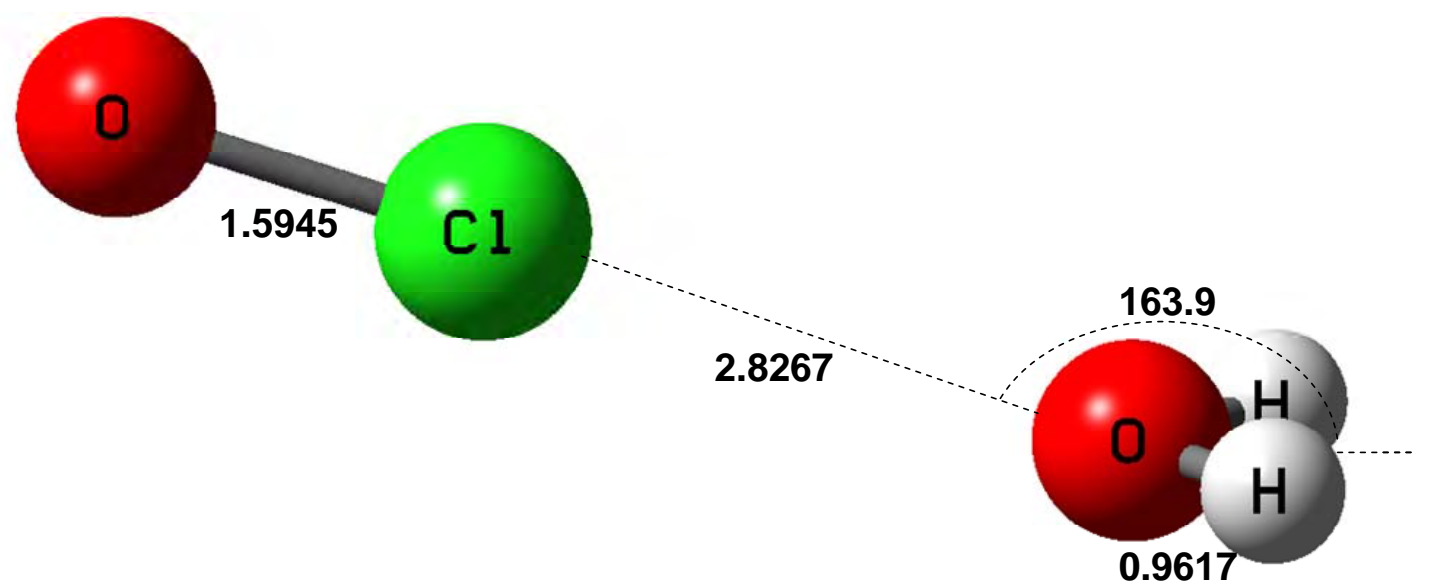

Fig 12. The geometry of the global minimum of $\mathrm{H}_{2} \mathrm{O} \cdot \mathrm{OCl}$ predicted by the mTTMC empirical potential. Distances are in angstroms and angles are in degrees.

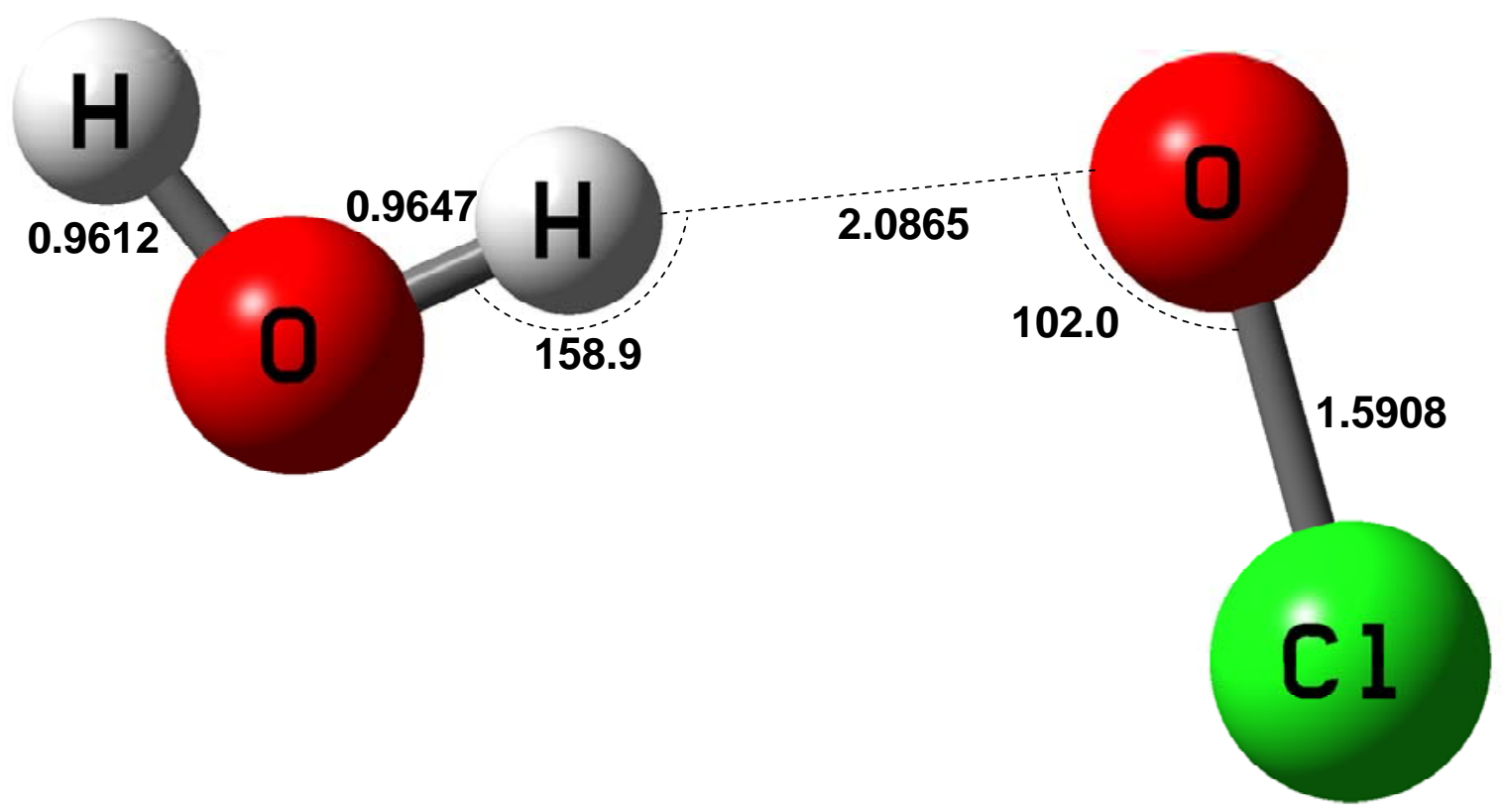

Fig 13. The geometry of the local minimum of $\mathrm{H}_{2} \mathrm{O} \cdot \mathrm{OCl}$ predicted by the mTTMC empirical potential. Distances are in angstroms and angles are in degrees. 
PNNL-16185 


\title{
Study of the inflammatory responses of Single-Walled Carbon Nanotubes (SWCNTs) On Murine Macrophage Cells (RAW 264.7)
}

\author{
Debamitra Dutta and Prof. Brij Mougil \\ Materials Engineering \\ University of Florida \\ Gainesville, FL \\ and \\ Dr. SK Sundaram \\ Pacific Northwest National Laboratory \\ Richland, WA
}

\section{Introduction}

Carbon nanotubes (CNTs) have been shown to possess some unique mechanical, physical and chemical properties that show potential for electronics, optics and biomedical applications [1-3]. Among the two types of carbon nanotubes, single-walled carbon nanotubes (SWCNTs) and multi-walled carbon nanotubes (MWCNTs), SWCNTs have drawn a lot of attention due to strong Van der Waals forces and self organizing properties $[4,5]$ that can give rise to 'rope like structures'. SWCNTs generally have a diameter between $0.5-2 \mathrm{~nm}$ with varying lengths that can be many thousand times larger than the diameter. Due to its excellent conductivity, SWCNT have become the candidate materials for future electronic miniatures smaller than micro-electromechanical devices.

It is obvious that the use of carbon nanotubes in the future can have a terrific impact on our society in every sphere of life. But before we move ahead from the stage of possibilities and potentials, it is imperative that we understand the effect of these amazing objects created by accident on our cells, organelles and tissues. It is important for us to study the potential hazards of inhalation and all other effects of the nanotubes in the physiological environment. Research approaches in the past decade have developed covalent and non-covalent methodologies to functionalize the insoluble nanotubes to solve the major problems of solubility [6, 7]. Although this has led to enhanced biocompatibility, a still better understanding of the toxicological aspects is necessary to realize the long-term fate of the nanotubes inside the living body.

Existing literature data on biological activity and toxicity assessments of carbon nanotubes are ambiguous. On one hand, there are several reports stating that purified CNTs when functionalized appropriately $[1,8,9]$ are completely non-toxic and can preserve the functionality of immune cells [10] and carbon nanotubes filled with DNA or peptide molecules can be utilized for molecular therapeutic applications [11-14]. These nanotubes have also been used to fabricate nanomotors for disease treatments. Cui et al have also shown that SWCNTs can induce the down-regulation expression of adhesion associated proteins and up-regulation of apoptosis related genes[15]. Various benefits and challenges associated with 
the manufacture of biocompatible carbon nanotubes has been discussed [16-18] to give insights to potential biological applications [19-22].

On the other hand, several investigators have reported the adverse effects of CNT on keratinocyte cells [23-26], growth inhibition behavior in the neuron cells of an embryonic rat brain [27], and granuloma formation in the mouse lung [4, 28-30]. The method of synthesizing the SWCNTs has a profound effect on the biological properties. For example, the presence of catalyst elements in SWCNTs like iron [4, 9, 31, 32] can result in inducing toxicity. Also, the presence of organics is associated with oxidative stress and the generation of free radicals. So organics if present in appreciable quantities, can interact with enzymes in the lung resulting in oxidative stress [33] via the production of superoxides [34] and finally lead to release of proinflammatory cytokines [35].

\section{Objective}

In the present study, we have examined the in vitro inflammatory behavior of SWCNTs (at concentrations $<100 \mu \mathrm{g} / \mathrm{ml}$ and incubation period of 24 hours) in murine macrophage cells (RAW 264.7) by studying the iNOS and COX-2 activity using western blotting techniques. Commercially available SWCNTs, containing impurities like nickel and yttrium were dispersed in cell media and added to the cells to stimulate any inflammatory reactions.

In a second phase, the goal of the research work was to study the effects of sonicating the dispersion medium (cell media) and attribute a mechanism to explain the variations in inflammation behavior. Experiments were designed to highlight the differences in the cytotoxic behavior of SWCNTs in presence and absence of sonication.

\section{Definitions}

It is important for us to know and understand the meaning of some biological terminologies that are associated with the current research work. As my major of study, was a non-biological one, and I have been involved in interdisciplinary subjects at every step of my work, I had to look up the internet to have a better understanding of these terms and then design experiments according. So I felt it might benefit readers in a similar situation, if these terminologies are explained before we proceed.

Immunoblotting or what is more popularly known as Western Blot technique (WB) is a powerful tool for protein characterization. It detects a wide range of proteins in samples of tissues, homogenates or extracts using gel electrophoresis technique to separate the denatured proteins on the basis of mass. It makes use of specific primary $\left(1^{\circ}\right)$ and secondary $\left(2^{\circ}\right)$ antibodies to detect proteins (antigen) immobilized on a matrix. It is customary to precede the process of detection by transfer onto a membrane to make even the smallest amount of proteins easily detectable. Additionally, it is also easier to handle membranes as compared to the gels. During migration through the gel, the proteins are separated on the basis of size and charge. The proteins having a smaller size are characteristically able to migrate faster than larger proteins through the gel.

Next, a monoclonal or polyclonal antibody capable of recognizing the protein of interest is chosen and can be either directly labeled or itself probed with a $2^{\circ}$ linked to a marker protein. For protein detection, the primary antibody is added at an appropriate dilution and incubated with the membrane. The specific antibody for a particular protein is directed towards the blotted protein and bind to it while other antibodies get washed away at the end of incubation. A secondary antibody - anti-immunoglobulin antibodies coupled to a reporter group is added to detect the bound antibodies which have bound. The excess secondary antibody is finally washed free of the membrane and a substrate is added that reacts with the conjugate and precipitates to give a visible band in the area where the primary antibody bound to the protein. We studied COX-2 and INOS protein expressions as stimulated by SWCNTs (any dose-dependent behavior) using western blot techniques and attempted to relate a potential biochemical mechanism to explain the observed activity in the cells.

Cyclooxygenase (COX) is an enzyme [36] in the metabolic pathway responsible for the formation of important biological mediators called prostanoids (including the prostaglandin, prostacyclin and thromboxane). There are two isoforms of COX, namely COX-1 and COX-2 [37]. COX-1 is a constitutive 
housekeeping gene found abundantly in most mammalian cells whereas COX-2 is a cytokine inducible isoform occurring abundantly in stimulated macrophages and other sites of inflammation. An increase in COX-2 expression has been associated with diseases like rheumatoid arthritis, seizures, ischemia and colon cancer.

An early immune response by the macrophages towards any foreign materials is generally associated with the production of nitric oxide (NO). NO plays an important role in signaling [38, 39] to different organelles and also functions as a regulatory molecule to generate immune response. It is generated by a soluble enzyme, inducible nitric oxide synthase (INOS) following exposure to certain cytokines. Apart from the immune system, INOS is also found in the cardiovascular system. In the last decade, there has been a lot of data generated on INOS expression in chronic inflammatory diseases of the human airways, kidney, heart, skin and teeth. Although sometimes the INOS reactivity is associated locally to macrophage cells, but more commonly it is related to the epithelial cells around the foci of inflammation. Thus, it has been argued that INOS expression is a secondary phenomenon indicative of an inflammatory disease. This essentially serves as an excellent biomarker for immune responses in vitro as well as in vivo instead of measuring the relative expression levels of numerous other cytokines.

Receptors are proteins found on the surface of the cell membrane or occurring within the cell nucleus or cytoplasm. It binds to a specific molecule called ligands (e.g. hormones) and initiates a cellular response in the system. A group of such receptors having the function of recognizing negatively charged macromolecules, and participating in the removal of any waste molecules from the body are referred to as scavenger receptors [40]. It has been shown that macrophage cells expressing scavenger receptors on their plasma membrane is able to uptake the low density lipoproteins deposited in the inner and outer linings of blood vessels and attain a foamy structure. During the uptake process, they also secrete a lot of inflammatory cytokines that lead to the development of atherosclerosis. A scavenger receptor antagonist (SRA) binds to the receptor and works to inhibit its normal physiological functioning.

Macrophages, being antigen presenting cells in living systems, primarily guard against all foreign body materials. Whenever these macrophage cells encounter any alien material like particulate matter or pathogens etc, they get activated and immediately phagocytose the foreign material and extrude certain chemical mediators of inflammation. In case of environmental particles [41], alveolar macrophages and the epithelial cells in the airway get stimulated to inhale the particles and trigger the release of reactive oxygen species. The RAW 264.7 murine macrophage cell line, an appropriate model in studying the macrophage signaling behavior was used in this research.

A dose-response curve is plotted for a system to express the correlative relationship between the exposure-characteristics and the spectrum of effects on the system due to the exposure. It is the most basic and integral concept in the science of toxicology. The shape of the dose-response curve has many implications in toxicity assessments of a system.

Glyceraldegyde 3-phsphate dehydrogenase (GAPDH) is an important enzyme used in glycolysis. Levels of GAPDH in the western blot experiment represent an internal control for the COX-2 and INOS bands.

Potentially toxic, natural compounds found inside pathogens like bacteria are known as endotoxins. Generally, the biological activity of endotoxin can be related to the lipopolysaccharide (LPS). The lipid component imparts toxicity while the polysaccharide components contribute towards immunogenicity.

\section{Materials and Methods}

\subsection{Materials}

The SWCNTs used for this study were purchased from Sigma-Aldrich Co. (Prod. No.519308). Bacterial LPS was purchased from Sigma Chemical (St Louis, MO). RPMI 1640, sodium pyruvate, FBS, Lglutamine, peniclillin and streptomycin were purchased from Invitrogen (Carlsbad, CA). All other chemicals used were purchased of cell-culture grade and better. 


\subsection{SEM-EDS Analysis}

Non-functionalized as-received SWCNTs were studied on a scanning electron microscope in energy dispersive spectrometry (EDS) mode to detect the compositional elements present along with the nanotubes. Aluminum stubs were smeared with silver paint, the nanotubes placed on the stubs and attached to copper tapes to make them conductive. The metallic stubs were then placed on the holder and introduced into the instrument at a vacuum of $10 \mathrm{kV}$.

\subsection{TEM Imaging}

Transmission Electron Microscopy (TEM) was used for the size measurement of SWCNTs. The images were taken on a Hitachi H-7000 microscope (Japan). TEM samples were prepared by placing a drop of SWCNTs solution (in denatured ethanol) directly onto a carbon-coated copper grid (200 mesh) and imaging was done at $10 \mathrm{kv}$.

\subsection{Cell Culture}

The cell line (American Type Culture Collection, Manassas, VA) used for this study was RAW 264.7 (murine macrophage cells). The cells were grown in RPMI 1640 supplemented with $10 \%$ fetal bovine serum, $2 \mathrm{mM}$ glutamine, $1 \mathrm{mM}$ sodium pyruvate and $100 \mathrm{U} / \mathrm{ml}$ penicillin and $0.1 \mathrm{mg} / \mathrm{ml}$ streptomycin in a humidified air containing $5 \% \mathrm{CO}_{2}, 95 \%$ air at $37{ }^{\circ} \mathrm{C}$. Cells were cultured in cell culture dishes $(60 \mathrm{~cm}$ diameter) (Fisher Scientific) and passaged using a cell scraper upon reaching 60-70\% confluency. The cell culture medium was exchanged once in two days.

For exposure to SWCNTs, cells were seeded into smaller cell culture dishes (30 cm diameter) at a seeding density of $4.2 \times 10^{5}$ cells per dish in a final volume of $3 \mathrm{ml}, 24 \mathrm{hrs}$ prior to addition of nanoparticles and incubated in humidified air containing $5 \% \mathrm{CO}_{2}, 95 \%$ air at $37{ }^{\circ} \mathrm{C}$. Prior to use on the next day, the culture medium was replaced with fresh media containing the added nanoparticles at the predefined concentrations. After incubation, the cells were washed 3 times with sterile PBS to remove all nonassociated nanoparticles from the cellular environment.

Two stock solutions of SWCNTs (at $1 \mathrm{mg} / \mathrm{ml}$ ) were prepared in sterile cell-media and sterile PL-127 (emulsifying agent) respectively. Pre-determined concentrations of the experimental SWCNT solutions at 25 to $100 \mu \mathrm{g} / \mathrm{ml}$ solution were prepared from the stock solution (either prepared normally or by serialized dilution method) using cell culture media, either sonicated or non-sonicated depending on the experimental sample. For sonicated samples, the cell culture media used for dilution was put into the ultra-sonication bath for a period of 15 minutes. An equal quantity of cell culture media (without any sample) sonicated for 15 minutes was treated as a control. All solutions (SWCNTs and controls) were vortexed well for one minute for better dispersion.

\subsection{Dose-Response Curves}

In order to generate a dose-response curve for the SWCNTs, macrophage cells were plated at the above-mentioned seeding density to a final volume of $3 \mathrm{ml}$ per cell culture dish and incubated with SWCNTs at predetermined doses (at 25 to $100 \mu \mathrm{g} / \mathrm{ml}$ ) for 24 hours.

Upon removal, the dishes were washed 3 times with ice-cold PBS. After the final wash, $1 \mathrm{ml}$ of sterile PBS was added and the cells were scraped carefully to remove all live cells. The cell-suspension ( $1 \mathrm{ml}$ in volume) was placed in centrifuge tubes. The number of live cells in each tube was then counted using a Coulter Cell Counter, utilizing dynamic light scattering principles.

A dose-response curve was generated by plotting the concentration of nanoparticles added, in the abscissa and the number of live cells counted, on the ordinate. The concentration range at the central portion of the dose response curve was selected to dose the macrophage cells in Western blot experiments.

\subsection{Western blot (WB) Analysis}

Raw 264.7 cells were plated and exposed to SWCNTs in cell-media and PL-127 (sonicated and nonsonicated) for 24 hours in the incubator similar to the experiments mentioned in the previous section (generation of dose-response curves). Upon removal, the dishes were washed with ice-cold sterile PBS and stored in the freezer at $-80^{\circ} \mathrm{C}$ for future processing. In due course of time, the cells were taken out and placed on a box of ice and $150 \mu \mathrm{l}$ ice-cold lysis buffer was added. The cells were then immediately scraped using a cell scraper and transferred to a homogenization assay and homogenized. $0.5 \mu \mathrm{l}$ spermine was added to the lysate (to prevent the formation of a viscous clump), transferred into an eppendorf vial and centrifuged at $4^{\circ}$ 
C, 18000 G (rcf*) for 5 minutes. After centrifugation, the supernatant was taken out very carefully and placed into freshly labeled eppendorf vials for the Protein Assay.

\subsubsection{Protein Assay}

The supernatant of the cell lysates prepared above were assayed for total protein content using the BSA protein assay at $582 \mathrm{~nm}$ in a multi-plate spectrophotometer against a BSA standard curve. Calculations were done to find the final concentration and quantity of sample that has to be loaded for gel-electrophoresis. The samples were then prepared using relevant amounts of $4 \mathrm{X}$ and $1 \mathrm{X}$ loading buffers and stored at room temperature for 1-d gel Electrophoresis.

\subsubsection{1-d gel Electrophoresis}

The different parts of a box for 1-d gel Electrophoresis are made to fit correctly and two $10 \mathrm{ml}$ SDS acrylamide gels (10 wells with $1.5 \mathrm{~mm}$ spacer) are taken out from the refrigerator. The cover is removed carefully along with the comb. The gels are then placed in the correct position inside the box. A buffer solution is prepared beforehand by adding $25 \mathrm{ml}$ a $20 \mathrm{X}$ buffer (NU PAGE MES SDS Running Buffer - 20X) to $475 \mathrm{ml}$ DI water. It is stirred for homogenizing the solution and poured into the inside chamber and made sure that the level comes to the very top. $50 \mu \mathrm{l}$ or some pre-decided quantities of the samples prepared above are loaded into each well. The See blue Plus 2 standard is loaded at the extreme left and the samples are placed to the right. The gel is then run on POWER PAC 300 till all bands are seen at the bottom.

While the gel is running, sufficient quantity of the transfer buffer is poured in 3 dishes to immerse the gel later. Once the gel is formed the POWER PAC 300 is put off and the buffer is emptied into the sink. The white box is unlocked and the rivets are broken using a scalpel. The side where the gel sticks at the bottom is placed below and the tip of the well and the portion where tape was removed is cut-off. The gel is submerged into the dish with transfer buffer and equilibrated for $10-15$ minutes.

\subsubsection{Preparing the membrane}

A nitrocellulose membrane (to drive the protein bands onto) is taken along with a pair of white PVDF filter papers. Some methanol is taken in a square plastic dish, into which the membrane is placed (using forceps) for a few seconds to make it hydrophobic. In the mean time, the WB membrane is placed into some transfer buffer, taken in a large plastic dish. The membrane is taken out of the methanol and placed onto the filter paper. The gel is then placed on it and sandwiched using the other filter paper and the whole set up is placed on the POWER/PAC 1000 machine for transfer, which generally takes between $60-70$ minutes.

\subsubsection{Blocking Buffer Using Carnation Milk}

The membrane is removed and washed in PBST solution twice with intermittent rocking for 5 minutes. The gel is then left to soak in a blocking buffer (5\% Carnation milk in PBS Tween Solution) prepared beforehand for one hour. After one hour, the gel is again washed three times using PBST and buffer equilibration and left for overnight rocking inside the cold storage.

\subsubsection{Conjugation of Primary $\left(1^{\circ}\right)$ and Secondary $\left(2^{\circ}\right)$ Antibodies}

The sample is taken out and all expient liquid is aspirated out. $8 \mathrm{ml}$ western blot buffer solution (50 $\mathrm{ml} 10 \mathrm{X}$ Tris Buffer Saline Solution $+450 \mathrm{ml}$ water $+10 \mathrm{~g}(2 \%)$ BSA + $100 \mathrm{mg}$ thimorosol (antibacterial) + $5 \mathrm{ml}(10 \%)$ Tween 20 (detergent and wetting agent)) is then added to the samples. The pre-selected $1^{\circ}$ antibody is added at the necessary dilutions and the sample is left to rock at slow speeds for an hour for continuous mixing, after which the membrane is washed using PBST for three times with intermittent rocking of 5 minutes each. The $2^{\circ}$ antibody (at correct dilution) is similarly added followed by four PBST solution washes. The detection agent (using West Pico Chemiluminescent solution) is prepared beforehand and the membrane is read on the Chemiluminescent instrument.

\subsection{Fluorescent Microscopic Imaging}

Cells were imaged using fluorescence microscopy to study the effect of scavenger receptor antagonists on the macrophage-uptake of SWCNTs. A thin monolayer of cells were plated in 4-well chamber slides (at approx. $3 \times 10^{3}$ cells per well) to a final volume of $500 \mu \mathrm{l}$ cell media. Prior to nanoparticle addition, the cell media was exchanged with serum free media and the cells were then exposed to alexafluor tagged SWCNTs at predetermined doses for 15 minutes. Upon removal, the dishes were washed with ice-cold PBS, fixed using glutaldehyde and watched using a fluorescent microscope at $647 \mathrm{~nm}$. 


\section{Experiments Performed}

Samples and the number of live cells counted on the Coulter Cell Counter for each sample in the dose-response curve experiments and details of samples used for the western blot experiments performed is given below.

\subsection{Dose-Response Curves}

As mentioned before, dose-responses curves were generated to study the effect of SWCNTs on murine macrophage cells. A representative curve for the dose-response curves generated has been shown in Figure 4. [Note: Dose-response curves for each Experiment have been recorded in the Laboratory Notebook at PNNL and a soft copy is available with Dr Thomas Weber at 331 Building, PNNL, Richland].

In order to generate a dose-response curve for cells incubated with cell media containing SWCNTs, an experiment (dose-response experiment I) was designed with particle concentrations from 12.5 to 100 $\mu \mathrm{g} / \mathrm{ml}$. As observed (Figure 4) there was a remarkable decrease in number of living cells with a decrease in particle concentration from $25 \mu \mathrm{g} / \mathrm{ml}$ to $12.5 \mu \mathrm{g} / \mathrm{ml}$. The next experiment (dose-response experiment II) was designed with particle concentrations varying between 12.5 to $25 \mu \mathrm{g} / \mathrm{ml}(12.5,15,20$ and $25 \mu \mathrm{g} / \mathrm{ml})$ to magnify any observed effects on the cell viability. Results (Table 2) indicated a slow decrease in cell growth with increasing particle concentration. The experiment was repeated for reproducibility (dose-response experiment III).

At this point, we realized the practical problem of not being able to re-use any leftover SWCNTs dispersed in the cell media for the subsequent experiment. It is known that cell media contains an enormous quantity of proteins which have a finite lifetime. Even cell media stored in the refrigerator at $-25{ }^{0} \mathrm{C}$ is not recommendable for use after a period of one week. In order to reduce the wastage of the SWCNTs, we decided to use PL-127 an emulsifying agent as the dispersion medium. Previous investigators have reported the advantages of the use of pluronic (1\% Pluronic F108) to stabilize and disperse single-walled nanotubes in toxicity studies [10].

In order to study any toxic effect of the emulsifier (pluronic PL-127) on the RAW 267.4 macrophage cells. So in the following experiment (dose- response experiment IV), we incubated the macrophage cells with sonicated and non-sonicated pluronic at emulsifier concentrations ranging from 0.25 to $1 \%$.

We concluded that there was no substantial toxic effect of the pluronic in the cells up to a pluronic concentration of $1 \%$. The nanotubes were observed to be more stable and better dispersed in 1\% PL-127 as compared to the use of cell media as the dispersion medium. A stock solution of $1 \mathrm{mg} / \mathrm{ml}$ of nanotubes in the emulsifier (1\%) was prepared and stored in the refrigerator for an indefinite period of time. In subsequent experiments, pre-determined concentrations of nanotubes were prepared from the stock solution using cellmedia as the diluent. Depending on the description of the sample desired, the diluent (cell-media) was sonicated (for SCM - sonicated cell media samples) for a period of 15 minutes on the ultra-sonication bath or not (for NSCM - non-sonicated cell-media samples).

In dose- response experiment $\mathbf{V}$, toxicity tests to study the differences in effect of SWCNTs in PL127 and cell media (sonicated and non-sonicated) were conducted. The effect of sonicating the cell media is observed in Table 5. In general, the number of cells counted in dishes containing samples in sonicated cell media was visibly higher than in non-sonicated cell media. 


\subsubsection{Experiments with SWCNTs}

\subsubsection{Dose-Response Experiment I}

Table 1: Experimental results of dose-response experiment I

\begin{tabular}{|c|c|c|c|c|}
\hline & Sample Details & No. of live cells & Avg. \# live cells & SWCNTs Conc \\
\hline \multirow[t]{3}{*}{1} & Naïve Control & 35751 & 34884 & - \\
\hline & & 35012 & & \\
\hline & & 33888 & & - \\
\hline \multirow[t]{2}{*}{2} & Sonicated Media & $\begin{array}{l}29644 \\
27935\end{array}$ & 31074 & $125, \mu \alpha / m l$ \\
\hline & & $\begin{array}{l}27935 \\
35642\end{array}$ & & $12.5 \mu \mathrm{g} / \mathrm{ml}$ \\
\hline \multirow[t]{2}{*}{3} & $12.5 \mu \mathrm{g} / \mathrm{ml} \mathrm{SWCNT}$ & 42580 & 33452 & \\
\hline & & $\begin{array}{l}39581 \\
18196\end{array}$ & & $25 \mu \mathrm{g} / \mathrm{ml}$ \\
\hline \multirow[t]{3}{*}{4} & $25 \mu \mathrm{g} / \mathrm{ml} \mathrm{SWCNT}$ & 13727 & 11562 & \\
\hline & & 10724 & & \\
\hline & & 10234 & & $50 \mu \mathrm{g} / \mathrm{ml}$ \\
\hline \multirow[t]{2}{*}{5} & $50 \mu \mathrm{g} / \mathrm{ml}$ SWCNT & $\begin{array}{l}12140 \\
11394\end{array}$ & 10877 & \\
\hline & & $\begin{array}{l}11394 \\
9097\end{array}$ & & $75 \mu \mathrm{g} / \mathrm{ml}$ \\
\hline 6 & $75 \mu \mathrm{g} / \mathrm{ml} \mathrm{SWCNT}$ & $\begin{array}{c}8875 \\
19311\end{array}$ & 12059 & \\
\hline \multirow[b]{2}{*}{7} & & 7992 & & $100 \mu \mathrm{g} / \mathrm{ml}$ \\
\hline & $100 \mu \mathrm{g} / \mathrm{ml} \mathrm{SWCNT}$ & $\begin{array}{l}11786 \\
11338\end{array}$ & 11562 & \\
\hline
\end{tabular}

5.1.1.2 Dose-Response Experiment II

Table 2: Experimental results of dose-response experiment II

\begin{tabular}{|c|c|c|c|c|c|}
\hline $\begin{array}{r}\text { Sl } \\
\text { No }\end{array}$ & Sample Details & $\begin{array}{c}\text { No. of live } \\
\text { cells } \\
\text { (Reading 1) }\end{array}$ & $\begin{array}{l}\text { No. of live cells } \\
\text { (Reading 2) }\end{array}$ & $\begin{array}{c}\text { Avg. \# } \\
\text { live cells }\end{array}$ & $\begin{array}{l}\text { SWCNT } \\
\text { Conc }\end{array}$ \\
\hline \multirow[t]{3}{*}{1} & \multirow[t]{3}{*}{ Sonicated Media } & 23138 & 23104 & \multirow[t]{3}{*}{24740} & \\
\hline & & 27387 & 26707 & & - \\
\hline & & 24116 & 23990 & & \\
\hline \multirow[t]{3}{*}{2} & \multirow[t]{3}{*}{$12.5 \mu \mathrm{g} / \mathrm{ml} \mathrm{SWCNT}$} & 21506 & 21876 & \multirow[t]{2}{*}{21946} & \multirow[t]{2}{*}{$12.5 \mu \mathrm{g} / \mathrm{ml}$} \\
\hline & & 22621 & 22257 & & \\
\hline & & 21726 & 21691 & \multirow{3}{*}{21294} & \multirow{2}{*}{$15 \mu \mathrm{g} / \mathrm{ml}$} \\
\hline \multirow[t]{3}{*}{3} & \multirow[t]{3}{*}{$15 \mu \mathrm{g} / \mathrm{ml} \mathrm{SWCNT}$} & 20289 & 20349 & & \\
\hline & & 21594 & 21785 & & \\
\hline & & 21893 & 21854 & \multirow{3}{*}{20226} & \multirow{2}{*}{$20 \mu \mathrm{g} / \mathrm{ml}$} \\
\hline \multirow[t]{3}{*}{4} & \multirow[t]{3}{*}{$20 \mu \mathrm{g} / \mathrm{ml} \mathrm{SWCNT}$} & 18547 & 18596 & & \\
\hline & & 20771 & 20501 & & \\
\hline & & 21279 & 21661 & \multirow{4}{*}{19346} & \multirow{4}{*}{$25 \mu \mathrm{g} / \mathrm{ml}$} \\
\hline \multirow[t]{3}{*}{5} & \multirow{3}{*}{$25 \mu \mathrm{g} / \mathrm{ml} \mathrm{SWCNT}$} & 20457 & 20271 & & \\
\hline & & 21463 & 20042 & & \\
\hline & & 16993 & 16849 & & \\
\hline
\end{tabular}


5.1.1.3 Dose-Response Experiment III

Table 3: Experimental results of dose-response experiment III

\begin{tabular}{|c|c|c|c|c|}
\hline $\begin{array}{c}\text { Sl } \\
\text { No }\end{array}$ & Sample Details & \# of live cells & $\begin{array}{c}\text { Avg. \# live } \\
\text { cells }\end{array}$ & $\begin{array}{c}\text { SWCNTs } \\
\text { Concentration }\end{array}$ \\
\hline 1 & Naïve Control & 15086 & 18283 & - \\
& (NSCM) & 19127 & & \\
2 & $12.5 \mu 636$ & 16608 & $12.5 \mu \mathrm{g} / \mathrm{ml}$ \\
& in NSCM & 16611 & & \\
3 & $25 \mu \mathrm{g} / \mathrm{ml} \mathrm{SWCNT}$ & 16120 & & $25 \mu \mathrm{g} / \mathrm{ml}$ \\
& in NSCM & 14864 & 15892 & \\
4 & $50 \mu \mathrm{g} / \mathrm{ml}$ SWCNT & 16645 & & $50 \mu \mathrm{g} / \mathrm{ml}$ \\
& in NSCM & 15846 & 15270 & \\
& & 15156 & & \\
\hline
\end{tabular}

\subsubsection{Experiments with PL-127}

5.1.2.1 Dose-Response Experiment IV

Table 4: Experimental results of dose-response experiment IV

\begin{tabular}{|c|c|c|c|c|c|}
\hline SI No & Sample Details & $\begin{array}{l}\text { \# live cells } \\
\text { Reading } 1\end{array}$ & $\begin{array}{l}\text { \# live cells } \\
\text { Reading } 2\end{array}$ & $\begin{array}{c}\text { Avg. \# } \\
\text { live cells }\end{array}$ & $\begin{array}{c}\text { Concentration } \\
\text { PL-127 }\end{array}$ \\
\hline \multirow[t]{3}{*}{1} & Naïve Control & 678 & 662 & 619 & - \\
\hline & & 543 & 539 & & \\
\hline & & 643 & 648 & & \\
\hline \multirow[t]{3}{*}{2} & $0.25 \%$ & 966 & 832 & 727 & $0.25 \%$ \\
\hline & PL-127 NSCM & 764 & 781 & & \\
\hline & & 504 & 513 & & \\
\hline \multirow[t]{3}{*}{3} & $0.5 \%$ & 770 & 794 & 802 & $0.5 \%$ \\
\hline & PL-127 NSCM & 812 & 772 & & \\
\hline & & 807 & 854 & & \\
\hline \multirow[t]{3}{*}{4} & $0.75 \%$ & 587 & 581 & 710 & $0.75 \%$ \\
\hline & PL-127 NSCM & 733 & 767 & & \\
\hline & & 826 & 765 & & \\
\hline \multirow[t]{3}{*}{5} & $1.0 \%$ & 694 & 652 & 747 & $1.0 \%$ \\
\hline & PL-127 NSCM & 847 & 853 & & \\
\hline & & 734 & 700 & & \\
\hline \multirow[t]{3}{*}{6} & $0.25 \%$ & 523 & 524 & 536 & $0.25 \%$ \\
\hline & PL-127 SCM & 557 & 589 & & \\
\hline & & 530 & 493 & & \\
\hline \multirow[t]{3}{*}{7} & $0.5 \%$ & 575 & 626 & 645 & $0.5 \%$ \\
\hline & PL-127 SCM & 582 & 566 & & \\
\hline & & 763 & 756 & & \\
\hline \multirow[t]{3}{*}{8} & $0.75 \%$ & 664 & 696 & 684 & $0.75 \%$ \\
\hline & PL-127 SCM & 656 & 635 & & \\
\hline & & 757 & 695 & & \\
\hline \multirow[t]{2}{*}{9} & $1.0 \%$ & 737 & 715 & 631 & $1.0 \%$ \\
\hline & PL-127 SCM & 609 & $\begin{array}{l}627 \\
538\end{array}$ & & \\
\hline
\end{tabular}


PNNL-16185

5.1.2.2 Dose-Response Experiment $V$

Table 5: Experimental results of dose-response experiment V

\begin{tabular}{|c|c|c|c|c|c|}
\hline $\begin{array}{r}\text { Sl } \\
\text { No }\end{array}$ & Sample Details & $\begin{array}{l}\text { \# live cells } \\
\text { Reading } 1 \\
\end{array}$ & $\begin{array}{l}\text { \# live cells } \\
\text { Reading } 2 \\
\end{array}$ & $\begin{array}{c}\text { Avg. \# } \\
\text { live cells }\end{array}$ & $\begin{array}{c}\text { SWCNTs } \\
\text { Concentration }\end{array}$ \\
\hline \multirow[t]{2}{*}{1} & Naïve Control & 38964 & 39874 & 36749 & - \\
\hline & & $\begin{array}{l}09404 \\
33891\end{array}$ & $\begin{array}{l}05002 \\
34266\end{array}$ & & \\
\hline \multirow[t]{3}{*}{2} & PL-127 NSCM & 14039 & 13882 & 47622 & - \\
\hline & & 55358 & 56455 & & \\
\hline & & 41929 & 36746 & & \\
\hline \multirow[t]{3}{*}{3} & $25 \mu \mathrm{g} / \mathrm{ml}$ & 31005 & 29558 & 30924 & $25 \mathrm{ug} / \mathrm{ml}$ \\
\hline & SWCNT in PL- & 30709 & 30319 & & \\
\hline & 127 in SCM & 32114 & 31836 & & \\
\hline \multirow[t]{3}{*}{4} & $50 \mu \mathrm{g} / \mathrm{ml}$ & 14370 & 14398 & 14875 & $50 \mu \mathrm{g} / \mathrm{ml}$ \\
\hline & SWCNT in PL- & 14375 & 14372 & & $s 0 \mu g / 1111$ \\
\hline & 127 in SCM & 15950 & 15783 & & \\
\hline \multirow[t]{3}{*}{5} & $100 \mu \mathrm{g} / \mathrm{ml}$ & 25920 & 25710 & 20426 & $100 \mu \sigma / m l$ \\
\hline & SWCNT in PL- & 16155 & 15862 & & \\
\hline & 127 in SCM & 19396 & 19512 & & \\
\hline \multirow[t]{3}{*}{6} & PL-127 sonicated & 12115 & 12255 & 10801 & \\
\hline & & 9901 & 15862 & & \\
\hline & & 10569 & 19512 & & \\
\hline \multirow[t]{3}{*}{7} & SCM & 42028 & 41806 & 22982 & \\
\hline & & 24823 & 25151 & & \\
\hline & & 21216 & 20736 & & \\
\hline \multirow[t]{3}{*}{8} & $25 \mu \mathrm{g} / \mathrm{ml}$ & 13563 & 13500 & 12979 & $25 \mu \mathrm{g} / \mathrm{ml}$ \\
\hline & SWCNT in SCM & 32230 & 32187 & & \\
\hline & & 12447 & 12405 & & \\
\hline \multirow{3}{*}{9} & $50 \mu \mathrm{g} / \mathrm{ml}$ & 14062 & 14798 & 34273 & $50 \mu \mathrm{g} / \mathrm{ml}$ \\
\hline & SWCNT in SCM & 29786 & 29731 & & \\
\hline & & 38846 & 38727 & & \\
\hline \multirow{3}{*}{10} & $100 \mu \mathrm{g} / \mathrm{ml}$ & 12849 & 13103 & 12343 & $100 \mu \mathrm{g} / \mathrm{ml}$ \\
\hline & SWCNT in SCM & 13463 & 13399 & & \\
\hline & & 10573 & 10673 & & \\
\hline
\end{tabular}

Use of sonication techniques was clearly having a negative effect on the toxicity studies of the system. In order to understand the effect of the contribution and understand the mechanism of action, further experiments with similar samples were conducted for western blot experiments.

\subsection{Western Blot Experiments}

Seven WB experiments (three different types) were conducted using SWCNT in PL-127 and two experiments were conducted using Scavenger receptor antagonists (Fucoidan and Polyinosinic Acid) to understand the mechanism of inflammation of SWCNT in the RAW 264.7 cells.

Details of all samples prepared for each western blot Experiment (using SWCNTs and SRA) is given in complete details below. Each western blot experiment was designed to get a better understanding of the activity of SWCNTs in the macrophage cells and its mechanism of action. Initially, a set of experiments, (enlisted below as Western Blot Experiment 5.2.1.1) was performed to study any difference in inflammatory 
PNNL-16185

behavior of SWCNTs in sonicated cell media as compared to the use of PL-127 as a dispersing media. The experiment was repeated nine times for reproducibility in results and we noticed the same trends in the protein up-regulation activity.

Thereafter, further experiments were designed to get a better understanding on the mechanism of action.

\subsubsection{Experiments with SWCNTs}

5.2.1.1 Type I (WB Experiment 2A, 3A, 6A, 6B, 6C)

Table 6: Details of samples for incubating macrophage cells for western blot experiments with SWCNTs Type I $(2 A, 3 A, 6 A, 6 B, 6 C)$

\begin{tabular}{|c|c|c|c|c|c|}
\hline $\begin{array}{l}\text { Sl } \\
\text { No. }\end{array}$ & Sample Details & $\begin{array}{c}\text { RPMI 1640 } \\
\text { (NSCM/ } \\
\text { SCM) }\end{array}$ & LPS & $\begin{array}{l}\text { PL- } \\
127\end{array}$ & $\begin{array}{c}\text { SWCNTs } 10 \\
\mathrm{mg} / \mathrm{ml}\end{array}$ \\
\hline $\begin{array}{l}1 \\
2 \\
3 \\
4 \\
5 \\
6 \\
7 \\
8 \\
9\end{array}$ & $\begin{array}{l}\text { Control (only cells) } \\
1 \% \text { PL-127 (sonicated) } \\
\text { SCM } \\
1 \text { ng/ml LPS } \\
1 \text { ng/ml LPS + SCM } \\
\text { LPS + SWCNT in PL127 } \\
\text { LPS+ SWCNT in SCM } \\
\text { SWCNT in PL-127 } \\
\text { SWCNT in SCM }\end{array}$ & \begin{tabular}{l}
$3 \mathrm{ml} \mathrm{NSCM}$ \\
\multicolumn{2}{c}{} \\
$3 \mathrm{ml} \mathrm{SCM}$ \\
$3 \mathrm{ml} \mathrm{NSCM}$ \\
$3 \mathrm{ml} \mathrm{SCM}$ \\
$3 \mathrm{ml} \mathrm{NSCM}$ \\
$3 \mathrm{ml} \mathrm{SCM}$ \\
$3 \mathrm{ml} \mathrm{NSCM}$ \\
$3 \mathrm{ml} \mathrm{SCM}$
\end{tabular} & $\begin{array}{l}3 \mu \mathrm{l} \\
3 \mu \mathrm{l} \\
3 \mu \mathrm{l} \\
3 \mu \mathrm{l}\end{array}$ & $30 \mu \mathrm{l}$ & $\begin{array}{c}50 \mu \mathrm{l} \text { in PL-127 } \\
50 \mu \mathrm{l} \text { in SCM } \\
50 \mu \mathrm{l} \text { in PL-127 } \\
50 \mu \mathrm{l} \text { in SCM }\end{array}$ \\
\hline
\end{tabular}

5.2.1.2 Type II (WB Experiment 4B)

Table 7: Details of samples in western blot experiment with SWCNTs/SRA Type II

\begin{tabular}{|c|c|c|c|c|c|c|}
\hline $\begin{array}{c}\text { Sl } \\
\text { No }\end{array}$ & Sample Details & $\begin{array}{c}\text { RPMI } 1640 \\
\text { (NSCM/ } \\
\text { SCM) }\end{array}$ & LPS & PL-127 & $\begin{array}{l}\text { Fucoidan } \\
\text { Stock } \\
\sim 1 \mathrm{mg} / \mathbf{m l}\end{array}$ & $\begin{array}{l}\text { SWCNTs } \\
\text { Stock } \\
\sim 10 \mathrm{mg} / \mathrm{ml}\end{array}$ \\
\hline $\begin{array}{l}1 \\
2 \\
3 \\
4 \\
5 \\
6 \\
7 \\
8\end{array}$ & $\begin{array}{l}\text { Control (only cells) } \\
1 \% \text { PL-127 (sonicated) } \\
1 \mathrm{ng} / \mathrm{ml} \text { LPS } \\
\text { LPS + } 50 \mu \mathrm{g} / \mathrm{ml} \mathrm{SWCNT} \\
\text { LPS }+25 \mu \mathrm{g} / \mathrm{ml} \text { Fucoidan } \\
\text { LPS }+25 \mu \mathrm{g} / \mathrm{ml} \text { Fucoidan } \\
\quad+50 \mu \mathrm{g} / \mathrm{ml} \mathrm{SWCNT} \\
25 \mu \mathrm{g} / \mathrm{ml} \mathrm{Fucoidan} \\
\quad+50 \mu \mathrm{g} / \mathrm{ml} \mathrm{SWCNT} \\
50 \mu \mathrm{g} / \mathrm{ml} \mathrm{SWCNT}\end{array}$ & $\begin{array}{c}3 \mathrm{ml} \text { NSCM } \\
3 \mathrm{ml} \text { SCM } \\
3 \mathrm{ml} \text { NSCM } \\
\quad, \\
\\
\quad, \\
\\
,\end{array}$ & $\begin{array}{l}3 \mu \mathrm{l} \\
3 \mu \mathrm{l} \\
3 \mu \mathrm{l} \\
3 \mu \mathrm{l}\end{array}$ & $30 \mu \mathrm{l}$ & $\begin{array}{l}15 \mu \mathrm{l} \\
15 \mu \mathrm{l} \\
15 \mu \mathrm{l}\end{array}$ & $\begin{array}{l}50 \mu \mathrm{l} \\
50 \mu \mathrm{l} \\
50 \mu \mathrm{l} \\
50 \mu \mathrm{l}\end{array}$ \\
\hline
\end{tabular}




\subsubsection{Type III (WB Experiment 7)}

Table 8: Details of samples in western blot experiment with SWCNTs/SRA Type III

\begin{tabular}{|c|c|c|c|c|c|}
\hline $\begin{array}{c}\text { Sl } \\
\text { No }\end{array}$ & Sample Details & $\begin{array}{c}\text { RPMI } 1640 \\
\text { (NSCM/ } \\
\text { SCM) }\end{array}$ & $\begin{array}{c}\text { LPS } \\
\sim\end{array}$ & $\begin{array}{l}\text { Fucoidan } \\
\text { Stock } \\
\sim 1 \mathrm{mg} / \mathrm{ml}\end{array}$ & $\begin{array}{l}\text { SWCNTs } \\
\text { Stock } \\
\sim 10 \mathrm{mg} / \mathrm{ml}\end{array}$ \\
\hline $\begin{array}{l}1 \\
2 \\
3 \\
4 \\
\\
5 \\
6 \\
7\end{array}$ & \begin{tabular}{l} 
Control (only cells) \\
$1 \mathrm{ng} / \mathrm{ml}$ LPS \\
$12.5 \mu \mathrm{g} / \mathrm{ml}$ Fucoidan \\
LPS $+12.5 \mu \mathrm{g} / \mathrm{ml}$ \\
\multicolumn{1}{c}{ Fucoidan } \\
$50 \mu \mathrm{g} / \mathrm{ml} \mathrm{SWCNT}$ in SCM \\
$\mathrm{LPS}+50 \mu \mathrm{g} / \mathrm{ml}$ SWCNT \\
in SCM \\
LPS $+50 \mu \mathrm{g} / \mathrm{ml} \mathrm{SWCNT}$ \\
in SCM $+12.5 \mu \mathrm{g} / \mathrm{ml}$ \\
Fucoidan
\end{tabular} & $\begin{array}{c}3 \mathrm{ml} \text { NSCM } \\
\text { ” } \\
\text { ” } \\
3 \mathrm{ml} \mathrm{SCM} \\
,\end{array}$ & $3 \mu \mathrm{l}$ & $\begin{array}{l}7.5 \mu \mathrm{l} \\
7.5 \mu \mathrm{l} \\
7.5 \mu \mathrm{l}\end{array}$ & $\begin{array}{c}50 \mu \mathrm{l} \text { in PL-127 } \\
50 \mu \mathrm{l} \text { in SCM } \\
50 \mu \mathrm{l} \text { in PL-127 }\end{array}$ \\
\hline
\end{tabular}

5.2.2 Experiments with Scavenger Receptor Antagonists

5.2.2.1 Type I (WB Experiment 3B)

Table 9: Details of samples in western blot experiment with SRA Type I

\begin{tabular}{|c|c|c|c|c|c|}
\hline $\begin{array}{c}\text { Sample } \\
\text { No. }\end{array}$ & Sample Details & $\begin{array}{c}\text { RPMI } 1640 \\
\text { (NSCM/ } \\
\text { SCM) }\end{array}$ & LPS & $\begin{array}{c}\text { PU Acid } \\
\text { Stock } \\
\sim 1 \mathrm{mg} / \mathrm{ml}\end{array}$ & $\begin{array}{c}\text { Fucoidan } \\
\text { Stock } \\
\sim 1 \mathrm{mg} / \mathrm{ml} \\
\end{array}$ \\
\hline $\begin{array}{l}1 \\
2 \\
3 \\
4 \\
5 \\
6\end{array}$ & $\begin{array}{c}\text { Control (only cells) } \\
1 \mathrm{ng} / \mathrm{ml} \text { LPS } \\
\text { LPS }+50 \mu \mathrm{g} / \mathrm{ml} \text { Fucoidan } \\
\text { LPS }+50 \mu \mathrm{g} / \mathrm{ml} \text { PU Acid } \\
50 \mu \mathrm{g} / \mathrm{ml} \text { PU Acid } \\
50 \mu \mathrm{g} / \mathrm{ml} \mathrm{Fucoidan}\end{array}$ & $\begin{array}{c}3 \mathrm{ml} \text { NSCM } \\
\text { “ } \\
\text { “ } \\
\text { “ } \\
\text { “ } \\
\text { “ }\end{array}$ & $\begin{array}{l}3 \mu \mathrm{l} \\
3 \mu \mathrm{l} \\
3 \mu \mathrm{l}\end{array}$ & $\begin{array}{l}30 \mu \mathrm{l} \\
30 \mu \mathrm{l}\end{array}$ & $30 \mu \mathrm{l}$ \\
\hline
\end{tabular}




\subsubsection{Type II (WB Experiment 4A)}

Table 10: Details of samples in western blot experiment with SRA Type II

\begin{tabular}{|c|c|c|c|c|}
\hline $\begin{array}{c}\text { Sample } \\
\text { No. }\end{array}$ & Sample Details & $\begin{array}{c}\text { RPMI } 1640 \\
\text { (NSCM/ } \\
\text { SCM) }\end{array}$ & LPS & $\begin{array}{c}\text { Fucoidan } \\
\text { Stock } \\
\sim 1 \mathrm{mg} / \mathrm{ml}\end{array}$ \\
\hline $\begin{array}{l}1 \\
2 \\
3 \\
4 \\
5 \\
6 \\
7 \\
8\end{array}$ & $\begin{array}{c}\text { Control (only cells) } \\
1 \mathrm{ng} / \mathrm{ml} \text { LPS } \\
50 \mu \mathrm{g} / \mathrm{ml} \text { Fucoidan } \\
25 \mu \mathrm{g} / \mathrm{ml} \text { Fucoidan } \\
12.5 \mu \mathrm{g} / \mathrm{ml} \text { Fucoidan } \\
\text { LPS }+50 \mu \mathrm{g} / \mathrm{ml} \text { Fucoidan } \\
\text { LPS }+25 \mu \mathrm{g} / \mathrm{ml} \text { Fucoidan } \\
\text { LPS }+12.5 \mu \mathrm{g} / \mathrm{ml} \text { Fucoidan }\end{array}$ & $\begin{array}{c}3 \mathrm{ml} \text { NSCM } \\
\text { " } \\
, \\
, \\
, \\
, \\
, \\
,\end{array}$ & $\begin{array}{l}3 \mu \mathrm{l} \\
3 \mu \mathrm{l} \\
3 \mu \mathrm{l}\end{array}$ & $\begin{array}{l}30 \mu \mathrm{l} \\
15 \mu \mathrm{l} \\
7.5 \mu \mathrm{l} \\
30 \mu \mathrm{l} \\
15 \mu \mathrm{l} \\
7.5 \mu \mathrm{l}\end{array}$ \\
\hline
\end{tabular}

To confirm the role of scavenger receptors in uptake process, experiments (Experiment 5.2.1.2) using SWCNTs in presence of SRAs (fucoidan and polyinosinic acid) were conducted to determine a more effective SRA (Experiment 5.2.2.1) and an optimum concentration of fucoidan (effective SRA) for complete inhibition activity. Finally, the COX-2 and INOS activity of SWCNTs in the presence of SRAs (fucoidan) on the cells were studied (Experiment 5.2.1.2 and 5.2.1.3). Presence of antagonists successfully negated all antagonist effects of LPS mediated inflammatory responses for inflammatory responses (Figure 12).

\subsection{Materials Analysis}

Table 11: Composition of the SWCNTs as procured from Sigma-Aldrich observed on SEMEDS at $10 \mathrm{kV}$ potential.

\begin{tabular}{|c|c|c|c|c|c|}
\hline $\begin{array}{c}\text { Weight \% by Element } \\
\text { Element }\end{array}$ & C K & O K & Si K & Y L & Ni K \\
Wt \% by element & 77.65 & 2.04 & 0.12 & 2.9 & 17.29 \\
Atomic \% by Element & C K & O K & SiK & Y L & NiK \\
Element & 93.37 & 1.85 & 0.06 & 0.47 & 4.25 \\
\hline
\end{tabular}


Figure 1: Spectroscopic analysis for composition of SWCNTs on SEM - EDS.

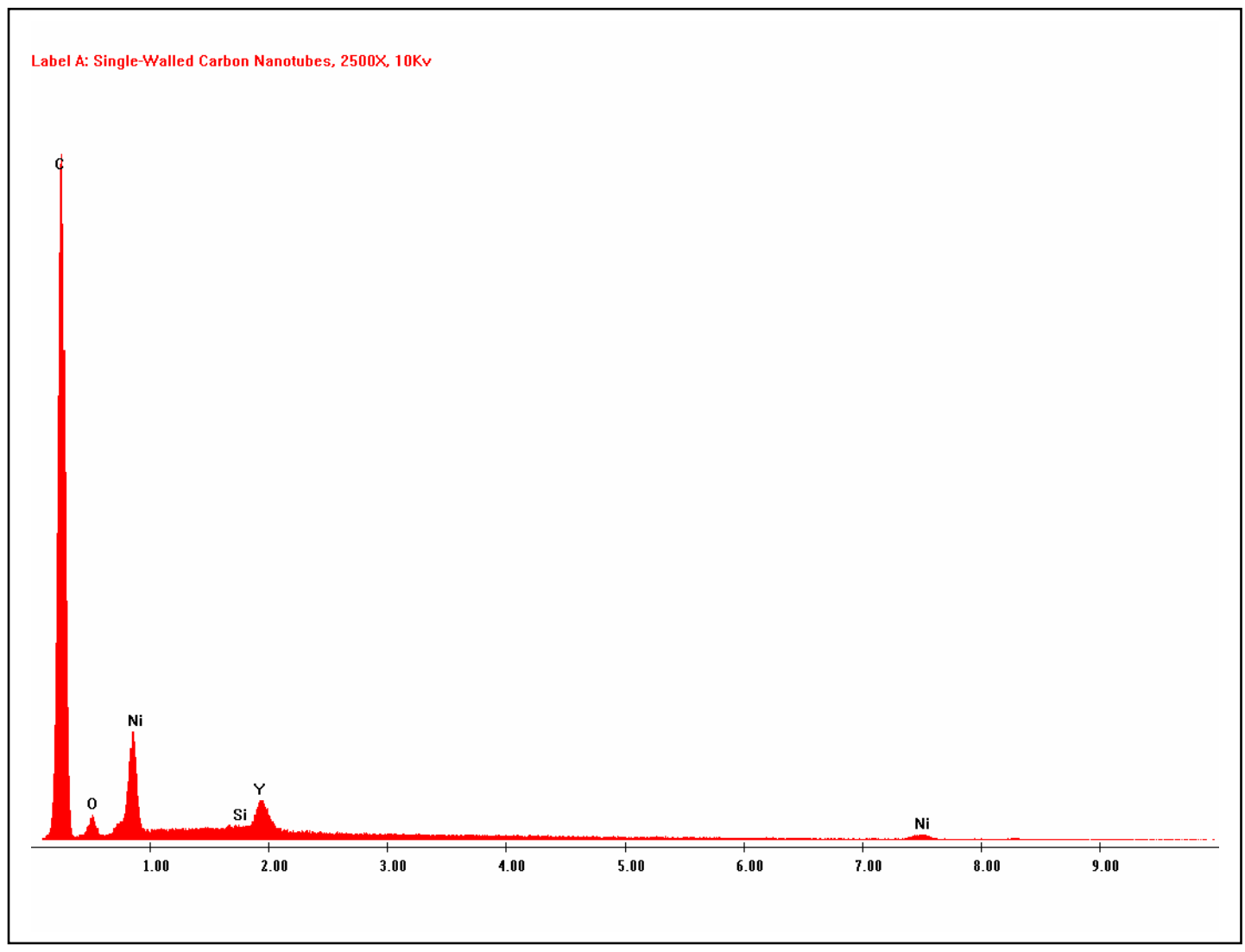

Table 12: Composition of white spots on SWCNTs structure observed on SEM EDS (10 kV potential).

\begin{tabular}{|c|c|c|c|c|c|}
\hline Element & C K & O K & Si K & Y L & Ni K \\
\hline Wt \% by element & 30.85 & 0.25 & 0.51 & 17.19 & 43.47 \\
\hline Atomic \% by Element & & & & & \\
\hline Element & C K & O K & Si K & Y L & Ni K \\
\hline Atomic \% by element & 71.89 & 0.43 & 0.51 & 5.41 & 20.72 \\
\hline
\end{tabular}


PNNL-16185

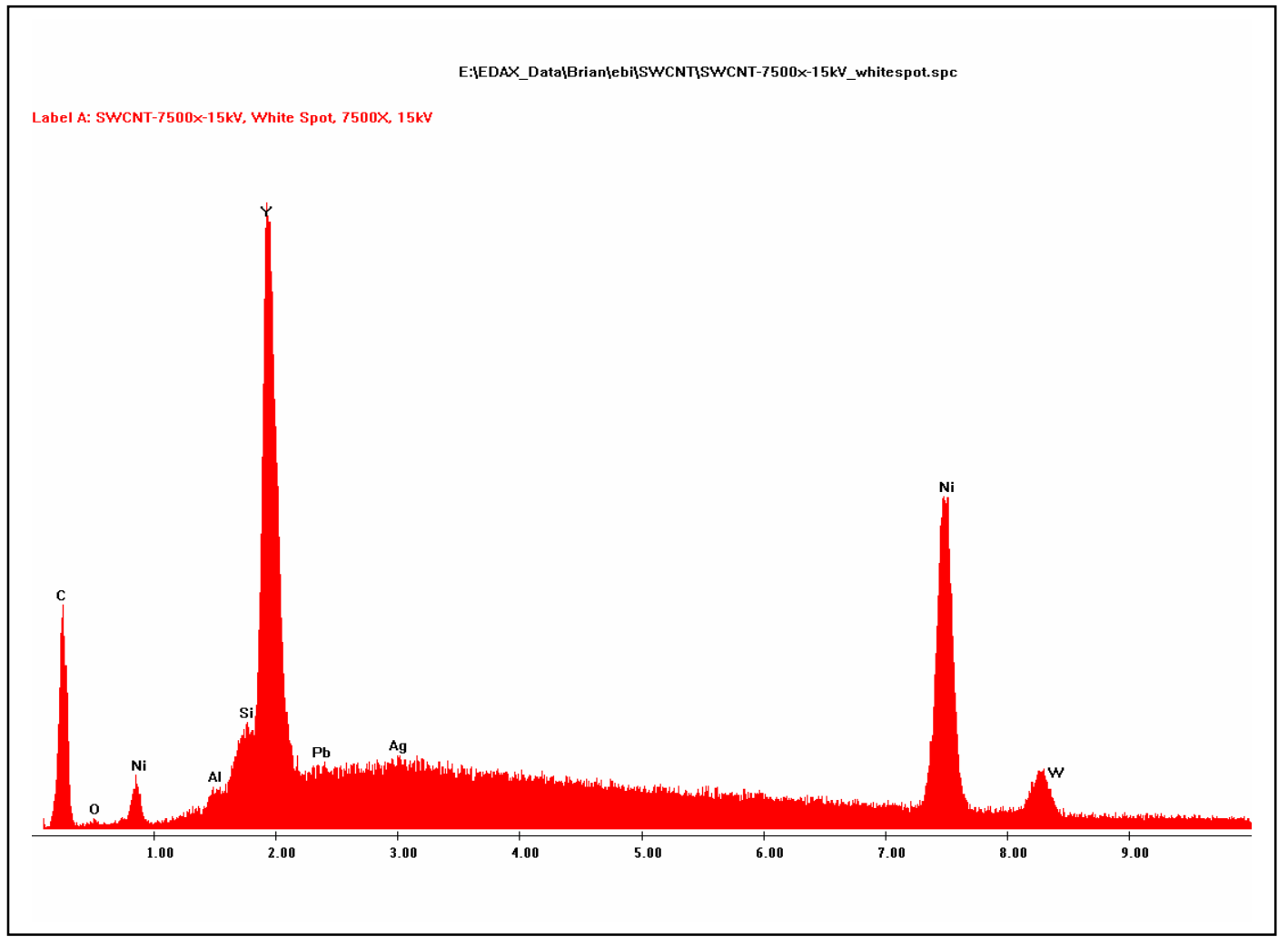

Figure 2: Spectroscopic analysis for composition of white spots of SWCNTs on SEM - EDS.

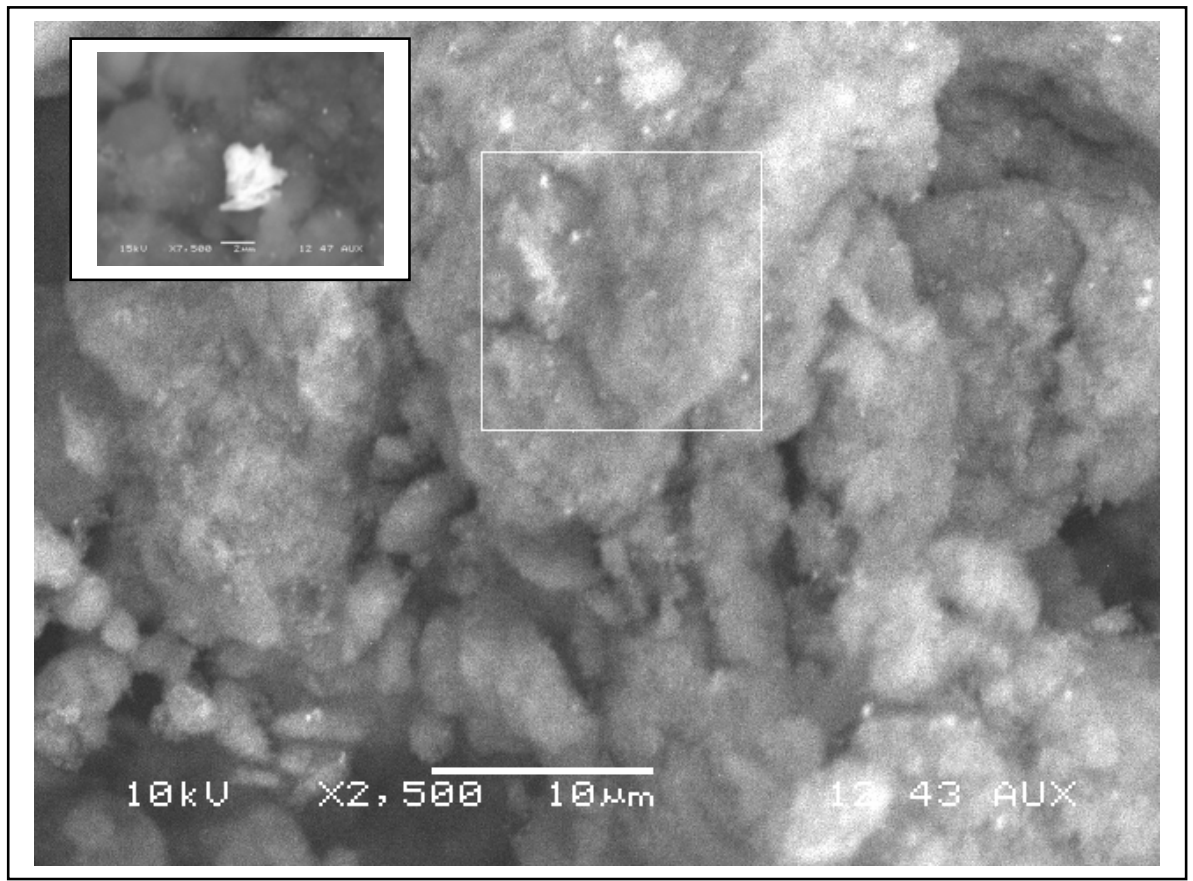

Figure 3: SEM image for SWCNTs and (inset)white spots observed on the structure of SWCNTs 


\subsection{Dose- response Curve}

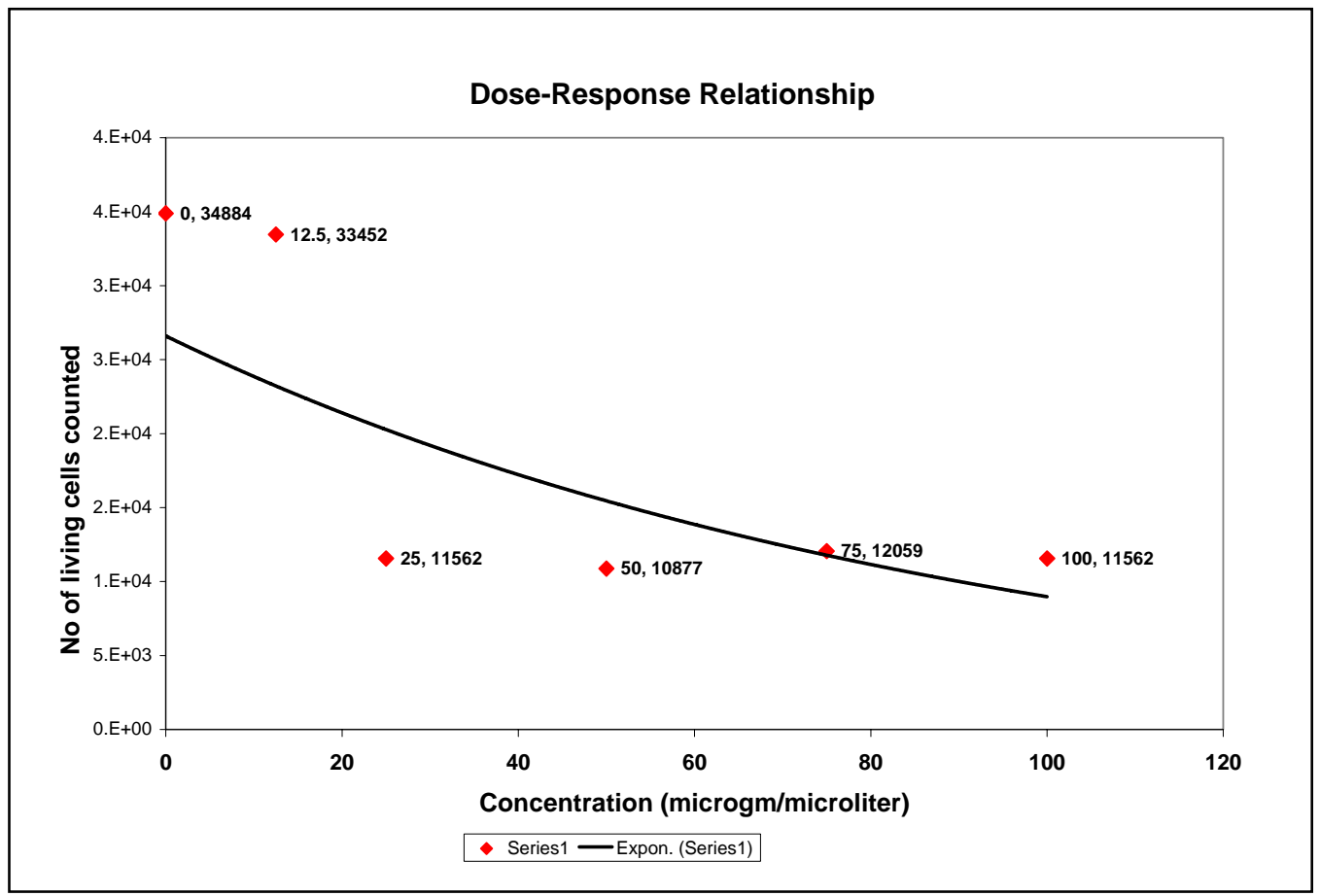

Figure 4: Representative Dose-Response Curve from Experiment 1

\subsection{Western Blot Analysis}

\subsubsection{Histograms for western blot analysis}

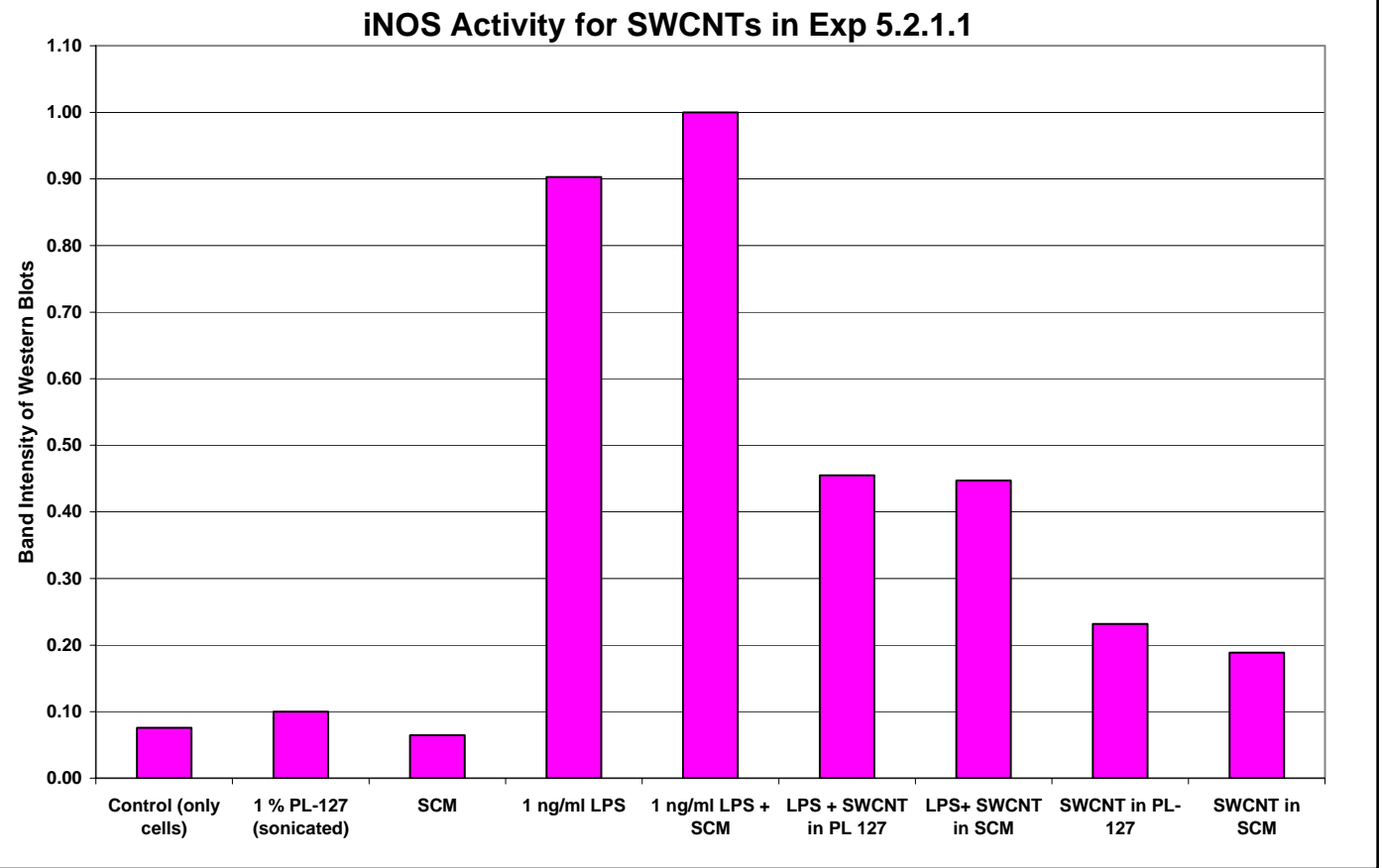

Figure 5: Histogram representing the iNOS activity of SWCNTs in Exp 5.2.1.1 


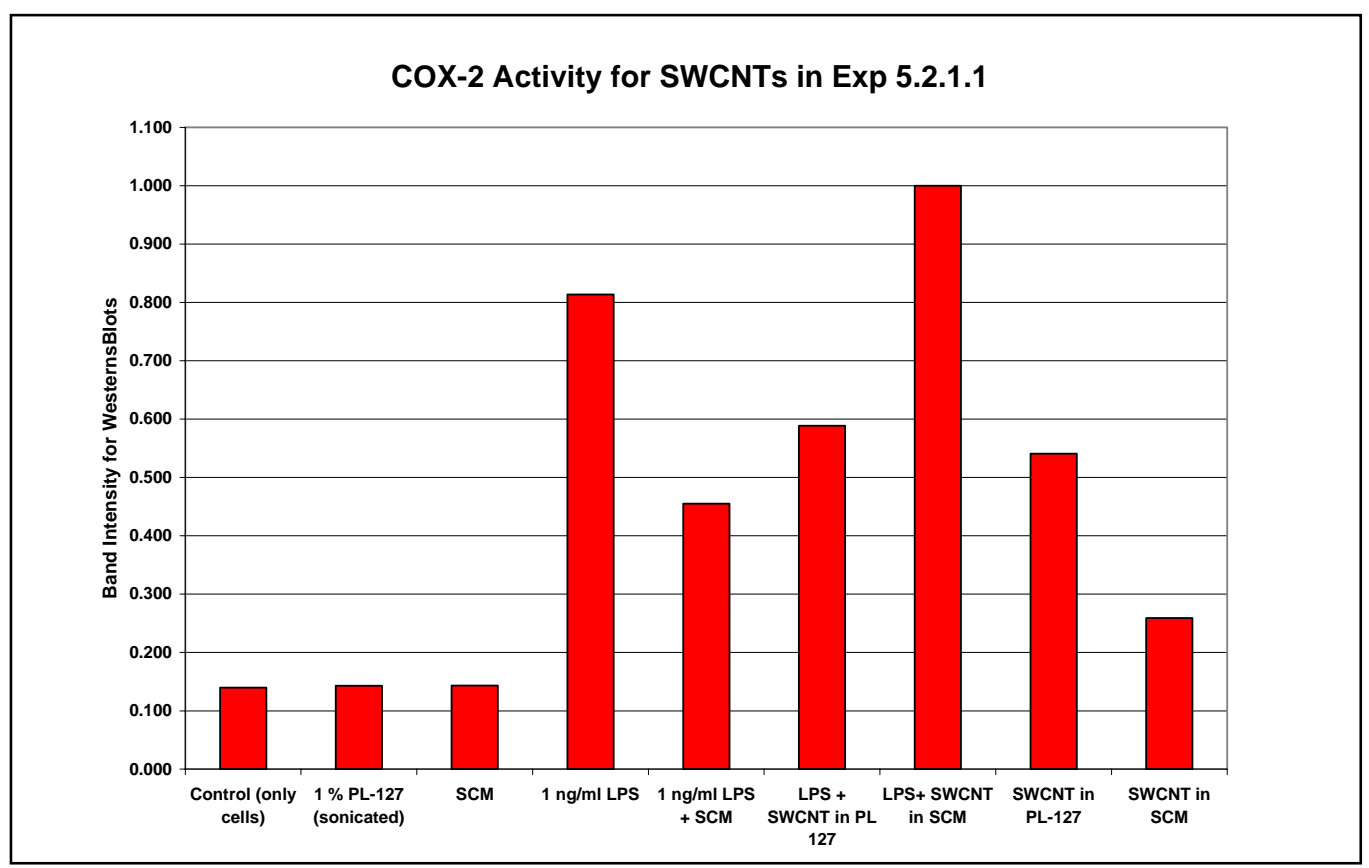

Figure 6: Histogram representing the COX-2 activity of SWCNTs in Exp 5.2.1.1

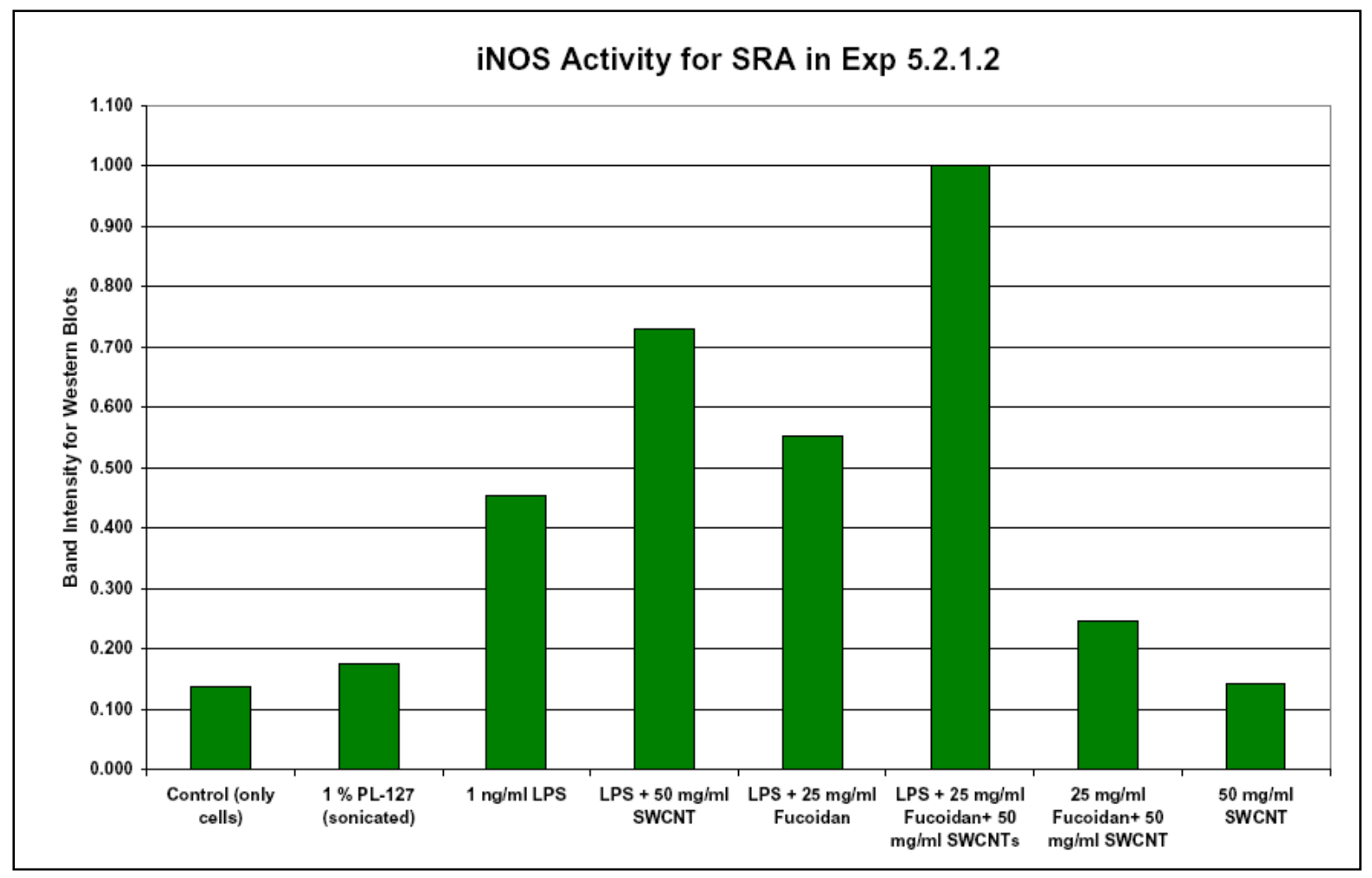

Figure 7: Histogram representing the iNOS activity of SWCNTs in Exp 5.2.1.2 
COX-2 Activity for SRA in Exp 5.2.1.2

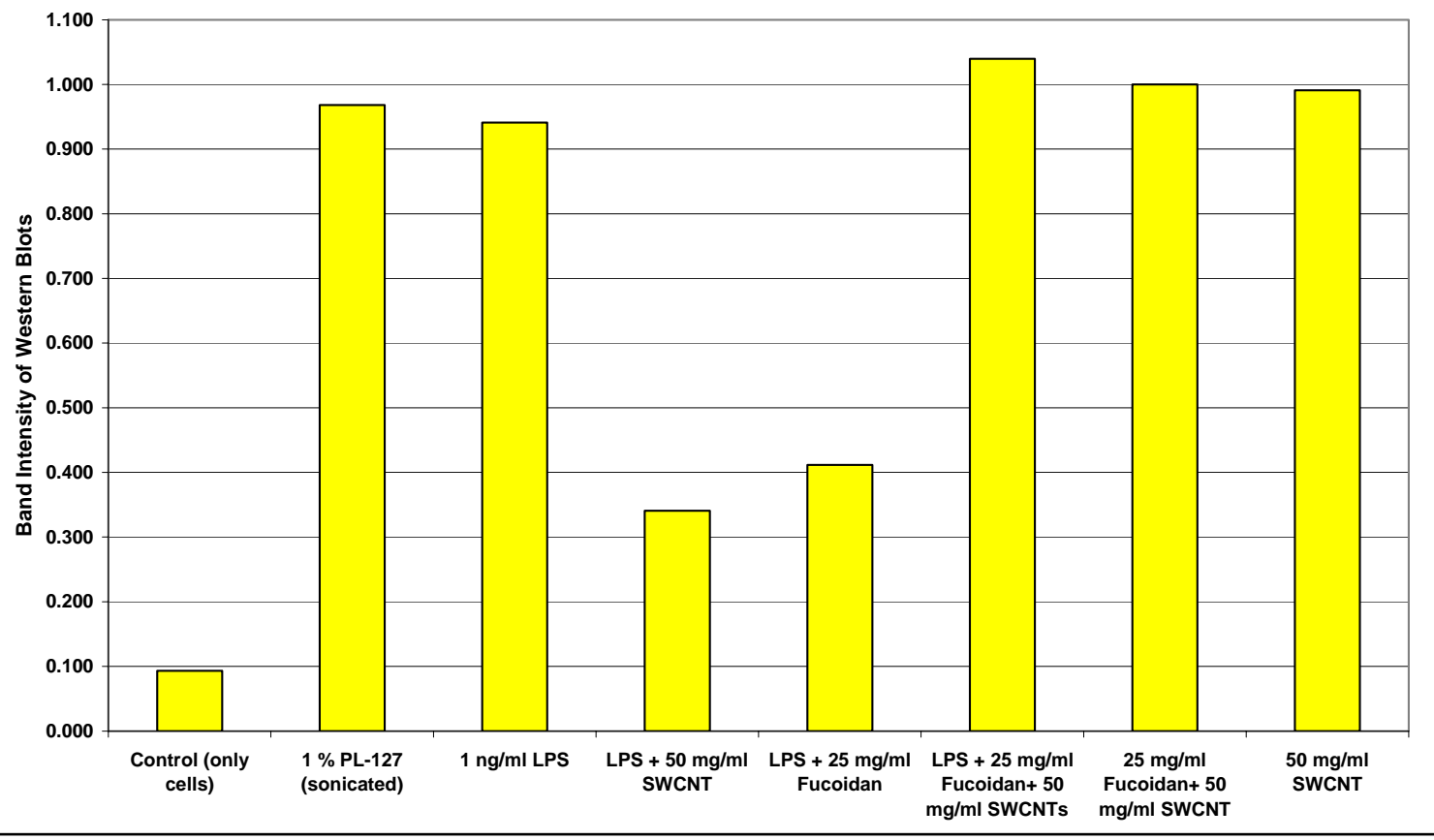

Figure 8: Histogram representing the COX-2 activity of SWCNTs in Exp 5.2.1.2

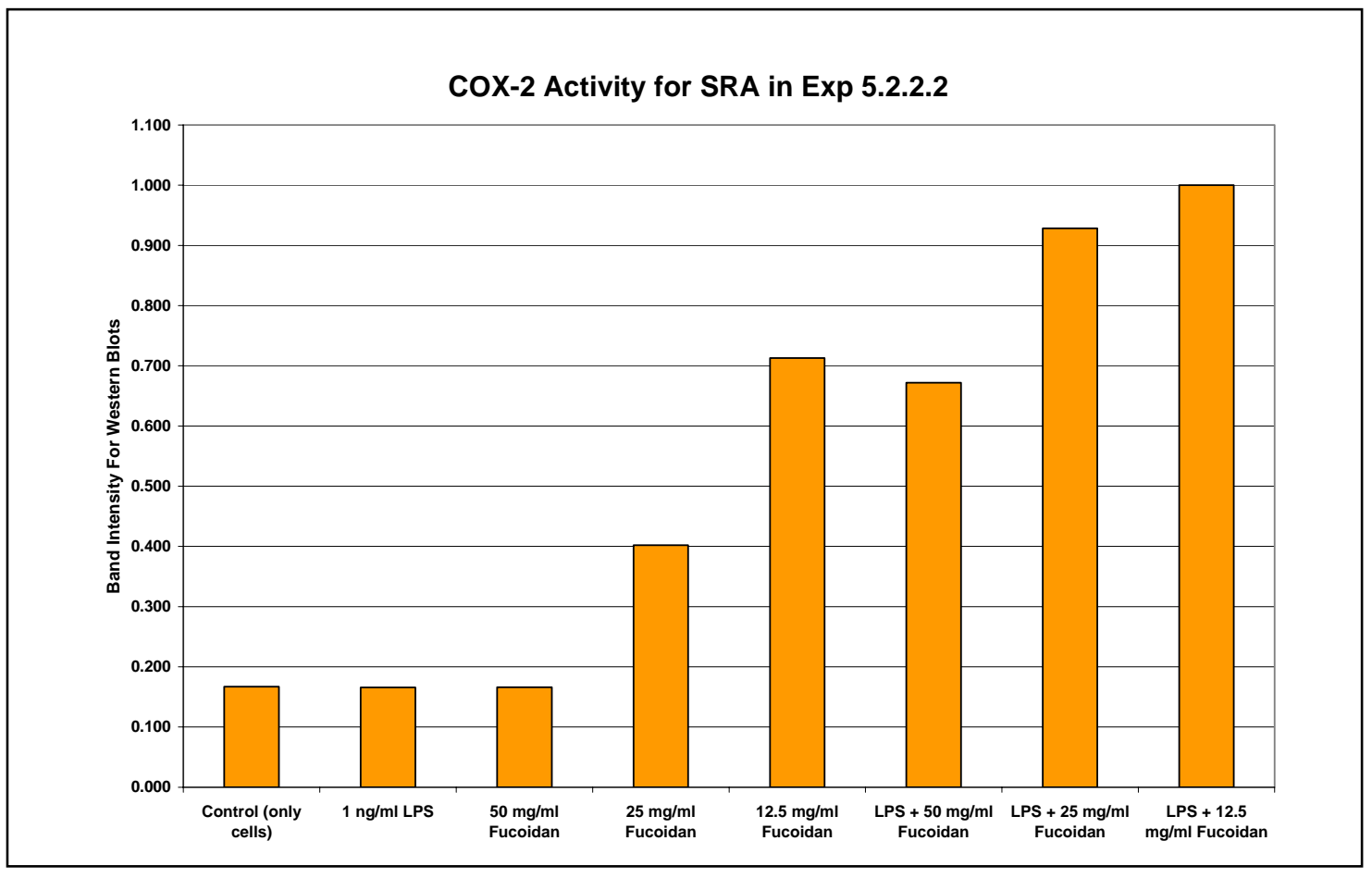

Figre 9: Histogram representing the iNOS activity of SRAs in Exp 5.2.2.2 


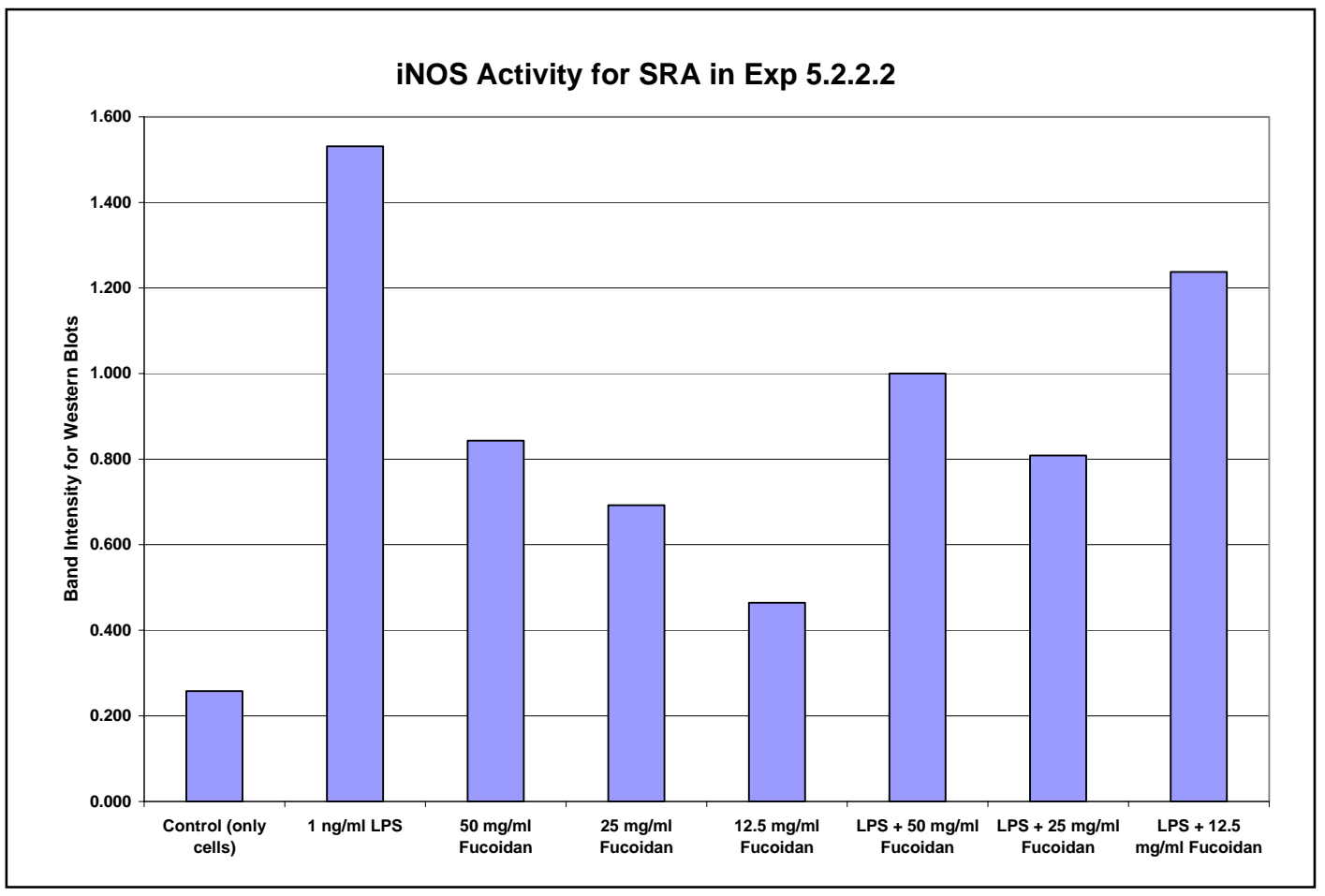

Figure 10: Histogram representing the COX-2 activity of SRAs in Exp 5.2.2.2

\subsubsection{Bands for western blot analysis}

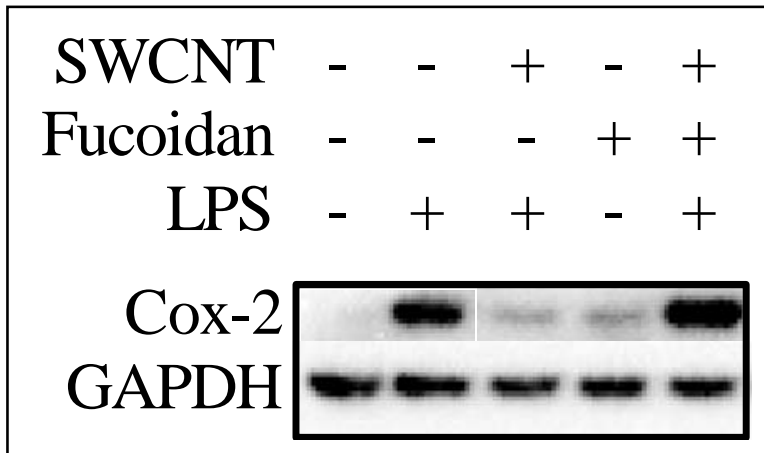

Figure 11: Representative western blot bands to show the functional role of Scavenger Receptors in the pro-inflammatory behavior of SWCNTs 


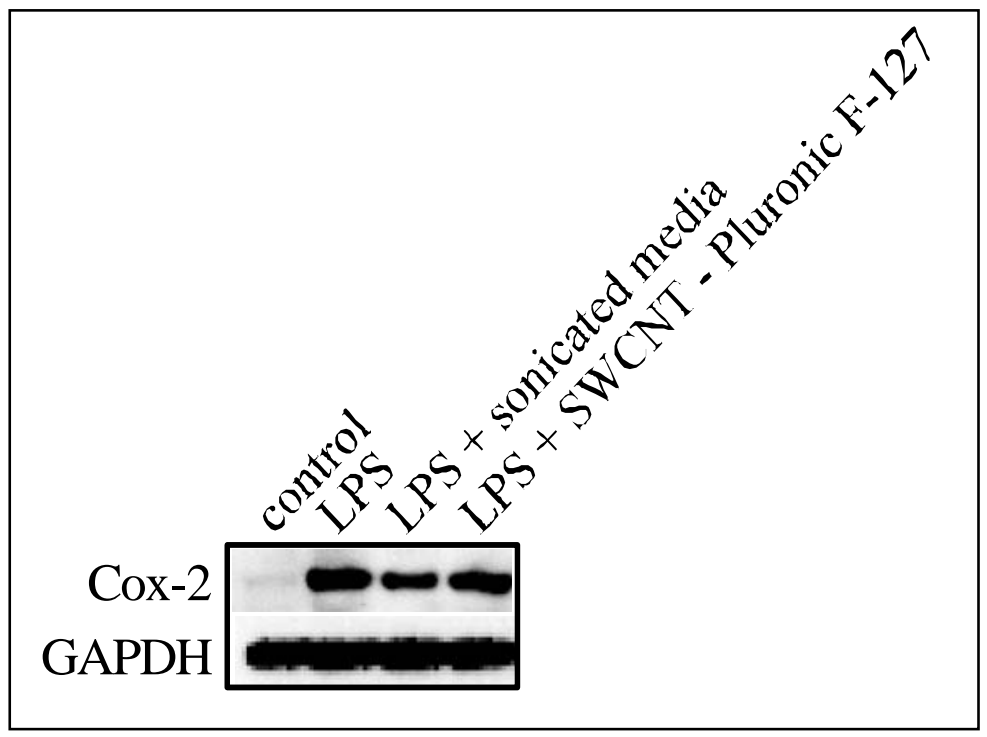

Figure 12: Western blot bands to show that the re-suspension of SWCNT in PL-127 does not cause any antagonism of LPS-Mediated Inflammatory Response

\subsection{Fluorescence Microscopic Imaging}
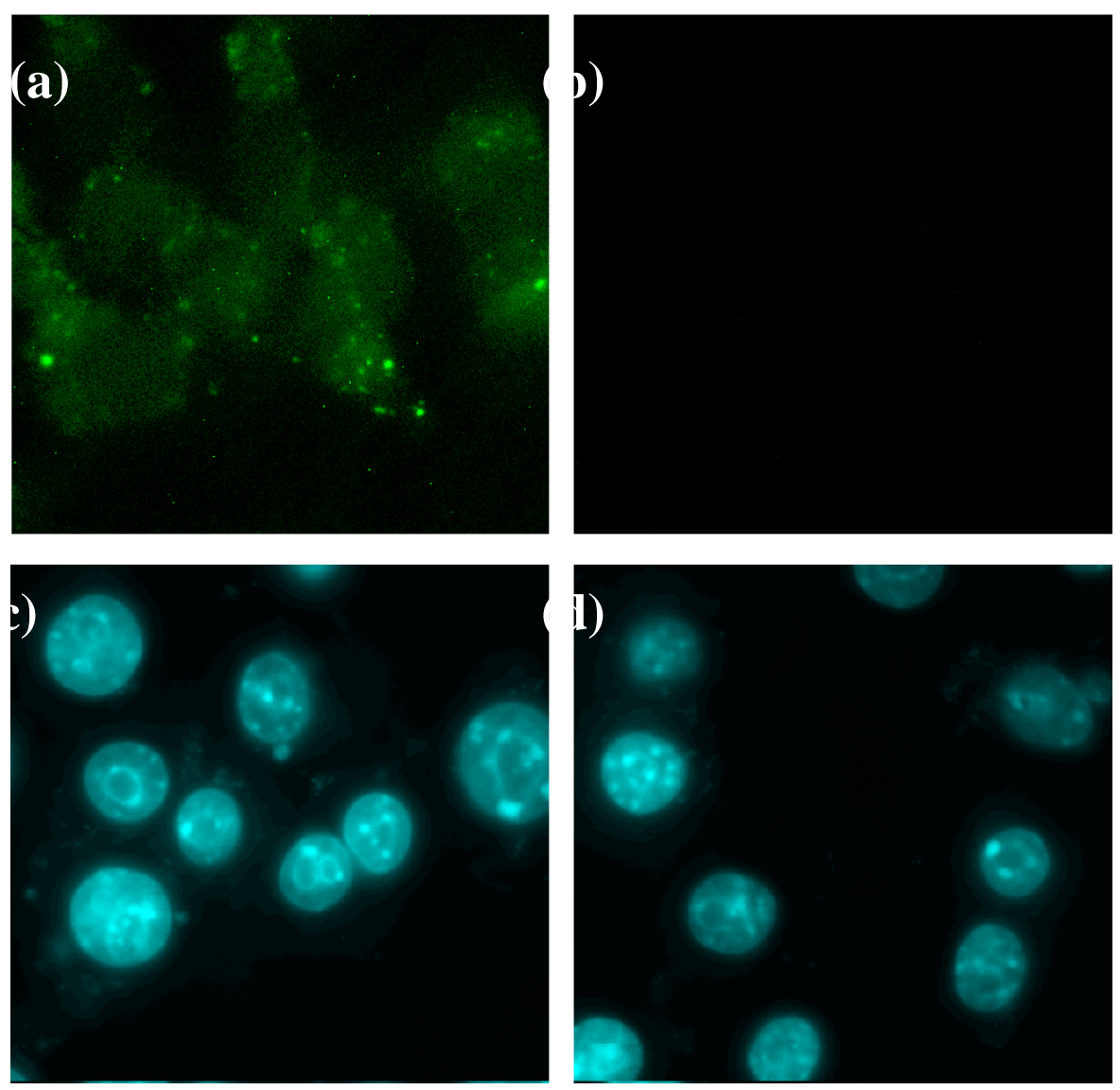

Figure 13: RAW 267.4 Cell experiment with SWCNTs. Fluorescent microscopic images of SWCNTs labeled with Alexafluor 647 in the (a) presence of Scavenger Receptor Antagonists (SRA) (b) absence of SRA (c) and (d) corresponding DAPI (to observe the nuclei) labeled cells for SWCNTs labeled with Alexafluor 647. Images clearly demonstrate the role of scavenger receptor in the SWCNTs uptake. 
The non-functionalized SWCNT sample used contained nickel and yttria as impurities along with carbon as observed from SEM-EDS results (Figure 1 and Table 11). The presence of impurities may play an inflammatory role in the macrophage cells. It is important to take some adequate steps to remove these elements (acid leaching or using purer samples of SWCNTs synthesized using Laser Ablasion etc) and verify observed effects attributed to the behavior of SWCNTs. (Note: This remained as incomplete work and is assigned as future work on this project). Certain white spots observed on the structures were recognized in SEM-EDS as areas of high impurity. The composition analysis (Figure 2 and Table 12) shows that the nickel and yttria content of these regions are comparatively higher than other areas of the SWCNT structure. SEM image of the structure of SWCNTs is shown in Figure 3 with a magnified image of the white spots on the SWCNT structure (inset).

Figure 4, as described before is the representative dose-response curve of Experiment 5.1.1. The points on the curve (representing the response of the cells to the nanotubes) indicate a drastic change in activity of cells for particle concentrations above $12.5 \mu \mathrm{g} / \mathrm{ml}$. At particle concentrations above $25 \mu \mathrm{g} / \mathrm{ml}$, the dose-response curve started attained a comparatively flat shape. To ensure that we were in the right window of concentration for dose-response analysis, we selected 25 and $50 \mu \mathrm{g} / \mathrm{ml}$ as the particle concentrations to dose the cells for western blot experiments.

Histograms in Figures 5-10 show iNOS and COX-2 activity of the macrophage cells incubated with corresponding SWCNT samples in the different experiments.

Figures 11-12 show representative bands from the western blot experiments indicating the role of scavenger receptors in nanoparticle uptake and the contribution of ultra-sonication techniques on the inflammatory behavior of SWCNTs respectively.

Macrophage activation when exposed to bacterial endotoxin (LPS) initiates inflammatory responses that can be observed from western blot bands. As expected and shown in Figure 11, a positive COX-2 activity is present in cells that were incubated with LPS and a very minimal COX-2 activity was observed when cells were incubated with SWCNT, but there was no activity when SWCNTs were added along with LPS.

Previous investigators have reported that scavenger receptors on the surface of mouse peritoneal macrophage cells can effectively internalize superparamagnetic iron oxide nanoparticle [42, 43] and modulate the cellular responses. It is possible that the SWCNTs are delivered into the murine macrophage cells in a similar fashion. It has been reported that SWCNTs can behave as generic intra-cellular transporters to deliver various proteins like bovine serum albumin (BSA) and cytochrome c [44]. The SWCNT-protein conjugate was observed to be released into the cell cytoplasm from the lysosomes. It is possible, that the nanotubes get associated with pieces of damaged proteins/albumin created during ultrasonication of the cell media. These pieces of damaged proteins enter the macrophage cells along with the SWCNTs, and exhibit an antagonistic effect of LPS mediated inflammatory (COX-2 and INOS) responses. As observed in Figure 12, re-suspending the SWCNTs in an emulsifying agent (PL-127) without any sonication exhibited no antagonism of LPS-mediated inflammatory responses in the cells.

The role of scavenger receptors in the uptake of SWCNTs was confirmed using fluorescent microscopy. Cells (DAPI labeled and non-labelled) were incubated with SWCNTs 
(similar to experimental sample) labeled with Alexa Fluor 647 along with scavenger receptor antagonists (fucoidan). Figure 13 shows the microscopic images of the the macrophage cells observed at $647 \mathrm{~nm}$. The absence of any fluorescent particles in Figure 13(b) indicates that no uptake was possible due to the non-availability of the scavenger receptors in the presence of fucoidan. Thus the process ofuptake of the nanotubes is clearly a receptor-mediated endocytotic uptake and hence the toxicity behavior can be correspondingly manipulated depending on the application of the nanoparticulate system in consideration.

[Note: Individual COX-2 and INOS bands for each western Blot Experiment has been recorded in the Laboratory Notebook at PNNL and a soft copy of some western Blot bands are available with Dr Thomas Weber at 331 Building, PNNL, Richland].

\section{Conclusions}

We have shown that non-functionalized SWCNTs display a variation in the inflammatory responses in macrophage cells depending on the dispersion medium of the nanotubes. Use of sonicated media as a dispersing medium led to antagonism of LPS mediated inflammatory responses whereas use of an emulsifier to disperse the nanotubes did not do so. This difference in results has tremendous implications in toxicity determination in nanoparticulate materials.

In addition, we have also shown that scavenger receptors play a functional role in the process of nanotube uptake, where SWCNTS behave as intracellular transporters to 'piggyback' proteins into the macrophage cells. By themselves, the SWCNTs to not show any substantial inflammatory effects, but in the presence of damaged proteins etc can exhibit COX2 and INOS responses on the RAW 264.7 cells

\section{Acknowledgments}

The author of this report would like to acknowledge the PNNL Summer Research Institute (SRI) in Interfacial and Condensed Phase Chemical Physics and the Supplement proposal to NIRT-NSF Grant (NSF Grant No. EEC-0506560) for financial support.

\section{References}

1. Pantarotto, D., et al., Translocation of bioactive peptides across cell membranes by carbon nanotubes. Chemical Communications, 2004(1): p. 16-17.

2. Bianco, A. and M. Prato, Can carbon nanotubes be considered useful tools for biological applications? Advanced Materials, 2003. 15(20): p. 1765-1768.

3. Baughman, R.H., A.A. Zakhidov, and W.A. de Heer, Carbon nanotubes - the route toward applications. Science, 2002. 297(5582): p. 787-792.

4. Warheit, D.B., et al., Comparative pulmonary toxicity assessment of single-wall carbon nanotubes in rats. Toxicological Sciences, 2004. 77(1): p. 117-125.

5. Warheit, D.B., What is currently known about the health risks related to carbon nanotube exposures? Carbon, 2006. 44(6): p. 1064-1069.

6. Shim, M., et al., Functionalization of carbon nanotubes for biocompatibility and biomolecular recognition. Nano Letters, 2002. 2(4): p. 285-288.

7. Bahr, J.L. and J.M. Tour, Covalent chemistry of single-wall carbon nanotubes. Journal of Materials Chemistry, 2002. 12(7): p. 1952-1958. 
8. Dumortier, H., et al., Functionalized carbon nanotubes are non-cytotoxic and preserve the functionality of primary immune cells. Nano Letters, 2006. 6(7): p. 1522-1528.

9. Kam, N.W.S., et al., Nanotube molecular transporters: Internalization of carbon nanotube-protein conjugates into mammalian cells. Journal of the American Chemical Society, 2004. 126(22): p. 6850-6851.

10. Sayes, C.M., et al., Functionalization density dependence of single-walled carbon nanotubes cytotoxicity in vitro. Toxicology Letters, 2006. 161(2): p. 135-142.

11. Cui, D.X. and H.J. Gao, Advance and prospect of bionanomaterials. Biotechnology Progress, 2003. 19(3): p. 683-692.

12. Gao, H.J., et al., Spontaneous insertion of DNA oligonucleotides into carbon nanotubes. Nano Letters, 2003. 3(4): p. 471-473.

13. Cui D, O.C., Ravindran S, Kong Y, Gao H., Encapsulation of pt-labelled DNA molecules inside carbon nanotubes. Mech Chem Biosyst., 2004. 1(2): p. 113-21.

14. Kam, N.W.S., Z.A. Liu, and H.J. Dai, Carbon nanotubes as intracellular transporters for proteins and DNA: An investigation of the uptake mechanism and pathway. Angewandte Chemie-International Edition, 2006. 45(4): p. 577-581.

15. Cui, D.X., et al., Effect of single wall carbon nanotubes on human HEK293 cells. Toxicology Letters, 2005. 155(1): p. 73-85.

16. Rojas-Chapana, J.A. and M. Giersig, Multi-walled carbon nanotubes and metallic nanoparticles and their application in biomedicine. Journal of Nanoscience and Nanotechnology, 2006. 6(2): p. 316-321.

17. Murakami, H. and N. Nakashima, Soluble carbon nanotubes and their applications. Journal of Nanoscience and Nanotechnology, 2006. 6(1): p. 16-27.

18. Liopo, A.V., et al., Biocompatibility of native and functionalized single-walled carbon nanotubes for neuronal interface. Journal of Nanoscience and Nanotechnology, 2006. 6(5): p. 1365-1374.

19. Liu, G.D. and Y.H. Lin, Carbon nanotube-tem plated assembly of protein. Journal of Nanoscience and Nanotechnology, 2006. 6(4): p. 948-953.

20. Ni, Y.C., et al., Chemically functionalized water soluble single-walled carbon nanotubes modulate neurite outgrowth. Journal of Nanoscience and Nanotechnology, 2005. 5(10): p. 1707-1712.

21. Perez, B., et al., Glucose biosensor based on carbon nanotube epoxy composites. Journal of Nanoscience and Nanotechnology, 2005. 5(10): p. 1694-1698.

22. Sinha, N., J.Z. Ma, and J.T.W. Yeow, Carbon nanotube-based sensors. Journal of Nanoscience and Nanotechnology, 2006. 6(3): p. 573-590.

23. Service, R.F., American Chemical Society meeting: Nanomaterials show signs of toxicity. Science, 2003. 300(5617): p. 243-243.

24. Shvedova, A.A., et al., Exposure to carbon nanotube material: Assessment of nanotube cytotoxicity using human keratinocyte cells. Journal of Toxicology and Environmental Health-Part A, 2003. 66(20): p. 1909-1926.

25. Monteiro-Riviere, N.A., Multi-walled carbon nanotube exposure in human epidermal keratinocytes: Localization and proteomic analysis. Abstracts of Papers of the American Chemical Society, 2005. 229: p. U911-U912.

26. Monteiro-Riviere, N.A., et al., Multi-walled carbon nanotube interactions with human epidermal keratinocytes. Toxicology Letters, 2005. 155(3): p. 377-384.

27. Mattson, M.P., R.C. Haddon, and A.M. Rao, Molecular functionalization of carbon nanotubes and use as substrates for neuronal growth. Journal of Molecular Neuroscience, 2000. 14(3): p. 175-182. 
28. Chan, H.C., et al., A phenylacetate derivative, SCK6, inhibits cell proliferation via G(1) cell cycle arrest and apoptosis. European Journal of Pharmacology, 2003. 467(1-3): p. 31-39.

29. Lam, C.W., et al., Pulmonary toxicity of single-wall carbon nanotubes in mice 7 and 90 days after intratracheal instillation. Toxicological Sciences, 2004. 77(1): p. 126-134.

30. Maynard, A.D., et al., Exposure to carbon nanotube material: Aerosol release during the handling of unrefined single-walled carbon nanotube material. Journal of Toxicology and Environmental Health-Part A, 2004. 67(1): p. 87-107.

31. Donaldson, K., P.H. Beswick, and P.S. Gilmour, Free radical activity associated with the surface of particles: A unifying factor in determining biological activity? Toxicology Letters, 1996. 88(1-3): p. 293-298.

32. Ghio, A.J., et al., Metals associated with both the water-soluble and insoluble fractions of an ambient air pollution particle catalyze an oxidative stress. Inhalation Toxicology, 1999. 11(1): p. 37-49.

33. Hiura, T.S., et al., The role of a mitochondrial pathway in the induction of apoptosis by chemicals extracted from diesel exhaust particles. Journal of Immunology, 2000. 165(5): p. 2703-2711.

34. Kumagai, Y., et al., Generation of reactive oxygen species during interaction of diesel exhaust particle components with NADPH-cytochrome P450 reductase and involvement of the bioactivation in the DNA damage. Free Radical Biology and Medicine, 1997. 22(3): p. 479-487.

35. Abe, S., et al., Diesel exhaust (DE)-induced cytokine expression in human bronchial epithelial cells - A study with a new cell exposure system to freshly generated DE in vitro. American Journal of Respiratory Cell and Molecular Biology, 2000. 22(3): p. 296-303.

36. Mitchell, J.A., S. Larkin, and T.J. Williams, Cyclooxygenase-2 - Regulation and Relevance in Inflammation. Biochemical Pharmacology, 1995. 50(10): p. 1535-1542.

37. Chien, P.S., O.T. Mak, and H.J. Huang, Induction of COX-2 protein expression by vanadate in A549 human lung carcinoma cell line through EGF receptor and p38 MAPK-mediated pathway. Biochemical and Biophysical Research Communications, 2006. 339(2): p. 562-568.

38. Kroncke, K.D., K. Fehsel, and V. Kolb-Bachofen, Inducible nitric oxide synthase in human diseases. Clinical and Experimental Immunology, 1998. 113(2): p. 147-156.

39. Chauhan, V., et al., Effects of ambient air particles on nitric oxide production in macrophage cell lines. Cell Biology and Toxicology, 2004. 20(4): p. 221-239.

40. Furumoto, K., et al., Hepatic uptake of negatively charged particles in rats: possible involvement of serum proteins in recognition by scavenger receptor. Journal of Controlled Release, 2004. 97(1): p. 133-141.

41. Nam, H.Y., et al., The role of nitric oxide in the particulate matter (PM2.5)-induced NF kappa B activation in lung epithelial cells. Toxicology Letters, 2004. 148(1-2): p. 95102.

42. Weissleder, R., et al., Superparamagnetic Iron-Oxide - Enhanced Detection of Focal Splenic Tumors with Mr Imaging. Radiology, 1988. 169(2): p. 399-403.

43. Stark, D.D., et al., Superparamagnetic Iron-Oxide - Clinical-Application as a Contrast Agent for Mr Imaging of the Liver. Radiology, 1988. 168(2): p. 297-301.

44. Kam, N.W.S. and H.J. Dai, Carbon nanotubes as intracellular protein transporters: Generality and biological functionality. Journal of the American Chemical Society, 2005. 127(16): p. 6021-6026. 
PNNL-16185 
PNNL-16185

\title{
A Fast Screening Technique to Evaluate Si Detector Response to Ions
}

\author{
Mikael Elfman \\ Engineering Nuclear Physics Research Scientist, Lund Institute of Technology, \\ University of Lund \\ Lund, Sweden \\ and \\ Yanwen Zhang \\ Pacific Northwest National Laboratory \\ Richland, WA
}

National security issues have recently prompted an urgent need for improved radiation detector materials. Existing materials do not meet the stringent requirements of nuclear nonproliferation and homeland security applications. Accelerated materials discovery efforts are needed to develop the next generation radiation detector materials with excellent energy resolution at room temperature. Fast screening techniques are, therefore, required to investigate numerous candidate materials to provide quick information about material properties relevant to detector performance.

The response generated in detector materials by radiation energy deposition is the fundamental basis for understanding material functionality as a radiation detector. Energy resolution and detection efficiency can provide a critical basis for identification of possible candidate materials. The current work demonstrates a fast screening technique in obtaining relevant quantitative data on the response of a silicon detector, which is a benchmark material. The experimental approach used in this work can be applied to quickly evaluate the candidates of radiation detector materials.

Silicon detectors are widely used for determining energies of neutral and charged particles in various ion beam analysis (IBA) techniques, radioactive ion beam applications, and satellite experiments. Despite their extremely widespread use for over four decades $\left[{ }^{1}\right]$ and limited approaches to calibration $\left[{ }^{2,3}\right]$, there exist no predictive calibrations for ions with a wide range of atomic number and energies. Silicon detector response to ions and the pulse height defect (PHD) for heavy particles have been reported over the years. However, contributions to PHD, such as non-ionization energy loss and inefficient electron-hole (e-h) collection in the detector active volume, are not fully analyzed in a quantitative manner, and variation of the average energy, $\varepsilon$, required to produce an e-h pair remains controversial. In the current study, the pulse height defect in a Si detector was extensively investigated over a wide energy region using a time-of-flight (TOF) telescope, and fundamental understanding of Si detector response to ions is addressed.

The experimental setup is shown in Fig.1. Energetic ion beams produced using a tandem accelerator are forward scattered by a bulk $\mathrm{Au}$ target into the TOF telescope, which is followed by the $\mathrm{Si}$ detector of interest. The energy of scattered ions ranges from a few hundreds keV to a few tens $\mathrm{MeV}$. The Si detector used in this study was an

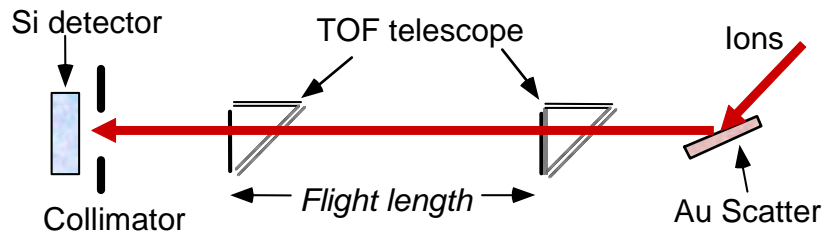

Fig. 1 Schematic diagram of the experimental 
ORTEC ultra ion-implanted-silicon detector with entrance contact made by an extremely thin boron implantation. The detector response is simultaneously measured over a wide energy range, for which the energy is determined from the TOF and isotopic mass prior to impinging on the Si detector. During the measurements, the output signals from the timing detectors were recorded in an inverse start-stop mode to minimize the dead time, and the Si detector response is delayed in order to coincide with the TOF signal. For each ion, the energy is determined as

$$
E(k e V)=\frac{1}{2} M \cdot\left(\frac{L}{T O F}\right)^{2}-\Delta E_{\text {foil }}=\text { slope } \cdot E(\text { ch })+\text { offset }
$$

where $M$ is the ion mass, TOF is the time for the ion to pass through the flight length $(L)$, and $\Delta E_{\text {foil }}$ is the energy loss in traversing the carbon foil in the second timing detector. The time calibration of the TOF telescope was done using both the signals of the ions that are scattered from the Au target surface and the timing calibrator (ORTEC module 462), with the two independent results confirming each other to achieve a reliable time calibration.

The detector response (the measured pulse height) for ions with atomic number between 1 and 79 is shown in

Fig. 2 versus particle energy. The results indicate that

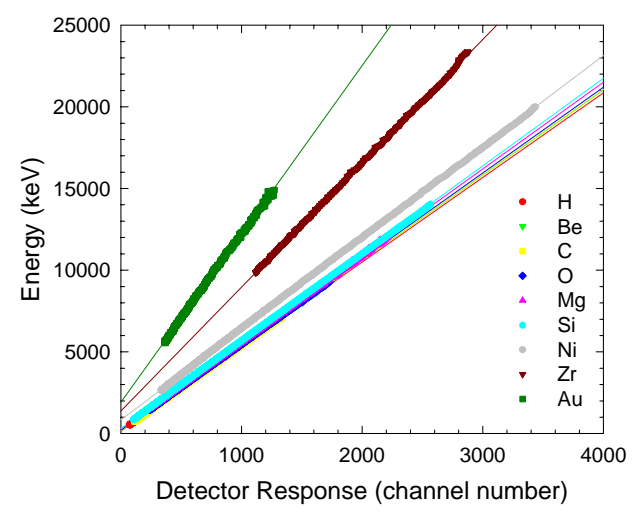

Fig. 2 True energy of various ions versus the pulse channel number from the Si detector. the pulse height-energy relation for each element can be described by a linear relationship (Eq. 1), where the mean slope (keV/channel) is the energy spanned by one channel and the mean offset $(\mathrm{keV})$ is mainly the energy loss in the entrance window and dead layer of the detector. Although the data closely followed a straight line relation, small systematic deviations of the data were observed, even for light particles, which will be discussed in more detail.

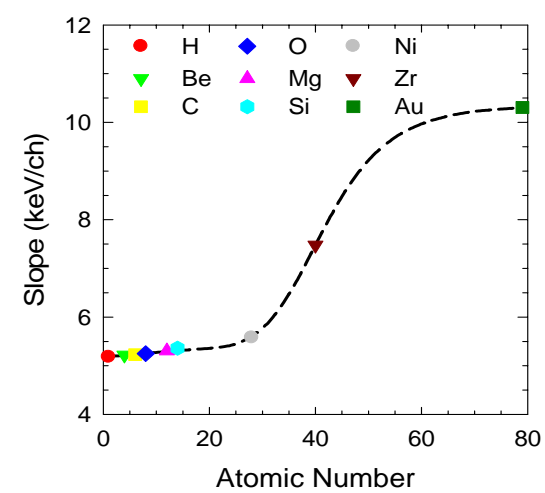

Fig. 3 Atomic number dependence of the mean slope and the mean intercept.

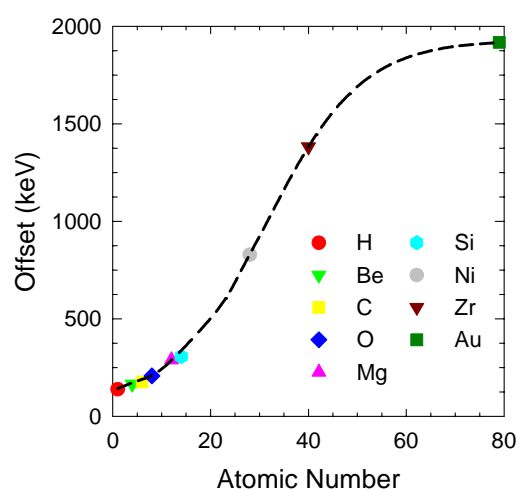

Fig. 4 Relative detection

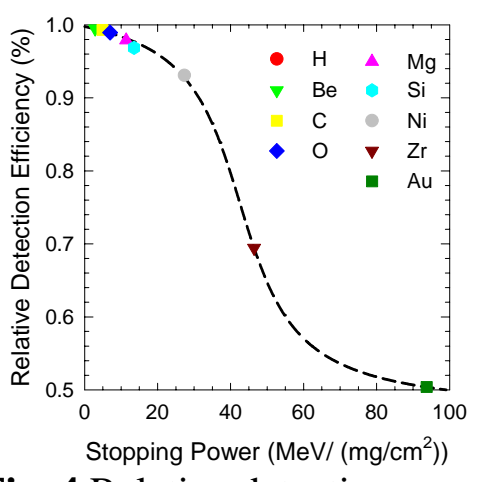

efficiency of e-h pair collection

The calibration parameters from Eq. 1 for a wide range of ions are shown in Fig. 3. A significant increase in the mean slope can be observed for heavy particles. The origin of this increase is unclear; however one might speculate that this is a consequence of non-linear effects and strong plasma recombination. It is important to note that the increase in the mean slope with atomic number is not a sufficient condition to determine if the effective electronic 
energy deposition to excite an e-h pair varies because the slope also depends on the probability of e-h pair collection. The efficiency of e-h pair collection is markedly affected by the production, migration and recombination of e-h pairs in the dense plasma created along the ion track. The relative detection efficiency derived from this study is shown in Fig. 4 as a function of stopping power for various particles at energy of $500 \mathrm{keV}$ per nucleon.
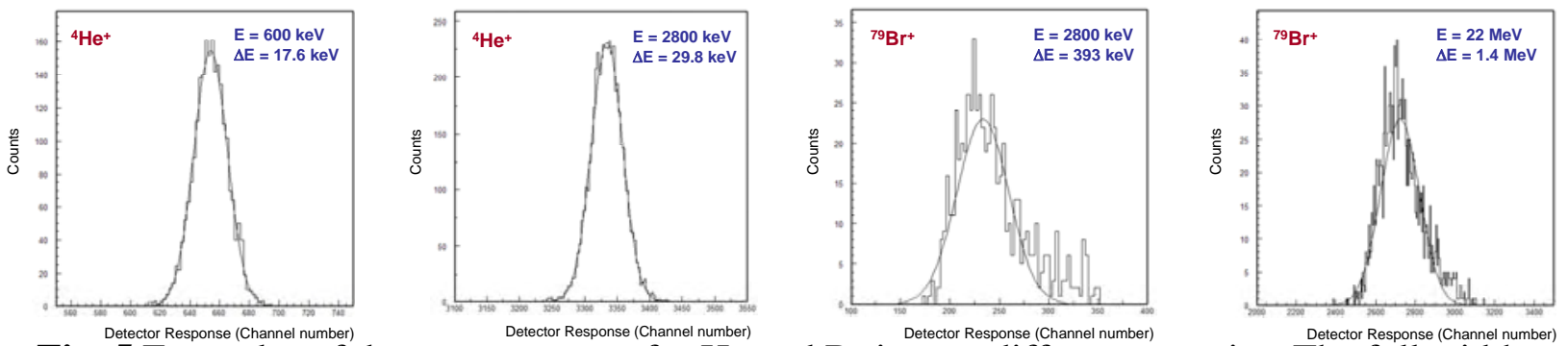

Fig. 5 Examples of detector response for He and $\mathrm{Br}$ ions at different energies. The full width at half maximum (FWHM) is illustrated in the corresponding plots.

The detector response for a given energy is shown in Fig. 5 using $\mathrm{He}$ and $\mathrm{Br}$ ions as examples. The peak broadening results from the intrinsic signal variance, together with the energy straggling in the carbon foil in the second timing detector and the detector contact/dead layers, as well as the uncertainties associated with the non-ionization part of the nuclear stopping. A new method of analysis of pulse height data is applied with corrections of the above mentioned uncertainties to obtain signal variance for silicon. The variation of the average energy for e-h pair creation is determined as a function of electronic stopping power.
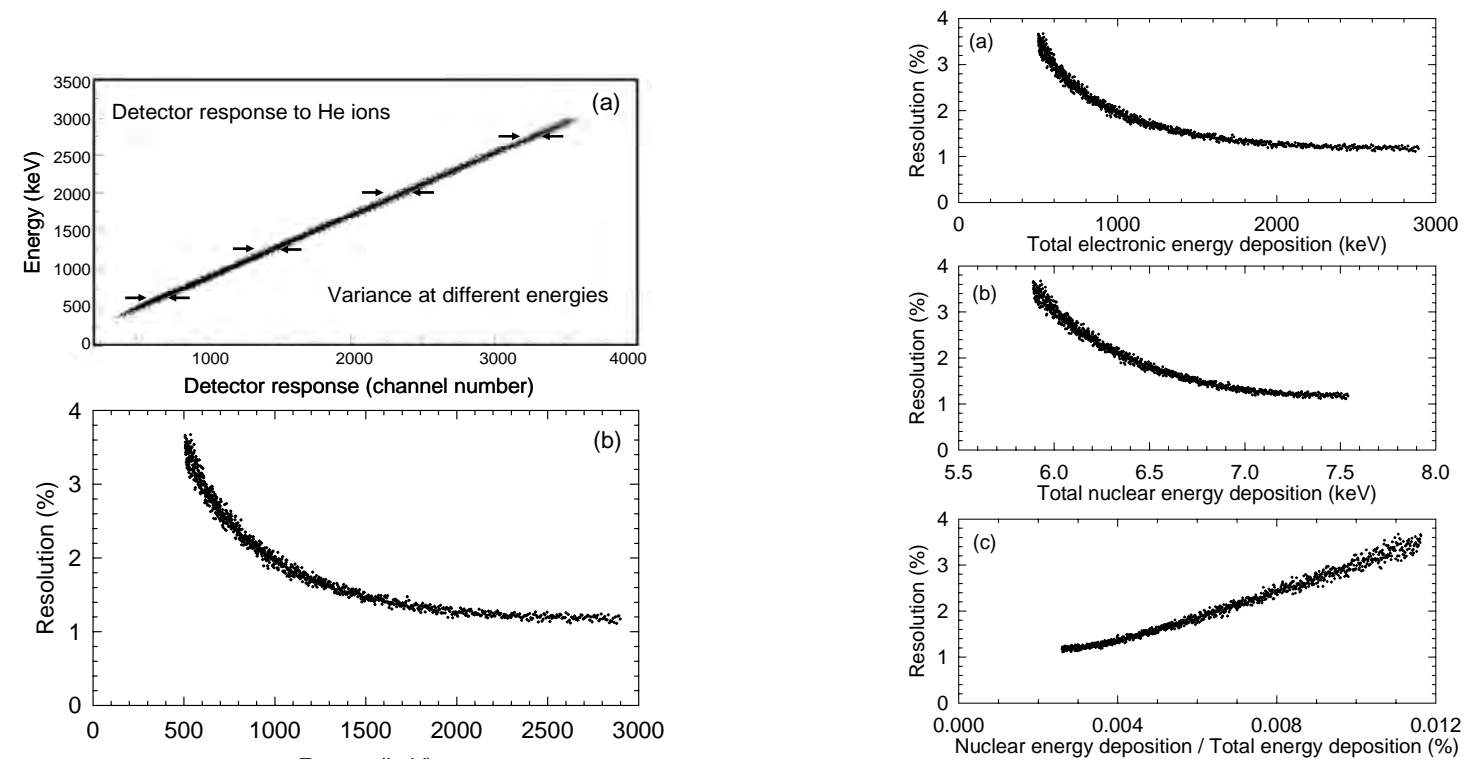

Fig. 6 (a) Detector response for He ions over a wide energy range, and (b) the measured energy resolution as a function of energy deposited in the detector.

Fig. 7 Detector resolution as a function of (a) electronic energy deposition, (b) nuclear energy deposition, and (c) the ratio of energy that does not produce e-h pairs to total energy deposition

To demonstrate TOF-ion approach as a fast screening technique, 3.0 MeV He ions forward scattered from the Au target were used to test the detector response. The electronic modules were optimized for processing the signals. During the measurement, more than 6 million He ions were detected by both the Si detector and TOF telescope in a coincident mode in 20 minutes. Fig. 6(a) shows the recorded detector response, for which the energy is determined by 
the TOF telescope. The signal broadening results from the statistical fluctuations in the number of charge carriers, together with the energy straggling in the carbon foil in the second timing detector and the Si detector contact layer, the limited timing resolution, the uncertainties associated with the non-ionization part of the nuclear stopping, as well as the electronic noise. The measured energy resolution in the current study is defined as the full width at half maximum (FWHM) of the peak normalized to its energy, and the results indicate a decrease with increasing particle energy, as shown in Fig. 6(b). The saturation level at $1.2 \%$ is the detector resolution for He ions including all sources of variances.

The measured energy resolution for He ions is shown in Fig. 7 as a function of electronic $\left[{ }^{4}\right]$ and nuclear energy deposition, as well as the ratio of non-detectitable to total ion energy. Similar behavior is observed from Figs. 6(b) and 7(a), since the majority of the e-h pairs is created from the electronic energy deposition. The broadening at lower energy ends, for both electronic and nuclear energy deposition, is attributed to statistical fluctuations due to relatively few charge carriers produced. Extrapolation of the results in Fig. 7(c) to zero should provide information about detector response when the radiation energy is deposited by electrons instead of ions.

For fast screening purposes, the candidates of radiation detector materials can be simply investigated using readily available energetic ions, such as He ions. The measured energy resolution observed in the candidate materials can be compared to the results from a standard detector, such as the Si detector in the current study. 


\title{
Progress Report: Attempting to investigate the effects of confinement on Organophosphorus Hydrolase from P. diminuta by Molecular Dynamics simulation.
}

\author{
Diego Enry Barreto Gomes \\ Universidad Federal do Rio De Janeiro, \\ Rio De Janeiro. Brazil \\ and \\ Dr. Thereza Soares, C\&ISD \\ Pacific Northwest National Laboratory \\ Richland, WA 99352
}

\section{Introduction}

Organophosphorous (OP) compounds are potent neurotoxic substances widely used as pesticides, insecticides and as chemical warfare nerve weapon. Because of the toxicity and general use of these neurotoxic compounds, several efforts focus on development methods to detect and detoxify these hazardous substances. Many analytical methods have been proposed ${ }^{1}$ to detect OP compounds but they lack of portability and fast response, have high cost and require of people capacitation. A promising alternative to an efficient, highly sensitive and fast way of detection of OP compounds are the enzymatic biosensors based on Organophosphorous Hydrolase (OPH; EC 3.1.8.1) a dimeric, binuclear metalloenzyme isolated from the soil bacterium Pseudomonas diminuta which is capable to selectively catalyze a hydrolytic reaction in the $\mathrm{P}-\mathrm{O}, \mathrm{P}-\mathrm{S}, \mathrm{P}-\mathrm{CN}$, and $\mathrm{P}-\mathrm{F}$ bonds present a large variety of $\mathrm{OP}$ compounds ${ }^{2}$. OPH biosensors have a potential use in Military, Industrial and Environmental applications as detection and even detoxification of contaminated samples. OPH-based biosensors take advantage of $\mathrm{OPH}$ responding to $\mathrm{OP}$ compounds as an enzyme substrate system, allowing a single-step direct method to measure enzyme activity ${ }^{3}$. By the end of the reaction the enzyme is reactivated and the biosensor can be reused for continuous monitoring or detoxification of OP compounds in the surrounding environment.

It has been show that confinement of OPH in a 2\% HOOC Functionalized Mesoporous Silica (HOOC-FMS) provided both high affinity for the protein molecules and a favored microenvironment that resulted in exceptionally high immobilization frequency with enhanced stability and increased enzyme specific activity by $200 \backslash \%{ }^{4}$ compared to OPH in solution, but the same effect in specific activity is not displayed with confinement in Unfunctionalized Mesoporous Silica. What happens to the enzyme in these confinement conditions to induce this outstanding increase in activity? How the nonspecific interactions between FMS molecules influence the vital reactions and events that define OPH activity?

In this report we investigate the effects produced by molecular confinement internal enzyme dynamics of $\mathrm{OPH}$ dimer in two simple models of confinement on the conformational dynamics of $\mathrm{OPH}$ dimmer from Pseudomonas diminuta and compare the results to $\mathrm{OPH}$ free in solution. We also would like to show how these effects may influence the interactions with soman as the substrate in order to produce a more efficient catalysis. We initiated this work based on the hypothesis that the adsorption of the enzyme to a circular array the oxygen atoms could mimic 
the effects of confinement on the structure and stability of proteins and could thereby provide a suitable model system for the effects of confinement in a 2\% HOOC-FMS.

\section{Computational Methods}

System setup: Four separate sets of MD simulations were undertaken, all using NWChem software ${ }^{5}$, each running on 72 Itanium-2 Madison cores. The four studies differed in their starting configuration but shared a very similar simulation protocol. The OPH dimmer was obtained from the crystal structure $1 \mathrm{HZY}^{6}$. Partial atomic charges for Soman and carbamylated Lys-169 were determined to reproduce the molecular electrostatic potential by using the restrained electrostatic potential (RESP) procedure ${ }^{7}$ applied to 6-31G* optimized B3LYP/DZVP structures, the bond and rotational parameters of a carboxylate were used for of Lys-169 and the bond, angle and rotational parameters involving central phosphorous in Soman were adapted from parameters involving the central phosphate group of nucleic acid. All other force field parameters were obtained from amber99 $\mathrm{ff}^{8}$.

Based on pKa calculation with propKa ${ }^{9}$ we assigned the proper protonation states to amino acid residues. Since propKa does not consider the contribution of charges from heteroatoms such as Zinc atoms, the assignment of the charges was dubious for HIS254. The four starting configurations were prepared as follows:

OPH-free and OPH-fix: These systems were simulated without any ligand and with HIS254 protonated only in chain B as proposed by propKa calculation. As an approach to represent confinement, $\mathrm{OPH}$-fix had all surface LysN $\varepsilon$ atoms fixed to simulate the effect of confinement due to electrostatic interaction of these charged.

s-OPH-free and s-OPH-fms: These systems were simulated with Soman ligand docked to the active site, in a non-covalent bound. s-OPH-fms was adsorbed to an array of atoms as described below.

Representation of s-OPH adsorbed to FMS: To represent the functionalized mesoporous silica (FMS) as described by Lei et $\mathrm{al}^{4}$ we used NANOPORE, our home made program, to design a $17 \mathrm{~nm}$ diameter, $13 \mathrm{~nm}$ length, quarter circle array of $1.0 \mathrm{~nm}$ separated oxygen atoms with enough room to fit the OPH dimmer as shown in Figure 1. To properly orient the OPH dimmer (OPHd) on the surface of FMS we used $\mathrm{APBS}^{10}$ to calculate the electrostatic surface OPHd and we used PyMol ${ }^{11}$ to orient OPHd to best fit the charge complementarily. 
PNNL-16185

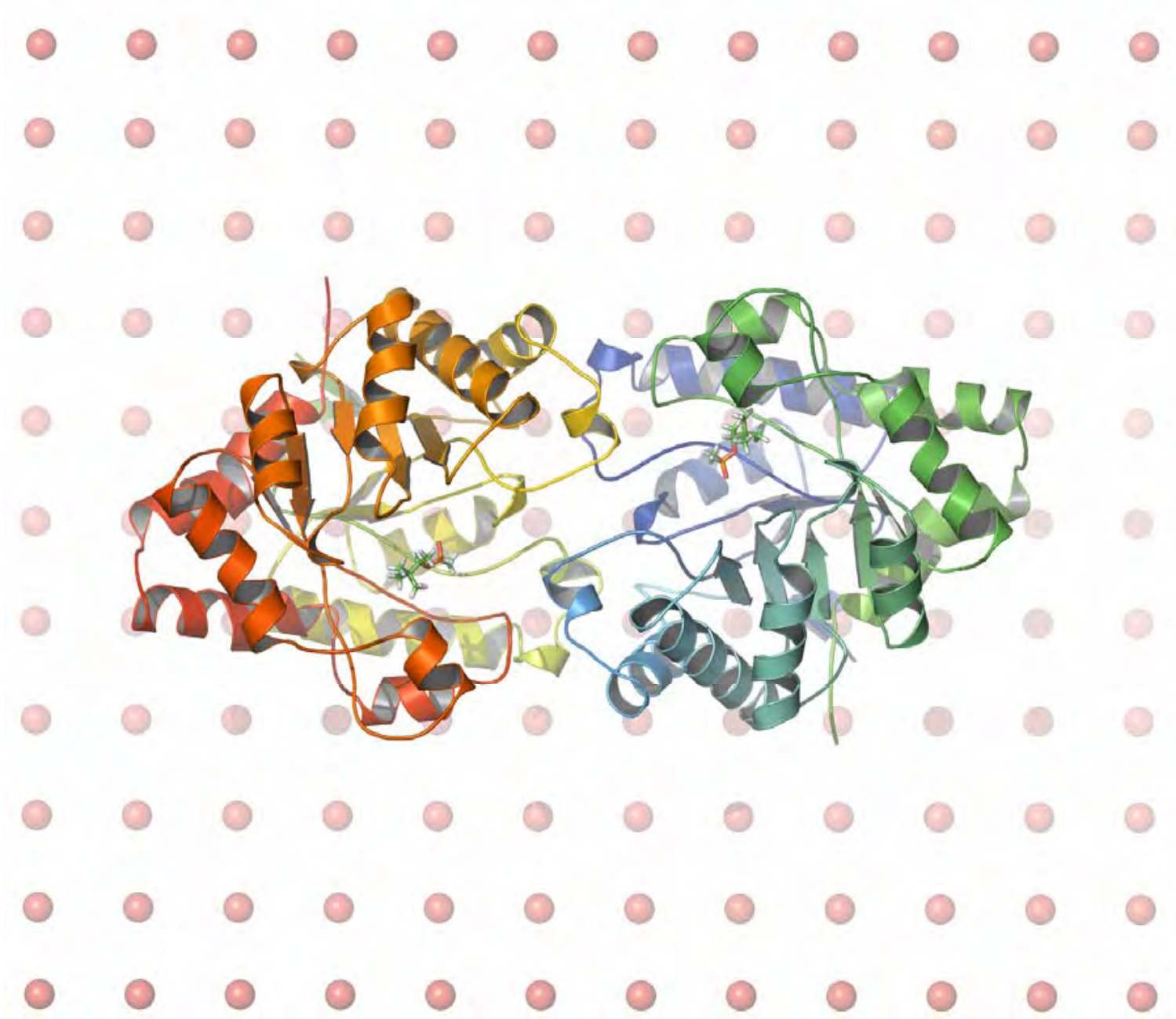

Figure 1 The Functionalized Mesoporous Silica represented as a 17nm diameter, 13nm length, quarter circle array of 1.0nm separated oxygen atoms (red spheres) with the OPH dimmer (ribbons) adsorbed.

PMF calculations: To estimate how the free energy of the interaction of OPH with Soman changes as a function of binding we applied the Potential Mean Force calculations with force constant ranging from $100 \mathrm{kj} / \mathrm{mol}$ to $800 \mathrm{kj} / \mathrm{mol}$, equilibrating for $50 \mathrm{k}-100 \mathrm{k}$ steps and sampling for $50 \mathrm{k}-100 \mathrm{k}$ steps, with $\lambda=0.01 \mathrm{~nm}$.

Simulation setup: The molecules simulated were solvated with SPCE $^{12}$ water model in a $13 \times 13 \times 14 \mathrm{~nm}$ box and counter ions were added to neutralize the charges. Non bonded interactions were considered to a cutoff of $1.0 \mathrm{~nm}$, updating the neighbor pair list every 10 steps, with time-step set to $2 \mathrm{fs}$. The electrostatic interactions were calculated with the Particle Mesh Ewald method ${ }^{13}$, and the SHAKE algorithm ${ }^{14}$ was used to constrain all bond lengths involving hydrogens. The systems were thermodynamically coupled to a $298 \mathrm{~K}$ bath and pressure coupling at 1 bar. An initial energy minimization was carried out followed by a 150 ps warm up simulation to equilibrate the systems before a production run of $5 \mathrm{~ns}$. The s-OPH-fms system was allowed to perform the minimization and warm up steps before adding the counter ions to the FMS grid and then repeat these two steps. 


\section{Results and Discussion}

pKa calculations: The protonation state of residues was determined according to the method implemented in propKa for $\mathrm{pH}$ 7.0. Figure 2 shows the histidines of the active site of OPH. Although propKa is a very fast and accurate method it was not able take into account the Zinc atoms and assigned of HIS 254 as protonate, in conflict with the positively charged environment. Considering this limitation of propKa we decided to simulate each of the two OPH dimmer chains with a different protonation for HIS 254: non protonated for chain A (HIE) and protonated (HIP) for chain B.

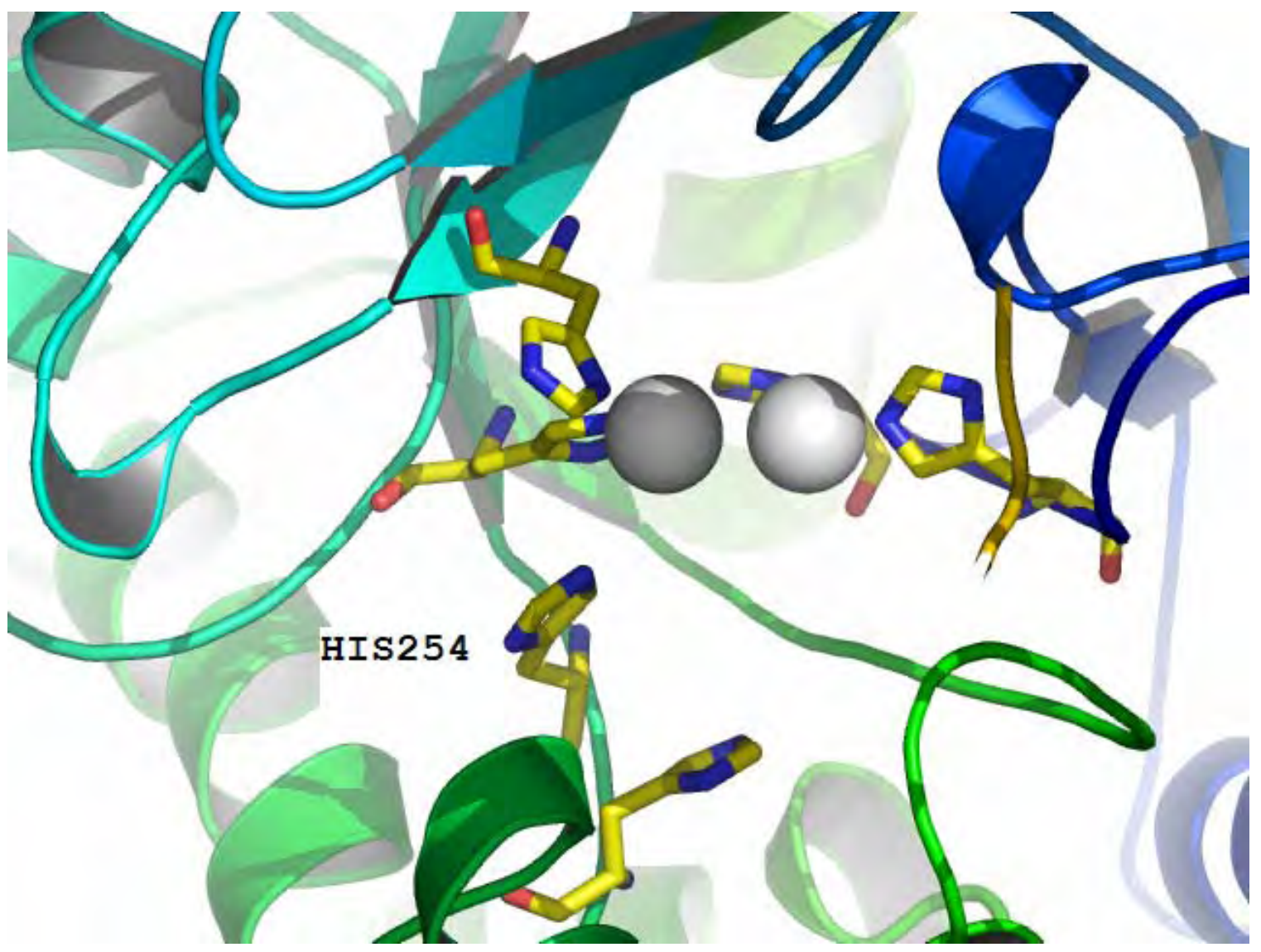

Figure 2 The histidines (sticks) of the active site of OPH (ribbons) and de Zinc atoms (gray spheres).

\subsection{MD simulations of OPH with or without LYS restraints.}

Trajectory stability: As a measure of structural stability we calculated the root-mean-square deviations (RMSD) for all backbone atoms in the OPH original file compared to each snapshot of the trajectory (Figure 3). We can see that the trajectory is stable after 2.0nsec. Energy and temperature remains steady over the course of the simulation (data not shown). 


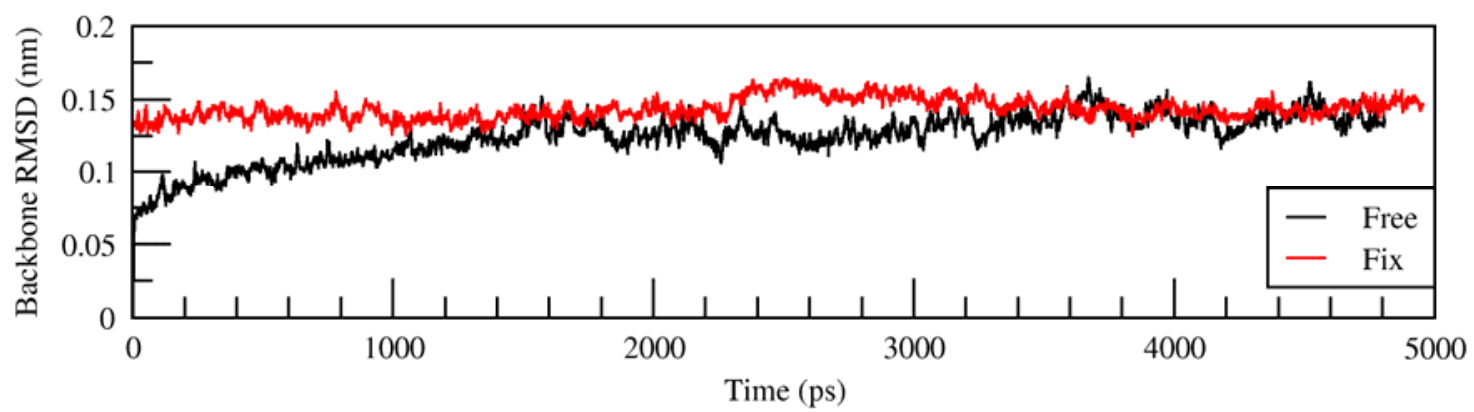

Figure 3 The Root Mean Squared Deviation to the first frame of molecular dynamics simulation for the backbone atoms for OPH-free (black) and OPH-fix (red).

Protein movements: To investigate if the effects of confinement on internal enzyme dynamics we performed the root-mean-square-fluctuation (RMSF) (Figure 4A and 4B)
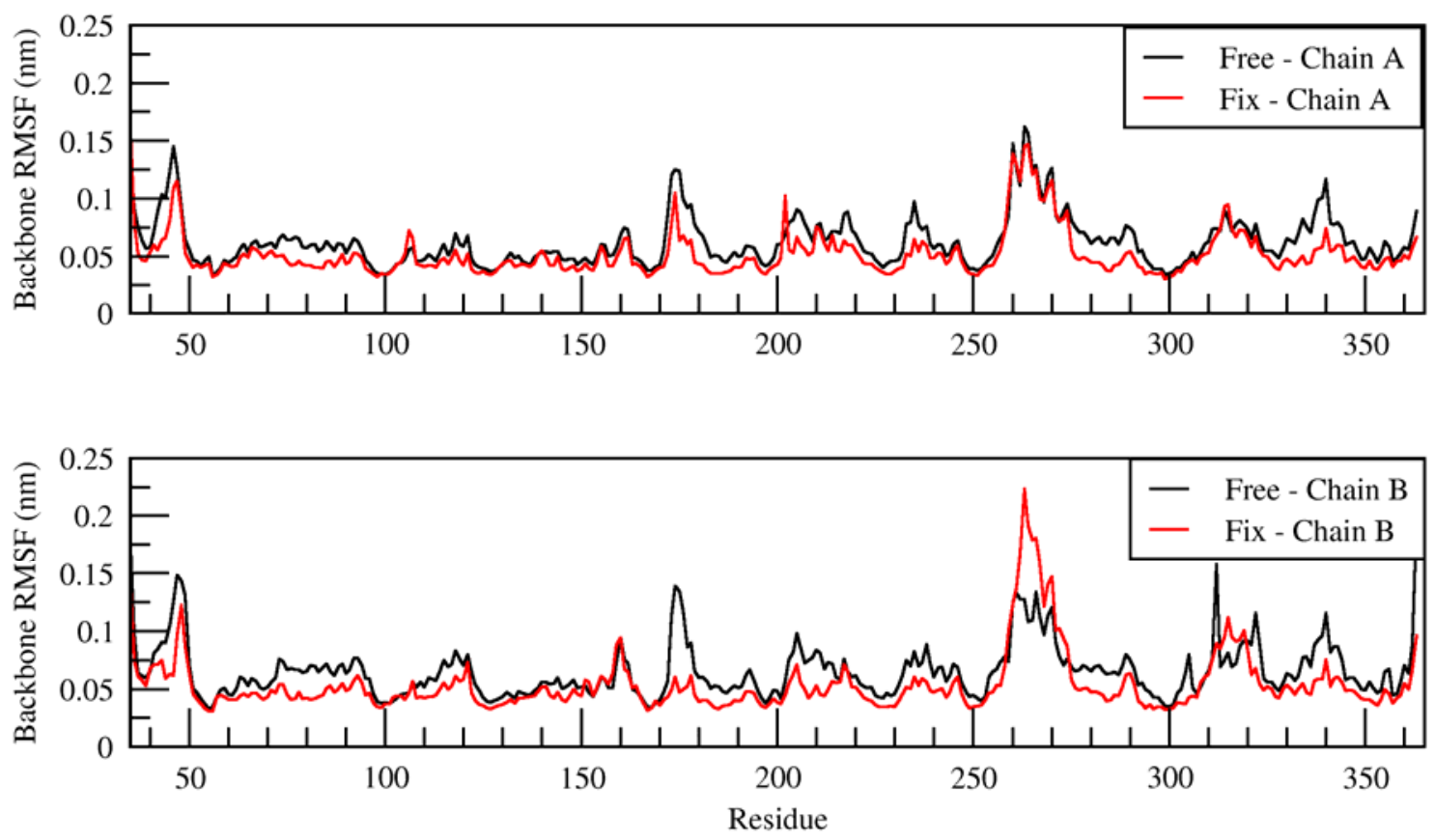

Figure 4 The Root Mean Squared Fluctuation to the mean structure calculated between 2000ps and 5000ps of molecular dynamics simulation for the backbone atoms for OPH-free (black) and OPH-fix (red) divided by chain A (top) and B (bottom). and analyzed the covariance matrix to extract the essential dynamics (ED) (Figure 5) of OPH free and $\mathrm{OPH} \_\mathrm{x}$. On both analyses we could observe some small discrepancies among the two systems and two specific hot spots on the RMSF and Eigenvector values located between protein residues 172-178, and residues 261-267 essentially on chain B. 

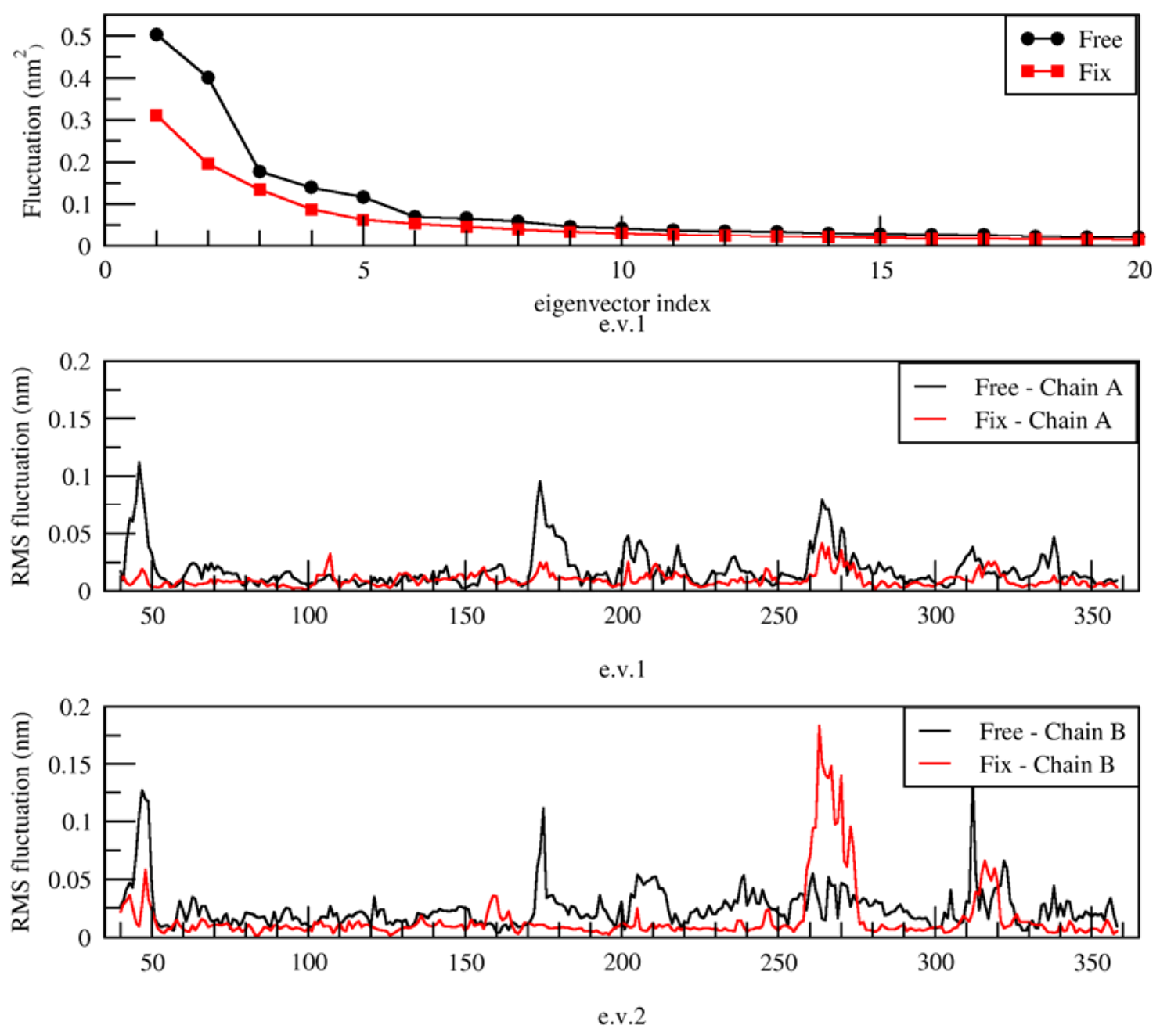

Figure 5 Eigenvalues for the first 20 eigenvectors (top). RMF fluctuation for the first eigenvector OPH-free (black) and OPH-fix (red) divided by chain A (middle) and B (bottom)OPH-free (black) and OPH-fix (red) divided by chain A (top) and B (bottom) \subsection\{Free energy tests\}

Figure 6A shows the 172-178 region, for these residues $\mathrm{OPH}-\mathrm{x}$ is stabilized compared to $\mathrm{OPH}$-free but the movement of these residues is more visible on chain $\mathrm{B}$. The restraint forced by fixing the $\mathrm{Nz}$ atom of the lysine near these residues is the most probable reason for producing this small stabilization on the fluctuation (RMSF) and the reduction on the RMS fluctuation of the first eigenvector (e.v.1). Figure 6B shows the 261-267 region where the opposite effect is observed also for chain B. For chain A, a small stabilization can be observe but for chain $\mathrm{B}$, instead of reducing the residues fluctuation compared to OPH-free, this particular portion of OPH-_x shows a more flexible behavior with the first eigenvector clearly showing an extended movement directed outside the active site. This awkward behavior of 261-267 residues in chain B, but not in chain A, may be caused by the different charge distribution as a result of protonated HIS254 which also causes constant clashes of it's N" hydrogen during the initial steps of molecular dynamics and probably transmits this stress to surrounding atoms. 


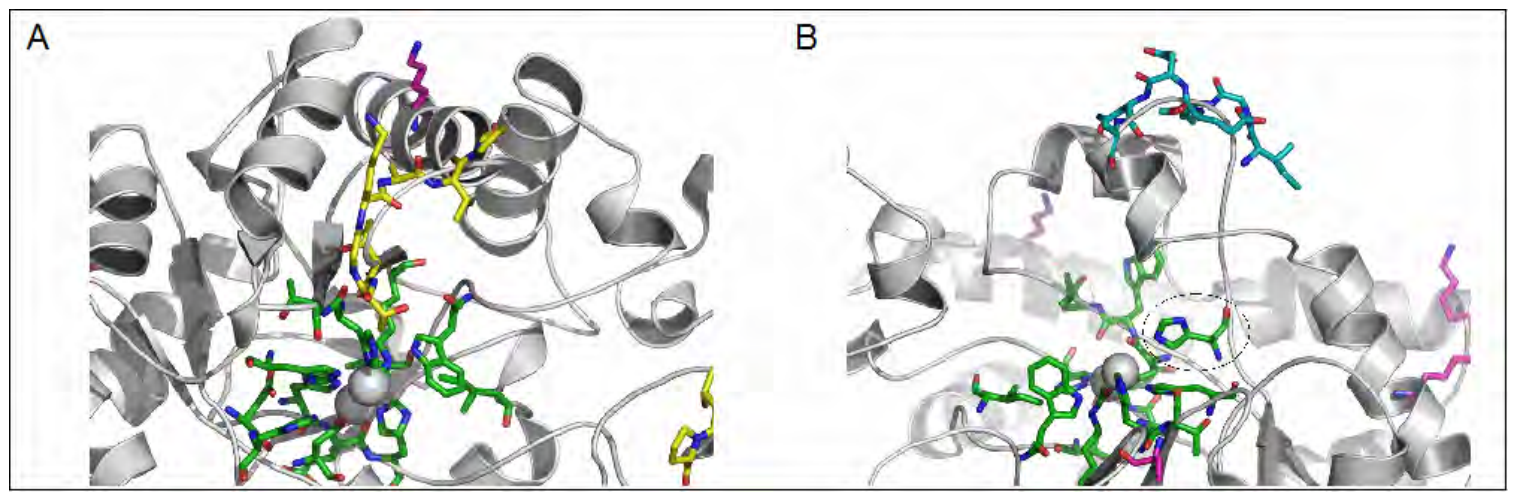

Figure 6 Hot spots on the RMSF and Eigenvector values. Residues of the active site are in green sticks, lysine residues in purple stick and protein in gray ribbons. Residues 172-178 are showed in yellow in figure 6A and residues 261-267 in blue in figure 6B. A and B are rotated by 180 degrees.

\subsection{Free energy tests}

We have run previous tests in order to estimate the free energy of binding of OPH with the organophophate nerve agent Soman. We performed a screening of different sets parameters for the Potential Mean Force calculations. The force constants were 100,200,400 and 800kJ/mol, equilibrations steps 50k and 100k steps and sampling rates were 50k and 100k steps. Figure 7 shows the different sampling windows of the PMF calculation with force $800 \mathrm{~kJ} / \mathrm{mol}$, equilibration 50k steps with sampling set to 100k steps, even with this high force we were not able to transport the organophosphate out of the active site, indicating that Soman is tightly bounded to $\mathrm{OPH}$, actually Soman is buried in the active site and embraced by the loops around the active site as showed in Figure 8.

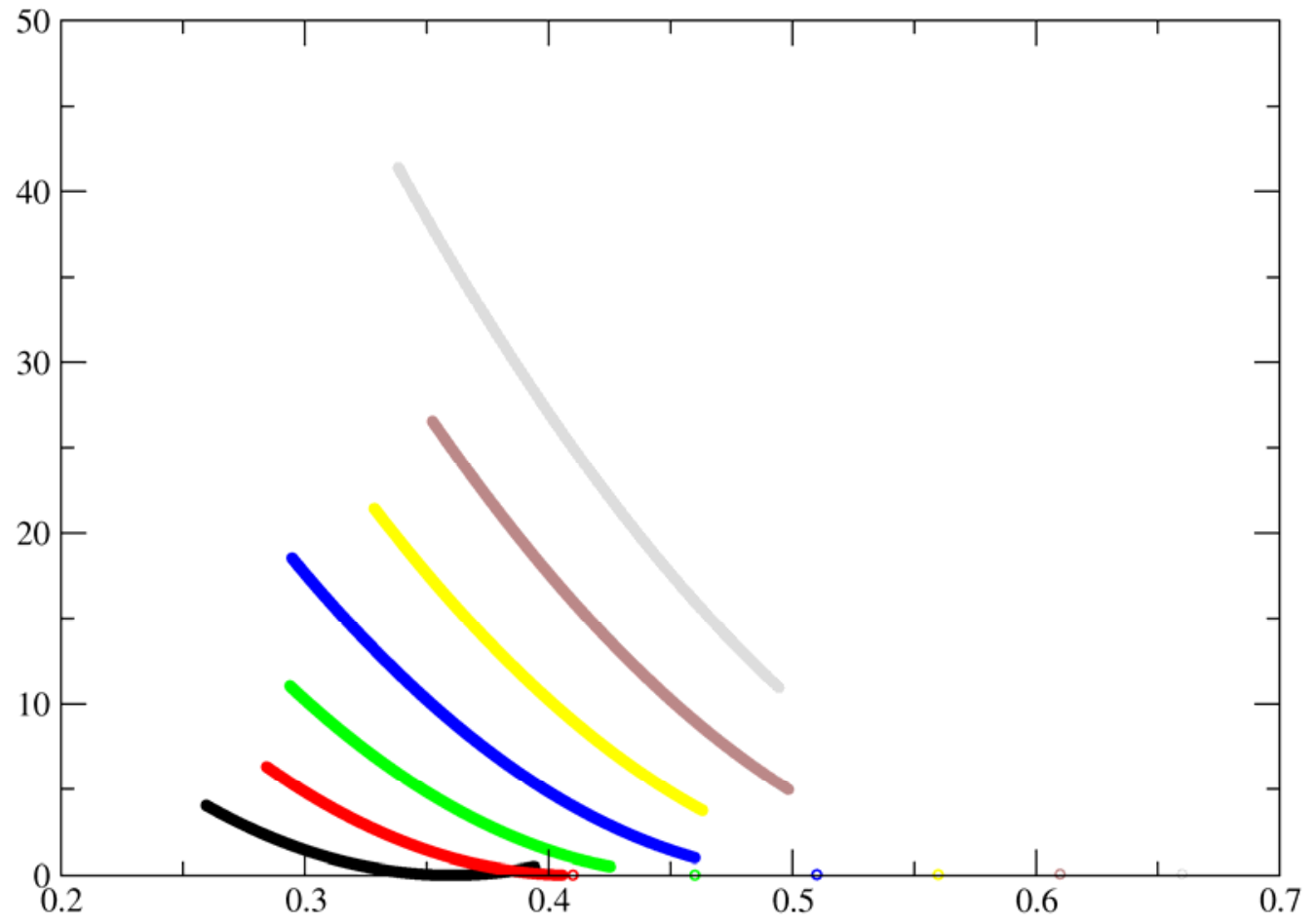

Figure 7 Sampling windows for the PMF calculation with force $800 \mathrm{~kJ} / \mathrm{mol}$, equilibration $50 \mathrm{k}$ steps with sampling set to 100k steps. Force in $\mathrm{Y}$ axis, distance between the centers of geometry Active site residues and Soman in $\mathrm{X}$ axis. 


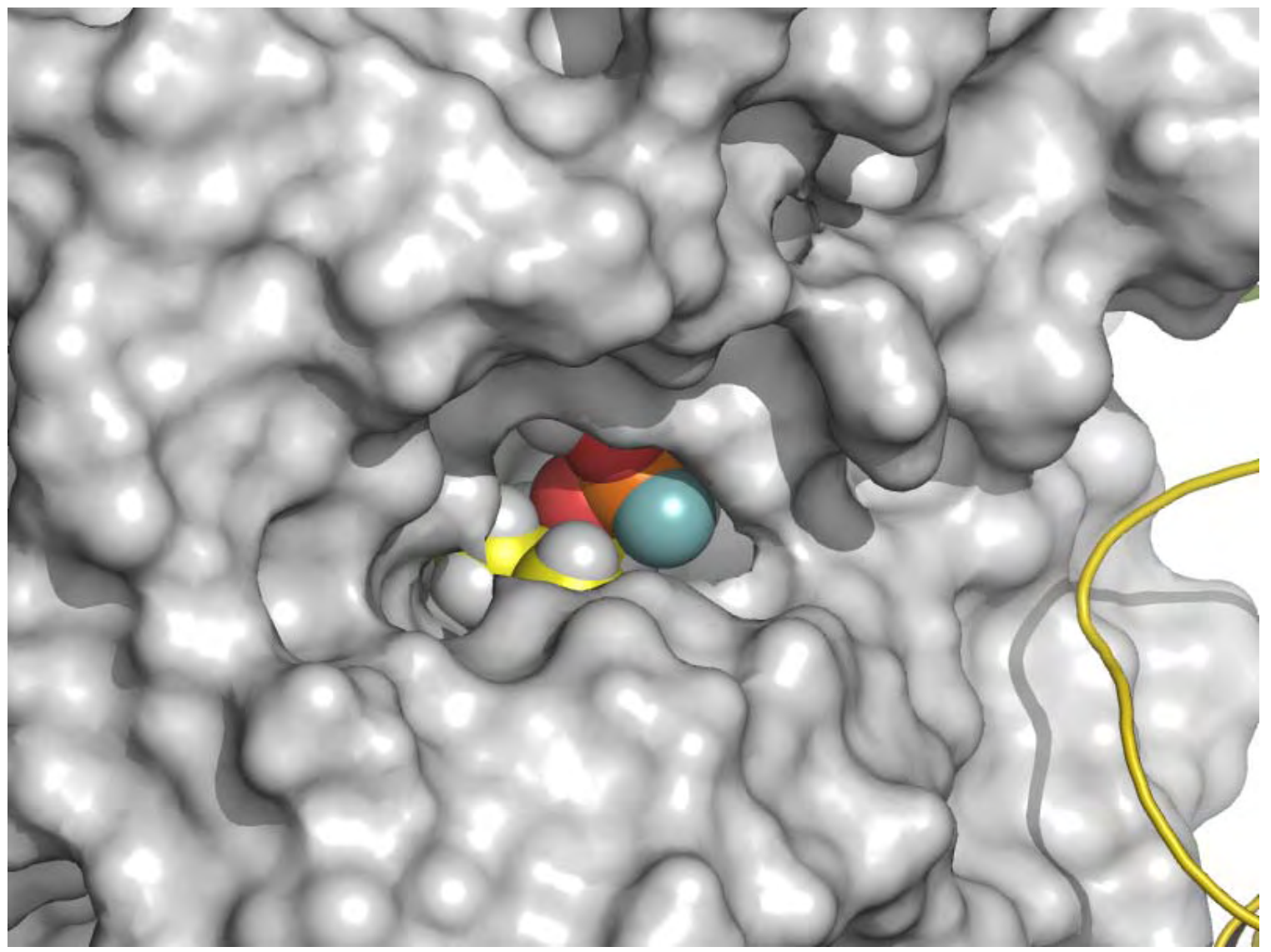

Figure 8 Soman buried in the active site and embraced by the protein loops.

\subsection{MD simulations of OPH with nanopore.}

An alternative model was proposed to represent the FMS was it's explicit representation as an array of charged Oxygen atoms, as showed in figure 1. According to the electrostatic potential surface, calculated with APBS as showed in Figure 9 the opposite side to the active site is more positively charged at $\mathrm{pH}$ 7.0. Using this as lead we adsorbed OPH to FMS and started the simulations. The simulations are still running and we expect this to be a more representative model for the confinement effects, which include the previous unconsidered charge effect attributed to HOOC functionalized silica.

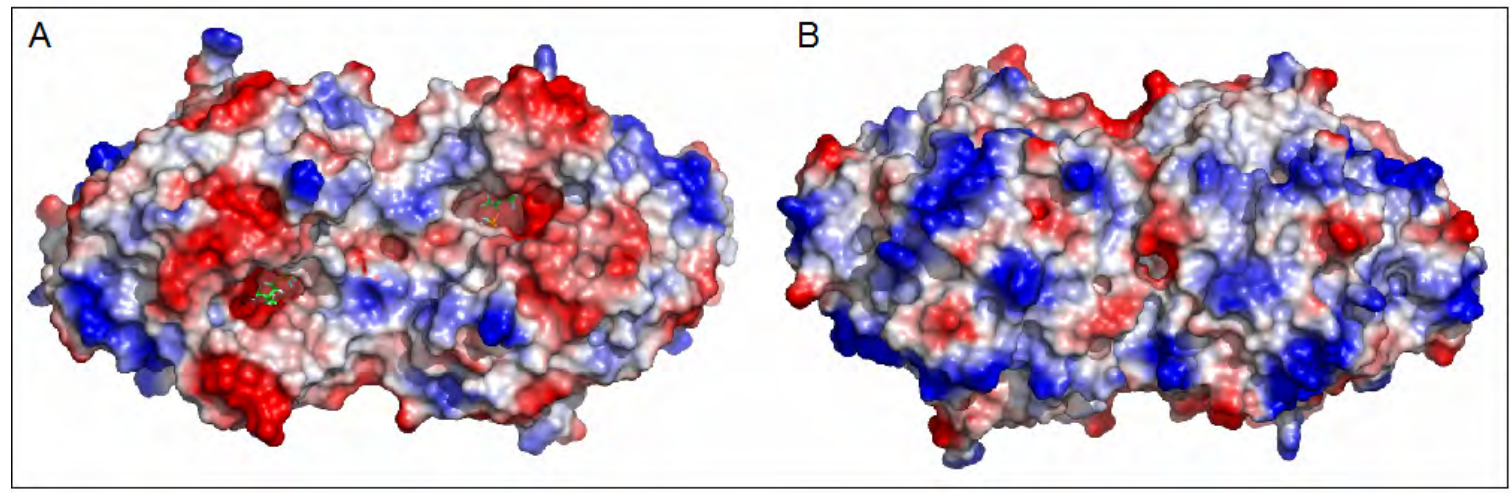

Figure 9 Electrostatic potential surface, calculated with APBS, blue is positive, red is negative, white neutral. The opposite side to the active site 9B is more positively charged than the active site side 9A of the protein at pH 7.0. 


\section{Concluding remarks}

Due to its central importance, there is intense interest in understanding how confinement modulates protein kinetic activity. Several studies have show that proteins are stabilized upon encapsulation in silica gel 15. Eggers and Valentine16 reported that molecular confinement influences protein structure and enhances thermal protein stability, which is probably because decrease of protein flexibility due to reduced mobility by the confined water molecules interacting with the exposed atoms of the macromolecule.

In our simulations with lysines fixed by their $\mathrm{Nz}$ atoms, we identified two hotspots in protein movement but only representative in chain B. The stabilization residues $172-178$ in the OPHfixed simulation may influence enzyme-substrate interaction by keeping the proper reaction coordination for a longer period. In contrast the opposite side of the active site, residues 261267, showed increased movement possibly as a direct effect of the protonation state of HIS254. Besides these two regions there is no considerable difference between internal enzyme dynamics of the two simulations that should explain the increased activity and stability of OPH in confined environment leading us to question if fixing the lysine $\mathrm{Nz}$, as a way to represent the binding of $\mathrm{OPH}$ the Functionalized Mesoporous Silica, would be enough to represent the confined environment of the silica gel.

We are continuing the project now correcting HIS254 protonation state and also simulating the HOOC Functionalized Mesoporous Silica represented explicitly as an array of Oxygen atoms and expecting this to be as more appropriate way to describe the effects due confinement in silica gel.

\section{References}

1)

(a) Mendoza, C. E. Thin-layer chromatography. In Pesticide Analysis; Dumas, K. G., Ed.; MarcelDekker: New York; 1981, pp 1-44. (b) Das, K. G.; Kulkarni, P. S. Gas-liquid chromatography. In Pesticide Analysis; Dumas, K. G., Ed.; Marcel Dekker: New York, 1981. (c) Hanks, A. R.; Colvin, B. M. High-performance liquid chromatography. In Pesticide Analysis; (d) Dumas, K. G., Ed.; Marcel Dekker: New York, 1981; pp 99-174. (e) Barcelo, D.; Lawrence, J. F. Residue analysis of organophosphorus pesticides. In Emerging Strategies for Pesticide Analysis; (f) Charins, T., Sherma, J., Eds.; CRC Press: Boca Raton, FL, 1992; pp 127-150.Palchetti, I.; Cagnini, A.; Del Carlo, M.; Coppi, C.; Mascini, M.; Turner, A. P. F. Anal. Chim. Acta 1997, 337, 315-321

2)

(a) Russell, R.; Pishko, M.; Simonian, A. S.; Wild, J. R. Anal. Chem. 1999, 71, 49094912. (b) Raushel FM. Bacterial detoxification of organophosphate nerve agents. Curr Opin Microbiol. 2002 Jun ; 5(3): 288-95

(a) Singh AK, Flounders AW, Volponi JV, Ashley CS, Wally K, Schoeniger JS. Development of sensors for direct detection of organophosphates. Part I: Immobilization, characterization and stabilization of acetylcholinesterase and organophosphate hydrolase on silica supports. Biosens Bioelectron. 1999 Dec ; 14(8-9): 703-13 (b) Lei Y, Mulchandani P, Wang J, Chen W, Mulchandani A. Highly sensitive and selective amperometric microbial biosensor for direct determination of p-nitrophenyl-substituted organophosphate nerve agents. Environ Sci Technol. 2005 Nov 15; 39(22): 8853-7 
(c) Mulchandani A, Chen W, Mulchandani P, Wang J, Rogers KR. Biosensors for direct determination of organophosphate pesticides. Biosens Bioelectron. 2001 Jun ; 16(4-5): 225-30 (d) Mulchandani P, Mulchandani A, Kaneva I, Chen W. Biosensor for direct determination of organophosphate nerve agents. 1. Potentiometric enzyme electrode. Biosens Bioelectron. 1999 Jan 1; 14(1): 77-85

4) Lei C, Shin Y, Liu J, Ackerman EJ. Entrapping enzyme in a functionalized nanoporous support J Am Chem Soc. 2002 Sep 25; 124(38): 11242-3

5) Kendall, R.A.; Aprà, E.; Bernholdt, D.E.; Bylaska, E.J.; Dupuis, M.; Fann, G.I.; Harrison, R.J.; Ju, J.; Nichols, J.A.; Nieplocha, J.; Straatsma, T.P.; Windus, T.L.; Wong, A.T. "High Performance Computational Chemistry: an Overview of NWChem a Distributed Parallel Application", Computer Phys. Comm., 2000, 128, 260-283

6) Benning, M.M., Shim, H., Raushel, F.M., Holden, H.M. High resolution X-ray structures of different metal-substituted forms of phosphotriesterase from Pseudomonas diminuta. Biochemistry v40 pp.2712- 2722, 2001

7) Christopher I. Bayly, Piotr Cieplak, Wendy D. Cornell and Peter A. Kollman, A WellBehaved Electrostatic Potential Based Method Using Charge Restraints for Deriving Atomic Charges: The RESP Model. J. Phys. Chem. 97 (1993) 10269-10280.

8) J. Wang, P. Cieplak, and P.A. Kollman. How well does a restrained electrostatic potential (resp) model perform in calculating conformational energies of organic and biological molecules. J. Comp. Chem., 21:1049-1074, 2000.

9) Hui Li, Andrew D. Robertson, and Jan H. Jensen "Very Fast Empirical Prediction and Interpretation of Protein pKa Values" Proteins, 2005, 61, 704-721.

10) Baker NA, Sept D, Joseph S, Holst MJ, McCammon JA. Electrostatics of nanosystems: application to microtubules and the ribosome. Proc. Natl. Acad. Sci. USA 98, 10037100412001

11) DeLano, W.L. The PyMOL Molecular Graphics System (2002) DeLano Scientific, San Carlos, CA, USA. http://www.pymol.org.

12) Berendsen HJC, Grigera JR, Straatsma TP, The missing Term in Effective Pair Potentials, Journal of Physical Chemistry 91 (1987) 6169-6271.

13) Darden T, Perera L, Li L and Pedersen L. (1999) "New tricks for modelers from the crystallography toolkit: the particle mesh Ewald algorithm and its use in nucleic acid simulations", Structure 7, R55-R60.

14) Ryckaert, J.P., Ciccotti, G. and Berendsen, H.J.C. (1977) J. Comp. Chem., 23, 327-341.

15) A.C. Pierre, (2004) The sol-gel encapsulation of enzymes. Biocatalysis and Biotransformation, 22, (3) 145-170.

16) Eggers DK, Valentine JS. Molecular confinement influences protein structure and enhances thermal protein stability. Protein Sci. 2001 Feb; 10(2): 250-61 


\title{
CHARACTERIZATION OF AEROSOL SAMPLES COLLECTED DURING THE MILAGRO- 2006 StUdy in the MeXico City MEtropolitan AREA
}

\author{
Kirsten Johnson and Dr. Mario Molina \\ Massachusetts Institute of Technology \\ Cambridge, MA \\ and \\ Rodrigo Gonzalez and Prof. Luisa Molina \\ Autonomous Metropolitan University \\ Mexico City, Mexico \\ and \\ Dr. Alexander Laskin and Dr. Yury Desyaterik \\ Pacific Northwest National Laboratory \\ Richland, WA
}

\section{INTRODUCTION}

Air pollution problems of mega-cities differ greatly and are influenced by a number of factors including topography, demography, meteorology, as well as the level and rate of industrialization and socio-economic development. The Mexico City Metropolitan Area (MCMA) is a rapidly growing urban area with a population of more than 18 million that suffers high pollutant emissions from a variety of area and mobile sources. It lies in an elevated basin which sits at near-tropical latitude and is surrounded by mountains on three sides. The complex terrain and meteorology, and their effects on the city's considerable air pollution problem have been the subject of many studies (e.g. Molina and Molina, 2002; de Foy et al., 2005; Fast and Zhong, 1998). A thorough understanding of particular matter $\leq 2.5 \mu \mathrm{m}$ in diameter $\left(\mathrm{PM}_{2.5}\right)$ is extremely important for quantification of aerosol climate effects, particulate emissions inventories, and human health effects.

The MILAGRO (Megacity Initiative: Local and Global Research Observations) campaign was carried out during March 2006, partially to build upon results from the MCMA-2003 Field Campaign during April 2003. A major objective of MILAGRO was to characterize and quantify pollutant emissions within the city and its environs to better understand the composition, transport, and reactivity of its atmospheric constituents.

Research during the PNNL Summer Research Institute involved characterization of the chemical and physical properties of aerosol samples collected during MILAGRO from various city and suburban locations.

\section{EXPERIMENTAL}

Particles were collected nearly continuously from multiple sites in order to investigate spatial and temporal variability of the particles' chemical and physical properties. Samples were collected at "T0" (Instituto Méxicano de Petróleo, IMP) in an industrial area within the city, as well as “T1” (Universidad Autónoma de Tecamac) and “T2” (Rancho la Biznaga) located 
$\sim 25 \mathrm{~km}$ and $\sim 50 \mathrm{~km}$ northeast of T0, respectively. These sites were chosen to study generation of pollution inside the city and subsequent transport towards the city boundaries.

For single particle studies, samples were collected with the Time-Resolved Aerosol Collector (TRAC) (Laskin et al., 2003) at a time resolution of 15 minutes within size range $0.3-2.0 \mu \mathrm{m}$ particle diameter. Samples were also collected with $8(0.18-18 \mu \mathrm{m})$ and 10 -stage $(0.056-$ $18 \mu \mathrm{m})$ Micro-Orifice Uniform Deposit Impactors (MOUDI) with collection times of 2-3 hours. MOUDI samples from T0 were provided by permission of Dr. R. Mamani-Paco and Dr. T. Castro, UNAM. Cu-supported microscopy grids with thin carbon and lacy carbon films were used as sample substrates for both the TRAC and the MOUDI devices. The primary analysis techniques used were Computer-Controlled Scanning Electron Microscopy with X-ray analysis (CCSEM/EDX), manual SEM/EDX, and Transmission Electron Microscopy with X-ray analysis (TEM/EDS). The majority of SEM images were recorded in secondary electron (SE) mode with back-scattering (BSE) and transmitted electron (TED) imaging used for greater contrast in specific cases. Particles were analyzed for elemental composition, size, shape, morphology, and mixing state.

Bulk aerosol samples were also collected at T0, T1 and T2 sites for quantitative information on the chemical composition of $\mathrm{PM}_{2.5}$. Samples were collected onto Teflon strips by means of a 3-stage DRUM impactor with the following cut-off sizes: Stage A $(1.15-2.5 \mu \mathrm{m})$, Stage B $(0.34-1.15 \mu \mathrm{m})$ and Stage $\mathrm{C}(0.07-0.34 \mu \mathrm{m})$. Analyses for concentrations of elements $\mathrm{Z}>$ $\mathrm{Na}$, hydrogen, and total mass were done in collaboration with Dr. V. Shutthanandan, PNNL, using Proton-Induced X-ray Emission (PIXE), Proton Elastic Scattering Analysis (PESA) and Scanning Transmission Ion Microscopy (STIM), respectively (Shutthanandan et al., 2002). The time resolution of the analyzed samples was $6 \mathrm{hr}$.

\section{RESEARCH OBJECTIVES}

Because more than 2000 samples were collected with thousands of particles per sample, a highly focused approach to single particle analysis was necessary. Preliminary information from the campaign including complimentary aerosol analyses and meteorological data was used to target the following areas:

- Evidence of aerosol processing (chemical reaction or physical mixing) from T0 to T1 sites through composition and morphological analysis

- The phenomenon of emissions of heavy metals and soot from overnight industrial activities at T0

- Internal structure and mixing characteristics of sulfur-containing particles

- Hygroscopic properties of mixed urban aerosol and susceptibility to wetremoval by washout/rainout

- Identification of major particulate emissions sources via PIXE/PESA/STIM and multivariate analysis

Investigations of aerosol processing from T0 to T1 was done primarily with MOUDI samples collected on 27 March 2006 in the following size categories: Stage $5(1.0-1.8 \mu \mathrm{m})$, Stage 6 $(0.56-1.0 \mu \mathrm{m})$, Stage $7(0.32-0.56 \mu \mathrm{m})$, and Stage $8(0.18-0.32 \mu \mathrm{m})$. CCSEM/EDX elemental composition for TRAC and MOUDI samples is expressed by ratio of element weight 
percent to carbon (S/C, O/C, N/C) in order to account for the thin substrate of the collection grids (Laskin et al., 2002).

\section{RESULTS}

A series of four MOUDI samples - two each from T0 and T1 - collected on 27 March 2006 were chosen for detailed morphological and compositional study (Table 1). Initial efforts were focused on samples A and B to study differences on a temporal scale. Companion data (c/o Dr. J. Wang, LBNL) from a cloud condensation nuclei (CCN) counter located at T0 suggested that sample B particles were more hydrophilic than those of sample A. While particles in sample A were collected during a maximum in total particle count (measured by a Scanning Mobility Particle Sizer, SMPS) likely related to morning rush-hour traffic, sample B particles were collected during a peak in number of active CCN occurring several hours later. Possible explanations for the offset include chemical reaction (e.g. $\mathrm{OH}$ or $\mathrm{O}_{3}$ oxidation) or physical processing (e.g. mixing with soluble inorganic salts) of the same type of particles, or emission of different types of particles altogether. SEM/EDX analysis confirmed both chemical and physical differences between the two. Whereas sample A (6-9am) contained a large number of irregular soot particles, sample B (11am - 2pm) showed marked differences in particle size and shape and a liquid-like appearance. CCSEM/EDX analysis showed that major elements for both were $\mathrm{C}, \mathrm{O}, \mathrm{S}, \mathrm{Si}, \mathrm{K}$ and $\mathrm{Na}$. However, sample B was found to have lower S/C and $\mathrm{O} / \mathrm{C}$ ratios, which was unexpected from the above hypotheses regarding chemical and physical processing which might lead to higher oxygen and sulfur content. Comparisons of samples A and $\mathrm{B}$ with $\mathrm{C}$ and $\mathrm{D}$ in relation to transport from $\mathrm{T} 0$ to $\mathrm{T} 1$ are in progress.

Table 1: Summary of MOUDI samples analyzed by CCSEM/EDX and SEM/EDX.

\begin{tabular}{|ccc|}
\hline Sample & Location & Collection time \\
\hline A & T0 & 6:00am - 9:00am \\
B & T0 & 11:00am - 2:00pm \\
C & T1 & 10:00am - 12:00pm \\
D & T1 & 1:00pm - 3:00pm \\
\hline
\end{tabular}
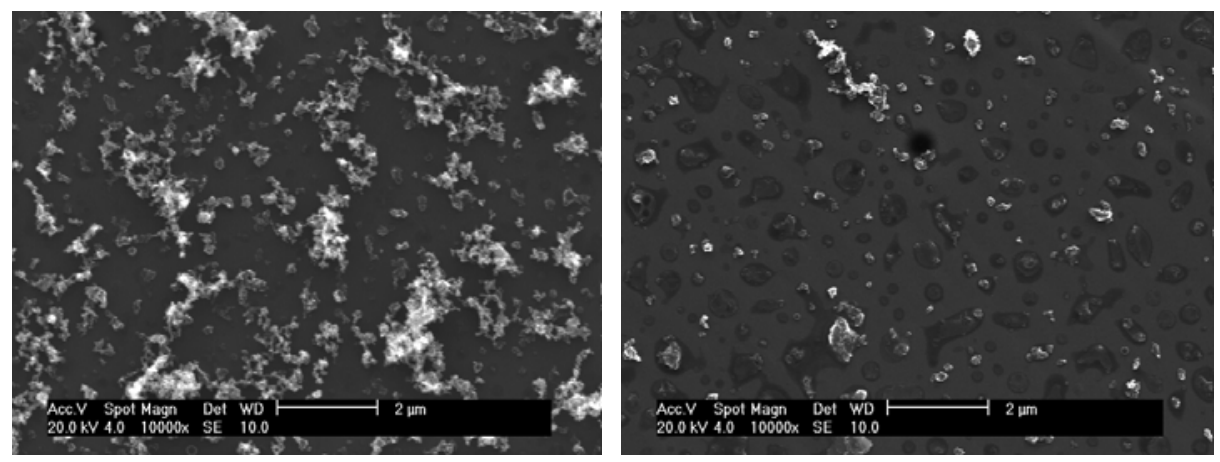

Figure 1. SEM images of MOUDI samples A (left) and B (right) from Stage $8(0.18-0.32 \mu \mathrm{m})$. 
Table 2. CCSEM/EDX statistics for MOUDI samples A and B.

\begin{tabular}{|l|cc|cc|}
\hline & \multicolumn{2}{|c|}{ Sample A } & \multicolumn{2}{c|}{ Sample B } \\
& Average & Median & Average & Median \\
\hline O/C & $7.8 \mathrm{e}-2$ & $4.7 \mathrm{e}-2$ & $4.9 \mathrm{e}-2$ & $3.6 \mathrm{e}-2$ \\
$\mathrm{~N} / \mathrm{C}$ & $7.4 \mathrm{e}-3$ & 0 & $9.0 \mathrm{e}-3$ & 0 \\
$\mathrm{~S} / \mathrm{C}$ & $6.1 \mathrm{e}-3$ & $3.7 \mathrm{e}-3$ & $4.4 \mathrm{e}-3$ & 0 \\
$\mathrm{Na} / \mathrm{C}$ & $1.3 \mathrm{e}-2$ & $5.6 \mathrm{e}-3$ & $1.4 \mathrm{e}-3$ & 0 \\
$\mathrm{Si} / \mathrm{C}$ & $5.0 \mathrm{e}-3$ & $4.2 \mathrm{e}-3$ & $2.4 \mathrm{e}-3$ & 0 \\
$\mathrm{~K} / \mathrm{C}$ & $9.0 \mathrm{e}-4$ & 0 & $2.0 \mathrm{e}-4$ & 0 \\
$\mathrm{O} / \mathrm{S}$ & 11.5 & 8.5 & 8.0 & 6.2 \\
\hline Diameter $(\mu \mathrm{m})$ & 0.229 & 0.182 & 0.263 & 0.208 \\
\hline \# Particles analyzed & \multicolumn{3}{|c|}{3329} & \multicolumn{3}{c|}{4084} \\
\hline
\end{tabular}

PIXE analysis during the MCMA-2003 campaign showed that overnight industrial emissions were important sources of metals that originated from northern sections of the city (Johnson et al., 2006). The location of the T0 site therefore provided a good opportunity for additional study on this phenomenon. Several early morning ( 1am) samples were studied which corresponded with peak values in various metals including $\mathrm{Zn}$ and $\mathrm{Pb}$ measured by PIXE. Figures 2 and 3 show fine needle-like Zn particles mixed with soot from a sample collected on 23 March at T0. Zn and soot were commonly observed, as well as occasional small $\mathrm{Pb}$ particles distinguishable in BSE mode imaging.
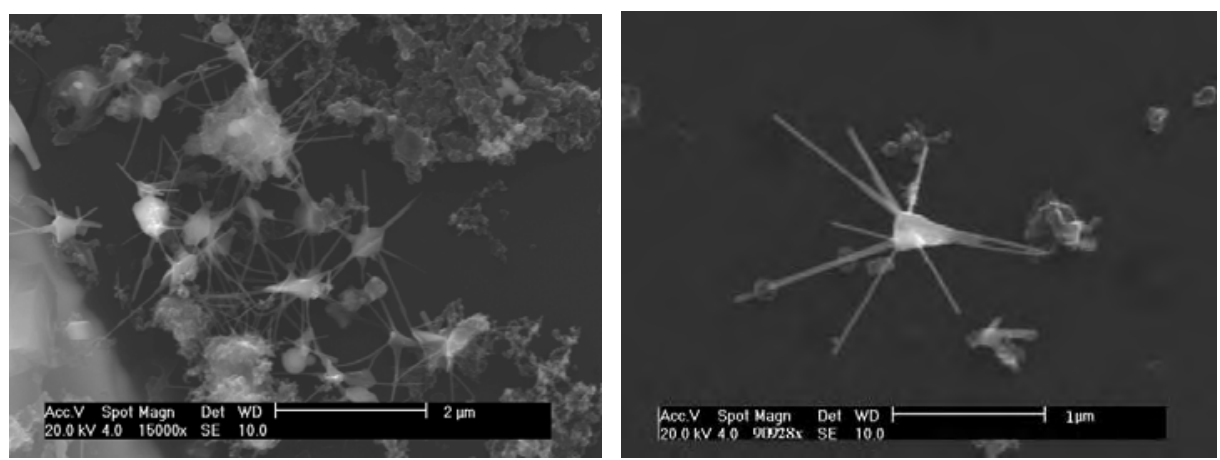

Figure 2: SEM images of samples collected on 23 March 1:30-1:45am (left) and 21 March 1:301:45am (right) at T0. 
PNNL-16185
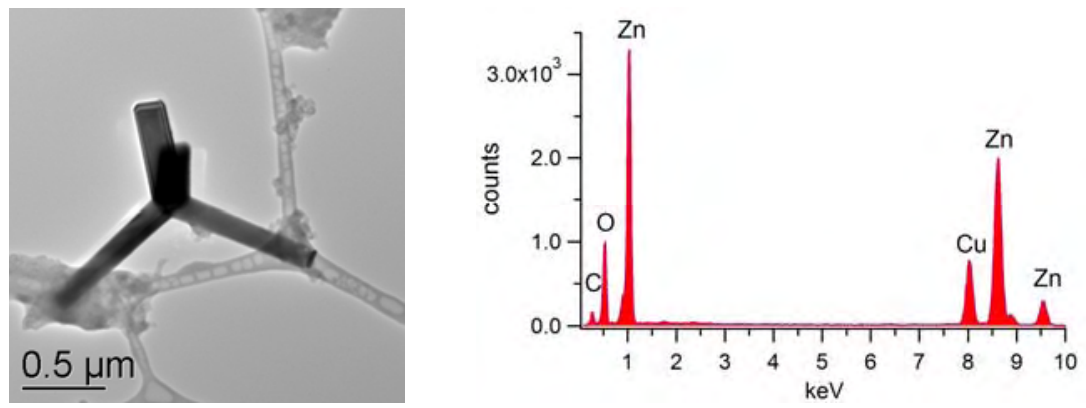

Figure 3: TEM image and X-ray spectrum of a Zn particle collected on 23 March (1:30-1:45am) at T0. $\mathrm{Cu}$ is an artifact of the substrate grid.

PIXE measurements at T0 indicate that the most abundant elements in non-volatile $\mathrm{PM}_{2.5}$ were $\mathrm{S}, \mathrm{Zn}$, soil-related elements ( $\mathrm{Si}, \mathrm{Al}, \mathrm{Fe}, \mathrm{Mg}, \mathrm{Ca}$ ), $\mathrm{K}, \mathrm{Pb}$, and $\mathrm{Cl}$. Soil elements track one another throughout the campaign (Fig. 4) as expected of a common emissions source. The concentration of $\mathrm{Zn}$ reaches peak values during early morning ( $1 \mathrm{am})$ hours (Fig. 5). Analysis of PESA/STIM data from T0 and PIXE/PESA/STIM from companion sites T1 and T2 is in progress, and multivariate analysis of these data is planned for identification of particulate emissions sources for comparison to MCMA-2003 (Johnson et al., 2006).
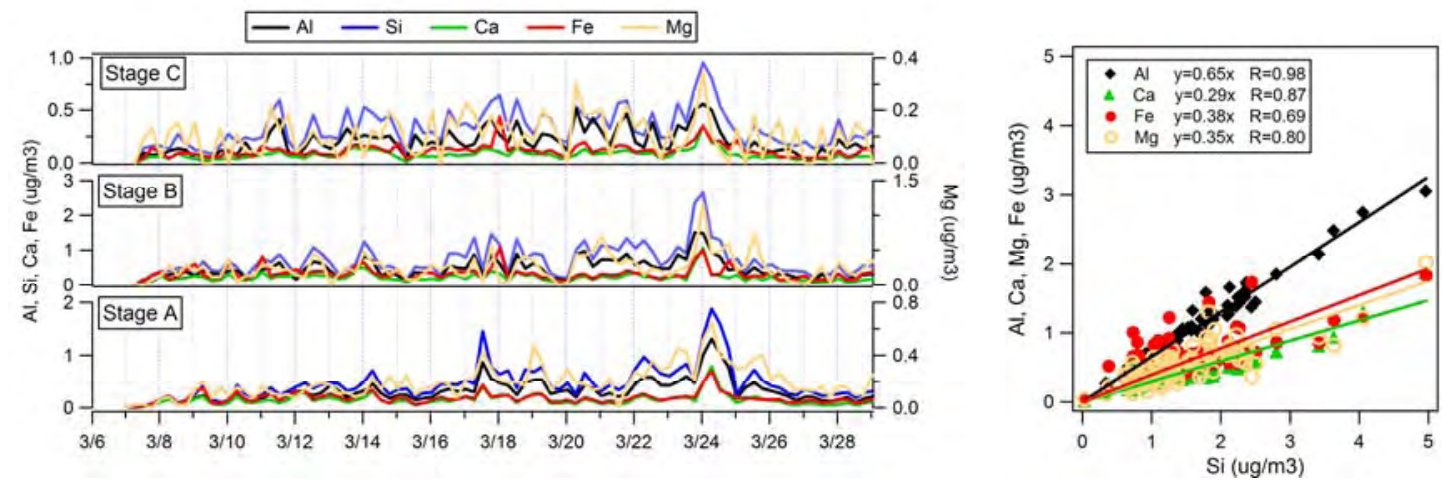

Figure 4. Time series of soil-related elements ( $\mathrm{Si}, \mathrm{Al}, \mathrm{Ca}, \mathrm{Fe}, \mathrm{Mg}$ ) at $\mathrm{T} 0$ from PIXE analysis in size categories Stage A $(1.15-2.5 \mu \mathrm{m})$, B $(0.34-1.15 \mu \mathrm{m})$ and C $(0.07-0.34 \mu \mathrm{m})$; correlation plot among these elements in $\mathrm{PM}_{2.5}$ (sum of Stages A, B, C).

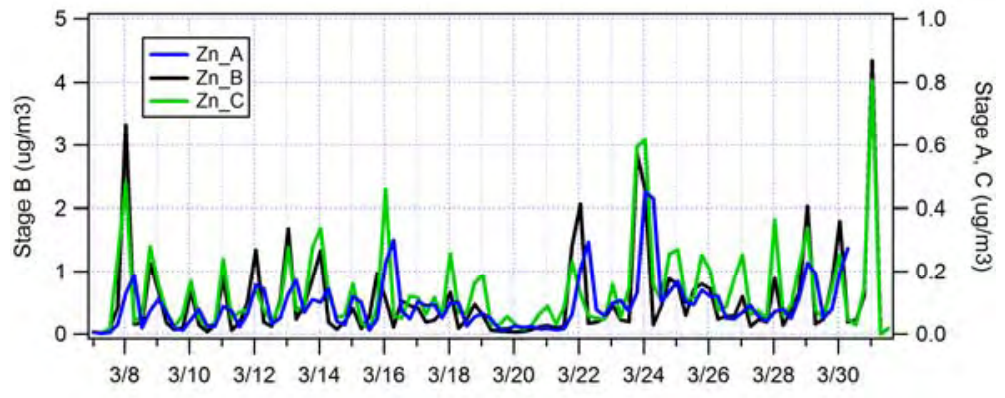

Figure 5. Time series of $\mathrm{Zn}$ at T0 by PIXE analysis in Stages A, B, C. 


\section{DISCUSSION}

Preliminary analysis of aerosol samples collected during the MILAGRO campaign confirm previous results from MCMA-2003. Specifically we found that soot and sulfur were major components of MCMA aerosol, and TEM analysis of spherical sulfur-containing (likely sulfate) particles showed that they were often internally mixed with soot. This information is essential to understanding particulate mixing and reactivity in order to better quantify particles' lifetimes and their effects on the environment. In addition, different types of sulfur-containing particles were collected during MILAGRO and suggest that $\mathrm{S}$ may be present in different chemical forms. While one type of sulfur-containing particles were characterized by a spherical shape, electron beam sensitivity (to damage), and mixing with other elements $(\mathrm{K}, \mathrm{Cl}$, $\mathrm{Sn}$ ) and soot particles, a second type had a liquid-like appearance, unaffected by the electron beam and were associated mostly with carbon only. Although SEM/EDX provides limited information on the chemical form of the elements present, analyses with Time-of-Flight Secondary Ion Mass Spectrometry (TOF-SIMS) at PNNL and with Scanning Transmission Xray Microscopy (STXM) with Near Edge X-ray Absorption Fine Structure spectroscopy (NEXAFS) in collaboration with Dr. M. K. Gilles at Lawrence Berkeley National Laboratory (LBNL) are possible means of additional information. TOF-SIMS and STXM studies of MOUDI samples A - D are underway and may be helpful in better understanding compositional differences as a result of aerosol processing.

PIXE/PESA/STIM data is complimentary to single particle analyses and useful in quantifying $\mathrm{PM}_{2.5}$ aerosol chemical composition. PIXE analysis from T0 samples confirms an abundance of S with average concentrations $0.066,1.568$, and $0.828 \mu \mathrm{g} / \mathrm{m}^{3}$ for Stages A, B, and C, respectively. Zn was also present in fairly high concentration (averages 0.093, 0.541, 0.136 $\mu \mathrm{g} / \mathrm{m}^{3}$ for $\mathrm{A}, \mathrm{B}, \mathrm{C}$ ) probably due to close proximity to industrial sources near T0. The strong correlation among elements related to soil/dust ( $\mathrm{Si}, \mathrm{Al}, \mathrm{Ca}, \mathrm{Fe}, \mathrm{Mg}$ ) parallels SEM/EDX analysis of individual soil/dust particles composed of the same elements.

\section{CONCLUSIONS}

Chemical and morphological analysis of aerosol samples collected during the 2006 MILAGRO campaign show that MCMA particles have a complex composition and are heavily mixed. Major types of particles include soot, dust and sulfur-containing particles, as well as metals associated with industry such as $\mathrm{Zn}$ and $\mathrm{Pb}$. Comparisons of differences in morphology and composition of particles collected within hours of one another are useful to understanding how they react and become mixed. More information is needed on the chemical form of the composition elements and related meteorology during the collection period to understand these differences, however. Further studies are in progress.

\section{REFERENCES}

de Foy, B., Caetano, E., Magana, V., Zitácuaro, A., Cárdenas, B., Martínez, A. P., Retama, A., Ramos, R., Reyes, R., Sosa, G., Molina, L. T., and Molina, M. J.: Mexico City basin wind circulation during the MCMA-2003 field campaign, Atmos. Chem. Phys., 5, 2267-2288, 2005. 
Fast, J. D., and Zhong, S.: Meteorological factors associated with inhomogeneous ozone concentrations within the Mexico City basin, J. Geophys. Res., 103, 18927-18946, 1998.

Johnson, K. S., Zuberi, B., Molina, L. T., Molina, M. J., Iedema, M. J., Cowin, J. P., Gaspar, D. J., Wang, C., and Laskin, A.: Processing of soot in an urban environment: Case study from the Mexico City Metropolitan Area, Atmos. Chem. Phys., 5, 3033-3043, 2005.

Johnson, K.S., B. de Foy, B. Zuberi, L.T. Molina, M.J. Molina, A. Laskin, Y. Xie, and V. Shutthanandan, "Aerosol composition and source apportionment in the Mexico City Metropolitan Area with PIXE/PESA/STIM and multivariate analysis”, Atmos. Chem. Phys. Discuss., 6, 3997-4022, 2006.

Laskin, A., Iedema, M. J., and Cowin, J. P.: Time-resolved aerosol collector for CCSEM/EDX single-particle analysis, Aerosol Sci. Tech., 37, 246-260, 2003.

Laskin, A., Iedema, M. J., and Cowin, J. P.: Quantitative time-resolved monitoring of nitrate formation in sea salt particles using a CCSEM/EDX single particle analysis, Environ. Sci. Tech., 36, 4948-4955, 2002.

Molina, M. J., and Molina, L. T., ed.: Air Quality in the Mexico Megacity: An Integrated Assessment. Kluwer Academic, 2002.

Shutthanandan, V., Thevuthasan, S., Disselkamp, R., Stroud, A., Cavanagh, A., Adams, E. M., Baer, D. R., Barrie, L. A., Cliff, S. S., Jimenez-Cruz, M., and Cahill, T. A.: Development of PIXE, PESA and transmission ion microscopy capability to measure aerosols by size and time, Nucl. Instr. and Meth.. B, 189, 284-288, 2002. 
PNNL-16185 
PNNL-16185

\title{
On the Structural Stability of Ridig-Rod $\beta$-Barrel Pores
}

\author{
Matteo Guglielmi, and Ursula Röthlisberger \\ Ecole Polytechnique Federale de Lausanne (EPFL), \\ Laboratory of Computational Chemistry and Biochemistry, \\ Institute of Basic Sciences, \\ Lausanne 1015, Switzerland \\ and \\ Roberto D. Lins \\ Pacific Northwest National Laboratory, \\ Computational Biology and Bioinformatics, \\ Richland, WA 99352, USA
}

Keywords: $\beta$-Barrel Pores, structural stability, molecular dynamics,

${ }^{*}$ Correspondence and requests should be addressed to RDL at the Pacific Northwest National Laboratory (e-mail: roberto.lins@pnl.gov) or UR at the Ecole Polytechnique Federale de Lausanne (e-mail: ursula.roethlisberger@epf.ch).

\section{Introduction}

Rigid-rod B-barrels pores are synthetically designed barrel-stave supramolecules developed to exploit the functional plasticity of their biological counterparts in biorganic chemistry [18]. They are constructed from elementary units made by p-octiphenyl staves connected to short peptide chains (usually 3-7 residues) [1] (Figure 1). The non structuralplanarity of each unit, due to the presence of a single stave, directs cylindrical self-assembly $\beta$ barrel formation via hydrogen bond pattern between the peptide chains from neighboring units [1]. Being the product of a synthesis process, the functionality of the rigid-rod $\beta$-barrels pores can be tailored to specific task like molecular recognition, molecular translocation and molecular transformation simply by variation of their peptide chains [1-15]. Due to the alternating orientation of neighboring amino acid residues of peptides in $\beta$-sheet conformation, both inner and outer surface of rigid-rod $\beta$-barrels can be functionalized in a flexible, precise and straightforward manner. This characteristic offers potential application on a wide variety of fields, e.g., detector for medical diagnosis, drug delivery, gene therapy, food industry and environmental bioremediation [19]. Facile access to transmembrane, large, and stable synthetic pores with variable internal active sites is, however, a necessary prerequisite for their practical applications [17]. 
Several factors contribute to stability issue of rigid-rod $\beta$-barrels pores. They can be divided in two mutually exclusive categories: "intrinsic" pore parameters ( $p$-octiphenyl length, peptide length and peptide sequence) and "environmental" factors ( $\mathrm{pH}$, ionic strength and membrane interaction). Previous experimental studies show that the changes in the $\mathrm{pH}$ are capable to tune the pore activity, i.e., activating/deactivating the macromolecular assembly by controlling the protonation levels of the internal dyads [2-15]. Such procedure aims to achieve a certain level of charge-charge repulsion in the inner barrel, which is considered to play an important role in terms of pore stability [6]. This effect has been named as internal change repulsion (ICR) model. The model states that intermediate ICR gives maximal pore activity, whereas insufficient and excessive ICR results in pore "implosion" and "explosion", respectively [6]. Additionally, the authors have also suggested the lateral pressure from the external membrane to play an essential role on barrel stability. Experimental observations led to the conclusion that the external membrane pressure (EMP) could trigger barrel implosion at low ICR while preventing barrel explosion at high ICR [16]. These findings have led to an update on the mechanism for rigid-rod $\beta$-barrel pores stability, the ICR-EMP model (Figure 2). While this is a sound model for such synthetic entities, biological pores can vary widely in their inner-pore net charge and dipole depending on the composition of the interacting molecule (ions, sugars, water, etc). On the other hand, proteins make use of a much more complex structural arrangement to interact with their medium (membranes), which might account for the same final balance. Therefore, if general, the ICR-EMP model would have an important impact in the understanding of the stability of biological pores.

In order to investigate the details and validity of these assumptions, a series of molecular dynamics simulations were carried out for two ICR-free $\beta$-barrel pores immersed into a biological membrane and in water. The pores are characterized by the peptide sequences GLNLNL and GLQLQL.

\section{METHODOLOGY}

\section{Systems Setup}

The GLNLNL and GLQLQL pores were modeled based on the experimental chemical data. The structures were studied immersed into a 148 phosphatidyl-ethonolanime lipid bilayer with two 18-carbon long aliphatic chains and free in water. A concentration molar of $0.1 \mathrm{M}$ $\mathrm{NaCl}$ was added to reflect the experimental setup of similar pores previously studied. The 
PNNL-16185

pore-lipid assembly was then solvated by SPC/E water molecules resulting in a system of $7.2 \mathrm{x}$ 8.2 x $8.2 \mathrm{~nm}$ in size. In water, higher ionic strength $(0.5 \mathrm{M})$ was also probed to completely abolish the possibility of any ICR (or ICR-like) effect.

\section{Molecular Dynamics Simulations}

All simulations were performed using the AMBER96 force field (Cornell et al., 1995) in conjunction with derived charges for the chemical groups not available in the force field (procedure described below) and implemented in the parallel version of the NWChem-4.7 program (Apra et al., 2005). The systems were equilibrated in a stepwise fashion. The systems were initially relaxed using up to 5000 steps of steepest descent energy minimization. It was followed by the system equilibration performed in successive 10-ps MD runs at 50, 100, 150, 200, 250, and $300 \mathrm{~K}$, with velocity reassignments every 0.1 ps. The production runs were carried out in the isothermal-isobaric ensemble for $20 \mathrm{~ns}$ for the system immersed into the lipid membrane and $10 \mathrm{~ns}$ for the pores in water. The temperature was maintained at $300 \mathrm{~K}$ by weak coupling the solute and solvent separately to a heat bath (Berendsen et al., 1984) with a relaxation time of $0.1 \mathrm{ps}$. The pressure was kept at $1.025 \times 10^{5}$ Pascal using weak coupling to a pressure bath (Berendsen et al., 1984) via isotropic coordinate scaling with relaxation time of 0.1 ps. Long-range electrostatic interactions were handled using the smooth particle mesh Ewald (SPME) method (Essmann et al., 1995) A cutoff $1.0 \mathrm{~nm}$ was used for the short-range non-bonded interactions (van der Waals and real-space electrostatic contribution). The SHAKE (Ryckaert et al., 1977) algorithm was applied to constrain all bonds involving a hydrogen atom, and a 2-fs timestep was used to integrate the equations of motion based on the leapfrog algorithm (Hockney, 1970). Trajectory frames were recorded every 0.2ps for analysis.

\section{Charge Model}

Based on the optimized geometry of the p-octiphenyl groups, Hartree-Fock calculations at the 6-31G* level followed by a restrained electrostatic potential (RESP) fit (Bayly et al., 1995) were performed using the NWChem4.7 program (Apra et al., 2005) to compute the partial charges. Values of 0.005 a.u. for the harmonic and 0.001 a.u. for the hyperbolic restraints, respectively, were used in the RESP fittings. Charge derivation by means of RESP fitting preserves the dipole moment of a molecule to its quantum-mechanical value, and should therefore lead to a realistic description of intermolecular interactions with identical as well as distinct molecular species during the simulations. The charge-derivation procedure was 
PNNL-16185

performed according to the AMBER96 force field philosophy (Cornell et al., 1995) in order to preserve force field compatibility. The final charge sets for the above-mentioned residues are shown in Figure 3. Since the p-octiphenyl staves present a weak molecular-wire character, a second set of charges was derived to take into account this effect and evaluate its influence on the evaluated quantities. Density functional theory at the B3-LYP/6-31G* level was used in this second charge set followed by a RESP fitting. A residual charge splitting at the $p$ octiphenyl staves termini producing the desired induced dipole. The resulting dipole along the stave was found to be ca. $4 \mathrm{Db}$ for charges derived without polarization, while the polarized charge set allowed a stave dipole of 7.5 Db. DFT B3-LYP/6-31G* was chosen over HF/631G* at this stage due to the apolar environment of the pores (membrane aliphatic chains). Additionally, comparison of point charges for an octiphenyl ring residue obtained with the two methods after a RESP fitting did not produce significant discrepancies, i.e., restricted to the second or third decimal points (data not shown). This is probably due to the apolar character of the studied chemical group.

\section{RESULTS AND DISCUSSION}

In order to evaluate the structural stability, we have monitored the inner pore dimensions (Figure 3), their corresponding conductances (Figure 4) and secondary structure stability (Figure 5) of the peptide chains as a function of the time series evolution. A higher ionic strength has also been probed for the pores free in aqueous solution. The systems have been given the following notation: 2ASN/2GLN (pores with non-polarized charge set immersed into the lipid membrane, $0.1 \mathrm{M} \mathrm{NaCl}$ ); $2 \mathrm{ASN}+/ 2 \mathrm{GLN}+$ (pores with polarized charge set immersed into the lipid membrane, 0.1M NaCl); 2ASN+-/2GLN+- (pores with polarized charge set free in water, $0.1 \mathrm{NaCl}$ ); and, $2 \mathrm{ASN}++/ 2 \mathrm{GLN}++$ (pores with polarized charge set free in water, $0.5 \mathrm{M} \mathrm{NaCl}$ ).

All the pores, except for 2ASN and 2ASN+ have collapsed into a "closed" conformation. Although the ICR effect seems to have an influence on pore stability, the presence of the membrane was able to hold these pores in their open state. It suggests that while ICR seems imperative for the stability of these systems in water, the presence of a lipid membrane can stabilize their open (biologically active) conformation depending on the peptide sequence chosen. The absence of the membrane has caused the pore dimensions and conductance of 2ASN/2ASN+ to diminish by almost half. Additionally, a decrease in stability 
PNNL-16185

of the secondary structure content is observed in all membrane-free simulations compared to the ones when the membrane is present.

These results are contrasting with a hypothetical model drawn from experimental observations. In reference 16, the authors have avoided the closure of the structure of a $\mathrm{pH}$ dependent pore, GLHLHL, at different $\mathrm{pH}$ values, by mutating the central leucine residue to an arginine, generating the sequence GLHRHL. This arginine, located outward the pore, would bind to functionalized external calixaarene ligands (Figure 2). These ligands of hydrophobic nature, (except for the functionalized group binding the arginine), were referred to act as "cushions" soothing the forces arising from external membrane pressure and the internal change repulsion. Our findings suggested that the presence of the membrane acts rather to hold the pore open, than applying an external closing pressure. Therefore, we hypothesize that their observations could be alternatively explained based on the fact on an increase of the total overall outer hydrophobic surface and consequently a maximization of the interaction with the lipid membrane.

\section{CONCLUSIONS}

We have studied the stability of ICR-free synthetic $\beta$-barrel pores via molecular simulations. We have investigated the influence of a double lipid membrane, and high ionic strength in the structural and functional stability of these systems. Our results indicate that the presence of the membrane not only stabilizes the open conformation of the pores, but also the secondary structure content; and, that the stability of a pore depends rather on its composition than on having an internal charge repulsion. The influence of an internal charge repulsion for these systems in water and immersed into a membrane will be assessed and shall soon be reported.

\section{ACKNOWLEDGMENTS}

The authors would like to thank the Swiss National Foundation and the Summer Research Institute of PNNL for their support and Prof. Stefan Matile for insightful discussions.

\section{References}

1) S. Matile, Chem. Soc. Rev., 2001, *30*, 158-167.

2) N. Sorde' and S. Matile, J. Supramol. Chem., in press.

3) G. Das and S. Matile, Proc. Natl. Acad. Sci. U. S. A., 2002, *99*, 5183-5188. 
PNNL-16185

4) G. Das, H. Onouchi, E. Yashima, N. Sakai and S. Matile, ChemBioChem, 2002, *3*, 1089-1096.

5) G. Das, P. Talukdar and S. Matile, Science, 2002, *298*, 1600-1602.

6) B. Baumeister, A. Som, G. Das, N. Sakai, F. Vilbois, D. Gerard, S. P. Shahi and S. Matile, Helv. Chim. Acta, 2002, *85*, 2740-2753.

7) B. Baumeister, N. Sakai and S. Matile, Org. Lett., 2001, *3*, 4229-4232.

8) A. Som, N. Sakai and S. Matile, Bioorg. Med. Chem., 2003, *11*, 1363-1369.

9) A. Som and S. Matile, Eur. J. Org. Chem., 2002, 3874-3883.

10) B. Baumeister and S. Matile, Macromolecules, 2002, *35*, 1549-1555.

11) G. Das, L. Ouali, M. Adrian, B. Baumeister, K. J. Wilkinson and S. Matile, Angew. Chem., Int. Ed., 2001, *40*, 4657-4661.

12) N. Sakai and S. Matile, J. Am. Chem. Soc., 2002, *124*, 1184-1185.

13) B. Baumeister, N. Sakai and S. Matile, Angew. Chem., Int. Ed., 2000, *39*, 1955-1958.

14) N. Sakai, B. Baumeister and S. Matile, ChemBioChem, 2000, *1*, 123-125.

15) N. Sakai, D.Houdebert and S. Matile, Chem. Eur. J., 2003, *9*, 223-232.

16) V. Gorteau, G. Bollot, J. Mareda, D. Pasini, D. Tran, A. N. Lazar, A. W. Coleman, N. Sakai and S. Matile, Bioorg. Med. Chem., 2005, *13*, 5171-5180.

17) S. Matile, The Chemical Record, 2001, 1, 162-172.

18) N. Sorde', Ph.D. thesis entitled: "Rigid-Rod B-Barrel Pores as Enzyme Sensors”, University of Geneve, 2004, \#3575

19) N. Sakai, J. Mareda and S. Matile, Accounts of Chemical Research, 38, 79-87

\section{FIGURES}

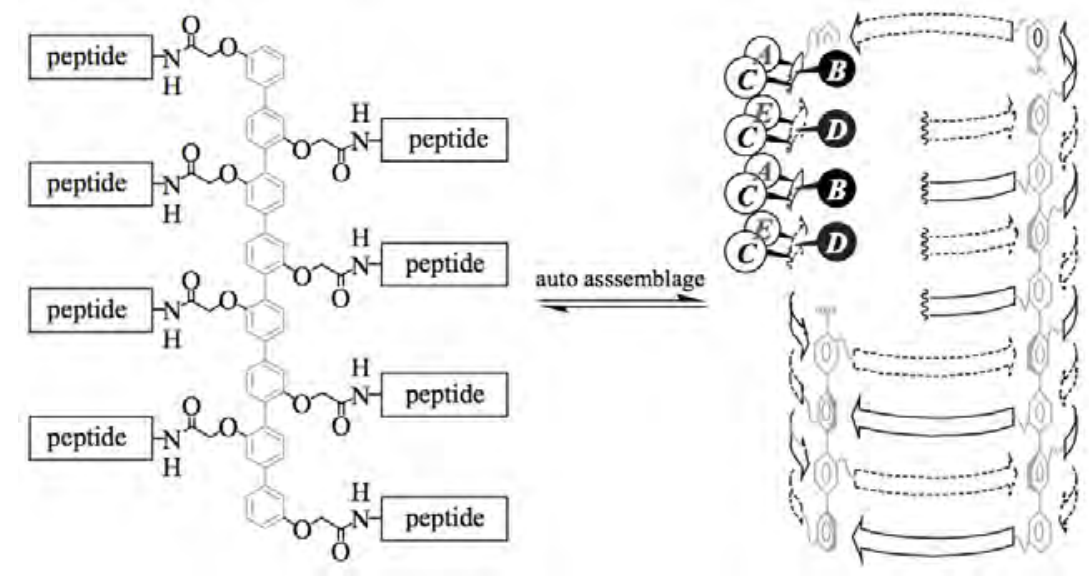

Figure 1. Schematic representation of the synthetic $\beta$-barrel pore unit (left) and assembly (right), as taken from reference 18. 
PNNL-16185

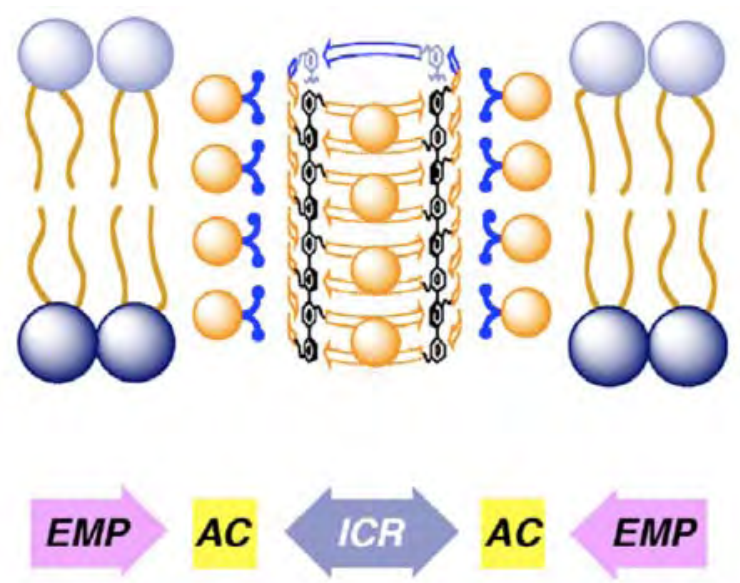

Figure 2. Schematic representation of the EMP-ICR model. The pore is shown with functionalized external calixaarene ligands that have been suggested to act as "cushions" between these two forces, as taken from reference 16.
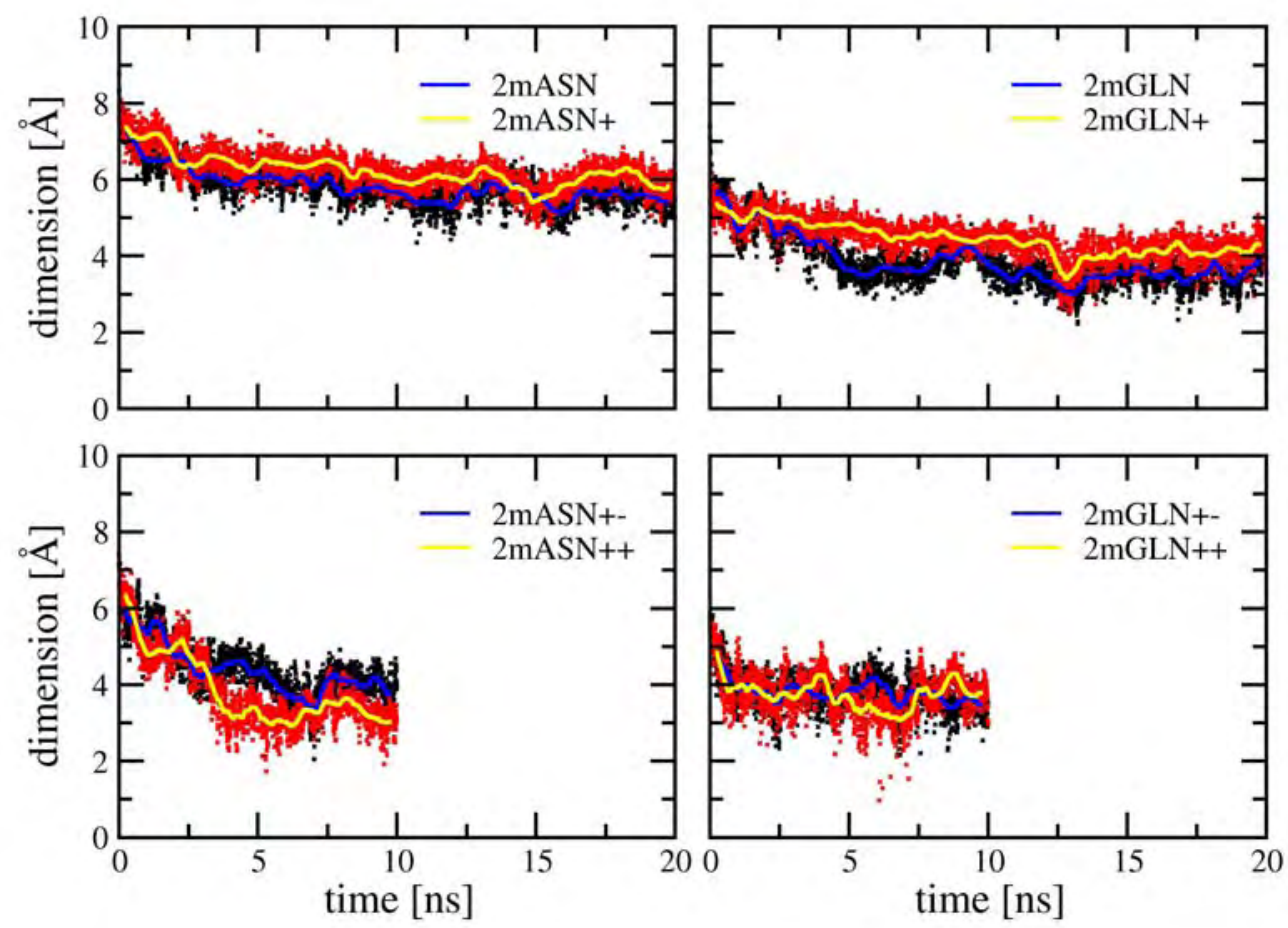

Figure 3. Inner pore dimensions as a function of time. 
PNNL-16185
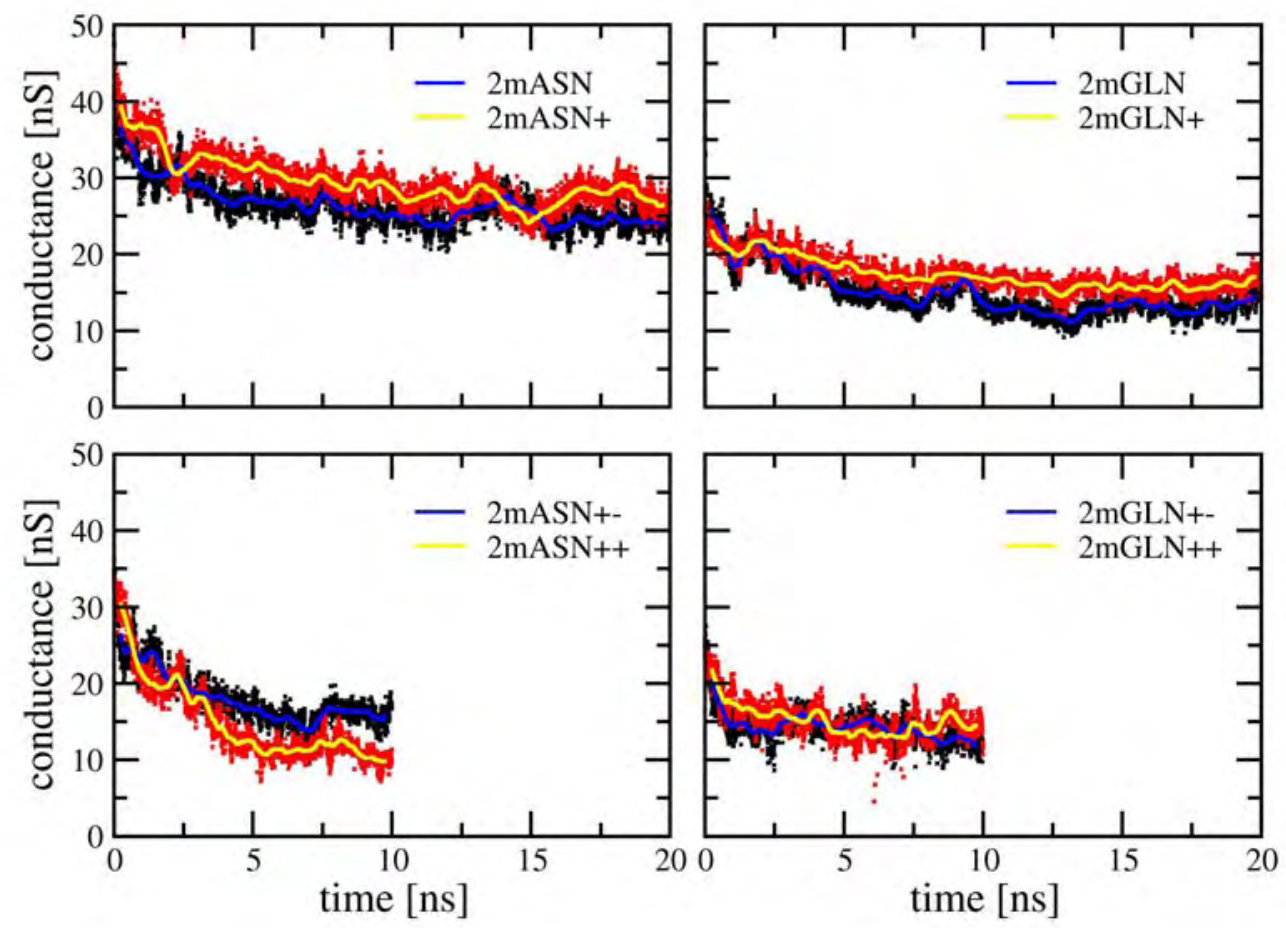

Figure 4. Pore conductance as a function of time. 
PNNL-16185

$2 \mathrm{mASN}$ socondary structure

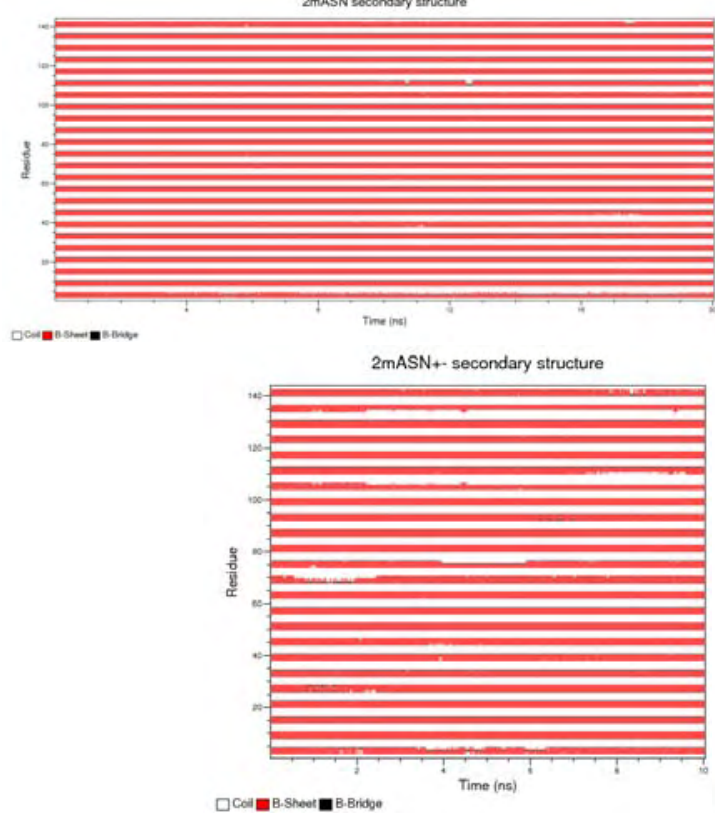

$\square$ Coll B-Sheor B-Bridge

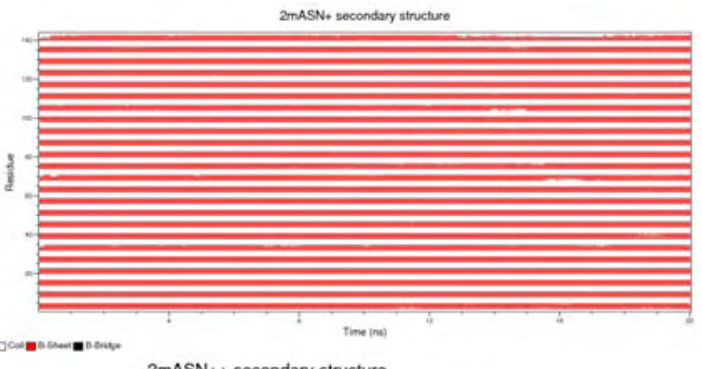

$2 \mathrm{mASN}++$ secondary structure

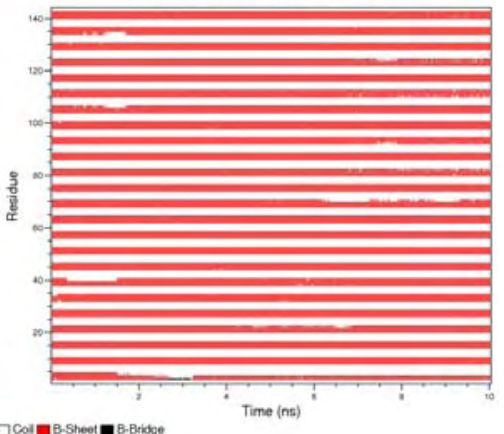

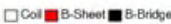

$2 m G L N$ secondary structure

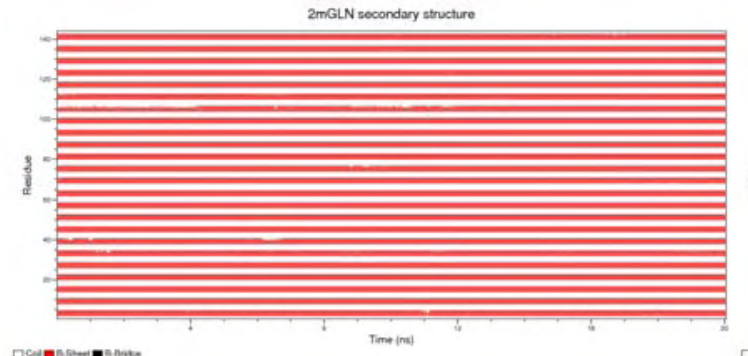

$2 m G L N+$ secondary structure

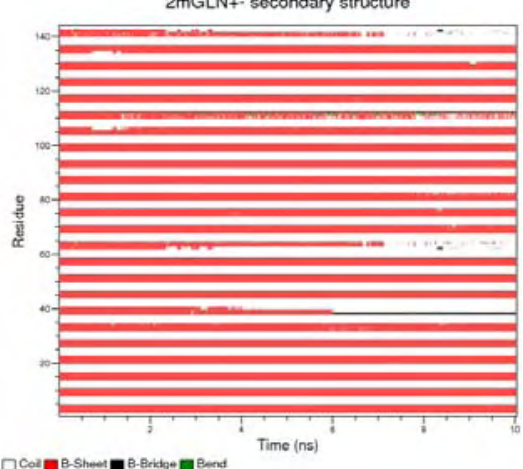

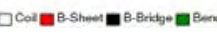

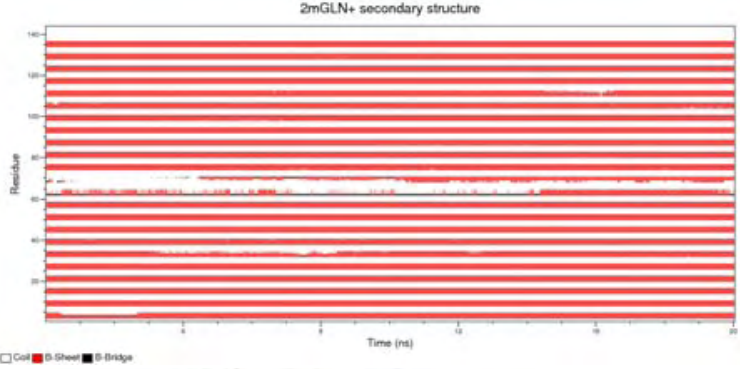

$2 \mathrm{mGLN}++$ secondary structure

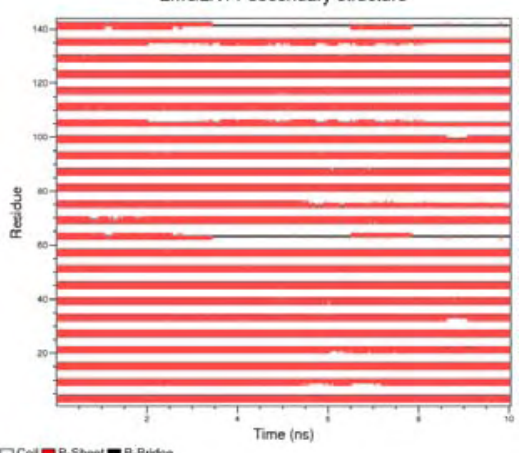

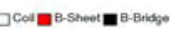

Figure 5. Secondary structure content as a function of time. 
PNNL-16185 
PNNL-16185

\title{
Chemical Speciation of Sulfer in Marine Aerosol Particles
}

\author{
Rebecca J Hopkins and Mary K. Gilles \\ Chemical Sciences \\ Lawrence Berkeley National Laboratory, \\ Berkeley, CA \\ and \\ Alexander Laskin \\ Pacific Northwest National Laboratory \\ Richland, WA
}

\section{Abstract}

Chemical speciation and apportionment of sulfur containing compounds in individual marine particles is facilitated by a complementary combination of microanalysis techniques. Chemical speciation of sulfur has provided insight into the atmospheric chemistry of marine particles collected in a field study conducted at Point Reyes National Seashore, CA. The results indicate separate formation of methylsulfonic acid (MSA) in cloud droplets and ammonium sulfate in smaller particles. These observations are consistent with two different pathways in the multiphase oxidation chemistry of dimethyl sulfide (DMS) in the marine boundary layer as shown in the recent modeling study of von Glasow and Crutzen. ${ }^{1}$

\section{Introduction}

Dimethyl sulfide (DMS) is the most important gaseous precursor for sulfate aerosols over the oceans. ${ }^{2}$ DMS is produced by phytoplankton and subsequently emitted to the atmosphere. Once in the marine boundary layer, it is oxidized via two main reaction pathways: addition of an $\mathrm{O}$ atom or abstraction of an $\mathrm{H}$ atom. The major DMS oxidation products fall into two classes: 1) those that form new particles and can act as new cloud condensation nuclei (CCN) such as $\mathrm{H}_{2} \mathrm{SO}_{4}$ and 2) those that condense onto existing particles increasing the particle diameter such as dimethylsulfoxide $\left(\mathrm{CH}_{3} \mathrm{SOCH}_{3}\right.$ or DMSO), methanesulfinic acid $\left(\mathrm{CH}_{3} \mathrm{~S}(\mathrm{O}) \mathrm{OH}\right.$ or MSIA), methanesulfonic acid $\left(\mathrm{CH}_{3} \mathrm{SO}_{3} \mathrm{H}\right.$ or MSA). ${ }^{1}$ The partitioning between $\mathrm{H}_{2} \mathrm{SO}_{4}$ and other DMS oxidation products is important because increasing the number of particles and increasing particle size can impact climate related properties of aerosols and cloud droplets differently, i.e. scattering and absorption of sunlight (direct effect) as well as changing the microphysical structure, lifetime, and surface area of clouds (indirect effects).

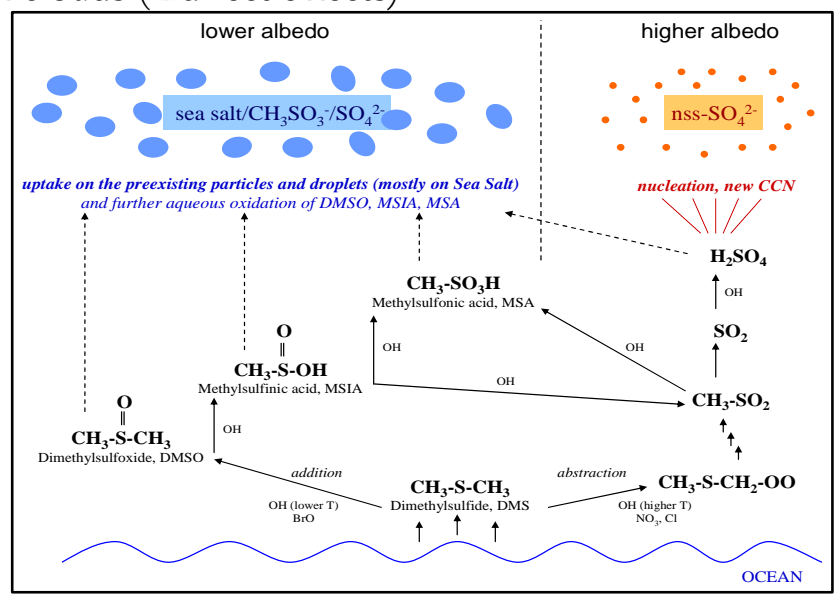

Figure 1 - Schematic diagram illustrating the different atmospheric oxidation pathways of DMS emitted from the ocean. 


\section{Results}

Collaborative work conducted at Pacific Northwest National Laboratory (PNNL) and Lawrence Berkeley National Laboratory (LBNL) has shown the presence of both mixed $\mathrm{CH}_{3} \mathrm{SO}_{3}{ }^{-}$ $/ \mathrm{nss}_{-} \mathrm{SO}_{4}{ }^{2-} / \mathrm{ss}_{-} \mathrm{SO}_{4}{ }^{2-}$ and separate nss- $\mathrm{SO}_{4}{ }^{2-}$ particles from atmospheric aerosols collected at $\mathrm{Pt}$ Reyes, CA. This confirms the existence of two oxidation pathways for DMS under cold, cloudy conditions. Scanning electron microscopy (SEM) images presented in Figure 2 illustrate the very different appearance of these particles. The mixed $\mathrm{CH}_{3} \mathrm{SO}_{3}{ }^{-} / \mathrm{nss}_{-}-\mathrm{SO}_{4}{ }^{2-} / \mathrm{ss}_{-} \mathrm{SO}_{4}{ }^{2-}$ particles display a crystalline morphology while the nss- $\mathrm{SO}_{4}{ }^{2-}$ are round in shape.

(a)

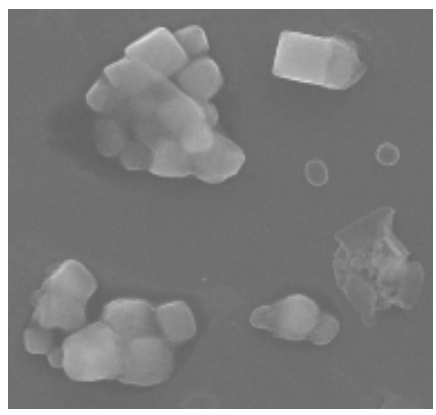

(b)

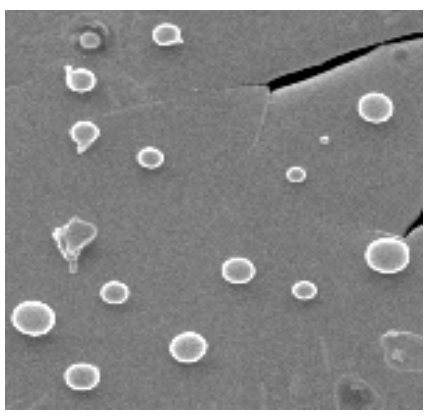

Figure 2 - SEM images of (a) crystalline mixed $\mathrm{CH}_{3} \mathrm{SO}_{3}{ }^{-} / \mathrm{nss}_{-} \mathrm{SO}_{4}{ }^{2-} / \mathrm{ss}^{-} \mathrm{SO}_{4}{ }^{2-}$ particles and (b) round nss- $\mathrm{SO}_{4}^{2-}$ particles.

Time-of-flight secondary ion mass spectrometry (TOF-SIMS) has been used to gain supplementary information in support of the observation of two particle types. TOF-SIMS ion maps show two types of externally mixed particles which can be attributed to $\mathrm{Na}^{+} / \mathrm{CH}_{3} \mathrm{SO}_{3}{ }^{-}$(or $\mathrm{CH}_{3} \mathrm{SO}_{3}{ }^{-} / \mathrm{nss}^{-} \mathrm{SO}_{4}{ }^{2-} / \mathrm{ss}_{-} \mathrm{SO}_{4}{ }^{2-}$ ) and $\mathrm{NH}_{4}{ }^{+} / \mathrm{SO}_{4}{ }^{2-}$ (or nss- $\mathrm{SO}_{4}{ }^{2-}$ ) particles. However, while this data supports observations from SEM data, it should be noted that TOF-SIMS is a surface sensitive technique and therefore additional complimentary information has been sought by analysing these aerosol particles using scanning transmission X-ray microscopy (STXM) coupled with near edge $\mathrm{X}$-ray absorption fine structure (NEXAFS) spectroscopy at LBNL.

(a)
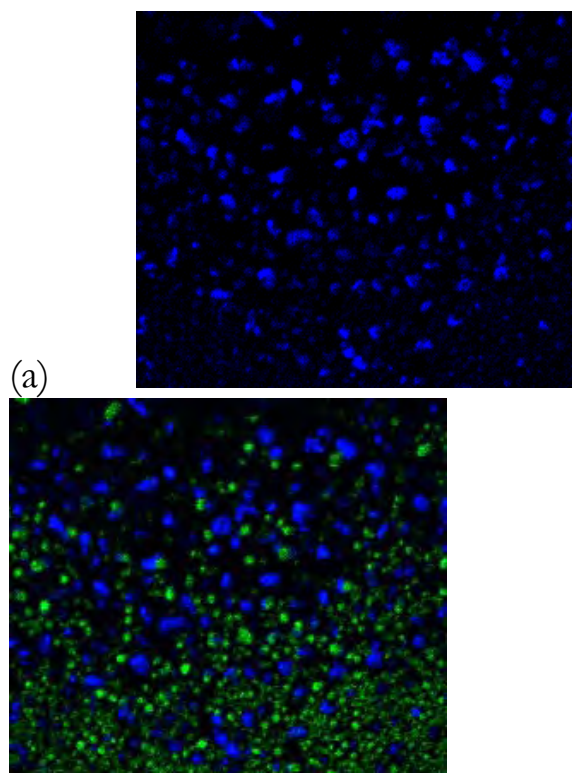

(b)

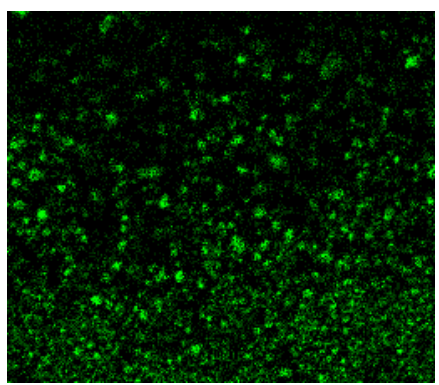

(c)

Figure 3 - TOF-SIMS ion maps of (a) $\mathrm{Na}^{+} / \mathrm{CH}_{3} \mathrm{SO}_{3}{ }^{-}$, (b) $\mathrm{NH}_{4}{ }^{+} / \mathrm{SO}_{4}{ }^{2-}$ and (c) images (a) and (b) overlaid. 
PNNL-16185

STXM/NEXAFS data has been used to calculate the partitioning of $\mathrm{CH}_{3} \mathrm{SO}_{3}{ }^{-}$/nss + ss$\mathrm{SO}_{4}{ }^{2-}$ in individual aerosol particles, ${ }^{3}$ but this result alone does not enable the branching ratio of the two oxidation pathways of DMS to be calculated. A combination of data collected using SEM/EDX at PNNL and STXM/NEXAFS at LBNL is needed to determine the $\mathrm{CH}_{3} \mathrm{SO}_{3}^{-}$/nss$\mathrm{SO}_{4}{ }^{2-}$ branching ratio. The $\left[\mathrm{S}_{\text {total }}\right] /[\mathrm{Na}]$ ratio has been measured for literally thousands of particles using SEM/EDX and when combined with the STXM/NEXAFS data which yields a mean $\mathrm{CH}_{3} \mathrm{SO}_{3}{ }^{-} / \mathrm{nss}+\mathrm{ss}_{-} \mathrm{SO}_{4}{ }^{2-}$ ratio of $34 / 66$ and the $\mathrm{ss}_{-} \mathrm{SO}_{4}{ }^{2-} / \mathrm{Na}$ ratio $\left(0.06\right.$ for $\left.\mathrm{ss}^{-} \mathrm{SO}_{4}{ }^{2-}\right)$ previously reported in the literature, ${ }^{4}$ it has been possible to calculate the $\mathrm{CH}_{3} \mathrm{SO}_{3}{ }^{-} / \mathrm{nss}-\mathrm{SO}_{4}{ }^{2-}$ ratio and therefore the branching ratio of the two oxidation pathways of DMS (see equations 1 and 2).

$\frac{[\mathrm{S}]_{\text {total }}}{[\mathrm{Na}]}=\frac{\left[\mathrm{SO}_{4}^{2-}\right]_{s S}}{[\mathrm{Na}]}+\frac{\left[\mathrm{SO}_{4}^{2-}\right]_{\text {nss }}}{[\mathrm{Na}]}+\frac{\left[\mathrm{CH}_{3} \mathrm{SO}_{3}^{-}\right]}{[\mathrm{Na}]}$

(1)

$\frac{[\mathrm{S}]_{\text {total }}}{[\mathrm{Na}]} \times \frac{0.34}{0.66}=\frac{\left[\mathrm{CH}_{3} \mathrm{SO}_{3}^{-}\right] /[\mathrm{Na}]}{\left[\mathrm{SO}_{4}^{2-}\right] /[\mathrm{Na}]}=\frac{\frac{\left[\mathrm{CH}_{3} \mathrm{SO}_{3}^{-}\right]}{\mathrm{Na}}}{0.06+\frac{\left[\mathrm{SO}_{4}^{2-}\right]_{\text {nss }}}{[\mathrm{Na}]}}$

Figure 4 illustrates the combined SEM/EDX and STXM/NEXAFS data which plots the $\mathrm{CH}_{3} \mathrm{SO}_{3}^{-} /$nss- $^{-} \mathrm{SO}_{4}{ }^{2-}$ ratio for five particle size ranges. It is evident that the branching ratio is size dependent, but an average value of 0.6 can be extracted. This branching ratio can now be used in atmospheric climate models to better understand the impact of sulfur chemistry on global climate change.

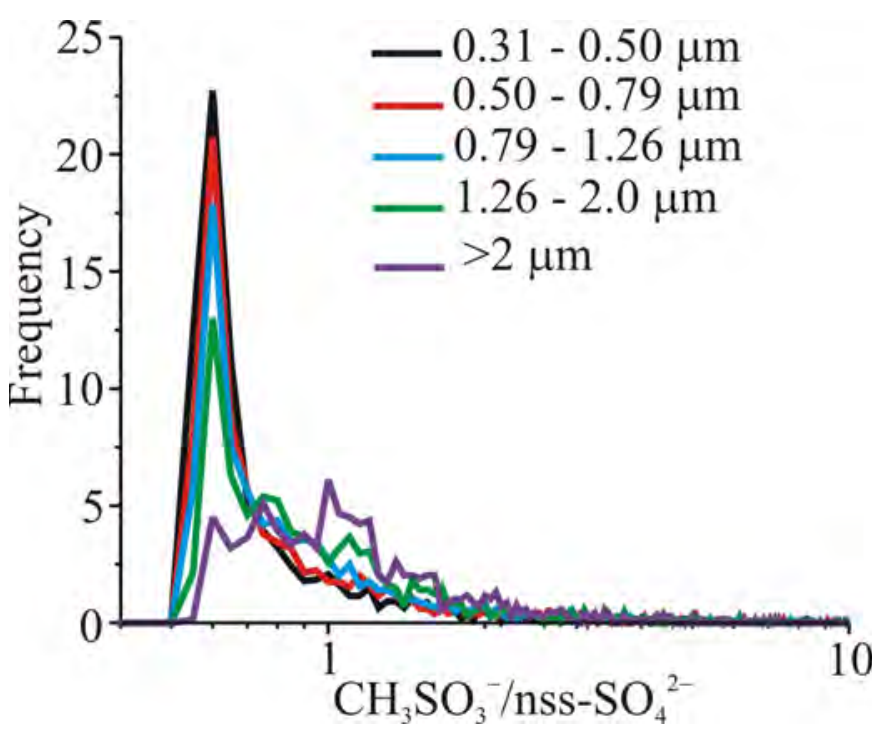

Figure 4 - Distribution of $\mathrm{CH}_{3} \mathrm{SO}_{3}{ }^{-}$to nss- $\mathrm{SO}_{4}{ }^{2-}$ present in particles with diameter in the range $0.31-0.5,0.5-0.79,0.79-1.26,1.26-2$ and $>2 \mu \mathrm{m}$. 
PNNL-16185

\section{Conclusion}

The branching ratio of two oxidation pathways of DMS which is emitted from the ocean into the atmosphere has been determined using the combined experimental data from three microanalysis techniques. SEM/EDX has been used to quantitatively assess the elemental composition of individual aerosol particles, TOF-SIMS has enables qualitative molecular speciation of sulfer containing compounds in individual particles and STXM/NEXAFS has facilitated quantitative assessment of $\mathrm{CH}_{3} \mathrm{SO}_{3}{ }^{-}$versus $\mathrm{SO}_{4}{ }^{2-}$ within individual particles. The combination of these results has yielded an average branching ratio of 0.6 for this important atmospheric chemical reaction which can now be used to model the impact of this sulfur chemistry on global climate change.

\section{References}

1. R. von Glasow, P. J. Crutzen,, Atmos. Chem. Phys., 2004, 4, 589.

2. R. J. Charlson, J. E. Lovelock, M. O. Andreae, S. G. Warren, Nature, 1987, 326, 655.

3. R. J. Hopkins, A. V. Tivanski, Y. Desyaterik, A. Laskin, M. K. Gilles, In preparation.

4. L. M. McInnes, D. S. Covert, P. K. Quinn, M. S. Germani, J. Geophys. Res., 1994, 99, 8257. 
PNNL-16185

\title{
Mechanistic and Energetic Studies of Radical \\ Peptides by means of FT-ICR SID MS
}

\author{
Name: Ngor Wai (Corey) Lam and Ivan Keung Chu \\ Department of Chemistry, \\ The University of Hong Kong, Hong Kong \\ and \\ Dr. Julia Laskin \\ Pacific Northwest National Laboratory \\ Richland, WA
}

Collective efforts (Jean, Julia and Ivan K. Chu) from the 2005-ICPCPSRI have lead to successful execution of the proposed project and result significant contribution toward understanding of the gas phase chemistry of novel radical cationic peptides. With the previous demonstration of kinetic studies with molecular radical angiotensin, we will extend our investigation on anionic radical cationic angiotensin III using similar methodologies. Studies of odd-electron radical cationic $\mathrm{M}^{\bullet+},[\mathrm{M}+\mathrm{H}]^{\bullet 2+}$, and anionic $[\mathrm{M}-2 \mathrm{H}]^{\bullet-}$ peptides can help to bridge the gap between small cations and protonated peptide ions and facilitate a better understanding on the role of hydrogen atom that governs radical peptide fragmentation. Our intended studies will utilize time- and energy-resolved SID, combined with an RRKM-based modeling approach, to determine the energetics of dissociation of medium-size molecular radical anionic oligopeptides, such as angiotensin III(RVYIHPF) and its derivatives. Our recent success at forming a wide variety of radical anions of oligopeptides should greatly expand the scope of investigations of molecular radical cations of oligopeptides. ${ }^{1}$ The ability to form peptide radical anions in the gas phase raises a question about their stability relative to those of the corresponding protonated species and smaller organic radical anions. Are the energetics of dissociation of these species similar to the dissociation energetics of smaller radical anions? Does conformational flexibility of these molecules have an effect on their gas phase stability? It is known that the bond dissociation energy for organic radical anions is in the range $2.5-6 \mathrm{eV},{ }^{2}$ while dissociation of protonated peptides requires only $0.8-2 \mathrm{eV} .^{3}$ This relative ease of dissociation of protonated peptides has been attributed to the presence of a proton that migrates along the backbone of the peptide and weakens the peptide bond in its 
PNNL-16185

vicinity-the "mobile proton model". ${ }^{4}$ Studies of $[\mathrm{M}-2 \mathrm{H}]^{\bullet-}, \mathrm{M}^{\bullet+}$ and $[\mathrm{M}+\mathrm{H}]^{\bullet 2+}$ can help to bridge the gap between small organic anions and protonated peptide ions and facilitate an understanding of the factors that govern bond strengths in ionic systems. This $\mathbf{M}^{\bullet+}$ and $[\mathrm{M}+$ $\mathrm{H}]^{\bullet 2+}$ species have a large number of vibrational degrees of freedom, which results in a slow dissociation that is ideally suited for kinetic studies on the timescale of our FT-ICR SID MS. An ion of almost identical molecular weight_protonated angiotensin III(RVYIHPF)—has been studied extensively and validated using the proposed methodologies.

For the upcoming program in the Pacific Northwest National Laboratory's Summer Research Institute in Interfacial and Condensed Phase Chemical Physics, we will conduct experiments on molecular radical anionic oligopeptides using aforementioned method. The advantages provided by SID include very fast ion activation, which eliminates possible discrimination against higher-energy dissociation pathways, and efficient “amplification” of small changes in the dissociation parameters. ${ }^{4}$ We will use this approach to address the question of stability of gas phase peptide radical anions. We will use a combination of experimental and computational approaches to conduct the first studies on the energetics of the dissociation of selected peptide radical anions and compare these results with the dissociation energetics of their corresponding cationic species. Collision energy-resolved studies provide important information on the appearance energies of different product ions and on the amount of energy required for dissociation of the precursor ions. Another dimension is added to SID experiments by conducting kinetic studies through varying the delay between the ion-surface collision and the analysis of the resulting products. Although studies of time- and energy-resolved spectra have been conducted so far for only a limited number of large ions, they have demonstrated a great potential for allowing the detailed elucidation of fragmentation energetics and mechanisms. $^{3}$ 
PNNL-16185

\section{References:}

1. (a) Chu, I. K.; Rodriquez, C. F.; Lau, T. C.; Hopkinson, A. C.; Siu, K. W. M. J. Phys. Chem. B 2000, 104, 3393-3397. (b) Chu, I. K.; Rodriquez, C. F.; Lau, T. C.; Hopkinson, A. C.; Siu, K. W. M. J. Am. Soc. Mass Spectrom. 2001, 12, 1114-1119. (c) Bagheri-Majdi, E.; Ke, Y.; Orlova, G.; Hopkinson, A. C.; Siu, K. W. M. J. Phys. Chem. B 2004, 108, 11170-11181.(d) Chu, I. K.; Siu, S. O.; Lam, C. N. W.; Chan, J. C. Y.; Rodriquez, C. F. Rapid Commun. Mass Spectrom. 2004, 18, 1798-1802. (e) Chu, I. K.; Lam, C. N. W. Manuscript in preparation.

2. McLafferty, F. W.; Turecek, F. Interpretation of Mass Spectra, $4^{\text {th }}$ Ed., University Science Books, 1993.

3. (a) Laskin, J. Eur. J. Mass Spectrom. 2004, 10, 259-267. (b) Schnier, P. D.; Price, W. D.; Jockush, R. A.; Williams, E. R. J. Am. Chem. Soc. 1996, 118, 7178-7189. (c) Schnier, P. D.; Price, W. D.; Strittmatter, E. F.; Williams, E. R. J. Am. Soc. Mass Spectrom. 1997, 8, 771-780.

4. (a) Laskin, J.; Denisov, E.; Futrell, J. H. J. Am. Chem. Soc., 2000, 122, 9703-9714. (b) Laskin, J.; Denisov, E.; Futrell, J. H. Int. J. Mass Spectrom. 2002, 219, 189-201. (c) Laskin, J.; Bailey, T. H.; Futrell, J. H. J. Am. Chem. Soc. 2003, 125, 1625-1632. (d) Laskin, J.; Bailey, T. H.; Futrell, J. H. Int. J. Mass Spectrom. 2004, 234, 89-99. 
PNNL-16185 
PNNL-16185

\title{
Tandem Accelerator and Ion Beam Techniques
}

\author{
Vu H. Lam and Kevin Coffey \\ Advanced Materials Processing and Analysis Center \\ University of Central Florida \\ Orlando, Florida \\ and \\ Theva Thevuthasan \\ Pacific Northwest National Laboratory \\ Richland, Washington
}

\section{Introduction}

When I joined Interfacial and Nanoscale Science Facility (INSF) at Environmental Molecular Sciences Laboratory (EMSL) as a Summer Research Intern (SRI) student intern, my project was to learn the ion beam capabilities commonly utilized in the accelerator facility and help the users in the laboratory. Several projects were identified for me to work on in collaboration with various users and EMSL scientists including; (1) learning the components of the accelerator, beam lines and end stations, (2) mastering the simulation packages such as SIMNRA and GUPIX along with the experimental capabilities, in particular, Rutherford backscattering spectrometry (RBS), nuclear reaction analysis (NRA), and proton induced x-ray emission (PIXE), (3) participate and contribute to the experiments conducted by a user from Harvard university, and (4) contribute to the maintenance of the instrumentation in the accelerator facility. During my stay at EMSL, there was a breakdown of the analyzing magnet due to cooling lines break down and as a result the lab was shut down for three weeks for intensive repair of the magnet cooling system and the high energy beam line electronics. This activity provided a very good opportunity to troubleshoot some of the instrumentation and, as a result, I could learn and obtain hand on experience in fixing some instrumentation in the laboratory. 
PNNL-16185

Overall, the visit to EMSL as a part of this program gave me an opportunity to learn new things. In addition, it provided valuable experience in running and maintaining some experimental systems which may not be widely available. This report summarizes the components and capabilities available in the accelerator facility. Finally, I sincerely appreciate the opportunity given to me to visit EMSL and work with the scientists at EMSL by the SRI administration.

\section{Ions Beam Materials Accelerator Laboratory (IBMAL)}

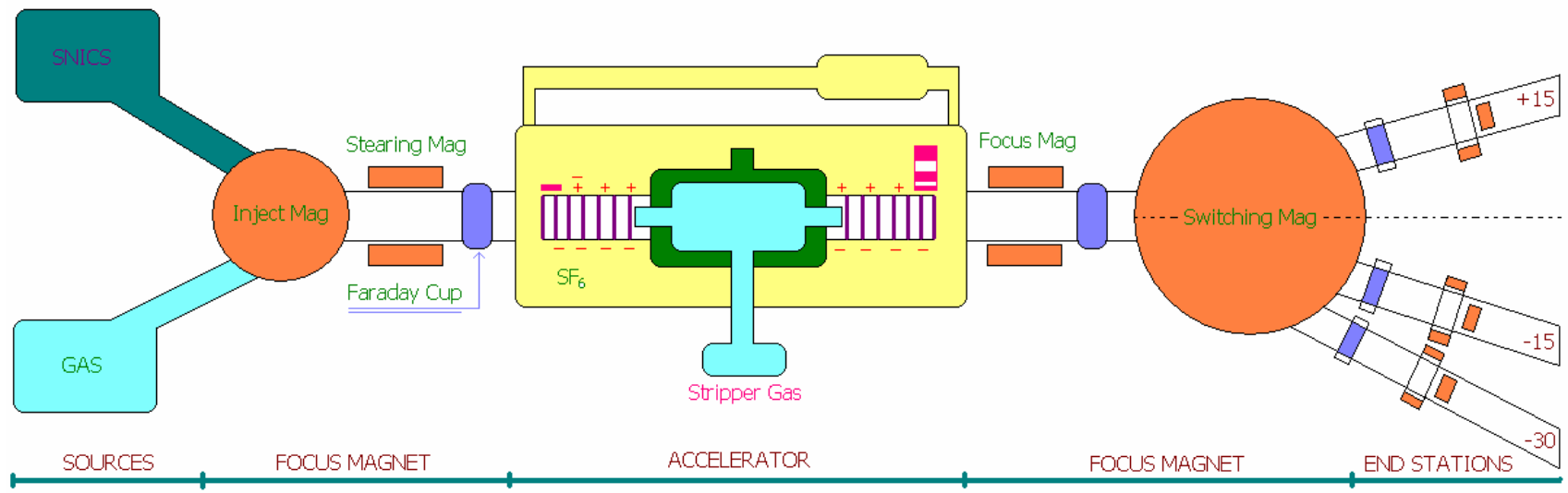

Fig 1: IBMAL

The accelerator lab can be break down to three main sections. The first section contains the sources of the ions beam. Second is the accelerator chamber and at last are the end stations. In general, the operation of the accelerator lab can be described as follow. First, negative ions will immerge from the source into the accelerator chamber. Right in the middle of the chamber consist of highly stored potential that was build up by using the Van de Graff method. From the beginning of the chamber to the middle region, the negative ions will attract by the positive potential and accelerate with the speed corresponding to the applied voltage. When the ions reach the middle regions, stripper gas will stripped off some of the electrons surrounding the particles and change the ions from negative to positive. The end region of the chamber will be grounded, in which creates a region that will attract the positive ions. From here, the positive ions that were created by the stripper gas will accelerate with the same potential as it first went from the beginning toward the middle of the tube. In general, the ions will accelerated twice as much of energy as the initial applied voltage. When the positive ions go out of the accelerator tank, it will then be focus down and steer to the end stations desired. There are three end 
PNNL-16185

stations; positive 15 degree, negative 15 degree, and negative 30 degree. Each station performs different experiments such as PIXE, NRA, RBS, and ERDA (Elastic Recoil Detection Analysis).

Each section of the IBMAL will be analyze separately and discuss in greater detail about its functionality and operations. Like the name indicated, the source provides the ions to be accelerate. Within the source section, there will be two distinct kind of source. One is considered to be a solid source and the other is gas. The solid source is called SNICS (Source of Negative Ion by Cesium Sputtering) source.

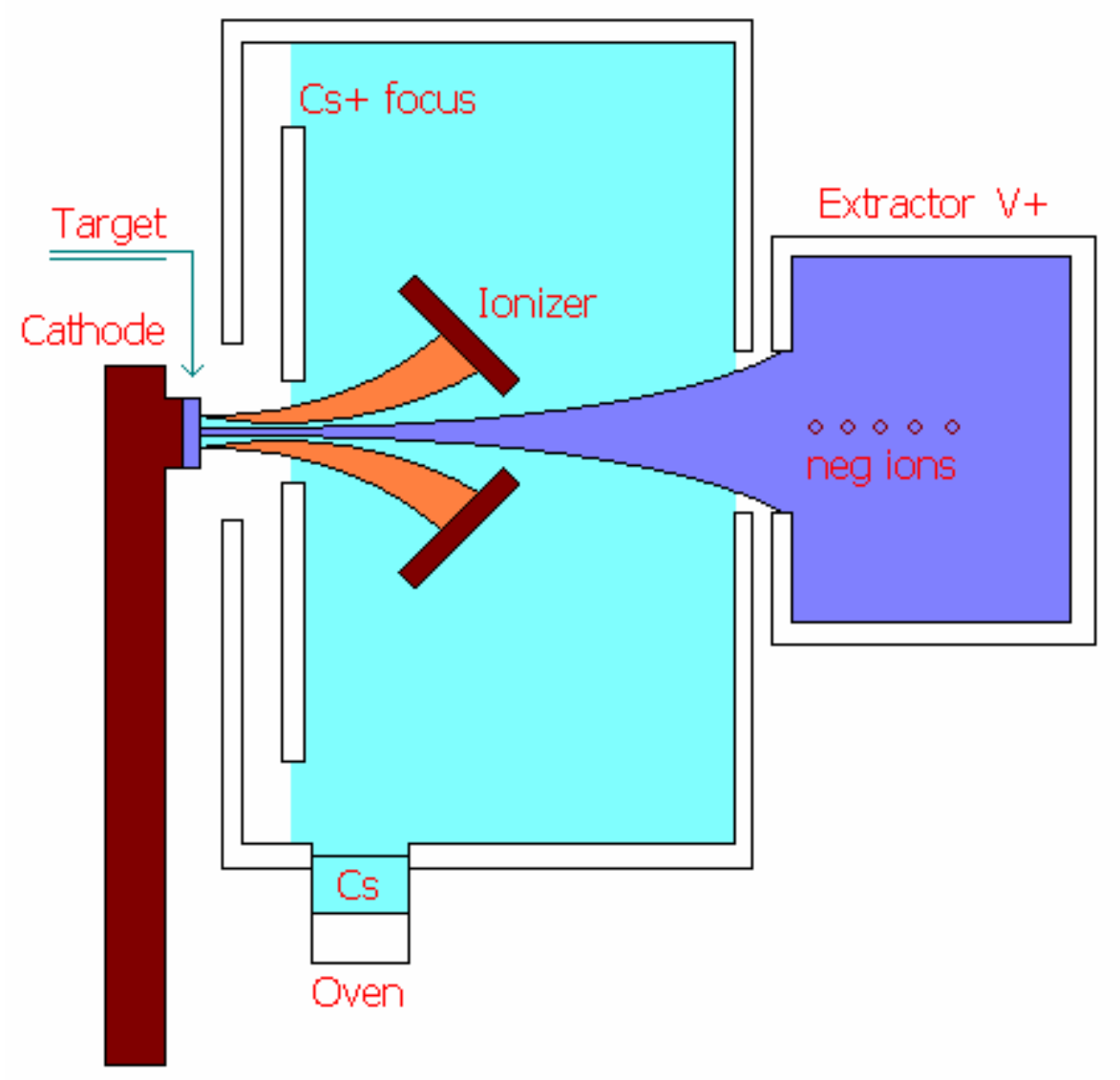

Fig 2: SNICS Source.

The target was hold by the cathode arm and was placed in the windows that can be access by the Cesium focus beam. The target can be any target that the user desired. The most common used target in the accelerator lab is gold (Au). The Cesium source was first placed in the oven chamber. The initial form of the Cesium source started as liquid. When the oven starts to heating up, the Cesium begins to vaporize. The temperature of the SNICS chamber increase 
PNNL-16185

gradually from room temperature to $150^{\circ} \mathrm{C}$. Cesium particles will then be ionized by the electric field created by the ionizer. Because the target was attached to the cathode, the positive Cesium ions will accelerate and focus on the target's surface. The Cesium ions started to sputter the target and kick out target particles. In this stage, surround the chamber will contain lots of target's electrons and holes pair created by the Cesium sputtering. The extractor has positive voltage applies at the end, therefore attract target electrons. In the end, the ions coming out of the SNICS source will be negative ions.

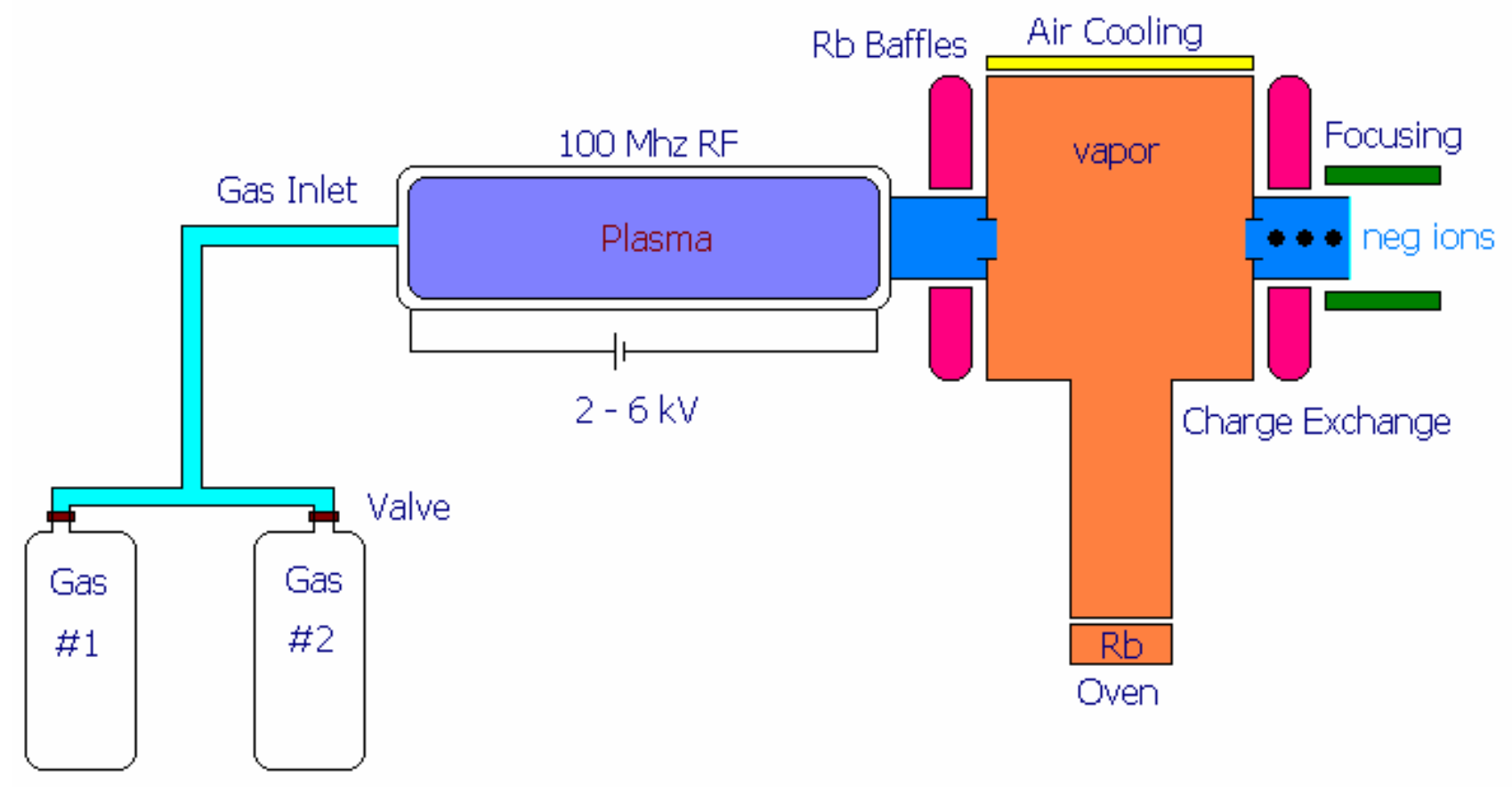

Fig 3: Gas Source.

Figure above illustrates the structure as well as the operations of the gas source. The gas source contains two separate gas tanks. One is Helium and the other is Hydrogen. The gas that most common used is Helium. The gas will enter the plasma chamber where they will be ionized. The plasma chamber has an applied potential of $6 \mathrm{kV}$. This potential has to increase slowly from 2 to $6 \mathrm{kV}$ during the warm up sequence. The frequency that is use for this chamber is 100 MHz. The RF (Radio Frequency) power supply will ionized the He gas and created plasma. The Helium ions will then enter the charge exchange chamber. In this chamber, the Rubidium source was heat from the oven below from room temperature to $250^{\circ} \mathrm{C}$. The Rubidium source starts as a liquid form then vaporize by the heat. This source will give away electrons and make the ions turning from positive to negative ions. 
PNNL-16185

Next stage is the focus magnet. In this region when the ions were injected from the source, the magnet was used to focus the size of the beam. The Faraday cup is also used in the area to control the beam before it enters the accelerator chamber.

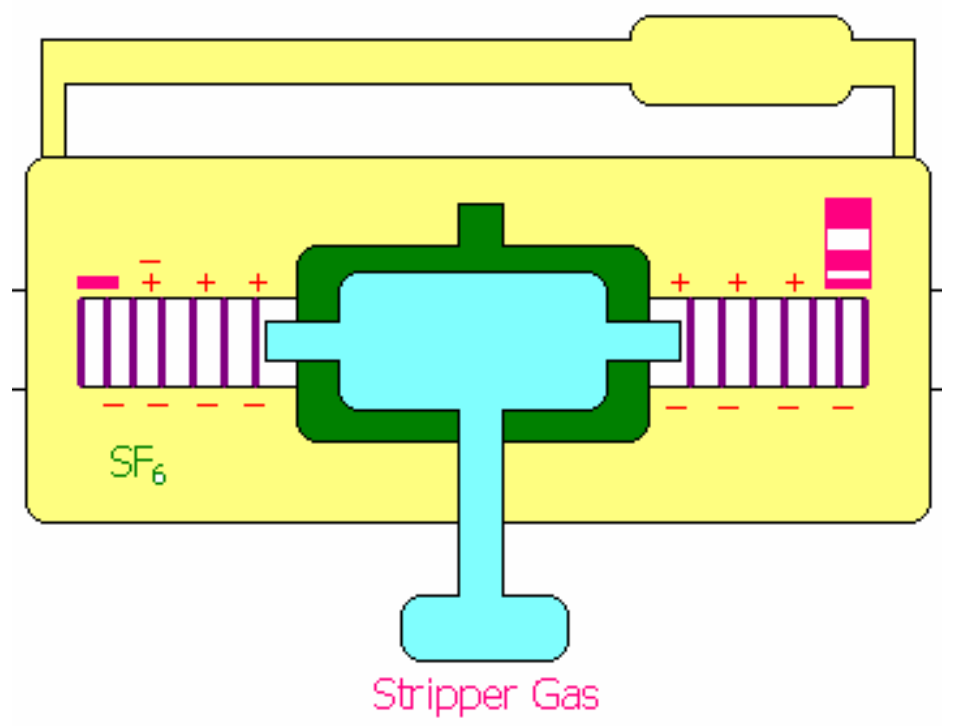

Fig 4: Accelerator Chamber.

There are many important components that inside the accelerator chamber. The first most important component is the applied potential. The energy of the ion beam can be defined in the equation below in which $\mathrm{E}$ is the ions energy, $\mathrm{q}$ is the charge of the ions and $\mathrm{V}$ is the terminal voltage.

$\mathrm{E}=(\mathrm{q}+1) * \mathrm{~V}$

The terminal voltage (V) has a voltage range from $0.5 \mathrm{MeV}$ to $3.0 \mathrm{MeV}$. By using the Van de Graff method, the potential can be build up from a $50 \mathrm{kV}$ source. 


\section{Applied Voltage}

\section{Terminal Voltage}

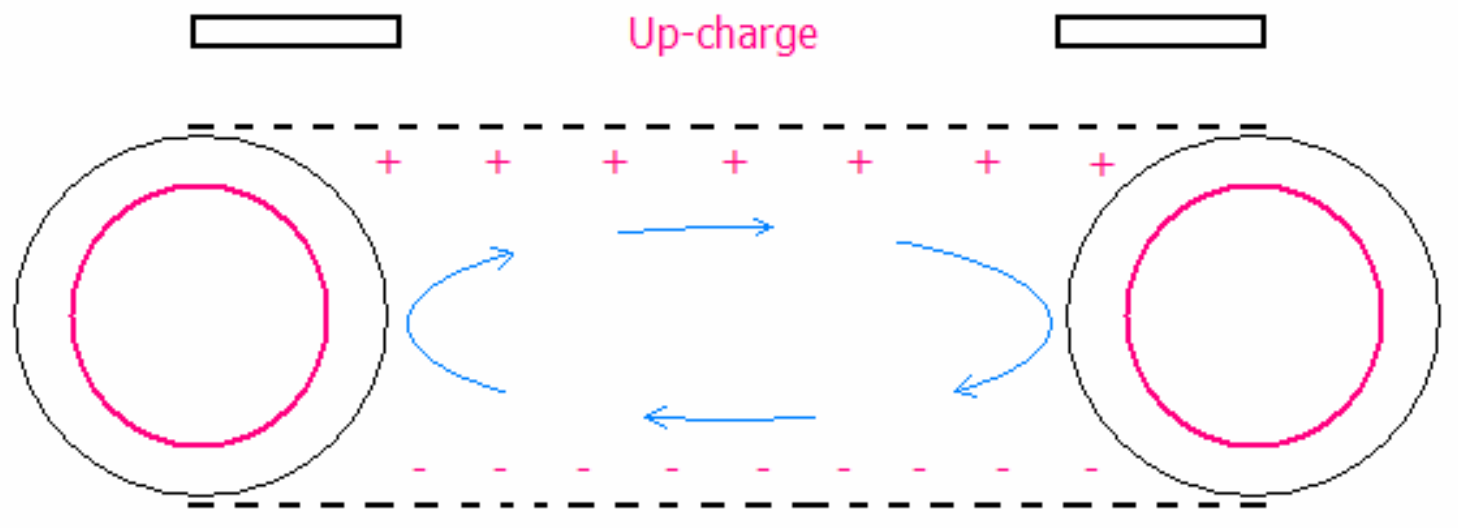

Down-charge

Fig 5: Van de Graff Method.

At the applied voltage region, negative charge will be applied in which attract the positive charge that will be localized on the surface of the chain. The voltage that applied at this region is about $50 \mathrm{kV}$. The chain will then carry the positive charge to the terminal voltage region where it was collect and store. This procedure keeps repeating until it reaches a desire potential. The charge of the ions (q) can be described as follow. When the negative ions enter the entrance of the accelerator chamber, it will accelerate toward the middle of the chamber where the terminal voltage applied. The middle region of the accelerator chamber will perform a special task called charge exchange. A gas tank is connect to the middle region of the chamber and contains either Nitrogen gas or Argon gas. These gases were used to strip off the electrons from the ions and converted it from negative ions to positive ions. During this interaction, the ions will obtain different charge such as $+1,+2,+3,+4,+5$, or +6 . This is the number that is used to calculate the energy obtained. The end of the accelerator chamber is grounded; therefore attract the positive ions in which make it accelerate toward the end of the chamber.

Between the chain in the middle of the chamber and the surround tube, the entire hollow region was filled by an insulating gas (SF6) to insulate the high potential created in the accelerator chamber. This is a dangerous gas due to its nature to consume Oxygen. The upper pipe on tope of the main tube is used to filter out the moisture of the gas to keep it more insulated. 
When the ions beam exit the accelerator chamber, it will then enter the focusing area. After focus the beam into smaller diameter, it was then steer it to a destination station by using the switching magnet. The angle of the outgoing beam was treated as a reference point and there are 3 end stations, one positive $15^{\circ}$, one negative $15^{\circ}$, and one negative $30^{\circ}$. Each branch consists of two basic instruments that use to aid the accuracy of the beam. One is the Steering Magnet and other is the Faraday Cup as figure 6 illustrate. Because the beam enter the branch is not guarantee to be right at the middle of the pipe, therefore the Steering Magnet was used to ensure that the beam is right on the middle and be able to arrive at the end station. The Faraday Cup is used to control the beam whether allow or not allow the beam to enter the vacuum chamber. One drawback is only one station can be access the beam once at a time.
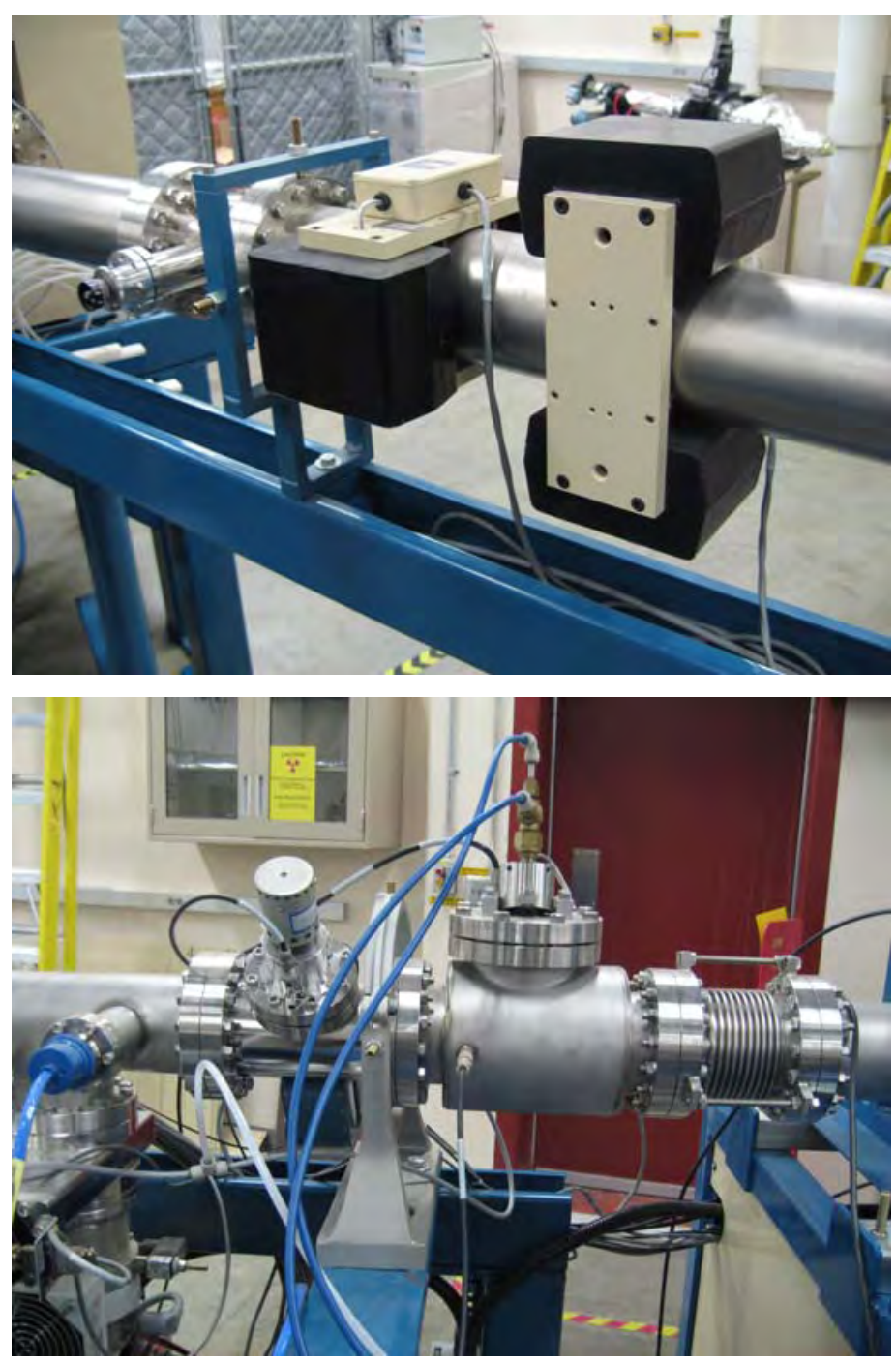

Fig 6: (a) Steering magnet, (b) Faraday cup. 
PNNL-16185

\section{Applications.}

Each station in the accelerator lab performs different experiments. The end stations with the branch of positive 15 degree can performs multiple experiments such as PIXE, PESA, STIM, RBS, and ERDA. Each experiment will be discussed briefly about its definition and its fundamental operation. The most common experiment that's using PIXE, PESA, and STIM is characterized aerosol samples. Aerosol samples are thin Teflon film with aerosol particles coating on its surface. The sample was collected in Mexico City to study about the pollution in the air. The samples are fairly thin so the beam can penetrate through. It is $2.5 \mu \mathrm{m}$ thick with particle deposits on its surface. PIXE is an experiment that using the ions beams to create and collect X-ray from the sample. When the beam hit the core electron of the atoms and has enough energy to kick out that electron, the next highest energy electron will fall down and filling the gap. When this electron moves down, it will emit X-ray. The X-ray was then detected and display with the corresponding energy of the ion beams. PIXE can be use to detect elements in which range from Sodium to Uranium. PESA (Photon Elastic Scattering Analysis) in the other hand is used to measure the Hydrogen quantity in the sample. The ions beams that use in PESA is Hydrogen ion. When the Hydrogen ions hit the sample, it will collide with the Hydrogen atoms inside the sample and scatter away at an angle. A detector will be place at a desire angle and will collect the scattering Hydrogen ions. The graph yields from the measurement represent the amount of Hydrogen inside the sample. In this experiment, PESA is not an ideals method to measure higher element such as Carbon, Oxygen, and Fluorine due to their close range of mass. However, PIXE can be use in this case to detect a while range of elements.

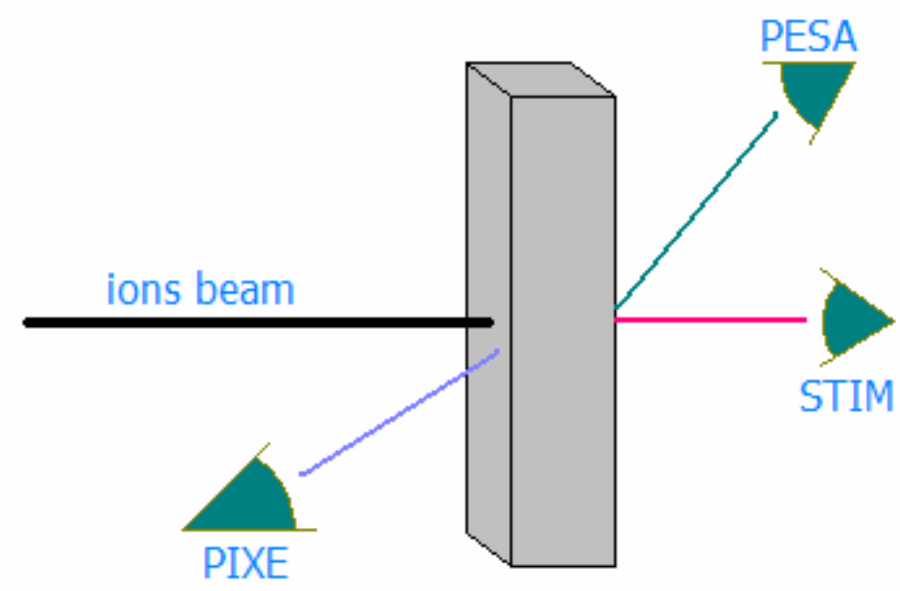

Fig 7: PIXE, TIM, and PESA. 
PNNL-16185

STIM (Scanning Transmission Ion Microscopy) is used to calculate the total mass of the aerosol particle deposited. The Hydrogen beam penetrated the thin sample and exit to the other side. Initially, the Hydrogen beam is coming in with a known energy (Eo). When the Hydrogen beam exits the sample, it will lose some of it energy due to collision inside the sample. This energy (E1) is measured and used to calculate the total mass of the aerosol collected based on this equation.

$\Delta \mathrm{E}=\mathrm{Eo}-\mathrm{E} 1$

RBS (Rutherford Backscattering Spectrometry) can be described in a similar way. For this method, user can find the composition of the material as well as its elements. As illustrate in Figure 8a, the ions beam coming in to collide with the sample surface. The sample atoms consist of different elements. Each element has it own mass and it can be heavier or lighter comparing to the mass of the ion beam. When the ion beam hit the sample atoms, it will lose some of its energy when bounding backward. The initial energy (Eo) will always be greater than the back scattering energy (Em). For heavy atom, the ion will bound back with much greater distance as well as energy compare to the atoms with lighter mass. When the ions pass through the first layer, it will lose some energy (E'o) and collide with the second layer in the sample. Through this, sample thickness can be illustrated.
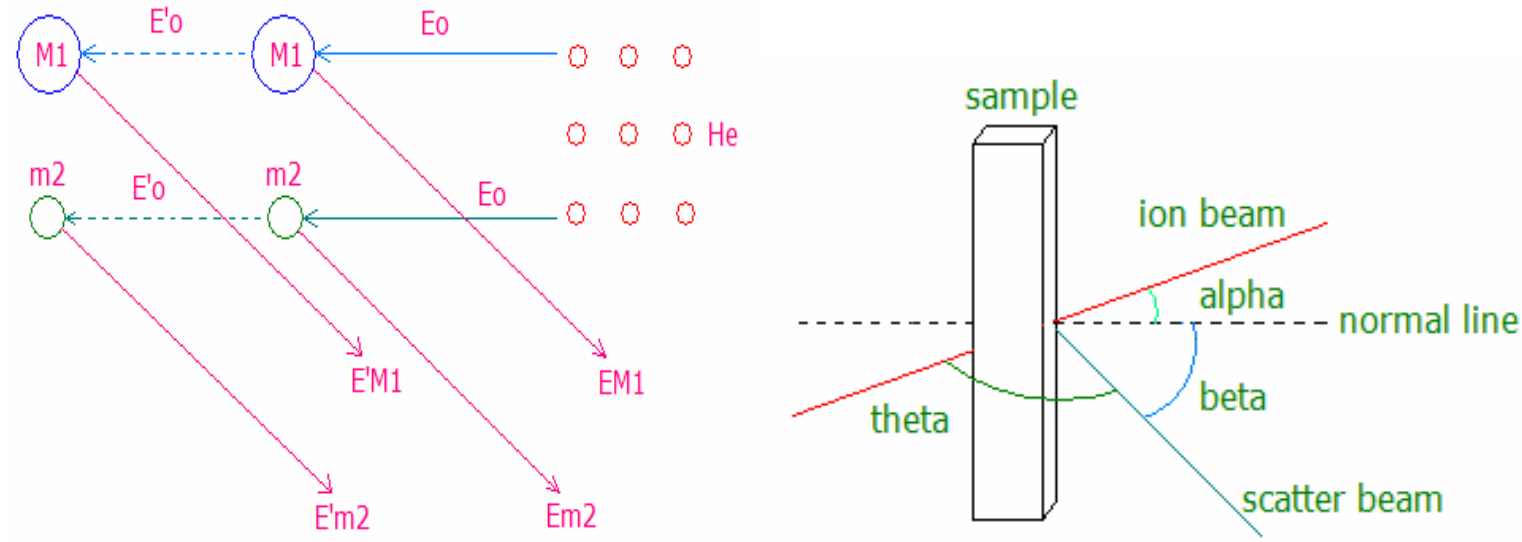

Fig 8: RBS (a) ions beam collision, (b) angles beam.

After achieve the graph of the RBS data, a simulation need to be performs to calculate the composition of the sample's elements. The simulation software called SIMNRA was used to analyze the data. In order to perform the simulation, certain information need to be provided 
such as alpha $(\alpha)$ angle, beta $(\beta)$ angle, and theta $(\theta)$ angle as Figure 8b. It also needs the initial guess of the elements and its composition. From there, user can change the parameter to simulate the graph so it can be identical to the experiment graph. After succeed the simulation, the software will provide the user the composition of the sample as well as the thickness. One drawback of RBS is it's not that sensitive to lighter elements.

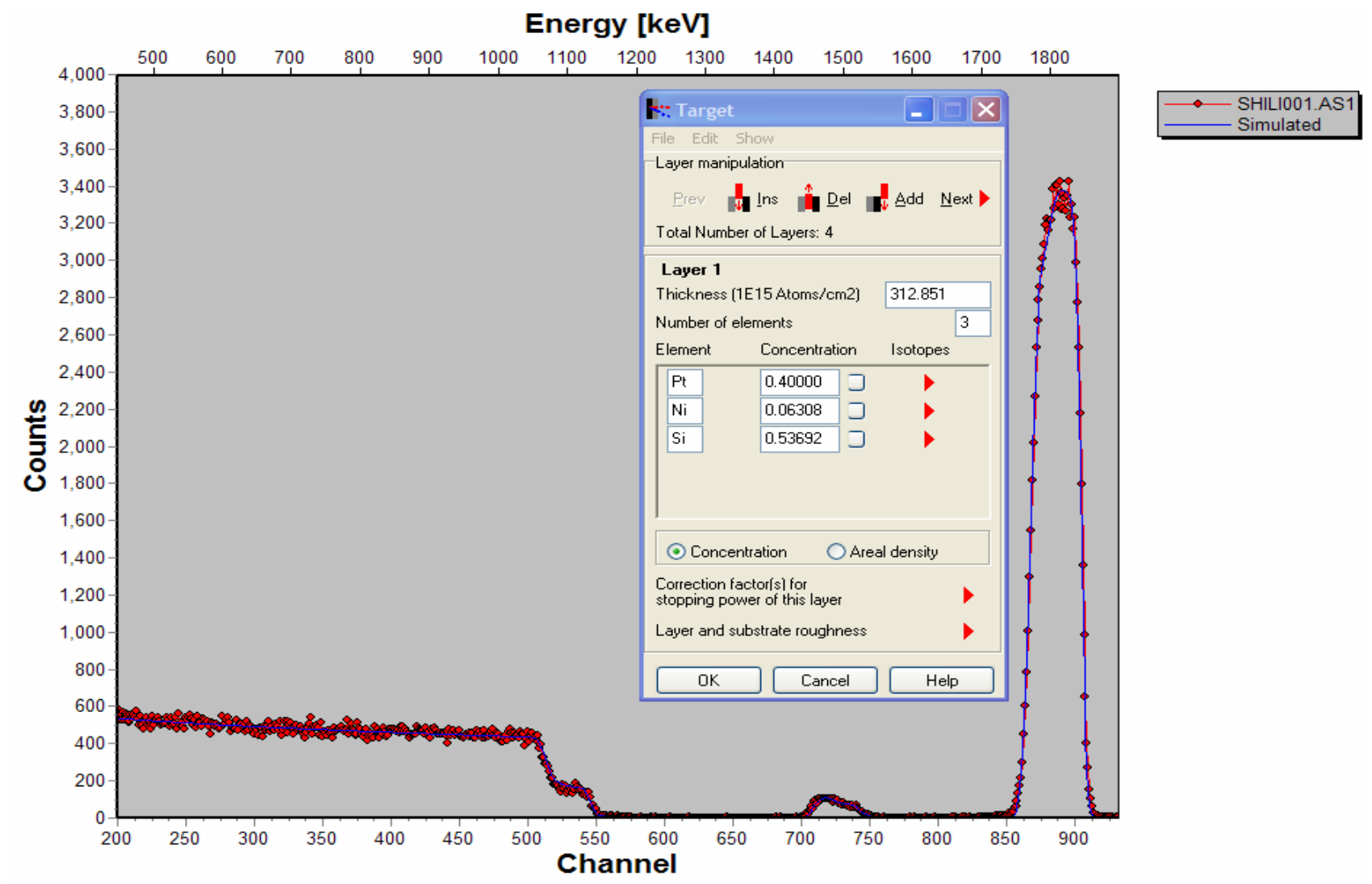

Fig 9: SIMNRA

ERDA (Elastic Recoil Detection Analysis) is mainly used to find material's surface sensitivity. It's also called Time-of-Flight ERDA. It's consists of few main components and first is the target. The target is defined by the user in which determine the ions that use for sensitivity testing. Second is the beam line. The ions beam for this experiment can be either Hydrogen or gold. Third is the detector station. There are two stations separate with a fix distance. Each station contains a carbon foil in which use for start and stop signal. At the end of the arm, another station is located to measure the energy of the beam line. There are two distinct tests that can be run by using the ERDA chamber. First is sensitivity sensor by using hydrogen ions beam line. The target that is used for this run is normally SiO2. Due to the lightness of 
PNNL-16185

hydrogen, when the beam hit the target, it will bounce toward the detectors. The ions will first hit the first carbon foil and produce secondary ions on the surface of the foil. The carbon foil's thickness is just about few micron meters; therefore ions beam can be easily pass through and very sensitive for detection. When the carbon foil creates ions on its surface, the detector will collect those ions by the applied potential and produce a pulse of signal that will recorded as a starting point. The hydrogen beam continues to travel toward the second detector where it again produce secondary ions and recorded as the stopping point. After passing through the second detector, it will collide with the last detector that located at the end of the arm. From here, the detector obtains the energy of the ions. Velocity (V) of the ions beam can be obtains by a simple equation where $\mathrm{D}$ is the fixed distance of the two detectors and $\mathrm{T}$ is the time measured when passing through the carbon foil.

$\mathrm{V}=\mathrm{D} / \mathrm{T}$

With the energy (E) obtain by the last detector, mass (M) can be calculated.

$\mathrm{E}=1 / 2 \quad \mathrm{M} * \mathrm{~V}^{\wedge} 2$

Hydrogen ions beam is very limited when dealing with sensitivity of other materials. Therefore the second method is using the gold ions beams for sensitivity sensor of other materials. The target for this test can be any films that the user desired. However, the common target that was used in the lab is SiO2. Unlike hydrogen beam line, the gold ions beam is much heavier in which can knock out the particles of the target. When the gold ions beam coming in, it collided with SiO2 target and sputter Silicon and Oxygen particles toward the detectors instead of the gold ions themselves. The measurement of the Silicon and Oxygen is identical to the Hydrogen ions when passing through the detectors. However, one advantage of this method offer is to create a broader range of materials that can be test for their mass and energy. 
PNNL-16185

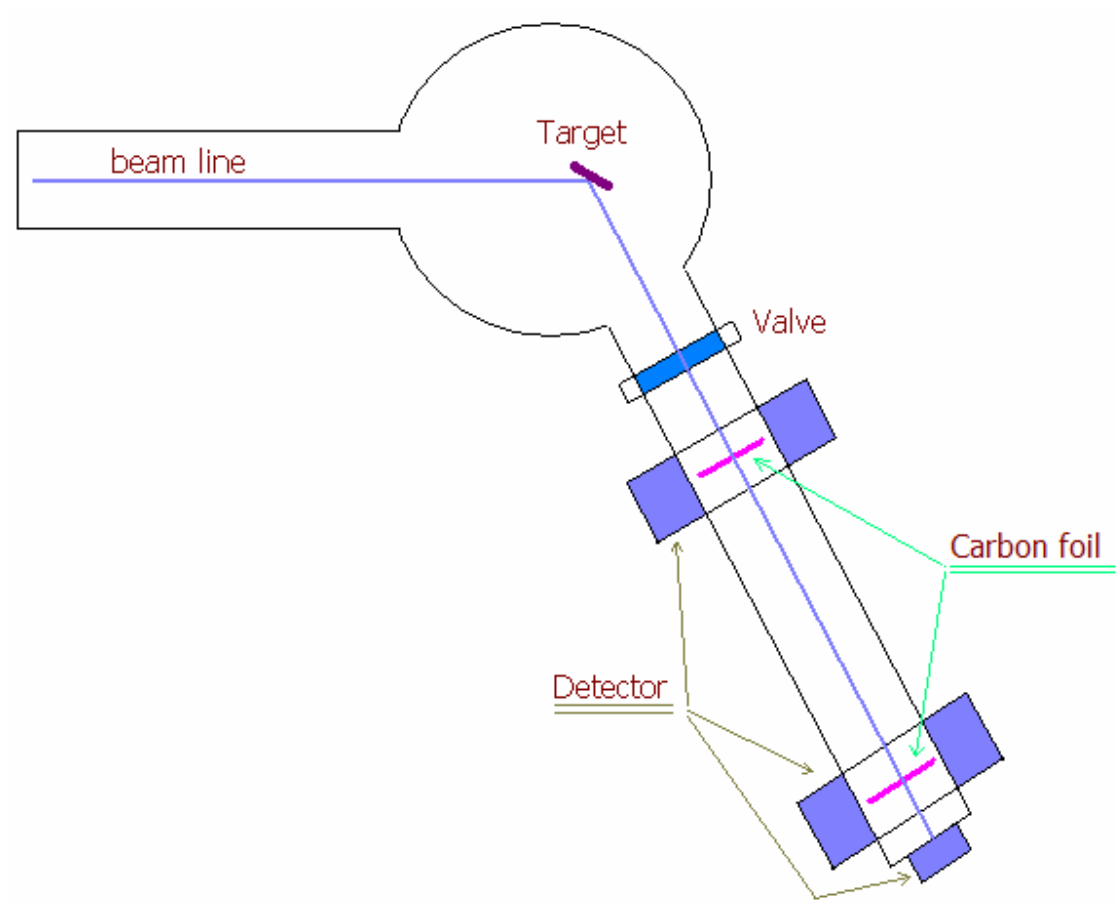

Fig 10: ERDA.

NRA (Nuclear Reaction Analysis) is an experiment that very similar to RBS. However, RBS is ideal for heavy elements and NRA is for light elements. When the ions beam collided with the sample, it will hit the nucleus and change its characteristic. During this interaction, the nucleus will emit gamma ray and particles. NRA also used to analyze crystal lattice structure and the damages of the interfaces between different materials. It's also known as channeling. In a crystal lattice structure, the atoms are formed in a specific orientation and created a hollow region between them. This hollow region is called a channel. When the ions beam hit the surface of the sample, it will collide with the first layer. If the crystal is perfectly perpendicular with the ions beam, only the first layer atoms will interact with the beam. The region when the beam hit the atoms will yield the scattering energy. However, when the beam hit the channel region, it will not collide with any atoms; therefore the scattering energy will be very small. By using this technique, scientist can determine the alignment between the channel and the ions beam. 

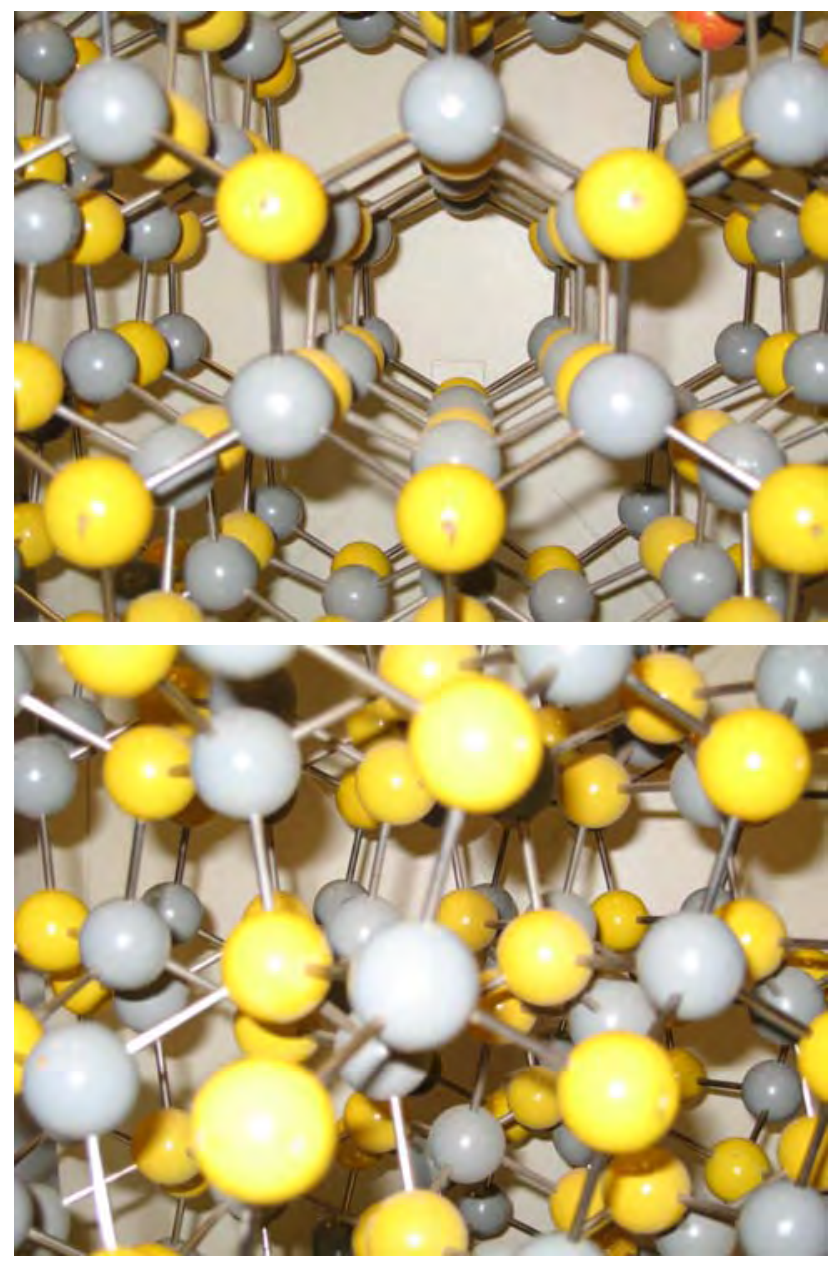

Fig 11: Crystal lattice structure. (a) Channel orientation, (b) Random orientation.

Before performing an alignment experiment in the crystal structure, a channel needs to be found. Finding a channel in the lattice requires multiple rotations of sample positions. Initially, place the sample as perpendicular to the beam as much as possible. The following step is to apply the ions beam to the sample. There are two main positions that need to be rotated. First position is along the X-axis. The sample will stay still at the middle region and only rotate at the end point along the X-axis. During this rotation, the ions beam will hit the surface of the sample and collect energy reading. A pattern needs to be recognized while recording the data. The energy will start high, then decreasing, and will increasing. This indicates that the middle region is the channel. To increase accuracy, second or a third run is recommended for the same axis. The second position is along the $\mathrm{Y}$-axis. This procedure will repeat until a channel is found. After obtained the coordinate of the $\mathrm{X}$ and $\mathrm{Y}$ axis, repeat the run for one more time to make sure the crystal lattice is align with the beam. There are several advantages by using NRA. First advantage of the channeling method is used to determine the lattice alignment between two films, the substrate and the film. When the channel is found, the beam will go 
PNNL-16185

through the channel and pass through each layers of the film. If the lattice structure of the substrate and the film are aligned, the scattering will yield very low and uniform energy. If not, the experiment proves that the film and the substrate crystals lattice structure are not aligned. Second advantage of this method is to see the defect of the interface layer. Even though the film and the substrate has their crystal lattice structure aligned, however the interface between them may distorted. This method provides a deeper understanding at the interface level between two surfaces. The third advantage is to test the uniformity of the film surface compare to the rest of the crystal lattice structure. The film itself and the substrate lattice structure maybe aligned; however, the surface can be distorted. The figure $12 \mathrm{~b}$ indicates, the beam will go through some region that the lattice align and collide with some region that the surface is distorted. With this method, scientist can learn about film quality on the surface level.
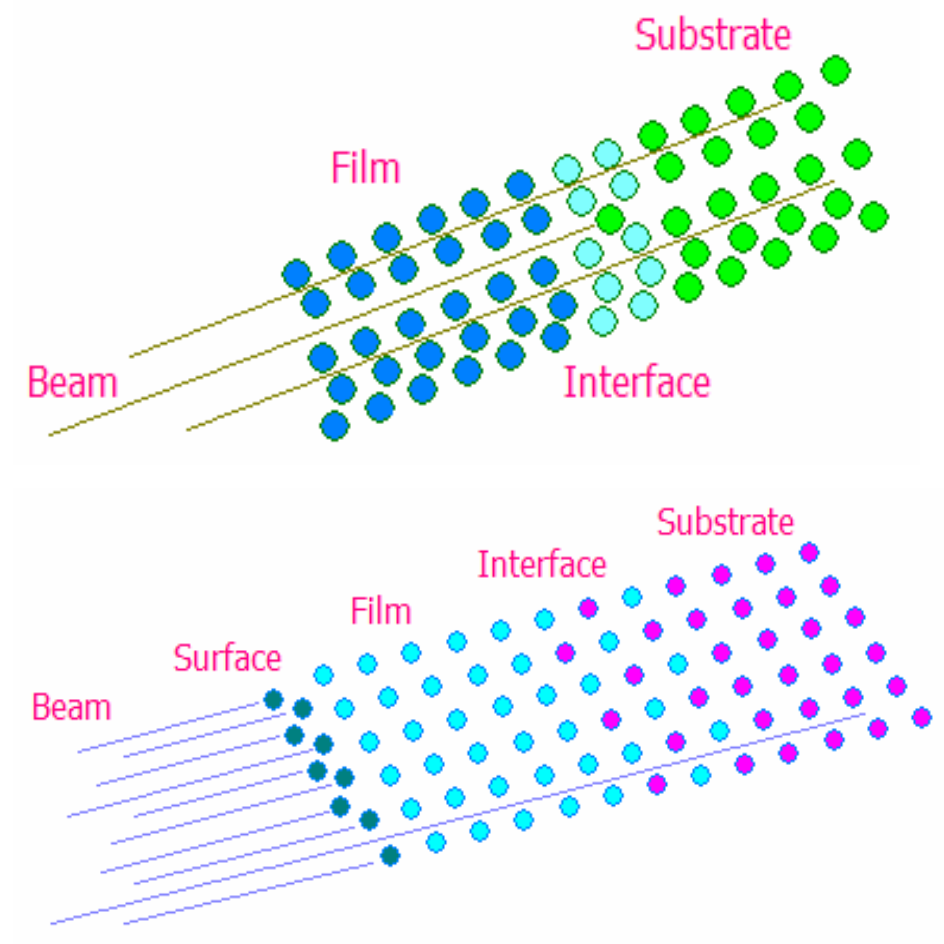

Fig 12: NRA (a) interface distorted, (b) surface distorted

\section{Acknowledgments.}

I would like to thank my host, Theva Thevuthasan, and the following scientists, Shuttha Shutthanandan and Yanwen Zhang, for their supports and guidance during my stay as a summer internship student at PNNL (Pacific Northwest National Laboratory). I would also like to thank Steve Barlow, Nikki Avery, Barbara Diehl, and Heather Bradshaw for their contribution to make this program possible. 


\title{
Thermal and Non-thermal Reactions at the Water/ $\mathrm{TiO}_{2}(110)$ Interface
}

\author{
Christopher Lane and Thomas Orlando \\ Department of Chemistry \& Biochemistry \\ Georgia Institute of Technology, \\ School of Chemistry and Biochemistry \\ Atlanta, GA \\ and \\ Dr. Greg Kimmel \\ Pacific Northwest National Laboratory \\ Richland, WA
}

Titanium dioxide is an environmentally abundant material that has become a lucrative industrial commodity for its uses and applications such as white pigments in paints and cosmetics, heterogenous catalyst, corrosion-protective coating, optical coating, and electric devices such as varistors. [1] Besides its applicability, $\mathrm{TiO}_{2}(110)$ is the most studied model transition metal oxide making it a standard surface to understand thermal and non-thermal reaction dynamics of water dissociation. Hydrogen production from a non-organic source is one route for manufacturing a fuel that is completely greenhouse gas emission free. Fujita et al. has demonstrated hydrogen production via the photocatalytic dissociation of water on the $\mathrm{TiO}_{2}$ surface. [2]

To understand the complete reaction landscape of the following reaction:

$2 \mathrm{H}_{2} \mathrm{O}(\mathrm{a}) \rightarrow 2 \mathrm{H}_{2}(\mathrm{~g})+\mathrm{O}_{2}$ (g)

where (a) is an adsorbed water species and (g) represents gas, we planned to study the direct interactions and non-thermal energy dissipation of water as well as hydrogen and oxygen on the $\mathrm{TiO}_{2}$ surface. Our experimental apparatus consists of a low-energy electron gun, a closedcycle helium cryostat, a molecular beamline for adsorbate deposition, an Auger electron spectrometer, and a quadrupole mass spectrometer (QMS) with an integrating cup. Typical base pressures for the system were $1 \times 10^{-10}$ Torr. The $1 \mathrm{~cm}$ diameter $\mathrm{TiO}_{2}(110)$ crystal was mounted in a resistively heated molybdenum base plate. K-type thermocouples attached to the $\mathrm{TiO}_{2}$ single crystal and to the molybdenum were used to monitor heating and cooling cycles in the range of $27-1050 \mathrm{~K}$. The amorphous solid water (ASW) ice films were deposited with the molecular beam $\left(\sim 2 \times 10^{14}\right.$ molecules $\left./ \mathrm{cm}^{2} \mathrm{~s}\right)$ at normal incidence to the $\mathrm{TiO}_{2}(110)$ surface at $100 \mathrm{~K}$. The molecular beam produced a film centered on the substrate having a diameter smaller than that of the substrate. The first monolayer (ML) coverage of water was defined as the desorption yield from the $\mathrm{Ti}^{+4}$ rows in the TPD spectra. During ESD experiments, the electron beam was oriented $35^{\circ}$ to the sample normal where the films were irradiated with variable energy $100 \mathrm{eV}$ incident electrons. The water films were uniformly irradiated by repeatedly scanning the electron beam in a grid pattern over an area slightly larger than the film. A typical instantaneous current density per scan is $\sim 2 \times 10^{15}$ electrons $/ \mathrm{cm}^{2} \mathrm{~s}$, and beam spot size is $\sim 1.5 \mathrm{~mm}$.

Prior to experiments, the $\mathrm{TiO}_{2}$ was annealed in vacuum. This process introduces intrinsic defects or oxygen vacancies into bulk crystal thus increasing the conductivity and greatly reducing charging effects during electron irradiation experiments. Before each experiment, the $\mathrm{TiO}_{2}(110)$ surface was cleaned by Ne ion sputtering for 3 min at $300 \mathrm{~K}$ then 
annealed for $10 \mathrm{~min}$ at $950 \mathrm{~K}$. From the integral of the high temperature recombinative TPD peak of $\mathrm{H}_{2} \mathrm{O}$, the surface has approximately $5 \%$ oxygen vacancies. [3,4]

As depicted in Fig. 1, the (110) surface is composed of corrugated rows of $\mathrm{O}^{-2}$ and $\mathrm{Ti}^{+4}$ in the [001] direction. Two types of oxygens exist on this surface, the threefold coordinated oxygen that resembles the bulk oxygen, and the twofold coordinated bridge bonding oxygen (BBO). When a BBO is thermally removed, an oxygen vacancy is created which is denoted by the white dashed circle in Fig. 1. Two electrons remain in this vacancy leaving two reduced $\mathrm{Ti}^{+3}$ ions. These defect sites drive much of the chemistry on this surface and are the subject of much debate in the literature. [1]

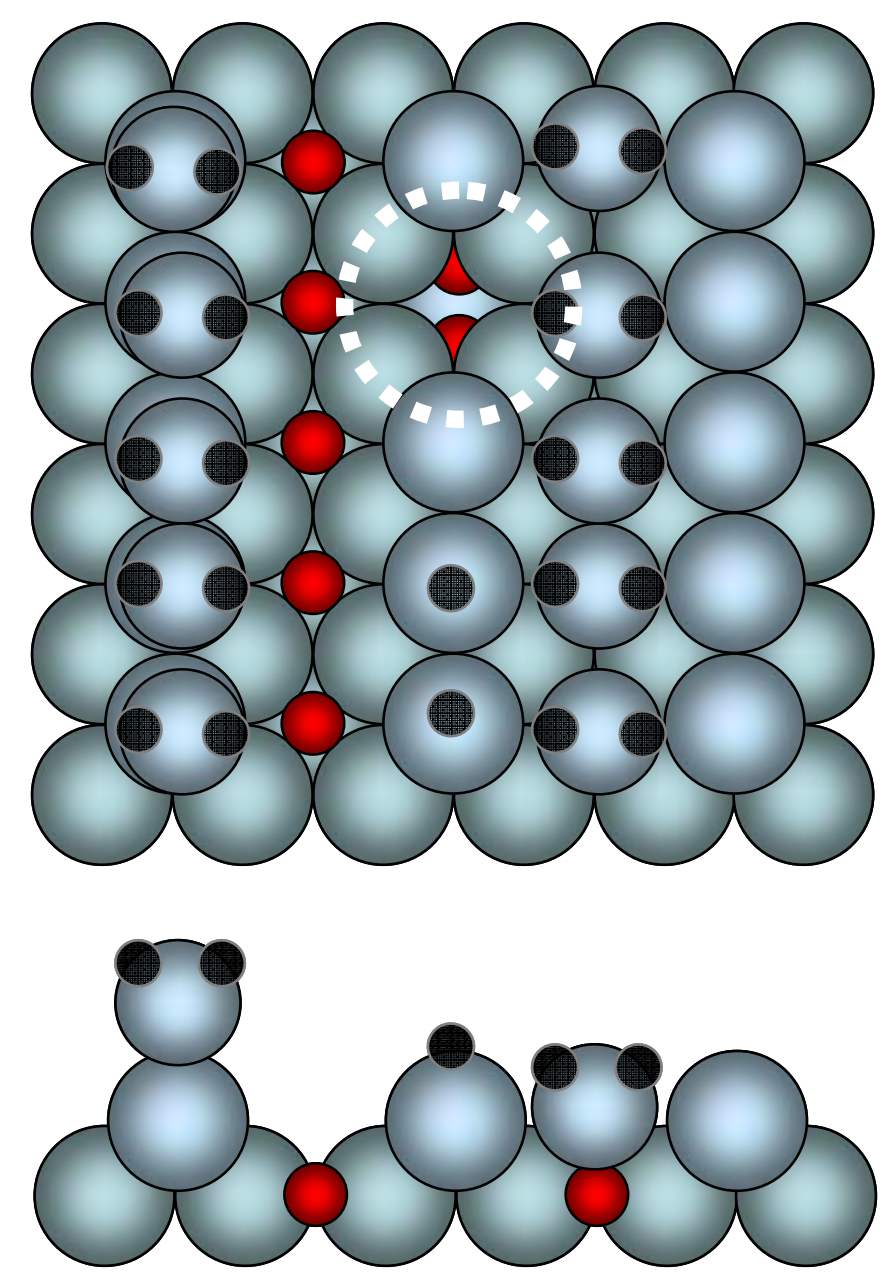

Figure 1. Ball model of the $\mathrm{TiO}_{2}(110)$ surface with adsorbed $\mathrm{H}_{2} \mathrm{O}$. Red balls represent titanium; Blue balls, oxygen; black balls, hydrogen. Water can adsorb on the bridge bonding oxygens (BBOs) and on the $\mathrm{Ti}^{+4}$ sites. An oxygen vacancy site is shown encircled by the white dashed line. When $\mathrm{H}_{2} \mathrm{O}$ reacts with an oxygen vacancy, two hydroxyls are produced side by side which is also depicted. [5]

We implemented temperature programmed desorption (TPD) and electron stimulated desorption (ESD) measurements to gain an understanding of how water interacts with the $\mathrm{TiO}_{2}(110)$ surface. Briefly, the TPD spectrum of water from the $\mathrm{TiO}_{2}$ (110) surface is composed of four peaks near $170 \mathrm{~K}, 200 \mathrm{~K}, 300 \mathrm{~K}$, and $500 \mathrm{~K}$ which have been attributed to the multilayer, water adsorbed on the bridge bonding oxygen, water in the $\mathrm{Ti}^{+4}$ rows, and water 
PNNL-16185

from the recombination of surface hydroxyls, respectively. [3] In our figures and discussion, 1 ML (monolayer) coverage of $\mathrm{H}_{2} \mathrm{O}$ refers to only the $\mathrm{Ti}^{+4}$ site saturation adsorption of $\mathrm{H}_{2} \mathrm{O}$ (5.2 $\mathrm{x} 10^{14}$ molecules $/ \mathrm{cm}^{2}$ ).

A summary of the observations and results for our first publication in preparation is given below. Our explanations for these results are still speculative. Other experiments not presented here are also in preparation for publication.

\section{Production of Oxygen Adatom Species Via Electron Irradiation of $\mathrm{H}_{2} \mathrm{O}$ on $\mathrm{TiO}_{2}(110)$}

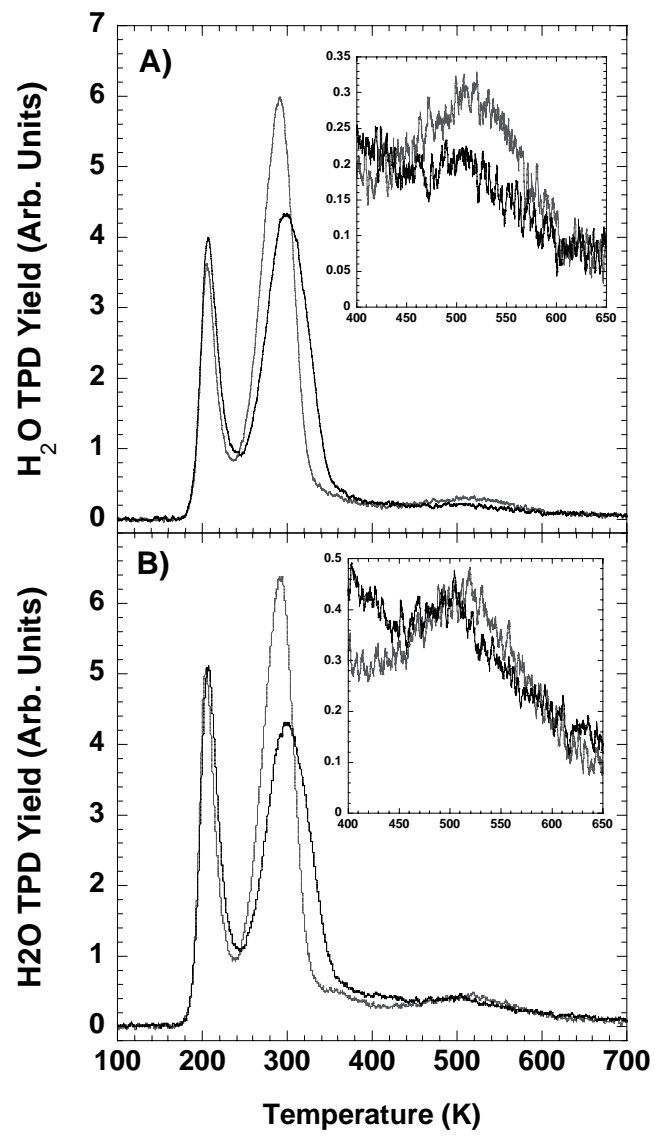

Figure 2. TPDs of $1.2 \mathrm{ML}$ coverage of $\mathrm{H}_{2} \mathrm{O}$ taken A) before (gray) and after (black) 700 Torr*s predose of $\mathrm{O}_{2}$ at $90 \mathrm{~K}$ and $\mathrm{B}$ ) before (gray) and after (black) $\mathrm{N}=500$ rasters of $100 \mathrm{eV}$ electrons of 1.2. The insets are enlargements of $500 \mathrm{~K}$ recombinative $\mathrm{H}_{2} \mathrm{O}$ TPD peak.

The $\mathrm{H}_{2} \mathrm{O}$ TPD spectra in both A) and B) frames of Fig. 2 depict the thermal desorption of 1.2 ML coverage of $\mathrm{H}_{2} \mathrm{O}$. In A), we reproduced an experiment done by Henderson et al. [6] where a new surface state of $\mathrm{H}_{2} \mathrm{O}$ was observed after predosing the surface with molecular oxygen. The gray TPD $\mathrm{H}_{2} \mathrm{O}$ spectrum in A) was taken from an annealed (110) surface having $\sim 5 \%$ vacancies, while the black TPD $\mathrm{H}_{2} \mathrm{O}$ spectrum was taken from a surface predosed with 700 Torr*s of $\mathrm{O}_{2}$. The new $\mathrm{H}_{2} \mathrm{O}$ surface state seen as a broadening and shift to higher temperature of the water desorbing from the $\mathrm{Ti}^{+4}$ rows appears in the $\mathrm{O}_{2}$ predosed $\mathrm{H}_{2} \mathrm{O}$ TPD. Henderson et al. attributed this to molecular oxygen reacting with the oxygen vacancy sites, thereby depositing an oxygen into the vacancy and an oxygen in the $\mathrm{Ti}^{+4}$ row. The oxygen adatom in the row further reacts with the adsorbed water producing either two hydroxyls 
directly or a precursor that leads to two hydroxyl groups in the $\mathrm{Ti}^{+4}$ row. [6] This mechanism explains the shift in the water TPD from the $\mathrm{Ti}^{+4}$ rows and the oxidation of the vacancy sites seen as a decrease in the high temperature recombinative peak show in the inset of A).

We have observed a similar water TPD state by simply irradiating the water film with $100 \mathrm{eV}$ electrons which is depicted in $\mathrm{B}$ ). The shift in the water TPD peak produced by electrons is experimentally similar to that produced by molecular oxygen. They are both dependent on the initial oxygen vacancy concentration and have similar annealing temperatures to remove the oxygen adatoms that affects the water TPD (data not shown). For our irradiation experiment, the shift in the water TPD is maximized when water saturates the $\mathrm{Ti}^{+4}$ row adsorption sites. Since our results are similar to Henderson's, we seem to be producing oxygen adatoms or a precursor leading to oxygen adatoms via electron irradiation.

Although water reacts with an oxygen vacancy site, the $\mathrm{Ti}^{+3}$ ions are not fully oxidized, and an electron remains delocalized beneath the hydroxyl groups. [7,8] This configuration is still a surface defect having an electron trap state approximately $1 \mathrm{eV}$ below the conduction band edge. $[9,10]$ When electron-hole pairs are created during irradiation whether from an excitation from the valence band to the conduction band in $\mathrm{TiO}_{2}$ or the $1 b_{1}$ level of water to the conduction band of $\mathrm{TiO}_{2}$, this hydroxyl defect configuration or electron trap site is conducive to electron-hole recombination. This explains the initial oxygen vacancy concentration dependence of the water TPD shift due to irradiation. When the electron and hole recombine, energy is distributed locally inducing dissociation and desorption of the neighboring water in the $\mathrm{Ti}^{+4}$ rows. An oxygen adatom or precursor to an oxygen adatom is a resultant from the dissociated water.

In conclusion we have reproduced similar results with irradiation of a water film on $\mathrm{TiO}_{2}$ (110) that Henderson et al. have observed with molecular oxygen predosing prior to dosing water on the $\mathrm{TiO}_{2}$ (110). Instead of non-thermally dissociating water to produce an oxygen adatom species on all the surface sites, we found that this event is dependent on the initial oxygen vacancy concentration, and occurs specifically at these sites. We agree with the literature that these sites are electron traps even after they react with water, and a source of chemical reactions due to their increased probability of electron-hole recombination.

\section{References}

[1] U. Diebold, Surf. Sci. Rep. 48 (2003) 53.

[2] A. Fujishima and K. Honda, Nature 238 (1972) 37.

[3] M.B. Hugenschmidt, L. Gamble, and C.T. Campbell, Surf. Sci. 302 (1994) 329.

[4] M. A. Henderson, Surf. Sci. 355 (1996) 151.

[5] S. Wendt, J. Matthiesen, R. Schaub, E.K. Vestergaard, E. Laegsgaard, F. Besenbacher, and B. Hammer, Phys. Rev. Lett. 96 (2006) 066107/1.

[6] M. A. Henderson, W.S. Epling, C.L. Perkins, C.H.F. Peden, and U. Diebold, J. Phys. Chem. B 103 (1999) 5328.

[7] C.D. Valentin and G. Pacchioni, (submitted) (2006).

[8] J. Zhao, B. Li, K.D. Jordan, J. Yang, and H. Petek, Phys. Rev. B 73 (2006) 195309.

[9] M. A. Henderson, W.S. Epling, C.H.F. Peden, and C.L. Perkins, J. Phys. Chem. B 107 (2003) 534.

[10] A.K. See and R.A. Bartynski, J. Vac. Sci. Technol., A 10 (1992) 2591. 
PNNL-16185

\title{
First-Principles Calculations of $\mathrm{La}_{(1-\mathrm{x})} \mathrm{Sr}_{(\mathrm{x})} \mathrm{Co}_{(\mathrm{y})} \mathrm{Fe}_{(1-\mathrm{y})} \mathrm{O}_{3}$ (LSCF) Solids and Surfaces
}

\author{
Chan-Woo Lee, Susan B. Sinnott, and Eric D. Wachsman \\ Department of Materials Science and Engineering \\ University of Florida \\ Gainesville, Florida 32611-6400 \\ and \\ Ram Devanathan \\ Pacific Northwest National Laboratory \\ Richland, WA
}

\section{Introduction}

As materials for electrolytes become more conductive and/or are manufactured as thin films, electrode polarization and heterogeneous kinetics at the electrode surface dominate its overall performance. In order to improve our understanding of heterogeneous electrocatalytic phenomena at the surface of ion conducting ceramics, it is essential to investigate the role of defects and charge transfer at these surfaces. The goal of the present work is to understand heterogeneous electrocatalytic phenomena at the surface of LSFC. As LSFC is single-phase mixed conductor [1-4], it conducts using both ionic and electronic conduction mechanisms. Thus, LSFC has a two phase boundary (2PB) plane where the electrolyte and cathode are contacted [4], as illustrated in Fig. 1a. In contrast, ordinary electronic conducting SOFC cathodes have a three-phase boundary (3PB) circumference where the cathode, electrolyte and gas meet [4], as shown in Fig. 1 b.
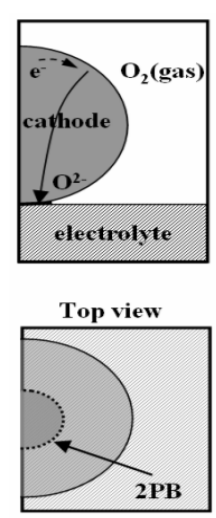

(a)
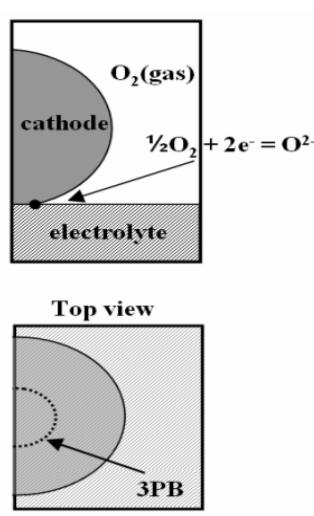

(b)

Fig.1 Schematic representation of (a) a two-phase boundary (2PB) plane and (b) a three-phase boundary (3PB) circumference. After figures 2 and 3 in Ref. 4. 
PNNL-16185

This 2PB increases the efficiency of ionic conduction of LSFC. In particular, it is expected that oxygen molecules readily dissociate to $\mathrm{O}^{2-}$ over a significant portion of the LSFC cathode surface. The oxygen ion then moves into the lattice and diffuses into the electrolyte by passing the 2PB area, as shown in Fig.1a. Thus, understanding the dissociation of $\mathrm{O}_{2}$ at the surface and the transport of $\mathrm{O}^{2-}$ through bulk LSFC, and documenting how these processes change depending on composition and surface structure is important. As a first step of the project, we start with the $\mathrm{LaFeO}_{3}$, system which is the basis material for LSCF. The system was optimized using the density functional theory (DFT) with consideration of magnetism. After that, the energetics of $\mathrm{LaO}, \mathrm{FeO}_{2}$ and $\mathrm{FeO}$ terminations of (001) plane were compared. As expected, $\mathrm{LaO}$ termination with fewer dangling bonds was relatively stable.

\section{Computational details}

The total energies of bulk and surface systems of $\mathrm{LaFeO}_{3}$ were calculated with the generalized gradient approximation (GGA) with the Perdew-Burke-Ernzerhof (PBE) functional [5] to DFT. Projected-augmented wave (PAW) [6,7] potentials which are implemented in the Vienna ab-initio simulation package (VASP) were used to describe each element in the system. A cutoff energy of 600 $\mathrm{eV}$ and $4 \times 4 \times 4 \mathrm{~K}$-points within Monkhorst-Pack scheme [8] were chosen for a bulk unit cell system containing $4 \mathrm{La}, 4 \mathrm{Fe}$, and $12 \mathrm{O}$ atoms. For La, Fe, and $\mathrm{O}$ bulk systems, K-points of $18 \times 18 \times 18$, $16 \times 16 \times 16$, and $8 \times 8 \times 8$ were chosen. In optimizing bulk and surface systems, magnetism was taken into account to have accurate total energies and geometries. Surface calculations of ceramic oxides usually need enough layers to describe the effect of long range reaction of ionic bonding. However, here, 5 layers were chosen for test calculations for different surface configurations. For surface calculations, a vacuum slab thickness of $10 \AA$ was chosen to describe a pseudo-vacuum condition and cutoff energy=600 eV and $K$-points=4×4×1 were used. All parameters including K-points, cutoff energy, and vacuum slab thickness were determined after extensive convergence tests.

\section{Results and discussion}

Table 1 shows calculated lattice constants and magnetic moment for $\mathrm{LaFeO}_{3}$ with paramagnetic and ferromagnetic conditions with the setting of cutoff energy $=600 \mathrm{eV}$ and $K$-point $=4 \times 4 \times 4$. Here, lattice constants calculated with the consideration of ferromagnetism showed better accuracy than those with non-magnetic calculations. Also, G-type antiferromagnetism was perfectly described [9]. Cell shape and volume changes in ferromagnetic calculations were due to magneto volume effect of Fe atoms and this effect was also observed in structure optimization of Fe bulk system. 
PNNL-16185

Table 1 Lattice constants and magnetic moment of DFT calculations of $\mathrm{LaFeO}_{3}$ bulk system compared with experimental data

\begin{tabular}{|c|c|c|c|}
\hline & Ferromagntic & Paramagnetic & Exp. [10] \\
\hline $\mathbf{a}(\AA)$ & $5.6856027 \mathbf{( 2 . 0 7 5 5 \% )}$ & $5.378 \mathbf{( - 3 . 4 4 7 0 \% )}$ & 5.570 \\
\hline $\mathbf{b}(\AA)$ & $7.8598143 \mathbf{( - 0 . 0 1 5 1 \% )}$ & $7.565(\mathbf{- 3 . 7 6 5 4 \% )}$ & 7.861 \\
\hline $\mathbf{c}(\AA)$ & $5.5672217 \mathbf{( 0 . 2 0 2 0} \%)$ & $5.433 \mathbf{( - 2 . 2 1 3 8 \% )}$ & 5.60 \\
\hline Moment $\left(\mu_{\mathrm{B}}\right)$ & $3.769 \mathbf{( - 1 8 . 0 7 \% )}$ &. & 4.6 \\
\hline
\end{tabular}

With this bulk unit-cell, among possible low miller index planes, $\{010\},\{110\},\{111\},\{011\}$ and $\{101\}$ [11], surface systems were developed by (010) plane with $\mathrm{LaO}, \mathrm{FeO}$, and $\mathrm{FeO}_{2}$ terminations.
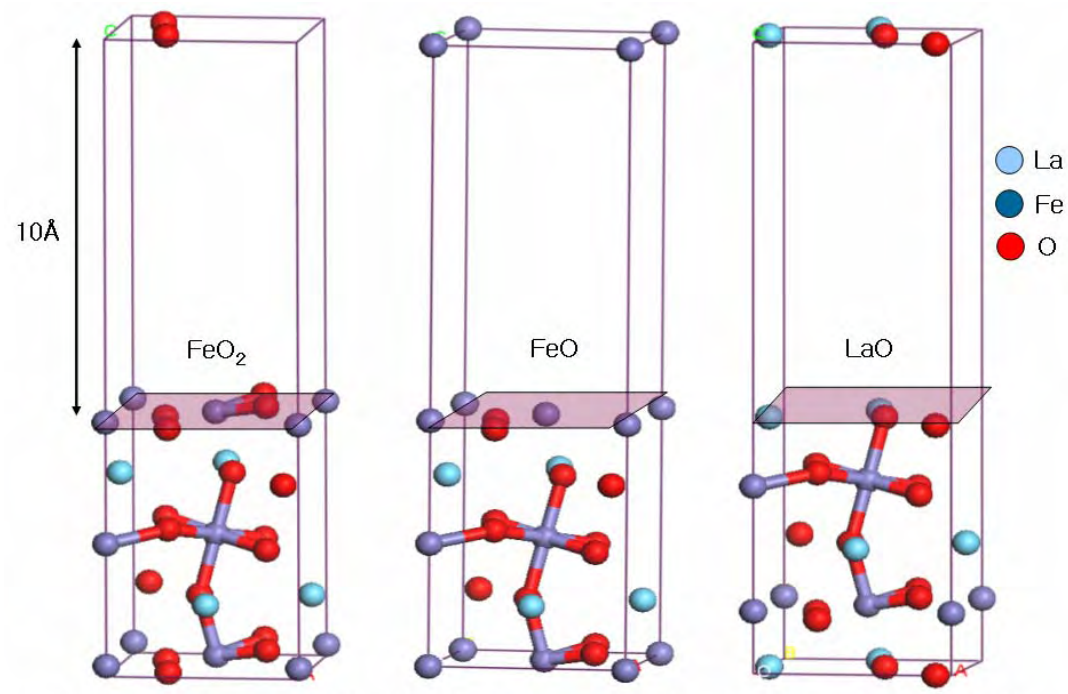

$\mathrm{La}=4, \mathrm{Fe}=6$, and $\mathrm{O}=16 \quad \mathrm{La}=4, \mathrm{Fe}=6$, and $\mathrm{O}=12 \quad \mathrm{La}=6, \mathrm{Fe}=4$, and $\mathrm{O}=14$

Surface area $=31.65301056 \AA^{2}$

Fig. 2 Schematic description of $\mathrm{LaO}, \mathrm{FeO}_{2}$, and $\mathrm{FeO}$ terminations of $\mathrm{LaFeO}_{3}(010)$ plane

As all surface systems in $\mathrm{LaFeO}_{3}$ are non-stochiometric, we can not compare bulk total energy and surface free energy directly. Hence, by using equation 1 formulated by Hagen et al.[12], we can compare relative stability of different configurations shown in Fig. 2.

$$
\sigma=\frac{1}{A_{s}}\left(G_{s}-\sum_{i} \mu_{i} N_{i}\right) \text { Eq.1 }
$$

Here, $G_{s}$ represents the total free energy of the supercell, $A_{s}$ is surface area of the supercell, index $i$ denotes the elements within the supercell: La, Fe, and O. $\mu_{i}$ denotes chemical potential of $i^{\text {th }}$ element and $N_{i}$ is the number of atoms of each element within the supercell. 
PNNL-16185

Table 2 Calculated surface free energies of $\mathrm{LaO}, \mathrm{FeO}$, and $\mathrm{FeO}_{2}$ terminations of $\mathrm{LaFeO}_{3}(010)$ plane

\begin{tabular}{|c|c|c|c|}
\hline & $\mathbf{L a O}$ & $\mathbf{F e O}$ & $\mathbf{F e O}_{2}$ \\
\hline $\begin{array}{c}\text { Surface free energy } \\
\left(\mathrm{eV} / \AA^{2}\right)\end{array}$ & -3.9207 & -2.7501 & -3.2150 \\
\hline
\end{tabular}

It is evident that the $\mathrm{LaO}$ termination is the most stable and $\mathrm{FeO}$ termination shows the highest instability. This can be explained by the possibility of having dangling bonds. For $\mathrm{LaO}_{2}$ termination, we have only one dangling bond. However, (010) plane terminated by $\mathrm{FeO}$ has three dangling bonds.

\section{Results and discussion}

Using DFT, $\mathrm{LaFeO}_{3}$ system was optimized by considering magnetic properties. Structural properties were predicted more accurately by this consideration and antiferromagnetism of $\mathrm{LaFeO}_{3}$ system was perfectly described. With this optimized system, surface free energies were calculated for (010) planes with different terminations: $\mathrm{LaO}, \mathrm{FeO}$, and $\mathrm{FeO}_{2}$. $\mathrm{LaO}$ terminated (010) plane has the lowest surface free energy which means the highest stability. This can be explained based on the number of dangling bonds.

\section{References}

1. J. Mizusaki, T. Sasamoto, W.R. Cannon, H.K. Bowen, J. Am. Ceram. Soc. 66 (1983) 247

2. J.E. ten Elshof, H.J.M. Bouwmeester, H. Verveij, Solid State Ion. 81 (1995) 97

3. J.E. ten Elshof, H.J.M. Bouwmeester, H. Verveij, Solid State Ion. 89 (1996) 81

4. S. B. Adler, Chem. Rev. 104 (2004) 4791

5. J. P. Perdew, K. Burke, and M. Ernzerhof, Phys. Rev. Lett. 77 (1996) 3865

6. G. Kresse and J. Furthmuller, Comp. Mater. Sci. 6 (1996) 15

7. G. Kresse and J. Furthmuller, Phys. Rev. B 54 (1996) 11169

8. H.J. Monkhorst, J.D. Pack, Phys. Rev. B 13 (1976) 5188

9. K. Ueda, H. Tabata, T. Kawai, Phys. Rev. Lett. 60 (1999) 561

10. I. Wærnhus, P.E. Vullum, R. Holmestad, T. Grande, K. Wiik, Solid State Ionics 176 (2005) 2783

11. M. Alfredsson, J.P. Brodholt, D.P. Dobson, A.R. Oganov, C.R.A. Catlow, S.C. Parker, G.D. Price, Phys. Chem. Minerals 31 (2005) 671

12. M. Hagen, M. W. Finnis, Philosophical Magazine A 77 (1998) 447 
PNNL-16185

\title{
Summary of research in the field of PVDF Polymers and Nanocomposites
}

\author{
Jiangyu Li \\ University of Washington \\ and \\ Leo Fifield \\ Pacific Northwest National Laboratory
}

Jiangyu Li visited PNNL twice in June and July, hosted by Leo Fifield. Each time he stayed one week there.

During Li's visit, Fifield and Li had extensive discussion on potential research collaboration in active polymers. The discussion has led to a joint white paper on PVDF polymers, submitted to DOE BES Materials Science program, which was invited to submit a full proposal. The joint proposal, entitled "Fundamental Studies on Phase Transition Characteristics and Electromechanical Behavior of Disordered Poly(vinylidene fluoride) Polymers Using Multiscale Modeling and In-situ Characterization,” was submitted to DOE BES in September.

In addition, research collaboration on conductive nanocomposites has been discussed, and a joint program involving Boeing, University of Washington, Tsinghua University, and Battel/PNNL is likely to be launched.

During Li's visit, preliminary studies on PVDF polymers have also been carried out. PVDF films have been spin coated, and IR spectrum has been measured. 
PNNL-16185 


\title{
A Photoelectron Spectroscopy and ab initio Computational Study of Boron Oxide Anions $\mathrm{BO}^{-}$and $\mathrm{BO}_{2}^{-}$
}

\author{
Si-Dian Li \\ Department of Chemistry \\ Xinzhou Teachers' University \\ Shanxi, China \\ and \\ Hua-Jin Zhai, Lei-Ming Wang, and Lai-Sheng Wang \\ Pacific Northwest National Laboratory \\ Richland, WA
}

\begin{abstract}
Combining photoelectron spectroscopy and $a b$ initio calculations, we have revisited the ${ }^{2} \Sigma^{+}$ground-state of $\mathrm{BO}$ neutral, determined the excitation energy from the ground - state ${ }^{2} \Sigma^{+} \mathrm{BO}$ to the first excited state of ${ }^{2} \prod \mathrm{BO}$ to be $2.96 \mathrm{eV}$, and obtained the electron affinity of $\mathrm{BO}_{2}$ at $4.49 \mathrm{eV}$ in this work. The first and second excited states of $\mathrm{BO}_{2}$ molecule are found to lie $2.23 \mathrm{eV}$ and $3.01 \mathrm{eV}$ above the ground-state. Our results indicate that density functional approach provides satisfactory predictions for the bond parameters, vibrational frequencies, and the vertical one-electron detachment energies of both boron monoxide and boron dioxide.
\end{abstract}

We performed a systematic combined PES and DFT investigation on boron oxides $\mathrm{B}_{\mathrm{x}} \mathrm{O}_{\mathrm{y}}$ $(x=1-10$ and $y=1-3)$ during the three months in Summer Research Institute. A brief summary on the smallest species of $\mathrm{BO}^{-}$and $\mathrm{BO}_{2}{ }^{-}$will be reported here.

Both boron monoxide $\mathrm{BO}$ and boron dioxide $\mathrm{BO}_{2}$ belong to the most important boron oxide clusters in gas phases. As the smallest boron oxide species, $\mathrm{BO}$ neutral and $\mathrm{BO}^{-}$anion have attracted much attention in both experiments and theory. ${ }^{1-8} \mathrm{BO}_{2}$ forms a typical linear Renner-Teller molecule ${ }^{9-11}$ with the bond length of $\mathrm{r}_{\mathrm{B}=\mathrm{O}}=1.2653 \AA$ and an estimated EA value of $4.288 \mathrm{eV}$ from equilibrium data. However, there has been no PES spectra reported for $\mathrm{BO}_{2}{ }^{-}$ anion to date.

\section{Experimental and computational procedures}

PES measurements. Cluster syntheses and PES measurements are carried out on a magnetic-bottle-type PES apparatus equipped with a laser vaporization supersonic cluster source which has been detailed elsewhere. $\mathrm{BO}^{-}$and $\mathrm{BO}_{2}^{-}$monoanions were produced by laser vaporization of high purity ${ }^{10} \mathrm{~B}$ target in the atmosphere of helium carrier gas seeded with 0.01 or $0.5 \%$ of ${ }^{16} \mathrm{O}_{2}$, mass-selected with a time-of-flight mass spectrometer, and finally photodetached at the laser energies of 355nm (3.493 eV), $266 \mathrm{~nm}(4.661 \mathrm{eV}), 193 \mathrm{~nm}(6.424 \mathrm{eV})$, or $157 \mathrm{~nm}(7.898 \mathrm{eV})$, depending on the intensities of the mass spectra and PES signals. The PES spectra of $\mathrm{BO}^{-}$and $\mathrm{BO}_{2}^{-}$at different detachment energies are presented in Fig.1 and Fig.2, respectively.

Density functional theory computations. DFT structural optimizations and frequency analyses were performed using the hybrid B3LYP procedure with the augmented Dunning's all-electron basis (aug-cc-pVTZ) implemented in Gaussian 03. Excited-state energies of the 
PNNL-16185

neutrals were calculated with time-dependent DFT (TDDFT) method at the ground-state structures of the anions. Both $\mathrm{BO}_{2}$ and $\mathrm{BO}_{2}{ }^{-}$strongly favor $\mathrm{D}_{\infty \mathrm{h}}$ linear structures over other bent geometries, similar to the well-known $\mathrm{CO}_{2}{ }^{+}$and $\mathrm{CO}_{2}$. Table 1 compares the measured and calculated one-electron vertical detachment energies of the two anions. Coupled cluster calculations $(\operatorname{CCSD}(\mathrm{T}))$ were performed to calculate the adiabatic detachment energies (ADEs) and vertical detachment energies (VDEs) of these small clusters at DFT structures. The valence orbital pictures of both closed-shell $\mathrm{BO}^{-}$and $\mathrm{BO}_{2}{ }^{-}$are shown in Fig.3 to facilitate spectrum assignments.

Franck-Condon factor simulations. The vibrational progressions of the well-resolved $\mathrm{X}$ ${ }^{2} \Sigma^{+}$BO band in Fig.1 (a) and $\mathrm{A}^{2} \Pi$ BO band in Fig.1 (c) have been modeled employing the Franck-Condon fitting procedure. The simulated results are compared with measured PES spectra in Fig. 4.

\section{Features of the PES spectra}

The $\mathrm{X}^{2} \sum^{+} \mathrm{BO}$ band in Fig. 1(a), which originates from the electron transition from ${ }^{1} \Sigma^{+}$ $\mathrm{BO}^{-}$to ${ }^{2} \Sigma^{+} \mathrm{BO}$, gives the one-electron detachment energies of $\mathrm{ADE}=2.505 \mathrm{eV}$ and $\mathrm{VDE}=2.505 \mathrm{eV}$ for ${ }^{10} \mathrm{BO}$. This defines an $\mathrm{EA}$ value of $2.505 \mathrm{eV}$ for ${ }^{10} \mathrm{BO}$ neutral, a value in nice agreement with the reported value of $\mathrm{EA}=2.508 \pm 0.008 \mathrm{eV}$ for ${ }^{11} \mathrm{BO}$. X band also clearly features with vibrational characteristics, with one hot band at $2.296 \mathrm{eV}$ and two vibrational peaks at $2.745 \mathrm{eV}$ and $2.986 \mathrm{eV}$, respectively,

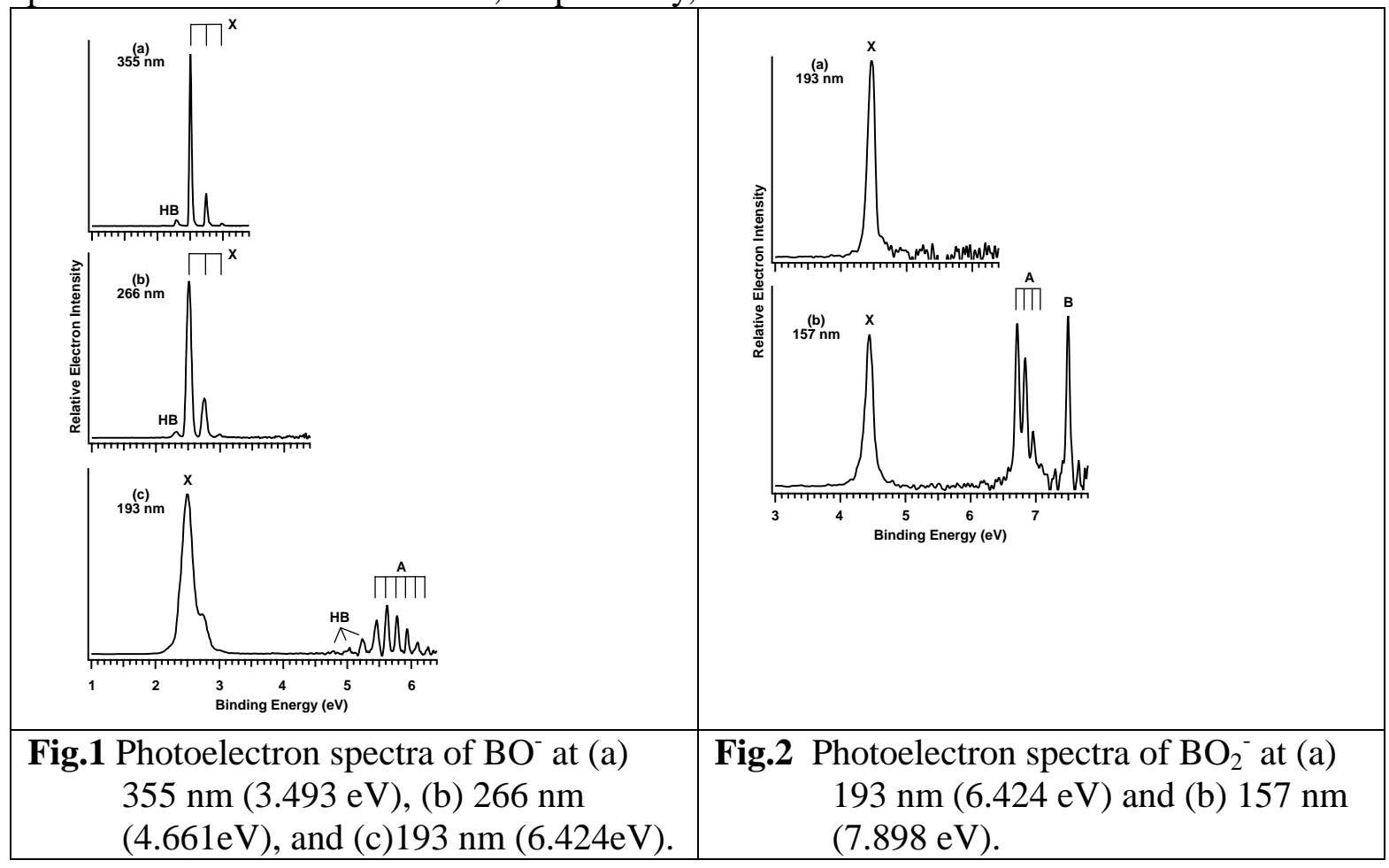


PNNL-16185

(a)
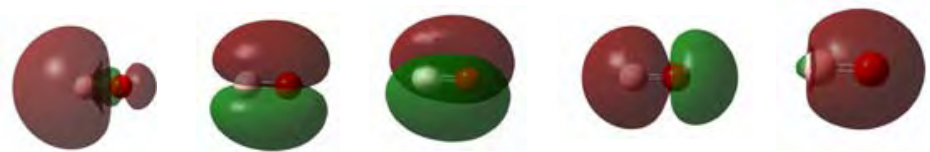

$\mathrm{HOMO}$

HOMO-1

HOMO-2

HOMO-3

(b)
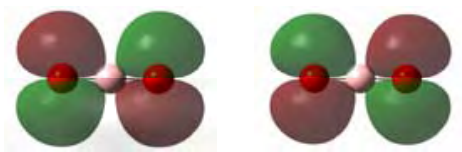

$\mathrm{HOMO}$
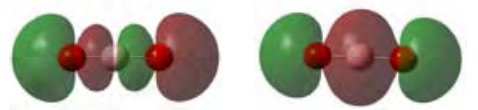

HOMO-3
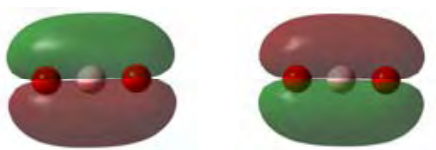

HOMO-1
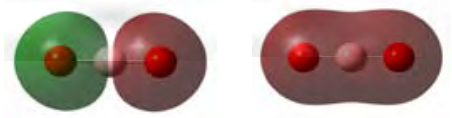

HOMO-4

HOMO-5

Fig. 3 Molecular pictures of $\mathrm{BO}^{-}$(a) and $\mathrm{BO}_{2}{ }^{-}$(b) at B3LYP level.

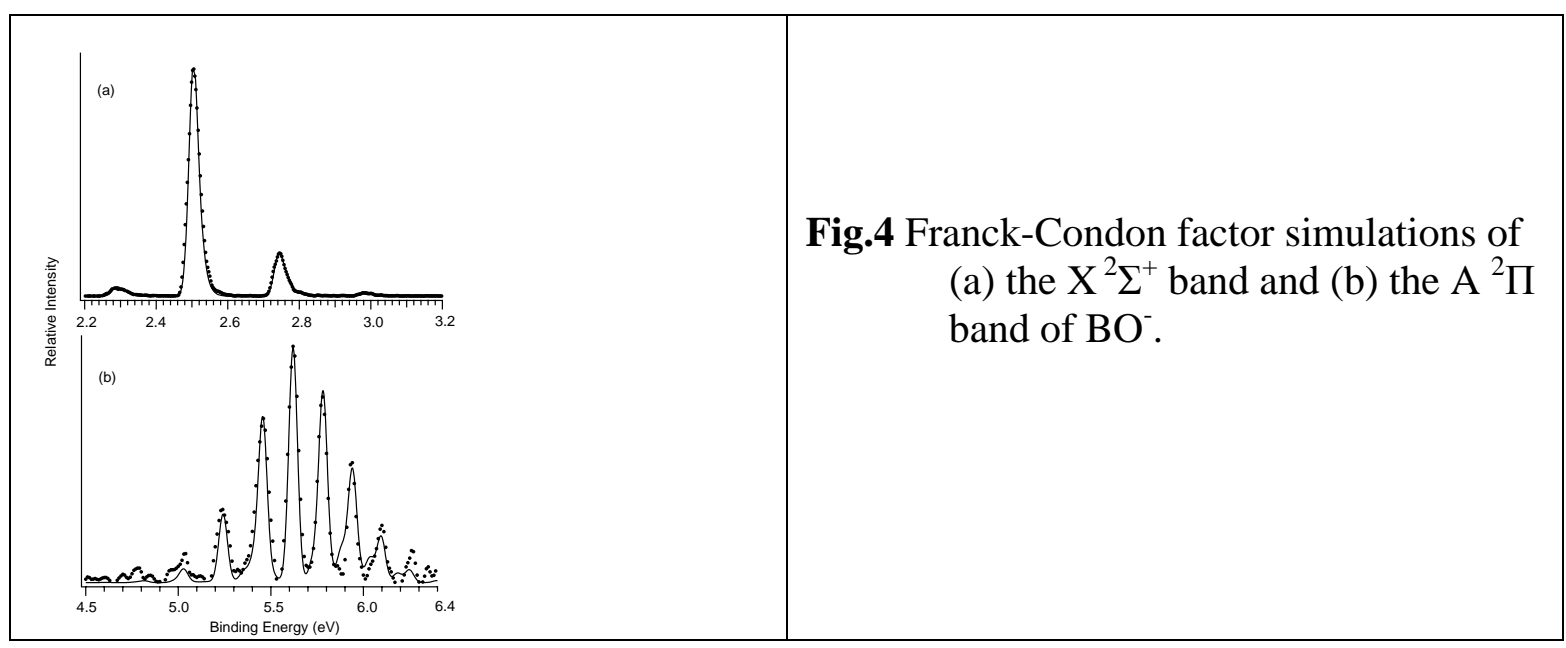

indicating a vibrational frequency of $\sim 1927 \mathrm{~cm}^{-1}$ for the ground-state of ${ }^{2} \sum^{+10} \mathrm{BO}$ neutral and a vibrational frequency of $\sim 1735 \mathrm{~cm}^{-1}$ for the ground-state of ${ }^{1} \Sigma^{+10} \mathrm{BO}^{-}$anion.

More interestingly, the second band $\mathrm{A}^{2} \Pi \mathrm{BO}$, which corresponds to a electron transition from ${ }^{1} \Sigma^{+} \mathrm{BO}^{-}$to $\mathrm{A}^{2} \Pi \mathrm{BO}$, exhibits a well-resolved vibrational progression with eight peaks (see Fig.1(c)), with two hot bands at $5.02 \mathrm{eV}$ and $5.24 \mathrm{eV}, \mathrm{ADE}=5.46 \mathrm{eV}, \mathrm{VDE}=5.62 \mathrm{eV}$, and four vibrational peaks at $5.78 \mathrm{eV}, 5.94 \mathrm{eV}, 6.10 \mathrm{eV}$, and $6.26 \mathrm{eV}$, respectively. This vibrational progression leads to a vibrational frequency of $\sim 1340 \mathrm{~cm}^{-1}$ for the first excited state of ${ }^{2} \Pi{ }^{10} \mathrm{BO}$ neutral and a vibrartional frequency of $\sim 1735 \mathrm{~cm}^{-1}$ for the ground-state of ${ }^{1} \Sigma^{+10} \mathrm{BO}^{-}$anion.

$\mathrm{BO}_{2}{ }^{-}$anion possesses a single feature in its $\mathrm{X}^{2} \Pi_{\mathrm{g}} \mathrm{BO}_{2}$ band with $\mathrm{ADE}=\mathrm{VDE}=4.49 \mathrm{eV}$, indicating an EA value of $4.49 \mathrm{eV}$ for $\mathrm{BO}_{2}$. At higher detachment energy of $157 \mathrm{~nm}$, both its $\mathrm{A}$ ${ }^{2} \Pi_{\mathrm{u}} \mathrm{BO}_{2}$ at $6.72 \mathrm{eV}$ and $\mathrm{B}^{2} \Sigma_{\mathrm{u}}{ }^{+} \mathrm{BO}_{2}$ at $7.50 \mathrm{eV}$ bands appeared to have high PES intensities (see Fig.2 (b)). 
Table 1 Experimental and calculated vertical detachment energies (eV) of $\mathrm{BO}^{-}$and $\mathrm{BO}_{2}^{-}$ anions

\begin{tabular}{|c|c|c|c|c|c|}
\hline $\begin{array}{c}\text { PES } \\
\text { Features }\end{array}$ & $\begin{array}{l}\text { VDE } \\
\text { (Exp.) }\end{array}$ & $\begin{array}{c}\text { Molecular } \\
\text { Orbital }\end{array}$ & Final State & $\begin{array}{c}\text { VDE } \\
\text { (TD- } \\
\text { B3LYP) }\end{array}$ & $\begin{array}{c}\mathrm{VDE} \\
(\mathrm{CCSD}(\mathrm{T}))\end{array}$ \\
\hline \multicolumn{6}{|c|}{$\mathrm{BO}^{-}$} \\
\hline $\begin{array}{l}\mathrm{X} \\
\mathrm{A}\end{array}$ & $\begin{array}{c}2.505 \\
5.62\end{array}$ & $\begin{array}{c}\mathrm{HOMO}\left(1 \sigma^{+}\right) \\
\text {HOMO- } \\
1\left(1 \pi_{\mathrm{u}}\right) \\
\end{array}$ & ${ }^{2} \sum^{+}{ }^{+}$ & $\begin{array}{l}2.627 \\
5.900\end{array}$ & 2.497 \\
\hline \multicolumn{6}{|c|}{$\mathrm{BO}_{2}^{-}$} \\
\hline $\begin{array}{l}X \\
A \\
B\end{array}$ & $\begin{array}{l}4.49 \\
6.72 \\
7.50\end{array}$ & $\begin{array}{c}\operatorname{HOMO}\left(1 \pi_{\mathrm{g}}\right) \\
\mathrm{HOMO}-1\left(1 \pi_{\mathrm{u}}\right. \\
) \\
\text { HOMO- } \\
2\left(1 \sigma_{\mathrm{u}}\right)\end{array}$ & ${ }^{2} \prod_{\mathrm{e}}^{2} \prod_{\mathrm{u}}{ }^{+}$ & $\begin{array}{l}4.321 \\
6.724 \\
7.419\end{array}$ & 4.466 \\
\hline
\end{tabular}

\section{Theoretical assignments}

At B3LYP level, the ground-states of ${ }^{10} \mathrm{BO}$ and ${ }^{10} \mathrm{BO}^{-}$have the bond lengths of $1.203 \AA$ and $1.234 \AA$ and bond energies of $8.41 \mathrm{eV}$ and $9.32 \mathrm{eV}$, respectively. These values are all in nice agreement with the previously reported values. Their vibrational frequencies turned out to be $1913 \mathrm{~cm}^{-1}$ and $1739 \mathrm{~cm}^{-1}$, well in line with the measured values of $1927 \mathrm{~cm}^{-1}$ and $1735 \mathrm{~cm}^{-1}$ obtained in our PES spectra, respectively. For BO ${ }^{-}$, the calculated DFT ADE $=2.588 \mathrm{eV}$ and $\mathrm{VDE}=2.627 \mathrm{eV}$. Nice agreement was achieved at $\operatorname{CCSD}(\mathrm{T})$ level, with $\mathrm{ADE}=2.484 \mathrm{eV}$ and $\mathrm{VDE}=2.497 \mathrm{eV}$. These $\mathrm{CCSD}(\mathrm{T})$ values almost exactly reproduce the experimental ADE and VDE, with a derivation smaller than $0.01 \mathrm{eV}$.

The valence electronic configuration of ${ }^{1} \sum^{+} \mathrm{BO}^{-}$ground-state is $\left(3 \sigma^{+}\right)^{2}\left(2 \sigma^{+}\right)^{2}(1 \pi)^{4}\left(1 \sigma^{+}\right)^{2}$. Detaching one electron from the HOMO $\left(1 \sigma^{+}\right)$of the anion produces the $\mathrm{X}^{2} \Sigma^{+} \mathrm{BO}$ band corresponding to the electron configuration of $\left(3 \sigma^{+}\right)^{2}\left(2 \sigma^{+}\right)^{2}(1 \pi)^{4}\left(1 \sigma^{+}\right)^{1}$. The HOMO $\left(1 \sigma^{+}\right)$of BO neutral with one unpaired electron is basically an anti-bonding orbital mainly concentrated on $\mathrm{B}$ side with the main contribution from $\mathrm{B} 2 \mathrm{p}_{\mathrm{x}}$. The anti-bonding nature of the HOMO is evidenced by both the increased bond lengths from neutral to the anion discussed above and the reduced Wiberg bond orders from $\mathrm{WBI}_{\mathrm{B}=\mathrm{O}}=1.79$ in $\mathrm{BO}$ to $\mathrm{WBI}_{\mathrm{B}=\mathrm{O}}=1.57$ in $\mathrm{BO}^{-}$. Removing one electron from the degenerate HOMO-1 $(1 \pi)$ of the anion (which has the main contribution from $\mathrm{O} 2 \mathrm{p}_{\mathrm{y}}$ and $\mathrm{O} 2 \mathrm{p}_{\mathrm{z}}$ ) generates the second band $\mathrm{A}^{2} \prod$ BO in Fig.1 (c), which corresponds to the first excited state of the neutral $\left(3 \sigma^{+}\right)^{2}\left(2 \sigma^{+}\right)^{2}(1 \pi)^{3}\left(1 \sigma^{+}\right)^{2}$.

At DFT level, linear $\mathrm{BO}_{2}$ and $\mathrm{BO}_{2}{ }^{-}$possess the $\mathrm{B}=\mathrm{O}$ bond lengths of $1.262 \AA$ and $1.264 \AA$ and the corresponding bond orders of 1.58 and 1.60, respectively, with $\mathrm{ADE}=\mathrm{VDE}=4.321 \mathrm{eV}$ for $\mathrm{BO}_{2}^{-}$. At $\mathrm{CCSD}(\mathrm{T})$ level, $\mathrm{ADE}=4.465 \mathrm{eV}, \mathrm{VDE}=4.466 \mathrm{eV}$ for $\mathrm{BO}_{2}^{-}$, both in excellent agreement with the experimental value of $4.49 \mathrm{eV}$. As a Renner-Teller molecule, the two degenerate bending vibrational modes of the $\mathrm{X}^{2} \Pi_{\mathrm{g}}{ }^{10} \mathrm{BO}_{2}$ ground-state loose their degeneracy: they possess two different vibrational frequencies at $417 \mathrm{~cm}^{-1}$ and $504 \mathrm{~cm}^{-1}$, respectively. The calculated $\mathrm{O}=\mathrm{B}=\mathrm{O}$ symmetric stretching modes turned out to have the frequencies of $1088 \mathrm{~cm}^{-1}$ and $1096 \mathrm{~cm}^{-1}$ for ${ }^{2} \Pi_{\mathrm{g}}{ }^{10} \mathrm{BO}_{2}$ ground-state and ${ }^{1} \Sigma_{\mathrm{g}}{ }^{+10} \mathrm{BO}_{2}^{-}$ground-state, respectively. These vibrational modes were not observed in our measured PES spectra. But our DFT ${ }^{2} \Pi_{\mathrm{g}}{ }^{10} \mathrm{BO}_{2}$ vibrational mode at $1088 \mathrm{~cm}^{-1}$ nicely fits the measured value of $\mathrm{v}_{1}=1071 \mathrm{~cm}^{-1}$ for ${ }^{2} \Pi_{\mathrm{g}}{ }^{11} \mathrm{BO}_{2}$.

The closed-shell ground-state of ${ }^{1} \Sigma_{\mathrm{g}}{ }^{+} \mathrm{BO}_{2}^{-}$possesses the electron configuration of 
$\left(2 \sigma_{\mathrm{g}}\right)^{2}\left(2 \sigma_{\mathrm{u}}\right)^{2}\left(1 \sigma_{\mathrm{g}}\right)^{2}\left(1 \sigma_{\mathrm{u}}\right)^{2}\left(1 \pi_{\mathrm{u}}\right)^{4}\left(1 \pi_{\mathrm{g}}\right)^{4}$. Detaching one electron from HOMO$\left(1 \pi_{\mathrm{g}}\right)$, HOMO- $1\left(1 \pi_{\mathrm{u}}\right)$, or HOMO-2(1 $\left(\sigma_{u}\right)$ produces the ground-state of $\mathrm{X}^{2} \Pi_{\mathrm{g}} \mathrm{BO}_{2}$, the first excited state of $\mathrm{A}^{2} \Pi_{\mathrm{u}} \mathrm{BO}_{2}$, or the second excited state of $\mathrm{B}^{2} \Sigma_{\mathrm{u}}{ }^{+} \mathrm{BO}_{2}$, respectively. DFT approach produced the vertical detachment energies of ${ }^{2} \Pi_{\mathrm{g}} \mathrm{BO}_{2}$ at $4.321 \mathrm{eV},{ }^{2} \Pi_{\mathrm{u}} \mathrm{BO}_{2}$ at $6.724 \mathrm{eV}$, and ${ }^{2} \Sigma_{\mathrm{u}}{ }^{+} \mathrm{BO}_{2}$ at $7.419 \mathrm{eV}$, all well in agreement with the corresponding measured VDE values of $4.49 \mathrm{eV}, 6.72 \mathrm{eV}$, and 7.50 $\mathrm{eV}$, respectively.

Franck-Condon simulation nicely reproduces both the $\mathrm{X}^{2} \Sigma^{+} \mathrm{BO}$ and $\mathrm{A}^{2} \prod \mathrm{BO}$ vibrational progressions (see Fig. 5 for details). The simulated $\mathrm{B}=\mathrm{O}$ bond length of the first excited state ${ }^{2} \Pi$ turned out to be $1.340 \AA$, a value $0.013 \AA$ shorter than the previously reported value of 1.353 $\AA .^{1}$ The high vibrational temperature above $1650 \mathrm{~K}$ indicates that $\mathrm{BO}^{-}$anions were not effectively cooled down in helium carrier gas under the our experimental conditions.

\section{Acknowledgments}

We thank Dr. Jun Li for inspiring discussions. S.-D Li gratefully acknowledges the financial support from both the Summer Research Institute and the Natural Science Foundation of China (No. 20573088).

\section{References}

1. K. P. Huber and G. Herzberg, Constants of Diatomic Molecules, Van Nostrand Reinhold, New York, 1979. pp. 102.

2. R. S. Mulliken, Phys. Rev. 25, 259(1925).

3. J. Almlof, A. V. Nemukhin, and A. Heiberg, Int. J. Quant. Chem. XX, 655(1981).

4. F. Melen, I. Dubois, and H. Bredohl, J. Phys. B 18, 2423 (1985).

5. P. Botschwina, Chem. Phys. 28, 231(1978).

6. A. V. Nemukhim, J. Almlof, and A. Heiberg, Chem. Phys. 57, 197(1981).

7. M. Tanimoto, J. Chem. Phys. 84, 1210(1986).

8. D. E. Jensen, J . Chem. Phys. 52, 3305(1970).

9. D. K. Russsell, M. Kroll, and R. A. Beaudet, J. Chem. Phys. 66, 1999(1977).

10. K. Kawaguchi, E. Hirota, and C. Yamada, Mole. Phys. 44, 509(1981).

11. T. R. Burkholder and L. Andrews, J. Chem. Phys. 95, 8697(1991). 
PNNL-16185 


\title{
Characterization of Microstructure and Composition of Magnetostrictive Nanobars as Bio-sensor Platform
}

\author{
Suiqiong Li and Zhongyang Cheng \\ Materials Engineering \\ Auburn University \\ Auburn, AL \\ and \\ Dr. Chongmin Wang \\ Pacific Northwest National Laboratory \\ Richland, WA
}

\begin{abstract}
The magnetostrictive nanobar and its array were recently induced as a high performance biosensor platform. In this research, the magnetostrictive Fe-B nanobars, which were synthesized using a template-based electrochemical deposition, were systematic characterized. The composition and microstructure, which are directly related to the performance of the nanobars, of the Fe-B nanobar were determined using scanning electron microscopy (SEM), transmission electron microscopy (TEM), x-ray photonelectron spectroscopy (XPS) as well as Auger electron spectroscopy (AES). Morphologically, nanobars are featured by very flat top and smooth cylindrical surface, which are critical factors for obtaining high performance as sensor platform. Structurally, XRD and electron diffraction reveal that the Fe-B nanobar is amorphous. AES analysis indicates that the nanobar shows no significant compositional variation along the length direction. HRTEM reveals that the nanobars were covered by an oxidization layer of a typical thickness of $\sim 10 \mathrm{~nm}$. It is believed that this oxidation layer is related to the passivation of nanobars in air. High temperature annealing and subsequent structural and compositional analysis indicate that the Fe-B nanobars possess a good thermal stability.
\end{abstract}

\section{INTRODUCTION}

We recently induced magnetostrictive particles (MSPs), such as strips and bars, as a new type of acoustic wave (AW) devices as a sensor platform. ${ }^{1}$ A extremely high sensitivity and a high Q value make the MSP much better sensor platform than the current platforms, such as microcantilevers. More importantly, the MSP technology provides a methodology to bring the target species to the sensor, which is a challenge for current technologies. All these make the MSP a great candidate for developing high performance biosensors.

The theoretical simulation and experimentally characterization indicated that, given the material parameters of the MSPs constant, the mass sensitivity of MSPs exponentially increases with the decrease of their size. ${ }^{1}$ To obtain extremely high mass sensitivity, such as the capability of detecting a single or several cells ( pg), the MSPs in size from nanometer to micrometer are required. In this research, amorphous Fe-B alloy was selected as the magnetostrictive material to fabricate nano-sized MSPs. The magnetostrictive nanobars and nanobar arrays, in diameter ranging from 50 to $200 \mathrm{~nm}$, were fabricated by electrochemically depositing amorphous Fe-B alloy into ion track-etched polymer membranes. Their 
morphology, microstructure, and composition were systematically studied by SEM, HRTEM and Auger electron spectroscopy.

\section{RESULTS AND DISCUSSIONS}

The morphology of the nanobars was observed using scanning electron microscopy (LEO 982 SEM), which is shown as figure 1. The SEM images showed that straight Fe-B nanobars of $50 \mathrm{~nm}-200 \mathrm{~nm}$ in diameter and 2-5 $\mu \mathrm{m}$ in length were fabricated in high density by the template-based synthesis. The nanobars in uniform length can be fabricated on a substrate (Figure 1. (a) and (c)) so that a sensor array can be fabricated; as well as can be released from the substrate to get individual nanobar (Figure1. (b)). The SEM images revealed that the nanobars have very flat end (Figure 1. (d)). The flat end of the nanobars is very favorable for obtaining high $\mathrm{Q}$ value when the nanobars are operated as a resonator. The SEM images indicated that there was no morphologic difference between the nanobars with different diameter/length and formed under different current density. Since the polymer template is relatively soft, the pore size is not strictly defined, which cause the diameter of deposited nanobars slightly increased along the pore axis.

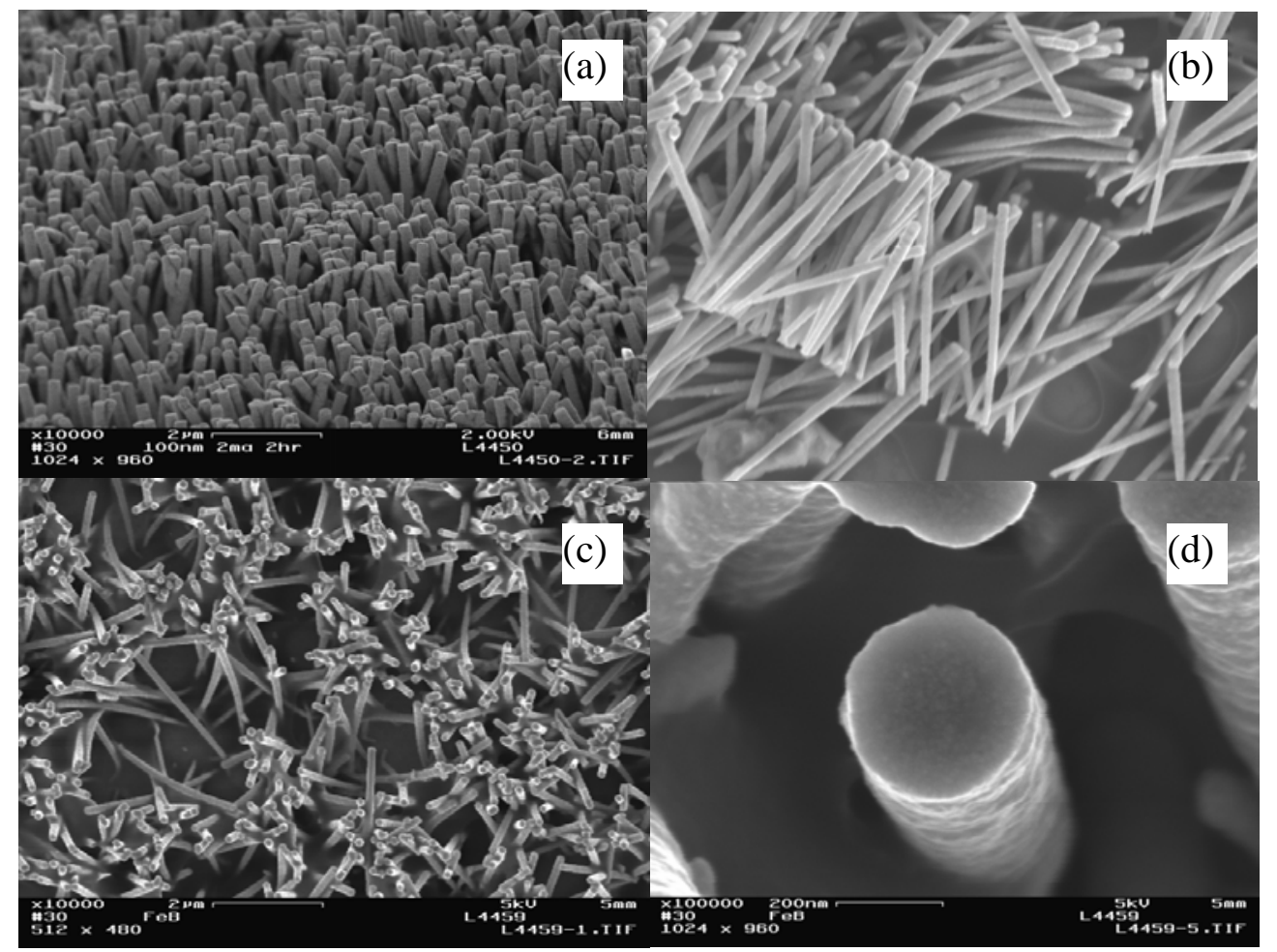

Figure 1. SEM image for (a) Fe-B nanobar array in diameter of 200nm, (b) \& (c) Fe-B nanobars in diameter of 50nm, and (d) top surface of the Fe-B nanobar in diameter of $100 \mathrm{~nm}$.

High-resolution transmission electron microscopy (JEOL 2010 HRTEM) was employed to characterize more details of the morphology and microstructure of the Fe-B nanobars. The Fe-B nanobars were placed on the $\mathrm{Cu}$ TEM grid coated with lacy carbon for the observation. Figure 2 showed the TEM image and the electron diffraction pattern of the Fe-B nanobar in diameter of $50 \mathrm{~nm}$. The electron diffraction pattern revealed that the microstructure of the 
nanobars is amorphous, which is as desired. The individual nanobar was characterized with the electron diffraction focused on the selected areas along the nanobar; the results confirmed that the Fe-B nanobar has uniform amorphous microstructure along the whole nanobar.
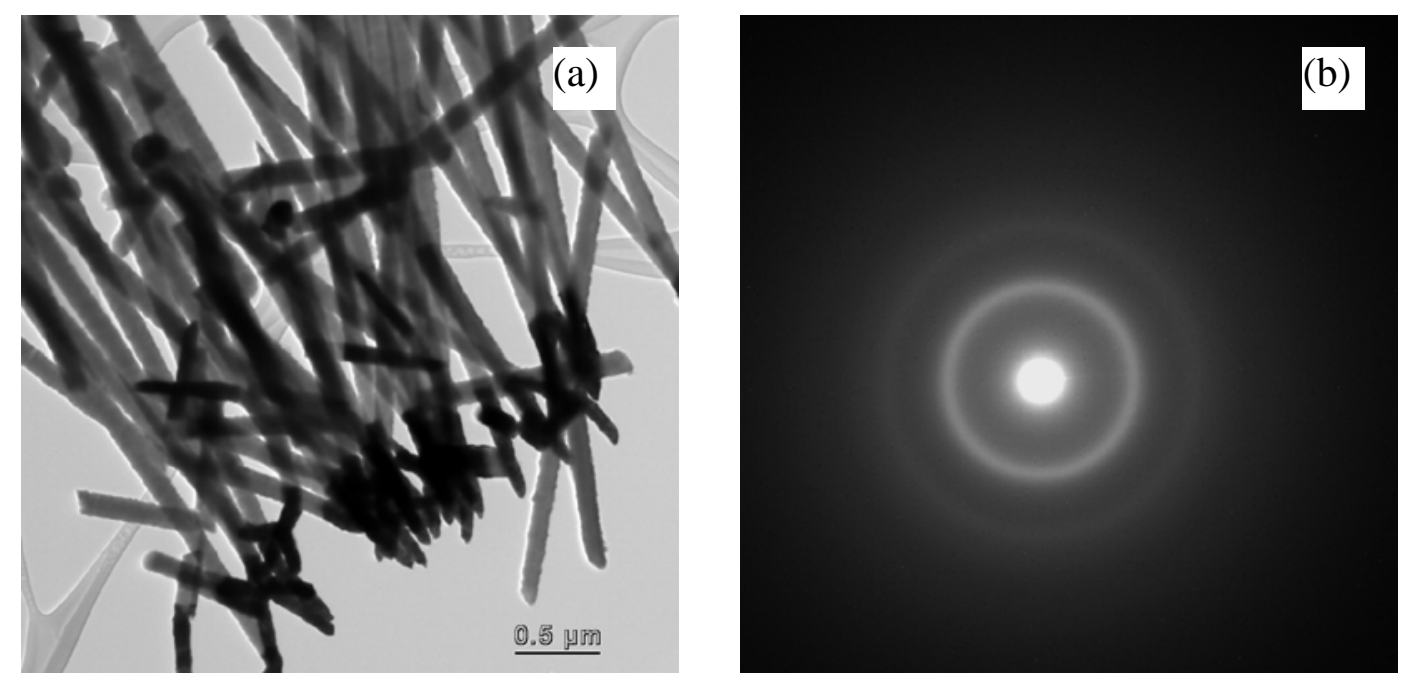

Figure 2. TEM image (a) and electron diffraction pattern (b) of the nanobars in diameter of $50 \mathrm{~nm}$

The details of the composition of the Fe-B nanobars were studied by Auger electron spectroscopy (Model 680 AES). Nanobars released from substrate were placed on a Cu TEM grid coated with lacy carbon. To study the composition of the nanobar along the length direction as well as along the diameter direction, 10 spots along the individual nanobar length were selected. The composition of each spot was analyzed by AES with the e-beam focused onto the spot with diameter of 20nm. After analysis along the length direction, certain amount material along the nanobar diameter direction was sputtered off by Ar ion-beam sputtering. The composition analysis was carried out repeatedly as the nanobar was sputtered off $5 \mathrm{~nm}, 10 \mathrm{~nm}$, $20 \mathrm{~nm}, 30 \mathrm{~nm}$ and $40 \mathrm{~nm}$. The analysis results showed that there are contamination and oxidation observed on the surface of the deposited nanobar. However, this layer is very thin, only about $10 \mathrm{~nm}$. After sputtered off $10 \mathrm{~nm}$, the contamination can not be observed anymore and only very small amount of the Oxygen can be detected. Except the contamination layer, the Fe and $\mathrm{B}$ composition along the nanobar diameter is uniform. The analysis results also revealed the uniform composition distribution along the nanobar length direction. The variation in the Fe and $\mathrm{B}$ composition along the nanobar length direction was less than $4 \%$. The analysis demonstrated that the whole nanobar exhibits uniform composition distribution with about $10 \mathrm{~nm}$ oxidation layer covered on the surface, which is believed that this oxidation layer is related to the passivation of nanobars in air. There is some amount of $\mathrm{C}$ was detected. It should not come from the nanobars. Even though the e-beam was focused on the nanowire, since the nanowire is thin, the background still contributed signals. Most of $\mathrm{C}$ detected should come from the lacy carbon. The AES analysis of the nanobars deposited under different current showed same uniform composition contribution.

The thermal stability of the Fe-B nanobars was studied by annealing the nanobars in the $\mathrm{N}_{2}$ environment. The nanobars were annealing at 200,300 , and $400{ }^{\circ} \mathrm{C}$ for 1 hour. The morphology and microstructure of the nanobars annealed at different temperature were characterized by HRTEM. The electron diffraction patterns showed that the nanobars still kept 
PNNL-16185

amorphous microstructure after annealed at $200{ }^{\circ} \mathrm{C}$ and $300{ }^{\circ} \mathrm{C}$. It was found that BCC iron crystal formed after annealed at $400{ }^{\circ} \mathrm{C}$. The Fe-B nanobars exhibit good thermal stability.

\section{CONCLUSIONS}

Magnetostrictive nanobars and nanobar arrays with uniform length and smooth morphology in diameter of $50 \sim 200 \mathrm{~nm}$ were synthesized by depositing amorphous Fe-B alloy into ion-track etched membranes. The SEM characterization of the nanobars demonstrated that the nanobars are featured by very flat end and smooth cylindrical surface. The template synthesized Fe-B nanobars exhibited uniform composition, amorphous microstructure and good thermal stability. They have great potential to be high performance AW sensor platform.

\section{ACKNOWLEDGMENTS}

The authors gratefully acknowledge financial support from USDA NRI-20053560315817 and USGS-01HQGR0106-ZYC5

The author also appreciates the financial support from DOE for the SEM, TEM and AES characterization carried out at Pacific Northwest National Lab (PNNL).

\section{REFERENCE}

1. Suiqiong Li, Lisa Orona, Liling Fu, Z.-Y. Cheng, "Electrosynthesis of Magnetostrictive Nanosensor Array”, Mater. Res. Soc. Symp. Proc. 900E, (2005), Nanoparticles and Nanostructures in Sensors and Catalysis, edited by Chuan-Jian Zhong, Nicholas A. Kotov, Wayne Daniell, Francis P. Zamborini, 0900-008-04 


\title{
Assembly of an Ion Trap Mass Spectrometer for LD-CI Experimentation
}

\author{
Julie A. Lloyd and Murray V. Johnston \\ Department of Chemistry \& Biochemistry \\ University of Delaware \\ Newark, DE 19716 \\ and \\ M. L. Alexander \\ Environmental Molecular Sciences Laboratory \\ Pacific Northwest National Laboratories \\ Richland, WA 99352
}

Although laser desorption and chemical ionization have both been commonly used in the field of mass spectrometry, the hyphenated LD-CI technique has not been studied as widely. ${ }^{1-6}$ A renewed interest in this technique has taken place in the field of aerosol mass spectrometry where laser desorption is well established. The new aspect of chemical ionization provides a soft ionization technique to help characterize polar, polyfunctional oxygenated organics and high molecular mass species found in aerosols. Therefore, the main objectives of this research are to 1) assemble an ion trap mass analyzer, 2) become trained on the use and operation of the ion trap and complementary components, and 3) conduct basic testing of the ion trap analyzer using different ionization techniques with a test compound.

An ion trap mass analyzer was assembled for laser desorption (LD) of analyte molecules followed by electron and chemical ionization (EI/CI) (Figure 1). The ion trap mass analyzer was controlled by existing electronics and software. ${ }^{7}$ The software was further developed in-house specifically for laser studies, but also for more efficient data analysis. Figure 2 shows a schematic of the laser set-up. LD-EI/CI experiments used a Continuum $\mathrm{Nd}$ :YAG laser at a wavelength of $10.6 \mu \mathrm{m}$ with a maximum energy output of $\sim 6 \mathrm{~mJ}$ per pulse ( 5 ns width). No focusing optics were used. The laser beam measured approximately $3 \mathrm{~mm}$ in diameter and hit the sample probe at a 90 degree angle. Sample probes were machined inhouse and varied in material used (i.e. stainless steel, aluminum, ceramic sleeved, etc.), type of surface (flat/grooved), and diameter of the probe. Perylene, a robust, nonvolatile PAH, was selected as a test compound to optimize the system parameters. A $2 \mathrm{mg} / \mathrm{mL}$ solution of perylene was prepared in benzene. The solution was applied to the sample probe in small aliquots and was allowed to dry before insertion in the vacuum chamber. For CI, acetonitrile was leaked into the vacuum chamber as the chemical ionization reagent. Experiments were conducted to optimize the ionization time, laser trigger delay, and laser energy. Buffer gas pressures, the concentration of perylene, CI agent, and the probe position were held constant.

Prelimilary results showed a combination of laser desorption and thermal desorption. A prolonged firing of the laser showed an increase in the analyte signal for both aluminum and stainless steel materials; however, the increase was more apparent using the aluminum probe. Several ionization processes other than electron ionization were also occurring within the system. With a high laser energy ( $>3 \mathrm{~mJ}$ ), the analyte underwent laser desorption ionization (LDI), thermal desorption laser ionization as well as electron ionization. The EI mass spectrum showed the most intense peaks at $252 \mathrm{~m} / \mathrm{z}\left(\mathrm{M}^{\cdot+}\right)$ and $250 \mathrm{~m} / \mathrm{z}\left(\mathrm{M}^{{ }^{+}}-2 \mathrm{H}\right)$ (Figure 3). Very few fragment ions at $224 \mathrm{~m} / \mathrm{z}$ and $126 \mathrm{~m} / \mathrm{z}$ were detected mainly due to the robust structure of perylene. Other lower mass peaks were observed and were attributed to the probe material and hydrocarbons from the turbomolecular pump oil. For CI experiments, a $3 \mathrm{~mJ}$ laser energy was 
used to eliminate other ionization processes as described above. The CI mass spectrum showed the most intense peak at $253 \mathrm{~m} / \mathrm{z}$ relating to $\mathrm{MH}^{+}$. Perylene fragment peaks were not present. Also, fewer background ions in the lower mass range were detected making the spectrum "cleaner".

Overall, the objectives of this research project were met; however, continued work is necessary to optimize the system. Increased mass range and sensitivity, continued software development, improved calibration methods, and noise elimination from electronics are all major factors that are required for improving the system.

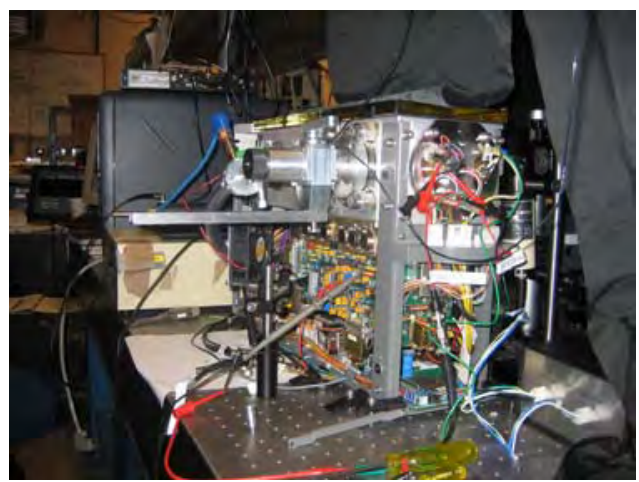

Figure 1. A snapshot of the assembled ion trap mass analyzer.

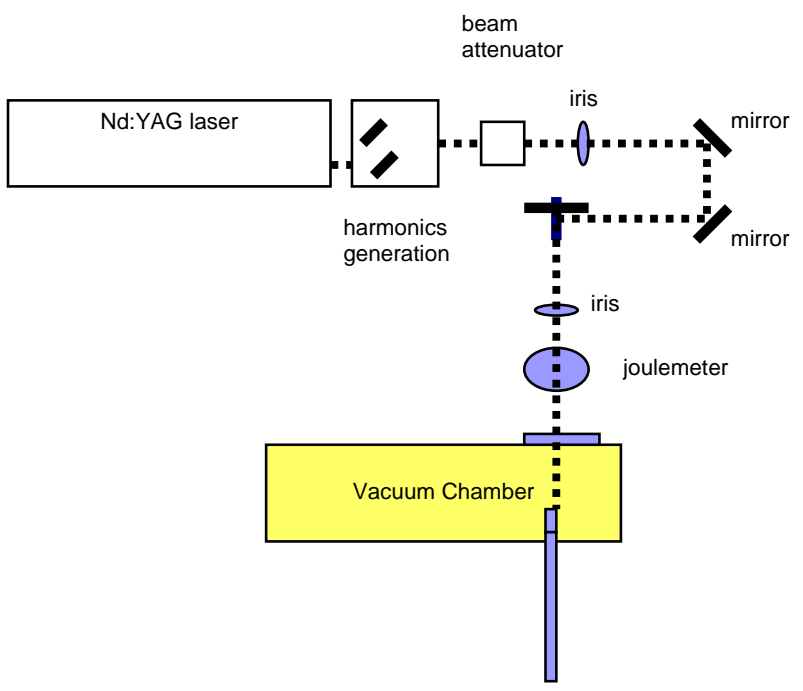

Figure 2. Schematic of the Nd:YAG laser set-up for laser desorption from the sample probe. 


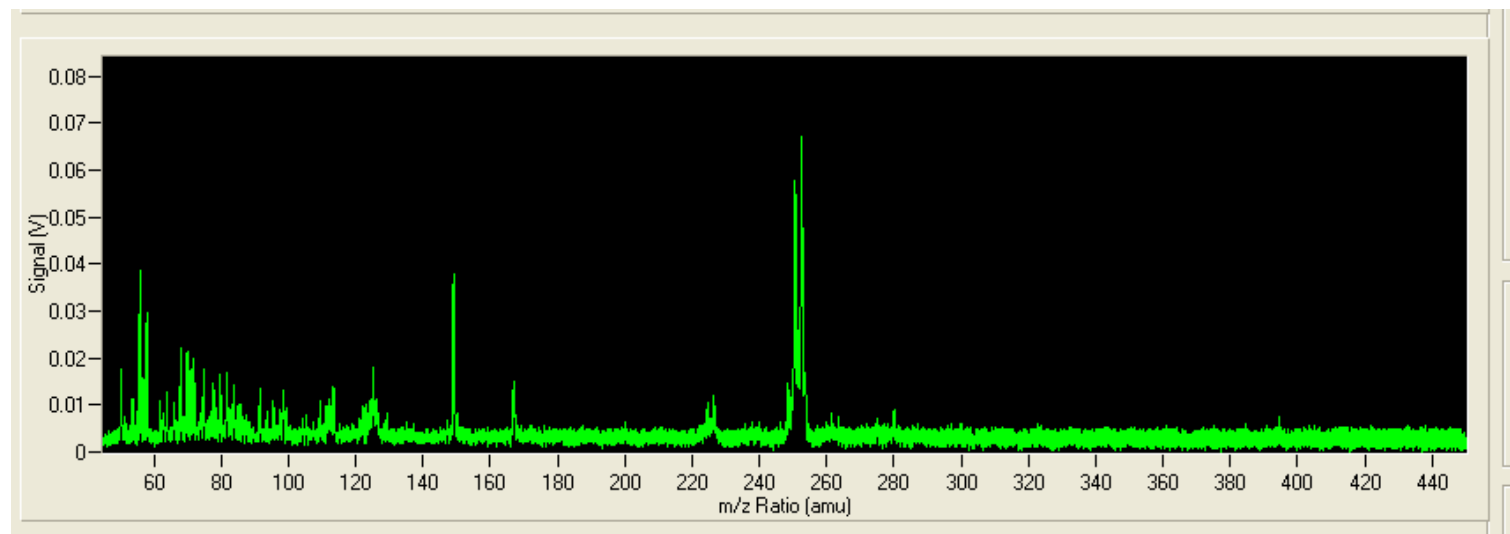

Figure 3. LD-EI mass spectrum of perylene on a stainless steel probe using $\sim 4.5 \mathrm{~mJ}$ laser energy.

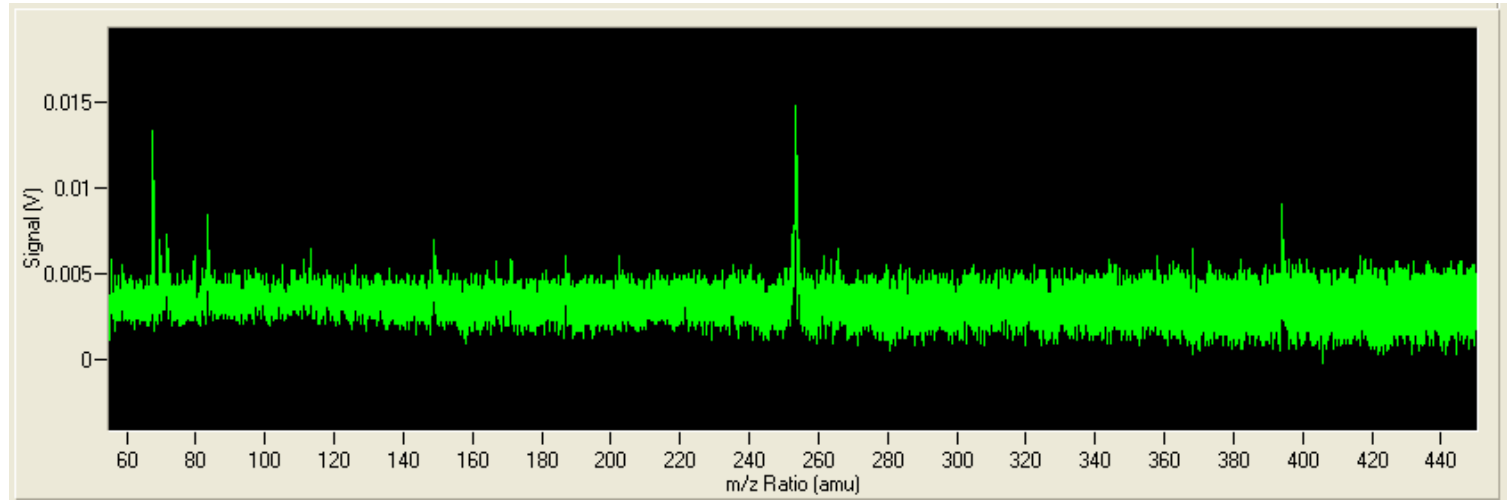

Figure 4. LD-CI mass spectrum of perylene on a stainless steel probe using $\sim 3 \mathrm{~mJ}$ laser energy.

\section{Literature Cited}

(1) Cotter, R. J. Anal. Chem. 1980, 52, 1767-1770.

(2) Heller, D. N.; Lys, I.; Cotter, R. J. Anal. Chem. 1989, 61, 1083-1086.

(3) Yost, R. A.; Wilder, B. J. Anal. Chem. 1983, 55, 2002-2005.

(4) Tronendle, F. J.; Reddick, C. D.; Yost, R. A. J Am Soc Mass Spectrom 1999, 10, 13151321.

(5) Speir, J. P.; Gorman, G. S.; Cornett, D. S.; Amster, I. J. Anal. Chem. 1991, 63, 65-69.

(6) Wood, T. D.; Marshall, A. G. J Am Soc Mass Spectrom 1991, 2, 299-304.

(7) Hopkins, D. F.; Alexander, M. L.; Swanson, K. R.; Buschbach, M. A. Scientific Computing and Instrumentation 2004, 18-20. 
PNNL-16185 
PNNL-16185

\title{
Investigation into the Effect of Milling Ammonia Borane with Magnesium Hydride
}

\author{
Rebecca Lowton and Peter Edwards \\ Inorganic Chemistry \\ University of Oxford, Worcester College, \\ Oxford, England \\ and \\ Thomas Autrey, \\ Pacific Northwest National Laboratory \\ Richland, WA
}

Introduction

One of the most pressing problems currently facing mankind is the search for alternative fuel sources. Taking into account the nature of the current political climate, the rate of global warming ${ }^{1}$ and diminishing fossil fuel reserves, it is clear that this is a problem which requires a rapid solution.

Amongst the many contenders as a replacement fuel are ethanol, liquid petroleum gas and methyl tertiary butyl ether. However, it is hydrogen that is widely believed to be the most promising future energy carrier in the transportation sector. Having a gravimetric energy density three times that of petroleum, the use of hydrogen in conjunction with fuel cell technology results in efficiencies of between 40 and 65\% (compared to gasoline in internal combustion engines which has an efficiency of only $10-15 \%)^{2}$. Hydrogen is also non-toxic, safe to breath, dissipates freely (meaning that an uncontained hydrogen leak would amount to nothing more than a waste of valuable fuel) and has an explosion limit 1/4 that of octane ${ }^{3}$. There are two major barriers to the wide - scale implementation of hydrogen as a fuel. 


\section{i) Production}

Molecular hydrogen $\left(\mathrm{H}_{2}\right)$ does not occur in large amounts naturally and therefore must be produced from another source. In order for hydrogen to be used as an energy carrier in the long term, it is necessary to find a way of producing large quantities of the gas from renewable energy sources (wind, wave, solar etc).

Several possibilities are currently under investigation. Some of the most promising include the nuclear powered electrolysis of water using polymer electrolyte membrane (PEM) electrolysers, the photoelectrolysis of water, and the gasification of biomass ${ }^{4},$. . Unfortunately, given the very low yields of $\mathrm{H}_{2}$ produced compared with processes using non-renewable sources (outlined below), these methods are currently far too expensive for large scale production, and so these procedures are still very much at the development stages.

At this time, the cheapest way of generating $\mathrm{H}_{2}$ is by gasification of coal over a metal catalyst ${ }^{6}$, or by steam reformation of natural gas ${ }^{7}$. Both of these methods derive their $\mathrm{H}_{2}$ from nonrenewable hydrocarbon-based sources and therefore suffer from many of the problems associated with the direct use of fossil fuels. These methods could, however, be used in the short term to assist in the transition to a hydrogen economy whilst methods using renewable sources are under further development. Not only is the efficiency of fossil fuels increased when used in this manner, but these methods also have the added advantage of localising the emissions of $\mathrm{CO}_{2}$ and other greenhouse gases.

\section{ii) Storage}

One of the most serious problems preventing the implementation of hydrogen technology is the lack of a suitable hydrogen storage medium. Whilst Hydrogen has an excellent energy storage capacity per unit mass, its storage capacity per unit volume is very poor indeed. For instance, the hydrogen required to travel the same distance as that travelled on the average full tank of petrol would occupy $49 \mathrm{~m}^{3}$ at room temperature and pressure ${ }^{8}$. The challenge facing researchers is to find a way of storing this large volume of gas in a vessel that will fit easily into the smallest car. 
PNNL-16185

Research into hydrogen storage materials for the transportation sector has several main focal points. These include compressed gas storage, cryogenic storage and chemical storage.

Compression of hydrogen in light weight, high strength cylinders such as those designed by Quantum Fuel Systems Technologies ${ }^{9}$ may seem like the simplest option for storage given that they have high gravimetric storage densities and are safe up to 800 bar pressure. However, there are many disadvantages associated with this method, the most notable of which is the large energy penalty on compression. The pressurization of the gas from standard pressure to 800 bar uses approximately 18\% of the latent energy stored within the hydrogen every time the cylinder is charged ${ }^{10}$ resulting in very low overall energy efficiency.

Cryogenic storage of hydrogen is possible only at very low temperatures as hydrogen has it's critical point at 33.2K. Approximately $1 / 3$ of the latent heat energy stored within the hydrogen is required for cooling the gas to this temperature and even then, due to the exothermic conversion of ortho - to para $-\mathrm{H}_{2}$, boil off at a rate of $\sim 5 \%$ per day is unavoidable. These problems combine to make this method of storage very expensive and inefficient.

The third and most extensive area of research is into chemical storage of hydrogen in metallic and complex hydrides and it is this area on which the remainder of this report will focus.

The criteria deemed necessary by the US Department of Energy for a suitable hydrogen storage material are listed below ${ }^{11}$. It is important to realize that these benchmarks are the values set for 2015 and are meant to be guidelines only.

- High storage capacity: minimum $6.5 \mathrm{wt} \%$ abundance of hydrogen and at least $65 \mathrm{~g}$ $\mathrm{L}^{-1}$ of hydrogen available from the material.

- A decomposition temperature, $\mathrm{T}_{\mathrm{dec}}=60-90{ }^{\circ} \mathrm{C}$, corresponding to the optimum working temperature of PEM fuel cells.

- Reversibility of thermal absorption / desorption cycle: low temperature of hydrogen desorption and low pressure of hydrogen absorption (a plateau pressure of the order of a few bars at room temperature), or ease of non - thermal transformation between substrates and products of decomposition.

- Low cost.

- Low toxicity of non - explosive and possibly inert (to water and oxygen) storage medium. 
PNNL-16185

The performance requirements of the materials will ultimately be determined by the market and are expected to increase over time.

Since it is necessary for a hydrogen storage material to have a high gravimetric storage capacity, the choice of elements on which to base such materials is very much limited to those elements with low atomic mass, at the beginning of the periodic table. Unfortunately, the vast majority of hydrides based on these light elements are ionic or covalent in nature and thus often have very high decomposition temperatures and can not simply be recharged by the application of $\mathrm{H}_{2}$ gas.

Much research focus has been given to ammonia borane $\left(\mathrm{NH}_{3} \mathrm{BH}_{3}, \mathrm{AB}\right)$ during the last few years as a potential hydrogen storage material. First prepared by Shore and Parry in $1956^{12}$, its decomposition was investigated in 1978 by Hu et al $^{13}$ and its potential as a hydrogen storage material first suggested by Baitalow et al. ${ }^{14}$ Thermal decomposition takes place in the range $77-137^{\circ} \mathrm{C}$ and complete decomposition occurs at $112^{\circ} \mathrm{C}$ releasing $1 \mathrm{~mol}$ of hydrogen per mole of complex.

This temperature is higher than the optimum working temperature of fuel cells (as water evaporation strongly decreases PEM conductivity at higher temperatures) and hence, this material would be a very promising hydrogen storage material if it's decomposition temperature could be lowered. This report will focus on attempts to lower this decomposition temperature by milling $\mathrm{AB}$ with magnesium hydride $\left(\mathrm{MgH}_{2}\right)$.

\section{Experimental Details}

Ammonia borane (Aviabor, 99\%) was mechanically milled with $\mathrm{MgH}_{2}$ (Alfa Aeser, 98\%) in proportions of $1: 1$ and 2:1 by Dr Chun Lu using a mechanical mill. The reactants were weighed out in a glovebox and placed into a container with several tungsten carbide balls and was then mounted onto a shaker mill. The mixture was milled for 2 minutes and then allowed to stand for 10 minutes in order to dissipate the generated heat. This process was repeated 8 times amounting to a total milling time of 16 minutes.

Calorimetric data was collected using a Setraram C80 calorimeter using a heating rate of $1 \mathrm{~K}$ $\min ^{-1}$. Volumetric data was collected using standard volumetric apparatus as shown in figures 1a and $1 \mathrm{~b}$. For the lower temperature experiment, the $1: 1$ mixture was heated to $80^{\circ} \mathrm{C}$ and this 
PNNL-16185

temperature was maintained until $\mathrm{H}_{2}$ release stopped. The temperature was then raised to $100^{\circ} \mathrm{C}$ and maintained until $\mathrm{H}_{2}$ release ceased. Corrections were made for the expansion of the gas in the apparatus.

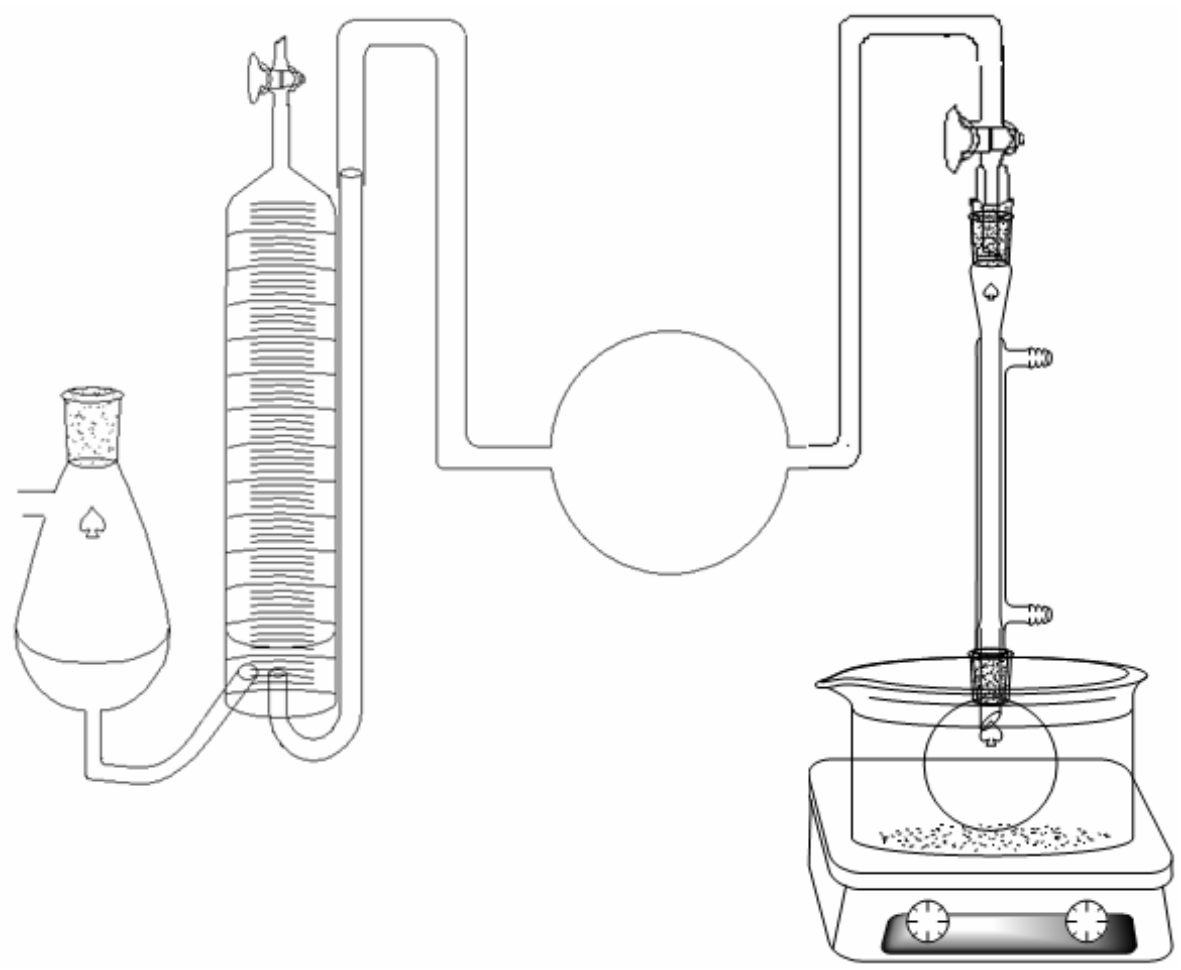

Figure 1a: Volumetric apparatus used for temperatures up to $100^{\circ} \mathrm{C}$

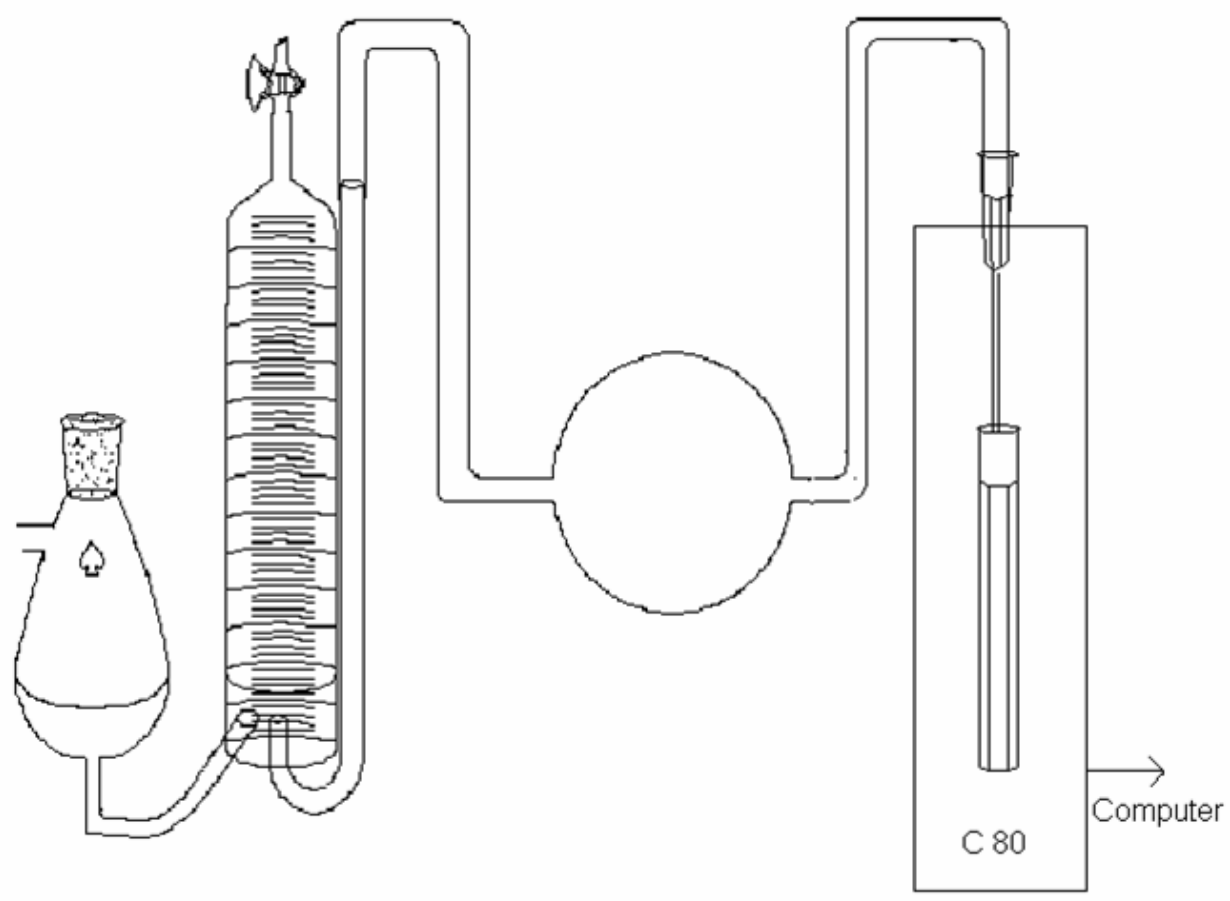

Figure 1b: Volumetric apparatus used for temperatures up to $200^{\circ} \mathrm{C}$ 
PNNL-16185

\section{Results}

Pure Ammonia Borane

Solid state ${ }^{11} \mathrm{~B}$ NMR of AB shows a peak at - 32ppm.

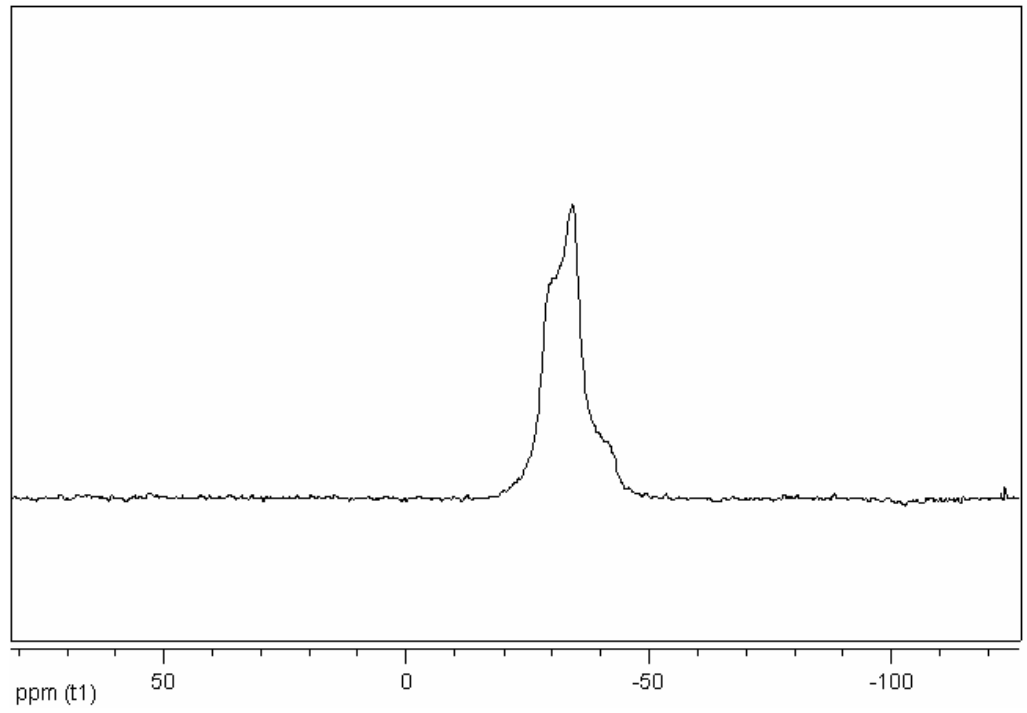

Figure $2:{ }^{11} \mathrm{~B}$ solid state NMR of $\mathrm{AB}$

Calorimetric data for $\mathrm{AB}$ collected on the C80 shows two exothermic effects during the heating of the compound to $250{ }^{\circ} \mathrm{C}$, the first beginning at $110^{\circ} \mathrm{C}$, peaking at $113^{\circ} \mathrm{C}$, and the second beginning at $137^{\circ} \mathrm{C}$ and peaking at $151^{\circ} \mathrm{C}$.

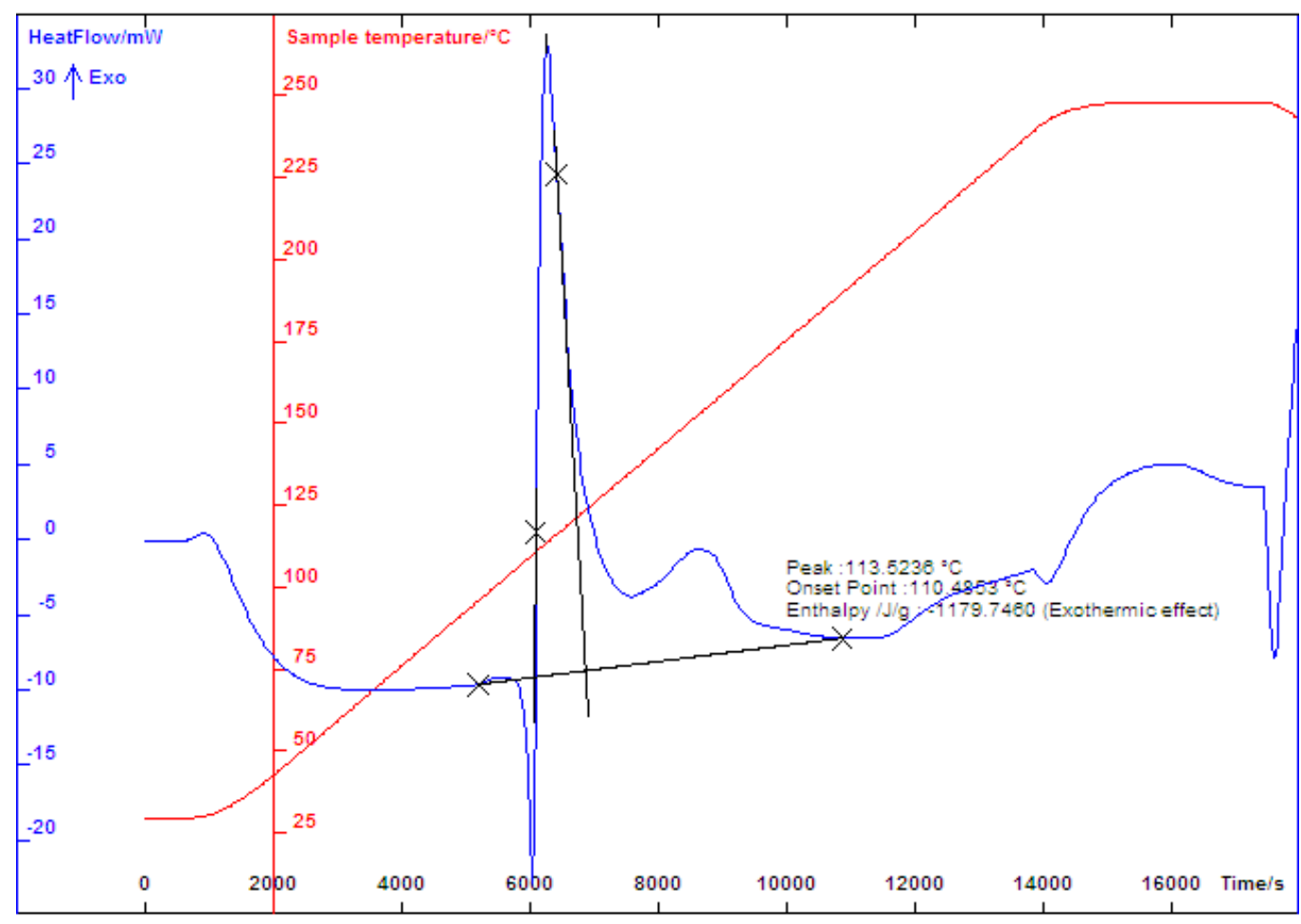

Figure 3: ${ }^{11} \mathrm{~B}$ solid state NMR of AB 


\section{$\underline{1: 1 \mathrm{AB}+\mathrm{MgH}_{2}}$}

${ }^{11} \mathrm{~B}$ solid state NMR of the product of the 1:1 milling reaction showed a shift in the AB peak from -32 ppm to $-29 \mathrm{ppm}$ and the development of a new second peak at $-40 \mathrm{ppm}$, showing the formation of a new material during the milling reaction.

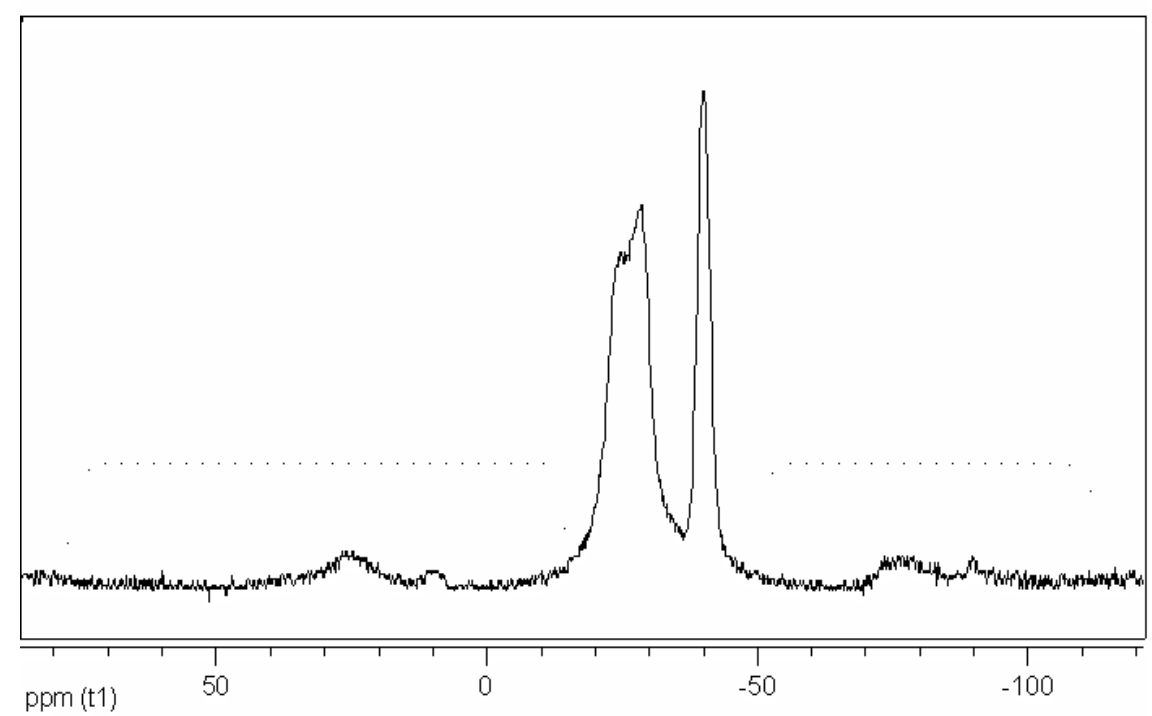

Figure $4:{ }^{11} \mathrm{~B}$ solid state NMR of $1: 1 \mathrm{AB}+\mathrm{MgH}_{2}$

The chemical shift of the peak corresponds well with $\mathrm{Mg}\left(\mathrm{BH}_{4}\right)_{2}$, however, unlike $\mathrm{Mg}\left(\mathrm{BH}_{4}\right)_{2}$, the material is not soluble in THF. Hence, the material is postulated to contain a borohydride unit of some description; however, further analysis will be needed to confirm this.

Heating the product formed to $250^{\circ} \mathrm{C}$ on the $\mathrm{C} 80$ gave the following data:

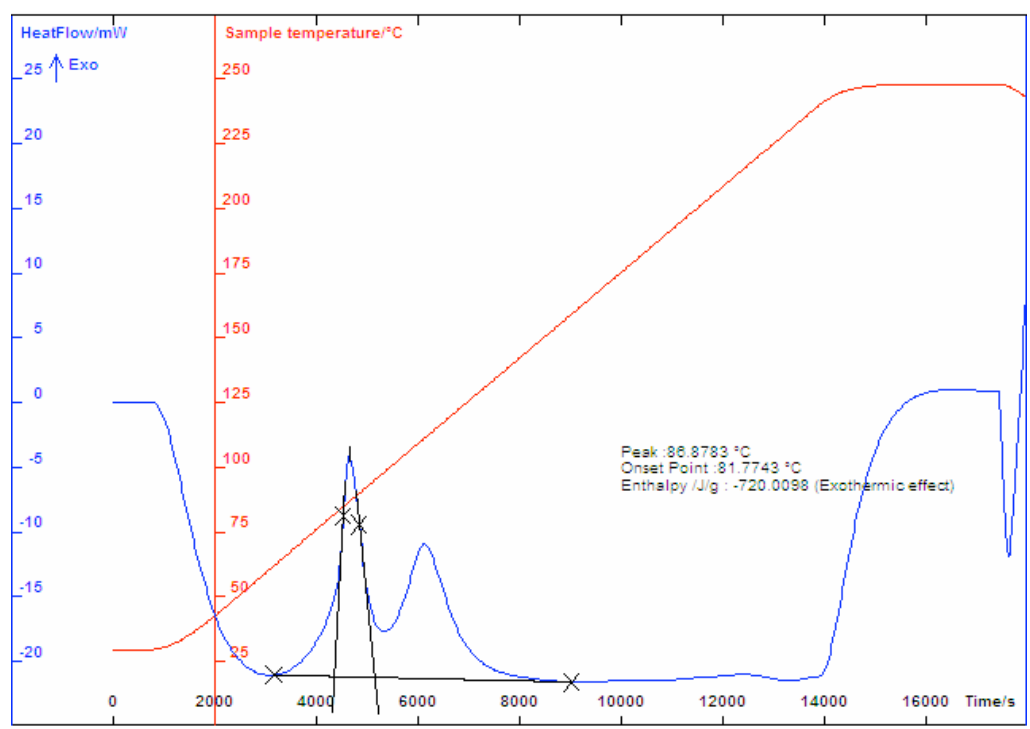

Figure 5: $\mathrm{C} 80$ plot of $1: 1 \mathrm{AB}+\mathrm{MgH}_{2}$ heated to $250{ }^{\circ} \mathrm{C}$ 
PNNL-16185

The temperature at which the first exothermic effect begins in the product of the $1: 1$ reaction is lower than that of pure $\mathrm{AB}$. The first effect begins at $82^{\circ} \mathrm{C}$ and peaks at $87^{\circ} \mathrm{C}$ (compare to $\mathrm{AB}$, peaking at $113^{\circ} \mathrm{C}$ ). There is considerable overlap between the first and second effects and therefore the point at which the $2^{\text {nd }}$ effect commences is unknown, however, it peaks at $111^{\circ} \mathrm{C}$. The total enthalpy of the change is calculated to be $-720 \mathrm{Jg}^{-}$ 1 .

The ${ }^{11} \mathrm{~B}$ solid state NMR of the product before and after desorption is shown below:
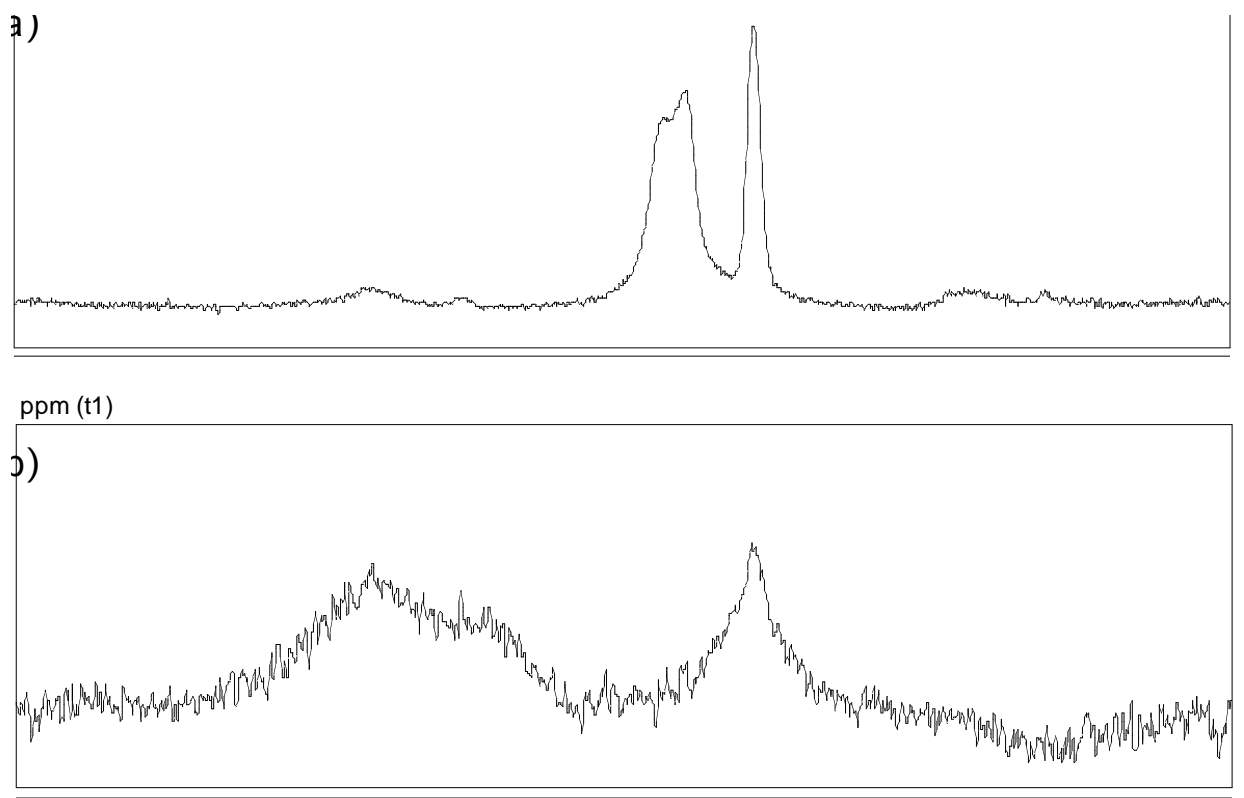

ppm (t1)

Figure 6: ${ }^{11} \mathrm{~B}$ solid state NMR of 1:1 $\mathrm{AB}+\mathrm{MgH}_{2}$ a) before and b) after desorption to $250^{\circ} \mathrm{C}$

It is clear that heating to $250^{\circ} \mathrm{C}$ results in decomposition of $\mathrm{AB}$ only, as the peak for the new material appears broadened, but essentially still in tact.

Volumetric studies give more detailed information regarding the release of hydrogen. 
PNNL-16185

Experiments show that up to $3 \mathrm{wt} \% \mathrm{H}_{2}$ is desorbed below $80^{\circ} \mathrm{C}, 5.4 \mathrm{wt} \%$ below $100^{\circ} \mathrm{C}$, and 8.4 wt $\%$ below $200^{\circ} \mathrm{C}$ :

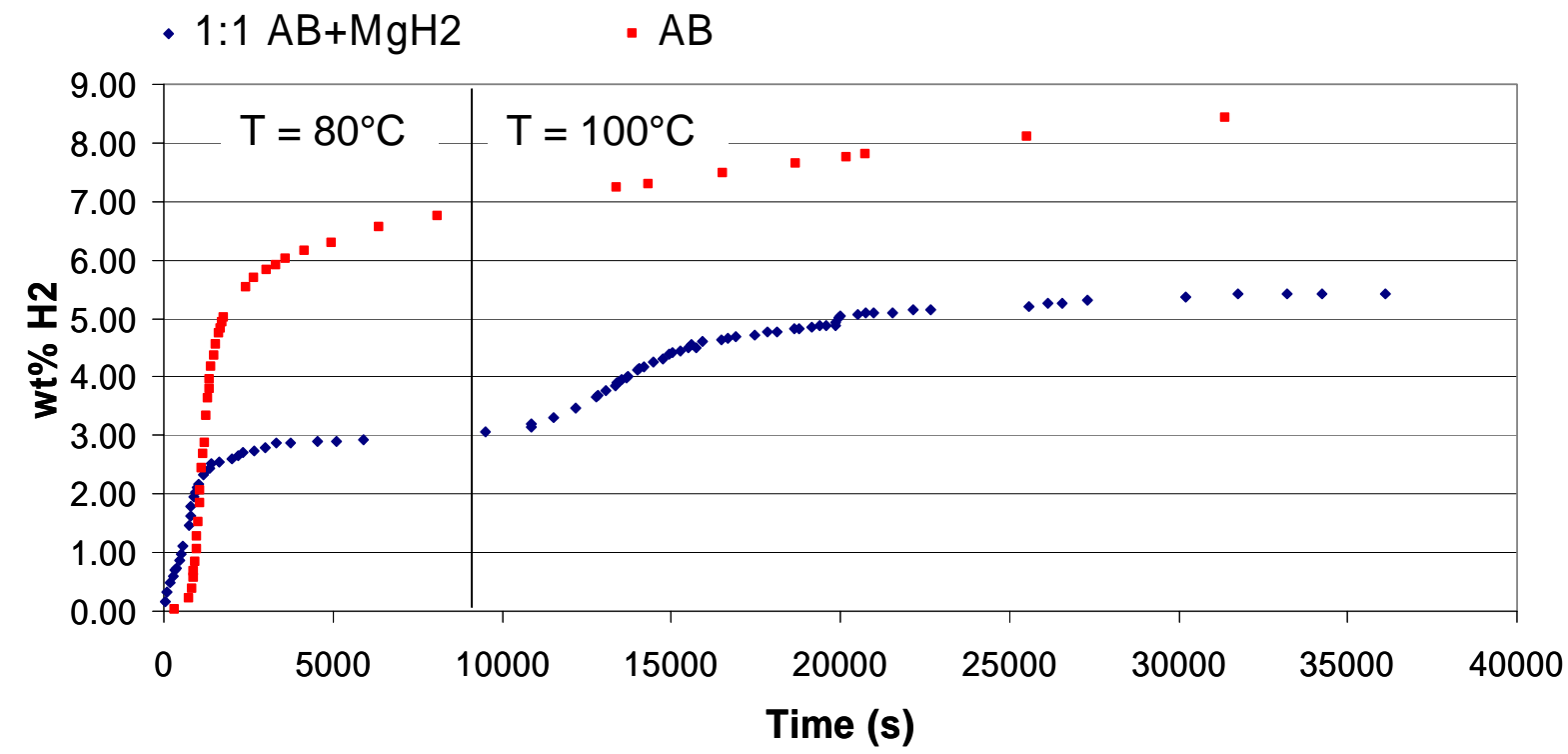

Figure 7a: $\mathrm{Wt} \% \mathrm{H}_{2}$ desorbed at $80{ }^{\circ} \mathrm{C}$ and $100{ }^{\circ} \mathrm{C}$ from $\mathrm{AB}$ and $1: 1 \mathrm{AB}+\mathrm{MgH}_{2}$

$5.4 \mathrm{wt} \%$ corresponds to 1.5 equivalents of $\mathrm{H}_{2}$ desorbed below $100^{\circ} \mathrm{C}$. Given that the rate of desorption ${ }^{15}$ at $100^{\circ} \mathrm{C}$ is approximately $1 / 4$ of that at $80^{\circ} \mathrm{C}$ (rates of $0.011 \mathrm{wt} \% \mathrm{~min}^{-1}$ and 0.041 $\mathrm{wt} \% \mathrm{~min}^{-1}$ respectively), it appears that the type of $\mathrm{H}_{2}$ released at the each of the temperatures may be from two different desorption processes. It could be that the $2^{\text {nd }}$ desorption step corresponds to the beginning of the second exothermal effect in the calorimetric data, and that if the temperature had been raised to $111^{\circ} \mathrm{C}$, the decomposition would have continued, releasing the full two equivalents. Heating to $200^{\circ} \mathrm{C}$ yields $8.4 \mathrm{wt} \% \mathrm{H}_{2}$ ( $2.2 \mathrm{H}_{2}$ equivalents).

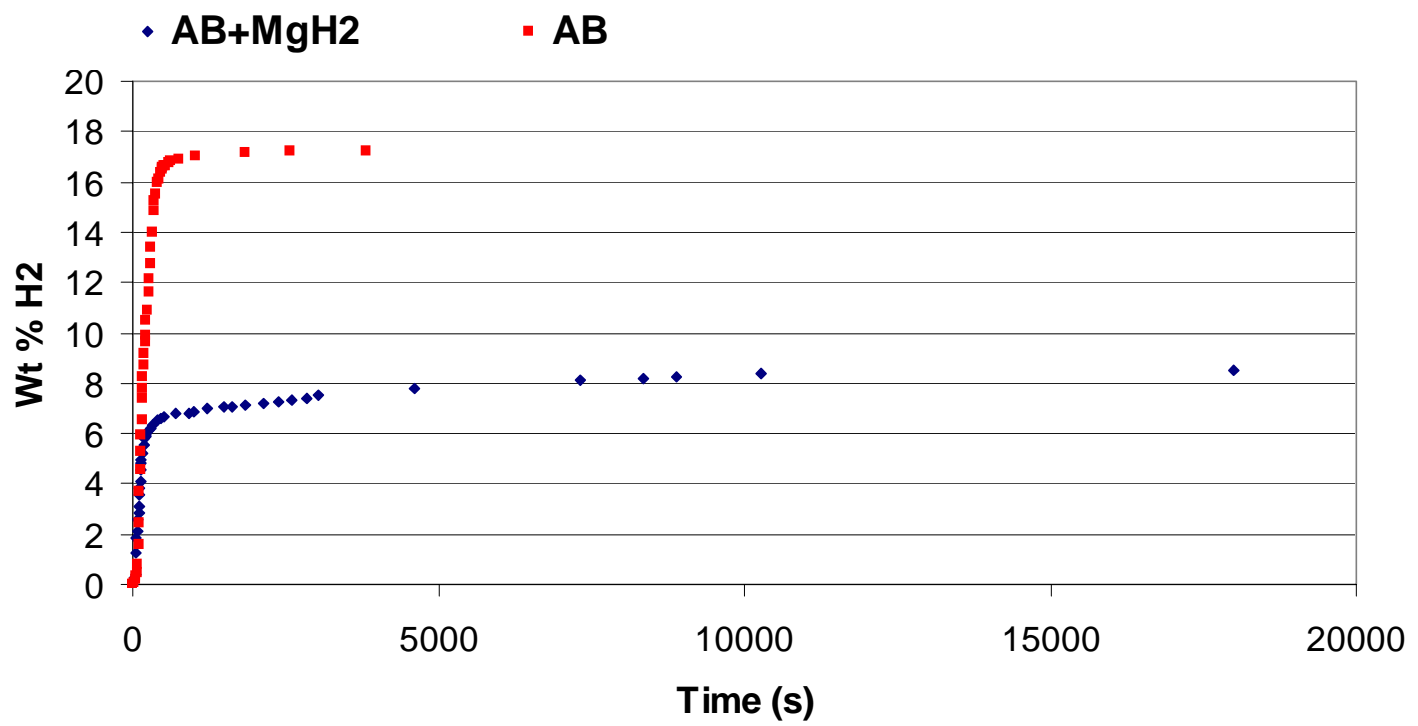

Figure 7b: $\mathrm{Wt} \% \mathrm{H}_{2}$ desorbed at $200{ }^{\circ} \mathrm{C}$ from $\mathrm{AB}$ and $1: 1 \mathrm{AB}+\mathrm{MgH}_{2}$ 
The ${ }^{11} B N M R$ spectrum of the products of decomposition of $A B$ and $1: 1 A B+M \mathrm{gH}_{2}$ to $100^{\circ} \mathrm{C}$ and $200^{\circ} \mathrm{C}$ shown in figures 7 and 8 , respectively. In both sets of spectra, the peak at - 40ppm in the decomposition product of the 1:1 mixture is still very much present, implying that all of the $\mathrm{H}_{2}$ formed comes from the $A B$ still present in the material after the milling reaction. This conclusion is supported by the fact that the amount of hydrogen liberated during the decomposition reaction of $A B$ is approximately twice that released from the $1: 1 \mathrm{AB}+\mathrm{MgH}_{2}$.
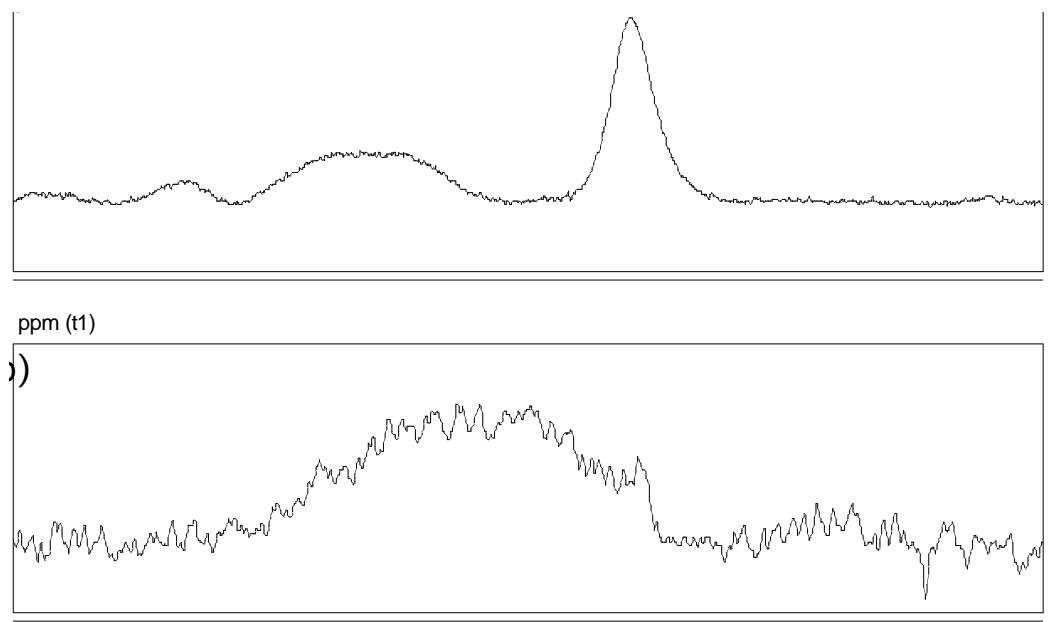

ppm (t1)

Figure 8: ${ }^{11} B N M R$ of products of thermal decomposition of a) $1: 1 \mathrm{AB}+\mathrm{MgH}_{2}$ and b) $A B$ at $100^{\circ} \mathrm{C}$

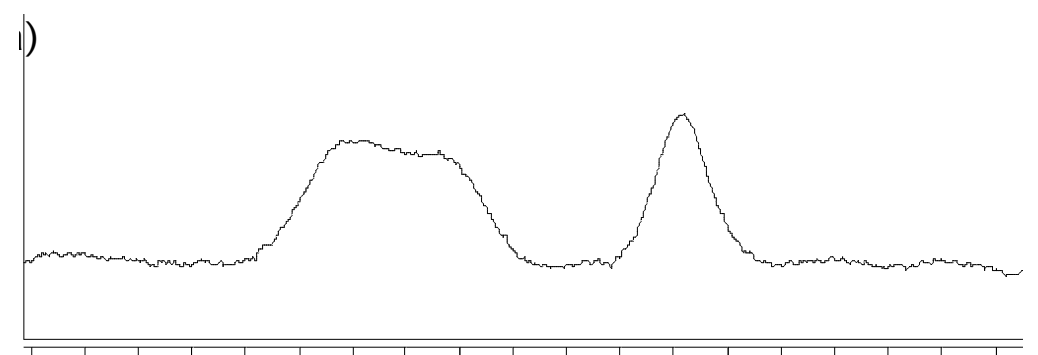

ppm (t1)

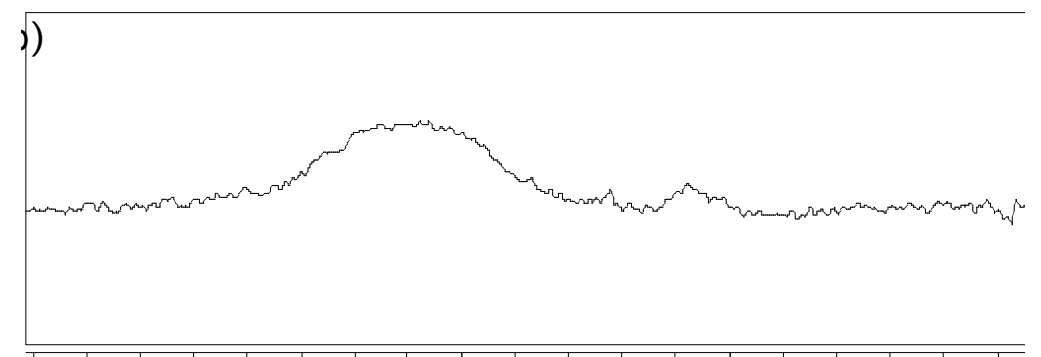

ppm (t1) 
PNNL-16185

Heating the $1: 1$ mixture at a rate of $1 \mathrm{~K} \mathrm{~min}^{-1}$ up to the end of the $2^{\text {nd }}$ exothermal effect $\left(168^{\circ} \mathrm{C}\right)$ gave the following results, showing a total $\mathrm{H}_{2}$ desorption of approximately $7 \mathrm{wt} \%$. Desorption clearly occurs in a 2 step process with each of the steps corresponding to an exothermal effect.

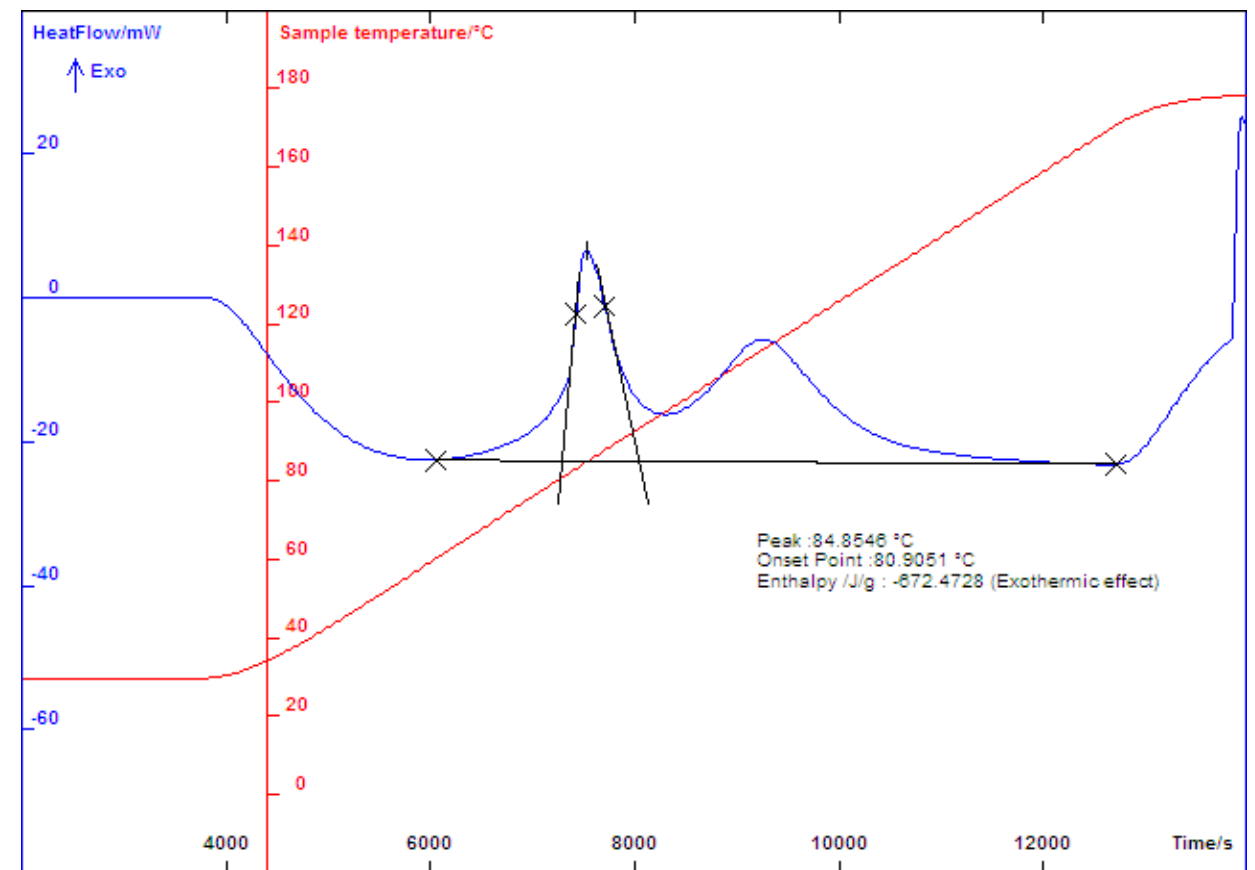

Figure 10: C80 Plot of 1:1 $\mathrm{AB}+\mathrm{MgH}_{2}$ quenched after the $2^{\text {nd }}$ exothermal effect

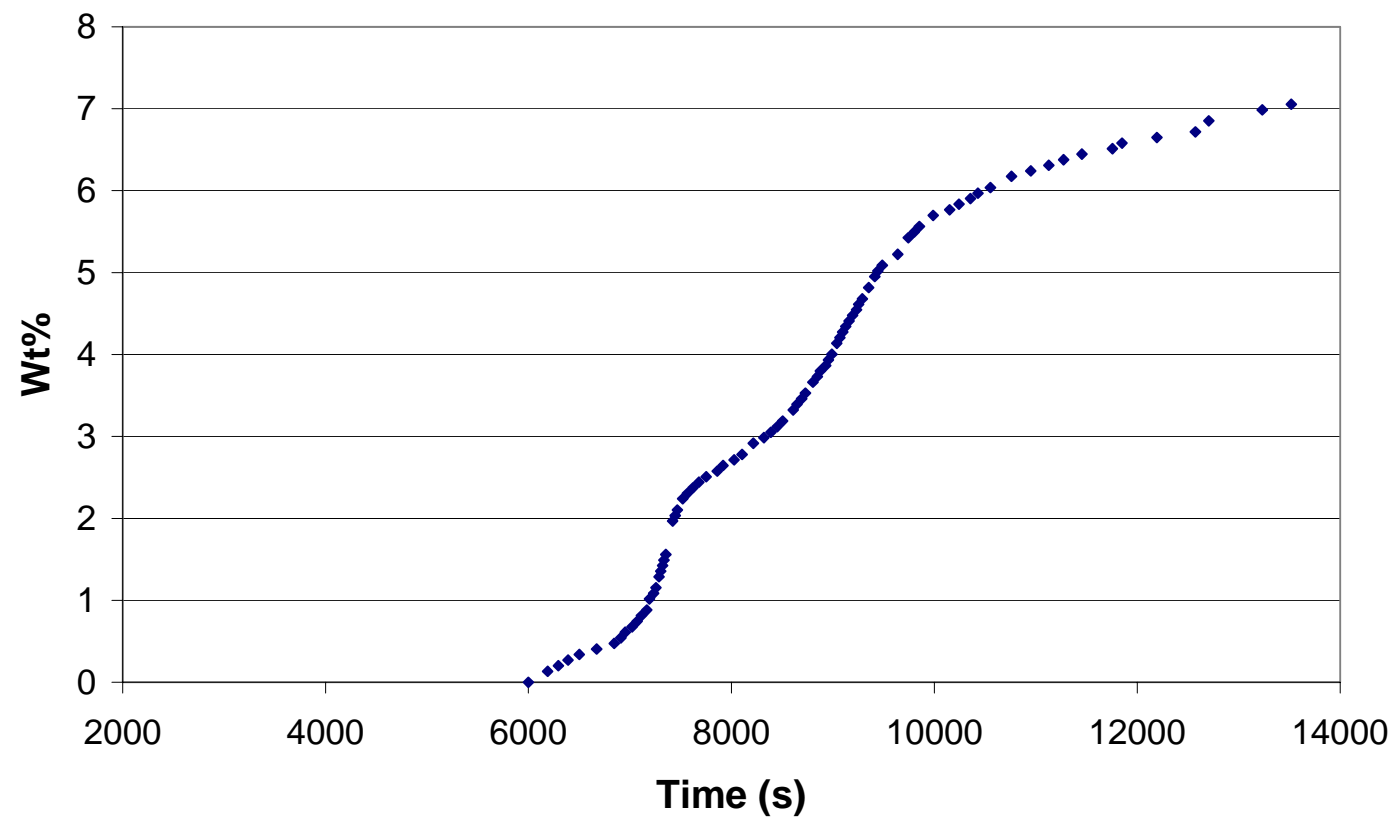

Figure 11: $\mathrm{Wt} \% \mathrm{H}_{2}$ desorbed from 1:1 $\mathrm{A}+\mathrm{MaH}_{2}$ up to the end of the $2^{\text {nd }}$ exothermic effect 
PNNL-16185

\section{$\underline{2: 1 \mathrm{AB}+\mathrm{MgH}_{2}}$}

The ${ }^{11} \mathrm{~B}$ solid state NMR spectrum shows only one peak corresponding to $\mathrm{AB}$, and hence, the product formed in the 1:1 reaction of $\mathrm{AB}$ with $\mathrm{MgH}_{2}$ does not form in the 2:1 reaction.

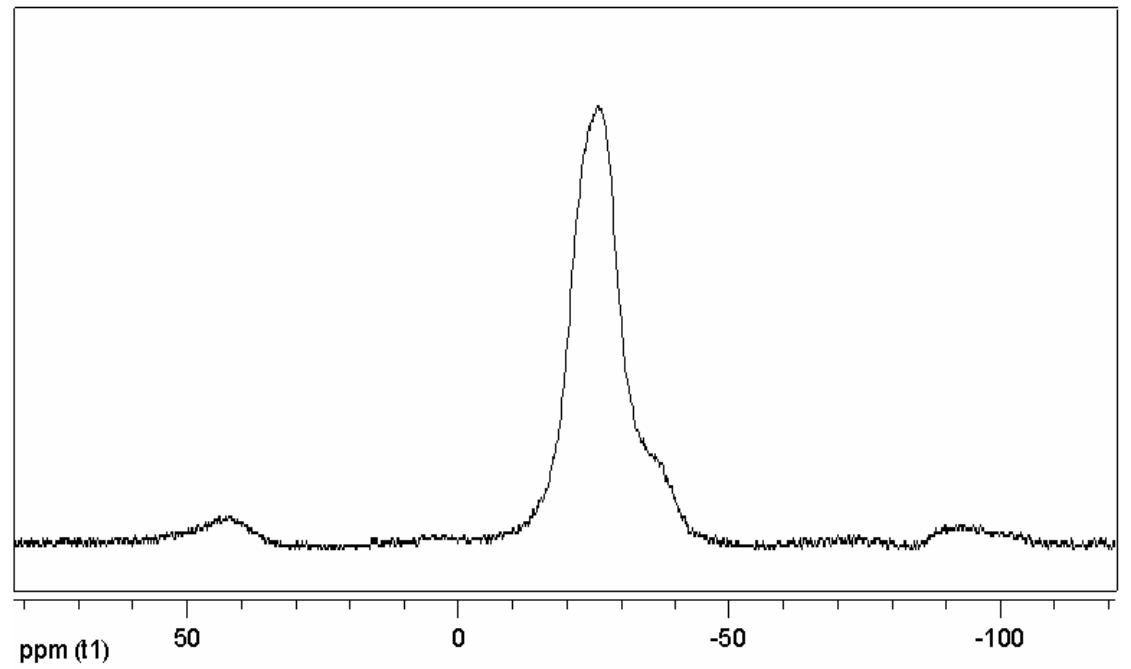

Figure 12: ${ }^{11} \mathrm{~B}$ NMR of product of 2:1 $\mathrm{AB}+\mathrm{MgH}_{2}$

The calorimetric data collected on the $\mathrm{C} 80$ during heating to $250^{\circ} \mathrm{C}$ gives very similar results to that of $\mathrm{AB}$ heated to $250^{\circ} \mathrm{C}$. The onset point of the exothermal effect is at $109^{\circ} \mathrm{C}$ ( $\mathrm{cf} \mathrm{AB}$ at $\left.110^{\circ} \mathrm{C}\right)$ and the effect peaks at $113^{\circ} \mathrm{C}\left(\mathrm{AB}\right.$ also at $\left.113^{\circ} \mathrm{C}\right)$.

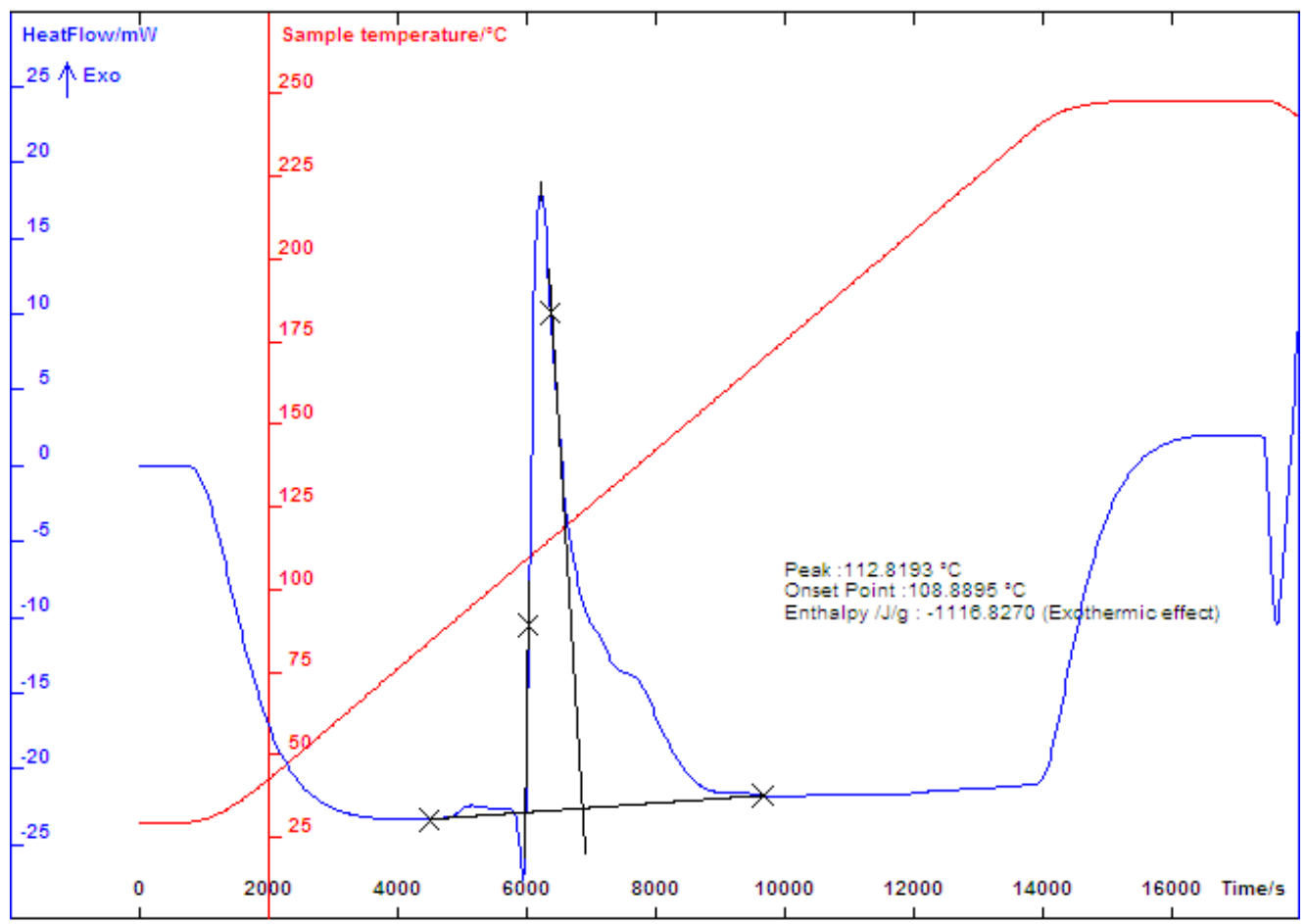

Figure 13: C80 Plot of 2:1 $\mathrm{AB}+\mathrm{MgH}_{2}$ 


\section{$\underline{\text { Conclusions }}$}

The mechanical milling of a one to one mixture of ammonia borane and magnesium hydride produces a new material which has the effect of lowering the decomposition temperature of the remaining ammonia borane from $113^{\circ} \mathrm{C}$ to $84^{\circ} \mathrm{C}$. The $\mathrm{H}_{2}$ storage capacity of the $1: 1$ mixture below $250^{\circ} \mathrm{C}$ is reduced by approximately $50 \%$ compared to that of pure ammonia borane. The 2:1 reaction of ammonia borane with magnesium hydride does not produce the new material and the product behaves in a similar way to pure ammonia borane.

\section{Acknowledgments}

Grateful thanks must go to Dr Tom Autrey for his kindness, support and expertise during my placement at PNNL. Thanks also to Dr. John Linehan for all of his help in the lab, and to Dave, Abi, Wendy, Suh-Jane and Rene for their assistance using the NMR, C80 and mass spec machines. Also, huge thanks to Dr. S Barlow, Nikki Avery, Rebecca Janosky and Pricilla Yamada for their help with bringing me to the US and to all of the PNNL staff involved in the summer internship program for all of their help and assistance.

I am very grateful to the SEE Program for covering my expenses for transportation and accommodation, and to the EPSRC for the remaining funding.

\section{References}

1. Choi, O., Fisher A., Climate Change, 2003; 58: 147

2. http://www.rmi.org/sitepages/pid540.php, 05/06/06

3. www.msdssearch.com

4. See www.bellona.no/en/energy/hydrogen

5. www-formal.stanford.edu/jmcprogress/hydrogen.html

6. Timpe, R.C., Kulas, R.W., Hauserman, W.B., Sharma, R.K., Olson, E.S., Willson, W.G., Int. J. Hydrogen Energy, 1997; 22: 487-492

7. Lipman, T.E., DeLucchi, M.A., Int. J. Vehicle Design, 1997; $17: 562$

8. $\mathrm{PV}=\mathrm{nRT}$

9. I. R. Harris, D. Book, P. A. Anderson, P.P. Edwards, Fuel Cell Rev., 2004 June/July: 17

10. L. Zhou, Renewable Sustainable Energy Rev., 2005; 9: 395

11. W. Grochala , P.P. Edwards, Chem. Rev., 2004; 104: 1283

12. Shore S.G., Parry W., J. Am Chem. Soc., 1956; 78: 503

13. Hu M. G., Greanangel R. A., Wendlandt W. W., Thermochim Acta.

14. Baitalow F. Baumann J., Wolf G., Jaenicke-Rößler K., Leitner G., Thermochim Acta, 2002; $391: 159$

15. The desorption rates were calculated as the ratio between the amount desorbed and the time taken to go from 20 to $80 \%$ of capacity measured at each temperature. 
PNNL-16185 


\title{
Molecular Modeling of the $\mathrm{N}$-acyl homoserine lactone hydrolase from Bacillus thuringiensis.
}

\author{
Wagner A. Lucena \\ Insect Molecular Biology Laboratory, \\ EMBRAPA Algodão, Campina Grande, PB 58107-720, Brazil \\ and \\ Roberto Lins \\ Pacific Northwest National Laboratory \\ Richland, WA
}
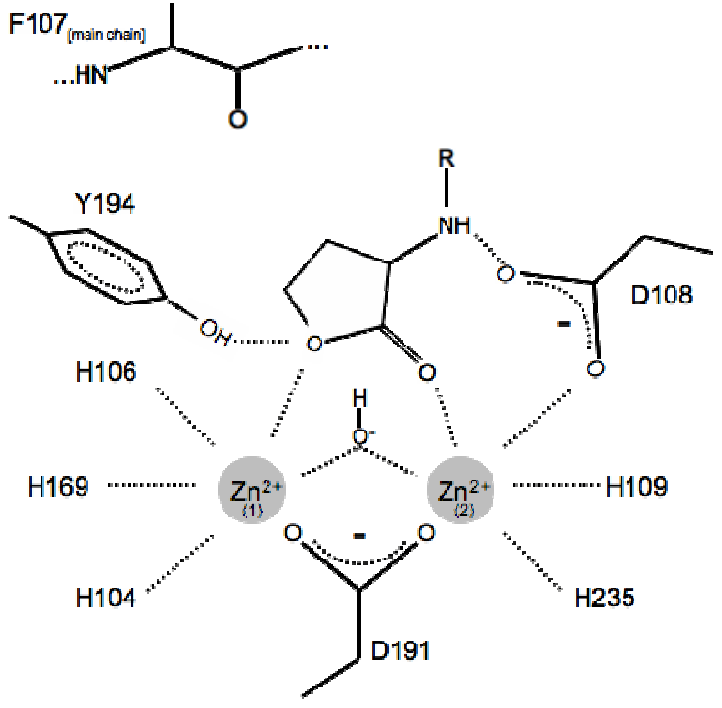
PNNL-16185

Influence of the $\mathrm{N}$-acyl group on the binding of homoserine lactones to the quorum-

quenching $\mathrm{N}$-acyl homoserine lactone hydrolase from Bacillus thuringiensis.

Wagner Lucena ${ }^{2}$ Tjerk P. Straatsma ${ }^{1}$ and Roberto D. Lins ${ }^{1, *}$

${ }^{1}$ Computational Biology and Bioinformatics, Pacific Northwest National Laboratory, Richland, WA 99352, USA

${ }^{2}$ Insect Molecular Biology Laboratory, EMBRAPA Algodão, Campina Grande, PB 58107-720, Brazil

*To whom the correspondence should be addressed. Phone: +1-509-375-2755; Fax: +1-509372-4720; E-mail: roberto.lins@pnl.gov.

\section{INTRODUCTION}

Quorum Sensing (QS) may be defined as the ability that some bacterial strains have to coordinate collective behavior using a regulatory mechanism that is dependent of expression of a group of genes and a signal transduction pathway. This phenomenon was first reported for the luminescent marine bacterium Vibrio fischeri (Naelson, 1970). Bacteria use QS mechanism to regulate a range of biological functions such as biofilm formation, bioluminescence, antibiotic production and virulence factor expression (Camilli, 2006). Since alterations on the QS can interfere in acute infections, devising ways to modulate QS can be a useful tool to coordinate the bacteria behavior via control of the population density, e.g., transgenic plants resistant to root diseases (Fray, 1999), biofilm formation in Pseudomonas aeruginosa (Davis, 1998; Dong, 2002), regulation of Ti plasmid in Agrobacterium tumenfasciens (Zhang, 1991; Hao and Burr, 2006). 
The general pattern is dependent of a production of small diffusible molecules namely autoinducers (AI) synthesized in low concentrations. When the signaling molecule concentration reach a threshold, it binds to a receptor and initiate a cascade signal that ends with the activation of a set of genes that are involved with a specific phenotype. While a peptide-based signal is typically used by Gram-positive bacteria (Spoering and Gilmore, 2006), $\mathrm{N}$-acyl homoserine lactones (AHLs) serve as the signal autoinducer molecules in Gramnegative type bacteria (Rasmussen, 2006). The homoserine lactones (HSLs) are synthesized by the family of LuxI homologue proteins, using acyl-carrier proteins as the major $\mathrm{N}$-acyl chain donor and S-adenosyl methionine to provide with the homoserine lactone moiety (Parsek et al., 1999 in Rasmussen, 2006). With exception of LuxLM from V. harveyi (Bassler et al., 1993), all HSL synthases are LuxI homologs and they usually produce HSLs with different N-acyl chains, which also cause different catalytic activity levels. Those enzymes are expressed in low cell densities and promote an accumulation of HSLs up to a certain level when binding to LuxR (DNA-binding proteins) occurs. They form a multimeric complex (TF-transcriptional factor) that recognize the Lux box DNA sequence and induce the expression of gene coordinated by lux operon, which includes LuxI (Val and Cronan, Jr. 1998).

A second group of enzymes, the $\mathrm{N}$-acyl homoserine lactone hydrolases, (lactonases), is responsible for the cleavage of the autoinducer homoserine ring (Dong et al., 2000), and therefore is known as a quorum-quenching (QQ) enzyme. The inactivation and/or absence of the QS messenger molecules can downshift the population density and inhibit biofilm formation. Recently, a 250-residue long N-acyl homoserine lactone hydrolase (AiiA) was isolated from Bacillus thuringiensis (BTK), a Gram-positive entomopathogenic soil bacterium (Dong et al., 2000). This enzyme family is spread with high similarity among isolates and strain of $B$. thuringiensis, B. cereus and B. mycoides but not in B. sphaericus and B. fusiformis (Dong et al., 2002). The BTK-AiiA enzyme presents a highly-conserved zinc-binding domain $\left({ }^{104} \mathrm{HXHXDH}{ }^{109} \approx \mathrm{H}^{169}\right)$ similar to the metallo- $\beta$-lactamase superfamily (Dong et al., 2002; Lee et al., 2002; Kim et al., 2005), and its metal-binding site is identical to the human glyoxalase II. The activity has been shown to be zinc-dependent through the use zinc chelators to decrease and reconstitute its catalysis (Thomas et al., 2005). BTK-AiiA is known to hydrolyze N-acyl(S)-homoserine lactone substrates whith acyl chains varying from 4-12 carbons long, revealing an acyl chain length preference with a maximum activity for C10-AHL. The overall hydrophobicity of the substrate is given by a balance between the hydrophilic homoserine lactone ring and the hydrophobic chain side. This amphipathy of the N-acyl-HSL allows them to navigate the phospholipid bilayer of the cell as well as the aqueous intracellular and extracellular environments (Fuqua et al., 2001). The overall structure of BTK-AiiA reveals a $\alpha \beta / \beta \alpha$ sandwich fold. The $N$-terminal repeat $(1-152)$ is formed by seven $\beta$-sheets, three $\alpha$ helices and six shorts $3_{10}$ helices. The C-terminal (153-250) contains five $\beta$-sheet strands and three $\alpha$-helices. Two loops are importantly evolved with the acyl-chain binding: H18-K29 and V58-I73. The crevice formed by the residues L16, V35, M53, P54, A57, L73, P75, M77, F107 and M138 acts as a pocket to the side-chain. Finally, the catalytic site is formed by H104, H106, D108, H109, H169, D191, Y194 and H235 (Liu et al., 2005; Kim et al., 2005). Although the enzyme is active against a range of N-acyl-HSL substrates it does not cleaves HSL itself. It has led to the suggestion that the amide linkage may be essential for proper substrate binding (Kim et al., 2005). Despite those data, analysis of the reaction products after incubation of racemic C6-HSL with AHL confirms that this enzyme hydrolyzes the lactone and not the amide bond of the substrate (Thomas et al., 2005).

One proposed catalytic mechanism is based on a nucleophilic attack by a water/hydroxide, bridging the two zinc ions, on the substrate's carbonyl carbon. The carbonyl bond would become more susceptible to the nucleophilic attack due to an enhanced 
polarization caused by the interactions with the zinc ions with the lactone ring and the carbonyl oxygens of the HSL. Both D108 and Y194 have been proposed to play a strong role in the catalysis (Liu et al., 2005; Kim et al., 2005). HSL binding to lactonases could possibly occur in two main distinctive configurations regarding the interaction of the lactone ring and carbonyl oxygen atom to the two zinc ions (Figure 1). Additionally, the positioning of the oxygens of the lactone ring can lean towards D108 or Y194 (si and re face, respectively) could lead to further insights into the catalytic mechanism. Y194 could either serve as a hydrogen-bonding partner stabilizing the tetrahedral adduct (Liu et al., 2005) or a general acid to protonate the leaving group (Liu et al., 2005; Kim et al., 2005). Alternatively, D108 has been proposed to act as a proton shuttle if the substrate binding takes place as in mode-2 and si face (Liu et al, 2005). Aiming to elucidate this problem Kim and co-workers reported the crystal structure of BTKAiiA complexed with a L-HSL molecule (Kim et al., 2005). In this study, the enzyme did not show any significant conformational change upon HSL binding (0.13 $\AA$ root-mean-square deviations for the protein $\mathrm{C}_{\alpha}$ atoms). HSL binding to the enzyme active site presented 0.75 occupancy with an average B-factor of $34.9 \AA^{2}$. The HSL carbonyl group was found to bind the Zn2 (see Figure 1) located in close proximity of the side chain of D108, while the ring oxygen is sandwiched between Zn-1 (see Figure 1) and the hydroxyl group of Y194 (mode-1 in Figure 1). The lactone nitrogen atom lies within at $3.3 \AA$ from D108-O $\delta 2$, hence suggesting the presence of a hydrogen-bond between these two groups. The authors naturally assume that the binding of the HSL molecule is representative of the substrate binding, however other possibilities for substrate binding orientation have been reasoned based on the crystal structure of BTK-AiiA complexed with glycerol (Liu et al., 2005).

Kinetic and substrate specificity measurements have showed that AiiA displays no or little residual activity to non-acyl lactones (Wang et al., 2004; Kim et al., 2005). Based on the fact that the presence of $\mathrm{N}$-acyl chain is sine qua non to the catalysis, N-formyl-L-homoserine lactone (NFH) was proposed to serve as the lactonase simplest substrate (Kim et al., 2005). The backbone chain amide groups of F107 and D108 have been suggested to possibly orient the acyl carbonyl helping the AHLs fitting into the active site pocket, while I73 and M138 have been proposed to take part in the $\mathrm{N}$-acyl chain binding (Kim et al., 2005). Those interactions between the substrate's N-acyl group and the enzyme would explain why HSL itself cannot be hydrolyzed.

In order to verify the influence of the $\mathrm{N}$-acyl group in the binding of the lactone ring to the lactonases, molecular dynamics simulations of the BTK-AiiA have been carried out for the enzyme free in solution, complexed with HSL and NFH (Figure 2). Our results show that the lactone ring can only bind to BTK-AiiA in mode- 1 and that the conformational variability of the lactone ring in the protein active is diminished upon attachment of a $\mathrm{N}$-formyl group to the ligand.

\section{METHODOLOGY}

\section{Molecular Systems}

The crystal structure of the homoserine lactone hydrolase (HSL) with Protein Data Bank (PDB) entry code 2A7M solved at $1.6 \AA$ resolution, (Liu et al., 2005) was used as a starting point for all simulations. Three systems were considered: i) AiiA: explicit-solvated protein with one hydroxyl and two zinc ions in the active site; ii) AiiA-HSL: AiiA complexed with a homoserine-lactone molecule (HSL); and iii) AiiA-NFH: AiiA complexed with Nformyl-homoserine lactone (NFH). Initial positions for HSL and NFH molecules were modeled by positioning their carbonyl and ring oxygen atoms in respect to the enzyme active site as described in the crystal structure of the AiiA complexed with HSL (Kim et al., 2005) (mode-1 in Figure 1). Although the latter, a $1.7 \AA$ resolution AiiA with PDB entry code 2BR6 have been 
resolved with the atomic positions for the homoserine lactone into the enzyme active site, the atomic positional coordinates of the protein residues 61-72 have not been determined. Therefore, HSL and NFH were modeled onto the coordinates for the PDB ID entry 2A7M (Liu et al., 2005) instead.

In all cases, simulations were performed under periodic boundary conditions based on a cubic box of initial edge lengths $7.8 \mathrm{~nm}$. The systems were solvated by filling the box with extended simple-point-charge (SPC/E) water molecules (Berendsen et al., 1987) imposing a minimum distance of $0.28 \mathrm{~nm}$ between water molecules and any solute atom, and preserving the coordinates of the 180 crystallographic water molecule oxygen atoms. The distances between the $\mathrm{Zn}^{2+}$ ions and their corresponding coordinating histidine nitrogen atoms were treated as a bonded-term with an equilibrium distance of $0.20 \mathrm{~nm}$ and $4.184 \times 10^{4} \mathrm{~kJ} / \mathrm{mol}$ force constant. Histidines 104, 109, 169, 235 were protonated on their $\delta$-nitrogen atom. All the other histidines displayed the proton at their $\varepsilon$-nitrogen atom. A total of 7 water molecules with the highest electrostatic potential at the oxygen site, and a minimum distance of $0.8 \mathrm{~nm}$ from any protein atom, were successively replaced by $6 \mathrm{Ca}^{2+}$ and $1 \mathrm{Na}^{+}$ions in order to neutralize the systems. This procedure resulted in the following systems: i) AiiA: 1 protein molecule (3,948 atoms), $2 \mathrm{Zn}^{2+}$ ions, 1 hydroxyl anion $\left(\mathrm{OH}^{-}\right)$, 7 counter ions, and 14,512 water molecules (47,495 atoms); ii) AiiA-HSL: 1 protein molecule (3,948 atoms), 1 homoserine lactone molecule (HSL), $2 \mathrm{Zn}^{2+}$ ions, $1 \mathrm{OH}^{-}, 7$ counter ions, and 14,509 water molecules (47,500 atoms); and iii) AiiA-NFH: 1 protein molecule (3,948 atoms), $1 \mathrm{~N}$-formyl homoserine lactone (NFH), $2 \mathrm{Zn}^{2+}$ ions, $1 \mathrm{OH}^{-}, 7$ counter ions, and 14,460 water molecules (47,358 atoms).

\section{Molecular Dynamics Simulations}

All simulations were performed using the AMBER96 force field (Cornell et al., 1995) in conjunction with newly-derived charges for the HSL and NFH molecules (procedure described below) and implemented in the parallel version of the NWChem-4.7 program (Apra et al., 2005). The systems were equilibrated in a stepwise fashion. First, the solvent and the substrates (when present) were relaxed using up to 5000 steps of steepest descent energy minimization, keeping the protein and ions fixed. This was followed by an additional 500 steps of minimization without solute constraints. System equilibration was continued by performing successive 10 ps of MD runs at 50, 100, 150, 200, 250, and $300 \mathrm{~K}$, with velocity reassignments every 0.4 ps. Following the equilibration time, production MD runs were carried out in the isothermal-isobaric ensemble for 4.0 ns. The temperature was maintained at $300 \mathrm{~K}$ by weak coupling the solute and solvent separately to a heat bath (Berendsen et al., 1984) with a relaxation time of $0.1 \mathrm{ps}$. The pressure was kept at $1.025 \times 10^{5}$ Pascal using weak coupling to a pressure bath (Berendsen et al., 1984) via isotropic coordinate scaling with relaxation time of 0.1 ps. Long-range electrostatic interactions were handled using the smooth particle mesh Ewald (SPME) method (Essmann et al., 1995) A cutoff $1.0 \mathrm{~nm}$ was used for the short-range non-bonded interactions (van der Waals and real-space electrostatic contribution). The SHAKE (Ryckaert et al., 1977) algorithm was applied to constrain all bonds involving a hydrogen atom, and a 2-fs timestep was used to integrate the equations of motion based on the leapfrog algorithm (Hockney, 1970). Trajectory frames were recorded every 0.2 ps for analysis.

\section{Charge Model}

The present simulations were carried out using the AMBER96 force field (Cornell et al., 1995) to describe the hydroxyl ion and the HSL and NFH substrate molecules, except for the charges, which were not readily available and hence needed to be derived. Based on the optimized geometry of the hydroxyl anion and the HSL and NFH substrates, Hartree-Fock calculations at the 6-31G* level followed by a restrained electrostatic potential (RESP) fit 
(Bayly et al., 1995) were performed using the NWChem4.7 program (Apra et al., 2005) to compute the partial charges. Values of 0.005 a.u. for the harmonic and 0.001 a.u. for the hyperbolic restraints, respectively, were used in the RESP fittings. Charge derivation by means of RESP fitting preserves the dipole moment of a molecule to its quantum-mechanical value, and should therefore lead to a realistic description of intermolecular interactions with identical as well as distinct molecular species during the simulations. The charge-derivation procedure was performed according to the AMBER96 force field philosophy (Cornell et al., 1995) in order to preserve force field compatibility. The final charge sets for the above-mentioned ion/molecules are shown in Table I.

\section{RESULTS AND DISCUSSION Protein Flexibility}

The root-mean-squared deviations (RMSD) of all protein atoms were calculated over 2 and 4 ns trajectories taking as a reference frame the crystallographic structure PDB ID 2A7M. The time evolution of the RMSD with respect to the 3D structure provides a measurement of the convergence of the dynamical properties of the protein. The RMSD ranges from 0.025 to $0.225 \mathrm{~nm}$ (Figure 3). All four studied systems presented RMSDs higher than the BTK-AiiA lactonase without substrates, suggesting that the homoserine lactone ring increases the displacement of BTK-AiiA lactonase. N-formyl homoserine lactone (mode 1) promotes a slightly higher RMSD even when was compared at 2 and 4 ns trajectories.

The root-mean-square atomic positional fluctuations (RMSF) of NFH and HSL (mode1) and HSL free systems showed wide deviations from the B-factor 3D crystallographic obtained, when the most flexible protein portions were observed (Figure-4). There are six flexible loops formed by the residues 22-28 (loop 1), 53-78 (loop 2), 125-138 ( $\alpha 3$ and loop 3), 175-186 (loop 4) 190-209 (loop 5) and 220-233 (loop 6). The loops 1 and 2 showed marked increase of RMSF; 0.7 and 0.8 , respectively. These loops are responsible to fit the $\mathrm{N}$-acyl substrate chain into the hydrophobic channel (Kim et al., 2005; date no shown).

Previous experimental data showed 0.75 occupancy and an average $B$-factor of $34.9 \AA^{2}$ for HSL. Our calculated $B$-factors from the 4 ns MD trajectory (equilibration excluded) for HSL and NFH were ca. $46 \AA^{2}$ and ca. $23 \AA^{2}$, respectively, suggesting that the NFH is more stable into the BTK-AiiA lactonase catalytic site.

\section{HSL and NFH binding modes}

Wang et al., 2004, using biochemical assays, presented specificity and kinetics data for BTK-AiiA lactonase testing several substrates. The lactonase showed low activity for the HSL. Others workers hypothesize that HSL is not a substrate indeed, but an inhibitor (Kim et al., 2005; Liu et al., 2005) and the N-acyl chain is necessary to the lactonase catalysis, although the enzyme cleaves at the carbon carbonyl of lactone ring.

We did measurements of the distances from zinc ions to the leaving group and carbonyl oxygens of the lactone ring (Figure 5).

The carbonyl oxygen distances of the HSLs to the nearby zinc are constant at ca. 0.2 $\mathrm{nm}$ in all simulated systems. The leaving group oxygen of the HSL (modes 1 and 2) also presented similar zinc distances, ranging from ca. $0.25 \mathrm{~nm}$ to ca. $0.6 \mathrm{~nm}$ through both trajectories. These results suggest that the HSL ring do not interact with the metal site. Comparing HSL and NFH trajectories, we observed that the HSL ring, both modes 1 and 2, showed tree configurations at the catalytic site, changing the position of the lactone ring plane related to the zinc site (data no shown). $\mathrm{NFH}$-mode 2 rotates its leaving group backwards to the metal site, presenting a zinc distance around $0.45 \mathrm{~nm}$ at the $2 \mathrm{~ns}$ simulation. Only NFH-mode-1 remains near to the zinc site with a leaving group oxygen distance of ca. $0.325 \mathrm{~nm}$, therefore 
binding the enzyme in a stable manner. These zinc ion/oxygen interactions are important to attach the lactone ring at the lactonase catalytic site.

All these results confirmed the presence of an $\mathrm{N}$-acyl chain is necessary to stabilize the substrate in the catalysis site, and probably contribute to the nucleophilic attack by interactions with the zinc atoms.

\section{Important residues in the binding of lactones}

The residues F107, I73, M138, F64, M53, D108 and Y194 have been point out as important to the binding and catalysis reaction. The behavior of these and other residues nearby the active site and the binding molecule have been monitored throughout the trajectories. The analyses for the residues of identified significant importance for HSL and NFH binding are presented below.

The analysis of the distance variation between C3 (carbonyl carbon of the lactone ring), N4 (HSL and NFH), O5 (carbonyl group of N-acyl chain) and C6 (methyl group) of the Nformyl chain and those residues showed some interesting aspects of AHL binding mode.

Kim et al. 2005 and Liu at al. 2005 postulated that the catalysis is mediated by D108 and Y194 residues, the HSL can present two substrate-binding orientations (modes 1 and 2) and that the carbonyl carbon of the lactone ring can place either its si or re face towards the zinc ions.

To point light over these questions, we measured the distances between the carbonyl carbon of the lactone ring (HSL and NFH mode 1) and the OD1 of the D108 and the OH of the Y194 (Figure 6).

The C3-HSL/OH-Y194 distance variation suggested that this residue do not interact with the lactone ring. In spite the fact that the C3-HSL/OD1-D108 distance varied around ca. $0.3 \mathrm{~nm}$ over almost the entire simulation, this supposed interaction is not enough to stabilize the HSL at the activity site and do not contribute to the discrepant configurations found to HSL inside of the catalytic site.

The OD1-D108 and OH-Y194 distances to C3-NFH obtained from the trajectories were ca. $0.35 \mathrm{~nm}$ and ca. $0.3 \mathrm{~nm}$, respectively. However, the C3-NFH/OD1-D108 distance ranged less than the Y194 measurements, suggesting that the N-formyl interact with D108 in a stable manner, therefore present a si face, with the leaving group and carbonyl oxygen of the AHL ring oriented towards the $\mathrm{D} 108$ residue.

The N4/D 108 distances showed different profiles, when the HSL and NFH were compared, trough the $4 \mathrm{~ns}$ trajectories (Figure-7). HSL distances varied from ca. $0.27 \mathrm{~nm}$ to ca. $0.67 \mathrm{~nm}$ and after $2.5 \mathrm{~ns}$ of simulation ranged widely, suggesting that D 108 do not interact with the N4. However, the N-formyl-HSL remains attached at the catalytic site by the interaction with D108, which distance continue constant around ca. $0.4 \mathrm{~nm}$.

The distances between the carbonyl oxygen of the N-acyl chain of NFH (modes 1 and 2) and the amide group of F107 were ca. $0.65 \mathrm{~nm}$ and $0.4 \mathrm{~nm}$, respectively. F107 amide group showed no apparent influence on the binding configuration of the ligand (Figure 8). The NFHmode 2 distance was lower, but probably due to the spatial positioning of the initial configuration submitted to the MD and not because these residue interacts strongly with the NFH.

Although carbonyl group of the $\mathrm{N}$-formyl chain in mode-1 seems remained fully solvated and does not interact with any residue of the protein throughout the entire trajectory, the methyl group of the NFH in mode- 1 was observed to interact within a $0.5 \mathrm{~nm}$ radius with I73, F64 and F107, flanking residues of the hydrophobic channel (data not shown).

Finally, to identify the most occurring substrate-binding configurations, we calculated the clusters for HSL and NFH (mode 1) (Figure 9). HSL presented seven clusters with two 
corresponding to $52.3 \%$ and $44.8 \%$ of total and NFH presented 12 clusters, with $92.9 \%$ concentrated at the two higher (69.3\% and $23.6 \%$, respectively). The frequency and position of these two major clusters of both systems suggest that $\mathrm{N}$-formyl binds to the catalytic site in a preferential manner. The analysis of the binding modes reveals that this preferential positioning is potentially more susceptible to the BTK-AiiA lactonase cleavage.

\section{CONCLUSION}

The biological functions regulated by AHLs are considerable scientific, economic, and medical importance (Dong et al., 2000). The disestablishment of such AHL-mediated QS systems has thus been targeted as a promising approach for a novel control technique of virulence gene expression in bacterial infections (Kim et al., 2005). Understanding the molecular basis of the phenomenon, i.e., the catalytic mechanism, is therefore of crucial importance. In the present report we have used molecular dynamics simulations to characterize the binding of homoserine lactones to the BTK-AiiA active site. The absence of the $\mathrm{N}$-acyl group in these molecules leads to an increased flexibility in the active site and to a non catalytic-competent binding mode. On the other hand, the substrate is held into a better-defined binding mode via a hydrogen bond between the D108 and its N-acyl group and hydrophobic interactions between its methyl group and the side chains of I73, F64 and F107. The placement of the NFH lactone ring lies towards Y194, suggesting this residue as the potential catalytic residue. However, given the intrinsic limitations of the classical approach, further implications on the issue cannot be made at this time.

In summary, we have shown that the presence of the $\mathrm{N}$-acyl group is essential for an efficient catalysis of the homoserine lactone molecules by BTK-AiiA; characterize the binding mode for the substrate; and, identified the key-player residues for substrate binding. The importance of long $\mathrm{N}$-acyl chains in the substrate binding will be further inferred and shall soon be reported. These findings aid the understanding of the first step into the molecular basis of the quorum-quenching regulation in B. thuringiensis and shed light into the devising the control of bacterial biofilm formation.

\section{ACKNOWLDGMENTS}

The authors would like to thank the PNNL-Summer Research Institute (SRI) for financial support and Dr. Constância Ayres for insightful discussions.

\section{REFERENCES}

Bassler BL, Wright M, Showalter RE, Silverman MR. Intercellular signalling in Vibrio harveyi: sequence and function of genes regulating expression of luminescence. Mol Microbiol. 1993. Aug; 9(4):773-86.

Camilli, A. and Bassler, B. 2006. Bacterial small-molecule signaling pathways. Science. 2006 Feb 24; 311(5764):1113-6.

Davies, D. G., M. R. Parsek, J. P. Pearson, B. H. Iglewski, J. W. Costerton, and E. P. Greenberg. The involvement of cell-to-cell signals in the development of a bacterial biofilm. Science. 1998. 280:295-298.

Dong YH, Gusti AR, Zhang Q, Xu JL, Zhang LH. Identification of quorum-quenching N-acyl homoserine lactonases from Bacillus species. Appl Environ Microbiol. 2002 Apr; 68(4):1754-9. 
Dong, Y.-H., J.-L. Xu, X.-C. Li, and L.-H. Zhang. AiiA, a novel enzyme inactivates acyl homoserine-lactone quorum-sensing signal and attenuates the virulence of Erwinia carotovora. Proc. Natl. Acad. Sci. 2000. USA 97:3526-3531.

Fray, R. G.; Throup, J. P.; Daykin, M.; Wallace, A.; Williams, P.; Stewart, G.S.A.B. and Grierson, D. Plants genetically modified to produce $\mathrm{N}$-acylhomoserine lactones communicate with bacteria. Nat Biotechnol. 1999 Oct; 17(10):1017-20.

Fuqua, C.; Parsek, M. R. Greenberg, P. Regulation of Gene Expression by Cell-to-cell Communication: Acyl-Homoserine Lactone Quorum Sensing.1991. Annual Review of Genetics. Vol. 35: 439-468.

Hao, G. and Burr, T. J. Regulation of Long-Chain N-Acyl-Homoserine Lactones in Agrobacterium vitis. J Bacteriol. 2006, Mar. p. 2173-2183.

Kim MH, Choi WC, Kang HO, Lee JS, Kang BS, Kim KJ, Derewenda ZS, Oh TK, Lee CH, Lee JK. The molecular structure and catalytic mechanism of a quorum-quenching N-acylL-homoserine lactone hydrolase. Proc Natl Acad Sci U S A. 2005 Dec 6;102(49):17606-11.

Lee, S.J.; Park, S-Y.; Lee, J-J.; Yum, D. Y., Koo, B.-T. and Lee, J.-K. Genes Encoding the NAcyl Homoserine Lactone-Degrading Enzyme Are Widespread in Many Subspecies of Bacillus thuringiensis Applied and Environmental Microbiology. August 2002, p. 39193924, Vol. 68, No. 8.

Liu, D.; Lepore, B. W.; Petsko, G. A.; Thomas,P. W.; Stone, E. M.; Fast, W. and Ringe, D. Three-dimensional structure of the quorum-quenching $\mathrm{N}$-acyl homoserine lactone hydrolase from Bacillus thuringiensis. Proc Natl Acad Sci U S A. 2005 Aug 16; 102. (33):11882-7.

Nealson, K.H.; Platt, T.; Hastings, J.W. Cellular control of the synthesis and activity of the bacterial luminescent system. J Bacteriol. 1970 Oct; 104(1):313-22.

Parsek, M.R.; Val D.L.; Hanzelka, B.L.; Cronan, J.E.; and Greenberg, E.P. Acyl homoserinelactone quorum-sensing signal generation. Proc. Natl. Acad. Sci. USA 96. 1999: 43604365.

Rasmussen TB, Givskov M. Quorum sensing inhibitors: a bargain of effects. Microbiology. 2006 152:895-904.

Spoering, A. L. and Gilmore, M. S. Quorum sensing and DNA release in bacterial biofilms. Current Opinion in Microbiology. 2006. Volume 9, Issue 2 , April 2006, Pages 133-137.

Thomas PW, Stone EM, Costello AL, Tierney DL, Fast W. The quorum-quenching lactonase from Bacillus thuringiensis is a metalloprotein. Biochemistry. 2005 May 24; 44(20):755969.

Val D.L. and Cronan, J.E. Jr. In vivo evidence that S-adenosylmethionine and fatty acid synthesis intermediates are the substrates for the LuxI family of autoinducer synthases. $\mathrm{J}$ Bacteriol.1998. May;180(10):2644-51.

Zhang, L.-H., Murphy P. J., Kerr, A. and Tate, M. E.. Agrobacterium conjugation and gene regulation by N-acyl-L-homoserine lactones. Nature. 1993.362:446-447.

Berendsen HJC, Grigera JR, Straatsma TP. The missing term in effective pair potential. J Phys Chem 1987; 91: 6269-6271.

Cornell WD, Cieplak P, Bayly CI, Gould IR, Merz KM, Ferguson DM, Spellmeyer DC, Fox T, Caldwell JW, Kollman PA. A 2nd generation force-field for the simulation of proteins, nucleic-acids and organic-molecules. J Am Chem Soc 1995; 117: 5179-5197.

Berendsen HJC, Postma JPM, van Gunsteren WF, DiNola A, Haak JR. Molecular dynamics coupling to an external bath. J Chem Phys 1984; 81: 3684-3690.

Esmann U, Perera L, Berkowitz ML, Darden T, Lee H, Pedersen LG. A smooth particle mesh Ewald method. J Chem Phys 1995; 103: 8577-8593. 
Ryckaert JF, Ciccotti G, Berendsen HJC. Numerical-integration of Cartesian equations of motion of a system with constraints - molecular-dynamics of n-alkanes. J Comp Phys 1977; 23: 327-341.

Hockney RW. The potential calculation and some applications. Methods Comput Phys 1970; 9: 136-211.

Apra E., Windus TL, Straatsma TP, Bylaska EJ, de Jong W, Kowalski K, Hirata S, Valiev M, Hackler MT, Zhao Y, Harrison RJ, Dupuis M, Smith DMA, Nieplocha J, Tipparaju V, Krishnan M, Auer AA, Brown E, Cisneros G, Fann GI, Fruchtl H, Garza J, Hirao K, Kendall R, Nichols JA, Tsemekhman K, Wolinski K, Anchell J, Bernholdt D, Borowski P, Clark T, Clerc D, Dachsel H, Deegan M, Dyall K, Elwood D, Glendening E, Gutowski M, Hess A, Jaffe J, Johnson B, Ju J, Kobayashi R, Kutteh R, Lin Z, Littlefield R, Long X, Meng B, Nakajima T, Niu S, Pollack L, Rosing M, Sandrone G, Stave M, Taylor H, Thomas G, van Lenthe J, Wong A, and Zhang Z, "NWChem, A Computational Chemistry Package for Parallel Computers, Version 4.7" (2005), Pacific Northwest National Laboratory, Richland, Washington 99352-0999, USA.

Kim MH, Choi WC, Kang HO, Lee JS, Kang BS, Kim KJ, Derewenda ZS, Oh TK, Lee CH, LEE JK. The molecular structure and catalytic mechanism of a quorum-quenching N-acylL-homoserine lactone hydrolase. Proc. Natl. Acad. Sci. 2005; 102 17606-17611.

Liu D, Lepore BW, Petsko GA, Thomas PW, Stone EM, Fast W, Ringe D. Threedimensional structure of the quorum-quenching N-acyl homoserine lactone hydrolase from Bacillus thuringiensis. Proc. Natl. Acad. Sci. 2005; 1023:11882-11887.

Wang L-H, Weng L-X, Dong Y-H, and Zhang LH (2004) Specificity and Enzyme Kinetics of the Quorum-quenching N-Acyl Homoserine Lactone Lactonase (AHL-lactonase). J. Biol. Chem. 279: 13645-13651.

\section{FIGURES}
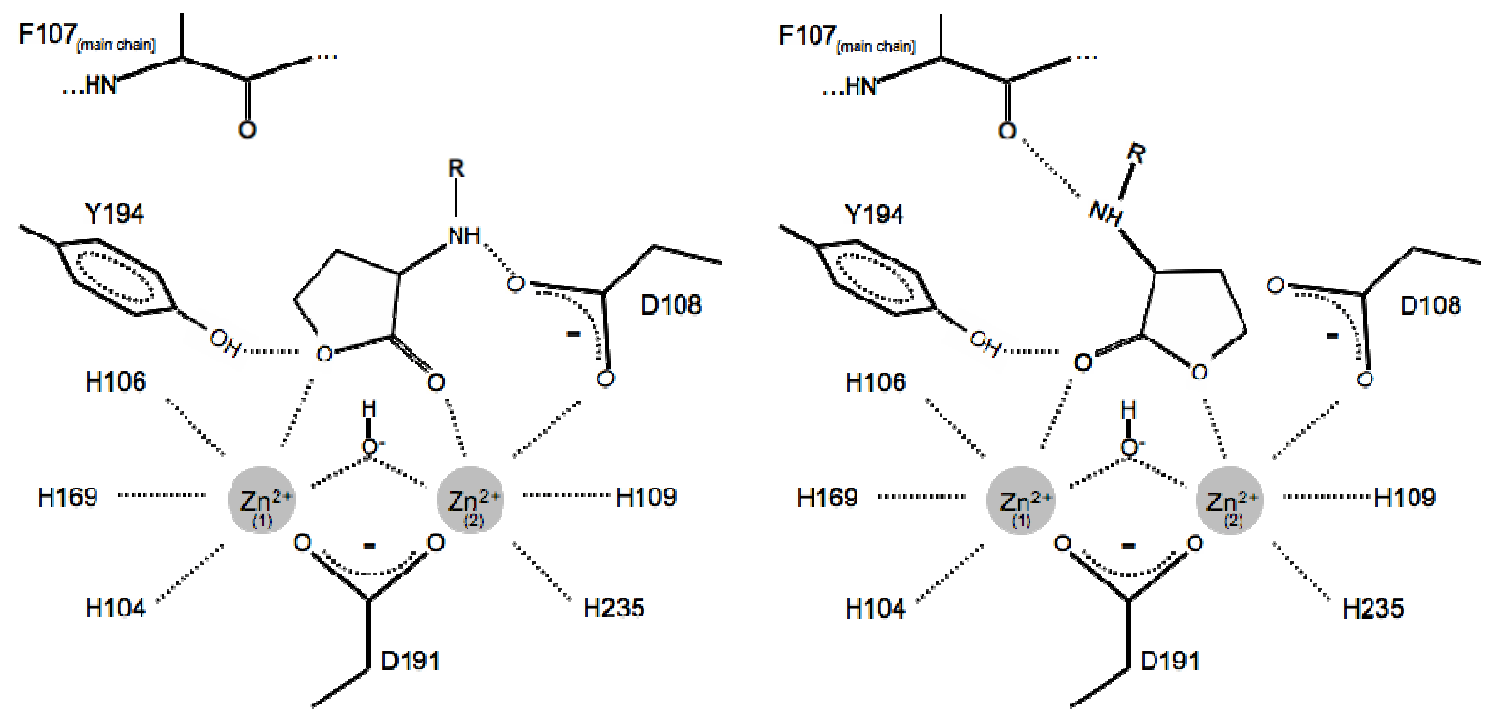

Figure 1. Hypothetical HSL binding modes. Mode 1 shown at left and 2 at right. (si and re faces involve a rotation orthogonal to the lactone ring in respect to D108 and Y194 residues; their representations are not shown here). 
PNNL-16185
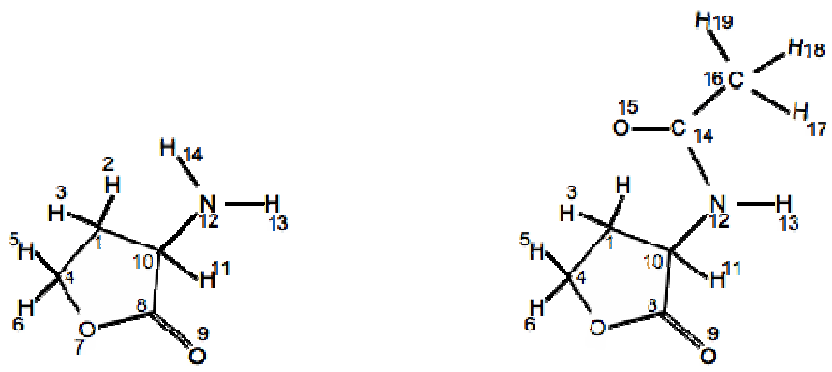

Figure 2. HSL (left) and NFH (right) molecules. (Atom numbers are for index purposes only).

\section{TABLES}

Table I. Atomic point charges for HSL and NFH. (Atoms are indexed according Figure

2).

\begin{tabular}{lcc}
\hline Index & HSL atoms $(e)$ & NFH atoms $(e)$ \\
\hline C1 & -0.199 & -0.199 \\
H2 & +0.062 & +0.062 \\
H3 & +0.062 & +0.062 \\
C4 & +0.240 & +0.240 \\
H5 & +0.014 & +0.014 \\
H6 & +0.014 & +0.014 \\
O7 & -0.447 & -0.447 \\
C8 & +0.716 & +0.716 \\
O9 & -0.605 & -0.605 \\
C10 & +0.395 & +0.395 \\
H11 & +0.024 & +0.024 \\
N12 & -0.815 & -0.815 \\
H13 & +0.360 & +0.360 \\
H14 $($ HSL) $/$ C14 $($ NFH) & +0.360 & +0.778 \\
O15 & & -0.599 \\
C16 & & -0.075 \\
H17 & & +0.025 \\
H18 & & +0.025 \\
H19 & & +0.025 \\
Net Charge & & $\mathbf{0 . 0 0 0}$ \\
\hline
\end{tabular}


PNNL-16185

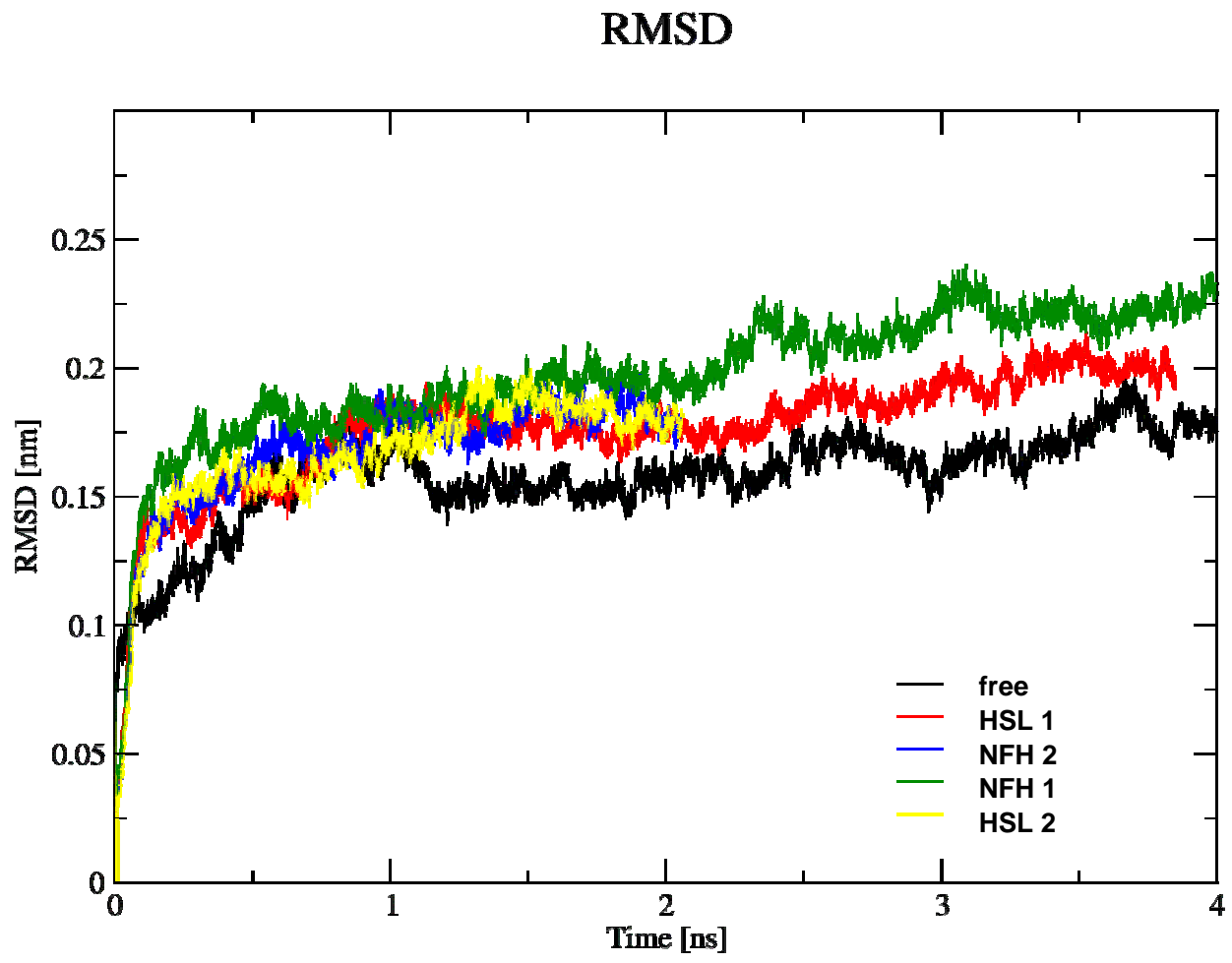

Figure-3: Root-mean-square atomic positional deviations (RMSD) from the AiiABTK crystallographic structure.

\section{RMSF}

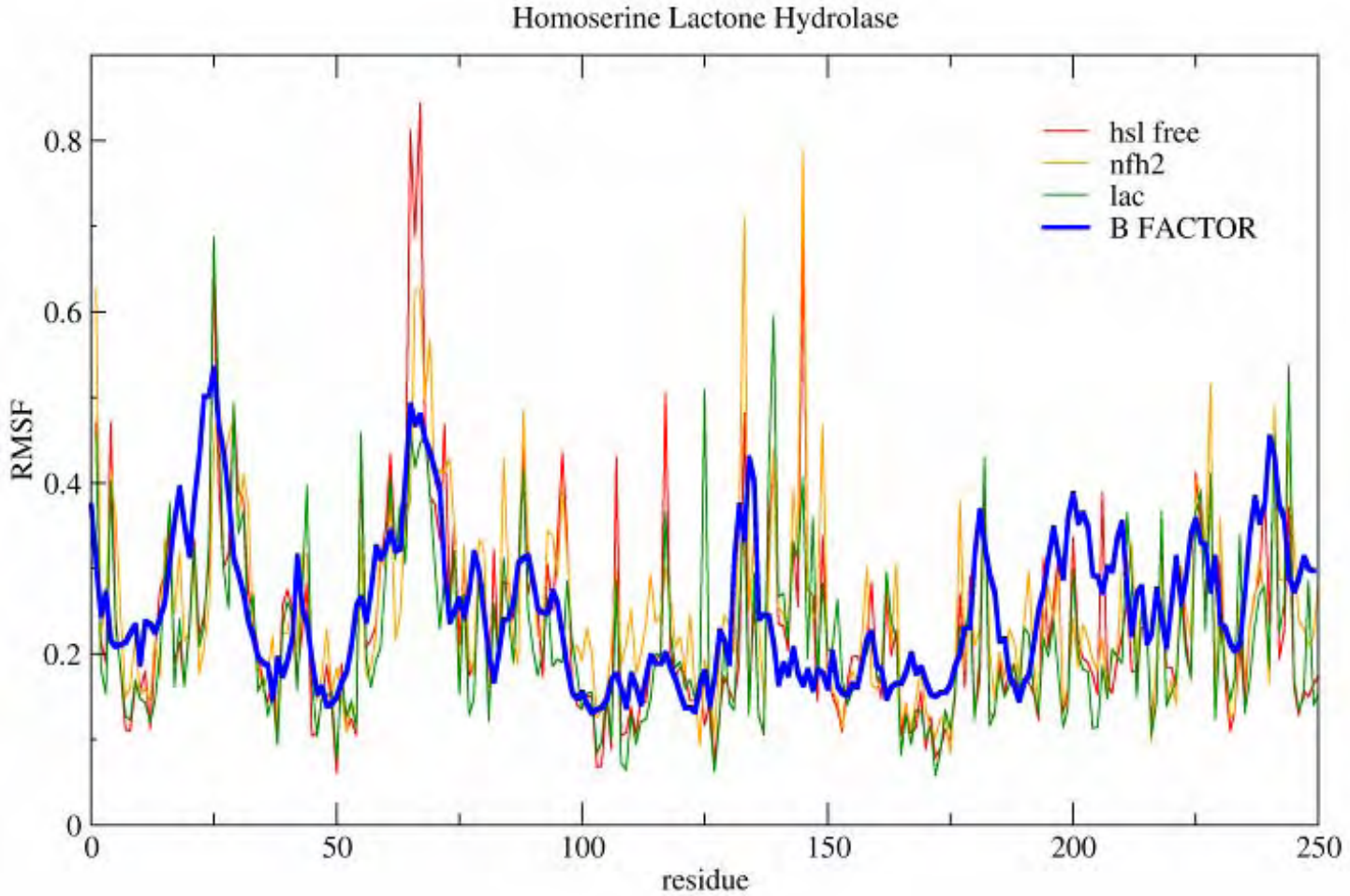

Figure-4: Root-mean-square atomic positional fluctuations (RMSF) 
PNNL-16185
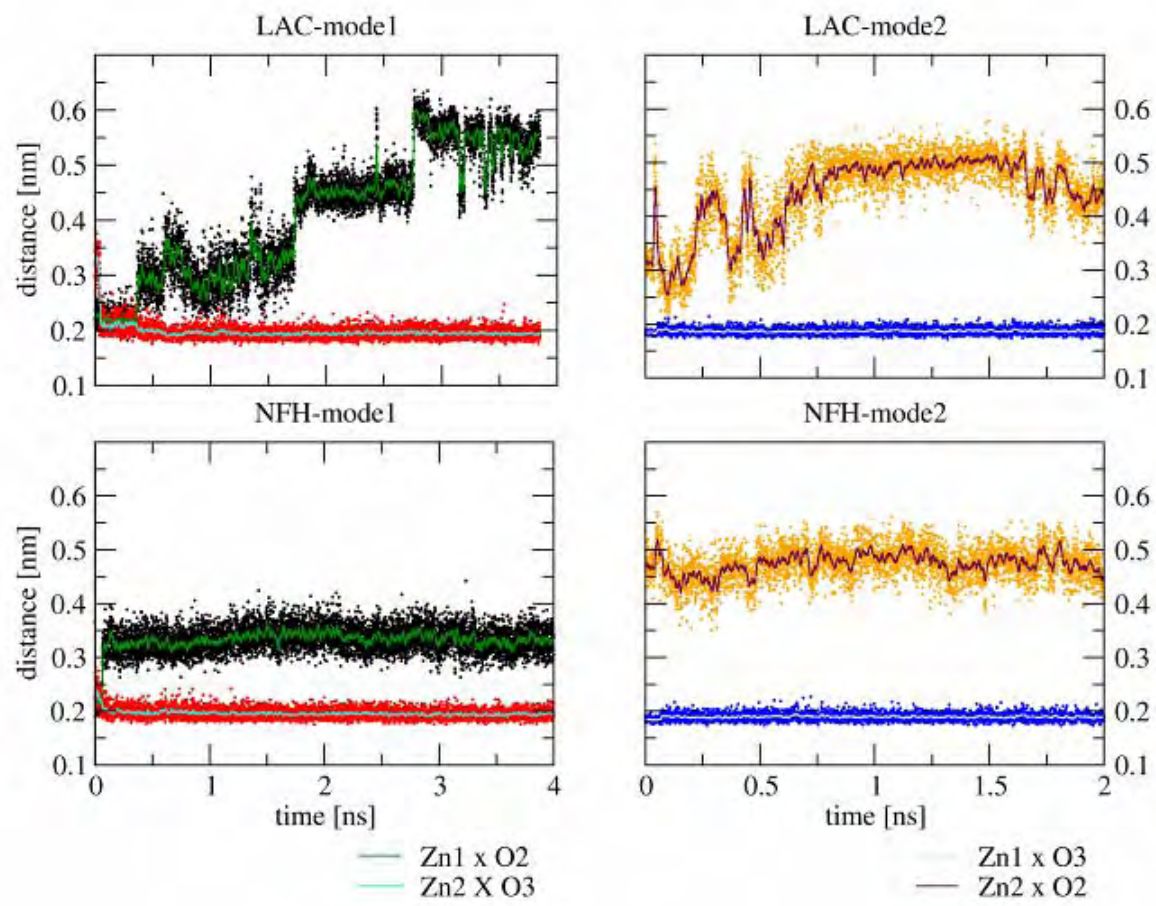

Figure-5: Distance variation between HSL oxygens and zinc atoms.
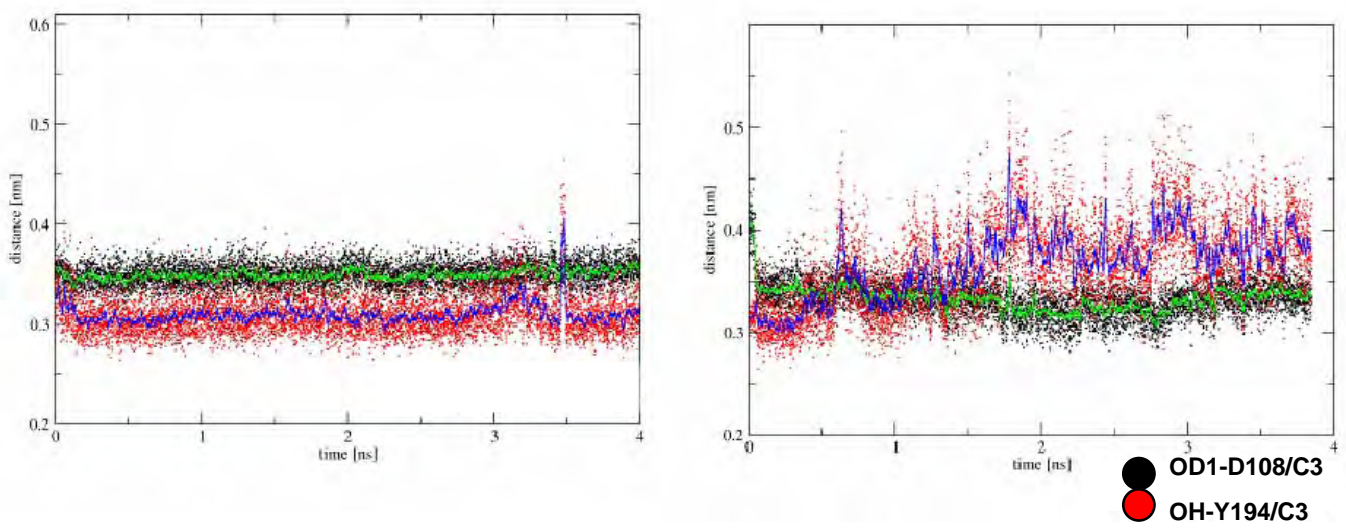

Figure- 6: Distance variation between the C3 of HSLs (mode 1) and two residues pointed out as responsible for the catalysis, OD1 of D108 and OH of Y194, respectively. A- N-formylhomoserine lactone. B- Homoserine lactone 
PNNL-16185

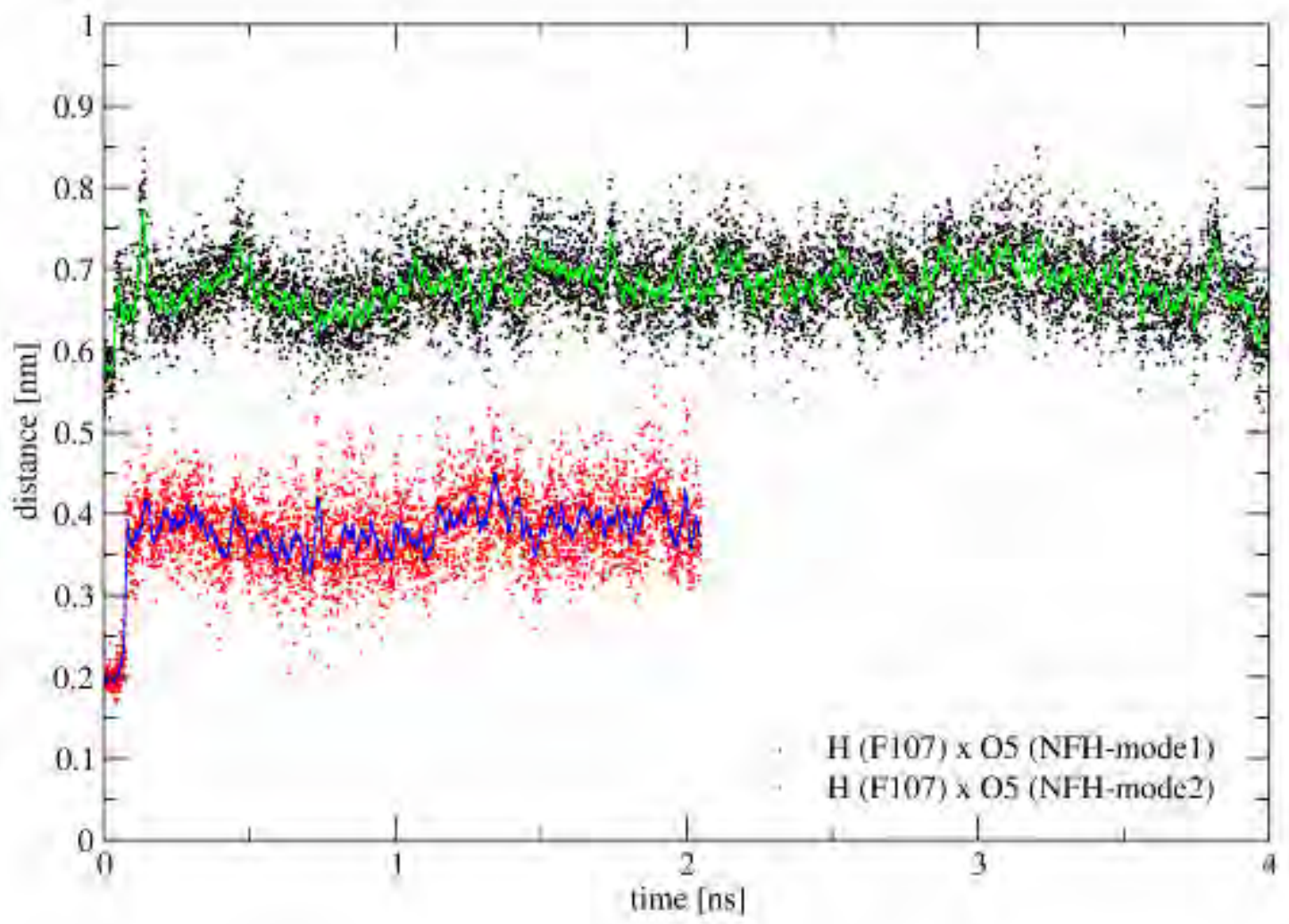

Figure-7: Distance variation between OD1 of the aspartic acid 108 and N4 of the HSLs mode-

1.

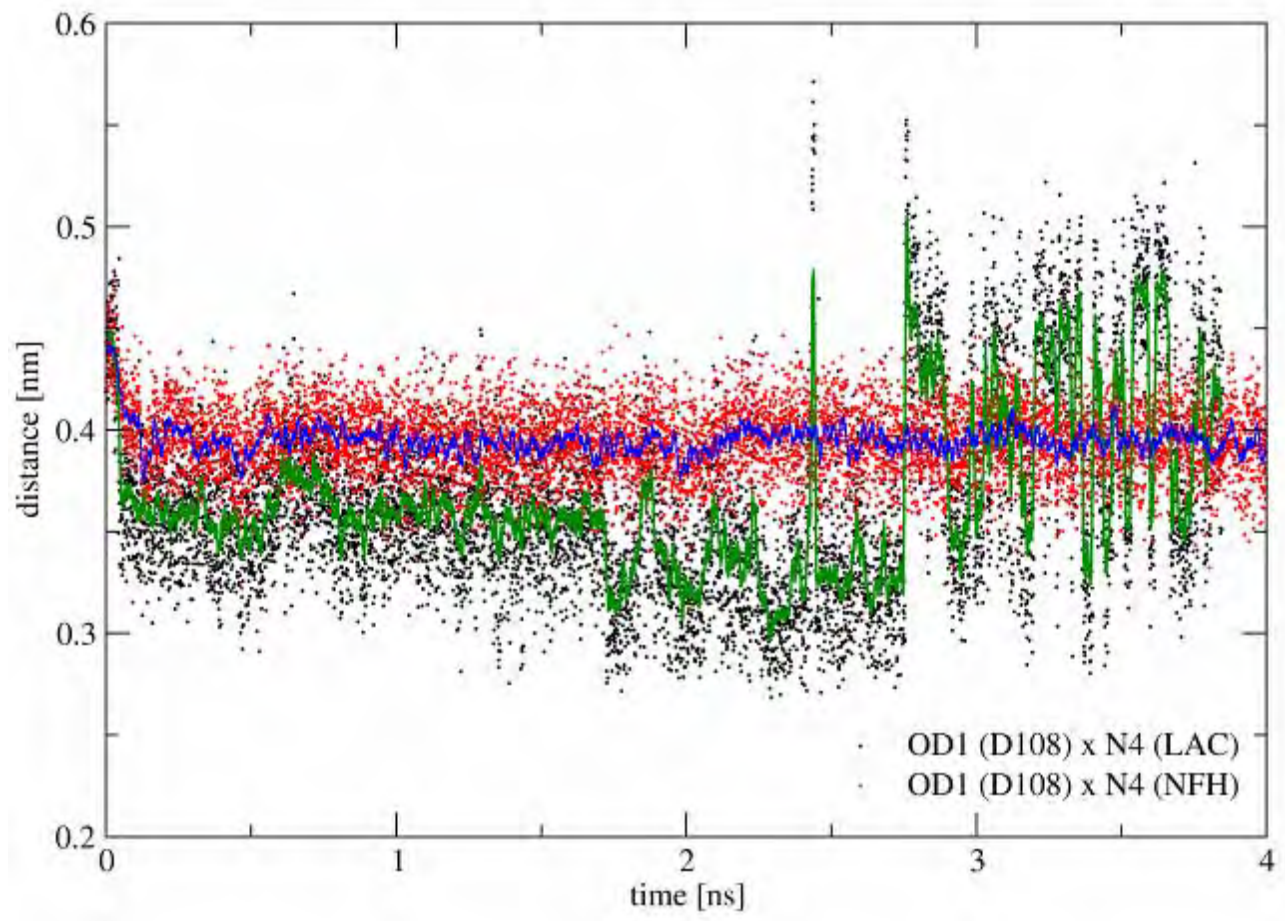

Figure-8: Distance variation between O5 ( $\mathrm{N}$-acyl chain) of $\mathrm{N}$-formyl homoserine lactone and F107 main chain amide group. 
PNNL-16185

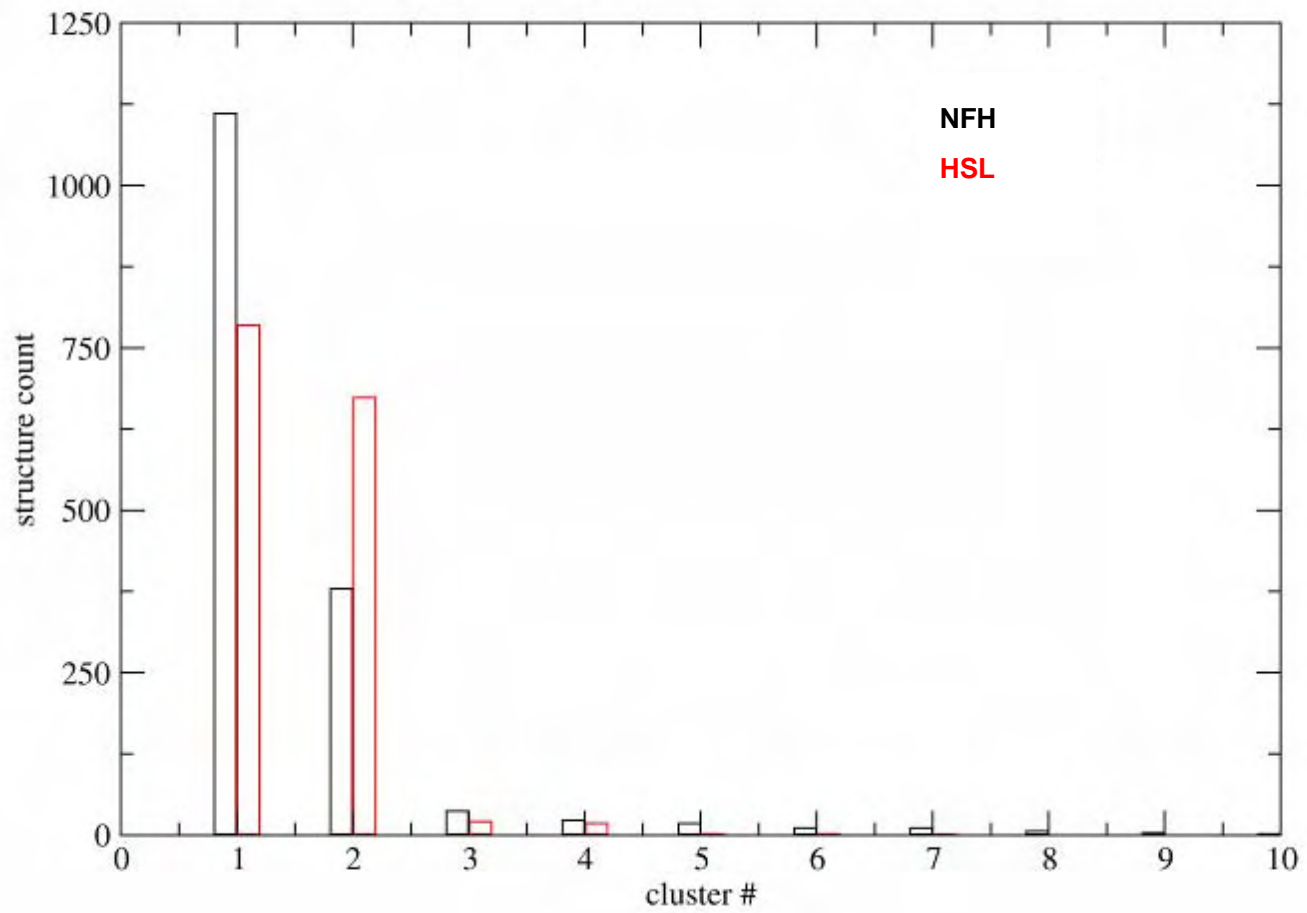

Figure-9: LAC and NFH most occurring configurations 
PNNL-16185 
PNNL-16185

\title{
Characterization of Polyaniline-Gold Nanocomposites
}

\author{
Amir Saheb \\ Mira Josowicz, Jiri Janata \\ Georgia Institute of Technology, School of Chemistry and Biochemistry \\ Atlanta, Georgia 30332 \\ and \\ Donald Baer \\ Pacific Northwest National Laboratory \\ Richland, WA 99354
}

amir.saheb@chemistry.gatech.edu Tel : 404.894.0589

mira.josowicz@chemistry.gatech.edu, jiri.janata@chemistry.gatech.edu

\section{Introduction}

The objective of this study was to explore the possibility of growing gold clusters containing low number of atoms, using the ability of PANI to switch from the conductive to insulating form and to use the ability of imine nitrogens to form strong chloroaurate complexes. Under normal electrochemical conditions as described in our paper the imine sites act as catalytic nucleation centers for forming the gold clusters containing large number of Au atoms ${ }^{1}$.

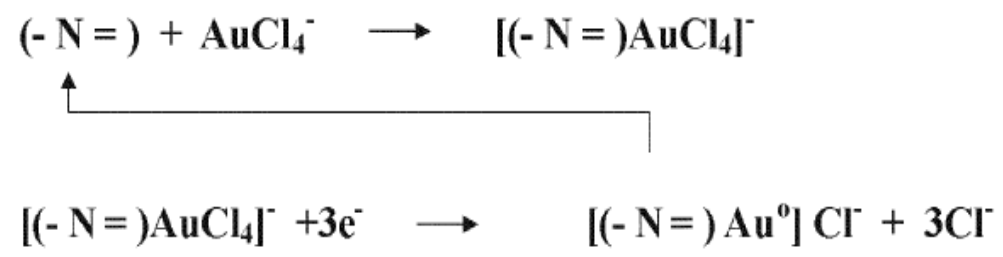


We applied novel synthetic conditions that should lead to the formation of lowatomic Au dispersion in PANI. We then conducted analysis that would help us to characterize the size, of the monodispersed Au atoms in the composite material.

\section{Experimental}

Three types of differently synthesized Au-PANI nano-composite materials were prepared using the following processes:

A. Fully electrochemical process.

A thin PANI film was deposited electrochemically on Pt-foil from solution containing 0.1 $\mathrm{M}$ aniline and $2 \mathrm{M} \mathrm{HBF}_{4}$. After the synthesis a cyclic voltammogram (CV) of the PANI film was recorded in $2 \mathrm{M} \mathrm{HBF}_{4}$. Then potential of $1 \mathrm{~V}$ vs. the $\mathrm{Ag} / \mathrm{AgCl}$ reference electrode in $0.1 \mathrm{M} \mathrm{KCl}$ solution was applied to the Pt-PANI electrode. As the potential was held at $1 \mathrm{~V}$ a $1 \mathrm{mM}$ solution of $\mathrm{KAuCl}_{4}$ in $0.1 \mathrm{M} \mathrm{HCl}$ was added to the PANI to form $\mathrm{AuCl}_{4} / \mathrm{PANI}$ complex. After that the cell was washed again with $0.1 \mathrm{M} \mathrm{KCl}$ for 12 minutes to remove excess $\mathrm{AuCl}_{4}{ }^{-}$and as the final step, a potential of $0.4 \mathrm{~V}$ was applied for 15 second to form $\mathrm{Au}(0)$. Samples with $\mathrm{n}=1,2,3,4,5$ and 8 repetitions of the above procedure were prepared, each containing $\mathrm{Au}_{\mathrm{n}}$ clusters. For comparison also PANI thin films on Pt-foils were prepared where the same treatment was done, but without $\mathrm{KAuCl}_{4}$ salt.

\section{B. Electrochemical-chemical process.}

Thin PANI films were electropolymerized using cyclic voltammetry (25 cycles) on Pt foils as described above and the background CV was obtained in $1 \mathrm{M} \mathrm{HCl}$. After that the chemical oxidation-reduction treatments of the PANI films were carried out chemically, using Ce(IV) and hydrazine, respectively. This process led to the formation of PANI-Au atomic clusters. After the preparation the PANI-Au materials were characterized by recording cyclic voltammograms (CV) in $1 \mathrm{M} \mathrm{HCl}$ solution. With the help of CVs the oxidation potential, $\mathrm{E}_{\mathrm{ox}}$, of $\mathrm{Au}$ to $\mathrm{AuCl}_{4}{ }^{-}$and the reduction potentials, $\mathrm{E}_{\text {red, }}$ of $\mathrm{AuCl}_{4}{ }^{-}$to $\mathrm{Au}$ as well as the charge under the oxidation, $\mathrm{Au}$ ox and reduction peaks, $\mathrm{Au}$ red were determined. The results are summarized in Table 1.

The solution used in chemical oxidation reaction were:

- $\quad 0.025 \mathrm{M} \mathrm{Ce}\left(\mathrm{SO}_{4}\right)_{2}$ in $1 \mathrm{M} \mathrm{HCl}$

- $\quad 110^{-5} \mathrm{M} \mathrm{KAuCl}_{4}$ in $1 \mathrm{M} \mathrm{HCl}$

- $\quad 0.1$ M Hydrazine dichloride

The first step consisted of a 20 sec treatment with $0.025 \mathrm{M} \mathrm{Ce}\left(\mathrm{SO}_{4}\right)_{2}$ in $1 \mathrm{M} \mathrm{HCl}$. This oxidized the PANI film from the emeraldine salt to the pernigraniline salt. Then the film was submerged in a $10^{-5} \mathrm{M} \mathrm{KAuCl}_{4}$ in $1 \mathrm{M} \mathrm{HCl}$ for 30 secs after which it was treated with a fresh solution of $0.025 \mathrm{M} \mathrm{Ce}\left(\mathrm{SO}_{4}\right)_{2}$ in $1 \mathrm{M} \mathrm{HCl}$ for 5 minutes washing out the unreacted gold. In addition, 5 mins of rinsing with deionized water was performed before the final $20 \mathrm{sec}$ treatment with $0.1 \mathrm{M}$ hydrazine dichloride to reduce $\mathrm{Au}^{3+}$ into metallic 
$\mathrm{Au}^{0}$. This would complete one cycle in the gold cluster growth at the imine site, further cycles would add one gold atom at a time.

C. Chemical process.

PANI-base of $5000 \mathrm{kD}$ obtained from Aldrich was used as a starting material. After treatment with $10 \mathrm{M} \mathrm{NH}_{4} \mathrm{OH}$, filtering and cleaning with methanol the material was converted to PANI salt using $1 \mathrm{M} \mathrm{HCl}$. After filtration the PANI was treated with the same chemical-oxidation solutions that were used in the above process (B). The samples were collected from the filtering funnel after the consecutive treatments $n=1,23,5,7$ and 10, washed with $1 \mathrm{M} \mathrm{HCl}$ and dried in desiccator overnight. It is expected that these materials will contain $\mathrm{PANI} / \mathrm{Au}_{\mathrm{n}}$ composites.

XPS measurements were then performed using a Physical Electronics Quantum 2000 Scanning ESCA Microprobe. This system uses a focused monochromatic Al Ka Xrays $(1486.7 \mathrm{eV})$ source and a spherical section analyzer. The instrument has a 16 element multichannel detector. The X-ray beam used was a $100 \mathrm{~W}, 100 \mu \mathrm{m}$ diameter beam that was rastered over a $1.4 \mathrm{~mm}$ by $0.2 \mathrm{~mm}$ rectangle on the sample. The X-ray beam is incident normal to the sample and the photoelectron detector was at $45^{\circ}$ off-normal. Wide scan data was collected using a pass energy of $117.4 \mathrm{eV}$. For the $\mathrm{Ag} 3 \mathrm{~d}_{5 / 2}$ line, these conditions produce FWHM of better than $1.6 \mathrm{eV}$. Narrow scan or high energy resolution data is collected using a pass energy of 46.95. For the $\mathrm{Ag} 3 \mathrm{~d}_{5 / 2}$ line, these conditions produced FWHM of better than $0.98 \mathrm{eV}$. The binding energy (BE) scale is calibrated using the $\mathrm{Cu} 2 \mathrm{p}_{3 / 2}$ feature at $932.62 \pm 0.05 \mathrm{eV}$ and $\mathrm{Au} 4 \mathrm{f}$ at $83.96 \pm 0.05 \mathrm{eV}$ for known standards.

Several other elemental analysis techniques were utilized including HRTEM, TOF-SIMS, Laser ablation-MS, and ICP-MS. However, due to the trace amounts and small size (angstrom level) of the gold clusters these proved ineffective in detecting the atomic clusters. In addition, PIXE (proton induced x-ray emission) is currently being conducted on the PANI-Au powder samples at EMSL as an alternative to XPS.

\section{$\underline{\text { Results }}$}

Both wide and narrow scan XPS, Figure 1 and 2, was performed on the PANI-Au film samples. However, only the narrow scan spectra was analyzed for quantitative atomic concentrations of $\mathrm{Au}, \mathrm{N}, \mathrm{C}, \mathrm{O}$, and $\mathrm{Cl}$.

XPS analysis of the electrochemically prepared (Procedure A) PANI-Au samples showed a correlation between the number of gold treatments and the atomic concentration of $\mathrm{Au}$ in the sample with respect to N, Figure 3. The consistency of the films were also probed by measuring the N:C atomic ratio as plotted in Figures 4 and 7. 
PNNL-16185

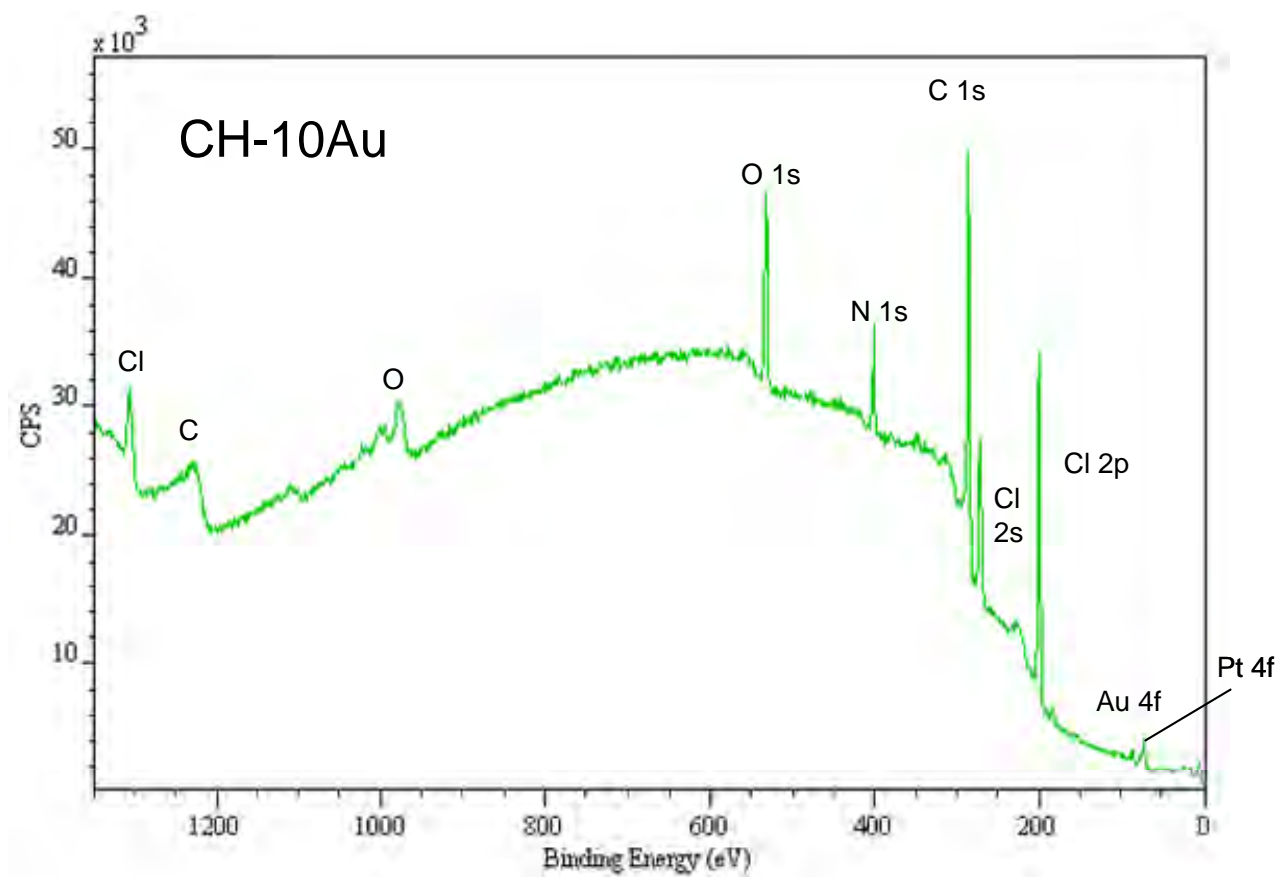

Figure 1. Wide scan XPS of PANI-Au films prepared chemically with 10 consecutive treatments of $\mathrm{AuCl}_{4}{ }^{-}$.

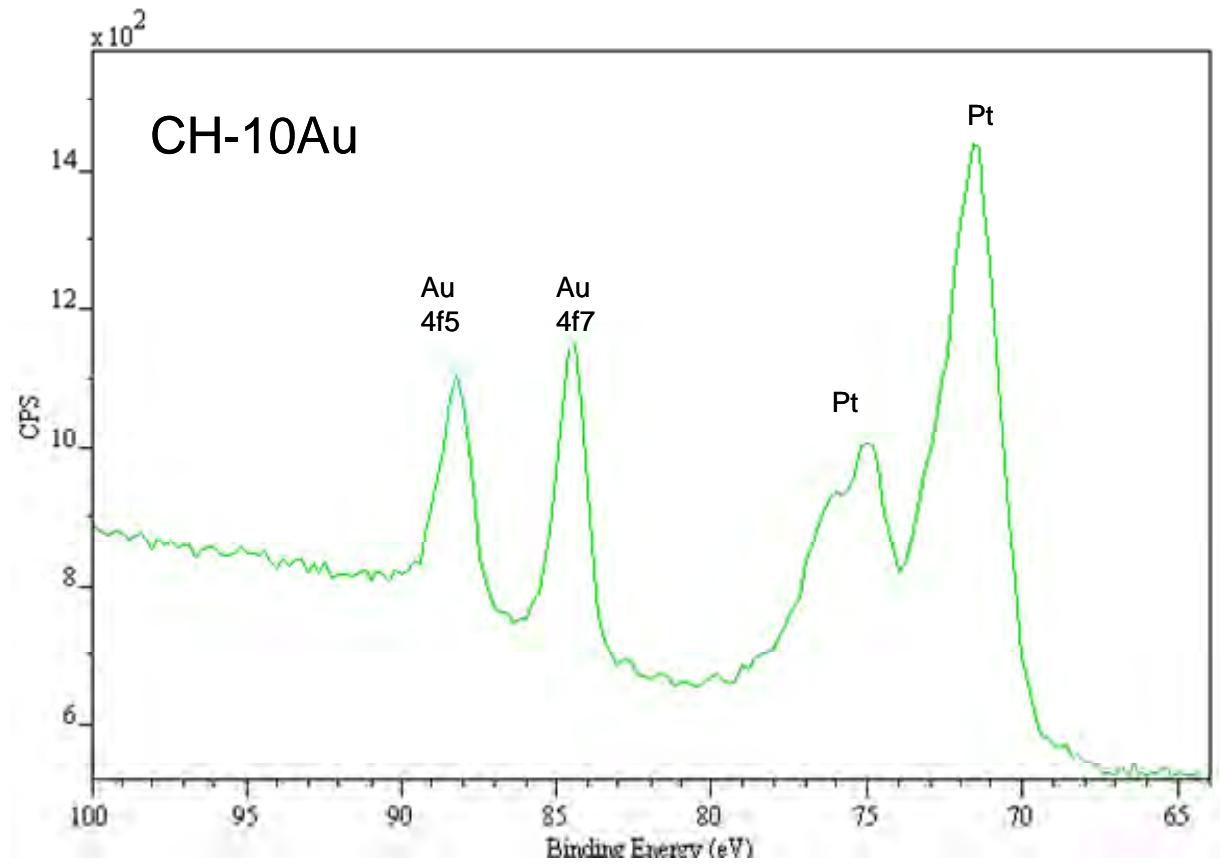

Figure 2. Narrow scan XPS of PANI-Au films prepared chemically with 10 consecutive treatments of $\mathrm{AuCl}_{4}^{-}$. 
PNNL-16185

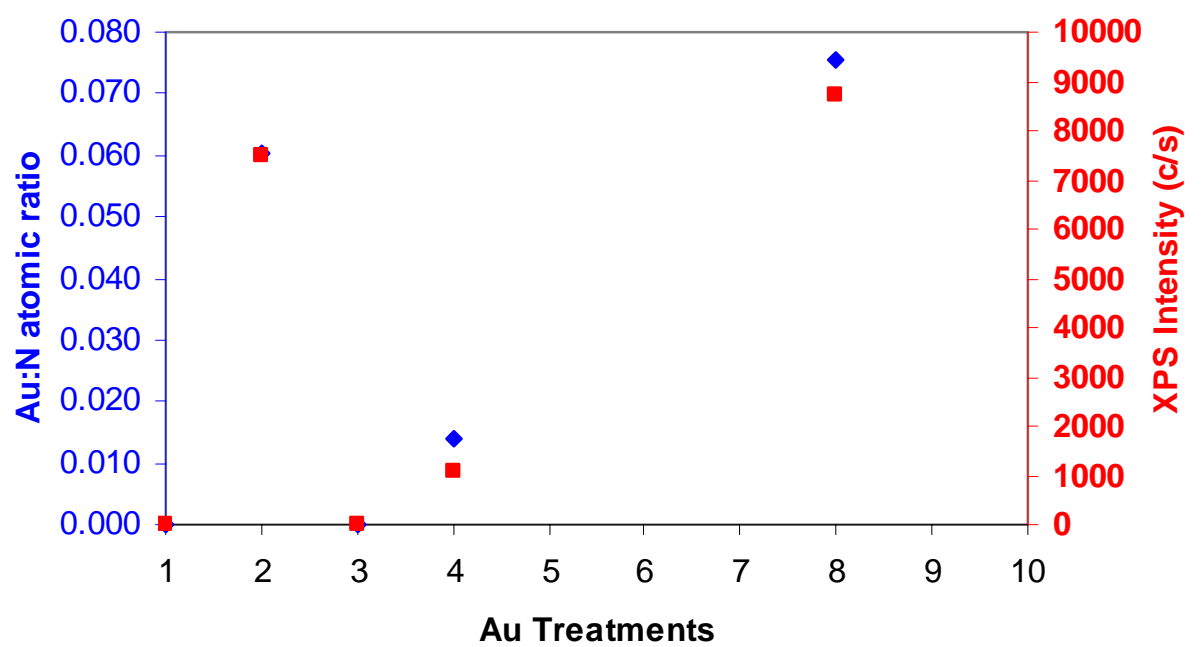

Figure 3. Changes of $\mathrm{Au} 4 \mathrm{f} 7: \mathrm{N}$ 1s atomic ratio with the number of Au treatments for the electrochemically prepared PANI-Au films (Procedure A).

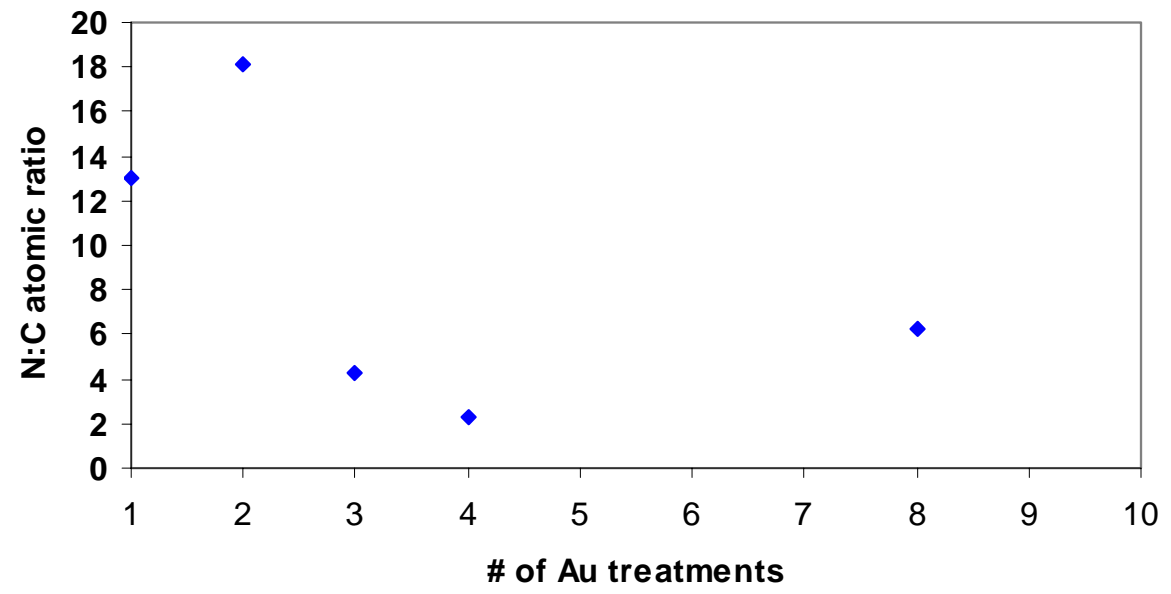

Figure 4. Dependence of $\mathrm{N}: \mathrm{C}$ atomic ratio on $\mathrm{AuCl}_{4}{ }^{-}$treatments of electrochemically prepared PANI-Au films (Procedure A).

With the exception of treatment \#2, each consecutive treatment resulted in an increase in the $\mathrm{Au}_{4 \mathrm{f} 7 / 2}$ peak. In addition, the binding energy decreased with more $\mathrm{Au}$ treatments as seen in Figure 5. All the binding energies are referenced to the $\mathrm{N} 1 \mathrm{~s}$ peak. 
PNNL-16185

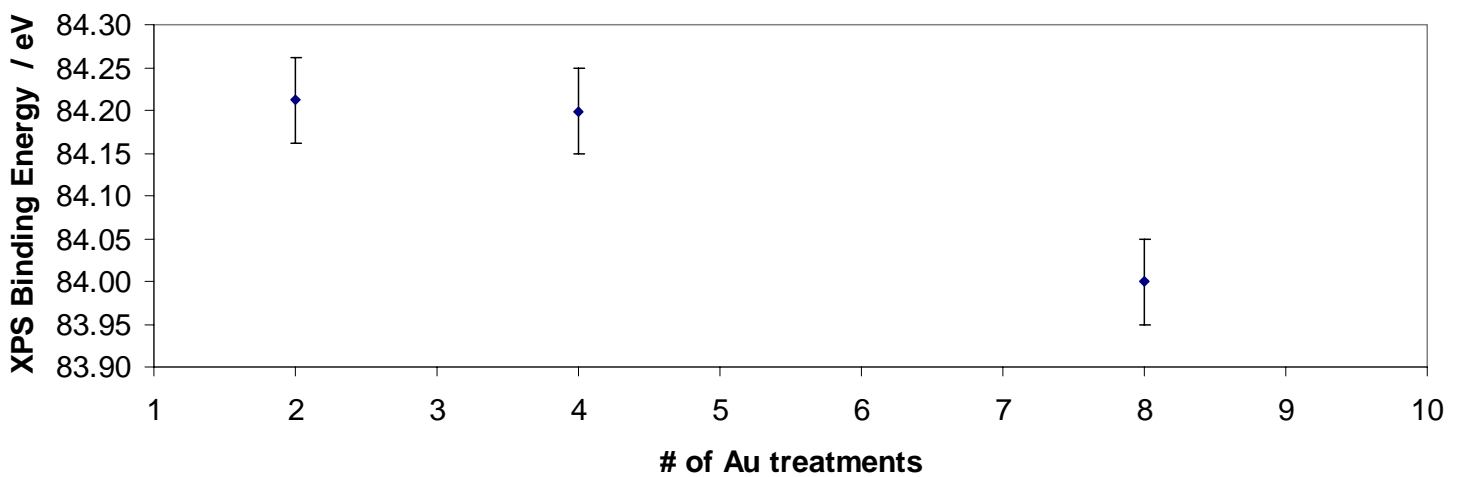

Figure 5. BE of the electrochemically prepared films as a function of Au treatements.

The chemically prepared PANI-Au films (Procedure B) also exhibited the same behavior, Figure 6 and 8 . It however, should greater consistency with regards to the N:C atomic ratio, Figure 7.

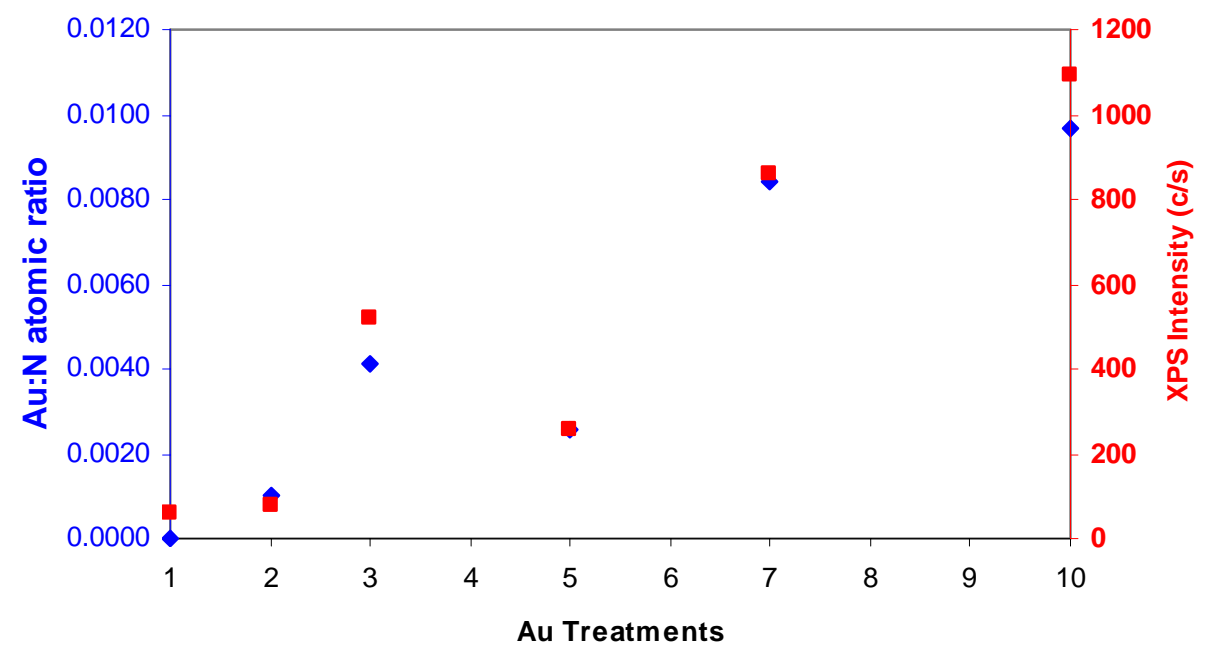

Figure 6. Changes of $\mathrm{Au} 4 \mathrm{f7}: \mathrm{N}$ 1s atomic ratio with the number of Au treatments for the chemically prepared PANI-Au films (Procedure B). 
PNNL-16185

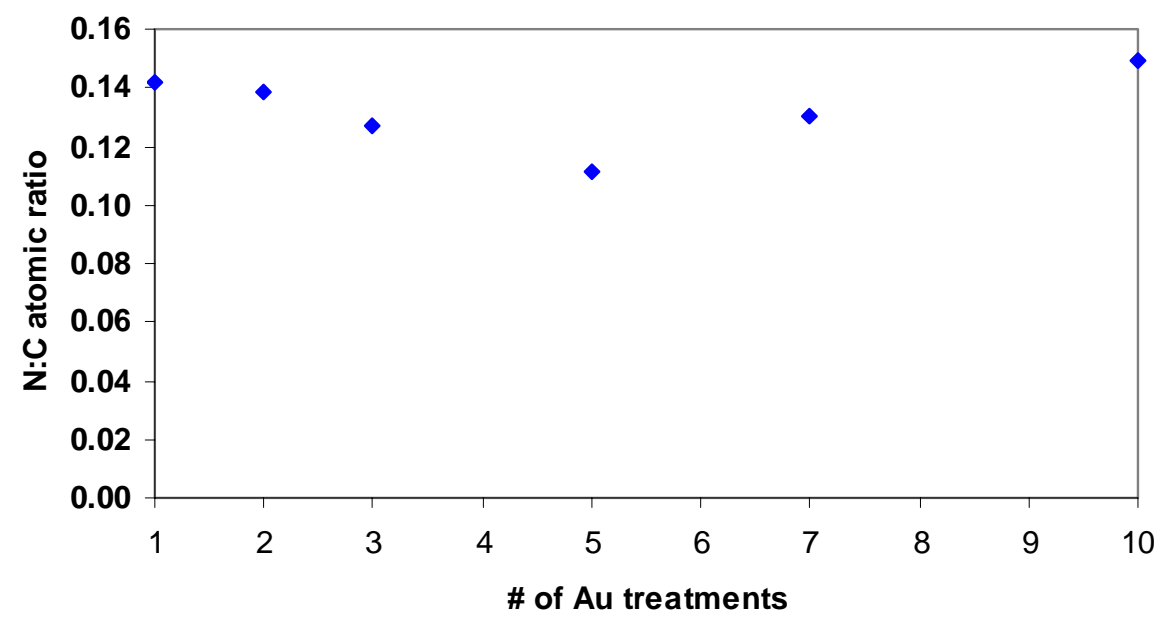

Figure 7. Dependence of the $\mathrm{N}: \mathrm{C}$ atomic ratio on $\mathrm{AuCl}_{4}{ }^{-}$treatments of the chemically prepared PANI-Au films (Procedure B).

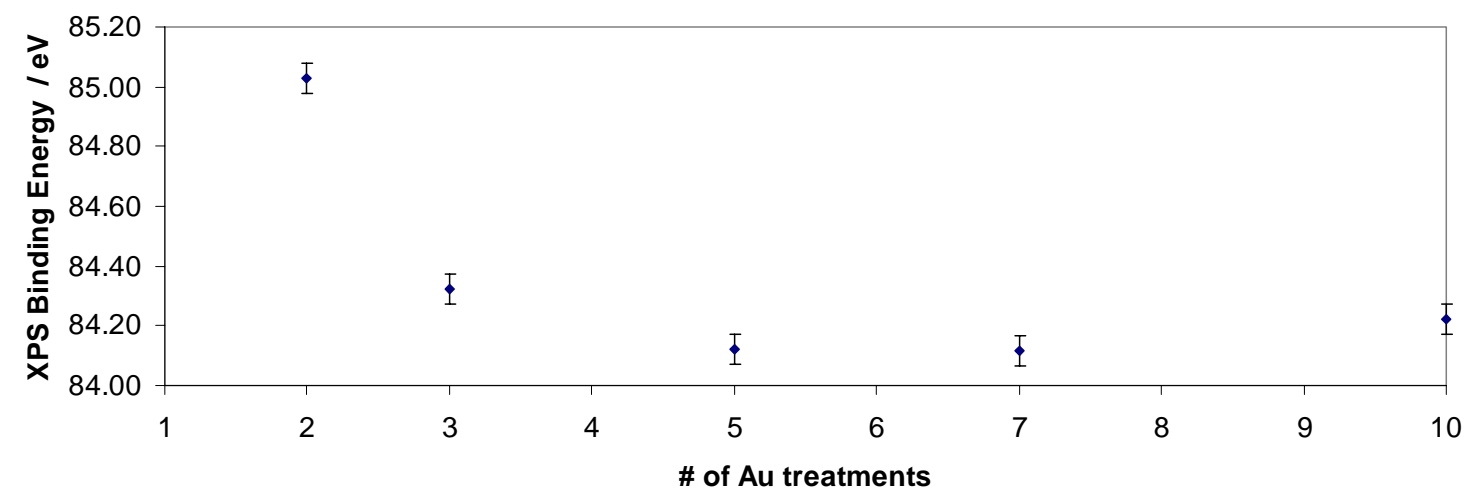

Figure 8. BE of the chemically prepared films as a function of Au treatements.

XPS analysis of the powder samples (Procedure C) showed no detectable gold. However, this may simply be a result of the sample itself which is in particulate form, thus having bulk properties as compared to the films. XPS is also not an appropriate technique for bulk analysis as it does not have the penetration depth required for larger particles.

\section{$\underline{\text { Discussion }}$}

Particles on the order of the de Broglie wavelength have size and shape dependent properties. In addition, the high surface to volume ratio of nanoparticles in the composite results in a large number of binding sites available for chemical sensing or catalysis ${ }^{2}$. This combination of conducting polymers with metallic nanoparticles yields materials with new properties.

Analysis of both the electrochemically and chemically prepared PANI-Au films on Pt-foil show a relationship between the shift in binding energy of Au in PANI and the number of Au treatments received. The shape of the binding energy curve is seen to 
follow the Coulomb interaction correction to the radius dependent ionization potential. The concept

of the effect of the contact potential is extended to show its contribution to the measured binding energy differences of the gold particle $\mathrm{Au}_{4 f 7 / 2}$ doublet as the particle size decreases.

\section{References}

1 J. A. Smith, M. Josowicz, M. Engelhard, D.R. Baer J. Janata, Phys. Chem. Chem. Phys., 2005, 7, 3619.

2 J. A. Smith, M. Josowicz and J. Janata, J. Electrochem. Soc., 2003, 150, E384.

\section{Acknowledgments}

I would like give special thanks to Mark Engelhard for making available his generous time and all the XPS knowledge I gained from him. In addition, I would like to thank Zihua Zhu (TOF-SIMS), Chongmin Wang (HRTEM), as well as, Mike Alexander and Matt Newburn (Laser ablation-MS) for all their help and assistance in the analysis process. 
PNNL-16185

\title{
Report on Interfacial and Condensed Phase Chemical Physics Summer Research Institute at PNNL Richland, WA, Sept 1-30, 2006
}

\author{
Andrzej L. Sobolewski \\ Institute of Physics \\ Polish Academy of Sciences \\ Warsaw, Poland \\ and \\ Sotiris Xantheas \\ Pacific Northwest National Laboratory \\ Richland, WA
}

Photochemistry of Liquid Waterand Radiation Induced Solvation

The photochemistry of liquid water and radiation induced solvation is a topic of considerable interest. The radiation-induced decomposition of water or electron detachment phenomenon plays an important role, for example, in nuclear reactor technology and in the radiation damage of living matter. However, the mechanisms of photoinduced ionization and dissociation of liquid water as well as photosolvation phenomena are, despite extensive experimental investigation, still poorly understood.

In a series of our recent papers a new paradigm of excited-state proton-transfer (PT) related reactivity (photoacidity) was proposed [1]. While PT in the electronic ground state is a charge-separation process (ion-pair formation), formation of a radical pair is the primary step in excited-state PT reactions. Other than ground-state PT, excited-state PT is not driven and stabilized by long-range polarization effects, but rather by nonadiabatic (curve-crossing) dynamics. As a consequence, cluster-size effects are less relevant in excitedstate hydrogen-transfer reactions than in ground-state PT processes. The primary mechanisms of excited-state hydrogen-transfer reactivity can thus be unraveled by spectroscopic and theoretical investigations of clusters.

One of the important issues of water photolysis is catalysation of the reaction by the UV/VIS absorbing chromophores. Aqueous clusters of metal cations have been the subject of considerable interest in this respect since they represent a first approximation to the phenomenon of aqueous solvation. In the following we discuss the preliminary results of 
ab initio study on the photophysics of the $\mathrm{Al}^{3+}\left(\mathrm{H}_{2} \mathrm{O}\right)_{6}$ cluster where the $\mathrm{Al}^{3+}$ ion is coordinated with the first solvation shell of water.

The optimal MP2/aug-cc-pVDZ geometry of the $\mathrm{Al}^{3+}\left(\mathrm{H}_{2} \mathrm{O}\right)_{6}$ cluster has the $\mathrm{T}_{\mathrm{h}}$ symmetry [2] (c.f. Fig. 1) with the Al-O bondlength of $1.936 \AA$. Water molecules are coordinated to the $\mathrm{Al}^{3+}$ ion in the $\mathrm{sp}^{2}$ hybrydization which means a significant charge transfer to the central ion. The lowest excited singlet states of the system calculated at the ground state geometry with the aid of the CC2/aug-cc-pVDZ method (Table 1) result from the $\mathrm{HOMO}\left(\mathrm{t}_{\mathrm{g}}\right) \rightarrow \mathrm{LUMO}\left(\mathrm{a}_{\mathrm{g}}\right)$ and $\mathrm{HOMO}-1\left(\mathrm{t}_{\mathrm{u}}\right) \rightarrow \mathrm{LUMO}\left(\mathrm{a}_{\mathrm{g}}\right)$ excitations. These excitations can be assigned as internal excitations of water (Fig. 1). Energy of these transitions is higher by about $3 \mathrm{eV}$ as compared to excitations of bare clusters of water [3] which once again indicate of a significant degree of ionization of water coordinated to the $\mathrm{Al}^{3+}$ ion.

Table 1. Vertical energy gap $(\Delta \mathrm{E})$ and oscillator strength $(f)$ of the $\mathrm{Al}^{3+}\left(\mathrm{H}_{2} \mathrm{O}\right)_{6}$ cluster calculated at the ground-state equilibrium geometry with the CC2/aug-cc-pVDZ method.

\begin{tabular}{|c|c|c|}
\hline State & $\Delta \mathrm{E} / \mathrm{eV}$ & $\mathrm{f}$ \\
\hline${ }^{1} \mathrm{~T}_{\mathrm{g}}$ & 10.37 & 0.0 \\
\hline${ }^{1} \mathrm{~T}_{\mathrm{u}}$ & 10.38 & 0.426 \\
\hline${ }^{1} \mathrm{~A}_{\mathrm{u}}$ & 12.53 & 0.0 \\
\hline${ }^{1} \mathrm{~A}_{\mathrm{g}}$ & 12.83 & 0.0 \\
\hline
\end{tabular}

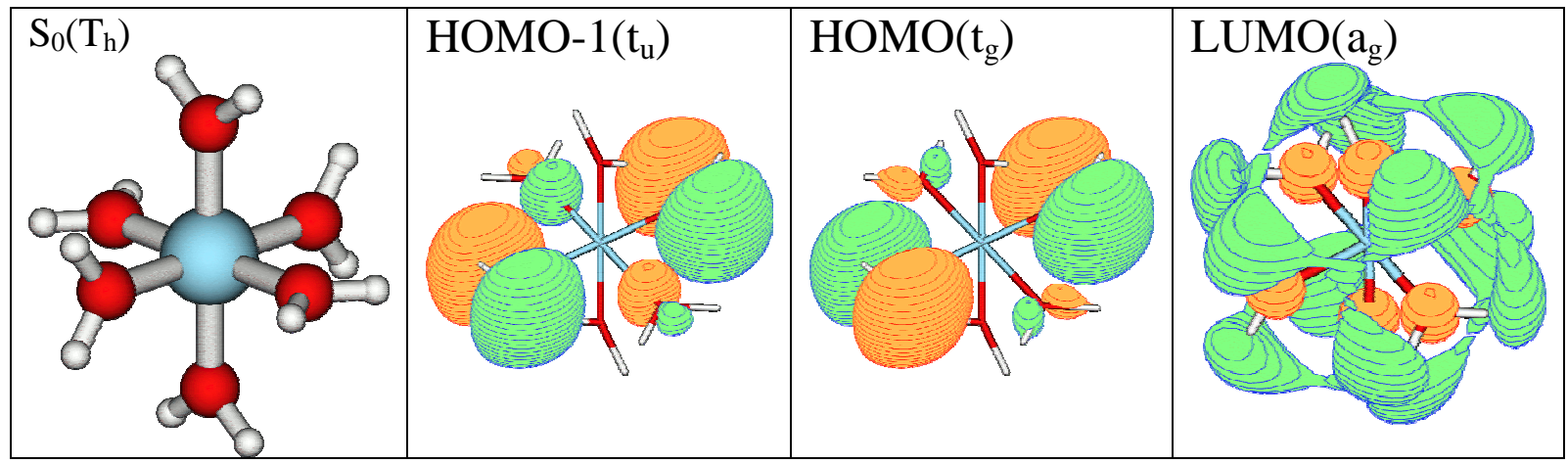

Fig. 1. The ground-state equilibrium geometry of the $\mathrm{Al}^{3+}\left(\mathrm{H}_{2} \mathrm{O}\right)_{6}$ cluster determined at the MP2/aug-cc-pVDZ level and the frontier molecular orbitals involved in the lowest optical excitations of the system. Only one component of the triply degenerated orbitals is shown. 
Since the lowest excited singlet states of the system are degenerated they subject to the Jahn-Teller effect which breaks the $T_{h}$ symmetry of the system upon optical excitation. Although, the resulting effects of the symmetry breaking need a more systematic study, in Fig. 2 we show preliminary results for a particular pathway of the excited-state geometry optimization at the CC2/aug(O)-cc-pVDZ level of theory presuming the $\mathrm{T}_{\mathrm{h}} \rightarrow \mathrm{C}_{2 \mathrm{v}}$ symmetry breaking pattern.

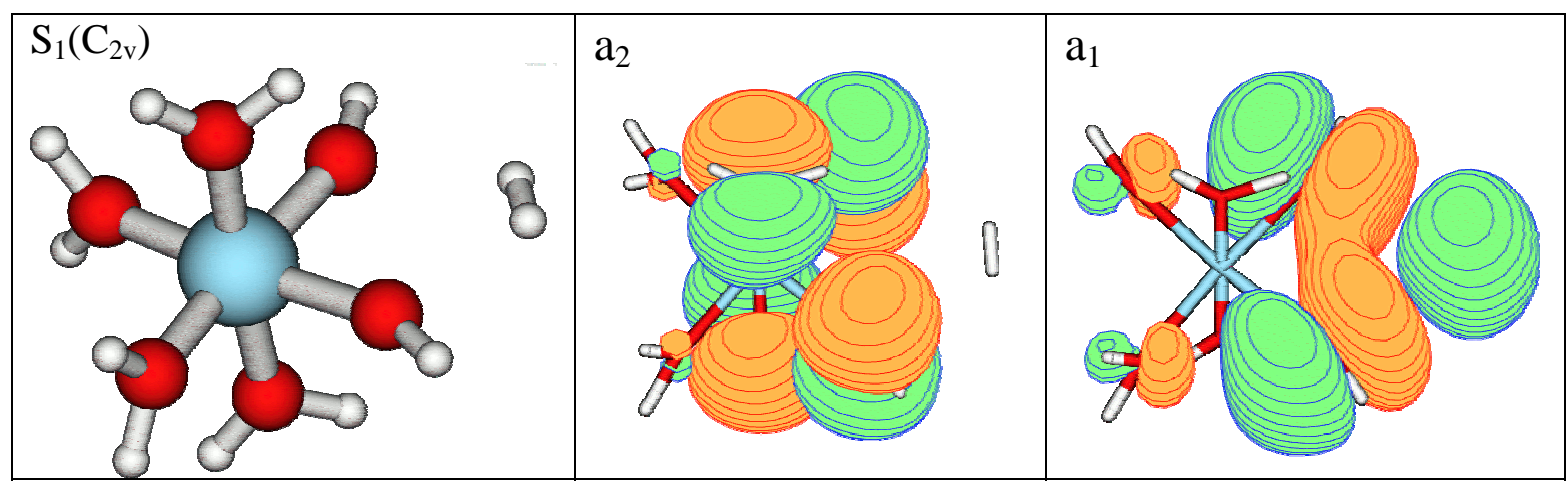

Fig. 2. The resulting structure of the CC2/aug(O)-cc-pVDZ optimization of the first excited singlet state of the $\mathrm{Al}^{3+}\left(\mathrm{H}_{2} \mathrm{O}\right)_{6}$ cluster upon the $\mathrm{T}_{\mathrm{h}} \rightarrow \mathrm{C}_{2 \mathrm{v}}$ symmetry breaking and the singly occupied molecular orbitals in the ${ }^{1} \mathrm{~A}_{2}$ state.

The interesting aspect of the theoretical results shown in Fig. 2 is that the $T_{h} \rightarrow C_{2 v}$ symmetry breaking of the $\mathrm{Al}^{3+}\left(\mathrm{H}_{2} \mathrm{O}\right)_{6}$ cluster does not result in dissociation of water molecules, but in formation of molecular hydrogen, instead. This effect can be explained in terms of nodal structure of the LUMO orbital (Figs. 1 and 2) which has a character of the Rydberg orbital of water with the nodes at the $\mathrm{OH}$ bonds.

The structure shown in Fig. 2 does not correspond to the equilibrium geometry. This is the last structure for which the CC2 iteration has converged in the course of optimization. At the next predicted geometry the ground state crosses the $S_{1}$ state and this precludes investigation of the system at the CC2 level. The strong non-adiabatic effects expected at the $\mathrm{S}_{1}-\mathrm{S}_{0}$ (conical) intersection drive the system to the ground state. Thus further study of the reaction is confined to the ground state. 
Preliminary results discussed above shown an important calatytic effect of the metal ion in the photolytic splitting of water. Further study should concentrate on elucidation of the physical mechanism which is beyond of the effect and on the search for other metallic or organic chromophores which can drive excitation energy of the cluster into the UV/VIS range.

1. W. Domcke and A. L. Sobolewski, Science, 302 (2003) 1693

2. E. Wasserman, J. R. Rustad, S. S. Xantheas, J. Chem. Phys. 106 (1997) 9769

3. J. N. Harvey, J. O. Jung, R. B. Gerber, J. Chem. Phys. 109, 8747 (1998); Y. Miller, E. Fredj, J. N. Harvey, R. B. Gerber, J. Phys. Chem. A 108, 4405 (2004). 


\title{
Molecular dynamics of aqueous interfaces:
}

\section{The surface propensity and thermodynamic properties of aqueous anions}

\author{
Jennie L. Thomas, Barbara J. Finlayson Pitts, and Douglas J. Tobias \\ Department of Chemistry, \\ University of California, Irvine \\ Irvine, CA \\ and \\ Liem X. Dang \\ Pacific Northwest National Laboratory \\ Richland, WA
}

Unique interface chemistry at the surface of sea-salt particles, comprised primarily of aqueous sodium chloride, has been postulated as an important source of photochemically active chlorine in the troposphere. ${ }^{1,2}$ Molecular chlorine then goes on to absorb sunlight and form atomic chlorine, a known oxidant of organics and an initiator of ozone formation in urban areas. ${ }^{3}$ A variety of oxides of nitrogen react with sea-salt particles, leading to replacement of chloride by nitrate ions as particles age..$^{4-8}$

There are a number of factors that could impact $\mathrm{Cl}_{2}$ production from aerosols comprised of a mixture of nitrate and chloride. If chloride and nitrate are equally available at the interface, then molecular chlorine production could drop as aerosols age. Alternatively, if nitrate resides in the bulk and pushes chloride to the interface, $\mathrm{Cl}_{2}$ production would stay more constant even as the chloride concentration in the aerosol decreases. Aerosol chamber experiments to characterize the amount of $\mathrm{Cl}_{2}$ produced from mixed $\mathrm{NaCl} / \mathrm{NaNO}_{3}$ aerosols are currently underway at University of California, Irvine. ${ }^{9}$ In coordination with these experimental studies, this work will focus on investigating aqueous $\mathrm{NaNO}_{3}$ as well as mixtures of $\mathrm{NaNO}_{3}$ and $\mathrm{NaCl}$ using classical molecular dynamics (MD) simulations.

MD simulations were used to investigate the behavior of aqueous $\mathrm{NaNO}_{3}$ in interfacial environments. A slab system ${ }^{10,11}$ was constructed containing 
condensed phase water and ions with a region of vacuum (representing the vapor phase) along the elongated z-axis. The surface potential aqueous $\mathrm{NaNO}_{3}$ and neat water slabs was calculated using the method described by Wick and Dang in $2006 .{ }^{12}$ The difference in the potential drop across the interface, from the condensed phase to the gas phase, between neat water and 1.5 and $6.8 \mathrm{M} \mathrm{NaNO}$ solutions was compared to experimental data summarized by Randles in $1977 .{ }^{13}$

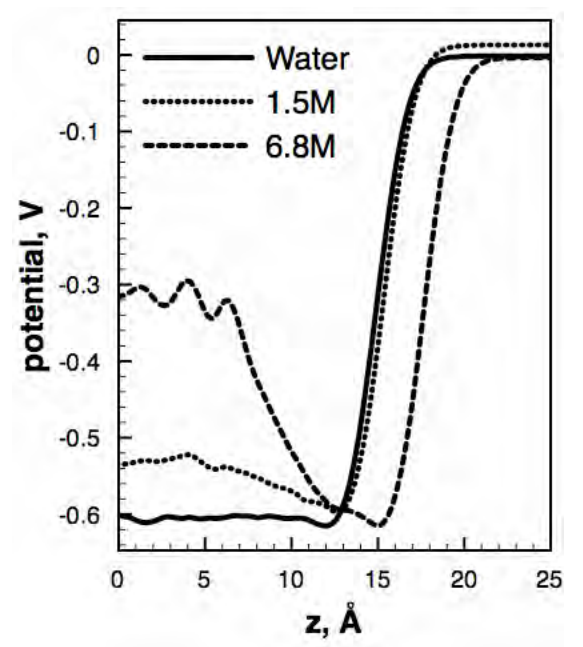

Figure 1. Calculated surface potential for $1.5 \mathrm{M}$ and $6.8 \mathrm{M} \mathrm{NaNO} \mathrm{Nampared} \mathrm{to}_{3}$ the surface potential water, calculations were completed using $2 \mathrm{~ns}$ of simulation data. The difference in the potential for $\mathrm{NaNO}_{3}$ solution and neat water is compared to experimental data.

Figure 1 shows potential as a function of $z$ coordinate in the slab for water, $1.5 \mathrm{M}$, and 6.8M NaNO $\mathrm{N}_{3}$. Subtracting the water potential in the center of the slab from the $\mathrm{NaNO}_{3}$ potential gives values that can be compared with measured data, which is always given as a difference from neat water. For an overview of the experimental techniques used in measuring surface potentials the reader is referred to Randles 1977 article. ${ }^{13}$ Experimental data is available for $1.5 \mathrm{M} \mathrm{NaNO}$, with a measured surface potential of $0.25 \mathrm{~V} .{ }^{13}$ The difference from water should increase with increasing concentration, which is reproduced by calculations.

A profile of the concentration of ions and water verses position in the slab was constructed for both pure $\mathrm{NaNO}_{3}$ and mixed $\mathrm{NaNO}_{3} / \mathrm{NaCl}$ in water. The goal is to characterize the interface of pure sodium nitrate solutions and mixed sodium 
nitrate/sodium chloride solutions, a model for aged sea-salt aerosols. Figure 2 shows density profiles for pure $\mathrm{NaNO}_{3}$ and mixed $\mathrm{NaNO}_{3} / \mathrm{NaCl}$ solutions.
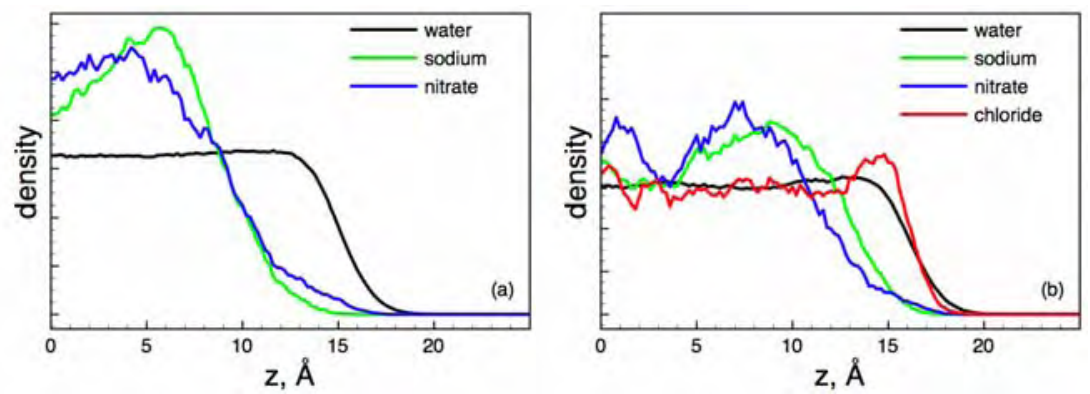

Figure 2. Density profiles for pure $1.5 \mathrm{M} \mathrm{NaNO}_{3}$ in water (a) and mixed $2 \mathrm{M} \mathrm{NaNO}_{3}$ with $2 \mathrm{M} \mathrm{NaCl}$ in water (b).

Clearly, nitrate resides below the air/water interface of both solutions. In the mixed system, chloride is available at the interface and may be important for reactions that occur on the surface of aged particles in the atmosphere.

An initial investigation of the behavior of one iodide ion in a water slab was completed at three temperatures. Recent studies show that constructing potential mean force curves (PMF) at three temperatures suggests interfacial ion solvation can be investigated using spatially resolved free energy, entropy, and enthalpy curves. ${ }^{14,15}$ This method was combined with Dang's work ${ }^{16}$ on ion binding at the liquid vapor interface, to investigate the behavior of iodide at the air/water interface. PMF curves were calculated at three temperatures using $1 \mathrm{~ns}$ of simulation data per PMF point. Figure 3 shows calculated PMF curves for iodide crossing the air/water interface at three temperatures.

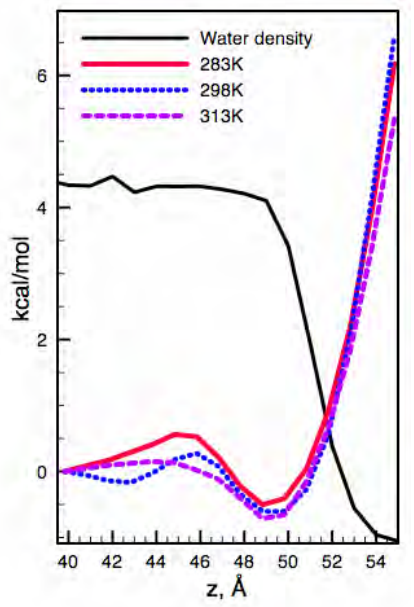

Figure 3. Calculated PMF curves for iodide crossing the air/water interface. 
However, it was discovered the behavior of these as a function of temperature was not appropriate for the proposed analysis. Alternative methods to calculate the entropic and enthalpic contributions to ion solvation are under investigation.

(1) Knipping, E. M.; Dabdub, D. J. Geophys. Res. 2002, 107.

(2) Knipping, E. M.; Lakin, M. J.; Foster, K. L.; Jungwirth, P.; Tobias, D. J.; Gerber, R. B.; Dabdub, D.; Finlayson-Pitts, B. J. Science 2000, 288, 301.

(3) Finlayson-Pitts, B. J. Chem. Rev. 2003, 103, 4801.

(4) Dall'Osto, M.; Beddows, D. C. S.; Kinnersley, R. P.; Harrison, R. M.; Donovan, R. J.; Heal, M. R. J. Geophys. Res. 2004, 109.

(5) Laskin, A.; Iedema, M. J.; Cowin, J. P. Environ. Sci. Technol. 2002, 36, 4948.

(6) Ro, C. U.; Oh, K. Y.; Kim, H.; Kim, Y. P.; Lee, C. B.; Kim, K. H.; Kang, C. H.; Osan, J.; De Hoog, J.; Worobiec, A.; Van Grieken, R. Environ. Sci. Technol. 2001, 35, 4487.

(7) Kerminen, V. M.; Teinila, K.; Hillamo, R.; Pakkanen, T. J. Aerosol Sci. 1998, 29, 929.

(8) Posfai, M.; Anderson, J. R.; Buseck, P. R.; Sievering, H. J. Geophys. Res. 1995, 100, 23063.

(9) Moskun, A.; Wingen, L.; Finlayson-Pitts, B. J., Personal communication.

(10) Benjamin, I. Journal of Chemical Physics 1991, 95, 3698.

(11) Wilson, M. A.; Pohorille, A. Journal of Chemical Physics 1991, 95, 6005.

(12) Wick, C. D.; Dang, L. X.; Jungwirth, P. Journal of Chemical Physics 2006, 125.

(13) Randles, J. E. B. Phys. Chem. Liq. 1977, 7, 107.

(14) MacCallum, J. L.; Tieleman, D. P. Journal of the American Chemical Society 2006, 128, 125.

(15) van der Vegt, N. F. A.; Lee, M. E.; Trzesniak, D.; van Gunsteren, W. F. Journal of Physical Chemistry B 2006, 110, 12852.

(16) Dang, L. X. Journal of Physical Chemistry B 2002, 106, 10388. 


\title{
The Investigation on: \\ I . The Solvation of $\mathrm{CN}\left(\mathrm{H}_{2} \mathrm{O}\right)_{\mathrm{n}}{ }^{-}$and $\mathrm{N}(\mathrm{CN})_{2}\left(\mathrm{H}_{2} \mathrm{O}\right)_{n}{ }^{-}$ \\ II. The Configuration of $\mathrm{MOOC}\left(\mathrm{CH}_{2}\right)_{\mathbf{n}} \mathrm{COO}-(\mathrm{M}=\mathrm{Na}, \mathrm{K})$ \\ III. Some Oxygen-rich Vanadate Anion Species by Combination \\ of Photoelectron Spectrometry and Electron-spray
}

\author{
Guanjun Wang and Mingfei Zhou \\ Department of Chemistry \\ Fudan University \\ Fudan, PRC \\ and \\ Dr. Lai-sheng Wang, Dr. Xuebin Wang, Dr. Hin-Koon Hoo \\ Pacific Northwest National Laboratory \\ Richland, WA
}

The combination of electron-spray with photo-electron spectrometry (PES) is a fruitful tool in the investigation on the solvation of anion and the multi-charge anion since it was introduced by Prof. Wang. A lot of novel phenomena was discovered such as the negative electron detachment energy in some multi-charge anion; the transition from linear to cyclic configuration by the interaction of water molecule in some aliphatic dicarboxylate dianion; and the hydrogen bond between $\mathrm{O}^{-}$and aliphatic C-H was discovered in $\mathrm{CH}_{3}\left(\mathrm{CH}_{2}\right)_{n} \mathrm{COO}^{-}(\mathrm{n}>5)$ molecule by lowtemperature PES firstly.

\section{I .The investigation on the solvation of $\mathrm{N}(\mathrm{CN})_{2}\left(\mathrm{H}_{2} \mathrm{O}\right)_{n}{ }^{-}$and $\mathrm{CN}\left(\mathrm{H}_{2} \mathrm{O}\right)_{n}{ }^{-}$}

The solvation effect of some anion have been investigated by PES in this group, such as $\mathrm{SO}_{4}{ }^{2-}, \mathrm{NO}_{3}{ }^{-}, \mathrm{N}_{3}{ }^{-},{ }^{-} \mathrm{OOC}\left(\mathrm{CH}_{2}\right)_{\mathrm{n}} \mathrm{COO}^{-}$. In the case of $\mathrm{SO}_{4}{ }^{2-}$ and ${ }^{-}$ $\mathrm{OOCCOO}^{-}$, the bare anion isn't stable in gas phase while the salvation affection of $\mathrm{H}_{2} \mathrm{O}$ molecule contributes to the stabilization of hydrate anion. 
We have investigated the hydrated $\mathrm{CN}^{-}$and $\mathrm{N}(\mathrm{CN})_{2}^{-}$by PES. The PES spectra were shown in Figure 1.1 and 1.2 respectively.

In the case of $\mathrm{N}(\mathrm{CN})_{2}{ }^{-}\left(\mathrm{H}_{2} \mathrm{O}\right)_{n}$, the number of water molecule can is up to $\mathrm{n}=12$ when the mass spectra signal of anion keep detectable. The spectra pattern is very similar for these all hydrated anions except that the peak get broaden with the increase of $\mathrm{H}_{2} \mathrm{O}$ molecules. The electron detachment energy of bare $\mathrm{N}(\mathrm{CN})_{2}{ }^{-}$anion is $4.12 \mathrm{eV}$. While the electron detachment energy increase to $4.52 \mathrm{eV}$ and $4.91 \mathrm{eV}$ respectively when first two $\mathrm{H}_{2} \mathrm{O}$ molecules were attached to anion. The shift is very similar, which mean that the first $\mathrm{H}_{2} \mathrm{O}$ molecule were trapped to the anion in the same way. The next two $\mathrm{H}_{2} \mathrm{O}$ molecules give 0.25 and 0.26 electron detachment energy shift respectively which mean these two guys were trapped to anion in a different way from the first two. Then all other $\mathrm{H}_{2} \mathrm{O}$ molecules bring smaller shift about $0.1 \mathrm{eV}$, so these $\mathrm{H}_{2} \mathrm{O}$ molecules should be capture as the second solvent shell by hydrogen bond with the first four ones. 


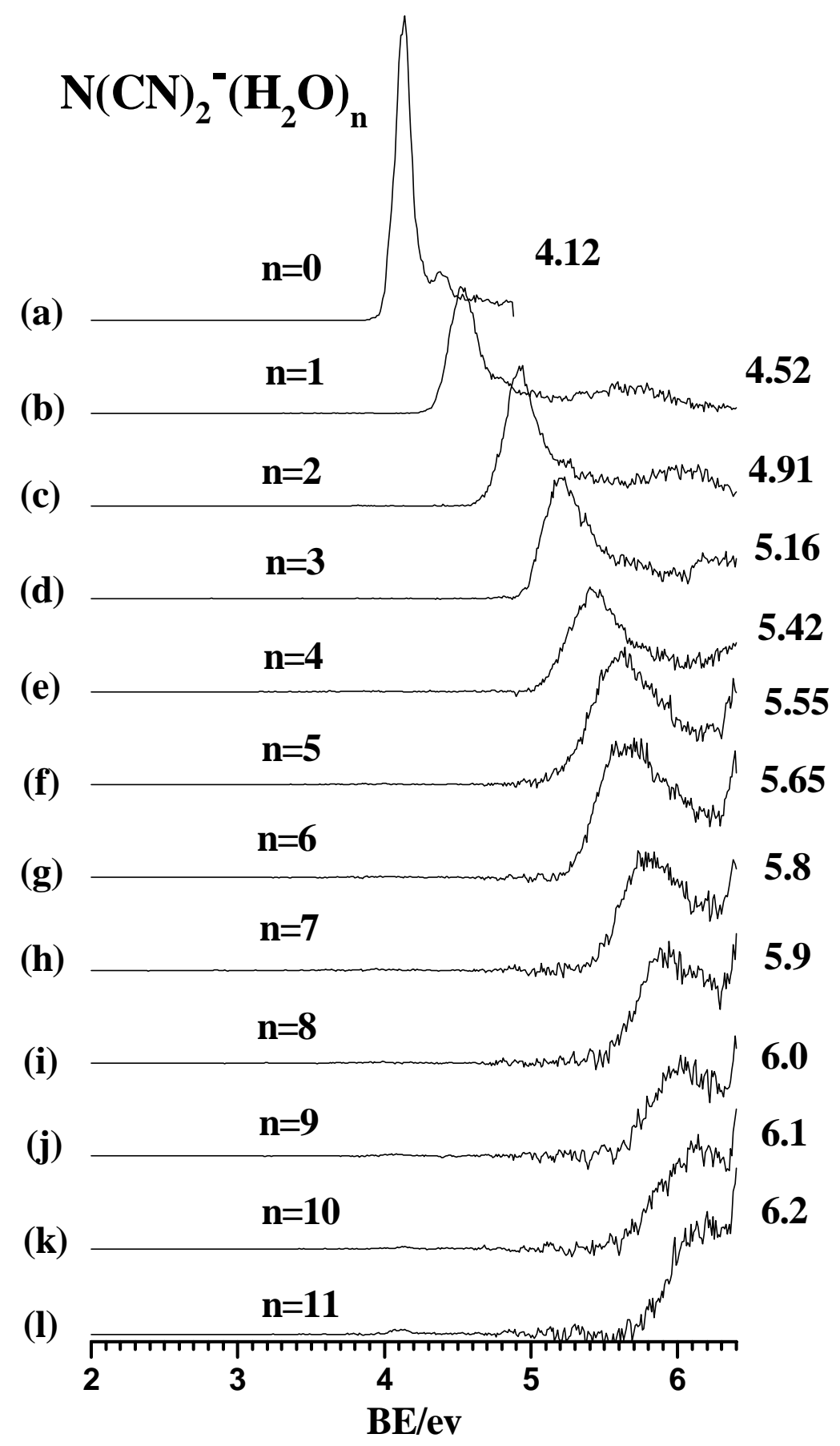

Figure 1.1 Photoelectron spectra of $\mathrm{N}(\mathrm{CN})_{2}{ }^{-}\left(\mathrm{H}_{2} \mathrm{O}\right)_{\mathrm{n}}(\mathrm{n}=0-11)$ measured at $193 \mathrm{~nm}(6.424 \mathrm{eV})$. The value list on right is the $\mathrm{VDE} / \mathrm{eV}$. 
In the case of $\mathrm{CN}\left(\mathrm{H}_{2} \mathrm{O}\right)_{n}{ }^{-}$, we can't get the signal of bare $\mathrm{CN}$ - anion in the mass spectra because the mass limitation of Quadrapole Ion-Guide. But the electron detachment energy of $\mathrm{CN}$ - has been measured in this group by the laser-ablation on the $\mathrm{CuCN}$ target, and the $\mathrm{VDE}$ is $3.85 \mathrm{eV}$. While the electron detachment energy increased to 4.35, 5.10 and 5.50 respectively when three $\mathrm{H}_{2} \mathrm{O}$ molecules were captured. We found that the second $\mathrm{H}_{2} \mathrm{O}$ molecule give larger electron detachment energy shift than first one. Then we assume that the second $\mathrm{H}_{2} \mathrm{O}$ was attached to the other end of $\mathrm{CN}$ - from the first one, so we guess that two $\mathrm{H}_{2} \mathrm{O}$ molecules maybe stabilized the anion synergeticlly. While the signal of $\mathrm{CN}\left(\mathrm{H}_{2} \mathrm{O}\right)_{n}{ }^{-}(\mathrm{n}>3)$ in mass spectra is too weak to detect.

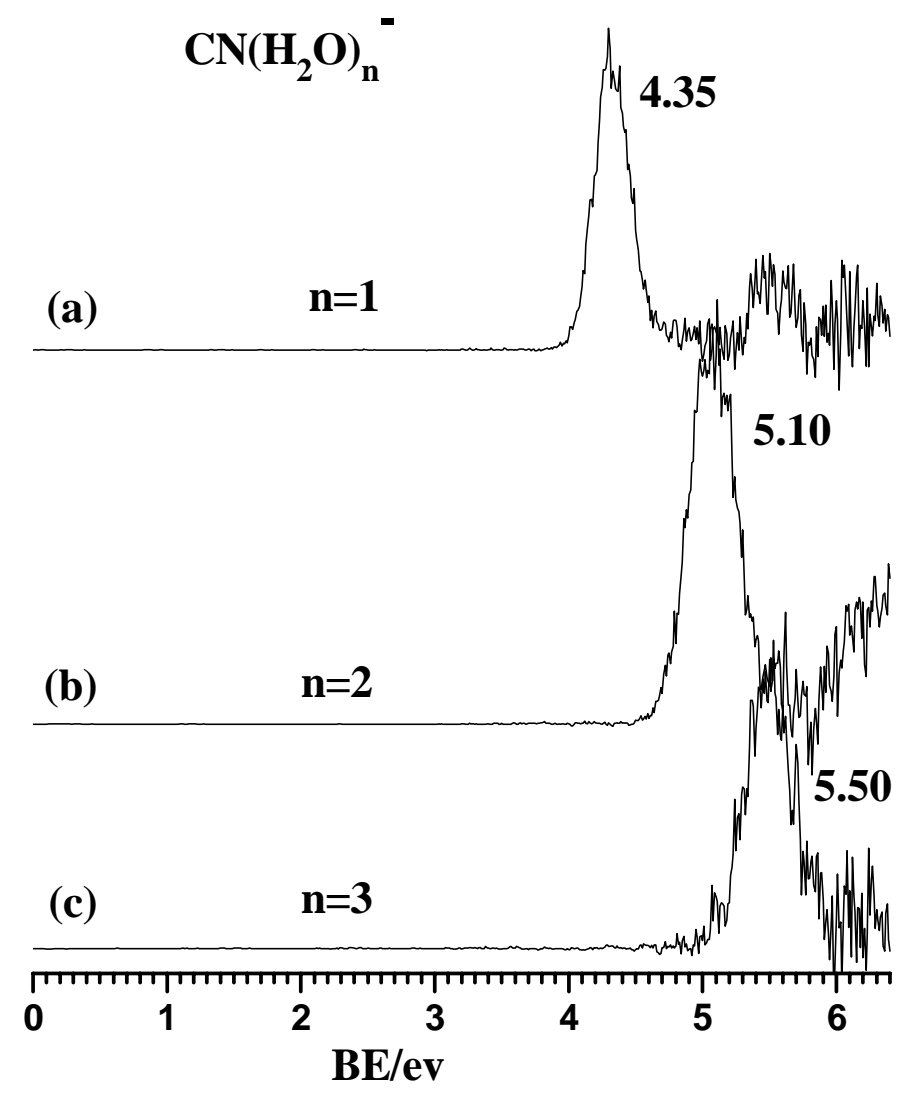

Figure 1.2 Photoelectron spectra of $\mathrm{CN}^{-}\left(\mathrm{H}_{2} \mathrm{O}\right)_{\mathrm{n}}(\mathrm{n}=1-3)$ measured at $193 \mathrm{~nm}(6.424 \mathrm{eV})$. The value list on right is the $\mathrm{VDE} / \mathrm{eV}$. 


\section{The transition of configuration in alkali dicarboxylate mono-anion} $\operatorname{MOOC}\left(\mathrm{CH}_{2}\right)_{\mathrm{n}} \mathrm{COO}^{-}(\mathrm{n}=1-6, \mathrm{M}=\mathrm{K}$ and $\mathrm{Na})$ molecule: from linear to cyclic

The PES spectra of aliphatic $\mathrm{MOOC}\left(\mathrm{CH}_{2}\right)_{\mathrm{n}} \mathrm{COO}^{-}(\mathrm{n}=1-6)$ were shown in figure $2.1(\mathrm{M}=\mathrm{K})$ and figure $2.2(\mathrm{M}=\mathrm{Na})$ respectively. All the peaks is relatively broad in both case and the ADE increase with the length of $\left(\mathrm{CH}_{2}\right)_{\mathrm{n}}$, Which is different from the $\mathrm{HOOC}\left(\mathrm{CH}_{2}\right)_{n} \mathrm{COO}^{-}$and $\mathrm{CH}_{3}\left(\mathrm{CH}_{2}\right)_{\mathrm{n}} \mathrm{COO}^{-}$that have been investigated in this group previously. The values were list in Table 2.1 for comparison. The ADE of $\mathrm{HOOC}\left(\mathrm{CH}_{2}\right)_{n} \mathrm{COO}^{-}$is about higher $1.0 \mathrm{eV}$ than $\mathrm{H}_{3} \mathrm{C}\left(\mathrm{CH}_{2}\right)_{\mathrm{n}} \mathrm{COO}^{-}$, because the former molecule is cyclic and is stabilized by the strong intra-molecule hydrogen bond. While it is found that the ADE of $\mathrm{MOOCCH}_{2} \mathrm{COO}^{-}(\mathrm{M}=\mathrm{Na}$ and $\mathrm{K})$ is approximate to the $\mathrm{H}_{3} \mathrm{CCH}_{2} \mathrm{COO}^{-}$in this experiment, which mean that the $\mathrm{MOOCCH}_{2} \mathrm{COO}^{-}$should be linear. And the molecule shape tend to cyclic with the increase of $\left(\mathrm{CH}_{2}\right)_{\mathrm{n}}$ length because the ADE increase.

Table 2.1 The VDE of $\mathrm{CH}_{3}\left(\mathrm{CH}_{2}\right)_{\mathrm{n}} \mathrm{COO}^{-}$, $\mathrm{HOOC}\left(\mathrm{CH}_{2}\right)_{\mathrm{n}} \mathrm{COO}^{-}$, $\mathrm{NaOOC}\left(\mathrm{CH}_{2}\right)_{\mathrm{n}} \mathrm{COO}^{-}$and $\mathrm{KOOC}\left(\mathrm{CH}_{2}\right)_{n} \mathrm{COO}^{-} \mathrm{n}=1-6$

\begin{tabular}{|r|c|c|c|c|}
\hline $\mathrm{n}$ & $\begin{array}{c}\mathrm{CH}_{3}\left(\mathrm{CH}_{2}\right)_{\mathrm{n}} \\
\mathrm{COO}^{-}\end{array}$ & $\begin{array}{c}\mathrm{HOOC}\left(\mathrm{CH}_{2}\right)_{\mathrm{n}} \\
\mathrm{COO}^{-}\end{array}$ & $\begin{array}{c}\mathrm{NaOOC}_{\left(\mathrm{CH}_{2}\right)_{\mathrm{n}}} \\
\mathrm{COO}^{-}\end{array}$ & $\begin{array}{c}\mathrm{KOOC}\left(\mathrm{CH}_{2}\right)_{\mathrm{n}} \\
\mathrm{COO}^{-}\end{array}$ \\
\hline 1 & 3.4 & 4.65 & 3.65 & 3.55 \\
\hline 2 & 3.4 & 4.64 & 4.00 & 3.80 \\
\hline 3 & 3.4 & 4.58 & 4.10 & 4.20 \\
\hline 5 & 3.4 & 4.46 & 4.15 & 4.30 \\
\hline 6 & 3.4 & 4.41 & 4.20 & 4.40 \\
\hline
\end{tabular}


It is notable that the $\mathrm{ADE}$ of $\mathrm{KOOCCH}_{2} \mathrm{COO}^{-}$increase more fast than the $\mathrm{NaOOCCH}_{2} \mathrm{COO}^{-}$with the length of $\left(\mathrm{CH}_{2}\right)_{n}$, because the radii of $\mathrm{K}^{+}$is larger than the $\mathrm{Na}^{+}$, which mean that the $\left(-\mathrm{O}^{-} \mathrm{K}^{+} \mathrm{O}-\right)$ fraction in cyclic molecule have more ability to adapt the ring tension.

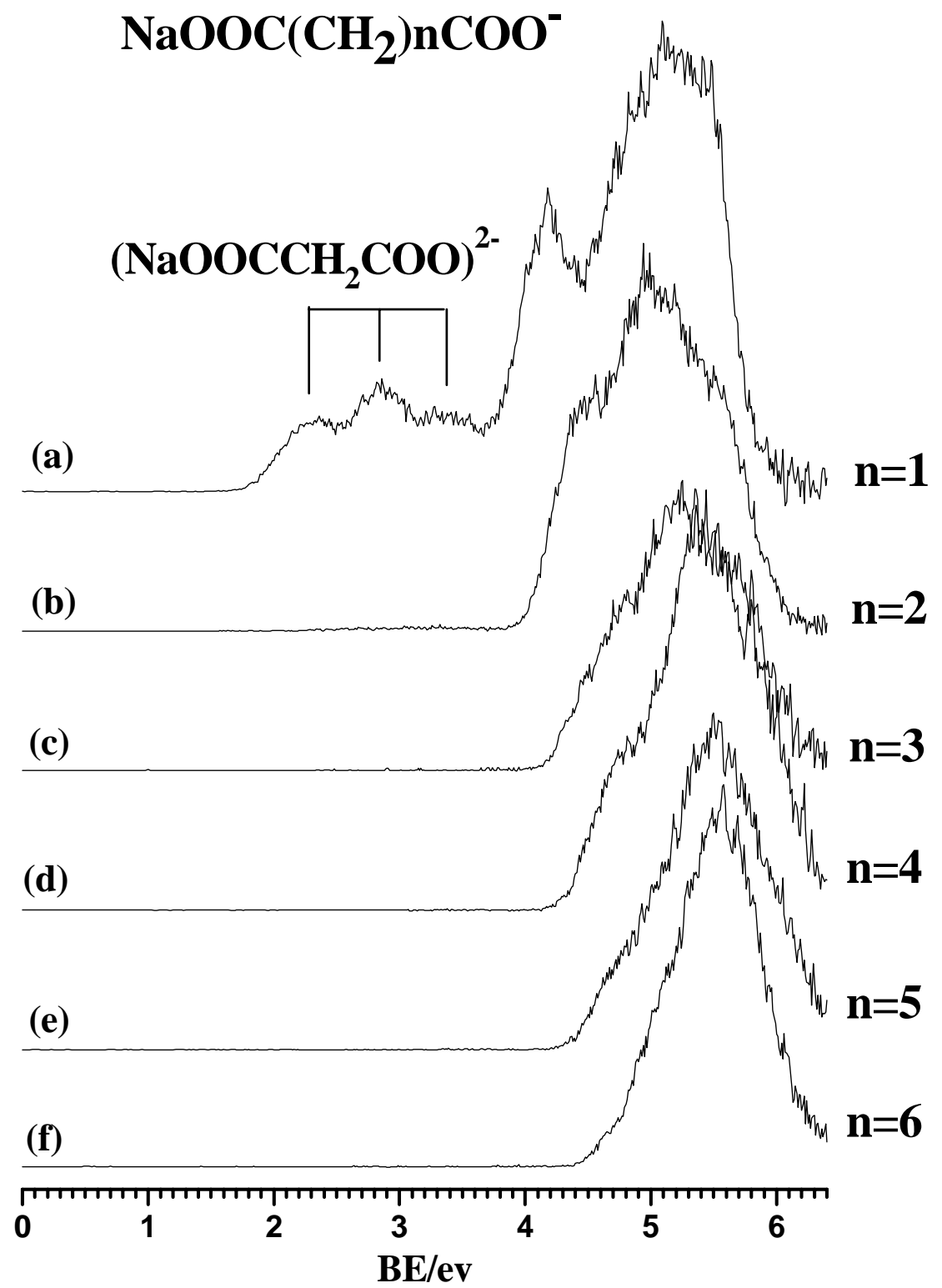

Figure 2.1 Photoelectron spectra of $\mathrm{NaOOC}\left(\mathrm{CH}_{2}\right)_{\mathrm{n}} \mathrm{COO}^{-}(\mathrm{n}=1-6)$ measured at $193 \mathrm{~nm}(6.424 \mathrm{eV})$. The value list on right is the VDE/eV. 
It is also notable that the peak of dimmer dianion $\left(\mathrm{NaOOCCH}_{2} \mathrm{COO}\right)_{2}{ }^{2-}$ is always very strong in the PES spectra of $\mathrm{NaOOCCH}_{2} \mathrm{COO}^{-}$although we have optimized the condition, we think the reason is that the $\mathrm{Na}^{+}$is stronger Lewis acid and can be coordinated by the $-\mathrm{O}^{-}$than $\mathrm{K}^{+}$, so the dianion is more preferable.

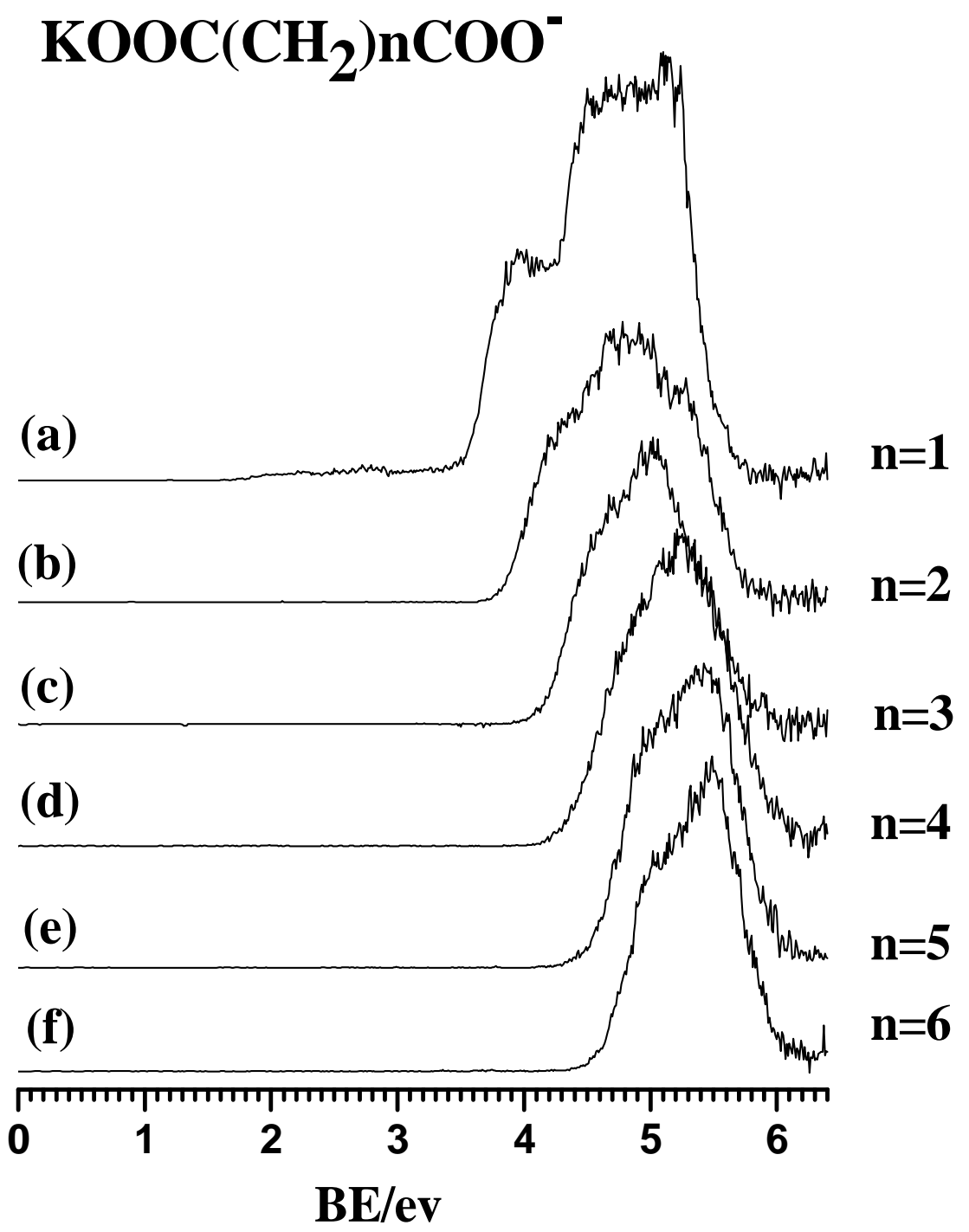

Figure 2.2 Photoelectron spectra of $\mathrm{KOOC}\left(\mathrm{CH}_{2}\right)_{n} \mathrm{COO}^{-}(\mathrm{n}=1-6)$ measured at $193 \mathrm{~nm}(6.424 \mathrm{eV})$. The value list on right is the $\mathrm{VDE} / \mathrm{eV}$. 


\section{Some oxygen-rich vanadate anion species prepared by the electro- spray of $\mathrm{NaV}_{2} \mathrm{O}_{6} / \mathrm{H}_{2} \mathrm{O}_{2} / \mathrm{H}_{2} \mathrm{O} / \mathrm{CH}_{3} \mathrm{CN}$ solution.}

We have done the electro-spray experiment with the solution of $\mathrm{NaV}_{2} \mathrm{O}_{6} / \mathrm{H}_{2} \mathrm{O}_{2} / \mathrm{H}_{2} \mathrm{O} / \mathrm{CH}_{3} \mathrm{CN}$. Some new oxygen-rich anions were found and identified, which will be discussed in detail.

Only the $\mathrm{VO}_{\mathrm{x}}{ }^{-}(\mathrm{x}=1-4)$ can be prepared by the laser-ablation on the vanadium target, while we found that $\mathrm{VO}_{5}{ }^{-}, \mathrm{VO}_{6}{ }^{-}$and $\mathrm{V}_{2} \mathrm{O}_{10}{ }^{2-}$ can also be prepared by electro-spray of solution.

The figure 3.1 show the PES spectra of $\mathrm{VO}_{5}{ }^{-}$and $\mathrm{VO}_{6}{ }^{-}$, the pattern is similar to the $\mathrm{VO}_{4}{ }^{-}$, because there is no electron in $d$-orbital of vanadium, all photo-electron are from the p-orbital of oxygen, and the ADE of $\mathrm{VO}_{5}{ }^{-}$is lower than the $\mathrm{VO}_{4}{ }^{-}$and $\mathrm{VO}_{6}{ }^{-}$, we think that the poorer delocalization of the extra electron ascribed to the odd oxygen atom in $\mathrm{VO}_{5}{ }^{-}$anion.

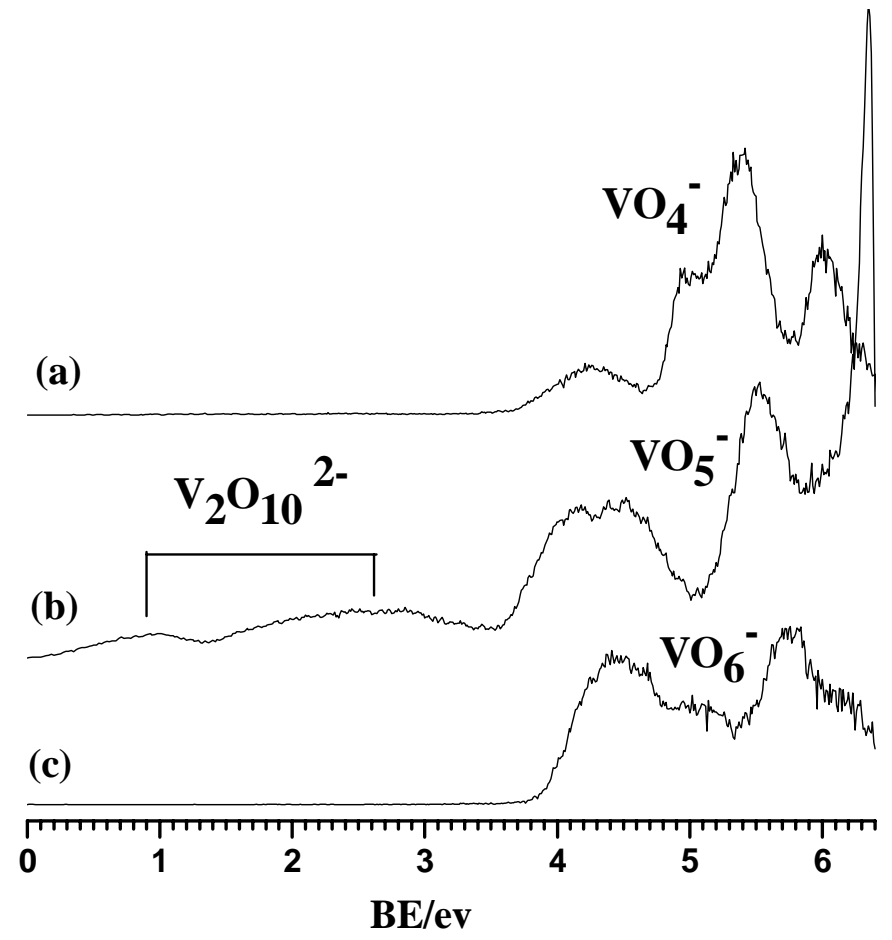

Figure 3.1 Photoelectron spectra of $\mathrm{VO}_{\mathrm{x}}(\mathrm{x}=4-6)$ measured at $193 \mathrm{~nm}$ $(6.424 \mathrm{eV})$. The value list on right is the VDE/eV. 
As shown in Figure 3.2, the dianion $\mathrm{V}_{2} \mathrm{O}_{10}{ }^{2-}$ can be prepared by adjusting the condition of electron-spray. The absence of some peak in the PES spectra with $266 \mathrm{~nm}$ and $193 \mathrm{~nm}$ laser is ascribed to the coulomb barrier which is the character of multiple-charge anion, which confirms the asignatin of these peaks to dianion $\mathrm{V}_{2} \mathrm{O}_{10}{ }^{2-}$, which was shown in figure 3.3.

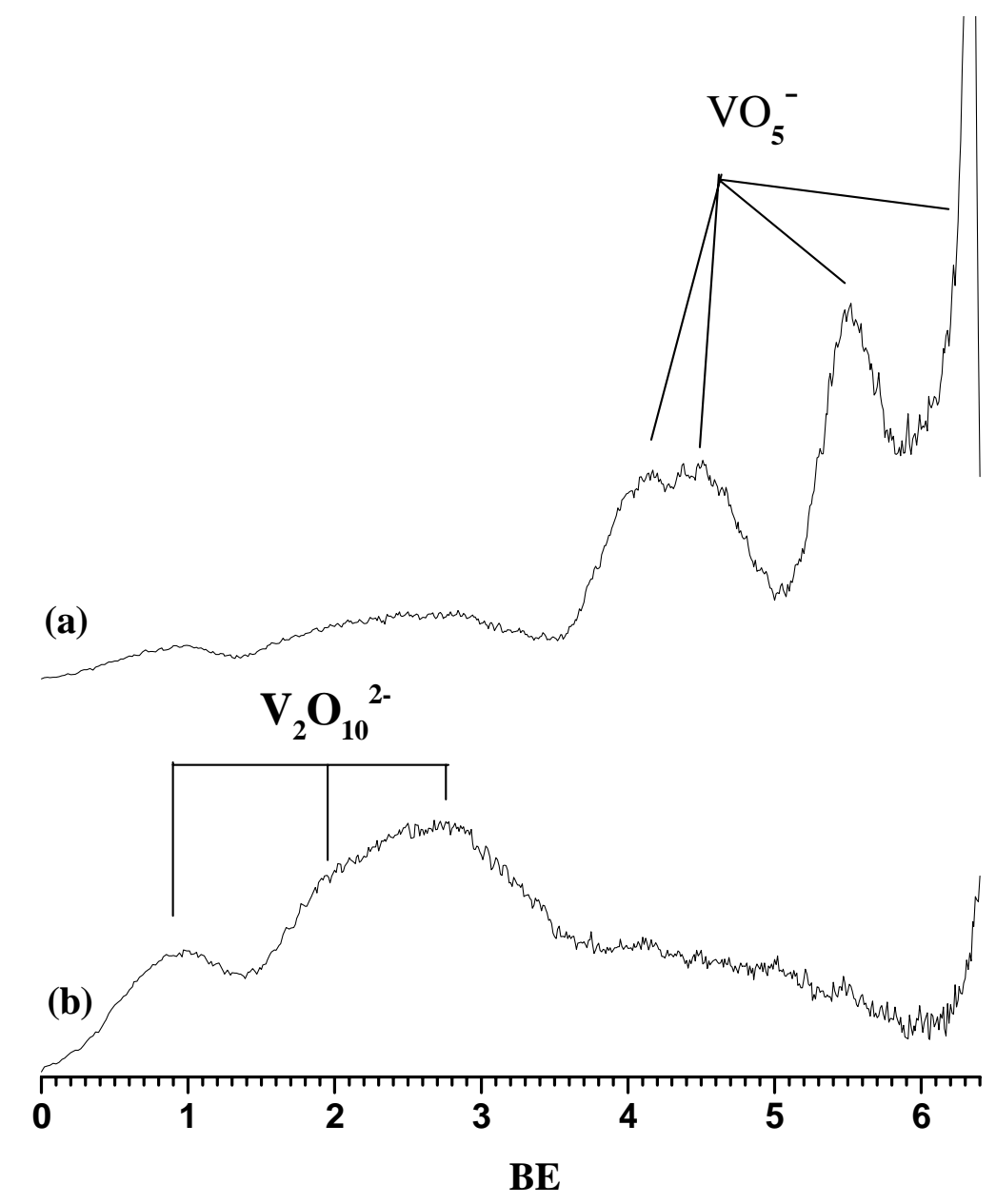

Figure 3.2 Photoelectron spectra of (a) $\mathrm{VO}^{5-}$, (b) $\mathrm{V}_{2} \mathrm{O}_{10}{ }^{2-}$ measured at $193 \mathrm{~nm}(6.424 \mathrm{eV})$. 


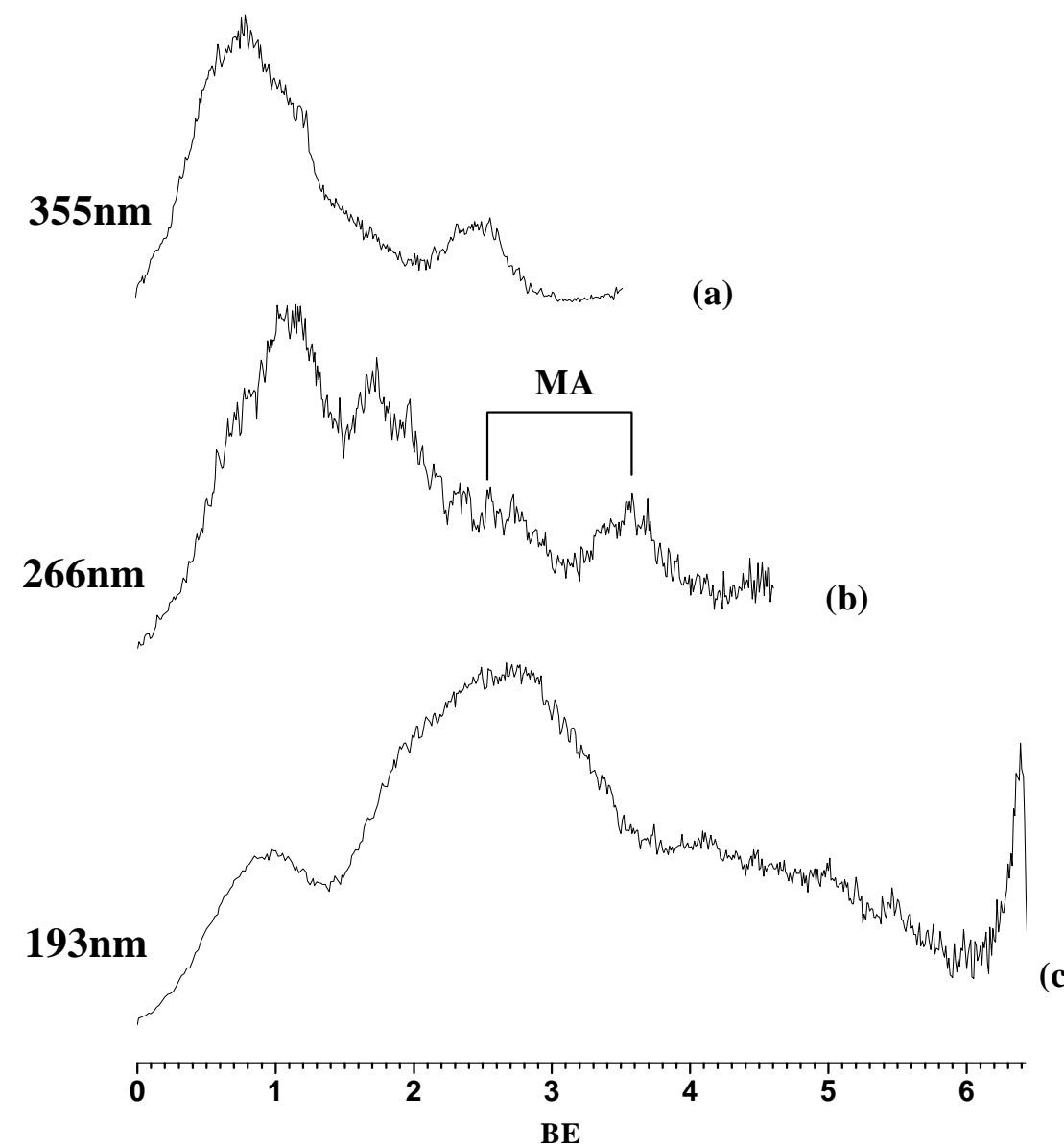

Figure 3.3 Photoelectron spectra of $\mathrm{V}_{2} \mathrm{O}_{10}{ }^{2-}$ measured at different laser wavelength (a)355 nm (3.5eV), (b)266 nm (4.662eV), (c)193 nm (6.424 eV).

It is very interesting that we have observed the $\mathrm{V}_{2} \mathrm{O}_{10}{ }^{2-}\left(\mathrm{H}_{2} \mathrm{O}_{2}\right)$, the solvated $\mathrm{V}_{2} \mathrm{O}_{10}{ }^{2-}$ by $\mathrm{H}_{2} \mathrm{O}_{2}$. The PES spectra was shown in Figure 3.4. It was showed that the pattern of two spectra is very similar except that the shift. The ADE of $\mathrm{V}_{2} \mathrm{O}_{10}{ }^{2-}$ is very low and approximate to $0.1 \mathrm{eV}$, while the ADE $\mathrm{V}_{2} \mathrm{O}_{10}{ }^{2-}\left(\mathrm{H}_{2} \mathrm{O}_{2}\right)$ increase to $0.9 \mathrm{eV}$, the shift is large than the attribution by one $\mathrm{H}_{2} \mathrm{O}$ molecule in the salvation of anion. So we suggest that the two O-H of $\mathrm{H}_{2} \mathrm{O}_{2}$ were attached to the $\mathrm{V}_{2} \mathrm{O}_{10}{ }^{2-}$ spontaneously. 


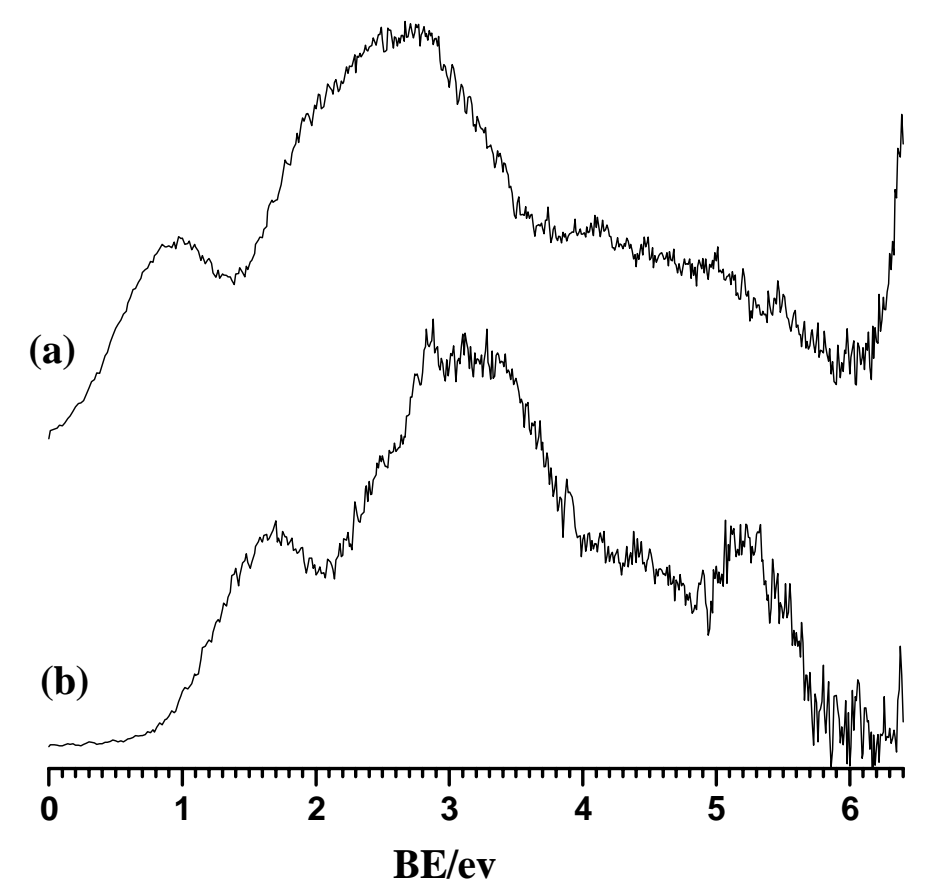

Figure 3.4 Photoelectron spectra of (a) $\mathrm{V}_{2} \mathrm{O}_{10}{ }^{2-}$, (b) $\mathrm{V}_{2} \mathrm{O}_{10}{ }^{2-}\left(\mathrm{H}_{2} \mathrm{O}_{2}\right)$ measured at $193 \mathrm{~nm}(6.424 \mathrm{eV})$.

References:

(1)Wang, X. B.; Yang, X.; Nicholas, J. B.; Wang, L. S. Science 2001, 294, 1332

(2)Wang, X. B.; Woo, H. K.; Kiran, B.; Wang, L. S. Angew. Chem. Int. Ed. 2005, 44, 4968

(3) Wang, X. B.; Woo, H. K.; Kiran, B.; Wang, L. S. Lau, K. C. J. Phys. Chem. A 2006, 110, 7801

(4) Wang, X. B.; Yang, X.; Nicholas, J. B.; Wang, L. S. J. Chem. Phys. 2003, 119, 3611

(5) Wu, H. B.; Wang, L. S. J. Chem. Phys. 1998, 108, 5310 
PNNL-16185 
PNNL-16185

\section{Appendix: The Survey and Responses}

\section{Interfacial and Condensed Phase Chemical Physics Summer Research Institute (ICPCPSRI): follow-up Questionnaire}

Dear Colleague;

From my perspective, the third year of PNNL's ICPCPSRI went well. However, your perspectives are much more important and will largely determine the fate of this program. Therefore, I encourage each of you to take a few minutes to answer some questions and to append any additional comments, suggestions, etc., to your reply. I would appreciate reply by 21 September, even if it is incomplete. If at a later time, you wish to add anything, please do so.

\section{Directions}

Not every question applies to everyone, and it is not necessary to answer everything that does apply. The more complete your answers, the more useful they are. I may wish to use your comments in follow-on documentation, please indicate whether you wish for me to attribute the comments to your or would prefer to remain anonymous.

•May I quote you with attribution?

\section{Section I. Contact Information}

Please provide me with current contact information

Name

Address

City, ST Zipcode, E-mail:

Are you willing to be contacted for further follow-up questions?

\section{Section II: Measures of success (or not)}

PNNL mentors and faculty advisors

1. Was this summer's activity part of an on-going collaboration?

2. How likely is this summer's experience to contribute to future collaboration outside the auspices of the summer program?

3. Did the work contribute to your research or project aims? How?

All

4. How likely is this work to appear in publication(s), presentation(s)? (If you are planning to publish or present, please provide some detail—where, when, etc. — approximately) 
5. Was the visit supported by the NSF?

6. (Grad students only) How likely is this work to contribute to your Ph.D. Thesis?

7. Were any new avenues of research opened?

8. Did your work here produce any intellectual property (IP) or is it likely to lead to IP development? (If yes, please give some description without disclosing the IP itself)

9. Would you recommend others to apply to or participate in this program?

Please add any additional comments you wish on the intellectual, technical or scientific success or shortcomings of the program.

\section{Section III. The experience}

All

1. What aspects of the program did you feel were particularly good?

2. What can we do to improve the program?

3. (Participants who visited PNNL) What difficulties did you encounter coming here or during your stay?

\section{Section IV. Comments}

Please take a moment to add any additional comments, opinions or anecdotes about the program, your visit, the research, etc.

Thank you,

Stephan E. Barlow

Director

ICPCPSRI

(509) 376-9051

se.barlow@pnl.gov 


\section{Survey Responses}

The following responses to the survey were elicited from our participants. Certain questions gave very little information and so are not included here. For example, two or three people indicated that the summer's work might lead to intellectual property, however nothing specific was reported, so those answers are omitted here. One visitor asked to remain anonymous and another reported some difficulties so that we judged it best to grant anonymity. In some case obvious spelling or grammatical errors have been corrected as well. The designation N/A here means "No Answer."

\begin{tabular}{|c|c|}
\hline $\begin{array}{l}\text { Faculty or } \\
\text { Supervisor }\end{array}$ & $\begin{array}{l}\text { II.1. Was this summer's activity part of an on-going } \\
\text { collaboration? }\end{array}$ \\
\hline William Arnold & $\begin{array}{l}\text { Yes. It provided Chan Lan Chun with additional experience and } \\
\text { skills that will enhance our ongoing research project on mineral } \\
\text { particle reactivity }\end{array}$ \\
\hline Zhongyang Cheng & $\begin{array}{l}\text { Yes. Dr. Wang has been collaborating with us on the } \\
\text { characterization of nano-scale magnetostrictive materials. The } \\
\text { activity in this summer program is a part of that collaboration. }\end{array}$ \\
\hline G. Barney Ellison & Yes. We are working with Dr. S. E. Barlow and Dr. A. Selenium \\
\hline Si-Dian Li* & Yes \\
\hline Jiri (Art) Janata & Yes \\
\hline $\begin{array}{l}\text { Faculty or } \\
\text { Supervisor }\end{array}$ & $\begin{array}{l}\text { II.2. How likely is this summer's experience to contribute to } \\
\text { future collaboration outside the auspices of the summer } \\
\text { program? }\end{array}$ \\
\hline William Arnold & Somewhat likely \\
\hline Zhongyang Cheng & $\begin{array}{l}\text { The summer's experience is great for our collaboration. This } \\
\text { experience helps us to look for future collaboration. }\end{array}$ \\
\hline G. Barney Ellison & $\begin{array}{l}\text { It is certain that we will continue to collaborate with EMSL and } \\
\text { Drs. Barlow and Zelenyuk }\end{array}$ \\
\hline Si-Dian Li* & It will help deepen further collaborations \\
\hline Jiri (Art) Janata & $\begin{array}{l}\text { We are hoping that the results obtained during the SRI will be } \\
\text { included in a joint publication and will also form a basis of joint } \\
\text { proposal to DOE }\end{array}$ \\
\hline
\end{tabular}

\begin{tabular}{|l|l|}
\hline $\begin{array}{l}\text { Faculty or } \\
\text { Supervisor }\end{array}$ & $\begin{array}{l}\text { II.3. Did the work contribute to your research or project } \\
\text { aims? How? }\end{array}$ \\
\hline William Arnold & $\begin{array}{l}\text { Yes. It provided Chan Lan Chun with additional experience and } \\
\text { skills that will enhance our ongoing research project on mineral } \\
\text { particle reactivity. }\end{array}$ \\
\hline Zhongyang Cheng & $\begin{array}{l}\text { Yes. The results obtained are highly needed for further } \\
\text { development of nano-biosensor. The results provide a solid } \\
\text { background and guideline for our investigation of nano-scale } \\
\text { magnetostrictive materials as sensor platforms }\end{array}$ \\
\hline
\end{tabular}




\begin{tabular}{|l|l|}
\hline G. Barney Ellison & $\begin{array}{l}\text { Yes, this summer's work contributed to our interests in organic } \\
\text { aerosols. Luis and I learned a great deal about the ability of } \\
\text { aerosols to take up water. }\end{array}$ \\
\hline Si-Dian Li* & $\begin{array}{l}\text { Yes. The results achieved during the program will form a new } \\
\text { foundation for future collaboration. }\end{array}$ \\
\hline Jiri (Art) Janata & $\begin{array}{l}\text { It provided experimental verification of our hypothesis that } \\
\text { metal/polyaniline composites can be built from the "bottom-up", } \\
\text { by atom-by-atom deposition. }\end{array}$ \\
\hline
\end{tabular}

\begin{tabular}{|c|c|}
\hline $\begin{array}{l}\text { Faculty or } \\
\text { Supervisor }\end{array}$ & $\begin{array}{l}\text { II.4. How likely is this work to appear in publication(s), } \\
\text { presentation(s)? (If you are planning to publish or present, } \\
\text { please provide some detail-where, when, etc.- } \\
\text { approximately) }\end{array}$ \\
\hline William Arnold & Unsure \\
\hline Zhongyang Cheng & $\begin{array}{l}\text { We will have articles published in Journals. We are preparing two } \\
\text { right now }\end{array}$ \\
\hline G. Barney Ellison & $\begin{array}{l}\text { Luis and Dr. Zelenyuk have one paper in press in Anal. Chem. } \\
\text { and they have submitted abstracts for the Dec. meeting of the } \\
\text { Am. Geophys. Union. When our aerosol mass spectrometer is } \\
\text { fabricated and operational, we will submit a paper to J. Phys. } \\
\text { Chem. }\end{array}$ \\
\hline Si-Dian Li* & $\begin{array}{l}\text { The first paper of our work is nearly finished and will be } \\
\text { submitted to the Journal of Chemical Physics. }\end{array}$ \\
\hline Jiri (Art) Janata & $\begin{array}{l}\text { As soon as we receive and analyze the final data we would like to } \\
\text { start writing a manuscript. Hopefully, the work will be of high } \\
\text { quality that would allow us to go to some top-tier journal. }\end{array}$ \\
\hline & II.7. Were any new avenues of research opened? \\
\hline William Arnold & 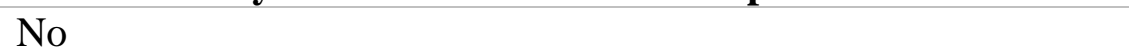 \\
\hline Zhongyang Cheng & Yes \\
\hline G. Barney Ellison & N/A \\
\hline Si-Dian Li* & Our work may open a new area of boronyl chemistry \\
\hline Jiri (Art) Janata & Yes \\
\hline $\begin{array}{l}\text { Faculty or } \\
\text { Supervisor }\end{array}$ & $\begin{array}{l}\text { II.9. Would you recommend others to apply to or participate } \\
\text { in this program? }\end{array}$ \\
\hline William Arnold & Yes \\
\hline Zhongyang Cheng & Yes \\
\hline G. Barney Ellison & This is an excellent experience and I recommend it to everyone. \\
\hline Si-Dian Li* & Without hesitation \\
\hline Jiri (Art) Janata & $\begin{array}{l}\text { Yes. I may recommend my students to apply the program next } \\
\text { year. }\end{array}$ \\
\hline
\end{tabular}




\begin{tabular}{|l|l|}
\hline $\begin{array}{l}\text { Faculty or } \\
\text { Supervisor }\end{array}$ & $\begin{array}{l}\text { III.1. What aspects of the program did you feel were } \\
\text { particularly good? }\end{array}$ \\
\hline William Arnold & $\begin{array}{l}\text { The level of support and collaborative opportunities offered. } \\
\text { The opportunity to graduate students to conduct experiments and } \\
\text { Whongyang Cheng }\end{array}$ \\
\hline G. Barney Ellison & N/A \\
\hline Si-Dian Li* & $\begin{array}{l}\text { Training on first class instrumentation; interaction with world } \\
\text { experts; }\end{array}$ \\
\hline Jiri (Art) Janata & Very good and useful \\
\hline
\end{tabular}

\begin{tabular}{|c|c|}
\hline $\begin{array}{l}\text { Faculty or } \\
\text { Supervisor }\end{array}$ & III.2. What can we do to improve the program? \\
\hline William Arnold & More widely advertise \\
\hline Zhongyang Cheng & N/A \\
\hline G. Barney Ellison & N/A \\
\hline Si-Dian Li* & Cut back on the red tape \\
\hline Jiri (Art) Janata & To further open the laboratory. \\
\hline $\begin{array}{l}\text { Faculty or } \\
\text { Supervisor }\end{array}$ & $\begin{array}{l}\text { III.3. What difficulties did you encounter coming here or } \\
\text { during your stay? }\end{array}$ \\
\hline William Arnold & $\mathrm{N} / \mathrm{A}$ \\
\hline Zhongyang Cheng & N/A \\
\hline G. Barney Ellison & N/A \\
\hline Si-Dian Li* & $\begin{array}{l}\text { Airfare to Richland is outrageously expensive; one of the co-PI's } \\
\text { (Mira Josowicz, former PNNL employee!!!!) encountered major } \\
\text { bureaucratic problems with arranging her visit, which had to be } \\
\text { cancelled. }\end{array}$ \\
\hline Jiri (Art) Janata & $\begin{array}{l}\text { Visiting other laboratories and going shopping were the biggest } \\
\text { difficulties }\end{array}$ \\
\hline $\begin{array}{l}\text { Faculty or } \\
\text { Supervisor }\end{array}$ & IV General comments \\
\hline William Arnold & N/A \\
\hline Zhongyang Cheng & N/A \\
\hline G. Barney Ellison & N/A \\
\hline Si-Dian Li* & $\begin{array}{l}\text { I used to be Associate Director of the group in which my student, } \\
\text { Amir Saheb, received his training and performed the experiments. } \\
\text { It has been particularly gratifying to see EMSL functioning as a } \\
\text { true user facility, precisely as it was initially set up. }\end{array}$ \\
\hline Jiri (Art) Janata & $\begin{array}{l}\text { I hope PNNL can further increase the scale of the program to } \\
\text { have more international students. }\end{array}$ \\
\hline & * Prof. Li was a visiting faculty participant \\
\hline
\end{tabular}




\begin{tabular}{|c|c|}
\hline PNNL Host & $\begin{array}{l}\text { II.1. Was this summer's activity part of an on-going } \\
\text { collaboration? }\end{array}$ \\
\hline Tom Autrey & No, it was to start a new one \\
\hline Don Baer & $\begin{array}{l}\text { I had two students. Both are parts of on going efforts. One is } \\
\text { formal and involves the Minnesota group active on one of my } \\
\text { research projects. One is long standing, but has no funding. }\end{array}$ \\
\hline Liem Dang & Yes \\
\hline Ram Devanathan & Yes \\
\hline Leo Fifield & $\begin{array}{l}\text { It was the beginning of what promises to be an ongoing } \\
\text { collaboration. }\end{array}$ \\
\hline Vanda Glezakou & I would say it was not, since this was my first time as a host. \\
\hline Julia Laskin & $\begin{array}{l}\text { This project provided an opportunity to follow up on our last } \\
\text { summer pilot study, resolve some open questions and expand the } \\
\text { range of systems to get a more general perspective on the gas- } \\
\text { phase chemistry of odd-electron peptide ions. }\end{array}$ \\
\hline Roberto Lins & $\begin{array}{l}\text { I had two students. One was part of an on-going collaboration, the } \\
\text { second one not. }\end{array}$ \\
\hline Thereza Soares & $\begin{array}{l}\text { No, although the ongoing work will result in a collaboration after } \\
\text { the summer student returns to his University }\end{array}$ \\
\hline Chongmin Wang & Yes \\
\hline Sotiris Xantheas & No, but it was aimed at establishing a future collaboration \\
\hline Alla Zelenyuk & Yes \\
\hline
\end{tabular}




\begin{tabular}{|c|c|}
\hline PNNL Host & $\begin{array}{l}\text { II.2. How likely is this summer's experience to contribute to } \\
\text { future collaboration outside the auspices of the summer } \\
\text { program? }\end{array}$ \\
\hline Tom Autrey & very likely \\
\hline Don Baer & Collaborations and interactions will continue \\
\hline Liem Dang & It is a good experience for students to be at the national lab \\
\hline Ram Devanathan & Quite likely \\
\hline Leo Fifield & $\begin{array}{l}\text { It has already led to submission of a joint proposal for outside } \\
\text { funding (BES). }\end{array}$ \\
\hline Vanda Glezakou & $\begin{array}{l}\text { The visitor decided she wanted to get more experience in } \\
\text { molecular simulations, so she worked more closely with my } \\
\text { colleague Liem Dang. Still, this interaction can potentially lead to } \\
\text { further collaboration of Liem and me with Professor Jordan's } \\
\text { group }\end{array}$ \\
\hline Julia Laskin & $\begin{array}{l}\text { There is no doubt that these collaborations will continue in the } \\
\text { future. }\end{array}$ \\
\hline Roberto Lins & $100 \%$ \\
\hline Thereza Soares & $\begin{array}{l}\text { I believe the summer student will continue the project as his } \\
\text { thesis work. I will also extend the research in collaboration with } \\
\text { Dr. Chenghong Lei here at PNNL }\end{array}$ \\
\hline Chongmin Wang & The collaboration will continue \\
\hline Sotiris Xantheas & Very likely \\
\hline Alla Zelenyuk & Very likely \\
\hline
\end{tabular}




\begin{tabular}{|c|c|}
\hline PNNL Host & $\begin{array}{l}\text { II.3. Did the work contribute to your research or project } \\
\text { aims? How? }\end{array}$ \\
\hline Tom Autrey & $\begin{array}{l}\text { not existing programs but helped to develop understand for future } \\
\text { programs }\end{array}$ \\
\hline Don Baer & $\begin{array}{l}\text { The Chan Lan Chun activities move our research forward and } \\
\text { will likely help define future work by the whole project team. } \\
\text { The research by Amir is still taking place and results are } \\
\text { unknown. }\end{array}$ \\
\hline Liem Dang & $\begin{array}{l}\text { It is contributed somewhat to my research, but I let the student } \\
\text { choose the projects to work on so that she can feel more familiar } \\
\text { with the subjects }\end{array}$ \\
\hline Ram Devanathan & $\begin{array}{l}\text { Not really. The SRI work was only slightly related to my } \\
\text { research }\end{array}$ \\
\hline Leo Fifield & $\begin{array}{l}\text { The visitor and I have complimentary expertise and the } \\
\text { combination strengthens our individual efforts }\end{array}$ \\
\hline Vanda Glezakou & It has not been assessed yet. \\
\hline Julia Laskin & $\begin{array}{l}\text { Yes. This area of research has been incorporated into my BES } \\
\text { program. The novel findings from this summer research will have } \\
\text { a significant impact on my program }\end{array}$ \\
\hline Roberto Lins & $\begin{array}{l}\text { Yes. The student who came as part of an existing collaboration } \\
\text { have generated, processed and analyzed data producing enough } \\
\text { material for a scientific article, which will be soon written. The } \\
\text { second student has learned the basic methodology will continue } \\
\text { the work. He has also asked me about the possibility of being his } \\
\text { Ph.D. co-supervisor }\end{array}$ \\
\hline Thereza Soares & It may contribute to possible grant proposal \\
\hline Chongmin Wang & Yes, this contributes our research interests in nano materials \\
\hline Sotiris Xantheas & $\begin{array}{l}\text { Yes. During the visit the faculty member interacted with several } \\
\text { PNNL staff members who are working in the general area of } \\
\text { excited electronic states of aqueous clusters both in the } \\
\text { experimental and theoretical fields. }\end{array}$ \\
\hline Alla Zelenyuk & Yes \\
\hline
\end{tabular}




\begin{tabular}{|c|c|}
\hline PNNL Host & $\begin{array}{l}\text { II.4. How likely is this work to appear in publication(s), } \\
\text { presentation(s)? (If you are planning to publish or present, } \\
\text { please provide some detail-where, when, etc.- } \\
\text { approximately) }\end{array}$ \\
\hline Tom Autrey & the work will be published next year \\
\hline Don Baer & Work will likely appear, location not yet certain. \\
\hline Liem Dang & $\begin{array}{l}\text { My summer student finished some calculations while she was } \\
\text { here, but I am not sure that will lead to publication. }\end{array}$ \\
\hline Ram Devanathan & Not likely right now. This work is in the initial stages \\
\hline Leo Fifield & $\begin{array}{l}\text { The work from this year will not be included in a presentation-- } \\
\text { not enough progress yet. }\end{array}$ \\
\hline Vanda Glezakou & Possibly \\
\hline Julia Laskin & $\begin{array}{l}\text { The results will be presented at the 22nd Asilomar Conference on } \\
\text { Mass Spectrometry, Pacific Grove, California, October } 20 \text { - 24, } \\
\text { 2006; 4th International UPPSALA Conference on ECD/ETD } \\
\text { Mass Spectrometry, Hong-Kong, China, December 12-15, 2006; } \\
\text { Gordon Research Conference on Gaseous Ions: Structures, } \\
\text { Energetics \& Reactions, Ventura, CA, Feb 25-Mar 2, 2007. } \\
\text { Several papers summarizing our results are in preparation }\end{array}$ \\
\hline Roberto Lins & $\begin{array}{l}\text { One or two scientific publications should appear in a } \\
\text { biophysics/biochemistry peer-review indexed journal mid/late } \\
2007\end{array}$ \\
\hline Thereza Soares & $\begin{array}{l}\text { I estimate that the work should lead to a scientific manuscript. I } \\
\text { expect it to be submitted for publication sometime in the summer } \\
2007 \text {. We don not know as of yet where we will send it for } \\
\text { publication, but I will gladly inform the SRI once the manuscript } \\
\text { is out. }\end{array}$ \\
\hline Chongmin Wang & A joint publication is under drafting \\
\hline Sotiris Xantheas & Very likely \\
\hline Alla Zelenyuk & $\begin{array}{l}\text { Very likely. An abstract was submitted to the Fall } 2006 \text { AGU } \\
\text { meeting (San Francisco, CA December } 2006\end{array}$ \\
\hline
\end{tabular}




\begin{tabular}{|c|c|}
\hline PNNL Host & II.7. Were any new avenues of research opened? \\
\hline Tom Autrey & Yes \\
\hline Don Baer & Started doped nano particle efforts with Chan Lan \\
\hline Liem Dang & $\mathrm{N} / \mathrm{A}$ \\
\hline Ram Devanathan & Yes. We used ab initio calculations to study oxide surfaces \\
\hline Leo Fifield & $\begin{array}{l}\text { In situ methods, not previously considered, were conceived of } \\
\text { and developed }\end{array}$ \\
\hline Vanda Glezakou & $\begin{array}{l}\text { Possibly, interns of more collaborations, getting to know the work } \\
\text { of other scientists, and making our work known to them. }\end{array}$ \\
\hline Julia Laskin & $\begin{array}{l}\text { Yes. Gas-phase chemistry of peptide radical anions is a } \\
\text { completely new unexplored area of research. Our study provides } \\
\text { a first very fundamental insight on the energetics and dynamics of } \\
\text { unimolecular fragmentation for these systems }\end{array}$ \\
\hline Roberto Lins & $\begin{array}{l}\text { Yes. The research project started by one of the students addressed } \\
\text { an important fundamental problem in the understanding of } \\
\text { bacterial biofilm formation and may provide material for future } \\
\text { grant applications. }\end{array}$ \\
\hline Thereza Soares & Possibly, but it is difficult to say just yet \\
\hline Chongmin Wang & Not yet, \\
\hline Sotiris Xantheas & N/A \\
\hline Alla Zelenyuk & Yes \\
\hline
\end{tabular}




\begin{tabular}{|l|l|}
\hline PNNL Host & $\begin{array}{l}\text { II.9. Would you recommend others to apply to or participate } \\
\text { in this program? }\end{array}$ \\
\hline Tom Autrey & yes this is a very good program \\
\hline Don Baer & $\begin{array}{l}\text { I am to the stage where I am not useful as a mentor because I am } \\
\text { not accessible. In all cases I need to line up others to do the } \\
\text { hands on effort. This does not always work equally well. }\end{array}$ \\
\hline Liem Dang & N/A \\
\hline Ram Devanathan & Yes \\
\hline Leo Fifield & Yes \\
\hline Vanda Glezakou & Absolutely \\
\hline Julia Laskin & Yes \\
\hline Roberto Lins & Definitely yes \\
\hline Thereza Soares & $\begin{array}{l}\text { Yes, I would certainly recommend either colleagues or students } \\
\text { to participate in the program. However, one should be aware that } \\
\text { this is a "free-time" activity since there are no work packages for } \\
\text { it. In other words, one must be really motivated about mentorship } \\
\text { to supervise a student in his/her free time }\end{array}$ \\
\hline Chongmin Wang & yes \\
\hline Sotiris Xantheas & N/A \\
\hline Alla Zelenyuk & Yes \\
\hline
\end{tabular}




\begin{tabular}{|l|l|}
\hline PNNL Host & $\begin{array}{l}\text { III.1. What aspects of the program did you feel were } \\
\text { particularly good? }\end{array}$ \\
\hline Tom Autrey & N/A \\
\hline Don Baer & N/A \\
\hline Liem Dang & N/A \\
\hline Ram Devanathan & I enjoyed mentoring the student. \\
\hline Leo Fifield & $\begin{array}{l}\text { The ability to meet face-to-face and work in the lab together } \\
\text { Discussions with the visitor, scientific and other, share } \\
\text { experiences and cultural information, help the visitor solve } \\
\text { specific problems }\end{array}$ \\
\hline Vanda Glezakou & $\begin{array}{l}\text { The program provided support to cover expenses that allowed } \\
\text { participants to spend a descent amount of time at PNNL }\end{array}$ \\
\hline Julia Laskin & $\begin{array}{l}\text { Organization and welcoming of the participants; flexibility and } \\
\text { understanding of the organizers to fit the needs of different } \\
\text { attendees. In particular, I would like to thank Ms. Nikki Avery for } \\
\text { the efficient, knowledgeable and friendly coordination of the } \\
\text { project. }\end{array}$ \\
\hline Roberto Lins & $\begin{array}{l}\text { I have appreciated the opportunity of mentoring a student which } \\
\text { is not readily available in the National Laboratories due to the } \\
\text { non-academic character of these Institutions. However, the nicest } \\
\text { aspect of the program is the freedom to choose a research project } \\
\text { that is not necessarily related to the mentor ongoing research. }\end{array}$ \\
\hline Thereza Soares & $\begin{array}{l}\text { Diversified research experience for students } \\
\text { The access of the visitors to several research areas and the } \\
\text { interaction with PNNL staff. The opportunity to attend meetings, } \\
\text { seminars etc and be exposed to several aspects of scientific } \\
\text { research } \\
\text { N/A }\end{array}$ \\
\hline Chongmin Wang
\end{tabular}




\begin{tabular}{|c|c|}
\hline PNNL Host & III.2. What can we do to improve the program? \\
\hline Tom Autrey & N/A \\
\hline Don Baer & N/A \\
\hline Liem Dang & N/A \\
\hline Ram Devanathan & It is fine the way it is \\
\hline Leo Fifield & N/A \\
\hline Vanda Glezakou & N/A \\
\hline Julia Laskin & $\begin{array}{l}\text { The program provided support to cover expenses that allowed } \\
\text { participants to spend a descent amount of time at PNNL }\end{array}$ \\
\hline Roberto Lins & $\begin{array}{l}\text { i) I think a short seminar series might help to stimulate discussion } \\
\text { and social interaction among the participants. The seminars, } \\
\text { scattered throughout the period so to fit everybody's schedule, } \\
\text { could be short ( } 15 \text { min) and would not have to involve results - } \\
\text { the student project plan would suffice. That would also inform the } \\
\text { other participants about the different projects being carried out at } \\
\text { PNNL by their colleagues; ii) Transportation aid. Some of the } \\
\text { participants (most of them I would think) were housed at the } \\
\text { PNNL housing facility. While the facility is an excellent resource, } \\
\text { transportation between the campus and the town is not quite } \\
\text { convenient. Having the bus to come up to the campus once or } \\
\text { twice a day or at least the possibility of a shuttle service once or } \\
\text { twice a week to the town (on a fixed-schedule basis) would help } \\
\text { the participants to organize themselves to go shopping for } \\
\text { groceries. }\end{array}$ \\
\hline Thereza Soares & $\begin{array}{l}\text { It is hard to say since I believe the program has a nice and } \\
\text { flexible format. However, I would suggest that every student } \\
\text { should give a talk about the work done before leaving. Certainly } \\
\text { it involves more work, but I think it will give a sense of } \\
\text { accountability to the students. Of course, I would gladly help with } \\
\text { the organization of such a thing if needed. }\end{array}$ \\
\hline Chongmin Wang & seems to be run seamless \\
\hline Sotiris Xantheas & $\begin{array}{l}\text { I felt that the program was running very smoothly thanks to the } \\
\text { SRI director and his staff. The environment was very friendly } \\
\text { and supportive. I cannot think of any further improvements to an } \\
\text { already successful program }\end{array}$ \\
\hline Alla Zelenyuk & To accommodate more participants \\
\hline
\end{tabular}




\begin{tabular}{|l|l|}
\hline PNNL Host & $\begin{array}{l}\text { III.3. What difficulties did you encounter coming here or } \\
\text { during your stay? }\end{array}$ \\
\hline Tom Autrey & N/A \\
\hline Don Baer & N/A \\
\hline Liem Dang & N/A \\
\hline Ram Devanathan & N/A \\
\hline Leo Fifield & N/A \\
Vanda Glezakou & $\begin{array}{l}\text { I think it is a very valuable part of the PNNL environment. } \\
\text { Everybody involved did an excellent job to integrate the visitors } \\
\text { here and provided a lot of help to the hosts in dealing with the } \\
\text { necessary paperwork }\end{array}$ \\
\hline Julia Laskin & N/A \\
\hline R. Lins & N/A \\
\hline T. Soares & N/A \\
\hline C, Wang & N/A \\
\hline Sotiris Xantheas & N/A \\
\hline Alla Zelenyuk & N/A \\
\hline PNNL Host & IV General comments \\
\hline Tom Autrey & N/A \\
\hline Don Baer & N/A \\
\hline Liem Dang & N/A \\
\hline Ram Devanathan & N/A \\
\hline Leo Fifield & N/A \\
\hline Vanda Glezakou & N/A \\
\hline Julia Laskin & Steve's barbeques were great! \\
\hline Roberto Lins & $\begin{array}{l}\text { I believe the program provides us with a good opportunity to } \\
\text { establish and/or to continue collaborative research with } \\
\text { Academia. }\end{array}$ \\
\hline Thereza Soares & I will leave this to the students .... \\
\hline Chongmin Wang & $\begin{array}{l}\text { Steve and his team are running a wonderful program and this } \\
\text { provides an eye opening opportunity for graduate student }\end{array}$ \\
\hline Alla Zelenyuk & $\begin{array}{l}\text { I think this is a highly valuable program both for the visitors and } \\
\text { PNNL staff. It is run quite efficiently and effectively and I hope } \\
\text { that it will be continued in the future for the mutual benefit of } \\
\text { both visitors and hosts }\end{array}$ \\
\hline N/A \\
\hline
\end{tabular}




\begin{tabular}{|l|l|}
\hline Visitor & $\begin{array}{l}\text { II.1. Was this summer's activity part of an on-going } \\
\text { collaboration? }\end{array}$ \\
\hline Jeremy Cain & Yes \\
\hline $\begin{array}{l}\text { Luis A. Cuadra- } \\
\text { Rodriguez }\end{array}$ & Yes \\
\hline Jun Cui & Yes \\
\hline Diego Gomes & N/A \\
\hline $\begin{array}{l}\text { Rodrigo González } \\
\text { Abraham }\end{array}$ & Yes \\
\hline Matteo Guglielmi & N/A \\
\hline Rebecca J Hopkins & $\begin{array}{l}\text { Yes. This was part of an active collaboration between the groups } \\
\text { of Alexander Laskin (PNNL) and Mary Gilles (LBNL) }\end{array}$ \\
\hline Ngor Wai Lam & N/A \\
\hline Chan-Woo Lee & Yes \\
\hline Suiqiong Li & N/A \\
\hline Rebecca Lowton & Yes \\
\hline Amir Saheb & Yes \\
\hline Guanjun Wang & N/A \\
\hline (name on file) & Yes \\
(name on file) & N/A \\
\hline
\end{tabular}




\begin{tabular}{|c|c|}
\hline Visitor & $\begin{array}{l}\text { II.2. How likely is this summer's experience to contribute to } \\
\text { future collaboration outside the auspices of the summer } \\
\text { program? }\end{array}$ \\
\hline Jeremy Cain & $\begin{array}{l}\text { Not much. We planned on collaborating together regardless of } \\
\text { what happened this summer. However, the results obtained this } \\
\text { summer helped with our collaboration, as we worked well } \\
\text { together. }\end{array}$ \\
\hline $\begin{array}{l}\text { Luis A. Cuadra- } \\
\text { Rodriguez }\end{array}$ & N/A \\
\hline Jun Cui & Very Likely \\
\hline Diego Gomes & N/A \\
\hline $\begin{array}{l}\text { Rodrigo González } \\
\text { Abraham }\end{array}$ & Very likely \\
\hline Matteo Guglielmi & N/A \\
\hline Rebecca J Hopkins & $\begin{array}{l}\text { This opportunity is likely to reinforce the existing collaboration. } \\
\text { A better appreciation of potential opportunities for research in the } \\
\text { future has been gained through this experience }\end{array}$ \\
\hline Ngor Wai Lam & N/A \\
\hline Chan-Woo Lee & $\begin{array}{l}\text { The experience at PNNL gave me a good idea about my research } \\
\text { and strengthen my potential as a researcher }\end{array}$ \\
\hline Suiqiong Li & N/A \\
\hline Rebecca Lowton & Very Likely \\
\hline Amir Saheb & Depending on the final results more characterization may follow. \\
\hline Guanjun Wang & N/A \\
\hline (name on file) & Very \\
\hline (name on file) & N/A \\
\hline
\end{tabular}




\begin{tabular}{|c|c|}
\hline Visitor & $\begin{array}{l}\text { II.3. Did the work contribute to your research or project } \\
\text { aims? How? }\end{array}$ \\
\hline Jeremy Cain & $\begin{array}{l}\text { Yes. The work we are collaborating on is essentially my doctoral } \\
\text { project. }\end{array}$ \\
\hline $\begin{array}{l}\text { Luis A. Cuadra- } \\
\text { Rodriguez }\end{array}$ & T J m t \\
\hline Jun Cui & $\begin{array}{l}\text { Yes, the skills I learned in PNNL will be used for my current and } \\
\text { future project. }\end{array}$ \\
\hline Diego Gomes & N/A \\
\hline $\begin{array}{l}\text { Rodrigo González } \\
\text { Abraham }\end{array}$ & $\begin{array}{l}\text { Yes: Helping on the Chemical and Physical characterization of } \\
\text { the suspended particles in the Mexico city atmosphere. }\end{array}$ \\
\hline Matteo Guglielmi & 型 \\
\hline Rebecca J Hopkins & $\begin{array}{l}\text { Yes. Significant experimental progress was made towards the } \\
\text { project aims. Samples collected during a scientific field mission } \\
\text { were examined using experimental techniques available at PNNL. } \\
\text { The results obtained from these experiments will determine the } \\
\text { direction of experiments conducted at LBNL. }\end{array}$ \\
\hline Ngor Wai Lam & N/A \\
\hline Chan-Woo Lee & $\begin{array}{l}\text { Yes. I learned my things about my research that I might miss if I } \\
\text { didn't attend SRI }\end{array}$ \\
\hline Suiqiong Li & $\mathrm{N} / \mathrm{A}$ \\
\hline Rebecca Lowton & $\begin{array}{l}\text { Partially. I am expecting to continue with my project here as is, } \\
\text { but also using analysis techniques developed at PNNL }\end{array}$ \\
\hline Amir Saheb & $\begin{array}{l}\text { Yes, samples prepared previously at my institution were } \\
\text { characterized using the latest elemental analysis techniques. }\end{array}$ \\
\hline Guanjun Wang & N/A \\
\hline (name on file) & $\begin{array}{l}\text { Yes. This research was a valuable follow-up on a previous } \\
\text { collaboration for chemical analysis of aerosol samples from the } \\
\text { Mexico City Metropolitan Area. We were able to build on } \\
\text { previous results of physical and chemical characteristics of } \\
\text { MCMA aerosol, as well as pursue several new research } \\
\text { directions. EMSL at PNNL has excellent research facilities, and } \\
\text { a unique capability to bring together results from different } \\
\text { analytical techniques to better understand a given question. }\end{array}$ \\
\hline (name on file) & N/A \\
\hline
\end{tabular}




\begin{tabular}{|c|c|}
\hline Visitor & $\begin{array}{l}\text { II.4. How likely is this work to appear in publication(s), } \\
\text { presentation(s)? (If you are planning to publish or present, } \\
\text { please provide some detail-where, when, etc.- } \\
\text { approximately) }\end{array}$ \\
\hline Jeremy Cain & Very likely to be published within a year \\
\hline $\begin{array}{l}\text { Luis A. Cuadra- } \\
\text { Rodriguez }\end{array}$ & $\begin{array}{l}\text { This is a continuation of ongoing collaboration with Dr. Alla } \\
\text { Zelenyuk where we have publish a paper on Analytical Chemistry } \\
\text { (available online). Also the work done during the summer is } \\
\text { submitted to be presented at the AGU meeting on Dec. } 2006\end{array}$ \\
\hline Jun Cui & $\begin{array}{l}\text { The project is under progress. We are expecting some } \\
\text { publications and presentations in the future. But now, we cannot } \\
\text { predict the exact time. }\end{array}$ \\
\hline Diego Gomes & $\begin{array}{l}\text { Yes, as soon as we get the final results, analyze and discuss them, } \\
\text { we would like to present this work at a conference for further } \\
\text { discussion and then publish in a specialized journal. }\end{array}$ \\
\hline $\begin{array}{l}\text { Rodrigo González } \\
\text { Abraham }\end{array}$ & Very likely, we do not have exactly dates yet. \\
\hline Matteo Guglielmi & $\begin{array}{l}\text { We are writing the paper and I'll present the results at the EPFL } \\
\text { (Switzerland) by the end of this year }\end{array}$ \\
\hline Rebecca J Hopkins & $\begin{array}{l}\text { This work was recently presented at two conferences - ACS in } \\
\text { San Francisco and AAAR in Minnesota. }\end{array}$ \\
\hline Ngor Wai Lam & $\begin{array}{l}\text { Part of the materials and results are presented in 54th ASMS in } \\
\text { Seattle by Dr. Julia Laskin this year (28May2006 to 1June2006) }\end{array}$ \\
\hline Chan-Woo Lee & Yes \\
\hline Suiqiong Li & $\begin{array}{l}\text { The work will be presented at } 2006 \text { MRS (Materials Research } \\
\text { Society) Fall Meeting, which will be held at Boston this } \\
\text { December. The proceeding will be published after the meeting. I } \\
\text { am also preparing a journal paper based on the work I did at SRI. } \\
\text { I plan to submit it to Journal of Nanoscience and } \\
\text { Nanotechnology. }\end{array}$ \\
\hline Rebecca Lowton & Unknown \\
\hline Amir Saheb & $\begin{array}{l}\text { It is likely to appear in a publication or presentation with relation } \\
\text { to chemistry or materials science journals or conferences }\end{array}$ \\
\hline Guanjun Wang & There are one or two potential publication on this work \\
\hline (name on file) & $\begin{array}{l}\text { Very likely to feature into a future publication on the MILAGRO- } \\
2006 \text { study and/or conference presentation. }\end{array}$ \\
\hline (name on file) & $\begin{array}{l}\text { This summer's work is actually fairly unlikely to appear in a } \\
\text { publication because the quality of the data is poor. Although the } \\
\text { idea is/was novel, a shortage of time and the early stage of } \\
\text { development of the instrumentation will not allow a quality } \\
\text { publication to result }\end{array}$ \\
\hline
\end{tabular}




\begin{tabular}{|c|c|}
\hline Visitor & $\begin{array}{l}\text { II.6. How likely is this work to contribute to your PhD. } \\
\text { Thesis? }\end{array}$ \\
\hline Jeremy Cain & $\begin{array}{l}\text { Absolutely. The work done this past summer will go into my } \\
\text { doctoral thesis. }\end{array}$ \\
\hline $\begin{array}{l}\text { Luis A. Cuadra- } \\
\text { Rodriguez }\end{array}$ & $\begin{array}{l}\text { The contributed in all aspects of my research, from design to } \\
\text { understanding the system. These are preliminary experiments that } \\
\text { had help us understand better particle generation and analysis, } \\
\text { which will help us design and build a new aerosol mass } \\
\text { spectrometer to study aqueous organic aerosols }\end{array}$ \\
\hline Jun Cui & $\begin{array}{l}\text { The work will be part of my continuing project as a postdoc in the } \\
\text { group. I also used the skills I learn in PNNL for my PhD. } \\
\text { Proposal. }\end{array}$ \\
\hline Diego Gomes & $\begin{array}{l}\text { The work is not intended to be part of the Ph.D. Thesis but the } \\
\text { same techniques and simulation approaches learned at PNNL will } \\
\text { be applied to another biological system. }\end{array}$ \\
\hline $\begin{array}{l}\text { Rodrigo González } \\
\text { Abraham }\end{array}$ & N/A \\
\hline Matteo Guglielmi & This will be one of the 23 topics of My Ph.D. Thesis. \\
\hline Rebecca J Hopkins & N/A \\
\hline Ngor Wai Lam & N/A \\
\hline Chan-Woo Lee & $\begin{array}{l}\text { The work will improve creativity and productivity of my Ph.D. } \\
\text { Thesis }\end{array}$ \\
\hline Suiqiong Li & $\begin{array}{l}\text { One of the chapters in my dissertation will be written based on } \\
\text { the work I did at SRI. }\end{array}$ \\
\hline Rebecca Lowton & Unknown \\
\hline Amir Saheb & $\begin{array}{l}\text { It will probably play a role in my thesis if we continue with the } \\
\text { work and extend it beyond the preliminary results. }\end{array}$ \\
\hline Guanjun Wang & This work is very helpful to my $\mathrm{Ph}$. D \\
\hline (name on file) & $\begin{array}{l}\text { Several aspects of the SRI summer research will contribute to my } \\
\text { thesis, although it primarily focuses on our previous collaboration } \\
\text { from } 2003\end{array}$ \\
\hline (name on file) & $\begin{array}{l}\text { This work will most likely be a small chapter of my Ph.D. thesis. } \\
\text { I feel that I gained more experience and general knowledge rather } \\
\text { than physical data and conclusions to contribute to my thesis }\end{array}$ \\
\hline
\end{tabular}




\begin{tabular}{|c|c|}
\hline Visitor & II.7. Were any new avenues of research opened? \\
\hline Jeremy Cain & $\begin{array}{l}\text { Quite possibly. In talking to researchers at the lab, I can get a } \\
\text { feel for what kind of new work could possibly be done }\end{array}$ \\
\hline $\begin{array}{l}\text { Luis A. Cuadra- } \\
\text { Rodriguez }\end{array}$ & $\begin{array}{l}\text { One other possibility opened where we could have two types of } \\
\text { particle generation that leads to different schemes for analysis. } \\
\text { This is going to be incorporated in the design }\end{array}$ \\
\hline Jun Cui & $\mathrm{N} / \mathrm{A}$ \\
\hline Diego Gomes & N/A \\
\hline $\begin{array}{l}\text { Rodrigo González } \\
\text { Abraham }\end{array}$ & Yes \\
\hline Matteo Guglielmi & $\begin{array}{l}\text { This work will contribute to the understanding of the } \\
\text { stability of pores immersed into biological membranes. }\end{array}$ \\
\hline Rebecca J Hopkins & $\begin{array}{l}\text { Yes. A possible further collaboration between LBNL and PNNL } \\
\text { was identified. }\end{array}$ \\
\hline Ngor Wai Lam & Yes \\
\hline Chan-Woo Lee & $\begin{array}{l}\text { I found that surface/interface science (experiments) are very } \\
\text { interesting }\end{array}$ \\
\hline Suiqiong Li & $\begin{array}{l}\text { This research introduced a novel magnetostrictive acoustic wave } \\
\text { sensor platform, which have great potential to be the high } \\
\text { performance biosensor for future biological analysis. }\end{array}$ \\
\hline Rebecca Lowton & Yes \\
\hline Amir Saheb & Depends on final analysis of the results \\
\hline Guanjun Wang & Yes \\
\hline (name on file) & $\begin{array}{l}\text { Yes, specifically we are investigating the subject of aerosol } \\
\text { processing and transport through samples collected from various } \\
\text { field sites in and surrounding the MCMA urban area. }\end{array}$ \\
\hline (name on file) & No \\
\hline
\end{tabular}




\begin{tabular}{|c|c|}
\hline Visitor & $\begin{array}{l}\text { II.9. Would you recommend others to apply to or participate } \\
\text { in this program? }\end{array}$ \\
\hline Jeremy Cain & $\begin{array}{l}\text { Yes. The facilities at PNNL are very nice and give a researcher } \\
\text { the ability to conduct excellent research. It is a great experience } \\
\text { because it shows the student what he/she will most likely be } \\
\text { doing in the years to come }\end{array}$ \\
\hline $\begin{array}{l}\text { Luis A. Cuadra- } \\
\text { Rodriguez }\end{array}$ & $\begin{array}{l}\text { I think the program is great. Is an excellent opportunity to meet } \\
\text { established scientists in the field and other fields and students } \\
\text { from all over the country as well. The facilities and help from } \\
\text { everyone in program were outstanding making research develop } \\
\text { smoothly. }\end{array}$ \\
\hline Jun Cui & Yes \\
\hline Diego Gomes & $\begin{array}{l}\text { Absolutely. It was a great opportunity to work with top quality } \\
\text { researchers and abundance of computational resources, together } \\
\text { with a very well organized institution it's a quite good place work } \\
\text { and produce high quality science. }\end{array}$ \\
\hline $\begin{array}{l}\text { Rodrigo González } \\
\text { Abraham }\end{array}$ & Yes \\
\hline Matteo Guglielmi & Absolutely. \\
\hline Rebecca J Hopkins & $\begin{array}{l}\text { Yes. I would recommend that others participate in this program. I } \\
\text { think that exposure to the wide range of research being conducted } \\
\text { at PNNL is very beneficial. }\end{array}$ \\
\hline Ngor Wai Lam & $\begin{array}{l}\text { Yes, I strongly recommend my colleagues to apply the program } \\
\text { in the coming year }\end{array}$ \\
\hline Chan-Woo Lee & Yes \\
\hline Suiqiong Li & Yes \\
\hline Rebecca Lowton & Yes \\
\hline Amir Saheb & Yes \\
\hline Guanjun Wang & Yes \\
\hline (name on file) & Absolutely. \\
\hline (name on file) & $\begin{array}{l}\text { Yes and no. I would say yes because the program itself is very well } \\
\text { organized and provides graduate students with an excellent opportunity } \\
\text { to work in a lab outside of their university, to have access to state-of- } \\
\text { the-art facilities, and to network with scientists who are well known in } \\
\text { their respective fields. The reason that I would say no relates to the } \\
\text { relationship with the mentors in the program. A student absolutely } \\
\text { must be in contact with the mentor before arriving at PNNL. There did } \\
\text { seem to be components built into the application process to ensure } \\
\text { contact with the mentor; however in reality, the program did not make } \\
\text { this a requirement. With only } 10 \text { short weeks to conduct research } \\
\text { within the walls of PNNL, the student and mentor need to set realistic } \\
\text { project goals, and the student must be better prepared on background } \\
\text { aspects of the project in order to be more productive during his/her stay. }\end{array}$ \\
\hline
\end{tabular}




\begin{tabular}{|c|c|}
\hline Visitor & $\begin{array}{l}\text { III.1. What aspects of the program did you feel were } \\
\text { particularly good? }\end{array}$ \\
\hline Jeremy Cain & $\begin{array}{l}\text { The program made the travel arrangements necessary. Also, I } \\
\text { think it is excellent that they cover a rental car costs for those } \\
\text { who wish to travel on the weekend. }\end{array}$ \\
\hline $\begin{array}{l}\text { Luis A. Cuadra- } \\
\text { Rodriguez }\end{array}$ & Facilities and organization \\
\hline Jun Cui & $\begin{array}{l}\text { I had a great chance to learn the research environment in PNNL, } \\
\text { and knew more undergoing research in my field. I also learned } \\
\text { the research skills for studying liquids with molecular dynamics. }\end{array}$ \\
\hline Diego Gomes & N/A \\
\hline $\begin{array}{l}\text { Rodrigo González } \\
\text { Abraham }\end{array}$ & $\begin{array}{l}\text { The access to the Facilities at any time and the provided support } \\
\text { for develop research. }\end{array}$ \\
\hline Matteo Guglielmi & $\begin{array}{l}\text { The possibility to have scientific exchange at PNNL and its } \\
\text { facilities. }\end{array}$ \\
\hline Rebecca J Hopkins & $\begin{array}{l}\text { I thought that the program was very well organized. The staff at } \\
\text { PNNL did an excellent job of making it possible to get the most } \\
\text { out of the experience. }\end{array}$ \\
\hline Ngor Wai Lam & $\begin{array}{l}\text { i) Visit the laboratory and facilities in EMSL. ii) The advance } \\
\text { instruments in PNNL open the new area in my research field and } \\
\text { development. }\end{array}$ \\
\hline Chan-Woo Lee & $\begin{array}{l}\text { Great opportunity of interacting with other researchers at } \\
\text { PNNL/Great mentoring system }\end{array}$ \\
\hline Suiqiong Li & $\begin{array}{l}\text { This program gave me the opportunity to have the hand-on } \\
\text { experience with the advantage facilities. Another great part is that } \\
\text { I was able to directly discuss my project with my mentor and } \\
\text { other experts in my research area (nanomaterials), and got advice } \\
\text { from them. This accelerated the progress of the project and } \\
\text { deepened my understanding in my research. }\end{array}$ \\
\hline Rebecca Lowton & $\begin{array}{l}\text { The approachability and friendliness of the staff at PNNL and the } \\
\text { organization of the internship as a whole }\end{array}$ \\
\hline Amir Saheb & $\begin{array}{l}\text { The ability to work alongside experts in the various fields and yet } \\
\text { have the flexibility to make independent decisions as to the } \\
\text { direction of the project }\end{array}$ \\
\hline Guanjun Wang & I know more friend from all kinds of science field \\
\hline (name on file) & $\begin{array}{l}\text { The ability to utilize different analytical techniques at the lab, the } \\
\text { openness to collaboration and mentorship provided. Also it was } \\
\text { very beneficial to the entire SRI program to meet other graduate } \\
\text { students and SRI participants. I really appreciated the welcome } \\
\text { BBQ }\end{array}$ \\
\hline (name on file) & $\begin{array}{l}\text { 1) The orientation - completion of appropriate training courses, } \\
\text { office set-up, knowing whom to ask about issues that may arise, } \\
\text { etc. 2) Outside the lab activities (i.e. BBQ and the lecture series) }\end{array}$ \\
\hline
\end{tabular}




\begin{tabular}{|c|c|}
\hline Visitor & III.2. What can we do to improve the program? \\
\hline Jeremy Cain & $\begin{array}{l}\text { Make it abundantly clear how the e-mail system works. I was } \\
\text { unable to check my e-mail because of the difficulty of it (and the } \\
\text { short time I was there) }\end{array}$ \\
\hline $\begin{array}{l}\text { Luis A. Cuadra- } \\
\text { Rodriguez }\end{array}$ & $\begin{array}{l}\text { Maybe have seminars every } 2 \text { weeks with program participants } \\
\text { where the students may present what they are proposing to do, } \\
\text { why and how. Also when they have some data they might present } \\
\text { that as well. Is always good to have people from different } \\
\text { disciplines to look at what your doing and vice versa }\end{array}$ \\
\hline Jun Cui & N/A \\
\hline Diego Gomes & $\begin{array}{l}\text { I would really enjoy attending more seminars in the specific area } \\
\text { I was here to work. }\end{array}$ \\
\hline $\begin{array}{l}\text { Rodrigo González } \\
\text { Abraham }\end{array}$ & $\begin{array}{l}\text { Promote a final presentation to the participants in order to discuss } \\
\text { the results or to inform the research realized. }\end{array}$ \\
\hline Matteo Guglielmi & Do it more than once a year. \\
\hline Rebecca J Hopkins & $\begin{array}{l}\text { Possibly cut down the amount of training needed. A lot of this } \\
\text { training seemed irrelevant. }\end{array}$ \\
\hline Ngor Wai Lam & $\begin{array}{l}\text { More outdoor activities for the participants to communicate with } \\
\text { each other }\end{array}$ \\
\hline Chan-Woo Lee & Housing problem...may be. \\
\hline Suiqiong Li & N/A \\
\hline Rebecca Lowton & $\begin{array}{l}\text { Ensure that applicants know who to approach with specific issues } \\
\text { IE for housing contact ......, for travel / visa information } \\
\text { contact.......... etc }\end{array}$ \\
\hline Amir Saheb & More social gatherings \\
\hline Guanjun Wang & Provide more information of adviser scientist on web \\
\hline (name on file) & $\begin{array}{l}\text { There were some initial delays as part of setting up computer } \\
\text { accounts and orientation. Specifically we could not use the Igor } \\
\text { analysis software for } 2-3 \text { weeks due to the need to set up a } \\
\text { different PNL user account. I understand the paperwork } \\
\text { involved, however. Also better coffee at the cafeteria would be } \\
\text { wonderful and very welcome! }\end{array}$ \\
\hline (name on file) & $\begin{array}{l}\text { 1) Ensure that contacts are made between mentors and students } \\
\text { before arriving at PNNL. 2) Hold other SRI participant activities - } \\
\text { i.e. lunch round tables - some other means of meeting the other } \\
\text { participants in the program and learning about their respective } \\
\text { projects. }\end{array}$ \\
\hline
\end{tabular}




\begin{tabular}{|c|c|}
\hline Visitor & $\begin{array}{l}\text { III.3. What difficulties did you encounter coming here or } \\
\text { during your stay? }\end{array}$ \\
\hline Jeremy Cain & $\mathrm{N} / \mathrm{A}$ \\
\hline $\begin{array}{l}\text { Luis A. Cuadra- } \\
\text { Rodriguez }\end{array}$ & So far none \\
\hline Jun Cui & None \\
\hline Diego Gomes & $\begin{array}{l}\text { In the beginning transportation was an issue, but once you get } \\
\text { used to the taxi feeder service everything works fine }\end{array}$ \\
\hline $\begin{array}{l}\text { Rodrigo González } \\
\text { Abraham }\end{array}$ & None \\
\hline Matteo Guglielmi & None \\
\hline Rebecca J Hopkins & None \\
\hline Ngor Wai Lam & No difficulties \\
\hline Chan-Woo Lee & Nothing \\
\hline Suiqiong Li & $\begin{array}{l}\text { I just felt a little inconvenient to do grocery shopping during my } \\
\text { stay since the guesthouse is far from downtown and I did not } \\
\text { have a car. }\end{array}$ \\
\hline Rebecca Lowton & $\begin{array}{l}\text { It was quite isolated at the guesthouse without access to a car and } \\
\text { difficult organizing supermarket trips etc. A weekly minibus trip } \\
\text { to the supermarket for all interns without access to a car would } \\
\text { have been useful }\end{array}$ \\
\hline Amir Saheb & Other than transportation, not many. \\
\hline Guanjun Wang & No \\
\hline (name on file) & $\begin{array}{l}\text { The computer account problems mentioned above. Also I was } \\
\text { shifted around several times for workspace. This was probably } \\
\text { because there were two of us on the same project and limited } \\
\text { desk space at EMSL }\end{array}$ \\
\hline (name on file) & $\begin{array}{l}\text { 1) Not having regular, effective contact with my mentor. 2) } \\
\text { Finding inexpensive transportation for evenings and weekends. }\end{array}$ \\
\hline
\end{tabular}




\begin{tabular}{|c|c|}
\hline Visitor & IV General comments \\
\hline Jeremy Cain & No additional comments. \\
\hline $\begin{array}{l}\text { Luis A. Cuadra- } \\
\text { Rodriguez }\end{array}$ & N/A \\
\hline Jun Cui & N/A \\
\hline Diego Gomes & N/A \\
\hline $\begin{array}{l}\text { Rodrigo González } \\
\text { Abraham }\end{array}$ & $\begin{array}{l}\text { It was one of the most interesting and exiting experience in mi } \\
\text { life. It was a great opportunity and means a big step in my } \\
\text { research career. }\end{array}$ \\
\hline Matteo Guglielmi & Vale di piu' un uovo oggi che una gallina domani! \\
\hline Rebecca J Hopkins & N/A \\
\hline Ngor Wai Lam & N/A \\
\hline Chan-Woo Lee & $\begin{array}{l}\text { The opportunity allowed me to have great turning point as a } \\
\text { researcher. I won't forget what I learned from SRI. I will also } \\
\text { strongly recommend the program if someone asks about the SRI. } \\
\text { I also want to thank Mrs. Barlow for great parties. }\end{array}$ \\
\hline Suiqiong Li & N/A \\
\hline Rebecca Lowton & $\begin{array}{l}\text { Generally, thank you to all who worked so hard to bring me over } \\
\text { to PNNL and were so helpful when I arrived. It was a wonderful } \\
\text { to experience work in a government based lab and to see how } \\
\text { research works outside of academia. It was a privilege to work } \\
\text { with Tom Autrey and the rest of the Molecular Interactions } \\
\text { group. }\end{array}$ \\
\hline Amir Saheb & N/A \\
\hline Guanjun Wang & N/A \\
\hline (name on file) & $\begin{array}{l}\text { Everyone was very welcoming and overall it was a good } \\
\text { experience }\end{array}$ \\
\hline (name on file) & $\begin{array}{l}\text { There were too many summer program participants (total 3) } \\
\text { working in my lab, especially with only two and a half "regular" } \\
\text { group employees. One of the summer program participants never } \\
\text { should have been chosen for his respective program (not SRI). } \\
\text { He was extremely immature and always disruptive to the group. } \\
\text { Therefore, focus and time were often taken away from the tasks } \\
\text { at hand with my project. }\end{array}$ \\
\hline
\end{tabular}

${ }^{1}$ A. Owens, A. Peacock, Nucl. Instr. Meth. A 531 (2004) 18.

${ }^{2}$ A. Lo Giudice, F. Fizzott, C. Manfredotti, E. Vittone, F. Nava, Appl. Phys. Lett. 87 (2005) 222105.

${ }^{3}$ Y. Zhang, G. Possnert, W.J. Weber, Appl. Phys. Lett. 80 (2002) 466

${ }^{4}$ Y. Zhang, W.J. Weber, H.J. Whitlow, Nucl. Instr. and Meth. B 215 (2004) 48. 

Stephan E. Barlow

Director

ICPCPSRI

(509) 376-2642

cpsi@pnl.gov

http://www.pnl.gov/si 